\title{
XXXV Brazilian Congress of Rheumatology (SBR 2018)
}

Rio de Janeiro, Brazil. 5 - 8 September 2018

Published: 21 August 2018

001

\section{AEROBIC CAPACITY IS IMPAIRED IN PATIENTS WITH SYSTEMIC} AUTOIMMUNE MYOPATHIES

Alexandre Moura dos Santos, Rafael Giovane Misse, Jean Marcos de Souza, Diego Sales de Oliveira, Fernanda Rodrigues Lima, Ana Lúcia de Sá Pinto,

Samuel Katsuyuki Shinjo

FACULDADE DE MEDICINA DA UNIVERSIDADE DE SÃO PAULO, SÃO

PAULO, SÃO PAULO, Brasil

Advances in Rheumatology 2018, 58(Suppl 1):O01

Background: Systemic autoimmune myopathies (SAM) are a heterogeneous group of diseases that cause chronic muscle inflammation and progressive muscle weakness. Reduced aerobic capacity has been documented in several autoimmune rheumatic diseases. However, few studies have assessed in SAM. Thus, the aim of the present study was to analyze aerobic capacity in SAM.

Materials and methods: This is a cross-sectional study, from 2017 to 2018, that compared 23 female patients with SAM (2017 EULAR/ACR classification criteria: 13 dermatomyositis, 4 polymyositis and also 6 antisynthetase syndrome) with 17 aged, gender and body mass index-matched healthy individuals. Aerobic capacity was evaluated by means of the treadmill maximum (Centurion 200, Micromed, Brazil) cardiorespiratory test, demonstrated through VO2max $(\mathrm{mL} /$ kg.min). Disease status was evaluated by International Myositis Assessment and Clinical Studies Group (IMACS) core set measures.

Results: Mean age of the patients was 47.9 years, with mean disease duration of 7.6 years. Patients had low disease activity according of IMACS parameters, using different immunosuppressive drugs and with daily prednisone $<10 \mathrm{mg}$. Median aerobic capacity was lower in patients with SAM, when compared to control group (18.0 vs. 28.5 $\mathrm{mL} / \mathrm{kg} . \mathrm{min}, \mathrm{P}=0.011$, respectively). In additional analysis, the reduced aerobic capacity was independent of type of SAM, disease duration, IMACS parameters and treatment.

Conclusions: Aerobic capacity was significantly reduced in SAM when compared to control group. These findings corroborate with literature data that aerobic capacity is reduced in several systemic autoimmune diseases. Recognition of this information is important in daily practice, as it may reflect in the difficulty of performing daily tasks, increasing level of sedentary lifestyle, besides being a prognosis of cardiovascular diseases.

Support by: FAPESP \#2016/23574-0 (RGM), \#2016/19771-5 (DSO), \#2017/13109-1 (SKS)
Table 1 (abstract 001). Comparison of aerobic capacity and demographic characteristics among patients with systemic autoimmune myopathies, healthy control

\begin{tabular}{|c|c|c|c|}
\hline 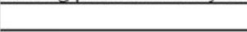 & SAM & $\mathrm{HC}$ & $P$ \\
\hline Age & $47,9 \pm 9,3$ & $47,8 \pm 4,4$ & 0,963 \\
\hline Disease duration (years) & $7,6 \pm 4,2$ & - & \\
\hline $\mathrm{BMI}\left(\mathrm{kg} / \mathrm{m}^{2}\right)$ & $29,4(26,1-33,2)$ & $32,5(27,7-29,1)$ & $0,290^{*}$ \\
\hline \multicolumn{4}{|l|}{ Aerobic capacity } \\
\hline $\mathrm{VO}_{2}$ peak (mL/kg/min) & $18.0(16-21)$ & $28.5(25.5-30.2)$ & $0.011^{*}$ \\
\hline
\end{tabular}

*: statistically significant; BMI: body mass index; $\mathrm{VO}^{2}$ peak: peak oxygen uptake

002

ANALYSIS OF COMT A/G GENE POLYMORPHISM IN WOMEN WITH AND WITHOUT FIBROMYALGIA

Luana Oliveira de Lima, Carlos Alexandre Martins Zicarelli, Andressa Saori Matsumura, Karen Barros Parron Fernandes, Layse Rafaela Moroti, Regina Célia Poli-Frederico

UNIVERSIDADE NORTE DO PARANÁ, LONDRINA, PR, Brasil

Advances in Rheumatology 2018, 58(Suppl 1):002

Background: Fibromyalgia syndrome (FS) is characterized by chronic generalized musculoskeletal pain also associated with symptoms such as fatigue, sleep disorders and psychological. Regarding its physiopathology, studies have been associating the syndrome with a genetic predisposition related to the serotoninergic, dopaminergic and catecholaminergic systems. The catechol-O-metiltransferase (COMT) enzyme acts inactivating the catecholamines and mutations in the gene encoding this protein have been related to the symptoms of fibromyalgia. The single nucleotide polymorphism (SNP) in this gene most studied is rs $4680 \mathrm{G} / \mathrm{A}$, and individuals carrying the allele A have a greater sensitivity to pain. However, other studies did not verify such association, evidencing that this investigation is necessary for a better understanding. The aim of this study was to analyze the relationship between polymorphism of the COMT G/A rs4680 gene in women with and without fibromyalgia.

Materials and methods: Participants in this cross-sectional study were 60 women over 18 years of age, 29 of the fibromyalgia group and 31 of the control group. Demographics and peripheral blood samples 
were collected for DNA extraction. Genotypic analyzes were performed using the PCR-SSP method followed by $1 \%$ agarose gel electrophoresis. Chi-square tests and the Kendall Tau-b correlation test were used for a possible association and correlation between the variables, establishing a $95 \%$ confidence interval and significance level of $p<0.05$.

Results and conclusions: A greater proportion of the study participants had GG genotype (58\%) and only $8.3 \%$ of the women had the AA genotype. There was a statistically significant association between genotypes and FS. Participants who were carriers of the AA or allele A genotype had FS $(p=0.01)$. In the present study, patients with AA and allele A were 4.07 times more risk to develop FS than those who did not carry this genotype/allele $(95 \% \mathrm{Cl} 1.37-12.14, \mathrm{p}=0.02 / 95 \%$ 1.65-10.27, $p=0.03$ ). There was an association between race and FS, demonstrating that the white race was 2.05 times more likely to develop the syndrome $(95 \% \mathrm{Cl} 0.93-4.53, \mathrm{p}=0.03)$. There was a proportional correlation between age and fibromyalgia patients $(\mathrm{rS}=0.812$; $p=0.001$ ). Therefore, the present study found that white women of greater age and carriers of the AA/A genotype/allele are at greater risk of developing FS.

\section{3}

ANGIOGRAPHIC PROGRESSION IN TAKAYASU'S ARTERITIS:

\section{INSIGHTS FROM A TERTIARY CENTER}

Matheus Vieira Gonçalves', Gustavo Guimarães Moreira Balbi²,

Camila Souto Oliveira', Ana Beatriz Santos Bacchiega',

Manuella Lima Gomes Ochtrop'

'DEPARTMENT OF RHEUMATOLOGY, HOSPITAL UNIVERSITÁRIO PEDRO

ERNESTO, UNIVERSIDADE DO ESTADO DO RIO DE JANEIRO, RIO DE

JANEIRO, BRAZIL, NITERÓI, RJ, Brasil; 'DEPARTMENT OF RHEUMATOLOGY,

HOSPITAL UNIVERSITÁRIO, UNIVERSIDADE FEDERAL DE JUIZ DE FORA, JUIZ

DE FORA, MINAS GERAIS, BRAZIL, JUIZ DE FORA, MINAS GERAIS, Brasil

Advances in Rheumatology 2018, 58(Suppl 1):003

Introduction: Takayasu's Arteritis (TA) is a large-vessel vasculitis, evolving primarily aorta and its major branches, with an insidious and frequently silent progression. Clinical and laboratorial assessment may be normal during flares, and even imaging modalities are not as sensible as needed to precisely document activity and though guide an accurate management. Routine angiographic evaluations are performed to detect vascular lesions and its accrual over time, with variable interval between imaging assessments. The aim of this study is to investigate vascular damage during follow-up and possible factors associated with its progression.

Materials and Methods: We included 30 consecutive outpatients that fulfilled the 1990 ACR classification criteria for TA and underwent at least two angiography examination over time, such as Doppler, computed tomography angiography or magnetic resonance angiography. Comparisons were made between the same imaging methods. Clinical, laboratorial and imaging data were collected from electronic medical records, since the first imaging modality registered. NIH criteria (Kerr et al., 1994, excluding new vascular damage) for active disease was calculated. Statistical analysis was performed by SPSS 22.0. Results: Thirty patients were analyzed, with 28 females (93\%), mean age at diagnosis $33.9+14.6$ years, $63.3 \%$ with angiographic type $V$ at diagnosis and mean follow-up period of $87.6+59.5$ months. A total of 37 flares were computed, resulting in angiographic progression in $60 \%$ of patients. Two patients changed their angiographic type over time to type $\mathrm{V}$. Right subclavian artery involvement was significantly higher among those who progressed (OR 7.54 95\% Cl 1.05-54.03; $\mathrm{p}=0.049$ ). Considering activity clinical features, excluding new vascular lesions from the $\mathrm{NIH}$ criteria, at least two of these features were present in $79 \%$ of patients who progressed vs. $46 \%$ of those who remained stable, with an OR $4.43(95 \% \mathrm{Cl} 1.02-19.27 ; \mathrm{p}=0.041)$.

Conclusion: The presence of 2 or 3 points in the NIH criteria with 3 variables correlated with progression of vascular damage; also, right subclavian artery involvement was more common in vascular progressors.
004

ANKRD1 EXPRESSION AS MARKER OF SKELETAL MUSCLE REMODELING IN NAIVE TREATMENT POLYMYOSITIS MUSCLE BIOPSIES

Marilda Guimarães Silva', Sueli Mieko Oba-Shinjo², Suely Kazue Nagahashi Marie ${ }^{2}$, Samuel Katsuyuki Shinjo²

${ }^{1}$ FACULDADE DE MEDICINA DA UNIVERSIDADE DE SÃO PAULO, SÃO PAULO, SP, Brasil; ${ }^{2}$ FACULDADE DE MEDICINA DA USP, SAO PAULO, SÃO PAULO, Brasil

Advances in Rheumatology 2018, 58(Suppl 1):004

Background: ANKRD1 codes for ankyrin repeat domain containing protein 1. It has an important role in myogenesis and myofibrillar assembly and has been considered as a marker of skeletal muscle pathological remodeling. To date, however, no study has assessed the ANKRD1 expression in adult polymyositis muscle biopsies or has correlated with histological changes in situ.

Materils and Methods: RNA was extracted from frozen muscle biopsies samples of 13 patients with untreatment defined polymyositis (Bohan and Peter, 1975; EULAR/ACR 2017 criteria). As a control group, 20 adult muscle biopsies from adult patients with noninflammatory myopathy diseases and no histological change were included. ANKRD1 transcript expression levels were determined by quantitative real time PCR. A visual analogue scale was included to score global degree of muscle inflammatory and general abnormalities from 0 (no inflammation or no abnormality) to 10 (most inflammation or most abnormal). ANKRD1 protein localization was analyzed by immunofluorescence technique.

Results: Average age of patients was 45.7 , with $69.2 \%$ of female gender. Median duration between diagnosis and symptom onset was 5.0 months, and all patients had objective, symmetric, predominantly proximal muscle weakness. Initial serum levels of creatine phosphokinase and aldolase were 3065 and $68.0 \mathrm{U} / \mathrm{L}$, respectively. Higher ANKRD1 relative expression levels were observed in polymyositis muscle relative to control group $(\mathrm{P}<0.001)$. Additionally, ANKRD1 expression levels correlated with serum level of creatine phosphokinase and aldolase $(r h o=0.879, P<0.001$ and $r h o=0.681, P=0.030$, respectively). ANKRD1 relative expression levels did not correlate with any clinical parameters or histological analysis (muscle inflammatory and general abnormalities). However, in immunofluorescence analysis, ANKRD1 was mainly observed in regenerating fibers.

Conclusions: The present data reinforce that ANKRD1 may be involved in skeletal muscle remodeling, including in muscle biopsies of naïve treatment patients with PM.

\section{5}

ANTI-INFLAMMATORY EFFECT OF RECOMBINANT CYSTATINS FROM FASCIOLA HEPATICA IN ANTIGEN-INDUCED ARTHRITIS MICE

\section{MODEL}

Thales Hein da Rosa ${ }^{1}$, Mirian Farinon ${ }^{1}$, Renata Ternus Pedó ${ }^{1}$, Martín Cancela', Henrique Bunselmeyer Ferreira', Ricardo Machado Xavier ${ }^{2}$

'UNIVERSIDADE FEDERAL DO RIO GRANDE DO SUL, PORTO ALEGRE, RS, Brasil; ${ }^{2}$ HOSPITAL DE CLÍNICAS DE PORTO ALEGRE, PORTO ALEGRE, RS, Brasil

Advances in Rheumatology 2018, 58(Suppl 1):005

Background: Rheumatoid arthritis (RA) is an autoimmune disease characterized by chronic inflammation of joints causing pain, edema, bone and cartilage degradation and frequently leading to joint destruction and disability. Fasciola hepatica is a trematode that parasites, in adult form, the bile ducts of different kinds of mammals. In addition, this worm is able to modulate the host immune response to a T helper (Th) 2 profile to survive. A Th2 response can suppress the pro-inflammatory Th1 response generated in several immunopathology, as RA. One of the mechanisms involved in this immune regulation is the secretion of cysteine proteases-inhibitors such as cystatins. In this study, we evaluated the role of recombinant cystatin 
1 and 3 from Fasciola hepatica in leukocyte migration and nociception in antigen-inducedarthritis (AIA) mice model.

Material and methods: Forty-two male BALB/C mice were subjected to AIA by subcutaneous injection of an emulsion containing methylated bovine serum albumin (mBSA) and Freund's complete adjuvant at day 0 . After, at days 7 and 14, mice received mBSA and Freund's incomplete adjuvant. At day 21, animals were challenged by an intraarticular (ia) joint injection of mBSA. Mice were randomized in five groups: vehicle (PBS), cystatin $1(100 \mu \mathrm{g} /$ dose and $150 \mu \mathrm{g} / \mathrm{dose})$ and cystatin $3(100 \mu \mathrm{g} /$ dose and $150 \mu \mathrm{g} /$ dose). Treatment was performed by intraperitoneal injections at $24 \mathrm{~h}$ and $30 \mathrm{~min}$ before ia injection of mBSA. Nociception was analyzed at $0,3 \mathrm{~h}, 6 \mathrm{~h}$, and $24 \mathrm{~h}$ after ia injection and leukocytes migration was analyzed $24 \mathrm{~h}$ after ia injection. Data are expressed as mean \pm SEM. Two-way ANOVA was performed for nociception and one-way ANOVA for leukocytes migration analyzes, both followed by Bonferroni's post-test.

Results: Treatment with both cystatins were noteffective in reduce nociception. However, cystatin $1(100 \mu \mathrm{g} / \mathrm{dose})$ was able to reduce leukocytes migration in $60 \%(51.17 \pm 2.94 \times 104$ leukocytes/cavity $)$ $(p<0.05)$ and cystatin $3(100 \mu \mathrm{g} /$ dose $)$ in $63 \%(48.06 \pm 10.04 \times 104$ leukocytes/cavity) $(p<0.01)$ compared with vehicle $(129.7 \pm 31.87 x 104$ leukocytes/cavity).

Conclusion: Although it was not able to reduce joint nociception, treatment with cystatin 1 and 3 improved acute experimental arthritis by attenuating leukocyte migration to knee joint. Based on this, cystatins 1 and 3 from Fasciola hepatica demonstrated a potential anti-inflammatory activity and this potential will be further investigated through a chronic mice model of collagen-induced arthritis.

\section{6}

ANTI-MI-2 AUTOANTIBODY IN A LARGE SAMPLE OF ADULT PATIENTS WITH DERMATOMYOSITIS: A RETROSPECTIVE COHORT STUDY

Maria Isabel Cardoso dos Passos Carvalho ${ }^{2}$, Samuel Katsuyuki Shinjo ${ }^{1}$ ${ }^{1}$ FACULDADE DE MEDICINA, UNIVERSIDADE DE SAO PAULO, SÃO PAULO, SP, Brasil; ${ }^{2}$ FACULDADE DE MEDICINA, UNIVERSIDADE DE SÃO PAULO, SÃO PAULO, SP, Brasil

Advances in Rheumatology 2018, 58(Suppl 1):006

Background. Anti-Mi-2 autoantibody is described in $2-45 \%$ of patients with dermatomyositis. This enormous prevalence range is due, in part, to scarcely available studies in the literature, as well as analyses based on a relatively small and/or heterogeneous sample of patients. In addition, most studies were limited to cross-sectional analysis. Therefore, in the present study, the prevalence, reactivity and impact of anti-Mi-2 was assessed in a large and homogeneous sample of patients with dermatomyositis.

Materials and methods. This is a longitudinal inception cohort study that initially included 177 consecutive adult patients with definite dermatomyositis (Bohan and Peter, 1975; 2017 EULAR/ACR classification criteria) from 2001 to 2017. Strict exclusion criteria were applied to achieve a homogeneous sample: overlap syndrome, neoplasia (previous or concomitant to dermatomyositis onset), pulmonary infections, chronic obstructive pulmonary disease, history of chronic smoking, clinically amyopathic dermatomyositis, patients with positivity to antisynthetase, anti-MDA-5, anti-PM/Scl or anti-Ku autoantibodies. Moreover, patients without collected serum/plasma were also excluded. Anti-Mi-2 analysis was performed using a commercial kit according to the manufacturer's protocol.

Results. After the exclusion criteria, 87 dermatomyositis cases were evaluated: 17 (19.5\%) anti-Mi-2(+) vs. 70 (80.5\%) anti-Mi-2(-). Mean age at the disease diagnosis onset was 42.4 years, with a predominance of female and white ethnicity. Median follow-up time was 4.3 years. Constitutional symptoms were present in $59.8 \%$ of cases, whereas cutaneous lesions in $100 \%$, involvement of gastrointestinal tract (high dysphagia) in $49.4 \%$, joint in $35.6 \%$ and pulmonary in $33.3 \%$. During follow-up, disease relapsing was observed in $19.5 \%$ of cases, whilst $13.8 \%$ of patients died and $4.6 \%$ presented neoplasia (post-dermatomyositis diagnosis). At the end of the present study, $31 \%$ patients were still receiving glucocorticoid therapy. Moreover, $33.3 \%$ had disease remission, $56.3 \%$ had complete clinical response and $10.3 \%$ had activity disease. In an additional analysis, all these parameters were compared among the patients with anti-Mi-2(+) vs. anti-Mi-2(-). There was no difference between the groups, except for the following parameters in patients with anti-Mi-2(+), all with $P<0.05$ : (a) elevated serum levels of muscle enzymes and lower frequency of pulmonary involvement at disease onset, (b) higher frequency of disease remission during the following up.

Conclusions. Anti-Mi-2 autoantibody was found in $19.5 \%$ of patients with dermatomyositis. This autoantibody was associated with lower occurrence of pulmonary involvement, higher frequency of disease in remission, and elevated levels of muscle enzymes. There was also no correlation regarding the frequency of disease relapsing or neoplasia development.

\section{Acknowledgements}

Support by FAPESP \#2011/12700-1 (SKS)

\section{7}

ANTI-RIBOSSOMAL P ANTIBODY IS ASSOCIATED WITH MOOD DISORDERS IN SYSTEMIC LUPUS ERYTHEMATOSUS

Beatriz Ricato Quental, Feranando Augusto Peres, Lilian Tereza Lavras

Costallat, Simone Appenzeller

UNICAMP, SP, SP, Brasil

Advances in Rheumatology 2018, 58(Suppl 1):007

Background:Systemic Lupus Erythematosus (SLE) is a chronic inflammatory autoimmune disease that can affect any organ or system. The SLE is triggered by an immunological imbalance associated with inflammation. Several autoantibodies have been described in SLE, including anti-ribossomal P (anti-P) antibody. However, in previous studies disagreement in relation to the association of the presence of anti-P and clinical manifestations was observed, moreover these studies demonstrate a percentage variation in positives patients for this antibody. Despite this fact, others works detected that ethnicity can influence the percentage of positive Anti-P patients. Therefore, the present study aims to correlate some clinic and laboratory manifestations of SLE with the Anti-P, besides analyzing the frequency of this antibody in the patients.

Methods: For this purpose, a group of 143 patients and 166 controls were selected for this research. These groups passed by clinic and neuropsychiatry evaluation, magnetic resonance image and the blood was collected in order to dose the Anti-P, following ELISA method.

Results and discussion: We observed anti-P in 12 (8.39\%) patients and in none of the control $(p<0.001)$. The presence of anti-P antibody was associated with higher disease activity $(p=0.007)$, the presence of depressive symptoms $(p=0.018)$ and the presence of anxiety symptoms $(p=0.047)$. No association of anti-P and other clinical, immunological and imaging feature was observed. In conclusion, anti-P is specific for SLE and associated with disease activity and mood disorders.

\section{8}

ARE LUPUS PATIENTS ADVISED TO UPDATE THEIR VACCINATION

CARD?

Maria Carolina Padovani Guerra, Rosa Weiss Telles, Maria Isabel Mendes Guedes, Maria Veloso Rocha Mameluque, Tiago Loredo e Silva, Cristina Costa Duarte Lanna, Fabiana de Miranda Moura dos Santos

UNIVERSIDADE FEDERAL DE MINAS GERAIS, BELO HORIZONTE, MINAS GERAIS, Brasil

Advances in Rheumatology 2018, 58(Suppl 1):008

Background: Systemic lupus erythematosus (SLE) is an autoimmune and systemic disease with high morbidity and mortality, in which infection is one of the main cause of death in Brazil. Factors associated with the disease and its treatment are related to the higher incidence of infection in this population. Vaccination, in this context, appears to be an important strategy to prevent infection. The aim of this study is to evaluate the knowledge about vaccination in lupus patients.

Material and methods: A cross-sectional study was performed and outpatients followed at the Lupus Clinic were included. The instrument used was a questionnaire developed by medical students on 
the knowledge about vaccines. The SPSS program was used to perform descriptive statistical analysis.

Results and Conclusions: One hundred and eight patients were included, 104 (96.3\%) women. The mean (DP) of age and of the disease duration were 43.6 (13.7) and 12.6 (8.3) years, respectively. Concerning the treatment, $75(70 \%)$ patients used immunosuppressant, 75 (77\%) antimalarials, 71 (67\%) prednisone and 3 (3\%) immunobiologicals. Sixty-nine $(63 \%)$ of them reported the vaccines were updated. Thirty five $(32.7 \%)$ out of 108 patients declared they have received orientation towards vaccination from their doctors, $30(28 \%)$ patients knew about special immunizations service of Reference Center for Special Immunobiologicals (Centro de Referência de Imunobiológicos Especiais- CRIE), 23 (21.5\%) knew about their right to receive free vaccines by government and 104 (97.2\%) accepted to receive the vaccines after the physician assistant recommendation. Our findings suggest that most patients did not receive orientation from his/her assistant physician to update their vaccination card before. The majority of them stated that they would get the vaccines if they have known about indication and rights. In addition, although being a tertiary center, only $28 \%$ had known about CRIE. Therefore, provide vaccination orientation during the attendance is a desired attitude of the health team in order to provide integral care.

\section{9}

ARTERIAL EVENTS IN PRIMARY ANTIPHOSPHOLIPID SYNDROME (PAPS): DON'T FORGET THE TRADITIONAL CARDIOVASCULAR RISK FACTORS

Gustavo Guimarães Moreira Balbi ${ }^{1,2}$, Flávio Signorelli ${ }^{3}$, Roger Abramino Levy ${ }^{3}$ ${ }^{1}$ HOSPITAL UNIVERSITÁRIO DA UNIVERSIDADE FEDERAL DE JUIZ DE FORA, JUIZ DE FORA, MG, Brasil; ${ }^{2}$ UNIVERSIDADE FEDERAL DE JUIZ DE FORA, JUIZ DE FORA, MG, Brasil; ${ }^{3}$ UNIVERSIDADE DO ESTADO DO RIO DE JANEIRO, RIO DE JANEIRO, RJ, Brasil

Advances in Rheumatology 2018, 58(Suppl 1):009

Background: Arterial events are a major concern when treating patients with isolated or primary (pAPS), and traditional cardiovascular risk factors may have an important role in increasing their occurrence. The aim of this study was to detect which of these factors were associated with increased risk of arterial events in our cohort of pAPS patients.

Methods: A cross-sectional study was performed in 114 outpatients who fulfilled thrombotic pAPS classification criteria (Sydney). Clinical and serologic features were compiled during visits and by chart review. Statistical analysis was performed using chi-square, MannWhitney U and Spearman's R tests, when applicable. Multivariate regression analysis included age, sex, ethnicity, and variables with $\mathrm{p}<0.10$ in the bivariate analysis.

Results: Fifty-three (46.5\%) patients had arterial events, $43(81.1 \%)$ were female, and 35 (66\%) Caucasian. The mean number of episodes of arterial thromboses was $1.72 \pm 1.12$ per patient. In a bivariate analysis, arterial events correlated with the presence of livedo $(p=0.029)$, dyslipidemia $(p=0.007)$, smoking $(p=0.006)$, and positive anti- $\$ 2$ glycoprotein I (aß2GPI) $(p=0.027)$. There was a tendency of higher rates of arterial thromboses in patients with hypertension $(p=0.052)$. Curiously, obese patients were less prone to develop arterial events in our cohort $(p=0.039)$. aGAPSS was higher in patients with arterial thromboses than in those with venous thromboses (median 9 (7-13) vs. $8(5-12) ; \mathrm{p}=0.010)$. In a multivariate analysis, dyslipidemia (OR 4.07; $95 \% \mathrm{Cl} 1,45-11,4 ; \mathrm{p}=0.008$ ), smoking (OR 2.97; 95\%Cl 1.08-8.102; $\mathrm{p}=0.034)$, and positivity to aß2GPI (OR 2,52; $95 \% \mathrm{Cl} 1,03-6,17)$ remained as risk factors forarterial events. Obesity was protective in our study (OR $0.25 ; 95 \% \mathrm{Cl} 0.085-0.72 ; \mathrm{p}=0.01)$. Regarding recurrence, 21 patients (40.3\% of arterial event patients) had more than one event. Both positive and persistently positive lupus anticoagulant (LA) correlated with thrombotic recurrence $(p=0.03$ and $p=0.039$, respectively). There was a trend of higher rates of recurrence in patients with hypertension $(p=0.061)$ and dyslipidemia $(p=0.061)$, however not reproduced in the multivariate analysis. No difference in aGAPSS was found between recurrent and not recurrent groups.

Conclusion: Traditional cardiovascular risk factors increase the risk of arterial events in pAPS patients and, therefore, their proper control should not be neglected. Patients with higher aGAPSS are prone to develop arterial events. The positivity and persistency of LA are associated with arterial thrombotic recurrence.

\section{0}

ASSOCIATION OF HLA CW18* WITH PSORIATIC ARTHRITIS IN BRAZILIAN PATIENTS

Sueli Coelho da Silva Carneiro', Flavia de Freire Cassia ${ }^{2}$, Luis Cristóvão Porto $^{3}$ 'UNIVERSIDADE FEDERAL DO RIO DE JANEIRO, RIO DE JANEIRO, RJ, Brasil; ${ }^{2}$ HOSPITAL FEDERAL DA LAGOA, RIO DE JANEIRO, RJ, Brasil; ${ }^{3}$ UNIVERSIDADE DO ESTADO DO RIO DE JANEIRO, RIO DE JANEIRO, RJ, Brasil

Advances in Rheumatology 2018, 58(Suppl 1):O10

Introduction: The association of HLA with psoriasis vulgaris and psoriatic arthritis is widely described in the literature. Reports on populations of diverse ethnical origins show different HLA markers in patients with psoriasis when compared to controls.

Objectives: To type HLA classes I (A, B e C) and II (DRB1 e DQB1) of Brazilian patients with psoriatic arthritis, from HUCFF outpatient unit, and compare them to controls pared in respect to ethnical origin, gender and age.

Materials and Methods: Twenty patients with clinical and/or histopathological diagnosis of psoriasis vulgaris and psoriatic arthritis, with at least 5 years of disease duration, were included in the study. They answered a questionnaire on ethnic background, family history, duration of disease, history of erythroderma, hospital internment and treatment. They were clinically evaluated in respect to distribution and morphology of skin lesions, joint involvement and disease activity and were submitted to laboratorial tests. One-hundred thirty four bone marrow donors served as controls after informed consent. Allelic typing of class I and II HLA genes were determined by PCR-SSP and PCR-SSO hybridization. Differences between the two groups were evaluated through chi-square or Fisher exact tests.

Results: The patients mean age was 47,25 years-old. There were $9 \mathrm{fe}-$ males and 11 males. HLA-B*57 and $\mathrm{HLA}^{*} \mathrm{CW}^{*} 18$ were significantly present in patients with arthritis $(p=0,00104, O R=6,6769$ e $p=0,00269$, $\mathrm{OR}=16,50$, respectively).

Conclusions: HLA-B*57 and HLA-CW*18 were significantly high in patients with psoriatic arthritis. HLA-C*18:01 is a rare allele in the European caucasian population and was associated with difficult-to-treat plaque psoriasis, but not with psoriatic arthritis yet.

\section{1}

ASSOCIATION OF TNF-A SYSTEM, ADIPOKINES AND ENDOTHELIAL ACTIVATION BIOMARKERS WITH TRADITIONAL RISK FACTORS FOR CARDIOVASCULAR DISEASE IN FEMALE PATIENTS WITH SLE PRELIMINARY ANALYSIS

Rosa Weiss Telles ${ }^{1,3}$, Ana Julia Furbino Dias Bicalho ${ }^{2}$, Diogo Couto Carvalho', Ihan Bruno Lopes Rabelo², Fernanda Oliveira Gomes², Luísa Lima Castro ${ }^{2}$, Fabiana de Miranda Moura Santos ${ }^{3}$, Antonio Lucio Teixeira $^{3}$, Cristina Costa Duarte Lanna ${ }^{3}$, Antonio Luiz Ribeiro ${ }^{3}$

'FACULDADE DE MEDICINA DA UFMG, BELO HORIZONTE, MG, Brasil; ${ }^{2}$ HOSPITAL DAS CLÍNICAS - UFMG, BELO HORIZONTE, MG, Brasil; ${ }^{3}$ FACULDADE DE MEDICINA - UFMG, BELO HORIZONTE, MG, Brasil Advances in Rheumatology 2018, 58(Suppl 1):011

Background: Atherosclerosis is an inflammatory process with immune cell activation and inflammation-driven plaque formation. Abnormalities in the function and release of cytokines, adipokynes and endothelial activators biomarkers have been identified in patients with SLE. Furthermore, traditional cardiovascular risk factors (RF) are common in SLE patients and the link between inflammation and RF in lupus has been poorly investigated.

Materials and Methods: A cross-sectional study involving 134 women with SLE (ACR 82/97) was developed. TNF-a and their soluble receptors (sTNFR1 and sTNFR2); adipokines (leptin, resistin and adiponectin) and endothelial activators biomarkers (sVCAM, sICAM, E-selectin, E-chaderin, VEGF) were measured. The traditional cardiovascular RF analyzed were body mass index (BMI), waist circumference (WC), 
systemic arterial hypertension (SAH), diabetes mellitus (DM), dyslipidemia (HDL-c $<50 \mathrm{mg} / \mathrm{dl}$ and/or LDL-c $\geq 130 \mathrm{mg} / \mathrm{dl}$ and/or total colesterol $\geq 200 \mathrm{mg} / \mathrm{dl}$ ), tryglicerides (TGL), smoking, postmenopausal status and Metabolic Syndrome (MetS). Spearman correlations or U-MannWhitney tests evaluated the univariate association between traditional RF and cytokines and endothelial activators biomarkers. Two-sided $p$ values $\leq 0.050$ were considered significant and $p$ values between 0.051 and $\leq 0.10$ were considered borderline.

Results and Conclusion: The mean age (SD) of the patients was 41.6(11.2) years. The median (IQR) disease duration was 121 (91-178) months. There was a positive correlation between obesity markers (BMI and WC) and ICAM, E-selectin and E-cadherin and an expected negative association with adiponectin. Regarding dyslipidemia, there was a significant positive association with STNFR1, STNFR2, ICAM and a negative association with adiponectin. $\mathrm{HDL}-\mathrm{c}$ was directly correlated to adiponectin and inversely correlated to sTNFR1, sTNFR2, ICAM, VCAM, Eselectin and VEGF. TGL levels were positively correlated to resistin, sTNFR1, sTNFR2, ICAM, VCAM, E-selectin and negatively with adiponectin. In addition, hypertrigliceridemia ( $\mathrm{TGL} \geq 150 \mathrm{mg} / \mathrm{dl}$ ) was associated positively with ICAM and E-selectin and negatively with adiponectin. Patients with DM had a lower level of adiponectin and a borderline higher level of ICAM, E-selectin and E-cadherin probably due to the small sample of diabetics in our study ( $n=13$ patients). Patients with $\mathrm{SAH}$ had a higher level of STNFR1, ICAM, E-selectin and E-cadherin. Smoking was positively related to resistin, E-selectin and E-cadherin and postmenopausal status was positively related to STNFR1, ICAM and E-cadherin. Considering Metabolic Syndrome, higher levels of leptin, sTNFR1, sTNFR2, ICAM, E-selectin and E-cadherin were observed, while lower levels of adiponectin were found. In conclusion, the panel of TNF system, adipokines as well as endothelial activators biomarkers showed a significant association with many traditional cardiovascular RF in patients with SLE, showing the dysregulation of the inflammation associated with atherosclerosis.

\section{2}

AUTOLOGOUS HEMATOPOIETIC STEM CELL TRANSPLANTATION FOR SYSTEMIC SCLEROSIS PATIENTS WITH CARDIAC

\section{INVOLVEMENT}

Daniela Aparecida de Moraes ${ }^{3}$, Juliana Bernardes Elias', Ana Beatriz Pereira Lima Stracieri', Luiz Guilherme Darrigo Junior ${ }^{1}$, Carlos Eduardo Setanni Grecco ${ }^{1}$, Marilia Cirioli Oliveira², Vanessa Leopoldoº ${ }^{2}$, Andreia Ferreira Zombrilli' ${ }^{2}$, Belinda Pinto Simões ${ }^{3}$, Maria Carolina de Oliveira ${ }^{3}$ ${ }^{1}$ FACULDADE DE MEDICINA DE RIBEIRÃO PRETO-USP, RIBEIRÃO PRETO, SP, Brasil; ${ }^{2}$ ESCOLA DE ENFERMAGEM DE RIBEIRÃO PRETO- USP, RIBEIRÃO PRETO, SP, Brasil; ${ }^{3}$ FACULDADE DE MEDICINA DE RIBEIRÃO PRETO- USP, RIBEIRÃO PRETO, SP, Brasil

Advances in Rheumatology 2018, 58(Suppl 1):012

Introduction: Autologous hematopoietic stem cell transplantation (AHSCT) is recommended as treatment for patients with rapidly progressive systemic sclerosis (SSc). The procedure aims to suppress autoreactivity, through installation of a renewed and tolerant immune system, promoting control of disease progression. High doses of cyclophosphamide (CY) and rabbit anti-thymocyte globulin (ATG) are commonly used. However, some patients who fulfill indication criteria present cardiac alterations secondary to SSc, that may increase cyclophosphamide-related cardiotoxicity.

Objectives: To assess the tolerability and efficacy of AHSCT for patients with severe forms of systemic sclerosis with cardiac involvement using an alternative conditioning regimen with fludarabine + melphalan + ATG (FLUMEL-ATG).

Patients and methods: This study included patients with diffuse skin involvement and/or interstitial lung involvement who had progression of the disease in the last 12 months, under standard treatment, with evidence of cardiac involvement in the pre-transplant evaluation. Patients underwent collection of autologous hematopoietic stem cells from the peripheral blood through leukapheresis.
Subsequently, they received Fludarabine $120 \mathrm{mg} / \mathrm{m} 2+$ Melphalan $140 \mathrm{mg} / \mathrm{m} 2$ + rabbit anti-thymocyte globulin (ATG), followed by infusion of previously collected autologous stem cells. After transplantation, patients were monitored for disease activity.

Results: From 2012 to 2018, nine patients with median age of 32 years (12-60) were transplanted using FLUMEL-ATG conditioning. Five were females and seven had been previously treated with monthly cyclophosphamide pulses. All patients presented pre-transplant cardiac abnormalities: non-sustained ventricular arrhythmias (3 patients), pulmonary artery trunk dilatation (3), systolic or diastolic ventricular dysfunction (2), and patchy cardiac fibrosis (2). During transplantation, all patients presented febrile neutropenia, and four developed bacterial pneumonia. One patient presented cardiotoxicity and 4 , pulmonary congestion. All patients were promptly treated and fully recovered. Five patients had cytomegalovirus reactivation after transplantation without clinical disease. There were no deaths. Median post-transplantation follow-up duration was 24 months (6-47). The mean (SD) of the cutaneous Rodnan score decreased significantly from 29 (11) before transplantation to $17(9)(p=0.001)$ at the last post-transplant evaluation. The mean (SD) predicted value of forced vital capacity increased non-significantly after the procedure, from $66 \%(12.2)$ to $74 \%$ (17.9) ( $p=0.068)$.

Conclusions: We believe AHSCT with FLUMEL + ATG transplant conditioning regimen is safe for SSc patients with mild cardiac involvement, with acceptable incidence of severe transplant-related cardiac events. This group of patients achieved long-term control of disease progression. Longer follow-up and larger number of patients are required to fully support these conclusions.

013

\section{AVALIAÇÃO DE ATIVIDADE EM PACIENTES COM ARTERITE}

TAKAYASU

Fabricia Fonseca Simil, Gilda Aparecida Ferreira

UFMG, BELO HORIZONTE, MG, Brasil

Advances in Rheumatology 2018, 58(Suppl 1):013

Objective To analyze the performance of FDG-PET/CT in the evaluation of the disease in patients with Takayasu's arteritis (TA), comparing clinical, biological and vascular data in angiotomography.

Methods: cross-sectional clinical trial, 19 patients with TA according to ACR/1990, laboratory (angiotomography and FDG); PET /CT with the 18F-FDG radiopharmaceutical). The FDG-PET/TC were used three parameters for mensuration the values of captating: visual scale, intensity of captation and value of captation maximum customized (SUVmax). Results and Conclusions: Of the 19 patients with TA, 95\% were female, with a mean (SD) age of 38 (8.3) years. Patients active according to $\mathrm{NIH}$ presented greater thickening in the left subclavian arteries $(p=0.017)$, ascending aorta $(p=0.038)$ and abdominal aorta $(p=$ $0.017)$ and double ring in the abdominal aorta $(p=0.05)$. Thickness greater than $2.86 \mathrm{~mm}$ in the aortic arch showed sensitivity of $75 \%$ and specificity of $72.5 \%$ (area below the ROC curve $=0.83,95 \% \mathrm{Cl}$ $0.63-1.0, p=0.017$ ) for prediction of disease activity, considering ITAS 2010 as a reference. Thickness greater than $2.9 \mathrm{~mm}$ in the ascending aorta artery, $100 \%$ sensitivity and specificity of $82.4 \%$ (the area below the ROC curve was 1 , with $95 \% \mathrm{Cl} 1.0-1.0, \mathrm{p}=0.02$ ) for predicting AT activity, ITAS VHS as a reference. The max SUV in the celiac trunk greater than 2.83 with $100 \%$ sensitivity and specificity $94 \%$ (the area below the ROC curve $0.971,95 \% \mathrm{Cl} 0.89-1.0, \mathrm{p}=0.034$ ) for activity prediction of AT, ITAS VHS as a reference. The visual scale of the FDG-PET / CT showed an association with double ring angiotomography in the following arteries: aortic arch $(p=0.001)$, brachiocephalic trunk $(P=0.001)$, right subclavian $(p=0.003)$, right carotid $(p=$ $0.003)$, left carotid $(p=0.003)$ and the descending thoracic aorta ( $p=$ 0.05 ). There was a correlation between the median abdominal aortic artery thickening and the median of the SUVmax of this region (1.3mm Var Min-max 0.05-4.4, $\mathrm{p}=0.019)$. Vascular alterations of angiotomography showed good performance to evaluate disease 
activity in this sample of TA patients compared to clinical criteria and the presence of double ring at angiotomography showed a significant association with FDG-PET/CT activity.

\section{4}

AXONAL DYSFUNCTION, NEURONAL MARKERS AND PROINFLAMMATORY CYTOKINES IN CHILDHOOD-ONSET SYSTEMIC LUPUS ERYTHEMATOSUS

Renan Bazuco Frittoli, Danilo Rodrigues Pereira, Mariana Postal, Aline Tamires Lapa, Roberto Marini, Gabriela Castellano, Fernando Cendes, Leticia Rittner, Simone Appenzeller

UNICAMP, CAMPINAS, SP, Brasil

Advances in Rheumatology 2018, 58(Suppl 1):014

Background: Involvement of the central nervous system is frequently observed in childhood-onset SLE patients (cSLE). Proton magnetic spectroscopy (1H-MRS) is an important non-invasive method of quantification of biological metabolites. Abnormalities in brain metabolites may predict future damage, such as lesions and atrophy in adults-onset SLE, however there are few studies in CSLE. The objective is to determine the presence of axonal dysfunction in CSLE and to determine clinical, laboratory and treatment features associated with its occurrence. To associate axonal dysfunction with sera Th1 (IL-12, TNF-a, IFN- $\gamma$ ), Th2 (IL-6 and IL-10), Th17 (IL-17) cytokines levels and antiribosomal $\mathrm{P}$ protein antibodies (anti-P) and S100 $\beta$.

Methods: We included 77 consecutive CSLE patients [median age 16 years (range 7-31)] from the Rheumatology outpatient unit and 66 healthy controls [median age 18 years (8-32)]. We performed multi voxel $1 \mathrm{H}-\mathrm{MRS}$ using point resolved spectroscopy sequence over the superior-posterior region of the corpus callosum (3T Phillips ${ }^{\circledR}$ scanner) and signals from $\mathrm{N}$-acetylaspartate compounds (NAA), choline-based compounds (Cho); creatine containing compounds ( $\mathrm{Cr}$ ) and lactate (Lac) were measured and metabolites $/ \mathrm{Cr}$ ratios were determined. A complete clinical, laboratory and neurological evaluation was performed in all subjects. Neurological manifestations were analyzed according to the ACR classification criteria. Mood and anxiety disorders were determined through Beck Depression and Beck Anxiety Inventory. SLE patients were further assessed for clinical and laboratory SLE manifestations, disease activity [SLE Disease Activity Index (SLEDAI)], damage [Systemic Lupus International Collaborating Clinics/American College of Rheumatology Damage Index (SDI)] and current drug exposures. Th1 (IL-12, TNF-a, IFN- - ), Th2 (IL-6 and IL-10), Th17 (IL-17) cytokines levels, S100 $\beta$ levels and anti-P were measured by ELISA using commercial kits. Data were compared by non-parametric tests.

Results: NAA/Cr ratio $(p=0.017)$ and $\mathrm{LaC} / \mathrm{Cr}$ ratio $(p=0.014)$ levels were significantly decreased and $\mathrm{Cho} / \mathrm{Cr}$ ratio levels $(p=0.038)$ was increased in CSLE patients when compared to healthy controls. We observed that $\mathrm{Cho} / \mathrm{Cr}$ ratio was associated with symptoms of anxiety ( $\mathrm{p}=0.02)$, cognitive decline $(p=0.03)$, corticosteroid use $(p=0.034)$ and correlated with IL-6 $(r=0.568 ; p=0.002)$. NAA/ $\mathrm{Cr}$ ratio was associated with disease activity $(p=0.01)$ and correlated with IL-4 $(r=0.375 ; p=0.024)$. Lac $/ C r$ ratio was associated with symptoms of depression $(p=0.016)$, presence of anti-P $(p=0.04)$ and correlated with IFN- $\gamma(r=0.611 ; p=0.004)$.

Conclusion: We observed significant axonal dysfunction in CSLE. Decreased NAA/Cr ratio was associated with disease activity and sera IL-4 levels and increased $\mathrm{Cho} / \mathrm{Cr}$ ratio was associated with neuropsychiatric manifestations, cumulative use of corticosteroids and IL-6, suggesting brain injury.

\section{6}

CLINICAL OUTCOMES OF PATIENTS WITH TAKAYASU ARTERITIS ON BIOLOGIC THERAPY: REAL-LIFE USE AND EXPERIENCE FROM A BRAZILIAN SINGLE-CENTER

Surian Clarisse da Costa Rocha Ribeiro, Mariana Ortega Perez, Leandro Lara do Prado, Samuel Katsuyuki Shinjo, Rosa Maria Rodrigues Pereira DISCIPLINA DE REUMATOLOGIA, HOSPITAL DAS CLINICAS DA

FACULDADE DE MEDICINA DA UNIVERSIDADE DE SÃO PAULO (FMUSP), SÃO PAULO, SP, Brasil

Advances in Rheumatology 2018, 58(Suppl 1):016
Background: Glucocorticoids and immunosuppressive drugs are the mainstay of therapy for Takayasu arteritis (TAK). However, in a significant proportion of patients, conventional therapies are unable to induce sustained remission. In this setting, biologic agents such as TNFa inhibitors and tocilizumab may be indicated. Our aim is to report the experience from a single-center with the use of biologic therapy in patients with TAK during the first 12 months of treatment. Methods: We consecutively studied a cohort of patients with TAK treated with TNF inhibitors and tocilizumab using a standard electronic protocol. TAK was classified by the 1990 American College of Rheumatology criteria. Disease activity was assessed according to the National Institutes of Health (NIH), Indian Takayasu's Arteritis Activity Score (ITAS 2010), Erythrocyte sedimentation rate (ESR) and C-reactive protein (CRP) at baseline, 6 and 12 months on biologic therapy. Response to treatment was categorized as complete response $(\mathrm{CR}): \mathrm{NIH}<2$ and prednisone $<10 \mathrm{mg} /$ day; partial response (PR): $\mathrm{NIH}<2$ and prednisone dosage decreased $\geq 50 \%$; or refractory disease (RD) in another situation. Damage was graded by Vasculitis Damage Index (VDI).

Results: Fourteen patients were evaluated, 92.8\% women, $61.6 \%$ Caucasian, $28.8 \pm 10.8$ years old and disease duration of $5.2 \pm 3.6$ years Biologics agents of choice were infliximab ( $n=12,85.7 \%)$, etanercept $(\mathrm{n}=1,7 \%)$ and tocilizumab $(\mathrm{n}=1,7 \%)$. After 6 months of treatment, CR was observed in $50 \%$ of patients, PR in $14.3 \%$ and RD in $35.7 \%$. After 12 months, CR in $50 \%$ patients, PR in $28.6 \%$ and RD in $21.4 \%$ (Table 1). Overall, $35.7 \%$ had moderate/severe infection during biologic treatment. Due to inadequate response, 3 patients in infliximab and 1 etanercept treatment performed switching to tocilizumab.

Conclusion: Our data show that, after 12 months, TNFa inhibitors and tocilizumab were associated with complete or partial response in a majority of patients $(78.6 \%)$, with decrease of inflammatory markers and prednisone dose.

Table 1 (abstract 016). Disease activity parameters, VDI and prednisone use from a cohort of patients with TA at baseline, 6 and 12 months on biologic therapy

\begin{tabular}{|l|c|c|c|r|}
\hline Parameters & Baseline & 6 months & 12 months & \multicolumn{1}{c|}{$p$} \\
\hline NIH & $2.78 \pm 0.69$ & $0.71 \pm 0.91$ & $0.5 \pm 0.65$ & $<0.001$ \\
\hline ITAS 2010 & $3.71 \pm 1.97$ & $0.42 \pm 0.64$ & $0.07 \pm 0.26$ & $<0.001$ \\
\hline ESR, mm & $46.07 \pm 23.34$ & $22.64 \pm 19.91$ & $20.64 \pm 14.20$ & 0.001 \\
\hline CRP, mg/L & $31.47 \pm 33.71$ & $11.43 \pm 22.47$ & $7.29 \pm 11.91$ & 0.02 \\
\hline VDI & $4.42 \pm 0.93$ & $4.42 \pm 0.93$ & $4.42 \pm 0.93$ & 1 \\
\hline Prednisone dose, $\mathrm{mg} /$ day & $20.0 \pm 11.88$ & $11.25 \pm 9.64$ & $7.32 \pm 6.75$ & 0.004 \\
\hline
\end{tabular}

017

COMPARISON OF CLINICAL FEATURES AND TREATMENT OUTCOMES BETWEEN PATIENTS WITH IMMUNE-MEDIATED NECROTIZING MYOPATHY AND OTHER SYSTEMIC AUTOIMMUNE MYOPATHIES

Samuel Katsuyuki Shinjo 1,3, Leonardo Santos Hoff', Jean Marcos de Souza $^{3}$

${ }^{1}$ FACULDADE DE MEDICINA, UNIVERSIDADE DE SAO PAULO, SÃO

PAULO, SP, Brasil; ${ }^{2}$ FACULDADE DE MEDICINA FMUSP, UNIVERSIDADE DE

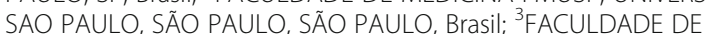
MEDICINA FMUSP, UNIVERSIDADE SAO PAULO, SÃO PAULO, SÃO PAULO, Brasil

Advances in Rheumatology 2018, 58(Suppl 1):017

Background. Differently from other systemic autoimmune myopathies (SAM), immune-mediated necrotizing myopathies (IMNM) generally present with clinical signs of severe proximal muscle weakness, rapid onset, very high levels of creatine phosphokinase, and usually 
refractory to conventional immunosuppressive therapies. IMNM is characterized histologically by necrotic and regenerating muscle fibers, in the presence of scarce or absent inflammatory infiltrates. The aim of the present study was to compare clinical features and treatment outcomes between patients with IMNM and other SAM.

Methods. A retrospective, single-center cohort study (2000 to 2017), included 153 consecutive patients with definite SAM: 14 (9.2\%) IMNM (10 anti-SRP, 3 anti-HMGCR, 1 seronegative), 89 (58.2\%) dermatomyositis, 20 (13.1\%) polymyositis and 30 (19.6\%) antisynthetase syndrome. Patients with clinically amyopathic dermatomyositis, inclusion body myositis and other myopathies were excluded. Patient data were extensively reviewed from electronic medical records, with prestandardized and parameterized information.

Results. Comparing to other SAM, patients with IMNM were significantly older (57.0 \pm 9.1 vs. $41.0 \pm 15.2$ years) and had a higher creatine phosphokinase at disease onset ( $14614 \pm 18532$ vs. $6252 \pm 9719$ U/L). In contrast, patients with IMNM were less likely to have interstitial lung disease (7.1\% vs. $41.2 \%)$. Gender distribution, degree of muscle weakness at disease onset, and frequency of upper dysphagia were similar between both groups. Distribution of frequency and time duration of patients that achieved a complete clinical response, time duration to glucocorticoid withdrawal were also comparable between both groups ( $P>0.05$ ). Severe infections occurred in $35.7 \%$ and $26.3 \%$ of patients with IMNM and other SAM, respectively $(P=0.659)$.

Conclusion. Our retrospective cohort study, patients with IMNM were older, had a higher creatine phosphokinase and had less interstitial lung diseases at disease onset, when compared to other SAM. However, other clinical manifestations and also treatment and disease outcomes were comparable between both groups. Therefore, in contrast to available studies in the literature, our patients IMNM had relatively a good outcome.

\section{8}

CYCLOPHOSPHAMIDE FOR NEUROLOGICAL MANIFESTATIONS OF BEHÇET DISEASE: PRELIMINARY RESULTS FROM A MONOCENTER COHORT STUDY

Priscila Dias Cardoso Ribeiro', Rywka Tenenbaum Medeiros Golebiovski², Flávia Maria Matos Melo Campos Peixoto², Pedro Matos², Pedro Paulo de Alcântara Pedro², Dennise de Oliveira Nogueira Farias², João Victor Campos de Oliveira², Eduarda Bonelli Zarur², Joice Moraes Faria Monteiro Belem², Alexandre Wagner Silva de Souza ${ }^{2}$

'UNIVERSIDADE FEDERAL DE SÃO PAULO, SÃO PAULO, SP, Brasil;

${ }^{2}$ UNIFESP, SÃO PAULO, SP, Brasil

Advances in Rheumatology 2018, 58(Suppl 1):018

Background. Neurological involvement, although uncommon (4 a $29 \%$ ), is one of the most serious causes of long-term morbidity and mortality in Behçet's disease (BD). There is a wide variety of neurological manifestations in BD that can be categorized in two major types: parenchymal (e.g. meningoencephalitis) and non-parenchymal (e.g. dural venous sinuses thrombosis and aseptic meningitis). To date, no controlled trials have assessed therapy for neuro-Behçet's disease (NBD). For acute or sub-acute parenchymal NBD attacks, 5-10 intravenous pulses of methylprednisolone and a course of high doses glucocorticoids are recommended, usually followed by a slowly tapering course. Azathioprine is the first-line maintenance therapy. Biologic agents such as IFNa or anti-TNF antibodies may be used in NBD patients refractory to immunosuppressive therapy. Cyclosporine A should be avoided as it is neurotoxic and can be considered a risk factor for developing NBD.

Objective. To describe outcomes of patients with NBD treated with intravenous pulses of cyclophosphamide followed by azathioprine compared with other therapeutic modalities including mainly highdose glucocorticoids and azathioprine.

Methods. A retrospective cohort study included 25 patients who fulfilled the International Study Group criteria for BD and presented neurological manifestations. NBD followed-up for a mean $91.5 \pm 51.6$ months after the onset of NBD manifestations. Relapses of NBD after starting therapy was the primary endpoint of this study.

Results. The mean age at study was $43.8 \pm 11.5$ years and $60 \%$ of them were females. Neurological manifestations were found at presentation in $52 \%$ of the cases, with parenchymal manifestations in $72 \%$. Only 1 patient used cyclosporine A at NBD onset. Intravenous pulses of cyclophosphamide were given as induction therapy for $68 \%$ and $12 \%$ of them had a failure to cyclophosphamide during induction therapy and had to be treated with a TNFa antagonists. The overall long-term relapse rate of NBD was $40 \%$ at a median of 60.0 months, while the NBD relapse rate with cyclophosphamide was $35.5 \%$ and $50.0 \%$ with other therapies ( $p=0.666$ ). Using the Kaplan Meier analysis, no significant differences were found between cyclophosphamide and other therapies regarding NBD relapses using the log-rank test $(p=0.767)$. The hazard ratio of cyclophosphamide to prevent relapses of NBD was $0.818(95 \%$ confidence interval: 0.217-3.080).

Conclusion. Preliminary data from this small open-label retrospective study do not show any benefit from intravenous pulses of cyclophosphamide to prevent long-term relapses of NBD.

\section{9}

DEVELOPMENT AND SEVERITY OF RHEUMATOID ARTHRITIS IS NOT RELATED TO CIITA (RS3087456) GENE POLYMORPHISM IN BRAZILIAN POPULATION

Isaura Isabelle Fonseca Gomes da Silva ${ }^{3}$, Suelen Cristina Lima ${ }^{3}$, Denise Queiroga de Nascimento ${ }^{3}$, Eliezer Rushansky ${ }^{1}$, Maria Helena Queiroz de Araujo Mariano ${ }^{1}$, José Artur Bogo Chies ${ }^{2}$, Sergio Crovella ${ }^{3}$, Paula Sandrin Garcia $^{3}$

'HOSPITAL UNIVERSITÁRIO OSWALDO CRUZ, RECIFE, PERNAMBUCO, Brasil; ${ }^{2}$ HOSPITAL DAS CLÍNICAS, UFRGS, PORTO ALEGRE, RIO GRANDE DO SUL, Brasil; ${ }^{3}$ UNIVERSIDADE FEDERAL DE PERNAMBUCO, RECIFE, PERNAMBUCO, Brasi

Advances in Rheumatology 2018, 58(Suppl 1):019

Background: Rheumatoid arthritis is an immunological disorder with etiology not fully understood. Genetics factors can contribute to development and severity of rheumatoid arthritis (RA) and can be targets of study to personalized medicine. Genes related to immune system as MHC locus has been associated to RA since plays a role in inflammation observed in the disease. Among these, the Class II Major Histocompatibility Complex Transactivator (CIITA) has been highlighted once that plays a role in the activation of the immune system by stimulating MHC II transcription. The polymorphism rs3087456 of CIITA is located at position -168 A/G has attracted attention since it can change your transcriptional level and plays a role in RA inflammation. Some studies in different populations had found controversial results and the relation between this polymorphism and RA in Brazilian population yet is unclear. Thus, our study aims to determine the potential association of rs3087456 CIITA polymorphism in development and severity of RA.

Methods: Blood samples were collected from 429 Brazilian patients with RA and 329 unrelated healthy control volunteers. All subjects were genotyped using TaqMan genotyping assay in ABI 7500 Real Time PCR platform. Statistical analysis was performed using $\mathrm{R}$ version 3.2.1 and Statistica version 8.0 softwares and Chi-square test was applied to compare allele and genotypes frequencies and to test their associations with RA severity it was applied one-way analysis of variance (ANOVA) test.

Results: The Chi-square test indicated no significant differences when comparing the allele frequency of RA patients and controls ( $p>$ 0.05 ). Similar outcomes was observed in genotype distribution using AA genotype as reference in a codominant model, suggesting that this polymorphism does not play a role in RA development in Brazilian population. Regarding to RA severity, genotypes were compared with RA disease activity indexes. The statistical analysis showed no difference among genotypes and disease activity indexes, DAS28, CDAl and HAQ $(p>0.05)$.

Conclusions: The genetic variant rs3087456 of CIITA gene does not appears to influence the RA susceptibility or severity in a Brazilian population.

Funding

CAPES, CNPQ, FACEPE. 
020

DIAGNOSTIC DELAY IN SPONDYLOARTHRITIS: HOW CAN WE DO

\section{BETTER?}

Gustavo Gomes Resende', Ricardo da Cruz Lage', Olivio Brito Malheiro', Déborah Lobato Guimarães ${ }^{1}$, Filipe Augusto Carvalho de Paula', Diogo Couto Carvalho', Fernanda Oliveira Gomes' ${ }^{1}$, Carlos Alexandre de Souza Bomtempo ${ }^{1}$, Marco Antonio Parreiras de Carvalho ${ }^{2}$

${ }^{1}$ HC UFMG, BELO HORIZONTE, MG, Brasil; ${ }^{2}$ FACULDADE DE MEDICINA UFMG, BELO HORIZONTE, MG, Brasil

Advances in Rheumatology 2018, 58(Suppl 1):O20

Background: Diagnostic delay, the gap between onset of musculoskeletal symptoms and diagnosis definition, is associated to worse prognostic and represents a major challenge to assistance of spondyloarthritis $(\mathrm{SpA})$ patients. It was historically reported to be 8-10 years. The aim of this study was to compare it between two different periods of inclusion in a same cohort and evaluate possible associations with SpA features.

Methods: Two cross-sectional analysis from the same database were made. The first included patients admitted to the SpA outpatient clinic up to 2002 (group A) and the second one included patients admitted between 2003 and 2013 (group B). ANOVA F-test and Mann-Whitney were used to compare the two different scenarios and to test the association between the dependent and explanatory variables. Effects with $p$-values $\leq 0.05$ were considered as evidence of statistical significance.

Results and conclusions: In group A ( $N=156)$ we found: $65.4 \%$ of males; mean age at onset of $27.7( \pm 11.6)$ years; $53.8 \%$ of HLA-B27 positivity and mean diagnostic delay of $8.1( \pm 0.68)$ years. In group $B(N=99)$ we found: $57.6 \%$ of males; mean age at onset of 31.0 $( \pm 13.0)$ years; $59.0 \%$ of HLA-B27 positivity; and mean diagnostic delay of $6.9( \pm 0.76)$ years. Analyzing all patients $(N=255)$, the subtype of SpA, gender, HLA-B27 status or presence of radiographic sacroiliitis (mNY criteria) were not associated to longer delay. Conversely, the presence of extra-articular manifestations - EAM (i.e. uveitis, psoriasis and/or inflammatory bowel disease) on admission was statiscally associated ( $p<0.0001)$ with longer delay (Fig. 1) and the age at onset was negatively correlated with diagnostic delay ( $r$ $=-0.28 p<0.0001)$. In conclusion, we have shown a reduction in diagnostic delay (mean difference of 1.3 years) over a decade. Possible explanations for that include the increased awareness amongst health-care professionals, the adoption of new classification criteria (ASAS 2009) and the incorporation of magnetic resonance imaging (MRI) as a diagnostic tool in SpA. The seen association between EAM presence and earlier disease onset with the longer diagnostic delay, could represent a greater difficulty of non-rheumatologists in identify inflammatory-type symptoms and unawareness of the EAM linkage with $\mathrm{SpA}$. As consequence, patients may not be referenced to rheumatologist in appropriate time and may have an inadequate investigation, delaying the diagnosis. Therefore, there is still a need for further targeted education of health-care professionals to address the issue.

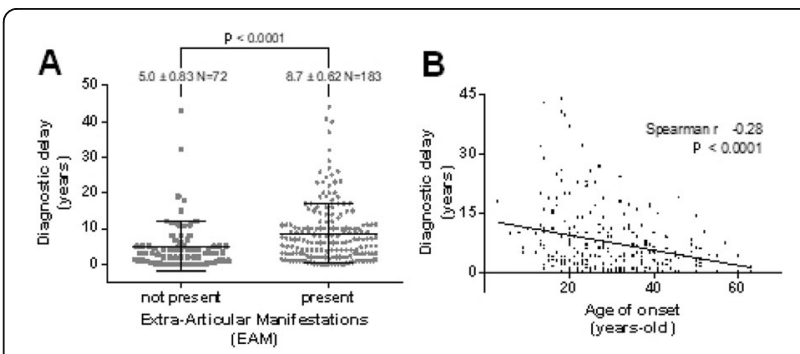

Fig. 1 (abstract 020). Longer diagnostic delay in SpA was associated with the presence of any extra-articular manifestation on amdission (a) and the younger age at onset (b).
021

\section{DIETETIC INTERVENTION IN PSORIATIC ARTHRITIS: THE DIETA}

TRIAL

Beatriz Leite ${ }^{1}$, Marcelo de Medeiros Pinheiro ${ }^{2}$

${ }^{1}$ ARACAJU, SE, Brasil; ' UNIVERSIDADE FEDERAL DE SÃO PAULO, SÃO

PAULO, SP, Brasil

Advances in Rheumatology 2018, 58(Suppl 1):O21

Background: Psoriatic Arthritis (PsA) is associated with multiple comorbidities, particularly metabolic syndrome (MetS). These findings brought up a potential link between adiposity and PsA, highlighting a potential fat-joint-skin axis mediated by oxidative stress and nutritional inadequacy. So, the aim is to evaluate if dietetic patterns changes or antioxidant supplementation or $5-10 \%$ weight loss could improve the disease activity in patients with PsA.

Materials and Methods: A total of 97 PsA patients were enrolled in a 12-week randomized, double-blinded, placebo-controlled trial. Patients were randomized into three groups: D - hypocaloric diet + placebo supplementation; D+S - hypocaloric diet + omega-3 supplementation ( $3 \mathrm{~g} /$ day); and $\mathrm{P}$ - placebo. Food intake (3-day registry, Healthy Eating Index $(\mathrm{HEl})$, and the Dietary Inflammatory Index (DII), body composition (whole-body DXA, weight and waist of circumference) and disease activity (BASDAl, DAS28-ESR, DAS28-CRP and MDA) were evaluated at baseline and after intervention. Statistical analysis used intention to treat approach. $\mathrm{P}$ level was set as below 0.05 .

Results and Conclusion: After 12 weeks, there was DAS28-CRP and BASDAI improvement, especially in the $D$ group $(-0.6 \pm 0.9 ; p=0.004$ and $-1.39 \pm 1.97 ; p=0.001$, respectively). In addition, higher proportion of patients achieved minimal disease activity in all groups (D: $34.3 \% ; D+S: 34.4 \% ; P: 27.3 \% ; p=0.006)$. The $D+S$ group had significant weight loss $(-1.79 \pm 2.4 ; p=0.004)$, as well as waist circumference $(-3.28 \pm 3.5, p<0.001)$ and body fat $(-1.2 \pm 2.2, p=0.006)$ reduction. There was no significant correlation between weight loss and disease activity improvement. Each 1-unit increase in HEl reduced the likelihood of achieving remission in 4\%. Also, each 100-calorie daily intake increase caused 3.4-fold DAS28-ESR impairment. In conclusion, a 12-week hypocaloric intervention provided suitable control of joint disease activity in patients with PsA, regardless of weight loss. Adding omega 3 supplementation caused relevant body composition changes.

\section{2}

DISEASE-MODIFYING ANTIRHEUMATIC DRUGS IN RHEUMATOID ARTHRITIS AND JUVENILE IDIOPATHIC ARTHRITIS: EFFECTS ON IMMUNIZATION COVERAGE AND VACCINE EFFICACY

Helena de Almeida Tupinambá', Laíssa Cristina Alves Alvino², Pedro Felipe de Almeida Vianna², Ana Beatriz Vargas-Santos², Manuella Lima Gomes Ochtrop ${ }^{2}$

${ }^{1}$ UNIVERSIDADE DO ESTADO DO RIO DE JANEIRO, RIO DE JANEIRO, RJ, Brasil; ${ }^{2}$ HOSPITAL UNIVERISTÁRIO PEDRO ERNESTO, RIO DE JANEIRO, RJ, Brasil Advances in Rheumatology 2018, 58(Suppl 1):O22

Background: The introduction of disease-modifying drugs (DMARDs) in the treatment of rheumatoid arthritis (RA) and juvenile idiopathic arthritis (JIA) was responsible for a dramatic change in the natural history of these diseases. However, the use of immunomodulatory agents also leads to increased risk of infections by common and opportunistic germs. Vaccination is the most important preventive action to reduce this risk. It is a worldwide recommendation that the vaccination schedule be updated in all patients who will be submitted to immunosuppression. This study aims to evaluate vaccine coverage and the impact of therapy on the efficacy of vaccination for Hepatitis B in a sample of patients with RA and JIA.

Methods: A cross-sectional study was carried out, with data derived from the review of medical records of patients with RA or JIA treated until March 2018 at the Biologic Outpatient Clinic of Tertiary Teaching Hospital in Rio de Janeiro. A total of 146 patients were selected for analysis. Vaccination coverage was studied using simple frequencies. To test factors related to the vaccine response to Hepatitis $B$, univariate logistic regressions were performed using Eviews. 
Results: Vaccination coverage was $65 \%$ for Influenza, $51 \%$ for Pneumococcal, 30\% Meningococcal, $64 \%$ for Tetanus, 3\% for Hepatitis A and $64 \%$ Hepatitis B. Of the 93 patients vaccinated for Hepatitis B, 40 had Anti Hbs measured after vaccination, with seroconversion in $38 \%$. Logistic regression used to search for relationship of Anti Hbs positivity and use of synthetic or biological DMARDs during vaccination resulted in an Odds Ratio (OR) of $0.17(95 \% \mathrm{Cl}$ 0.04-0.7) for use of biological DMARDs (bDMARD) and 1,55 (95\% Cl 0.33-7.23) for synthetic DMARDs (sDMARD).

Discussion: The vaccination coverage was superior compared to data from the US for influenza and pneumococcal, but it's still not ideal yet. The vaccine response to hepatitis $B$ appears to be negatively influenced by the use of bDMARDs, with a chance of seroconversion of only 1 in every 5.9 immunized found in our study. Conclusions: Increasing attention has been given to vaccination of patients using bDMARDs. It's important to have in mind, that an attempt to get it done before starting the treatment will probably lead to better results in efficacy.

\section{3}

DISPENSATION OF IMMUNOBIOLOGICALS IN MODEL OF ASSISTED THERAPY IN THE SUS REDUCES COSTS WITH IMMUNOBIOLOGICAL IN ANKYLOSING SPONDYLITIS

Lorenza Rosa Silverio Scomparin, Carla Gonçalves Schahin Saad, Fernando Henrique Carlos de Souza, Renata Miossi, Ana Cristina de Medeiros Ribeiro, Mariana Gioieli Waisberg, Karina Rossi Bonfiglioli, Leandro Lara do Prado, Vanessa Teich, Eloisa Bonfa, Julio Cesar Bertacini de Moraes

FACULDADE DE MEDICINA DA UNIVERSIDADE DE SAO PAULO, SAO PAULO, SP, Brasil

Advances in Rheumatology 2018, 58(Suppl 1):023

Introduction: In the 2000s, immunobiologicals were introduced as a treatment option in various rheumatologic conditions, including Ankylosing Spondylitis (AS). The incorporation of these medications by the Unified Health System (SUS) brought important clinical advances in the effective control of the inflammatory process and the quality of life of this population. With the increase in indications and the volume of patients attended, the impact on the SUS budget has increased, requiring a rational and efficient dispensation process.

The predominant model in the supply of medication in the SUS is the direct dispensing to the patient. This model presents several weaknesses, given that the immunobiologicals are injectable and thermolabile, it has lead to an important interruption in the proper conservation and transport, and also in the safety of the medication application.

To improve this process, an assisted dispensing model was created in 2007 to ensure safety application, monitor effectiveness, and rationalize use by combating resource wastage.

Objectives: To compare the model of assisted therapy with that of direct dispensing of SUS for immunobiological drugs for, in terms of reducing the volume of use of immunobiological drugs and their financial impact according to the acquisition costs for each medication during the study period.

Methods: All the visits were included in the center responsible for the model of assisted therapy, with medication provided by the Ministry of Health for patients presenting in the year 2015. In each of the consultations were recorded: medication, number of bottles, prescribed dose, dose received, cancellations, faults and estimates of bottles that would have been dispensed by the direct system. The financial value, in Reais, was calculated according to the acquisition value by the Ministry of Health for each immunobiological in 2015. Results: A total of 1688 consultations were performed for patients with AS in 2015 by the model of assisted therapy. 669 (23.26\%) bottles of a total volume of 2876 prescribed vials of all medications intended for the treatment of AED were no longer used, reducing expenses by $R \$ 470,289.15$ (23.78\%). Based on the same savings percentage of the model in question for the whole SUS and according to data from DATASUS for AS in 2015, the reduction of immunobiological expenses for the treatment of AS would be R $\$ 49,467,109,97$.

\section{4}

DISPENSATION OF IMMUNOBIOLOGY IN MODEL OF ASSISTED THERAPY IN SUS REDUCES COSTS WITH IMMUNOBIOLOGICAL IN YOUTH IDIOPATHIC ARTHRITIS

Lorenza Rosa Silverio Scomparin, Carla Gonçalves Schahin Saad, Fernando Henrique Carlos De Souza, Renata Miossi, Ana Cristina De Medeiros Ribeiro, Mariana Gioieli Waisberg, Karina Rossi Bonfiglioli, Leandro Lara Do Prado, Vanessa Teich, Eloisa Bonfa, Julio Cesar Bertacini De Moraes

FACULDADE DE MEDICINA DA UNIVERSIDADE DE SAO PAULO, SAO PAULO, SP, Brasil

Advances in Rheumatology 2018, 58(Suppl 1):024

Introduction: Immunobiologicals were introduced as a treatment option for several diseases, including Juvenile Idiopathic Arthritis (JIA) by the Unified Health System (SUS), which brought up important advances, especifically as a new alternative capable of modifying the course of the disease. Given this, the number of indications is a growing process, as well as its budget, since such drugs, by the common SUS model, are dispensed directly to the patient. Given the fact that the medications are injectable and thermolabile, there is an important gap in the return, transport and application of the medications. In 2007 a model of dispensation was created, which values the assisted therapy and safety application, thus combating waste and rationalizing the use of medicines.

Objectives: To compare the direct dispensing model of SUS with that of assisted therapy, in terms of volume reduction of immunobiological drugs and their financial impact.

METHODS: All the visits were included in the center responsible for the model of assisted therapy, with medication provided by the Ministry of Health for patients with JIA in the year 2015. In each service were recorded: medication, number of bottles, prescribed dose, dose received, cancellations, shortages and estimation of bottles received by the direct dispensing system. The financial value was calculated according to the acquisition value by the Ministry of Health for each immunobiological in 2015.

Results: A total of 1898 consultations were performed for patients with JIA in 2015 under the model of assisted therapy. 941 (26.19\%) bottles of a prescribed total volume of all medications of 3593 bottles were no longer used, reducing expenses by $\mathrm{R} \$ 341,205.63$ (25.21\%). Extrapolating the percentage of savings of the model in question for the whole SUS, based on the SUS expenditures on data released by DATASUS for the year 2015, the reduction of immunobiological expenses destined for treatment of JIA would be the amount of $\mathrm{R} \$ 6,141.370 .1$.

Conclusion: The model of assisted therapy considerably reduces the volume of dispensed bottles compared to the model of direct dispensing bringing significant reduction of expenses to the SUS.

\section{5}

DNA COPY NUMBER VARIATIONS AND FAMILIAL RECURRENCE IN PATIENTS WITH CHILDHOOD-ONSET SYSTEMIC LUPUS

\section{ERYTHEMATOSUS}

Nailu Angelica Sinicato ${ }^{1}$, Luciana de Oliveira', Vera L Gil-da-Silva-Lopes ${ }^{1}$, Iscia Lopes-Cendes ${ }^{1}$, Roberto Marini ${ }^{1}$, Timmothy Niewold ${ }^{2}$, Simone Appenzeller ${ }^{1}$

'UNICAMP, CAMPINAS, SP, Brasil; ${ }^{2}$ NEW YORK UNIVERSITY, NEW YORK, NY, Estados Unidos

Advances in Rheumatology 2018, 58(Suppl 1):O25

Background: The genetic predisposition in Systemic Lupus Erythematosus (SLE) encompasses multiple genes that may trigger or increase risks for the development of the disease. Especially in autoimmune diseases, the understanding of the copy number variations (CNVs) in the development of diseases is still little understood. Our main objective was to investigate the familial recurrence of SLE and the profile of CNVs in patients with CSLE.

Materials and methods: We included consecutive patients with CSLE followed up at the Pediatric Rheumatology Unit of UNICAMP between 2013/2016. CNVs were evaluated through the CytoScan HD 
Affymetrix ${ }^{\circledR}$. Recurrence analysis was performed using conventional heredograms of three family generations and analyzed visually. Results: We evaluated 112 patients with CSLE, 96 (85.7\%) female, with a mean age of 21 years (SD \pm 4.8 years) and had mean disease duration of 7.8 years $(S D \pm 6.5)$. Forty patients with CSLE have family members with autoimmune diseases (except SLE), the most common were hypothyroidism 21 (52.5\%), hyperthyroidism 9 (22.5\%) and rheumatoid arthritis $3(7.5 \%)$. In relation to the SLE family recurrence, we observed 10 first-degree relatives (recurrence rate: 25.3), 10 second-degree relatives (recurrence rate: 8.3 ), and 3 third-degree relatives (recurrence rate: 1,3 ) with SLE. We did not observe statistical difference between familial and sporadic SLE in relation to demographic, clinical, laboratorial and treatment data. In the CNVs analysis, we analyzed 107 patients and observed CNV in relevant genes [genes: MECP2 (duplications), BANK1 (deletions), DNASE1 and TRAP1 (deletions), IKBKG (deletions) and CFHR4 (4 deletions and 2 duplications)]. We do not observe association between the presence of CNV and family recurrence of SLE and the clinical, laboratory and treatment profile.

Conclusions: We conclude that there is a greater recurrence of SLE in first-degree relatives and we observed the presence of CNVs in 10 genes previously related to SLE. No association between the presence of CNV and family recurrence was observed. The interest in genetic factors in SLE brings new challenges in order to understand its role in the development and progression of SLE. Founding grants: Fapesp and Capes.

\section{6 \\ DO PATIENTS REALLY UNDERSTAND THE PRESCRIPTION? ASSESSING LUPUS PATIENT'S COMPREHENSION OF PHYSICIAN PRESCRIPTION - A PILOT STUDY \\ Mariana Luiza Lewergger Borges, Maurício Petroli, Luiza Andrade Mussi, Lilah Ferreira Fontenelle Ribeiro, Rafael da Cunha Cancela, Sabrina Fausto de Lima, João Pedro Simão de Mello, Nycholas da Costa Tavares, Tamara Felzenszwaibe Waga, Marlon de Carvalho Ferreira Ribeiro, Ricardo Araujo Rauneitti, Mirhelen Mendes de Abreu \\ UFRJ, RIO DE JANEIRO, RJ, Brasil \\ Advances in Rheumatology 2018, 58(Suppl 1):O26}

Background: Communication skills and patients understanding of the prescription play an important role in adherence to the treatment program and affects the outcome of the disease. Identifying how patients understand the prescription may contribute to develop patient's education strategies that will be fundamental for shared decision-making process.

Methods: Designed as a cross-sectional study, this survey interviewed SLE patients (ACR, 2012) older than 18 years old, from a tertiary outpatient facility. Data were collected using a questionnaire that consisted of modules on socio-economic characteristics, clinical and therapeutically profile. The interview occurred after the consultation, on a face-to-face interview. Afterwards, questions regarding the prescription received during the patient-physician encounter were applied. Comprehension was defined according to the right answers related to the questions and to the followed score: great comprehension (from 90 to 100\%); very good comprehension (from 70 to $89 \%$ ); good comprehension (from 50 to 69\%); weak comprehension (from 30 to $49 \%$ ) e no comprehension (0 to $29 \%$ ). Finally, a retrospective analysis of the medical chart (last two years) to add the analysis. Descriptive statistics were employed using central tendency and dispersion measurements. Also, we analyzed the correlation between comprehension's scores, self-assessment score, educational level, and clinical aspects.
Results and conclusions: Thirty-seven patients were analyzed. Of them, $89 \%$ were female, $66 \%$ had between 10 to 15 years of disease; and $34 \%$ declared 10 to 15 years of schooling. Most of them (65\%) were hospitalized at least once a year, and SLEDAI score was 13 (SD \pm 8 ). The average of medicines per day used was 9 (SD \pm 4 ). Patients' comprehension score was full for $24 \%$ of the sample, partial for $43 \%$; weak was found in $20 \%$ patients and no comprehension was observed in $13 \%$ of them. The variable that influenced most in the comprehension score was level of schooling $(p=0.050)$. SLEDAI score was also relevant but not significant to influence comprehension $(p=0.065)$. We observed that, in this sample, there is a regular prescription's comprehension. Future studies can help to understand the factors related with the quality of comprehension so that assertive strategies for patient's education can be outlined.

\section{7}

DOES GENERAL SELF-EFFICACY MODERATE THE RELATIONSHIP BETWEEN KNEE SYMPTOMS AND FUNCTION? THE ELSA-BRASIL MUSCULOSKELETAL STUDY

Rosa Weiss Telles ${ }^{1,3}$, Daniela Castelo Azevedo ${ }^{3}$, Luciana Andrade Machado $^{2}$, Sandhi Maria Barreto ${ }^{3}$

${ }^{1}$ FACULDADE DE MEDICINA DA UFMG, BELO HORIZONTE, MG, Brasil; 2ELSA-BRASIL MUSCULOESQUELÉTICO, BELO HORIZONTE, MG, Brasil; ${ }^{3}$ FACULDADE DE MEDICINA - UFMG, BELO HORIZONTE, MG, Brasil Advances in Rheumatology 2018, 58(Suppl 1):027

Background: Self-efficacy is the perceived belief in one's capabilities to organize and execute the tasks required to achieve a desired outcome. It is a psychosocial construct known to modify pain expression and coping and it also appears to mediate the relationship between pain and physical function in multiple musculoskeletal disorders. The purpose of this study was to investigate whether self-efficacy levels modify the association between chronic knee pain and knee function in Brazillian adults. We hypothesized that this association would be stronger among adults with low self-efficacy, as compared with those with moderate to high self-efficacy.

Material and Methods: This cross-sectional study used data from baseline assessments of ELSA-Brasil Musculoskeletal Study, which consists of an ancilary study from the Brazilian Longitudinal Study of Adult Health (ELSA-Brasil). Knee symptoms were identified by the report of knee pain, discomfort or stiffness in the previous twelve months, lasting for at least six months. Knee function was subjectively and objectively assessed by the WOMAC index (WOMAC-function subscale) and the five-times sit-tostand test (5T-STS test), respectively. Function outcomes were entered in the models as dichotomous variables (highest quintile, meaning worst function). Low self-efficacy was defined as the lowest quintile of self-efficay measured by the General Self-efficacy scale. An interaction term was included in logistic regressions to assess the influence of low self-efficacy on the relationship between knee pain and both measures of function. All models were adjusted for potential confounders (sex, age, depressive symptoms, body mass index, and occupational nature). Results and Conclusions: 1735 participants were included in the analysis with mean age equal to 56.0 (8.9) years, $53.4 \%$ were women. In univariate analyses, chronic knee symptoms and low self-efficacy were both significantly associated with low knee function (WOMACfunction: OR: $10.32 ; 95 \% \mathrm{Cl} 8.15-13.07$ and OR: $1.32 ; 95 \% \mathrm{Cl} 1.05-1.66$; 5T-STS test: OR: $1.89 ; 95 \% \mathrm{Cl} 1.54-2.33$ and OR:1.27; $95 \% \mathrm{Cl} 1.03-1.57$, respectively). However, after adjustments, self-efficacy was neither associated with knee function nor altered the association between knee symptoms and function. Using measures of knee function as continuous variables did not change the results in none of the adjusted linear regression models tested. In conclusion, in this cross- 
sectional study of Brazillian adults low self-efficacy was neither associated, nor dit it moderate the association between knee symptoms and function.

\section{Funding}

Brazilian Ministry of Health (Science and Technology Department), of Science and Technology (FINEP, CNPq) and of educations (CAPES).

\section{8}

EFFICACY OF A MULTIDISCIPLINARY REHABILITATION SERVICE IN THE TREATMENT OF CHRONIC SEQUELAE AFTER CHIKUNGUNYA FEVER: REDUCTION OF PAIN AND IMPROVEMENT OF FUNCTIONALITY Denise Leite Dos Santos ${ }^{2}$, Erika Guerrieri Barbosa ${ }^{2}$, Fernanda de Oliveira Ferreira', Raphael Avelar Nunes², Gulia Delmaschio Cypriano², Hevilla Souper ${ }^{2}$ 'UNIVERSIDADE FEDERAL DE JUIZ DE FORA, GOVERNADOR VALADARES, $M G$, Brasil; ${ }^{2}$ SECRETARIA MUNICIPAL DE SAÚDE, GOVERNADOR VALADARES, MG, Brasil

Advances in Rheumatology 2018, 58(Suppl 1):O28

Background: In 2017 the municipality of Governador Valadares suffered a Chikungunya fever outbreak, presenting 13,000 notifications. This situation demanded a differentiated confrontation to assist users in the chronic phase, since it is estimated that almost $30 \%$ of the cases become chronic. A Center for rehabilitation in arboviruses was structured, with the objective of monitoring chikungunya fever chronic users. Given the diversity of clinical aspects, the service was structured with a multidisciplinary team, acting with procedures many times different from the current protocol, since it was clinically necessary to make adjustments to obtain clinical improvement of symptoms.

Objective: to present the effectiveness of the multidisciplinary rehabilitation service in the treatment of chronic sequelae after chikungunya fever in the municipality of Governador Valadares.

Methods: We analyzed the medical records of 181 patients, with chronic sequelae after chikungunya fever, who sought care at the Center for Rehabilitation in Arboviroses. Participants were assessed at the first consultation, before treatment, and after the treatment. We analyzed the pain reported by patients (Visual Analog Pain Scale), functionality and quality of life (HAQ Scale), data that were registered in the medical records. The medical rheumatologist used a standardized protocol of evaluation, identifying the clinical profile of each patient, verifying the presence of symptoms such as fever, skin changes, diarrhea, vomiting, nausea, dizziness, fatigue and neuropathies such as cramps, paresthesias and tingling. The treatment involved a specialist rheumatologist with extensive experience in the field, a physiotherapist who specializes in manual therapies; an occupational therapist and a psychologist with a specialty in cognitive behavioral therapy. The results of the EVA and HAQ scales were compared before and after treatment using the Wilcoxon test, considering $95 \%$ confidence.

Results: The clinical profile revealed a high prevalence of neuropathies $(77.8 \%)$, the most frequent symptom being paraesthesia $(87.2 \%)$, followed by cramps (12\%) and tingling was reported by only $1(0,8 \%)$ participant. The data revealed a significant decrease in pain levels and improvement of functionality after treatment $(p<0.001)$. Conclusions: The study demonstrates that the most frequent clinical repercussion of the chikungunya fever was neuropathy, which requires propaedeutics and differentiated rehabilitation. The treatment used was effective to reduce pain and improve functionality for the cases, making necessary the development of more detailed studies to investigate such procedures not yet described in the clinical protocols and literature.

\section{9}

ENDOTHELIAL FUNCTION FEATURES AND STRUCTURAL PROPERTIES OF LARGE ARTERIES IN SYSTEMIC AUTOIMMUNE MYOPATHIES

Rafael Giovane Missé2, Isabela Bruna Pires Borges², Valéria Aparecida Costa Hong ${ }^{1}$, Luiz Aparecido Bortolotto', Samuel Katsuyuki Shinjo ${ }^{2}$ 'INSTITUTO DO CORAÇÃO DO HOSPITAL DAS CLÍNICAS DA FACULDADE DE MEDICINA DA UNIVERSIDADE DE SÃO PAULO, SÃO PAULO, SÃO PAULO, Brasil; ${ }^{2}$ FACULDADE DE MEDICINA DA UNIVERSIDADE DE SÃO PAULO, SÃO PAULO, SÃO PAULO, Brasil

Advances in Rheumatology 2018, 58(Suppl 1):O29
Background. High frequency of cardiovascular diseases and theirs risk factors have been described in systemic autoimmune myopathies (SAM). These comorbidities can be the cause of endothelial dysfunction and arterial stiffness, as shown in several other systemic autoimmune diseases. In this aspect, the aim of the present study was to assess the endothelial function and arterial stiffness in patients with SAM.

Materials and Methods. This cross-sectional study included 19 patients with SAM (14 dermatomyositis and 5 anti-Jo-1 antisynthetase syndrome) from 2017 to 2018. As a control group, 14 aged, gender and body mass index-matched were engaged. Flow-mediated vasodilation (FMD) analysis was achieved from brachial arterial above the antecubital fossa (Doppler ultrasound), whereas carotid-femoral vessels pulse wave velocity (PWV) evaluation was performed by Complior system. Disease status was assessed by the International Myositis Assessment \& Clinical Studies Groups (IMACS) core set measures.

Results. Mean age of the patients was 45.0 years, with disease duration of 5.0 years. Patients had a median Manual Muscle Testing (MMT-8) of 80, Health Assessment Questionnaire (HAQ) of 0.70 , patient Visual Analogue Score (VAS) of 2.4, physician VAS of 1.5, Myositis Disease Activity Assessment VAS of 0.0 , serum level of creatine phosphokinase of $97 \mathrm{U} / \mathrm{L}$. Patients were using different immunosuppressive drugs plus prednisone $<10 \mathrm{mg} /$ day. The FMD (\%) measurement was $8.3 \pm 4.8$ vs. 4.1 $\pm 4.0 \quad(\mathrm{P}<0.029)$ in $\mathrm{SAM}$ and control group, respectively. Analyzing individually the diseases, $F M D(\%)$ was $8.9 \pm 4.3$ vs. $4.1 \pm 4.0(P<0.013)$ in dermatomyositis and control group, whereas FMD (\%) was $6.4 \pm 5.5$ vs. $4.1 \pm 4.0(P=0.432)$ in antisynthetase syndrome and control group. The PWV was comparable between SAM and control group (7.0 \pm 0.8 vs. 7.0 $\pm 1.0 \mathrm{~m} / \mathrm{s} ; \mathrm{P}=0.905$, respectively).

Conclusion. Although there is no structural properties of large arteries, patients with clinical and laboratory stable SAM (specifically dermatomyositis) have significant endothelial dysfunction when compared to control group. More studies are necessary to confirm our data and to analyze the possible impact of this vessel dysfunction in disease pathogenesis.

\section{Funding}

Support by FAPESP \#2016/23574-0 (RGM), \#2016/20371-1 (IBPB), \#2017/ 13109-1 (SKS)

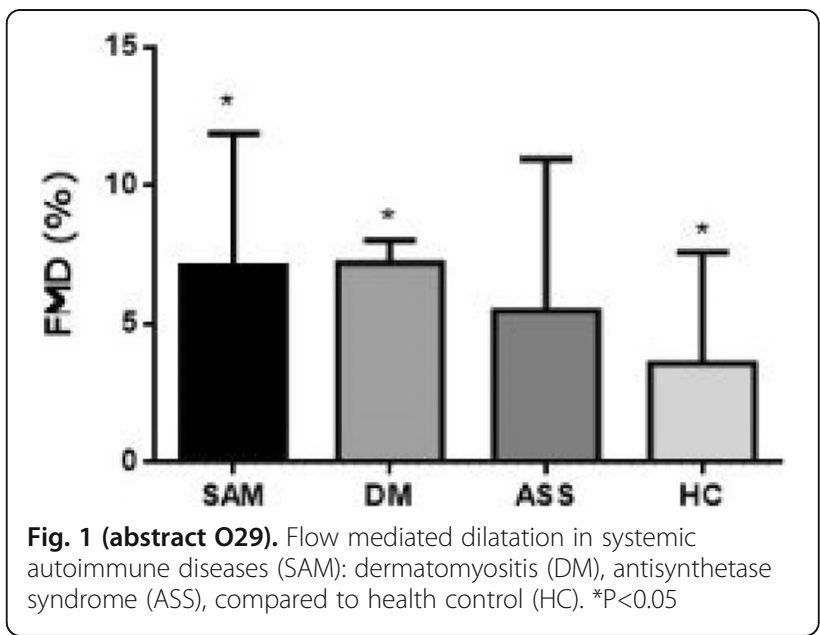

030

EPIDEMIOLOGICAL ANALYSIS OF DEATHS BY DISSEMINATED INTRAVASCULAR COAGULATION IN BRAZIL BETWEEN 2010 AND

2015

Érica Alves Nascimento, Andressa Borelli Santos, Mariana Bomfim

Menezes, Débora Larissa Montarroyos Leite

UNIVERSIDADE TIRADENTES, ARACAJU, SERGIPE, Brasil

Advances in Rheumatology 2018, 58(Suppl 1):O30 
Introduction: Disseminated intravascular coagulation is a syndrome in which there is generalized activation of coagulation, with systemic fibrin deposition and consequent consumption of coagulation factors and platelets, with hemorrhage. It is initiated after conditions such as sepsis and trauma and can be acute or chronic, with two different phenotypes, a thrombotic and a hemorrhagic. It is the phenotype that will determine the treatment of the syndrome.

Objectives: To access the prevalence of deaths due to disseminated intravascular coagulation in Brazil, between 2010 and 2015.

Materials and methods:

Retrospective study demonstrating all cases of death due to disseminated intravascular coagulation, from 2010 to 2015, in Brazil. Epidemiological data were collected through the database of the Mortality Information System (SIM).

Results:

The relationship between the number of deaths and age does not follow an exponential increase. It is observed a peak in children under one year, which soon decreases progressively, until the age group of 10 to 14 years, increasing exponentially until the age group of 70 to 79 years. In the regions of Brazil, the one that had the highest number of deaths registered was the Southeast, with $40.8 \%$ of the cases. The Cento-Oeste was responsible for the fewest cases, with only $7.8 \%$ of the cases. The number of deaths was higher in women, but not significantly. They accounted for $51.1 \%$ of all deaths. In relation to the number of deaths per year, there was a nonexponential decrease in this number between 2010 and 2015, going from 205 to 185 registered cases.

Conclusion: It was possible to observe that there is a great affection in children under one year, showing that this condition is an important death rate in these children. It is triggered by sepsis, complications in childbirth, hypoxia, hypothermia, acidosis, enterocolitis, among others. Thus, early treatment in both adults and children is of paramount importance. It is also important to remove the triggering factor from this condition.

\section{1}

EPIDEMIOLOGICAL ANALYSIS OF THE PROFILE OF HOSPITALIZED PATIENTS WITH ACUTE RHEUMATIC FEVER, IN BRAZIL, BETWEEN 2010 AND 2017

Érica Alves Nascimento, Andressa Borelli Santos, Mariana Bomfim

Menezes, Débora Larissa Montarroyos Leite

UNIVERSIDADE TIRADENTES, ARACAJU, SERGIPE, Brasil

Advances in Rheumatology 2018, 58(Suppl 1):O31

Introduction:

Rheumatic fever is an inflammatory, systemic disease that occurs in genetically predisposed people. It is a consequence of a pharyngotonsillitis caused by Group A $\beta$-hemolytic Streptococcus. Rheumatic heart disease is the main consequence of acute rheumatic fever, which also has other manifestations, like arthritis, chorea, subcutaneous nodules and marginate erythema.

Objective:

To access the prevalence of acute rheumatic fever treated as hospitalization between 2010 and 2017.

Materials and methods:

Retrospective study demonstrating all cases of hospitalization for acute rheumatic fever, from 2010 to 2017, in Brazil. Epidemiological data were collected through the SUS Hospital Information System (SIH/SUS) database.

Results:

From 2010 to 2017 , there was a $62,5 \%$ decrease in the 31,595 registered cases, mainly due to socioeconomic improvement and more investments in health. From 2012 to 2013, however, there was an increase, decreasing again in 2014. The Northeastern region accounts for $40 \%$ of cases of hospitalization, contrasting with the South region, responsible for only $8.6 \%$ of the cases, which shows socioeconomic and health care differences in the country. The highest incidence occurs in the age groups of 40 to 69 years. There is a higher prevalence in women, accounting for $51.45 \%$ of the cases. Regarding the character of hospitalization, $86 \%$ were hospitalized as a matter of urgency.
Conclusion:

The study shows that there was a decrease in the number of hospitalizations for acute rheumatic fever in Brazil. However, this disease should not be neglected, since improvements, especially in basic care, may prevent some cases of rheumatic fever.

\section{2}

EPIDEMIOLOGICAL AND CLINICAL PATTERN OF JUVENILE SJÖGREN'S SYNDROME IN A COHORT OF 30 PATIENTS

Blanca E R G Bica', Mauricio Petroli², Ana Carolina Lopes Santiago ${ }^{2}$

Gabriel Alves Oliveira De Pinho², Juliana Carvalho De Mello², David Hong Kang $^{2}$, Ricardo Mannato Bolelli ${ }^{2}$

'UNIVERSIDADE FEDERAL DO RIO DE JANEIRO, RIO DE JANEIRO, RJ, Brasil; ${ }^{2}$ UFRJ, RIO DE JANEIRO, RJ, Brasil

Advances in Rheumatology 2018, 58(Suppl 1):032

Background: Sjögren's Syndrome (SS) is a chronic autoimmune disease, rare in childhood and adolescence, characterized mainly by xerostomia and xerophthalmia, which compromises exocrine glands causing inflammatory response that leads to glandular hyposecretion. SS is probably underdiagnosed in pediatric range due to differences in presentation regarding adults with this condition, causing low recognition of the disease in children. The authors describe demographic, clinical, laboratory and treatment profiles of a cohort of children with primary SS attended at a university center.

Methods: Retrospective analysis of 30 selected patients' medical records between 2005 and 2017 allowed collection of various data. Laboratory and additional investigations included documentation of ocular dryness (Schirmer test, Rose-Bengal stain); evidence of parotid involvement (scintiscan, sialometry); and histological evidence of lymphocytic infiltration of the minor salivary glands or other organs. Results and Conclusions: 30 patients diagnosed with juvenile SS were selected: 22 girls (73\%) and 8 boys (27\%) with an average age of 11 years. The clinical characteristics were: parotid enlargement as the initial manifestation of the disease in 11 patients (37\%), and recurrent episode in 4 patients $(13,3 \%) .12$ patients $(40 \%)$ had xerostomia and $17(57 \%)$ xerophthalmia. One patient $(3,3 \%)$ presented with leukocytoclastic vasculitis in lower limbs as first manifestation of the disease in association to recurrent parotid swelling. Two $(6,6 \%)$ patients presented neurological symptoms ( 1 peripheric sensory neuropathy and 1 with dysautonomic manifestations). Positive Schirmer test was observed in 11 patients (37\%), Rose Bengal stain in 10 (33\%). 76\% of patients had abnormal salivary glands scintigraphy. In 8 patients (30\%) the salivary gland biopsy revealed compatible with Sjogren syndrome. 9 patients (30\%) presented positive RF, 14 patients $(46 \%)$ anti-Ro/SSA, 10 patients (33\%) anti-La/SSB, 28 patients (93\%) had $+v e$ ANA. $50 \%$ received glucocorticoid. Hydroxychloroquine was the drug most often used in 25 patients (83\%), followed by methotrexate in 12 patients $(40 \%)$, azathioprine in 4 patients $(13 \%)$ and cyclophosphamide in 3 patients (10\%). Only one patient required the use of human immunoglobulin and one leflunomide (3.3\%). Two patients (6.6\%) received rituximab. The present study demonstrated the demographic, clinical aspects, laboratory and treatment in a series of patients with primary juvenile Sjögren's syndrome, a relatively rare condition, presenting an overview of this population in our hospital.

\section{3}

EPIDEMIOLOGY AND MANAGEMENT PRACTICES FOR CHILDHOODONSET SYSTEMIC LUPUS ERYTHEMATOSUS PATIENTS: A SURVEY IN LATIN AMERICA

Clovis Artur Silva', Juliana C. O. A. Ferreira', Vitor C. Trindade ${ }^{1}$ Graciela Espada' ${ }^{2}$ Zoilo Morel ${ }^{3}$, Eloisa Bonfa', Claudia S. Magalhães ${ }^{4}$ ${ }^{1}$ FMUSP, SAO PAULO, SP, Brasil; ${ }^{2}$ HOSPITAL DE NIñOS DR RICARDO GUTIERREZ, BUENOS AIRES, ARGENTINA, SAO PAULO, SP, Argentina; ${ }^{3}$ HOSPITAL DE CLINICAS, UNIVERSIDAD NACIONAL DE ASUNCION, PARAGUAY, ASUNCION, Paraguai; ${ }^{4}$ UNESP, SAO PAULO, SP, Brasil Advances in Rheumatology 2018, 58(Suppl 1):033

Background: Two groups have reported data focused on epidemiology, clinical and laboratorial features of CSLE patients in LA: BRACSLE (Brazilian Childhood-onset SLE Registry Group) and GLADEL 
(Grupo Latino Americano De Estudio del Lupus). However, to the best of our knowledge, epidemiology and management of CSLE based on LA Pediatric Rheumatologists (LAPR) were not carried out. Therefore, the objective to our study was to assess epidemiology and management practices of LAPR about childhood-onset systemic lupus erythematosus (CSLE).

Methods: A cross-sectional study was performed in 288 LAPR PANLAR members based on online survey about CSLE practices.

Results: The response rate of web-based survey by LAPR was 170/ $288(59 \%)$ and the majority worked in University Hospitals (63\%). The most five countries of surveyed specialists was: Brazil $n=85(52.5 \%)$, Argentina $n=22(14 \%)$, Mexico $n=22(14 \%)$, Colombia $n=11(7 \%)$, Chile $n=6$ (3.7\%). The ACR and/or SLICC classification criteria (99\%) and disease activity tools (97\%) were almost universally used by LAPR, whereas damage index (70\%) and CHAQ (58\%) instruments were less frequently used. Laboratory exams, diagnostic imaging and biopsies were generally available ( $>75 \%)$, however low availability for densitometry (66\%). Drug access was excellent for the most common prescribed medications $(>75 \%)$, except for belimumab (11\%). Emerging mosquito-borne diseases were also reported: dengue (20\%), Chikungunya (11\%) and Zika (8\%). Groups were further divided in two, according to the number of CSLE patients followed by LAPR in the last year: group A ( $\geq 25$ patients) and group B ( $<25$ patients). Frequencies of condom in combination with other contraceptive methods were significantly higher in group A than $B(p=0.01)$. The frequencies of reported pregnancy $(p<0.001)$ and non-adherence to therapy were significantly higher in group $A(p=0.023)$. Alcohol intake $(p=0.004)$ and illicit drug use $(p=0.007)$ were also reported more frequently by LAPR of group $A$ in at least one CSLE patient.

Conclusion: This first large web-based survey demonstrated an overall excellent access for diagnosis and therapy by LAPR, probably related to their high rate of practices in tertiary care of University Hospitals. Adherence to therapy, pregnancy and substance abuse were identified as major challenges in this population, particularly in larger centers.

\section{4}

EVALUATION OF CORTICAL MICROARCHITECTURE, BONE STIFFNESS AND BONE REMODELING IN PATIENTS WITH ATYPICAL FEMORAL FRACTURE

Mariana Ortega Perez², Diogo S Domiciano², Luciene M dos Reis', Vanda Jorgetti ${ }^{1}$, Rosa Maria Rodrigues Pereira ${ }^{2}$

'DISCIPLINA DE NEFROLOGIA, HOSPITAL DAS CLÍNICAS DA FACULDADE DE MEDICINA DA UNIVERSIDADE DE SÃO PAULO (FMUSP), SÃO PAULO, SP, Brasil; ' ${ }^{2}$ IISCIPLINA DE REUMATOLOGIA, HOSPITAL DAS CLÍNICAS DA FACULDADE DE MEDICINA DA UNIVERSIDADE DE SÃO PAULO (FMUSP), SÃO PAULO, SP, Brasil

Advances in Rheumatology 2018, 58(Suppl 1):034

Background: Atypical femoral fractures (AFF) are low energy femoral fractures and subtrochanteric/diaphyseal localization that have been related to long-term bisphosphonate therapy. Patients with AFF exhibit cortical thickening in femoral shaft, suggesting that AFF may occur due to cortical stress. Analysis of cortical bone microarchitecture, biomechanical properties of bone, and bone remodeling in AFF are poorly explored in the literature. Simultaneous evaluation of these parameters may be useful in understanding the mechanisms of this type of fracture. Our aim is to evaluate patients with AFF including: 1) cortical bone microarchitecture and bone stiffness by high resolution peripheral quantitative computed tomography (HR-pQCT) and 2) cortical microarchitecture and bone remodeling parameters by bone histomorphometry of iliac crest.

Materials and methods: Eighteen patients with AFF by the American Society of Bone Mineral Research. Cortical bone parameters (cortical volumetric bone mineral density: $\mathrm{Ct.vBMD}, \mathrm{mgHA} / \mathrm{cm} 3$ and cortical thickness: Ct.Th, $\mathrm{mm})$ and bone stiffness $(\mathrm{S}, \mathrm{kN} / \mathrm{mm})$ were studied in tibia and distal radius by HR-pQCT and compared to healthy controls matched for sex and age. Cortical thickness $(\mathrm{Ct.Th}, \mu \mathrm{m})$ and bone remodeling (bone formation rate; surface, volume and thickness osteoid; eroded surface; osteoblastic surface) were assessment (Osteomeasure software ${ }^{\oplus}$ ) and compared with healthy individuals matched for sex and age.
Results: The mean age of the patients was $64.9 \pm 13.3$ years old, $94.4 \%$ women and $72.2 \%$ Caucasian. Seventeen used bisphosphonates $(5.8$ \pm 2.7 years) and $83.3 \%$ alendronate at the time of fracture. One patient was on denosumab, but had received bisphosphonate for 6 years. Presence of rheumatic disease was observed in $50 \%$ of the patients, most of them with rheumatoid arthritis and $44.4 \%$ of the patients used oral glucocorticoid. All fractures were diaphyseal, of which $16(88.8 \%)$ were complete and 4 (22.2\%) bilateral. HR-pQCT $(n=12)$ in tibia showed decreased/normal Ct.vBMD in $83.3 \%$ and decreased/normal Ct.Th in $91.6 \%$ of the patients. Similar findings were observed in distal radius. Seventy-five percent of the patients had decreased bone stiffness in tibia and $58.3 \%$ in radius. Bone histomorphometry $(n=6)$ exhibited $\mathrm{Ct}$.Th decreased in $66.6 \%$ and normal in $33.3 \%$ of the cases. All patients had suppressed bone remodeling. Conclusion: Unlike the femoral diaphysis, most of our patients with AFF presented decrease in the cortical density and cortical thickness in tibia and distal radius. Histomorphometric analysis was concordant with these findings. Our data suggest that impairment of cortical microarchitecture associated with decreased bone stiffness and suppression of bone remodeling would explain bone fragility in these patients.

\section{6}

EVALUATION OF THE EFFECT OF FASCIOLA HEPATICA EXTRACT AS AN ANTI-INFLAMMATORY THERAPY IN EXPERIMENTAL ARTHRITIS

Renata Ternus Pedó ${ }^{\text {, Mirian Farinon }}{ }^{1}$, Thales Hein da Rosa ${ }^{1}$

Martín Pablo Cancela Sehabiague', Henrique Bunselmeyer Ferreira',

Patricia Gnieslaw de Oliveira ${ }^{2}$, Ricardo Machado Xavier'

'UNIVERSIDADE FEDERAL DO RIO GRANDE DO SUL, PORTO ALEGRE, RS,

Brasil; ${ }^{2} U N I V E R S I D A D E$ DA CALIFÓRNIA, SAN DIEGO, SAN DIEGO,

CALIFÓRNIA, Estados Unidos

Advances in Rheumatology 2018, 58(Suppl 1):O36

Background: Rheumatoid arthritis (RA) is an autoimmune disease where the chronic inflammation and subsequent cartilage and bone erosion lead to joint destruction. Fasciola hepatica (F. hepatica) is a parasite that regulates the immune response of its hosts through excretory-secretory products (ESPs) and tegument antigens. ESPs are able to suppress the immune response via T helper 1 (Th1) and both ESPs and tegument antigens of F. hepatica are able to suppress the activation of dendritic cells by toll-like receptors. Based on this, in this study we aimed to evaluate the effect of F. hepatica extract in two animal models of arthritis.

Material and Methods: Male BALB/C mice $(n=21)$ had antigeninduced arthritis (AIA) with methylated bovine serum albumin (mBSA) and were divide into negative control, vehicle (PBS) and F. hepatica extract $(200 \mu \mathrm{g} /$ dose $)$ groups. Intraperitoneal treatment was performed $24 \mathrm{~h}$ and $30 \mathrm{~min}$ before intraarticular (ia) injection of mBSA. Was evaluated paw nociception in $0,3,6$ and $24 \mathrm{~h}$ and leukocytes migration into knee joints $24 \mathrm{~h}$ after ia injection of mBSA. Male DBA/1 J mice $(n=8)$ had collagen-induced arthritis $(C I A)$ with bovine collagen type II and were divided into prophylactic F. hepatica extract treatment $(200 \mu \mathrm{g} /$ dose) and vehicle (PBS) groups. During the treatment period (day 18 from 46) was evaluated clinical articular score, nociception, paw edema and body weight. Statistical analysis was assessed with ANOVA, followed by Turkey. The data are presented as mean \pm SEM

Results: In AIA, treatment with F. hepatica extract reduced nociception in $3(7,46 \pm 0,34 \mathrm{~g}), 6(5.7 \pm 0.27 \mathrm{~g})$ and $24 \mathrm{~h}(6.37 \pm 0.37 \mathrm{~g})$ compared with vehicle $(24 \mathrm{~h}: 3.81 \pm 0.44 \mathrm{~g})(\mathrm{p}<0.001)$ and inhibited leukocytes migration to inflammatory site $(40 \pm 7.76 \times 10 \wedge 4$ leukocytes/cavity) compared with vehicle $(90.90 \pm 12.87 \times 10 \wedge 4$ leukocytes/cavity) $(p<0.01)$. In a preliminary $\mathrm{CIA}$, prophylactic treatment did not improve analyzed parameters. However, treatment group presented a later beginning of clinical signs of arthritis (day 33) compared to vehicle (day 25). Additionally, while vehicle group presented a reduction of $3.4 \%$ of body weight, treatment group gained $2.4 \%$ of body weight at the end of experimental period $(p=0.0509)$.

Conclusions: Treatment with F. hepatica improved acute experimental arthritis attenuating nociception and leukocytes migration to knee 
joint. In a preliminary experiment, prophylactic treatment with $\mathrm{F}$. hepatica presented no effect over analyzed parameters in chronic experimental arthritis, but delayed the clinical manifestation of arthritis and seemed to prevent the body weight loss observed in this model. Further studies will be performed to clarify the effect of F. hepatica extract in chronic experimental arthritis.

\section{7}

EVALUATION OF THE EFFECTIVENESS OF A PROGRESSIVE RESISTANCE TRAINING PROGRAM FOR PATIENTS WITH FIBROMYALGIA: A RANDOMIZED CONTROLLED TRIAL

Jamil Natour, Mariana Vassalli, Raphael Vilela Timoteo da Silva, Anamaria Jones

EPM/UNIFESP, SÃO PAULO, SP, Brasil

Advances in Rheumatology 2018, 58(Suppl 1):037

Background: Fibromyalgia (FM) is a chronic pain syndrome, not inflammatory, characterized by the presence of diffuse pain and painful points. Commonly, it is linked to other symptoms such as fatigue, sleep disorders, morning stiffness; and psychological disorders such as anxiety and depression. The medical treatment of FM brings benefits in the short term. For long-term benefits it is usually associated with non-medicated treatment, such as patient education, physical conditioning, rehabilitation and psychological therapy. In this study, we used the progressive resistance training, which is muscle strengthening performed through the gradual increase of load during the training period.

Objectives: To evaluate the impact of a global progressive resistance training program on pain, quality of life, functional capacity and muscular strength in patients with fibromyalgia

Methods: Sixty patients were randomized into2 groups: experimental group and control group. Patients in the experimental group underwent a progressive resistance training program, performed twice a week for 12 weeks. The charge intensity was progressively increased from $40 \%$ to $80 \%$ of $1 \mathrm{RM}$. The following muscle groups were worked: trunk flexors and extensors, elbow flexors and extensors, knee flexors and extensors, hip abductors and adductors and shoulder abductors. In addition to strength training, the experimental group also received a structured education program in one hour class once a week for five weeks. Patients in the control group received the same education program.

Results: After the intervention, significant improvements were observed in the experimental group in comparison with control group over time for the following parameters: pain $(p=0.004)$, FIQ ( $p=$ 0.021 ), quality of life (with statistically significant improvement for all the SF-36 domains), functional capacity, assessed by the 6-minute walk test $(p=0.045)$, and muscle strength (with statistically significant improvement for all muscle groups trained). The intergroup and intragroup comparisons were showed in Table 1.

Conclusions: The progressive resistance training program was effective in improving pain, quality of life, functional capacity and muscular strength of patients with fibromyalgia.

Table 1 (abstract 037). Intergroup and intragroup comparisons

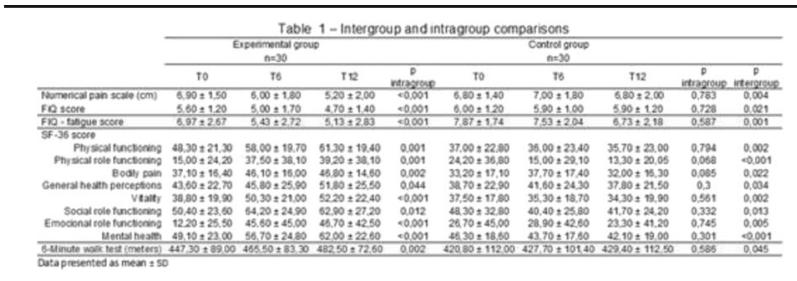

039

EXPOSURE FACTORS ASSOCIATED WITH SYMPTOMATIC OSTEONECROSIS IN PATIENTS WITH SYSTEMIC LUPUS ERYTHEMATOSUS

Yara Mariana Alvim Santos, Ubiratan Brum de Castro, Wilson Tavares Campos, Cristina Costa Duarte Lanna, Gilda Aparecida Ferreira UFMG, BELO HORIZONTE, MG, Brasil

Advances in Rheumatology 2018, 58(Suppl 1):039

Background: The aim of this study was to evaluate the exposure factors associated with symptomatic osteonecrosis $(\mathrm{ON})$ in patients with Systemic Lupus Erythematosus (SLE).

Methods: this is a case-control study, with 139 SLE patients according to ACR/1997 criteria, 33 with symptomatic ON, matched for sex and cumulative dose of glycocorticoid with 106 patients with no known diagnosis of ON. An extensive clinical and laboratory evaluation using a standard protocol established since 2004 was carried out. Results and Conclusions: Of the 139 patients, 93\% were female, the mean (SD) age was $40.8(12.1)$ years and the median (min-max) of disease duration was 102 (6-372) months. The SLICC-DI/ACR score (excluding the ON variable at the end of the study) ranged from 0 to 9 (median of 1). Considering the 33 patients with SLE and ON, at the time of ON diagnosis, the mean (SD) age was $33(11)$ years, the mean (SD) of the disease duration was 75.7 (62.4) months and the median (min-max) of the prednisone daily dose was $10(0-60) \mathrm{mg}$. The median (min-max) of modified SLEDAI-2K prior to the diagnosis of osteonecrosis (mean score of three consecutive appointments per year over a three-year period) was $5(0-11)$. The most affected joints were hips (78.8\%) and knees (45.5\%), bilateral in $48.5 \%$ of cases and in multiple sites in $9 \%$. Patients with SLE and ON were younger $(p<0.001)$, had lower age at SLE diagnosis $(p=0.021)$, higher frequency of livedo reticularis $(p=0.025)$, nephritis $(p=0.009)$ and higher score of modified SLEDAI-2K prior to ON diagnosis $(p<0.001)$ compared to non-ON patients. In the binary logistic regression analysis, disease activity $(\mathrm{OR}=1.4,95 \% \mathrm{Cl}: 1.187-1.728, \mathrm{p}=0.000)$ and livedo reticularis $(\mathrm{OR}=8.3,95 \% \mathrm{Cl}: 1.305-53.215, \mathrm{p}=0.025)$ were independently associated with ON. The modified SLEDAI-2K in the three previous years of ON diagnosis was a good predictor of ON development (area under the ROC curve $=0.791,95 \% \mathrm{Cl}: 0.697-0.885$ ) and the score $>3.5$ was the most appropriate cut off for the prediction of ON development. In this group of lupus patients, higher disease activity score was associated with symptomatic ON and was a good predictor of ON development independently of the glycocorticoid use. In addition, younger age at ON diagnosis and at onset of SLE, the presence of livedo reticularis and of nephritis, and a higher damage score after exclusion of ON variable, were other factors associated with symptomatic ON.

\section{0}

HIGHER CUMULATIVE DOSE OF CYCLOPHOSPHAMIDE AND

VASCULITIS DAMAGE INDEX AS MAIN RISK FACTORS OF INFECTIONS IN SYSTEMIC VASCULITIS PATIENTS DURING HOSPITALIZATION

Felipe Mendonça De Santana, Carlos Emílio Insfran Echauri, Lorenza Rosa Silvério Scomparin, Maíra Luciana Marconcini de Lacerda, Rafael Pontes Andreussi, Adriana Coracini Tonacio de Proença, Lissiane Karine Noronha Guedes, Eduardo Ferreira Borba Neto, Rosa Maria Rodrigues Pereira FACULDADE DE MEDICINA DA UNIVERSIDADE DE SÃO PAULO, SÃO PAULO, SP, Brasil

Advances in Rheumatology 2018, 58(Suppl 1):040

Background: Systemic vasculitis encompasses a heterogeneous group of diseases distinguished by the presence of an inflammatory response within the vessels wall. Its treatment often requires the use of immunosuppressives. Hence, infections are fairly common in 
vasculitis patients. However, discerning acute infection from disease relapse is not straightforward. We searched for clinical differences between vasculitis patients admitted due to severe infections and those admitted due to other conditions.

Methods: A cross-sectional study was performed using standard electronic medical records of a rheumatology tertiary center. Data from consecutive inpatients admitted on rheumatology Clinics from 2015 to 2017 were revised. Those with confirmed systemic vasculitis were included. Twenty-six patients (most of them with Granulomatosis with poliangiitis) were considered eligible for analysis, comprising 37 admissions. These were classified regarding the presence or absence of infection at entry. Data collected during hospitalization was used to ascertain whether the baseline clinical condition was indeed confirmed as infection, including cultures and radiologic findings. Baseline variables were compared between patients admitted due to infection (Infection group) and those admitted for other conditions (Non-infection group). The infection group consisted of 14 admissions and the non-infection group of 23 admissions. Respiratory infections were most common (12 of the total of 14). The Non-infection group comprised mainly of patients admitted due to vasculitis activity. $X 2$ test and Fisher exact test were used for categorical variables and Welch $t$ test, Student $\mathrm{t}$ test and Mann-Whitney test for quantitative variables, where appropriate. The alpha level was set at 0.05 .

Results: Patients admitted due to infection had significantly higher previous cumulative cyclophosphamide (PCCD) dose (mostly intravenous) (7.4 [IQ 7.0-15.8] vs. 0.0 [IQ 0.0-9.4] g, $\mathrm{p}=0.038$ ), a higher baseline Vasculitis Damage Index (VDI) (6.07 [SD \pm 3.73 ] vs. 3.52 [SD \pm 2.20 ], $\mathrm{p}=0.032$ ) and lower Birmingham Vasculitis Activity Score (BVAS) (0.0 [IQ 0.0-2.25] vs. 6.0[IQ 2.0-10.0], $\mathrm{p}=0.006$ ). No significant differences regarding age, sex, cumulative dose of glucocorticoid during the last year, days of admission and comorbidities were identified. No patients used biologic therapy during the admission. No death was observed.

Conclusions: Infection was closely related with previous damage of the disease but not with activity scores at admission. Supporting this observation, cumulative cyclophosphamide dose was also a main factor for infection. Further studies are necessary to confirm the role of these findings.

\section{1}

IMPACT OF DIFFERENT CLASSES OF LUPUS NEPHRITIS IN MATERNAL AND FETAL OUTCOMES OF PATIENTS WITH SYSTEMIC LUPUS ERYTHEMATOSUS

Evandro Mendes Klumb ${ }^{1,2}$, Marcela Ignacchiti Lacerda ${ }^{2}$, Bruna Costa Rodrigues $^{2}$, Camila Castro Silva ${ }^{2}$, Camila Pitasi Argueles ${ }^{2}$, Flavia Cunha dos Santos ${ }^{2}$, Guilherme Ribeiro Ramires de Jesús ${ }^{2}$, Nilson Ramires de Jesús ${ }^{2}$, Roger Abramino Levy ${ }^{2}$

'UNIVERSIDADE DO ESTADO DO RIO DE JANEIRO, RIO DE JANEIRO, RJ,

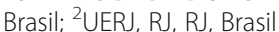

Advances in Rheumatology 2018, 58(Suppl 1):O41

Background: Systemic lupus erythematosus (SLE) is a multisystemic autoimmune disease that has variable clinical course, ranging from mild to severe forms. Lupus nephritis (LN) occurs in $60 \%$ of patients and is the most common indication for immunosuppression and hospitalization, leading to the highest morbidity and mortality among SLE manifestations. History of $L N$ is associated with increased risk of adverse maternal outcomes such as hypertensive disorders, which are even more frequent when nephritis is active at conception. There is also increased fetal morbidity when LN occurs during pregnancy. The aim of the study was to compare SLE activity during pregnancy, maternal complications and gestational outcomes between SLE patients with $L N$ and without $L N$, also analysing the differences between $\mathrm{LN}$ subclasses.

Materials and Methods: This is a cohort study nested in a Cohort of autoimmune pregnancies followed from 2011 to 2016 with singleton gestations and delivery after 22 weeks. Patients were classified according to the presence of nephritis (including subclasses), SLE nonrenal and renal activity, association with antiphospholipid antibodies/ syndrome, permanent damage related to SLE and medication at conception and during pregnancy. The main outcomes were SLE nonrenal and renal activity; SLICC/ACR damage index (SDI); maternal complications; adverse fetal outcomes; general clinical complications.
Results and conclusions: Patients with LN had more frequently systemic flares $(p=0.004)$, used more often prednisone $>20 \mathrm{mg} /$ day $(p=0.02)$ and azathioprine $(p=0.007)$, had more hospitalization related to SLE $(p=0.01)$ and not related to SLE $(p=0.002)$. When only patients with proliferative glomerulonephritis (classes III/IV) were compared to patients without LN and other classes of nephritis (II and V), the findings remained similar. History of nephritis and SLE activity at conception were significant risk factors for hypertensive disorders ( $R R=4.4$, $\mathrm{p}<0.001$; RR=4.1, $\mathrm{p}=0.001$, respectively) and placental insufficiency ( $R R=9.8, p<0.001 ; R R=2.86, p=0.007$, respectively). Patients with $L N$ and permanent damage $(\mathrm{SDI} \geq 1)$ had significantly more disease activity than patients without $L N(p=0.02)$. SLE reactivation during pregnancy, hospitalization and greater need for immunosuppression were more frequent in patients with $L N$, especially when proliferative nephritis was present. Patients with LN had a higher frequency of permanent damage related to SLE and this was also associated with a higher frequency of adverse maternal and fetal outcomes.

Consent for publication

The authors declare that they have obtained informed written consent from the patient's tutors for publication

\section{2}

IMPACT OF STATINS IN PATIENTS WITH CLINICAL AND

LABORATORY STABLE SYSTEMIC AUTOIMMUNE MYOPATHIES

Isabela Bruna Pires Borges, Samuel Katsuyuki Shinjo

FACULDADE DE MEDICINA - USP, SÃO PAULO, SP, Brasil

Advances in Rheumatology 2018, 58(Suppl 1):042

Background. Recent studies have shown a high prevalence of dyslipidemia and others cardiovascular risk factors in patients with systemic autoimmune myopathies (SAM). However, little is known about impact of statins in these patients, what motivated to assess data to perform the analyses of the present study.

Materials and methods. This is a retrospective study, from 2004 to 2018, in which 440 patients with SAM (2017 EULAR/ACR classification criteria) were initially evaluated. Among them, 22 patients were selected: dyslipidemia (total cholesterol $>200, \mathrm{HDL}<40, \mathrm{LDL}>130$ and/or triglyceride $>150 \mathrm{mg} / \mathrm{dL}$ ), clinical and laboratory stable MAS, underwent to statins. Patients with clinically amyopathic dermatomyositis and immune-mediated necrotizing myopathies were excluded.

Results. Data form 22 consecutive patients (14 dermatomyositis, 1 polymyositis and 7 anti-Jo-1 antisynthetase syndrome) were assessed. Mean age of cases was 50.4 years and $68.2 \%$ were female. Median time between diagnosis and disease symptoms onset was 3.5 months; mean duration of disease of 5.0 years; initial serum levels of creatine phosphokinase was $1406 \mathrm{U} / \mathrm{L}$. When statins were introduced, creatine phosphokinase was $116 \mathrm{U} / \mathrm{L}, 50 \%$ patients were not using glucocorticoid, whereas the other half used a median dose of $2.5 \mathrm{mg} /$ day. Moreover, 2 patients were using any immunosuppressive drugs. Ten patients received simvastatin $(10-60 \mathrm{mg} /$ day), 11 atorvastatin $(20-40 \mathrm{mg} / \mathrm{day})$, and 1 atorvastatin $(10 \mathrm{mg} / \mathrm{day})$ which was later replaced by simvastatin $(20 \mathrm{mg} /$ day). Median time of statin exposition was 26 months. During the follow-up, in addition to the improvement of the lipid profile, no disease relapsing or clinical and laboratory intercurrence was observed (Table 1). Furthermore, glucocorticoids were totally suspended $(P=0.008)$.

Conclusions. Regardless of the small sample, our data show efficacy and safety of statin specifically in patients with clinical and laboratory stable SAM and with dyslipidemia. Further studies with larger sample and including patients with different disease activity degree are necessary to corroborate our results.

Support by FAPESP \#2016/20371-1 (IBPB) and \#2017/13109-1 (SKS).

\section{3}

IMPACT OF TREAT-TO-TARGET APPROACH IN THE MUSCLE MAGNETIC RESONANCE IMAGING OF PATIENTS WITH IMMUNEMEDIATED NECROTIZING MYOPATHIES

Jean De Souza, Samuel Katsuyuki Shinjo

HCFMUSP, SÃO PAULO, SP, Brasil

Advances in Rheumatology 2018, 58(Suppl 1):043 
Background. Immune-mediated necrotizing myopathies (IMNM) are rare autoimmune myopathies usually characterized by muscle disability, atrophy and also fat replacement. Moreover, there is currently no standardized or specific treatment for IMNM. In the present study, the authors assessed thigh magnetic resonance imaging (MRI) of IMNM patients who underwent an early-goal induction of remission with methylprednisolone and/or intravenous human immunoglobulin pulse therapies.

Materials and methods. This inception cohort study, from 2013 to 2018, included 7 consecutive patients with IMNM (4 with anti-signal recognition particle autoantibody and 3 with anti-hydroxy-methylglutaryl coenzyme A reductase), according to the Myositis Study Group/119th European Neuromuscular Centre workshop criteria classification. Immediately at diagnosis, patients received methylprednisolone and/or intravenous human immunoglobulin pulse therapies, associated posteriori with immunosuppressant or immunomodulatory drugs (methotrexate, azathioprine, mycophenolate mofetil, isolated or in combination, or also rituximab). After data collection, middle third thigh MRI was assessed to evaluate muscle edema, atrophy and fat replacement, according to a semi-quantitative scale: mild $(<25 \%)$, moderate $(25-50 \%)$ or severe $(>50 \%$ of the cross-sectional area). Disease improvement assessment, evaluated by the International Myositis Assessment and Clinical Studies Group (IMACS) core set measures, was performed before immunosuppression and by the time of MRI acquisition.

Results. Current mean age of patients was 48 years, with female gender predominance $(70 \%)$. Median duration from symptoms onset to diagnosis was 4 months, whereas from disease diagnosis to MRI was 38 months. The IMACS total improvement score (comparing disease onset to MRI assessment) showed a major response improvement. At the time of MRI, all patients were using at least one immunosuppressive or immunomodulatory drug, but only 3 out of 7 patients were still using prednisone $(5-15 \mathrm{mg} /$ day). Regarding thigh MRI abnormality features, 3 out of 7 patients had (mild) muscle edema, one (mild) muscle atrophy and 5 fat replacement ( 4 mild and 1 moderate).

Conclusions. In contrast to scarcely available studies in the literature, our patients had significantly more benign MRI features accompanied with good clinical outcome. This may be a consequence of the treat-to-target induction approach applied in the present study. Although more studies are necessary to corroborate our data, this scenario supports a decisive role of treat-to-target therapy in IMNM.

\section{Consent for publication}

The authors declare that they have obtained informed written consent from the patient's tutors for publication

\section{4}

INCREASED SPERM DNA FRAGMENTATION IN MALE SYSTEMIC LUPUS ERYTHEMATOSUS PATIENTS

Bruno Camargo Tiseo', Gabriela Araújo Munhoz ${ }^{2}$, Eloisa Bonfa²,

Eduardo F. Borba' ${ }^{2}$, Guilherme J. A. Wood ${ }^{3}$, Miguel Srougi ${ }^{3}$,

Clovis A. Silva ${ }^{4}$, Marcello A. S. Cocuzza

${ }^{1}$ DISCIPLINE OF UROLOGY - HOSPITAL DAS CLINICAS DA FACULDADE

DE MEDICINA DA USP, SÃO PAULO, SP, BraSil; ${ }^{2}$ RHEUMATOLOGY DIVISION, HOSPITAL DAS CLINICAS HCFMUSP, FACULDADE DE MEDICINA, UNIVERSIDADE DE SAO PAULO, SP, BRAZIL, SÃO PAULO, SP, Brasil; ${ }^{3}$ DISCIPLINE OF UROLOGY, HOSPITAL DAS CLINICAS HCFMUSP, FACULDADE DE MEDICINA, UNIVERSIDADE DE SAO PAULO, SP, BRAZIL, SÃO PAULO, SP, Brasil; ${ }^{4}$ PEDIATRIC RHEUMATOLOGY UNIT, CHILDREN'S INSTITUTE, HOSPITAL DAS CLINICAS HCFMUSP, FACULDADE DE MEDICINA, UNIVERSIDADE DE SAO PAULO, SP, BRAZIL, SÃO PAULO, SP, Brasil

Advances in Rheumatology 2018, 58(Suppl 1):044

Objective: To evaluate sperm DNA fragmentation analysis in nonazoospermic male SLE (Systemic Lupus Erythematosus) patients. Methods: Twenty-eight consecutive male SLE patients (ACR criteria) and 34 healthy controls were evaluated for demographic/exposures data, urologic evaluation, hormone profile and sperm analysis (including sperm DNA fragmentation). Clinical features, disease activity/ damage scores and treatment were also evaluated.
Results: The median age [33 (20-52) vs. 36.5(25-54) years, $\mathrm{p}=0.329]$ and frequency of varicocele $(25 \%$ vs. $32 \%, p=0.183)$ were similar in SLE patients and healthy controls. Sperm DNA fragmentation showed significantly higher levels of cells class III [44 (9-88) vs. $16.5(0-80) \%, p=0.001]$ and cell class IV [10.5(3-86) vs. $7(0-36) \%, p=0.039$ ] in SLE. Sperm DNA fragmentation Index was also significantly higher in SLE patients [62 (31-97) vs. 25.5(0$100) \%, p<0.001]$. Conventional sperm parameters (including sperm count, motility and morphology) were similar in both groups. In SLE patients no correlations were observed between sperm DNA fragmentation index and age, disease duration, SLEDAI-2K and SLICC/ACR-DI scores, and cumulative dose of prednisone, hydroxychloroquine, intravenous cyclophosphamide (IVCYC), methotrexate, azathioprine and mycophenolate mofetil $(p>0.05)$. Further analysis of SLE patients treated with and without IVCYC showed that total sperm motility was significantly lower in the former group $[64 \%(15-83)$ vs. $72 \%(57-86), \quad p=0.024]$. Sperm DNA fragmentation index was alike in both groups [52.5(31-95) vs. 67.5(34-97)\%, $\mathrm{p}=0.185]$.

Conclusions: To our knowledge, this is the first demonstration that male non-azoospermic SLE patients have increased sperm DNA fragmentation without evident gonadal dysfunction. IVCYC does not seem to be a major determinant for this abnormality. Future prospective study is necessary to determine the impact of this alteration in these patients' fertility.

\section{5}

INFLUENCE OF PHYSIOTHERAPEUTIC INTERVENTION ON DISEASE ACTIVITY, FUNCTIONALITY AND PAIN IN SUBJECTS WITH SPONDYLOARTHRITIS: A SYSTEMATIC REVIEW OF LITERATURE

Ana Gabriela De Lima, Mariana Bogoni Budib, Silvio Assis de Oliveira Junior, Izaias Pereira da Costa, Paula Felippe Martinez

UNIVERSIDADE FEDERAL DE MATO GROSSO DO SUL, CAMPO GRANDE,

MS, Brasil

Advances in Rheumatology 2018, 58(Suppl 1):045

Background: Spondyloarthritis are distinct diseases with common characteristics, including clinical aspects (axial pain of inflammatory, association of arthritis of large joints and peripheral enthesopathies), radiological and laboratory findings. This set of diseases include ankylosing spondylitis, psoriatic arthritis, reactive arthritis, enteropathic arthropathy and undifferentiated spondyloarthritis. Conservative treatment has shown to be fundamental for the improvement of disease activity, pain and functionality. In this context, it is necessary to systematize evidence in the search for scientific support on physiotherapeutic methods suitable for the rehabilitation of subjects with spondyloarthritis. The aim of this study is to verify the influence of physical therapy on disease activity, functionality and pain in subjects with spondyloarthritis.

Materials and methods: Systematic search was conducted using PubMed, PEDro and SciELO electronic databases based on the research strategies recommended by the Preferential Reports for Systematic Analysis and Meta-Analysis (PRISMA) items using combinations of Medical Subject Heading descriptors Terms (MeSH): Exercise; Rehabilitation; Exercise Therapy; Physical Therapy Modalities; Spondylarthropathies. As criteria of inclusion, randomized and nonrandomized clinical trials published between 2013 and 2018 were considered, which integrated protocols with physical exercises and electrotherapy for the treatment of individuals with spondyloarthritis. First, titles and abstracts of all the articles identified by the research strategy were evaluated in isolation and in duplicate by two reviewers (A.G.L and M.B.B). In the second step, the same reviewers evaluated the full articles, independently and together. Thus, 14 studies were selected.

Results and conclusions: Stretching exercises combined with aerobic exercise reflect on softening pain, improving functionality and activity of the disease. Resistance and aerobic exercises associated with stretching presented positive results for functionality and disease activity. To soften pain and to improve functionality, the use of electrotherapy associated with resistance exercises was more effective than electrotherapy applied in isolation. Alternative methods of exercise, 
such as exergames and the McKenzie method, have softened the pain, improved functionality and activity of the disease when compared to other therapeutic modalities. In addition, this research revealed the low effectiveness of educational interventions not associated with kinesiotherapy. Similarly, the hydrotherapy presented results similar to the exercises performed in soil for the functionality, pain and activity of the disease. Physical therapy has a significant improvement in pain, function and disease activity. However, there is a gap regarding the type of exercise, volume and intensity appropriate to each stage of the rehabilitation process of subjects with spondyloarthritis.

\section{7}

IS THERE STILL A ROLE FOR NSAIDS IN THE TREATMENT OF RHEUMATOID ARTHRITIS? DATE FROM A LARGE COHORT IN BRAZIL Ana Paula Monteiro Gomides ${ }^{1}$, Licia Maria Henrique da Mota ${ }^{2}$, Geraldo da Rocha Castelar Pinheiro ${ }^{4}$, Cleandro Pires de Albuquerque ${ }^{3}$, Ana Beatriz Vargas-Santos ${ }^{4}$, Manoel Barros Bertolo ${ }^{5}$, Alisson Aliel Vigano Pugliesi ${ }^{5}$, Letícia Rocha Pereira ${ }^{4}$, Eduardo de Almeida Macedo ${ }^{5}$, Paulo Louzada Filho ${ }^{6}$ Maria de Fátima $L$ da Cunha Sauma ${ }^{7}$, Marcel Lobato Sauma, Júlia Brito de Medeiros ${ }^{8}$, Claiton Viegas Brenol ${ }^{9}$, Ivanio Alves Pereira ${ }^{10}$, Sebastiao

Radominski ${ }^{11}$, Maria Fernanda Resende ${ }^{12}$, Maria Raquel Costa Pinto ${ }^{12}$, Gustavo Gomes Resende ${ }^{12}$, Karina Bonfiglioli ${ }^{13}$, Henrique Carriço ${ }^{13}$, Rina Dalva Neubarth Giorgi ${ }^{14}$, Nathalia de Carvalho Sacilotto ${ }^{15}$

${ }^{1}$ UNB/UNICEUB, BRASÍLIA, DF, Brasil; ${ }^{2}$ BRASÍLIA, DF, Brasil; ${ }^{3} U N B$, BRASÍLIA, DF, Brasil; ${ }^{4}$ UERJ, RIO DE JANEIRO, RJ, Brasil; ${ }^{5}$ UNICAMP, CAMPINAS, SP, Brasil; ${ }^{6} U S P$ RIBEIRÃO PRETO, RIBEIRÃO PRETO, SP, Brasil; ${ }^{7}$ UF DO PARÁ, BELÉM, PA, Brasil; ${ }^{8}$ UF PARÁ, BELÉM, PA, Brasil; ${ }^{9}$ UFRGS, PORTO ALEGRE, RS, Brasil; ${ }^{10}$ UFSC, FLORIANÓPOLIS, SC, Brasil; ${ }^{11}$ UF PARANÁ, CURITIBA, PARANÁ, Brasil; ${ }^{12}$ UFMG, BELO HORIZONTE, MG, Brasil; ${ }^{13}$ USP, SÃO PAULO, SP, Brasil; ${ }^{14} \mathrm{HSPE}-\mathrm{SP}$-, SÃO PAULO, SP, Brasil; ${ }^{15} \mathrm{HSPE}-\mathrm{SP}$, SÃO PAULO, SP, Brasil

Advances in Rheumatology 2018, 58(Suppl 1):047

Introduction: Non-hormonal anti-inflammatory drugs (NSAIDs) have always been used in the treatment of rheumatoid arthritis, but with the emergence of new therapeutic classes, which allow better control of the disease, has been reducing the need for long-term use. In addition, the potential risk in cardiovascular diseases and adverse effects on the gastrointestinal tract, kidneys and possible drug interactions, make the use of this therapeutic class better indicated only in phases of exacerbation and for the shortest possible time. The objective of this study is to evaluate the current use of NSAIDs in RA patients in a large real life cohort in Brazil.

Materials And Methods: A multicenter study was conducted with RA patients from 11 centers in different states of Brazil. Participants should have a written diagnosis of RA and regular follow-up in the referral services of the public network. Clinical and complementary evaluation and registry analysis were performed. The present study is a cross section referring to the initial evaluation of the patients of this project.

Results and Conclusions: A total of 1117 patients with an average disease time of 12.6 years were analyzed. $78.73 \%$ had a positive rheumatoid factor. The median DAS was 3.52 and the CDAI of 9,1022 (90.84\%) used conventional synthetic MMCDs (isolated or in combination) and $406(36.09 \%)$ used biological MMCDs.

122 patients (10.9\%) were currently using NSAIDs at the time of evaluation. The most commonly used NSAIDs were naproxen $(54 \%$ of all patients). Table 1 shows all types of drugs as well as doses in use. When questioned about previous use of NSAIDs during RA treatment, 743 patients (66.58) reported having used this class of therapy.
Considering the above, we found a low rate of current use of NSAIDs in this Brazilian population, data that should be confirmed in other studies.

\section{9 \\ MALIGNANCY IN CHILDHOOD ONSET -SYSTEMIC LUPUS}

\section{ERYTHEMATOSUS: REAL-LIFE DATA FROM A NATIONWIDE SERIES}

Barbara Geane Alves Fonseca ${ }^{1,2}$, Manuela Pacifico Segredo (FMB-Unesp) ${ }^{2}$,

Glaucia Novak ${ }^{3}$, Beatriz Molinari ${ }^{3}$, Ana Paula Sakamoto ${ }^{4}$, Virginia Paes Leme Ferriani ${ }^{5}$, Ana Maria Soares Rolim ${ }^{6}$, Adriana Fonseca7, Adriana Maluf Elias Sallum ${ }^{3}$, Rosa Maria Rodrigues Pereira ${ }^{8}$, Maria TeresaTerreri ${ }^{4}$, Ana Raquel Feitosa ${ }^{9}$, Eunice Mitiko Okuda ${ }^{10}$, Eloisa Bonfá ${ }^{8}$, Clovis A. A. $^{2}$ Silva $^{8}$, Claudia Saad-Magalhaes ${ }^{2}$

${ }^{1}$ UNESP, BOTUCATU, SP, Brasil; ${ }^{2}$ FMB-UNESP, BOTUCATU, SP, Brasil; ${ }^{3}$ ICRFMUSP, SÃO PAULO, SP, Brasil; ${ }^{4} E P M-U N I F E S P, S \tilde{O}$ PAULO, SP, Brasil; ${ }^{5}$ FMRP- USP, RIBEIRÃO PRETO, SP, Brasil; ${ }^{6}$ OBRAS SOCIAIS IRMÃ DULCE, SALVADOR, BA, Brasil; ${ }^{7} I P P M G-R J$, RIO DE JANEIRO, RJ, Brasil; ${ }^{8}$ FMUSP, SÃO PAULO, SP, Brasil; ${ }^{~} \mathrm{HOSPITAL}$ GERAL DE FORTALEZA, FORTALEZA, CE, Brasil; ${ }^{10} \mathrm{FM}$ - SANTA CASA SP, SÃO PAULO, SP, Brasil

Advances in Rheumatology 2018, 58(Suppl 1):049

Background: Malignancy surveillance in childhood-onset SLE (cSLE) is needed and background risk is important for safe treatment planning. A descriptive report of malignancy outcome, in a nationwide series of c-SLE, is presented.

Methods: A retrospective data collection was conducted, looking for rare C-SLE manifestations, in 27 Brazilian pediatric rheumatology centers. Cases fulfilling 1997-ACR criteria, onset before 18 years, who had a comprehensive 3-time-point-clinical assessments, at c-SLE diagnosis, follow up, and last visit and additionallythe results of abiopsy-proven malignancy manifestation, were selected, at any time point of follow up. This was a convenience sample, where the end of follow up was defined as the date of cancer diagnosis, allowing inclusion of young adults.

Results: There was a total of 1,555 valid c-SLE cases reported; of those, 6had cancer diagnosis, seen in the same service during cSLE follow up. Their mean age was 15.6 y(12-25 years), mean follow up duration 4.6 years ( 0.5 to 14years). Malignancy diagnoses and clinical presentation were hematologic $(n=3)$ and non hematologic $(n=3)$. Of the hematologic, $(n=1)$ was diagnosed withHodgkin lymphoma, presenting with fever, weight loss and enlarged lymphonodes, 5 month after c-SLE onset; $(n=1)$ had nonHodgkin lymphoma presenting with anemia, fever and arthritis, all concomitant to c-SLE onset; $(\mathrm{n}=1)$ had an orbital mass withproptosis, diagnosed as a MALT lacrimal glands lymphoma, after 2 years of c-SLE onset. Of the other types of malignancy $(n=3),(n=1)$ hadHPV-related anal-carcinoma after genital condyloma, it was diagnosed after 14 years from c-SLE onset; $(n=1)$ had a testicle mass with germinal cells carcinoma, 4-years after c-SLE onset and $(n=1)$ had a brain tumor, oligodendroglyoma type, presenting with headaches and seizures, 1.3 yearafter c-SLE onset All had active disease status, their mean SLEDAI scores were 14 (range 2-32). The SLICC-SDI scored at least 1 point for malignancy. We did not observe multiple malignancies. CONCLUSIONS: Thefrequency estimates of malignancy in our c-SLE population was $0.4 \%$. Despite being a very rare outcome, it is is a challenge, demanding awarenessbecause signs and symptoms of hematologic malignancy may also mimic c-SLE manifestations. Also, this outcome may be related to disease factors or due to the treatment with glucocorticoids and cytotoxic drugs. 


\section{0}

MICROSTRUCTURAL CHANGES IN CORPUS CALLOSUM IN SYSTEMIC LUPUS ERYTHEMATOSUS PATIENTS: DIFFUSION TENSOR STUDY

Beatriz Lavras Costallat ${ }^{12}$, Aline Tamires Lapa ${ }^{2}$, Lilian Tereza Lavras Costallat ${ }^{2}$, Fernando Cendes ${ }^{2}$, Willian Javier Garcia Herrera ${ }^{3}$, Letícia Rittner ${ }^{3}$, Simone Appenzeller ${ }^{2}$

${ }^{1}$ UNICAMP, CAMPINAS, SP, Brasil; ${ }^{2}$ FACULDADE DE CIÊNCIAS MÉDICAS

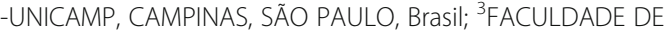

ENGENHARIA ELÉTRICA E DA COMPUTAÇÃO- UNICAMP, CAMPINAS, SÃO PAULO, Brasil

Advances in Rheumatology 2018, 58(Suppl 1):050

Background: The aim of this study was to determine the microstructural changes of the corpus callosum in patients with systemic lupus erythematosus by the diffusion tensor technique (DTI).

Material and method: The study evaluated 116 patients with SLE (110 women, mean age 40 years) and 48 healthy controls (40 women, mean age 35.5 years). All patients fulfilled 4 or more American College of Rheumatology (ACR) SLE criteria and neuropsychiatric manifestations were analyzed based on the ACR diagnostic criteria for neuropsychiatric impairment, classified as active, inactive, or absent on the date of inclusion of the study. SLEDAI and SLICC were evaluated. All patients and controls underwent cranial MRI examination using diffusion tensor technique (DTI) and fractional anisotropy $(F A)$, mean diffusivity $(M D)$, axial diffusivity $(A D)$ and radial $(R A)$ values were calculated in the corpus callosum (CC).

Results: Patients with SLE presented lower values of AF $(0.629$ \pm 0.0612 vs $0.67781 \pm 0.0154, p<0.001)$ and significantly higher values of MD $(0.0023 \pm 0.0036$ vs $0.0010 \pm 0.0001, p=0.020)$, and $A D$ $(0.0018 \pm 0.0001$ vs. $0.0017 \pm 0.0002, p=0.040)$ and $R D(0.0008 \pm 0.0006$ vs. $0.0005 \pm 0.0001, p=0.024)$ when compared to controls. Patients with active NPSLE presented lower values of AF and greater MD when compared to inactive NPSLE [FA $(0.590 \pm 0.072$ vs $0.661 \pm 0.021)$ and $\mathrm{MD}(0.0044 \pm 0.005$ vs $0.0010 \pm 0.0001)] ;(0.590 \pm 0.072$ vs 0.668 $\pm 0.015)$ and MD $(0.0044 \pm 0.005$ vs $0.001 \pm 0.001)$ and compared to controls FA $(0.590 \pm 0.072$ vs. $0.668 \pm 0.015)$ and $\mathrm{MD}(0.0044 \pm 0.005$ vs $0.001 \pm 0.0001)$. NPSLE patients had higher AD values when compared to non-NPSLE [FA $(0.0018 \pm 0.001$ vs $0.0017 \pm 0.00009)$ ] and also when compared to controls [FA $(0.0018 \pm 0.001$ vs. $0.0017 \pm$ 0.0002)]. The inactive NPSLE patients had higher RD values than controls [FA $(0.0006 \pm 0.001$ vs $0.0011 \pm 0.0006)$ ]. The SLEDAI presented a negative correlation with FA $(r=-0.346, p<0.001)$ and positive with $M D(r=0.239, p=0.010), A D(r=0.223, p=0.016)$ and RD 0.018). The SLICC presented a negative correlation with FA $(r=-0.314, p=0.001)$ and positive with MD $(r=0.275, p=0.002)$ and $A D(r=0.193, p=0.03)$.

Conclusion: Our study indicates that there is microstructural impairment in patients with active NPSLE and patients with systemic disease activity. The DTI MRI study may provide elements for a more comprehensive analysis of the microstructure of the white matter in patients with NPSLE.

\section{1}

NON-CRITERIA MANIFESTATIONS, ANTIPHOSPHOLIPID PROFILE AND THEIR ASSOCIATION IN PRIMARY ANTIPHOSPHOLIPID SYNDROME (PAPS)

Gustavo Guimarães Moreira Balbi ${ }^{1,3}$, Flávio Signorelli², Roger Abramino Levy ${ }^{2}$ ${ }^{1}$ HOSPITAL UNIVERSITÁRIO DA UNIVERSIDADE FEDERAL DE JUIZ DE FORA, JUIZ DE FORA, MG, Brasil; ${ }^{2}$ UNIVERSIDADE DO ESTADO DO RIO DE JANEIRO, RIO DE JANEIRO, RJ, Brasil; ${ }^{3}$ UNIVERSIDADE FEDERAL DE JUIZ DE FORA, JUIZ DE FORA, MG, Brasil

Advances in Rheumatology 2018, 58(Suppl 1):051

Background: Antiphospholipid syndrome (APS) is associated with different clinical and laboratorial manifestations that may impact treatment, but were not included in the current classification criteria (Sydney). The aim of this study is to evaluate the frequency of noncriteria manifestations in our population and their correlation with demographic and antiphospholipid antibodies (aPL) profile.
Methods: We performed a cross-sectional study with 120 outpatients who fulfilled pAPS classification criteria (Sydney). Clinical and serologic features were obtained during visits and by chart review. Statistical analysis was performed using chi-square, Mann-Whitney $\mathrm{U}$ and Spearman's R tests, when applicable.

Results: All patients were included in the analysis (114 thrombotic with or without pregnancy morbidity and 6 obstetric with no thrombotic manifestations). One hundred and three (85.8\%) were female and $72(60 \%)$ Caucasian. The mean age at the time of analysis was $42.6 \otimes 12.9$. We found the following frequency of non-criteria manifestations: migraine $(47.5 \%)$, livedo (24.2\%), Raynaud's phenomenon $(24.2 \%)$, thrombocytopenia (10\%), valvopathy $(7.5 \%)$, cutaneous ulcers $(7.5 \%)$, seizures $(5.8 \%)$, hemolytic anemia $(0.8 \%)$, nephropathy $(0.8 \%)$, and white matter lesions $(0.8 \%)$. Regarding the aPL profile, $94 \%$ were positive to lupus anticoagulant, $35.8 \%$ to anticardiolipin $(\mathrm{aCL})(21 \%$ with high titers $>80 \mathrm{MPL}$ or $\mathrm{GPL}), 5 \%$ to $\mathrm{aCL} \operatorname{lgA}(\mathrm{N}=95)$, $44 \%$ to anti-ß2-glycoprotein I, and $21 \%$ were triple positive. In a bivariate analysis, livedo correlated with Caucasian race $(p=0.006)$ and thrombocytopenia with persistently positive $\mathrm{aCL}(\mathrm{p}=0.012), \mathrm{aCL}$ titers $>80(0.027)$, and triple positivity $(p=0.009)$. Raynaud's phenomenon and migraine were more frequent in women $(p=0.013$ and $p=0.001$, respectively). There was a tendency of increased probability of hemolytic anemia in patients with persistently positive $\mathrm{aCL}(p=0.057)$ and nephropathy in men $(p=0.013)$, but the number of events was very low in both groups.

Conclusion: Migraine was the most common observed non-criteria manifestation, followed by livedo and Raynaud's phenomenon. Caucasians were more prone to present livedo, as women were more prone to complain of Raynaud's phenomenon and migraine. Thrombocytopenia was associated with persistently positive $\mathrm{aCL}$, high titers of $\mathrm{aCL}$, and triple positivity.

\section{2}

\section{NORMAL TENSION GLAUCOMATOUS DAMAGE IN PATIENTS WITH} SYSTEMIC SCLEROSIS

Cecilia Victoria Agapito Tito, Juliana Silvatti, Izabela Negrão Frota de Almeida, Elise Vivan Taniguchi, Augusto Paranhos Junior, Tiago dos Santos Prata, Cristiane Kayser

UNIVERSIDADE FEDERAL DE SÃO PAULO, SÃO PAULO, SP, Brasil Advances in Rheumatology 2018, 58(Suppl 1):052

Background: Systemic sclerosis (SSc) is characterized by peripheral vasospasm and structural abnormalities of the microcirculation and small vessels. A number of evidences suggested that vascular abnormalities are involved in the pathogenesis of normal tension glaucoma (NTG), as ocular vasospasm may induce optic nerve head damage. In this study, we investigated the presence of normal tension glaucomatous damage in SSc.

Methods: In this single-center, cross-sectional study, 39 patients (3 male, 36 female) with SSC (2013 ACR/EULAR classification criteria) and 18 age- and sex-matched controls were included. All participants underwent complete ophthalmological examination, including visual acuity, intraocular pressure (IOP) measurement with a Goldmann applanation tonometer, slit-lamp biomicroscopy, and gonioscopy. Retinal nerve fiber and optic disc morphology were evaluated by means of spectral-domain optic coherence tomography (SD-OCT) in which peripapillary retinal nerve fiber layer (pRNFL) thickness and vertical cup-disc ratio (VCDR) were obtained. Visual field examination was performed in all participants. NTG was defined as follows: IOP $\leq 21 \mathrm{~mm} \mathrm{Hg}$, evidence of glaucomatous optic neuropathy (GON) (defined as VCDR $\geq 0.6$, asymmetry of the cup-to-disc ratio $\geq 0.2$ between eyes, presence of localized retinal nerve fiber layer and/or neuroretinal rim defects, and disc haemorrhages), and visual field defects.

Results: A total of 36 eyes of normal subjects, and 72 eyes of SSC patients were evaluated. The mean IOP in SSC patients was of 14.52 $\pm 2.63 \mathrm{mmHg}$ and of $15.12 \pm 2.46 \mathrm{mmHg}$ in controls $(p=0.271)$. The average pRNFL was significantly thinner in SSc patients compared to controls $(106.54 \pm 11.34 \mu \mathrm{m}$ versus $113.11 \pm 17.71 \mu \mathrm{m}$, respectively $\mathrm{p}$ $<0.05$ ). An excavated disc with a cup-to-disc ratio $\geq 0.6$ was observed in 31 (43\%) eyes of SSc patients and in 17 (47\%) eyes of the controls $(p=0.774)$. The average VCDR assessed by SD-OCT was of $0.53 \pm 0.19$ 
in SSc patients and of $0.62 \pm 0.16$ in the control group $(p<0.05)$. Visual field defects were observed in $42 \%$ of SSc patients and in $65 \%$ controls $(p=0.077)$.

Conclusion: A significant thinning of the pRNFL and decreased VCDR were observed in SSc patients compared with healthy subjects, suggesting that subclinical GON abnormalities are present in SSc. Since reduced pRNFL thickness has been demonstrated in patients with glaucomatous optic neuropathy and might precede visual field loss and optic nerve head defects, prospective studies to detect potential risks for developing glaucomatous damage in patients with SSc are warrant.

\section{3}

\section{OCCURRENCE OF AUTOIMMUNE DISEASES IN RELATIVES OF PATIENTS WITH SYSTEMIC VASCULITIS AT AN UNIVERSITY HOSPITAL: A CASE-CONTROL STUDY}

Alessandra De Sousa Braz ${ }^{1,2}$, João Calvino Soares de Oliveira², Matheus Vieira Falcão², Alexia Lavínia Holanda Gama², Jade Arruda de Carvalho Motta ${ }^{2}$, Maria Roberta Melo P. Soares ${ }^{2}$, Karla Valéria M. De Campos ${ }^{2}$ Eutilia Andrade M. Freire ${ }^{2}$

${ }^{1}$ UNIVERSIDADE FEDERAL DA PARAÍBA, JOÃO PESSOA, PB, Brasil; ${ }^{2}$ UFPB, JOÃO PESSOA, PB, Brasil

Advances in Rheumatology 2018, 58(Suppl 1):053

Background: Vasculitis is a general term for a group of rare diseases that have in common inflammation of blood vessels. There are many types of vasculitis, and the different diseases may vary significantly in terms of symptoms, severity, and duration. There are very few studies that aim to analyze the occurrence of autoimmune diseases (AID) among relatives of patientes with diagnosis of vasculitis, despite the support of the scientific community affirming that genetics is one of the great responsibles for the origin of autoimune disturbs. The purpose of the research is to verify the occurrence of AID in first-degree relatives of patients diagnosed with vasculitis at an University Hospital. Materials and Methods: It was performed a case-control study, observational and tranversal, in the rheumatology sector at an University Hospital. The participants were selected by a non-probabilistic way, through active recruit of patients. The control-group were selected at the hematology, endocrinology and pneumology sectors at the same hospital. The inclusion criteria for the case-group were been diagnosed with Systemic Vasculitis (according to the Chapel Hill Consensus Conference) and not having any cognitive deficit, while for the control-group was not been diagnosed with Vasculitis and not having cognitive deficit. At the descriptive statistics, the relative and the absolut frequencies regarding the qualitative variables were determined. Concerning the inferencial statistics, the Mann-Whitney test were applied regarding the ordinal variables. The significance level to all the inferential procedures was $5 \%$. The data was analyzed using the statistician program SPSS version 20.0. Results and

Conclusions: There were 169 participants of the research, 19 from the case-group and 150 from the control-group. Among the patients with Vasculitis, $63 \%$ were female and $47,3 \%$ had first-degree relatives with AID, presenting Rheumatoid Arthritis - RA (44,4\%), Systemic Eritematous Lupus - LES (22,2\%), Kawasaki $(22,2 \%)$ and Hashimoto Thyroiditis (11\%). Among the control-group, $58,6 \%$ were female and $4,6 \%$ had first-degree relatives with AID, presenting RA $(71,4 \%)$ and LES $(28,6 \%)$. The occurrence of AID in the case-group has shown statistical significance, when compared to the control-group. It can be noticed that in the case-group occurred a greater frequency of AID, such as RA and LES. The result found shows the need of more research in the field of familiar genetics of vasculitis, since it's still a subject not very present in scientific studies.

\section{4}

ONE YEAR 24H-PROTEINURIA: SIMILAR RESULTS WITH BOTH CYCLOPHOSPHAMIDE AND MYCOPHENOLATE MOFETIL FOR LUPUS NEPHRITIS INDUCTION IN REAL LIFE SITUATION

Maíra Luciana Marconcini De Lacerda, Gabriela Araujo Munhoz, Luciana Parente Costa Seguro, Eduardo Ferreira Borba Neto, Eloisa Silva Dutra de Oliveira Bonfa HC FMUSP, SÃO PAULO, SP, Brasil

Advances in Rheumatology 2018, 58(Suppl 1):054
Background/Purpose: One year 24h-proteinuria is the best single predictor for long term renal prognosis in lupus nephritis, as demonstrted in two trials and also in real life situation. Our aim was to compare one-year $24 \mathrm{~h}$-proteinuria in patients with lupus nephritis treated with with low-dose intravenous cyclophosphamide (Eurolupus) or Mycophenolate mofetil (MMF)-based regime as induction therapy in real life situation.

Methods: Retrospective analysis using an electronic chart database of consecutive lupus patients (SLICC 2012) with active lupus nephritis treated as induction therapy with either Euro-lupus $(500 \mathrm{mg}$, every 15 days for 3 months, followed by MMF or azathioprine) or MMF (3g/ day). All patients were followed at the Lupus Outpatient Clinic at the Tertiary University Hospital. Clinical outcomes and laboratory results were evaluated at baseline and after 12 months. Exclusion criteria included pregnancy and creatinine clearance $<10 \mathrm{~mL} / \mathrm{min}$.

Results: 36 patients received Euro-lupus and 54 patients received MMF. Euro-lupus and MMF groups had comparable age (34.69 \pm 10.73 vs. $38.18 \pm 11.11$ years, $p=0.143$ ), female gender ( 86.1 vs. $85.2 \%, p=1.0)$, white race (77.8 vs. $66.7 \%, p=0.343$ ) and disease duration ( $6.06 \pm 5.95$ vs. $6.30 \pm 6.33$ years, $p=0.859)$. Baseline laboratory parameters, SLEDAI and glucocorticoid therapy data are shown in Table 1. The frequency of previous nephritis $(p=0.514)$, systolic $(p=0.891)$ and diastolic $(p=0.668)$ blood pressure levels were comparable in the two groups. After 12 months, 24h-proteinuria was similar in both groups ( $0.71 \pm 0.68$ vs. $0.79 \pm 0.78 \mathrm{~g} / \mathrm{day}, \mathrm{p}=0.525)$, as also the frequency of patients with proteinuria $<0.8 \mathrm{~g} / 24 \mathrm{~h}(66.6 \%$ vs. $61.1 \%, p$ 0.659), creatinine levels ( 0.81 vs. $0.87 \mathrm{mg} / \mathrm{dL}, \mathrm{p}=0.537)$ and prednisone daily dose $(13.3 \pm 11.3$ vs. $10.7 \pm 7.9 \mathrm{mg} /$ day, $p=0.195)$.

Conclusion: Euro-lupus and MMF protocols were effective as induction therapy for active lupus nephritis with a comparable frequency of patients achieving the proteinuria target at 12 months, which is the best single predictor for long term renal prognosis.

Table 1 (abstract 054). Baseline parameters of lupus nephritis patients

\begin{tabular}{|c|c|c|c|}
\hline $\begin{array}{l}\text { Table 1. Baseline parameters of lupus } \\
\text { nephritis patients. }\end{array}$ & $\begin{array}{l}\text { Eura-lupus } \\
\text { (n-36) }\end{array}$ & $\begin{array}{l}\text { MMF } \\
(n=54)\end{array}$ & $p$ \\
\hline $\mathrm{scr}[\mathrm{mg} / \mathrm{dll}]$ & $0.97 \pm 0.53$ & $0.85 \pm 0.34$ & 0.234 \\
\hline Alb $[\mathrm{mg} / \mathrm{dll}]$ & $3.15 \pm 0.53$ & $3.31 \pm 0.60$ & 0.278 \\
\hline Positive anti-dsDNA & $29(80.6 \%)$ & $37(68.5 \%)$ & 0.233 \\
\hline C3 [me/dl] & $61.69 \pm 29.35$ & $71.12 \pm 29.38$ & 0.139 \\
\hline $\mathrm{C} 4 \mathrm{Img} / \mathrm{dll}$ & $9.25 \pm 7.81$ & $11.35 \pm 8.29$ & 0.232 \\
\hline PTU24h[R/YOOI] & $2.71 \pm 2.12$ & $2.22 \pm 1.38$ & 0.234 \\
\hline SLEDAI & $13.63 \pm 4.56$ & $12.18 \pm 6.04$ & 0.223 \\
\hline Prednisone dose $[\mathrm{mg} / \mathrm{dax}]$ & $42.77 \pm 16.01$ & $37.13 \pm 15.94$ & 0.104 \\
\hline IV methwlprednisolone & $31(86.1 \%)$ & $37(68.5 \%)$ & 0.008 \\
\hline
\end{tabular}

\section{5}

OVERWEIGHT AND OBESITY VERSUS CHRONIC MUSCULOSKELETAL SYMPTOMS: IS THERE A CONNECTION?

Rosa Weiss Telles ${ }^{1,3}$, Aline Barbara Pereira Costa ${ }^{3}$, Luciana Andrade Machado $^{2}$, Sandhi Maria Barreto 3

${ }^{1}$ FACULDADE DE MEDICINA DA UFMG, BELO HORIZONTE, MG, Brasil; ELSA-BRASIL MUSCULOESQUELÉTICO, BELO HORIZONTE, MG, Brasil; ${ }^{3}$ FACULDADE DE MEDICINA - UFMG, BELO HORIZONTE, MG, Brasil Advances in Rheumatology 2018, 58(Suppl 1):055

Background: The aim of the study was to investigate the independent association between markers of overweight/obesity and chronic musculoskeletal symptoms in participants of ELSA-Brasil Musculoskeletal Study.

Material and Methods: Overweight (BMI 25-29.9kg/m2) and obesity $(\mathrm{BMl} \geq 30 \mathrm{~kg} / \mathrm{m} 2)$ were ascertained using data from current height and weight, and weight at age 20 (for overweight/obesity at age 20). Three mutually exclusive overweight $(B M I \geq 25 \mathrm{~kg} / \mathrm{m} 2)$ trajectories were identified: absence of excess weight; excess weight at one point in time; excess weight at two points in time. Waist circumference (WC) was used to identify levels of abdominal obesity. Chronic 
musculoskeletal symptoms (pain, discomfort and/or stiffness $\geq 6$ months during the preceding 12 months) were evaluated in nine body sites, and three dependent variables were used: presence of symptoms; number of sites of symptoms (none, 1-2 sites, $\geq 3$ sites), and region of symptoms (upper limbs, axial skeleton, lower limbs). Associations between each overweight/obesity marker and the dependent variables were investigated with multinomial logistic regressions adjusted by potential confounders.

Results and Conclusions: 2,821 participants, mean age 56.0(8.94), were included in the analysis. At the time of evaluation, $63.4 \%$ were overweight, $54.9 \%$ have chronic musculoskeletal symptoms and $18.8 \%$ reported symptoms in $\geq 3$ sites. Lower limbs were the most prevalent region of symptoms (36.0\%). Adjusted analyzes indicated a significantly higher chance of chronic symptoms among those who were obese at age 20 (OR 2.23; $95 \% \mathrm{Cl} 1.01-4.91)$ or currently (OR $1.60 ; 95 \% \mathrm{Cl} 1.30-1.97)$. The same was observed for symptoms in $\geq 3$ sites: obesity at age 20 OR $2.83 ; 95 \% \mathrm{Cl} 1.11-7.21$; current obesity OR 2.06; $95 \% \mathrm{Cl} 1.55-2.73$. Models on excess weight trajectories indicated a risk-accumulation behavior; i.e. the chance of chronic symptoms was increased by $\sim 30 \%$ when excess weight was present at one point in time and by $\sim 50 \%$ when it was present at two points in time. The same was observed for the association between symptoms in $\geq 3$ sites and excess weight trajectories: one point in time OR 1.61; $95 \% \mathrm{Cl} 1.27-2.04$; two points in time OR $1.73 ; 95 \% \mathrm{Cl} 1.11-2.70$. Abdominal obesity showed the same pattern of association with chronic symptoms that BMl, adding none explanation to the study. Overweight/obesity markers were found to be independently associated with chronic musculoskeletal symptoms, with stronger effects being observed among adults experiencing symptoms in multiple sites and/or with longer exposures to excess weight during lifetime.

Funding

Brazilian Ministry of Health (Science and Technology Department), of Science and Technology (FINEP, CNPq), and of Education (CAPES).

\section{6}

PEDANAM AS AN INSTRUMENT OF COGNITIVE EVALUATION IN NEUROPSYCHIATRIC SLE

Jaqueline Cristina de Amorim', Simone Thiemi Kishimoto', Clovis A Silva $^{2}$, Roberto Marini ${ }^{1}$, Lilian Tereza Lavras Costallat', Paula Teixeira Fernandes ${ }^{1}$, Simone Appenzeller ${ }^{1}$

${ }^{1}$ UNICAMP, CAMPINAS, SP, Brasil; ${ }^{2}$ INSTITUTO DA CRIANÇA USP, SAO PAULO, SP, Brasil

Advances in Rheumatology 2018, 58(Suppl 1):056

Background: Validation and use of a computerized battery for cognitive evaluation of patients with systemic lupus erythematosus (SLE) as part of an investigation of neuropsychiatric manifestations is useful and allows multicenter studies.

Materials and methods: This is a quantitative transversal study with control group conducted in pediatric and adult rheumatology clinics. The computer test batteries ANAM (Automated Neuropsychological Assessment Metrics) or PedAnam (Pediatric version) consists of 10 subtests: Sleepiness scale, simple reaction time, procedural reaction time, code substitution (learning phase), logical relations, spatial processing, running memory continuous performance test, mathematical processing matching grids, matching to sample, memory search, code substitution (delayed phase) and simple reaction time (delayed phase). We included 98 SLE patients and healthy age-matched individuals. All individuals underwent an evaluation through the battery tests taking 30 minutes on average to solve the problems.

Results and conclusions: We included 98 SLE patients 69 female and 29 male, the age with a minimum of 6 and a maximum of 68 and mean of 28.6 years. While the control group with 84 people had a mean age of 25 years with a minimum of 6 and a maximum of 65 years, 73 female and 11 male. For the evaluation was used the Performance Validity Index score, that provides a performance indicator, between 0 and 14, for someone with good effort, or above for someone outside the range of that expected for someone providing good effort. Patients presented an average performance of 8.05 , with a minimum of 0 and a maximum of 33, while the control group had a mean of 4.4, minimum 0 and maximum of 27. As the smaller score results in a better effort, it is possible to notice meaningful differences between the groups $(p<0.05)$. Cognitive difficulties are often observed in SLE and practical tools like ANAM and PedAnan should be used to measure the cognitive loss that patients may have; these losses should be monitored more closely if there are other neuropsychiatric symptoms.

\section{7}

POLYMORPHISM OF MICRORNA REGION IN THE TNFA GENE IN CHILDHOOD-ONSET SYSTEMIC LUPUS ERYTHEMATOSUS

Jessica Fernandes Vivaldo ${ }^{1,2}$, Mariana Postal ${ }^{2}$, Nailú Angélica Sinicato², Roberto Marini ${ }^{2}$, Timothy B. Niewold ${ }^{3}$, Simone Appenzeller ${ }^{4}$

'UNICAMP, CAMPINAS, SÃO PAULO, Brasil; ${ }^{2}$ UNIVERSIDADE ESTADUAL DE CAMPINAS, CAMPINAS, SÃO PAULO, Brasil; ${ }^{3}$ NEW YORK UNIVERSITY, NOVA IORQUE, NOVA IORQUE, Estados Unidos; ${ }^{4}$ UNIVERSIDADE DE CAMPINAS, CAMPINAS, SÃO PAULO, Brasil

Advances in Rheumatology 2018, 58(Suppl 1):057

Background: Studies have implicated microRNAs (miRNAs) in the pathogenesis of systemic lupus erythematosus (SLE). miRNAs regulate approximately $90 \%$ of the protein-encoding genes and play a central role in various biological processes, including impairment of cell differentiation and proliferation e apoptosis. Polymorphism in miRNA regions of TNFA gene may account for the variations observed in the clinical. The aim of this study was to investigate the presence of polymorphisms of miRNA regions of TNFA gene associated to clinical and laboratory profile of these SLE patients.

Methods: Consecutive childhood-onset SLE (CSLE) patients followed at Pediatric Rheumatology Unit of the Unicamp were enrolled in study. Healthy volunteers with were included as control group. A complete clinical, laboratory and neurological was performed in all subjects. CSLE patients were further assessed for clinical and laboratory SLE manifestations, disease activity [SLE Disease Activity Index (SLEDAI)], damage [Systemic Lupus International Collaborating Clinics/American College of Rheumatology Damage Index (SDI)] and current therapy. Total dose of corticosteroids and other immunosuppressant medications used since the onset of disease were calculated by data obtained by careful review of the medical charts. We investigated miRNA region of the TNFA gene in CSLE compared to healthy volunteers using DNA Sequencing by Capillary Electrophoresis. Data were compared by non-parametric tests.

Results: We included 110 cSLE patients [83 women (75.4\%)]. The mean disease duration was $13.18 \pm 4.32$ (1-20 years). We included as a control group 60 healthy individuals [52 women (86.7\%)] recruited from the local community. The mean score of cumulative SLEDAI was $2.88 \pm 2.20(0.09-12.52)$. The mean of total corticosteroid dose was $23437.54 \pm 16656.55 \mathrm{mg}$. We identified polymorphism rs3093665 (c.*77 A>C) in 8 (7.3\%) cSLE patients, polymorphism rs3093666 (c.*419 C>T) in $3(2.7 \%)$ patients and polymorphism rs3093667 (c. $\left.{ }^{*} 453 \mathrm{G}>\mathrm{T}\right)$ in $2(1.8 \%)$ cSLE patients. Polymorphism rs3093665 is located in a region of interest, into a miRNA region-binding site of miR-452 and polymorphism rs3093667 into a miRNA region-binding site of miR-19a. In the control group, we identified polymorphism rs3093665 in only 1 control. We observed an association between polymorphism rs3093665 and organic brain syndrome $(p=0.001)$, an association between polymorphism rs3093666 and thrombocytopenia $(p=0.02)$ and an association between polymorphism rs3093667 and 
hematuria $(p=0.024)$. Conclusion: To our knowledge, this is the first study to evaluate polymorphisms in miRNA regions of TNFA gene in SLE. We identified polymorphism rs3093665 and rs 3093667 , both into miRNA region-binding sites. Alterations contribute to the development of pathological conditions and clinical disorders in SLE.

\section{8}

POLYPHARMACY IN RHEUMATOID ARTHRITIS: DATA OF A LARGE COHORT IN BRAZIL

Ana Paula Monteiro Gomides' ${ }^{1}$ Licia Maria Henrique da Mota², Geraldo Rocha Castelar ${ }^{3}$, Cleandro Pires de Albuquerque ${ }^{2}$, Ana Beatriz VargasSantos ${ }^{3}$, Letícia Rocha Pereira ${ }^{3}$, Manoel Barros Bertolo ${ }^{4}$, Alisson Aliel Vigano Pugliesi ${ }^{4}$, Eduardo de Almeida Macedo ${ }^{4}$, Paulo Louzada Filho ${ }^{5}$, Maria de Fátima L da Cunha Sauma ${ }^{6}$, Marcel Lobato Sauma ${ }^{6}$, Júlia Brito de Medeiros ${ }^{6}$, Claiton Viegas Brenol ${ }^{7}$, Ivanio Alves Pereira ${ }^{8}$, Sebastiao Radominski ${ }^{9}$, Maria Fernanda Resende ${ }^{10}$, Maria Raquel Costa Pinto ${ }^{10}$, Gustavo Gomes Resende ${ }^{10}$, Karina Bonfiglioli ${ }^{11}$, Henrique Carriço ${ }^{11}$, Rina Dalva Neubarth Giorgi ${ }^{12}$, Nathalia de Carvalho Sacilotto ${ }^{12}$

'UNB/UNICEUB, BRASILIA, DF, Brasil; ${ }^{2} U N B$, BRASÍLIA, DF, Brasil; ${ }^{3} U E R J$, RIO DE JANEIRO, RJ, Brasil; ${ }^{4}$ UNICAMP, CAMPINAS, SP, Brasil; ${ }^{5}$ USP RIBEIRÃO PRETO, RIBEIRÃO PRETO, SP, Brasil; '́ UF DO PARÁ, BELEM, PA, Brasil; ${ }^{7}$ UFRGS, PORTO ALEGRE, RS, Brasili; ${ }^{8}$ UFSC, FLORIANÓPLIS, SC, Brasil; ${ }^{9}$ UF PARANÁ, CURITIBA, PARANÁ, Brasil; ${ }^{10}$ UFMG, BELO HORIZONTE, MG, Brasil; ${ }^{11}$ USP, SÃO PAULO, SP, Brasil; ${ }^{12} \mathrm{HSPE}-\mathrm{SP}$, SÃO PAULO, SP, Brasil Advances in Rheumatology 2018, 58(Suppl 1):O58

Introduction: Polypharmacy is a much-discussed problem in medicine in general. In rheumatology, few studies have evaluated this problem, which may hinder medical treatment with the appearance of drug interactions with altered mechanisms of drug action, new symptoms, poor adherence and treatment abandonment. Some studies in the literature suggest a higher rate of hospitalizations and higher mortality in patients using multiple drugs and probably with multiple comorbidities. This study evaluated, unprecedented in Brazil, polypharmacy in patients with rheumatoid arthritis (RA).

Material And Methods: A multicenter study was conducted with the participation of public services in different states of Brazil. The patients were evaluated through a complete clinical examination, analysis of complementary exams and records of medical records with a history of the disease since the onset of symptoms. The present study was a cross section with the initial evaluation of the participants.

Results And Conclusions: 1117 RA patients were evaluated in the total, with the majority of women ( $89 \%$ of the total) with a median age of 56.6 years. $78.73 \%$ had positive rheumatoid factor and $55.2 \%$ had erosive disease. The median ADHD was DAS 28 found was $3.52 \%$ and DAS 28 was 3.52 .

When the drugs for RA were analyzed, we saw that patients used on average 2.8 and at most 5 drugs of different therapeutic classes. Regarding the medications for comorbidities beyond RA, the mean was 2.7 and the maximum of 9 different types of medications. Evaluating the drugs in total (for RA and comorbidities) the average was 5.5 different drugs and the maximum 11 different drugs.

The most used therapeutic classes in this sample can be seen in Table 1.

Information on this important problem is essential for rheumatologists and discussions on the subject may favor improved management of patients with RA and diverse comorbidities.

Table 1 (abstract 058). Medications used in patients with RA

\begin{tabular}{lll}
\hline MEDICATION & NUMBER OF PATIENTS & \% OF PATIENTS \\
\hline FOLIC ACID & 682 & 61.1 \\
CALCIUM & 637 & 57.0 \\
D VITAMIN & 713 & 26.5 \\
DRUGS FOR DISLIPIDEMIAS & 313 & 28 \\
ANTI HYPERTENSIVE & 524 & 47 \\
HYPOGLYCLEANTS & 145 & 13 \\
LEVOTHYROXINE & 129 & 11 \\
\hline
\end{tabular}

059

PREVALENCE AND OUTCOME OF SERIOUS INFECTIONS IN PATIENTS WITH SYSTEMIC AUTOIMMUNE MYOPATHIES: A RETROSPECTIVE COHORT STUDY

Samuel Katsuyuki Shinjo ${ }^{1,2}$, Leonardo Santos Hoff ${ }^{2}$

${ }^{1}$ FACULDADE DE MEDICINA, UNIVERSIDADE DE SAO PAULO, SÃO

PAULO, SP, Brasil; ${ }^{2}$ FACULDADE DE MEDICINA FMUSP, UNIVERSIDADE

SAO PAULO, SÃO PAULO, SÃO PAULO, Brasil

Advances in Rheumatology 2018, 58(Suppl 1):059

Background. Serious infections are a major cause of morbidity and mortality in patients with systemic autoimmune myopathies (SAM). Herein, the authors describe the prevalence, outcome, and characteristics of serious infections in our large sample of patients with SAM.

Material and Method. A retrospective, single-center cohort study involving 162 consecutive patients with SAM, followed between 2000 and 2017, was conducted. Serious infections were defined as infections requiring hospital admission, intravenous antimicrobial therapy and/or tuberculosis. Patient data were extensively reviewed from electronic medical records, with pre-standardized and parameterized information.

Results. Forty-four (27.2\%) out of 162 patients had serious infections during to mean follow-up of 62 months. Patients had mean age of 45.2 years, with $59.1 \%$ female gender. Thirty patients had dermatomyositis, 5 polymyositis, 5 antisynthetase syndrome, and 4 immune-mediated necrotizing myopathy. Thirty-four (77.3\%) out of 44 patients had a severe clinical onset, with upper dysphagia, lung disease, vasculitis and/or significant muscle weakness. Sixteen patients had more than one episode of serious infection. Out of 81 episodes of serious infections, $23.5 \%$ were aspiration pneumonia, $18.5 \%$ bacterial pneumonia, $16 \%$ skin infections, $8.6 \%$ pyelonephritis, $8.6 \%$ herpes-zoster, $7.4 \%$ tuberculosis, $3.7 \%$ infectious diarrhea, and $13.6 \%$ other causes. Ten patients died during to follow-up, and 7 deaths were related to the infection. Infections associated with death included: 2 bacterial pneumonia, 2 skinrelated sepsis, 1 aspiration pneumonia, 1 complicated elective cholecystectomy and 1 retropharyngeal abscess. Only 2 patients died in the first 6 months of follow-up; all the 5 remaining infection-related deaths occurred after 1 year of following-up.

Conclusion. Serious infections are observed in $27.2 \%$ of patients with SAM. Aspiration pneumonia was the most common type of infection. Therefore patients with dysphagia should be followed carefully. Unfortunately, death is a common complication of infection and usually occurs in patients after 1 year of follow-up.

\section{0}

PREVALENCE OF ACUTE AND CHRONIC ARTHRITIS IN A POPULATION IN BUÍQUE/PERNAMBUCO

Tania Caroline Monteiro De Castro, Daniela Gerent Petry Piotto, Simone Andrade Lotufo, Cecilia Harumi Tomizuka, Alessandra Haddad Segato, Fernanda Maria Miyamoto Barreto, Lucila Camargo Lopes de Oliveira, Denise Faria do Amaral, Marcia Wehba Esteves Cavichio

GRUPO FLEURY, SÃO PAULO, SÃO PAULO, Brasil

Advances in Rheumatology 2018, 58(Suppl 1):060

Background: A non-governamental organization (ONG) Amigos do Bem operates in 115 villages with the lowest levels of human development (IDH) in Brazil through actions that promote access to food, health, education and the generation of employment and income. Since 1993, this ONG acts in order to guarantee medical and dental care of all those assisted by the institution. Monthly, doctors, dentists and other health professionals voluntarily attend the population of this Northeastern community. Arthritis, whether acute or chronic, is a diagnostic challenge, because of the wide variety of pathologies that can occur with arthritis.

The purpose of the study was to determine the prevalence of acute and chronic arthritis in children and adolescents attended at the Catimbau, Buique, Pernambuco.

Materials and Methods: In 2017, during three days, seventeen doctors and health professionals volunteers (radiologists, pediatricians, otorhinolaryngologists and nutritionists) at Fleury Group, performed consultations, clinical, laboratory and diagnostic exams. A total of 
676 patients, aged between 2 months and 21 years were attended by pediatricians. The children with rheumatologic complaints were directed to an evaluation with one of the three specialists in pediatric rheumatology who participated in the medical team, on the same day.

Results: A total of $58(9 \%)$ cases of musculoskeletal pain were observed in this community, with the mean age of 9.6 years, $37(63.8 \%)$ female. Thirty-seven children (5.7\%), mean age of 9.1 years, and 34 (65\%) females reported complaints of arthralgia. Only five children $(0.78 \%)$ presented arthritis at physical examination. Of these, two were acute monoarthritis and three, chronic arthritis. Among the acute arthritis, one was a teenager with probable septic arthritis in the left knee, the other was a 12-year-old boy with a right shoulder monoarthritis after trauma. He reported having previously dislocated this shoulder (two episodes) and was referred to the orthopedist. Regarding chronic arthritis, three patients presented with knee arthritis, confirmed by ultrasonography. These patients were referred to the Federal University of Pernambuco for diagnostic investigation: a chronic monoarthritis due to tuberculosis (had a history of tuberculosis in the family), and two juvenile idiopathic arthritis already presenting limitations to the movements. Two children (0.3\%) had chronic arthralgia after Chinkungunya.

Conclusion: This work shows the importance of medical care, especially in the most needy communities, in order to reduce the damages resulting from arthritis pathologies that are not diagnosed at an early stage.

\section{1}

PREVALENCE OF ARTICULAR AND PERIARTICULAR ULTRASONOGRAPHIC MANIFESTATIONS IN PATIENTS WITH INFLAMMATORY BOWEL DISEASE

Miriam Kuster Huber ${ }^{1}$, Érica Vieira Serrano', José Alexandre Mendonça², Raquel Ionne Küster', Valéria Valim', Maria Bernadete Renoldi de Oliveira Gavi ${ }^{1}$

${ }^{1}$ HUCAM/UFES, VITÓRIA, ES, Brasil; ${ }^{2}$ UNICAMP, CAMPINAS, SP, Brasil Advances in Rheumatology 2018, 58(Suppl 1):061

Background Arthritis and periarthritis are the most common extraintestinal manifestations in patients with inflammatory bowel disease (IBD), however subjective complaints may be non-specific or subclinical manifestations may exist. Detailed anamnesis and clinical examination associated with musculoskeletal ultrasonography (MEUS) may increase this potential diagnostic. The objective of this study was to evaluate the prevalence of musculoskeletal manifestations confirmed by MEUS.

Materials And Methods: Cross-sectional study of patients with IBD from a University Hospital submitted to ASAS classification for axial and peripheral spondyloarthritis. Those patientes with some clinical suspicion were invited to physical examination with MASES search, clinical synovitis screening by DAS 28 and evaluation by MEUS. Two experienced rheumatologists performed independent and blind evaluations. We used Esaote My Lab 70, linear transducer L12-18, power doppler (PD) of $12 \mathrm{MHz}$ and PRF $750 \mathrm{MHz}$, wall filter 1. They were analyzed by MEUS: sacroiliac by $P D$, with resistance index (RI); 12 sites of enthesis by MASEl; vitreous by mode B and PD, clinically symptomatic joints and tendons by mode $B$ and PD.

Results: 118 patients with IBD were interviewed and 40 (33\%) presented complaints such as inflammatory arthralgia and / orNarthritis and / or inflammatory low back pain and / or ocular symptoms and / or psoriasis and/or enthesitis. Of this group, 34 patients with IBD Were evaluated clinically and by MEUS: 25 women, mean age of 43 years, mean diagnosis time of 11 years. Of these $59 \%$ had inflammatory low back pain, $56 \%$ had inflammatory arthralgia, $21 \%$ had arthritis and $68 \%$ had at least one MASES site. The MASEl sites were clinically palpated and presented positivity in 50\%. Of the 442 enthesis of MASES evaluated: $27 \%$ with clinical enthesitis. Ocular symptoms occurred in $50 \%$. At MEUS: $24 \%$ with sacroiliitis (mean RI: $0.65), 23 \%$ with vitreitis, $35 \%$ with synovitis (15 joints in mode B and 1 in PD) and 14\% with tenosynovitis. At least one ultrasound enthesitis in $100 \%$ patients. Mean of MASEl was 17.79. From the 408 enthesis evaluated, 280 (68\%) were altered and 14 (41\%) patients presented MASEI greater than or equal to 18. Interobserver agreement was statistically significant $(p<0.1)$ in intraclass correlation coefficients - for synovitis (CCl: 0.795), tenosynovitis (CCl: 0.609) and MASEI (CCl: 0.741).

Conclusions Musculoskeletal symptoms were the most common extra intestinal manifestations and the MEUS confirmed enthesitis in $100 \%$ of patients with IBD. MEUS is an important complementary method for the diagnosis of spondyloarthritis in IBD.

\section{2}

\section{QUALITY OF LIFE AND COGNITIVE FUNCTION IN SYSTEMIC LUPUS} ERYTHEMATOSUS

Cátia Maria Geralda dos Santos Nascimento, Paula Teixeira Fernandes, Lilian Tereza lavras Costallat, Simone Appenzeller

UNICAMP, SP, SP, Brasil

Advances in Rheumatology 2018, 58(Suppl 1):062

Introduction: Quality of life (QoL) is worse in SLE patients. The objective was to determine if cognitive dysfunction influences QOL life in SLE patients.

Methods: In this cross-sectional study, we included 226 consecutive SLE patients [average age 33.63 years ( $D P \pm 11.88$ ); average schooling 11.53 years $(\mathrm{DP} \pm 3.9)]$ from the Rheumatology outpatient unit and 134 healthy controls [average age 33.54 years (DP \pm 13.6 ); average schooling 12.6 years (DP \pm 3.04)]. Cognitive dysfunction was evaluated using the Montreal Cognitive Assessment test (MoCA). ShortForm Health Survey questionnaire (SF-36) evaluated the quality of life. Fatigue Severity Scale questionnaire (FSS) evaluated presence of symptons fatigue. Symptons of depression and anxiety were determined through Beck Depression and Beck Anxiety Inventory. SLE patients were further assessed for clinical and laboratory SLE manifestations, disease activity [SLE Disease Activity Index (SLEDAI)], damage [Systemic Lupus International Collaborating Clinics/American College of Rheumatology Damage Index (SDI)] and current drug exposures. Chi-square test was used to compare the categorical variables. Mann-Whitney test was used to compare numerical variables. For correlation between numerical variables, the Pearson correlation test was used. The level of significance adopted for this study was $5 \%$.

Results: In approximately $65 \%$ SLE patients were observed dysfunction cognitive and in $41 \%$ healthy control $(p<0.001)$. Presence of anxiety symptoms were observed in $63.3 \%$ SLE patients and in $44.8 \%$ healthy controls ( $p=0.004$ ); depression symptoms in $36.7 \%$ patients and in 19.4\% control group ( $p=0.003$ ); fatigue symptoms in $61.9 \%$ patients and in $30.6 \%$ control group $(p<0.001)$. All SF-36 domains, physical and mental components and total score were significantly lower in the SLE patients compared to healthy controls. Low quality of life was associated with presence symptons depression, anxiety and fatigue. Scores of cognitive dysfunction were significantly correlated only with mental health domain $(p=0.021 ; r=0.153)$. Scores of depression, anxiety and fatigue symptons apresented inverse correlation with all SF-36 domains. Age at onset of disease and age at diagnosis of the disease were significantly correlated with all domains. However, disease duration apresented inverse correlation with domains: functional capacity $(p=0.035 ; r=-0.14)$, physical aspects limitation $(p=0.026 ; r=-0.149)$ and pain $(p=0.032 ; r=-0.143)$. Presence of damage was associated low physical aspects score domain $(p=0.037)$. Conclusion: We observed depression, anxiety and fatigue symptons are associated with lower SF-36 physical and mental components in SLE patients. Cognitive dysfunction compromises mental health and, disease damage increases the limitation by physical aspects of patients.

\section{3}

\section{QUALITY OF LIFE AND SOCIAL PHOBIA IN SYSTEMIC LUPUS} ERYTHEMATOSUS

Cátia Maria Geralda dos Santos Nascimento, Paula Teixeira Fernandes, Lilian Tereza Lavras Costallat, Simone Appenzeller

UNICAMP, CAMPINAS, SP, Brasil

Advances in Rheumatology 2018, 58(Suppl 1):063

Background: SLE patients presented lower scores quality of life than healthy controls. Neuropsychiatric manifestations compromise the quality of life of patients with lupus. Anxiety disorders and mood are commonly reported in lupus. Anxiety when intense presents itself in 
many ways, becoming anxiety disorders. Among the various anxiety disorders, it has social phobia, which is characterized by intense and persistent fear of social situations. In social phobia, there is functional impairment and quality of life of the subject. The objective is to determine the frequency of social phobia and to avaluate the quality of life in patients with Systemic Lupus Erythematosus (SLE) and to determine the associations with neuropsychiatric manifestations (NPM), disease activity, disease damage, and use of corticosteroids (CE). Methods: We include 81 SLE consecutive patients [mean age 38.41 years (DP \pm 9.49) from the Rheumatology outpatient unit and 63 healthy controls [mean age 35.31 years (DP \pm 10.3)]. Short-Form Health Survey (SF-36) assessed quality of life. Liebowitz Social Anxiety Scale (LSAS) assessed presence of symptoms of social phobia. Mood and anxiety disorders were determined through Beck Depression and Beck Anxiety Inventory. SLE patients were further assessed for clinical and laboratory SLE manifestations, disease activity [SLE Disease Activity Index (SLEDAI)], damage [Systemic Lupus International Collaborating Clinics/American College of Rheumatology Damage Index (SDI)] and current drug exposures. NPM were classified according to the criteria established by the American College of Rheumatology (ACR). Data were compared by non-parametric tests.

Results: Social phobia was observed in $52(64.2 \%)$ SLE patients and in $2(3.2 \%)$ healthy controls $(p<0.001)$. Presence of anxiety symptoms were observed in 62 (76.5\%) patients and in 20 (31.7\%) healthy controls $(p<0.001)$. Depressive symptoms were observed in $39(48.1 \%)$ patients and in $4(6.3 \%)$ healthy controls $(p<0.001)$. There was significant association between social phobia and anxiety disorders $(p=0.017)$ and mood disorders ( $p=0.004)$. All SF-36 domains, physical and mental components scores were significantly lower in SLE patients than healthy controls. Pain $(p=0.022)$, vitality $(p=0.002)$ and social aspects $(p=0.026)$ domains were associated with the presence social phobia. There was no significant association between social phobia and cumulative damage, activity disease, current prednisone dose or other neuropsychiatric manifestations.

Conclusion: We observed a high frequency of social fobia related to mood disorders and worse quality of life. Social phobia should be screened routinely it can influence the quality of life of patients.

\section{4}

QUALITY OF LIFE, BODY COMPOSITION AND PSYCHOLOGICAL ASPECTS IN SYSTEMIC LUPUS ERYTHEMATOSUS (SLE)

Simone Thiemi Kishimoto, Catia Maria Geralda dos Santos Nascimento, Lilian Tereza Lavras Costallat, Paula Teixeira Fernandes, Simone Appenzeller

UNICAMP, CAMPINAS, SP, Brasil

Advances in Rheumatology 2018, 58(Suppl 1):064

Background: In systemic Lupus Erythematosus (SLE) an increase in factors related to physical inactivity, such as obesity, mood swings, anxiety and depression, increasing the risk of mortality and worse patient's quality of life has been observed. The objective of this study was to evaluate the body composition, psychological and quality of life in SLE.

Methods: For this study we included 250 SLE patients [mean age 24.1 years ( $S D \pm 6.4)$ ], 150 healthy controls [mean age 24.6 years (SD \pm 13.5)]. To evaluate the body composition, the vertical electric bioimpedance apparatus (Omron HBF-514C) was used. Quality of life was assessed through the questionnaire SF-36 (Short-Form Health Survey). For the evaluation of the psychological aspects related to the symptoms of anxiety, depression and self-esteem were used the questionnaires: Beck Anxiety Inventory (BAl), Beck Depression Inventory (BDI) and Rosenberg Self-Esteem Scale. SLE patients were further assessed for clinical and laboratory SLE manifestations, disease activity (SLEDAI), damage [Systemic Lupus International Collaborating Clinics/American College of Rheumatology Damage Index (SDI)] and current drug exposures.

Results: Related to body composition, we observed better body mass indexes $(p<0.0001)$ and total fat $(p=0.0002)$ in the control patients compared to the SLE group and the skeletal muscle ( $p<0.0001)$ was higher in the control group compared to SLE. Related to psychological aspects, the control group showed better self-esteem indexes $(p<0.0001)$ and lower rates of depressive symptoms ( $p<0.0001)$ compared to the SLE group. The anxiety symptoms $(p=0.0383)$ were better in the control group compared to SLE. Quality of life related to functional capacity $(p<0.001)$, physical aspects $(p<0.0001)$, pain $(p<0.0001)$, general health $(p<0.0001)$, vitality $(p<0.0001)$ and mental $(p=0.0002)$ showed to be better in the control group compared to the SLE group.

Conclusion: In this study we observed better indexes of body composition, psychological and quality of life in the control group in relation to SLE. We emphasize the importance of alternative methods of complementary drug treatment, such as the practice of physical activities, thus helping to control obesity, psychological improvement and quality of life in these patients.

\section{6}

SAFETY AND IMMUNOGENICITY OF THE QUADRIVALENT HPV VACCINE IN GIRLS WITH JUVENILE SYSTEMIC LUPUS ERYTHEMATOSUS AND DERMATOMYOSITIS

Gecilmara S. Pileggi ${ }^{1}$, Natalia B. F. Pinto ${ }^{2}$, Aline L. Oliveira ${ }^{2}$, Ingrid H. R. Grein ${ }^{3}$, Flavio R. Sztajnbok ${ }^{4}$, Juliana O. Sato ${ }^{5}$, Blanca Bicas ${ }^{6}$, Rozana G. Almeida ${ }^{7}$, Luciana Paim ${ }^{8}$, Nadia E.Aikawa ${ }^{9}$, Simone Appenzeller ${ }^{10}$, Melissa M. Fraga ${ }^{11}$, Aline C. Fraga ${ }^{12}$, Cássia M. P. L. Barbosa ${ }^{13}$, Maria C. dos Santos $^{14}$, Aline Islabão ${ }^{15}$, Teresa C. M. V. Robazzi ${ }^{16}$, Marcia Bandeira ${ }^{3}$, Sheila Knupp Feitosa de Oliveira ${ }^{7}$, Claudia Saad Magalhães ${ }^{17}$, Virgínia Paes Leme Ferriani ${ }^{1}$

'FACULDADE DE MEDICINA DE RIBEIRÃO PRETO - FMRP-USP, Brasil; ${ }^{2}$ FACULDADE DE MEDICINA DE RIBEIRÃO PRETO - FMRP-USP., Brasil; ${ }^{3}$ HOSPITAL PEQUENO PRÍNCIPE, CURITIBA, Brasil; ${ }^{4}$ NÚCLEO DE ESTUDOS DA SAÚDE DO ADOLESCENTE (NESA) - UERJ., Brasil; ${ }^{5}$ FACULDADE DE MEDICINA DE BOTUCATU - UNESP., Brasil; ${ }^{6}$ HOSPITAL UNIVERSITÁRIO

CLEMENTINO FRAGA FILHO - UFRJ, Brasil; ${ }^{7}$ INSTITUTO DE

PUERICULTURA E PEDIATRIA MARTAGÃO GESTEIRA - UFRJ., Brasil;

${ }^{8}$ HOSPITAL INFANTIL ALBERT SABIN - HIAS., Brasil; ${ }^{9}$ INSTITUTO DA

CRIANÇA DO HOSPITAL DAS CLÍNICAS DE SÃO PAULO - FMUSP, Brasil;

${ }^{10}$ UNIVERSIDADE ESTADUAL DE CAMPINAS - UNICAMP., Brasil;

${ }^{11}$ HOSPITAL DARCY VARGAS DE SÃO PAULO, Brasil; ${ }^{12}$ HOSPITAL

INFANTIL NOSSA SENHORA DA GLÓRIA DE VITÓRIA, Brasil;

${ }^{13}$ UNIVERSIDADE FEDERAL DE SÃO PAULO - UNIFESP, Brasil;

${ }^{14}$ FACULDADE DE MEDICINA DA SANTA CASA DE SÃO PAULO, Brasil;

${ }^{15}$ INSTITUTO DO CÂNCER INFANTIL E PEDIATRIA ESPECIALIZADA DO

HOSPITAL DA CRIANÇA DE BRASÍLIA., Brasil; ${ }^{16} \mathrm{HOSPITALAR} \mathrm{PROFESSOR}$

EDGARD SANTOS (HUPES) - UFBA, Brasil; ${ }^{1{ }^{1}}$ FACULDADE DE MEDICINA

DE BOTUCATU - UNESP, Brasil

Advances in Rheumatology 2018, 58(Suppl 1):066

Autoimmune diseases, such as systemic lupus erythematosus (SLE) are more prevalent in women in the same age range of higher risk of papilomavírus (HPV) infection. Patients with SLE have a 4-times high risk for developing HPV carcinogenic infection. However, the safety of HPV vaccine in SLE patients are a matter of debate. The aims of this study were to assess the safety and effectiveness of HPV vaccination in girls and adolescents with Juvenile SLE (JSLE) and Dermatomyositis (JDM). It was a multicenter prospective intervention study, involving 14 Brazilian pediatric rheumatology centers. The inclusion criteria: female with age 9-20 years with confirmed diagnosis of JDM or JSLE. Control group: health girls in the same age group. Exclusion criteria: Being or intention to be pregnant at the beginning of the study. The vaccine used was quadrivalent HPV in the 3 doses schedule (0-2-6 months). Safety end points: occurrence of adverse events following vaccination (AEFV) was assessed by a questionnaire handed out to all vaccinated patients and controls, to be filled for 14 consecutive days following each dose of the HPV vaccine; disease activity was assessed before and after each dose, using the Systemic Lupus Erythematosus Disease Activity Index (SLEDAI) for patients with JSLE, Childhood Myositis Activity Score (CMAS) and Manual Muscle Test (MMT) for JDM, medications and exams were recorded. The algorithm conditional inference trees was used to verify the possible influence of the variables (number of doses, age, disease activity, medication) on the occurrence of AEFV. Luminex immunoassay was used to evaluate the response.

Results: We have included 222 patients with JSLE, 48 with JDM and 41 health controls. The frequency of AEFVs was similar in patients 
and controls, lasting range 1-5 days; none variable assessed did have showed influence on the occurrence of AEFVs ( $p>0.05)$ and none severe AEFV was reported. Disease remained inactive after vaccination in $73.7 \%$ JSLE and $91.5 \%$ JDM. In 90\% JSLE and 96\% JDM continued the therapy and the lab exams remained stable in $84 \%$ JSLE and $82 \%$ JDM when we compared before and after each dose of the vaccine. Among them 236 patients was vaccinated with the 3 doses, only $26(11 \%)$ not responded to the vaccine, none variable was related to them.

Conclusion: The HPV4 vaccine has shown to be safe, well tolerated and immunogenic in girls and adolescents with JSLE and JDM and should be recommended for this group of patients at high risk to develop carcinogenic lesions of HPV.

\section{7}

SEASONAL EVALUATION OF VITAMIN D AT A TERTIARY HOSPITAL IN SAO PAULO

Matheus Xavier Guimarães', Fernanda Pulcheri Ramos², Andrey Tonetto Barbosa ${ }^{2}$, Raissa Barbosa de Souza ${ }^{2}$, Caroline Almeida Oliveira ${ }^{2}$, Kioko

Takei $^{2}$, Daniela Crema², Rina Dalva Neubarth Giorgi ${ }^{2}$, Elaine de Azevedo ${ }^{2}$ ${ }^{1}$ HOSPITAL DO SERVIDOR PUBLICO ESTADUAL, SÃO PAULO, SÃO PAULO, Brasil; ${ }^{2}$ HOSPITAL DO SERVIDOR PUBLICO ESTADUAL DE SÃO PAULO, SÃO PAULO, SÃO PAULO, Brasil

Advances in Rheumatology 2018, 58(Suppl 1):067

Introduction: It's well recognized that vitamin D status in the body is one of the main determinants of bone integrity. The main source of vitamin D is UVB-catalyzed skin synthesis, and it can also be found at low levels of concentration in foods such as eggs and fish. Its deficiency is a prevailing condition in several places of the world, including in tropical countries like Brazil and in big cities like São Paulo (SP). The serum concentration of its metabolite, 25-hydroxyvitamin D $(25 \mathrm{OH}-\mathrm{D})$, is the method of choice that defines its levels, being little active and stored in adipose tissue, thus directly reflecting the bodies reserves of this vitamin. The objective of this study was to evaluate $25 \mathrm{OH}-\mathrm{D}$ levels in different age groups and their variation according to the seasons of the year.

Methods: A cross-sectional study that analyzes $25 \mathrm{OH}-\mathrm{D}$ results collected from January to December 2016 in a tertiary hospital in SP, regardless of socio-cultural habits, historical supplementation, solar exposure and diseases. Groups were established according to the season of the year in which the laboratory was collected. The seasons were divided in autumn (March to June), winter (June to September), spring (September to December) and summer (January to March and December). Statistical analysis included mean and standard deviation, frequencies, chi-square and Fisher exact. It was considered statistically significant $p<0.05$.

Results: A total of 32.535 vitamin D results were analyzed, 8.529 of which were collected during the autumn, 7.709 during the winter, 5.883 during the spring and 10.408 during the summer. The media of $25 \mathrm{OH}-\mathrm{D}$ in summer $(30,36 \pm 11,22 \mathrm{ng} / \mathrm{dL})$ was bigger than autumn $(28,74 \pm 11,07 \mathrm{ng} / \mathrm{dL})$, spring $(24,4 \pm 8,73 \mathrm{ng} / \mathrm{dL})$ and winter $(25,08$ $\pm 10,10 \mathrm{ng} / \mathrm{dL})(\mathrm{p}<0,0001)$. Considering the values above $30 \mathrm{ng} / \mathrm{dL}$ as sufficiency status, we found a higher prevalence of this result in the sum of summer and autumn vs spring and winter $(\mathrm{N}=8033 ; \mathrm{N}=3096$; $p<0,0001$, respectively). On the other hand, in all age groups, results below $20 \mathrm{ng} / \mathrm{dL}$ were more frequent in sum of winter andspring $(\mathrm{N}=4194 \mathrm{p}<0.0001)$ when compared with the other seasons $(\mathrm{N}=2895)$. Conclusion: This study shows lower levels of $25 \mathrm{OH}-\mathrm{D}$ in the winter and spring months, which is associated with less sun exposure. The data above prove that is necessary to create protocols of adequate $25 \mathrm{OH}-\mathrm{D}$ supplementation in the months of less sun exposure, whitout the absolute necessity of serial dosing of this hormone, since its seasonal insufficiency or deficiency is known, reducing unnecessary expenses.
068

SEGMENTATION AND MICROSTRUCTURAL ANALYSES OF CORPUS CALLOSUM IN SYSTEMIC LUPUS ERYTHEMATOSUS PATIENTS

Beatriz Lavras Costallat ${ }^{1}$, Beatriz Lavras Costallat ${ }^{2}$, Aline Tamires Lapa ${ }^{2}$,

Lilian Tereza Lavras Costallat ${ }^{2}$, Fernando Cendes ${ }^{2}$, William Javier Garcia Herrerra $^{3}$, Letícia Rittner ${ }^{3}$, Simone Appenzeller ${ }^{2}$

${ }^{1}$ UNICAMP, CAMPINAS, SÃO PAULO, Brasil; ${ }^{2}$ FACULDADE DE CIÊNCIAS MÉDICAS - UNICAMP, CAMPINAS, SÃO PAULO, Brasil; ${ }^{3}$ FACULDADE DE ENGENHARIA ELÉTRICA E DA COMPUTAÇÃO- UNICAMP, CAMPINAS, SÃO PAULO, Brasil

Advances in Rheumatology 2018, 58(Suppl 1):O68

Background: The aim of this study was determine the microstructural changes in each segment of the corpus callosum (CC) in patients with systemic lupus erythematosus by magnetic resonance diffusion tensor technique (DTI).

Material and method: The study evaluated 116 patients with SLE and 48 healthy controls. All patients fulfilled 4 or more American College of Rheumatology (ACR) SLE. SLEDAI and SLICC were evaluated. All patients and controls underwent cranial MRI and the CC was segmented into 5 sub-regions, according to validated classification. The fractional anisotropy (FA), mean diffusivity (MD), axial diffusivity (AD) and radial (RA) values were calculated.

Results: Patients with SLE presented lower values of FA $(0.5969 \pm$ 0.9569 vs $0.6696 \pm 0.3812 ; p<0.001)$ and higher of $A D(0.0018 \pm$ 0.0002 vs $0.0015 \pm 0.0003 ; p<0.001)$ in parcel 1 of CC when compared with controls.

In parcel 5 of CC, patient with SLE presented lower values of RD $(0.0007 \pm 0.0002$ vs $0.0008 \pm 0.0003 ; p=0.040)$ when compared with controls. The SLEDAI score presented a negative correlation with FA in parcel $1(r=-0.333, p<0.001)$ and positive with $A D(r=0.235, p=$ $0.011)$ and $R D(r=0,190 ; p=0.042)$ in parcel 2 and with $M D(r=0.276$, $p=0.003), A D(r=0.327, p<0.001)$ and $R D(r=0.330, p<0.001)$ in parcel 3. The SLICC score presented a negative correlation with FA ( $r=$ $-0.184, p=0.048)$ and positive with $M D(r=0.188, p=0.043)$ and $A D$ $(r=0.246, p=0.008)$ in parcel 1 . In parcel 3 , the SLICC score presented a positive correlation with $\mathrm{MD}(\mathrm{r}=0.218 ; \mathrm{p} 0,019)$. In parcel 1 , patients with active NPSLE presented lower values of FA when compared to inactive NPSLE $(0.533 \pm 0.0980$ vs $0.619 \pm 0.862 ; \mathrm{p}<0.001)$ and to non-NPSLE $(0.533 \pm 0.0980$ vs $0.619 \pm 0.0606 ; p<0.001)$. In parcel 3, patients with active NPSLE presented higher values of MD when compared to non-NPSLE $(0.0013 \pm 0.0002$ vs $0.0009 \pm 0.0001$; $\mathrm{p}=0.027$ ) In parcel 1, patients with active NPSLE presented higher values of $A D$ when compared to controls $(0.0018 \pm 0.0002$ vs 0.0014 $\pm 0.0003 ; p<0.001)$. In parcel 3 , patients with active NPSLE presented lower values of $A D$ when compared to non-NPSLE $(0.0012 \pm 0.0003$ vs $0.0016 \pm 0.0001 ; p=0.003$ ).

Conclusion: The anterior parcels were those that presented microstructural alteration. The DTI may provide elements for a more comprehensive analysis of the microstructure of the CC in patients with NPSLE.

069

SENSITIVITY AND SPECIFICITY OF INTERLEUKIN-22 IN DISTINGUISHING RHEUMATOID ARTHRITIS (RA) PATIENTS FROM NON-RA PATIENTS AND HEALTHY INDIVIDUALS

Laurindo Ferreira Da Rocha Jr', Moacyr Jesus Barreto De Melo Rego², Adson Belém Ferreira Da Paixão일 Kamila De Melo Vilar ${ }^{2}$, Andrea Tavares Dantas', Pablo Ramon Gualberto², Hugo Deleon De Lima², Henrique De Ataíde Mariz', Ivan Da Rocha Pitta ${ }^{2}$, Maira Galdino Da Rocha Pitta ${ }^{2}$,

Angela Luzia Branco Pinto Duarte

'HOSPITAL DAS CLÍNICAS - UNIVERSIDADE FEDERAL DE PERNAMBUCO, RECIFE, PE, Brasil; ${ }^{2}$ LINAT/NUPIT-SG/UNIVERSIDADE FEDERAL DE

PERNAMBUCO, RECIFE, PERNAMBUCO, Brasil

Advances in Rheumatology 2018, 58(Suppl 1):069 
Background: The interleukin (IL)-22 is a member of the IL-10 family and has been implicated in the pathogenesis of rheumatoid arthritis (RA). In the last few years IL-22 has been reported to be higher in RA sera compared to other subjects. This work aimed to evaluate the sensitivity and specificity of IL-22 in distinguishing RA among healthy subjects (HS) and patients with other rheumatic diseases.

Material and Methods: The serum levels of IL-22 was measured by ELISA in 135 patients with RA, 74 with Systemic Lupus Erythematous (SLE), 60 with systemic sclerosis (SSc), 48 with osteoarthritis (OA), 29 with fibromyalgia (FM) and 68 healthy subjects (HS). Associations of serum IL-22 levels among distinct groups were analyzed by univariate comparisons using Mann-Whitney test. Optimum cut-off values were calculated to optimize sensitivity and specificity. Receiveroperating characteristic (ROC) curves were plotted and the areas under the ROC curves (AUC) were calculated to assess the performance of IL-22 to distinguish RA from the subjects studied.

Results and Conclusions: RA patients had significantly increased serum median IL-22 levels 118 (IQR 31.29 - 429.5) pg/ml compared with non-RA patients 31.25 (IQR $31.25-108.3$ ) pg/ml and HS, 31.25 $\mathrm{pg} / \mathrm{ml}, \mathrm{p}<0.0001$. The sensitivity of IL-22 in differentiating patients with RA from non-RA patients and from healthy controls was of $74,81 \%(95 \% \mathrm{Cl} 66.6-81.9)$. The AUC of IL-22 ability to distinguish RA from all the subjects of the study demonstrated was $0.780(95 \%$ $\mathrm{Cl} 0.736-0.819)$. The yielded sensitivities, specificities and positive likelihood ratios (LR) of IL-22 to distinguish RA from each disease evaluated as well as the optimal cut-off values found are featured on the Table 1. Serum IL-22 levels might have the potential to be used as a diagnostic biologic marker in RA. Further studies with larger number of patients, early RA and naïve patients are necessary in order to better understand IL-22 role as a diagnostic tool in RA.

Table 1 (abstract 070). Diagnostic performance of serum IL-22 in distinguishing RA patients from non-RA patients and healthy controls

\begin{tabular}{|c|c|c|c|c|c|}
\hline & Subjects tested & $\begin{array}{c}\text { Optimal } \\
\text { cut-off values }(\mathrm{pg} / \mathrm{ml})\end{array}$ & $\begin{array}{l}\text { Sensitivity } \\
\text { (95\% C) }\end{array}$ & $\begin{array}{l}\text { Specificity } \\
\text { (95\% a) }\end{array}$ & LR \\
\hline SLE & 74 & 173.25 & $\begin{array}{c}44.44 \\
(35.9-53.2)\end{array}$ & $\begin{array}{c}89.19 \\
(79.8-95.2)\end{array}$ & 4.11 \\
\hline SSC & 60 & 31.25 & $\begin{array}{c}74.81 \\
{[66.6-81.9\}}\end{array}$ & $\begin{array}{c}93.33 \\
(83.8-98.2)\end{array}$ & 11.22 \\
\hline$O A$ & 48 & 31.25 & $\begin{array}{c}74.81 \\
(66.6-81.9)\end{array}$ & $\begin{array}{c}83.33 \\
(69.8-92.5)\end{array}$ & 4.49 \\
\hline FM & 29 & 31.25 & $\begin{array}{c}74.81 \\
(66.6-81.9)\end{array}$ & $\begin{array}{c}86.21 \\
(68.3-96.1)\end{array}$ & 5.42 \\
\hline NON-RA & 211 & 31.25 & $\begin{array}{c}74.81 \\
(66.6-81.9)\end{array}$ & $\begin{array}{c}71.56 \\
(65-77.5)\end{array}$ & 2.63 \\
\hline HS & 68 & 31.25 & $\begin{array}{c}74.81 \\
(66.6-81.9)\end{array}$ & $\begin{array}{c}98.53 \\
(92.1-100)\end{array}$ & 50.87 \\
\hline Overall & 279 & 31.25 & $\begin{array}{c}74.81 \\
(66.6-81.9)\end{array}$ & $\begin{array}{c}78.14 \\
(72.8-82.8)\end{array}$ & 3.42 \\
\hline
\end{tabular}

\section{0}

\section{SERUM INTERLEUKIN-17 IS A POTENTIAL BIOMARKER OF}

\section{DERMATOMYOSITIS ACTIVITY}

Marilda Guimarães Silva', Walcy Paganelli Rosolia Teodoro², Samuel Katsuyuki Shinjo²

'FACULDADE DE MEDICINA DA UNIVERSIDADE DE SÃO PAULO, SÃO

PAULO, SP, Brasil; ${ }^{2}$ FMUSP, SÃO PAULO, SP, Brasil

Advances in Rheumatology 2018, 58(Suppl 1):070

Background. The increased serum level of the interleukin-17 (IL-17) has been observed in several systemic autoimmune diseases. However, the IL-17 relevance is scarcely described in dermatomyositis, with conflicting data. Therefore, the aim of the present study was to assess the IL-17 serum levels from patients with dermatomyositis in large cohort and to correlate with disease-related clinical data.

Materials and method. This cross-sectional single center study included 81 patients with adult dermatomyositis (Bohan and Peter, 1975; EULAR/ACR criteria, 2017). As a control group, 98 healthy individuals matched by gender, age and ethnicity were included. IL-17 serum levels were assessed by Milliplex Map kit. The disease activity was based on the International Myositis Assessment and Clinical Studies Group (IMACS) core set. The histological analysis was performed using the immunohistochemistry technique, using a monoclonal antibody IL-17.

Results. Mean age of patients was 45.9 years, with predominance of female gender $(70.4 \%)$ and white ethnicity $(69.4 \%)$. Median duration of disease was 6 years. Median IMACS core set were: HAQ of 0.90 , MMT-8 of 80, patient's VAS of 3.0, physician's VAS of 2.0, creatine phosphokinase of $136 \mathrm{U} / \mathrm{L}$, aldolase of $4.8 \mathrm{U} / \mathrm{L}$. Moreover, $58.0 \%$ were using prednisone (median dose of $5 \mathrm{mg} /$ day) and $58.0 \%$ were in use at least one immunosuppressive drugs. Serum level of IL-17 was $0.49 \mathrm{vs} .0 .35 \mathrm{pg} / \mathrm{mL}$ $(\mathrm{P}=0.009)$, respectively, in dermatomyositis and control group. In addition, serum level of IL-17 correlated inversely with MMT-8 (rho=0.408 ; Spearman correlation), and positively with MYOACT ( $\mathrm{rho}=0.381$ ), creatine phosphokinase $(\mathrm{rho}=0.447)$, aldolase $(\mathrm{rho}=0.412)$, patient ${ }^{\prime}$ VAS (rho=0.324), physician' VAS (rho $=0.326), \mathrm{P}<0.005$ for all analysis. In addition, IL-17 was detected in inflammatory cell infiltration around perimysium blood vessels by immunohistochemistry.

Conclusions. IL-17 serum levels of dermatomyositis are not only increased, but also correlate with disease activity parameters of patients. These data suggest that IL-17 may be not only a potential marker of dermatomyositis activity, but also as a possible target for treatment.

071

SEXUAL FUNCTION EVALUATION BY VALIDATED INSTRUMENT IN FEMALE JUVENILE IDIOPATHIC ARTHRITIS PATIENTS

Gabriela Ribeiro Viola Ferreira', Renato Bussadori Tomioka², Ana Claudia Grunspun Pitta ${ }^{1}$, Daniela Mencaroni Rodrigues Lourenço', Katia Kozu', Nadia Emi Aikawa', Adriana Maluf Elias Sallum', Lucia Maria Arruda Campos', Carmita Helena Najjar Abdo², Rosa Maria Rodrigues Pereira², Eloisa Bonfá2, Clovis A. Silva'

${ }^{1}$ ICR-HC-FMUSP, SÃO PAULO, SP, Brasil; ${ }^{2} \mathrm{HC}-\mathrm{FMUSP}$, SÃO PAULO, SP, Brasil Advances in Rheumatology 2018, 58(Suppl 1):071

Background: Juvenile idiopathic arthritis (JIA) is a painful and disable disease occurs mainly in females during the adolescence and young adulthood, and may affect sexual performance. There are, however, no studies evaluated sexual function with validated sexual instrument in female adolescent and young adults with JIA.

Material and methods: A cross-sectional study was performed in 21 consecutive JIA (ILAR criteria) and 25 healthy controls from two Pediatric Rheumatology services. The Sexual Quotient Questionnaire for Females (SQQ-F) score was applied and sexual dysfunction was defined with score less then 62 points in SQQ-F. Demographic data, gynecological disturbances, number of sexual activity were also evaluated.

Results and conclusions: The median age [26.5 (17-38.1) vs. 29.3 (19.7-35.8) years, $\mathrm{p}=0.7)]$ and SQQ-F score [75.9 (50-92) vs. 78.2 (5894), $p=0.529$ ] were similar in JIA patients and controls. Sexual dysfunction (particularly desire/sexual fantasies and desire/interest disorders) was observed in $14 \%$ of JIA patients (RF-negative polyarticular), with median of disease activity score-28 (DAS-28) of 1.36 (1.05-3.41). No differences were evidenced of sexual dysfunction between JIA patients and healthy controls $(14.4 \%$ vs. $12 \%, p=1.0)$. The median of number of intercourses, as well as desire/sexual fantasies, desire/ interest, arousal/foreplay, arousal/lubrication, arousal/in tune with partner, penetration/relaxation, pain/penetration, desire/involvement, orgasm and general satisfaction scores were alike in both groups $(p>0.05)$. In conclusion, to our knowledge this was the first study to analyze validated score (SQQ-F), including major domains of female sexual function. In spite chronic disease, sexual function seems to be not affected in young female JIA.

\section{2}

\section{SURVIVABILITY ANALYSIS WITHIN A COHORT OF ELDERLY WITH} FEMUR FRACTURE DUE TO BONE FRAGILTY

Viviane Cristina Uliana Peterle', Natalia Mariana Diogenes Silva de Albuquerque $^{2}$, Amanda Cristina de Souza 2 , Regina Alice Fontes Von Kirchenheim ${ }^{3}$, Ana Paula Monteiro Gomides ${ }^{4}$

${ }^{1}$ SECRETARIA DE ESTADO E SAÚDE DO DF, BRASÍLIA, DF, Brasil;

${ }^{2}$ UNICEUB, BRASÍLIA, DF, Brasil; ${ }^{3}$ SESDF, BRASÍLIA, DF, Brasil; ${ }^{4} U N B /$

UNICEUB, BRASÍLIA, DF, Brasil

Advances in Rheumatology 2018, 58(Suppl 1):072 
Background: Hip fracture is correlated with high mortality rates. Considering that the fracture risk fluctuate in-between populations, the study's goal was to analyze the factors associated with in-hospital mortality due to femur fracture and bone fragility when correlating to survival time.

Materials and Method: The sample was analyzed at a public hospital throughout 7 years (January 2010 to January 2017), and it was composed by a cohort of 349 patients older than 60 years with record of hip fractures caused by minimum trauma at the moment of admission. The chosen variables were taken from hospital records of medical charts. In order to evaluate which variables have any impact on the death outcome and the odds ratio of selected predictors, a logistic regression model was built. The R2 of the Nagelkerke model represented 0.80 , thus explaining $80 \%$ of the answer's variability. Furthermore, Kaplan-Meier's survivability estimator with log-rank test and Cox regression were made.

Results and Conclusion: The sample had a predominance of women $(n=229)$ with a mean of $79.7 \pm 0.5$ years. When observing the logistic regression analysis adjusted for age and sex [IC95\%], the variables that explained mortality through the model $(p<0.05)$ were due to the presence of infection $O R=5.7(p 0,026)$, Respiratory Infection $O R$ $=7.38(\mathrm{p} 0.006)$, time at ICU OR $=1.10(\mathrm{p} 0.004)$ and Arterial Hypertension $O R=4.05(p 0.003)$. The surgical procedures were also selected by the COX regression model. Patients who underwent osteosynthesis had a lower survival time [HR $=2.1$ IC95\% 1.03-4.41; $p=0.005$ ] when compared to those who underwent arthroplasty. The Kaplan Meier's estimator verified that since the patient's hospitalization, the mean survival time was 62.7 days [IC95\% 47.8-77.6]. Considering only patients who underwent surgery and the post-operative time, it was observed that the average survival time was 39.2 days. [IC95\% 26.3453.33;]. Therefore, it is noticeable the importance of identifying the factors associated with fracture pre, peri and post-operative and correlate them with the event of mortality. Thereby, Osteoporosis constitute an alarming public health issue because of its association with age-related fractures, thus being vital to incorporate interventions in the health system planning to mitigate the various levels of risk.

\section{5}

SYMPTOMATIC POLYAUTOIMMUNITY AT DIAGNOSIS OF 1,463 CHILDHOOD-ONSET LUPUS: A BRAZILIAN MULTICENTER STUDY

Debora N. Setoue ${ }^{1}$, Ana Claudia Grunspun Pitta ${ }^{2}$, Fernanda J. Fiorot ${ }^{2}$ ${ }^{1}$ HC-FMUSP, SÃO PAULO, SP, Brasil; ${ }^{2}$ ICR-HC-FMUSP, SÃO PAULO, SP, Brasil

Advances in Rheumatology 2018, 58(Suppl 1):075

Background: Childhood-onset systemic lupus erythematosus (CSLE) is a chronic autoimmune illness with a broad clinical and laboratory spectrum, which may involve any organs and systems. Polyautoimmunity (PA), is defined according to the presence of more than one autoimmune diseases (AD) in each patient and multiple autoimmune syndrome (MAS) was defined as three or more AD. However, to our knowledge the prevalence of PA in CSLE in a large series has not been studied. The objective of study was to evaluate symptomatic PA and MAS at CSLE diagnosis.

Material and methods: A multicenter retrospective study was performed in 1,463 CSLE (ACR criteria) patients from 27 Pediatric Rheumatology services. Symptomatic PA was defined according to the presence of more than one concomitant autoimmune disease (AD) and symptomatic multiple autoimmune syndrome (MAS) was defined as three or more AD. An investigator meeting was held to define the protocol. Demographic data, SLICC classification criteria and SLEDAI-2K were evaluated.
Results and conclusions: At CSLE diagnosis symptomatic PA was observed in 144/1,463 (9.8\%) and symptomatic MAS occurred in solely $10 / 1,463(0.7 \%)$. In the former group the more frequently observed associated $A D$ were Hashimoto thyroiditis $n=42 / 144(29 \%)$, antiphospholipid syndrome $n=42 / 144$ (29\%), autoimmune hepatitis $n=26$ / $144(18 \%)$ and type 1 diabetes mellitus $n=23 / 144$ (15.9\%). Further comparisons between CSLE patients with and without PA showed a higher median age $(p=0.016)$ and lower mean SLICC criteria $(p=0.039)$ in those with PA. Additionally, these CSLE patients had less renal involvement ( $35 \%$ vs. $44 \%, p=0.038$ ) and red blood cell cast $(6 \%$ vs. $12 \%, p=0.042)$ and more antiphospholipid antibodies $(29 \%$ vs. $15 \%, p<0.0001$ ). Approximately $10 \%$ of cSLE had symptomatic PA at diagnosis, particularly endocrine autoimmune disorders and antiphospholipid syndrome. Lupus was characterized by a mild disease onset and MAS was infrequently evidenced. Further studies are necessary to determine if this subgroup of cSLE patients have a distinct genetic background with a less severe disease and a better longterm outcome.

\section{6}

SYSTEMIC LUPUS ERYTHEMATOSUS AFTER CHIKUNGUNYA FEVER:

\section{TRIGGER OR COINCIDENCE?}

Claudia Diniz Lopes Marques', Henrique Ataíde Mariz', Illana Costa²,

Camila Lucena ${ }^{2}$, Lays Martins ${ }^{2}$, Lilian Valadares ${ }^{3}$, Eutilia Freire ${ }^{4}$, Izaias

Pereira da Costa ${ }^{5}$, Jose Fernando Verztman ${ }^{6}$

Angela Luzia Branco Pinto Duarte ${ }^{2}$

1 UNIVERSIDADE FEDERAL DE PERNAMBUCO, RECIFE, PE, Brasil;

${ }^{2}$ HOSPITAL DAS CLÍNICAS - UNIVERSIDADE FEDERAL DE PERNAMBUCO,

RECIFE, PE, Brasil; ${ }^{3}$ HOSPITAL GETULIO VARGAS, RECIFE, PE, Brasil;

${ }^{4}$ HOSPITAL UNIVERSITARIO LAURO WANDERLEY- UNIVERSIDADE

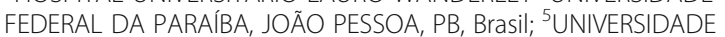

FEDERAL DO MATO GROSSO DO SUL, CAMPO GRANDE, MS, Brasil;

${ }^{6}$ HSFE-RJ, RIO DE JANEIRO, RJ, Brasil

Advances in Rheumatology 2018, 58(Suppl 1):076

Background: Virus infection has been implicated in the initiation of autoimmune disease (DAl) through an immune response, critical for viral clearance. Immune regulatory mechanisms may fail, culminating in the breakdown of self-tolerance, resulting in immune-mediated attack directed against both viral and self-antigens. Different viruses have been implicated in systemic lupus erythematosus (SLE) pathogenesis. However, there are no case reports in the literature of SLE development after Chikungunya virus (CHIKV) infection. The aim of this study is to describe the clinical characteristics of patients who evolved with SLE after CHIKV infection.

Material and Methods: A cross-sectional retrospective case series study, of patients diagnosed with SLE after CHIKV infection confirmed by lgM or IgG serology.

Results and conclusions: Nine patients previously healthy were included, all women, with a mean age of 42.3 ( \pm 10.41$)$ years. Regarding Chikungunya (CHIK) symptoms, all had fever and joint pain in the acute phase and $75 \%$ had arthritis and rash; $66.7 \%$ had used corticosteroids before the diagnosis of DAl. The median time between the acute infection and the onset of autoimmune symptoms was 6 months (IQR = 6), but one patient, it was 7 days after the onset of CHIK symptoms. The SLE criteria observed at the time of diagnosis can be seen in Table 1. The ANA titers ranged from $1 / 320$ to $1 / 1280$, with a homogeneous pattern in $45 \%$ of cases. The median time to onset of nephritis after diagnosis was 2 months (IQR $=4.5)$. All patients used prednisone at the start of treatment; hydroxychloroquine was used for $66.7 \%$ of the cases; azathioprine in $55.0 \%$ and mycophenolate mofetil in $33.3 \%$., pulsotherapy with methylprednisolone 
in $44.4 \%$ and cyclophosphamide in $22.2 \%$. Although it is not possible to state that CHIKV was a trigger for the development of SLE with this study, it is rational to imagine that, as with other infections, it has been the trigger for autoimmunity, since there is a close temporal relationship with the infection. Unlike patients who develop chronic arthritis similar to rheumatoid arthritis, this would be another form of evolution following CHIKV infection.

Table 1 (abstract 076). SLE criteria observed at the time of diagnosis

\begin{tabular}{lll}
\hline Criteria & $\mathrm{n}$ & $\%$ \\
\hline Subacute cutaneous lupus & 5 & 55.6 \\
Malar rash & 6 & 66.7 \\
Discoide rash & 1 & 11.1 \\
Arthritis & 8 & 88.9 \\
Arthralgia & 9 & 100.0 \\
Alopecia & 7 & 77.8 \\
Oral ulcers & 5 & 55.6 \\
Hemolytic anemia & 6 & 66.7 \\
Leukopenia & 6 & 66.7 \\
Lymphopenia & 6 & 66.7 \\
Thrombocytopenia & 3 & 33.3 \\
Serositis & 3 & 33.3 \\
Neurologic & 2 & 22.2 \\
Nephritis & 5 & 55.6 \\
ANA positive & 9 & 100.0 \\
Anti-DNA positive & 5 & 55.6 \\
Anti-Sm positive & 0 & 0.0 \\
Anti-cardiolipine positive & 2 & 22.2 \\
Low complement & 7 & 77.8 \\
Coombs positive & 5 & 55.6 \\
\hline & & \\
\hline & &
\end{tabular}

\section{7}

TIME OF USE AND CAUSES OF INTERRUPTION OF SYNTHETIC MEDICATIONS IN RHEUMATOID ARTHRITIS: REAL LIFE DATA IN BRAZIL

Ana Paula Monteiro Gomides ${ }^{1}$, Licia Maria Henrique da Mota², Geraldo Rocha Castelar ${ }^{3}$, Cleandro Pires de Albuquerque ${ }^{2}$, Ana Beatriz VargasSantos ${ }^{3}$, Letícia Rocha Pereira ${ }^{3}$, Manoel Barros Bertolo ${ }^{4}$, Alisson Aliel Vigano Pugliesi ${ }^{4}$, Eduardo de Almeida Macedo ${ }^{4}$, Paulo Louzada Filho ${ }^{5}$, Maria de Fátima L da Cunha Sauma ${ }^{6}$, Marcel Lobato Sauma ${ }^{7}$, Júlia Brito de Medeiros ${ }^{7}$, Claiton Viegas Brenol ${ }^{8}$, Ivanio Alves Pereira ${ }^{9}$, Sebastiao Radominski ${ }^{10}$, Maria Fernanda Resende ${ }^{11}$, Maria Raquel Costa Pinto ${ }^{11}$, Gustavo Gomes Resende ${ }^{11}$, Karina Bonfiglioli'12, Rina Dalva Neubarth Giorgi $^{13}$, Nathalia de Carvalho Sacilotto ${ }^{13}$, Henrique Carriço ${ }^{12}$

${ }^{1}$ UNB/UNICEUB, BRASILIA, DF, Brasil; ${ }^{2}$ UNB, BRASÍLIA, DF, Brasil; ${ }^{3} U E R J$, RIO DE JANEIRO, RJ, Brasil; ${ }^{4}$ UNICAMP, CAMPINAS, SP, Brasil; ${ }^{5}$ USP RIBEIRÃO PRETO, RIBEIRÃO PRETO, SP, Brasil; ${ }^{6} U F$ DO PARÁ, BELÉM, PA, Brasil; ${ }^{7}$ UF PARÁ, BELEM, PA, Brasil; '8UFRGS-, PORTO ALEGRE, RS, Brasil; ' $U F S C$, FLORIANÓPOLIS, SC, Brasil; ; UF PARANÁ, CURITIBA, PARANÁ, Brasil; ${ }^{11}$ UFMG, BELO HORIZONTE, MG, Brasil; ${ }^{12}$ USP, SÃO PAULO, SP, Brasil; ${ }^{13}$ HSPE-SP, SÃO PAULO, SP, Brasil

Advances in Rheumatology 2018, 58(Suppl 1):077

Background: The treatment of rheumatoid arthritis (RA) has undergone significant changes in the last decade. Despite this, conventional synthetic disease-modifying drugs (cs-DMARD) remain essential and first choice, most often for naive patients. Given the scarcity of epidemiological data in Brazil, knowledge of the practical use of these drugs in the country is of fundamental importance. The objective of this study was to evaluate the frequency and time of use of cs-DMARD in a large Brazilian population with RA, as well as the main reasons for interruption.

Materials and Methods: A multicentric cohort of RA patients from 11 public treatment centers in different regions of Brazil was performed. The participants underwent clinical evaluation, analysis of laboratory tests and medical records from the beginning of their medical care. The present study contains data from the initial evaluation of patients who were followed up for 1 year.

Results and Conclusions: The total sample included 1125 patients, with $89.5 \%$ being women. $78.73 \%$ had a positive rheumatoid factor. The median DAS was 3.52 and the CDAI was 9.1022 (90.84\%) used conventional cs-DMARDs (isolated or in combination) and 406 (36.09\%) used biological disease-modifying drugs (bDMARDs). The most used therapeutic regimen was the combination MTX + leflunomide, with or without corticosteroids (142/12.6\%). The most used csDMARD and their time of use can be seen in Table 1. The general causes of drug discontinuation can be seen in Table 2 and the medical causes of suspension in Table 3. Given these real-life data, we can conclude that there is a high frequency of use of cs-DMARDs in Brazil, both in monotherapy and in combination, which are often used for long periods. Knowledge of drug discontinuation motifs and adequate monitoring of side effects may favor the use of these therapeutic classes, which are known to be effective and low cost.

\section{0}

\section{USE OF PSYCHOTROPICS IN PATIENTS WITH RHEUMATOID} ARTHRITIS: DATA OF AN IMPORTANT MULTICENTRIC COHORT IN BRAZIL

Ana Paula Monteiro Gomides ', Licia Maria Henrique da Mota' ${ }^{2}$ Geraldo Rocha Castelar ${ }^{3}$, Cleandro Pires de Albuquerque 2 , Ana Beatriz VargasSantos ${ }^{3}$, Manoel Barros Bertolo ${ }^{4}$, Alisson Aliel Vigano Pugliesi ${ }^{4}$, Eduardo de Almeida Macedo ${ }^{4}$, Paulo Louzada Filho ${ }^{5}$, Maria de Fátima $L$ da Cunha Sauma ${ }^{6}$, Marcel Lobato Sauma ${ }^{6}$, Júlia Brito de Medeiros ${ }^{6}$, Claiton Viegas Brenol $^{7}$, Ivanio Alves Pereira ${ }^{8}$, Sebastiao Radominski ${ }^{9}$, Maria Fernanda Resende ${ }^{10}$, Maria Raquel Costa Pinto ${ }^{10}$, Gustavo Gomes Resende ${ }^{10}$, Karina Bonfiglioli ${ }^{11}$, Henrique Carriço ${ }^{11}$, Rina Dalva Neubarth Giorgi ${ }^{12}$, Nathalia de Carvalho Sacilotto ${ }^{12}$, Letícia Rocha Pereira ${ }^{3}$

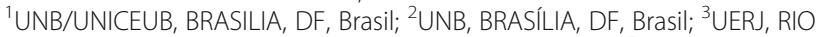
DE JANEIRO, RJ, Brasil; ${ }^{4} U N I C A M P$, CAMPINAS, SP, Brasil; ${ }^{5}$ USP RIBEIRÃO PRETO, RIBEIRÃO PRETO, SP, Brasil; ' UF DO PARÁ, BELÉM, PA, Brasil; ${ }^{7}$ UFRGS, PORTO ALEGRE, RS, Brasil; ${ }^{8}$ UFSC, FLORIANOPOLIS, SC, Brasil; ' $9 F$ PARANÁ, CURITIBA, PARANÁ, Brasil; ${ }^{10}$ UFMG, BELO HORIZONTE, MG, Brasil; ${ }^{11}$ USP, SÃO PAULO, SP, Brasil; ${ }^{12}$ HSPE-SP, SÃO PAULO, SP, Brasil Advances in Rheumatology 2018, 58(Suppl 1):080

Introduction: Indications for use of psychotropic drugs (antidepressants, anxiolytics and mood stabilizers) in rheumatoid arthritis (RA) range from the treatment of psychiatric comorbidities concomitant with the disease (mainly anxiety and depression) to as adjuncts in chronic pain management. If on the one hand they can favor a better balance of patients globally, on the other hand they can trigger new symptoms due to side effects and drug interactions. Data on the actual frequency of use of these therapeutic classes in RA are unknown in the literature and this work, in a pioneering way, intends to analyze the subject in a large "real life" population in Brazil.

Material And Methods: A multicenter study was conducted in Brazil with the participation of 11 public hospitals in different states. Patients should be undergoing treatment for RA and evaluated through clinical examination and analysis of complementary exams. Medical records with a history of the disease since diagnosis were analyzed.

This study was a cross section corresponding to the initial evaluation of the participants.

Results and Conclusions: 1117 RA patients were evaluated in total. $89 \%$ were women with a median age of 56.6 years. $78.73 \%$ had positive rheumatoid factor and $55.2 \%$ had erosive disease. The median DAS 28 was $3.52 \%$ and the HAQ median was 0.87 .

94 patients $(8.4 \%)$ used some psychotropic drugs at the time of the evaluation, and 15 (1.34\%) used 2 or more drugs of these therapeutic classes concomitantly. Antidepressants were the most commonly 
used drugs with 86 patients $(7.7 \%)$, the most used being shown in Table 1.16 patients (1.4\%) reported using anxiolytics and only 1 $(0.09 \%)$ were using a mood stabilizer.

Changes in mental health are known to be frequent in patients with chronic diseases, especially when there are persistent painful pictures. Rational treatment of psychiatric symptoms is desirable for RA patients and may impact the response to treatment of the underlying disease. The knowledge of how it has been done to approach these patients in medical practice, avoiding excessive use of medications but also underdiagnostics, is of fundamental importance in rheumatology. This paper provides information on a large Brazilian population treated in public referral centers in the treatment of RA. Further studies should be performed to confirm these data.

Table 1 (abstract 080). Antidepressants most used in the sample

\begin{tabular}{lll}
\hline DRUG & N & $\%$ \\
\hline FLUOXETINE & 40 & 46.5 \\
AMITRIPTYLINE & 26 & 30.2 \\
SERTRALINE & 13 & 15.1 \\
CITALOPRAM & 5 & 5.8 \\
PAROXETINE & 3 & 3.5 \\
DULOXETINE & 3 & 3.5 \\
\hline
\end{tabular}

\section{1}

VACCINAL COVERAGE AMONG SLE PATIENTS AND REASONS FOR

\section{NON ADHERENCE}

Gabriela Amorim Mattos, Raquel de Melo Legal Alqusto

UNIVERSIDADE DO ESTADO DO RIO DE JANEIRO, RIO DE JANEIRO, RJ, Brasil

Advances in Rheumatology 2018, 58(Suppl 1):081

Background: Systemic lupus erythematosus (SLE) is an autoimmune disease that is associated with multiple infections due to immune system compromise and/or immunosuppressors (IS) use both causing high morbimortality. Vaccines are the main preventive procedure in this scenario and are strongly recommended by most medical societies. However vaccination coverage has been reported to be insufficient even in developed countries and its reasons are not well defined which comprehension may allow interventions as a window of opportunity to increase immunization coverage.

Methods: This is a cross-sectional observational study performed at a university hospital that included adults patients with SLE (ACR criteria). A semi-structured questionnaire was applied to identify vaccination coverage according to the recommendations of the National Brazilian Immunization Program (PNI-MS) and the Brazilian Society of Immunizations (SBIM). Socioeconomic variables and reasons for not receiving the vaccines were identified.

Results: We studied 174 lupus of whom 147 were receiving steroid and/or imunossupressors (IS) and were classified as immunosuppressed. The vaccinal coverage according to the PNI-MS was found to be $2,7 \%$ for SLE patients on IS and of $14,8 \%$ among those not receiving these agents. According to the SBIM recommendations, no patient (with or without IS) had a complete vaccinal coverage. There was no association of years of formal education (12 years at school) and vaccine coverage $(p>0,05)$. The main patient referred reason for not having received the vaccines were lack of medical recommendation which varied from $60 \%$ (MMR) up to $100 \%$ (Meningococcus C). For Pneumococcus, lack of medical recommendation was reported by $88 \%$ of patients. Other factors that also negatively influenced vaccination coverage were lack access, fear of adverse events, medical contraindication and not paying attention to medical prescription.

Discussion and Conclusions: In the present study we found a very low vaccination coverage for immunosuppressed patients that is inferior to other previously reported in the general population, and it may contribute for the high rates of infection found among these patients. At the same time, one of the main reasons for not adherence to vaccination protocols seems to be lack of knowledge and/or medical fear of complications that is in fact a paradox, since infections are frequent causes of morbimortality. The present data should stimulate interventions among patients and their physicians to improve immunizations.

Consent for publication

The authors declare that they have obtained informed written consent from the patient's tutors for publication

\section{2}

VITAMIN D DOWNREGULATES PRO-FIBROTIC GENE EXPRESSION: IS IT A POTENTIAL MOLECULE FOR TREATMENT OF SYSTEMIC SCLEROSIS?

Anderson Rodrigues De Almeida', Andréa Tavares Dantas ${ }^{2}$, Marina Ferraz Cordeiro', Michelly Cristiny Pereira ${ }^{3}$, Rafaela Silva Guimarães Gonçalves²,

Moacyr Jesus Barreto De Melo Rego ${ }^{3}$, Angela Luzia Branco Pínto

Duarte $^{2}$, Maira Galdino Da Rocha Pitta ${ }^{3}$

'LINAT/NUPIT-SG/UNIVERSIDADE FEDERAL DE PERNAMBUCO, RECIFE, PE, Brasil; ${ }^{2}$ HOSPITAL DAS CLIINICAS - UNIVERSIDADE FEDERAL DE

PERNAMBUCO, RECIFE, PERNAMBUCO, Brasil; ${ }^{3}$ LINAT/NUPIT-SG/

UNIVERSIDADE FEDERAL DE PERNAMBUCO, RECIFE, PERNAMBUCO, Brasil Advances in Rheumatology 2018, 58(Suppl 1):082

Background: Fibrosis is the major mechanisms of systemic sclerosis (SSc) that contributes to increased mortality and decreased quality of life of patients. Transforming growth factor beta (TGF- $\beta$ ) is the main pro-fibrotic cytokine involved in the development of fibrosis in SSc, promotes the activation of fibroblasts and leads to an increase in the expression of molecules that act favoring the development of pathological fibrosis, mainly type I collagen and alpha smooth muscle actin (a-SMA). Thus, molecules involved in the fibrotic process may represent important therapeutic targets in SSc. This study aimed to evaluate the effects of 1,25-Dihydroxyvitamin $D$ on the gene expression of ACTA2, IL6 and COL1A1 in healthy skin and lung fibroblasts stimulated with TGF- $\beta$.

Material and Methods: Healthy fibroblast lines of skin (HFF-1) and lung (MRC-5) were obtained from Rio de Janeiro Cell Bank (BCRJ). Cells were plated in six well plates $(1 \times 104$ cells/well), stimulated with $5 \mathrm{ng} / \mathrm{mL}$ of recombinant TGF- $\beta 1$, and treated with $1 a, 25$-Dihydroxyvitamin D3 at the concentration of $100 \mathrm{nM}$. After six hours of incubation, the total RNA was extracted using TRI Reagen (Sigma-Aldrich, St. Louis, MO, USA) and CDNA synthesis was performed from $500 \mathrm{ng}$ using GoScriptTM Reverse Transcription Mix (Promega, Madison, WI, USA). Quantitative analysis of the ACTA2, COL1A1 and IL6 transcripts was performed by Real Time PCR. The $18 \mathrm{~S}$ ribosomal gene was used as reference gene. The calculation of relative gene expression was performed using the 2- $\Delta \Delta \mathrm{Ct}$ methods.

Results and Conclusions: We observed that treatment with vitamin $D$ promoted a significant reduction in the gene expression of ACTA2 $(p<0.05)$, but did not significantly alter the expression of COL $1 A 1$ and 
IL6 in healthy skin fibroblasts (Fig. 1a). Additionally, vitamin D downregulated ACTA2, COL1A1 and IL6 expression in lung fibroblasts, but these results were not statistically significant (Fig. 1b). In conclusion, the results showed that treatment with vitamin $D$ promoted the reduction in the expression of ACTA2 in skin fibroblasts, suggesting an antifibrotic effect. However, further studies are needed to better understand the real role of vitamin D in SSc treatment.

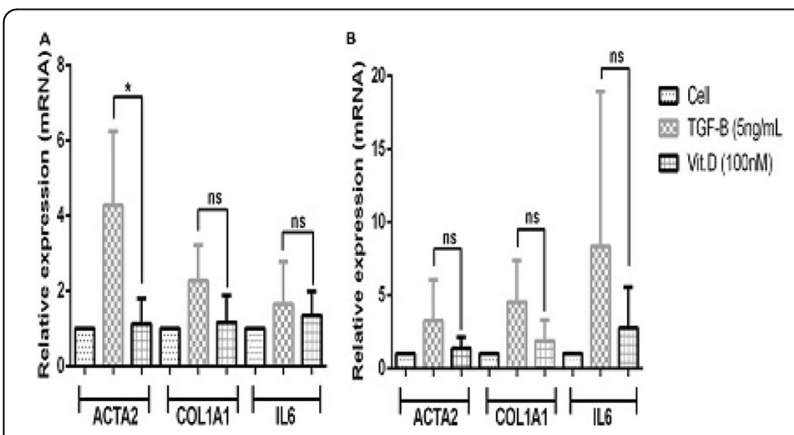

Fig. 1 (abstract 082). Evaluation of 1,25(OH)2D3 treatment on the gene expression of ACTA2, COL1A1, and IL6 in healthy skin (HFF-1) (a) and lung (MRC-5) (b) fibroblasts. Values of relative quantification after normalization with reference gene $18 \mathrm{~S}$ through application

\section{P001}

5-YEAR EFFICACY AND SAFETY OF APREMILAST TREATMENT IN SUBJECTS WITH PSA: A POOLED ANALYSIS OF THE 3 PHASE III STUDIES

Priscila Magalhaes Reis Nakasato

CELGENE, Estados Unidos

Advances in Rheumatology 2018, 58(Suppl 1):P001

Long-term efficacy and safety of APR treatment were evaluated up to 5 years in subjects with active psoriatic arthritis (PsA) from the phase III PALACE 1-3 studies.

Subjects were randomized (1:1:1) to placebo (PBO), APR $30 \mathrm{mg}$ twice daily (APR30), or APR $20 \mathrm{mg}$ twice daily (APR20). PBO subjects were re-randomized (1:1) to APR30 or APR20 at Week 16 (early escape) or Week 24. Double-blind APR treatment continued to Week 52; subjects could continue APR during an open-label, long-term treatment phase up to 5 years. Safety was assessed at each study visit.

1,493 subjects were randomized and received $\geq 1$ dose of study medication (PBO: $n=495 ;$ APR30: $n=497 ;$ APR20: $n=501$ ). Of those randomized to APR30 at baseline, Week 16, or Week 24, 45.9\% (331/ 721) completed 260 weeks of study. At Week 52, ACR20/ACR50/ ACR70 response rates were $55.3 \% / 26.1 \% / 11.9 \%$ for APR30 subjects, regardless of when APR was started (baseline, Week 16, or Week 24); sustained response rates of $67.2 \% / 44.4 \% / 27.4 \%$ were observed with continued APR30 treatment at Week 260. Marked SJC improvements were seen, with mean percent reduction of $82.3 \%$ at Week 260; TJC reduction was $72.7 \%$. At Week 260, 62.4\% (136/218) of APR30 subjects with baseline enthesitis achieved a Maastricht Ankylosing Spondylitis Enthesitis Score of 0; 80.9\% (114/141) with baseline dactylitis achieved a dactylitis count of 0. A HAQ-DI MCID of $\geq 0.35$ was achieved by $52.6 \%$ of APR30 subjects at Week 260, and $60.4 \%$ achieved low disease activity and remission, defined as a Clinical Disease Activity in Psoriatic Arthritis score $\leq 13$. Sustained responses in psoriatic skin involvement were observed with continued treatment at Week 260 in APR30 subjects with $\geq 3 \%$ psoriasis body surface area involvement at baseline, with response rates of $65.8 \%(98 / 149)$ and $43.6 \%(65 / 149)$ in those achieving PASI-50 and PASI-75 responses, respectively. Consistent efficacy results were seen across the individual studies. No new safety concerns were identified with APR up to 260 weeks. Adverse events (AEs) in $\geq 5 \%$ of APR30 subjects entering the 5th year of APR exposure were nasopharyngitis (6.6\%) and upper respiratory tract infection (5.8\%); most AEs were mild/moderate in severity. Among APR30-exposed subjects, serious AEs occurred in 5.8\% in Weeks $>208$ to $\leq 260 ; 5$ subjects discontinued due to an AE.

APR demonstrated sustained, clinically meaningful improvements in PsA signs/symptoms, physical function, and associated psoriasis in subjects continuing treatment over 5 years. APR continued to demonstrate a favorable safety profile and was generally well tolerated at 5 years.

\section{P003}

A CASE OF REFRACTORY PSORIATIC ARTHRITIS WITH EXCELLENT RESPONSE TO THE FOURTH IMMUNOBIOLOGICAL DRUG ATTEMPT

Lucas Androczevecz Silva, Diogo Cunha Lacerda, Leonardo Michaelis Schmidt, Rodrigo Zuber Maciel, Rubia Carla Cappellari Tolentino, Leonardo Trovo Zilotti, Giovana Balbinot, Rafaela Sorpile Araújo, Polyana Klomfass Piati, Hugo Ogassawara Bioni CENTRO UNIVERSITÁRIO FAG, CASCAVEL, PARANÁ, Brasil Advances in Rheumatology 2018, 58(Suppl 1):P003

Background: Psoriatic arthritis is an inflammatory disease, soronegative, associated to cutaneous psoriasis and has variable presentations and clinical courses. Nowadays, the treatment normally consists by the use of DMARDS and anti-TNF medications. In refractory situations to the conventional therapy with DMARDS, some other drugs must be considered, like the use of anti-TNF drugs, the use of ustequinumabe (which is a human monoclonal antibody that binds to the IL12 and IL-23 citokynes) and the use of secuquinumabe (which is an antibody against IL-17 citokyne).

Case Report: Female patient, 43 years old, farmer, diagnosed with psoriasis when she was only 8 months old. By the age of 12 her hands and knees started to be affected by arthritis. When she was first biochemically evaluated, inflammatory markers were high, RF was negative and HLAB-27 was positive. Simple radiography showed no sacroiliac involvement. Methotrexate was started 10 years ago and, 2 years ago, was used etanercept, which resulted in secondary failure (arthritis and cutaneous lesions continued to progress). Taking that in consideration, etanercept was exchanged by ustequinumabe (which has a different mechanism of action), but it resulted in a worsening of the cutaneous and joint condition. Therefore, ustequinumabe was exchanged by a third immunobiological drug: infliximab. The clinical response to the induction dose of infliximab was satisfactory, however the disease continued to progress after a while (even after the dose was increased and the space between them was reduced). Motivated by the unsuccessful clinical response of three immunobiological medications, a fourth and last adalimumabe was attempted and the patient showed excellent response. After three months of adalimumabe, the dermatological lesions and the arthritis demonstrated fantastic answer to the treatment.

Conclusion: The way of treating psoriatic arthritis may vary according to presentation and severity that the disease manifests. In cases which the condition is refractory to DMARDS, immunobiological therapy must be considered. The advent of this new therapeutic modality (biological drugs) emerged bringing hope of adequate treatment in situations which DMARDS weren't enough. In the present case, the expected therapeutic response was only reached after attempts with 1 DMARDS (methotrexate) and 4 biological drugs. Therefore, the exposed situation exalts that the discrepancy between the clinical response to different immunobiological agents may be notable, and the persistence of the therapy on the look out for the right treatment can be of extreme benefit to the patient.

\section{Consent for publication}

The authors declare that they have obtained informed written consent from the patient's tutors for publication 
P006

A CROSS-SECTIONAL EVALUATION OF A BRAZILIAN SPONDYLOARTHRITIS SINGLE-CENTER TERTIARY COHORT: CLINICAL AND TREATMENT DATA

Andrea Shimabuco, Julio Bertacini de Moraes, Percival Sampaio-Barros, Claudia Goldenstein-Schainberg, Celio Roberto Gonçalves, Carla Gonçalves Schahin Saad

DISCIPLINA DE REUMATOLOGIA DA FACULDADE DE MEDICINA DA

UNIVERSIDADE DE SÃO PAULO, SÃO PAULO, SP, Brasil

Advances in Rheumatology 2018, 58(Suppl 1):P006

Background: The use of synthetic DMARDS (sDMARDs) in spondyloarthritis (SpA) has been increasingly questioned and restricted to peripheral disease, on the other hand, the use of immunobiological agents for the treatment of $\mathrm{SpA}$ has been further improved by the release of new drugs with other mechanisms than anti-TNF, such as secukinumab (anti-IL17, SEC) and ustekinumab (anti-IL12/23, UST). The objective of our study is to describe clinical and treatment data of a SpA patients cohort followed at the outpatient clinic of a Brazilian single center.

Methods: 516 SpA patients evaluated from January 2017 to January 2018. Data from electronic medical records assessed including diagnosis, disease characteristics, treatment and disease activity at the last visit.

Results: Among all patients 195 (37.8\%) were classified as Ankylosing Spondylitis (AS), 198 (38.3\%) psoriasis arthritis (PsA), 66 (12.8\%) axial non-radiographic or peripheral SpA, 42 (8.1\%) SpA related to inflammatory bowel disease and $15(3.0 \%)$ as reactive arthritis patients. From all SpA patients 190 (36.8\%) have no axial disease, with isolated peripheral arthritis. Regarding treatment, $321(62.2 \%)$ patients used sDMARDs as monotherapy or in association [156/321 (48.6\%) methotrexate (MTX); $125 / 321$ (38.9\%) sulfasalazine (SSZ); 53/321 (10.3\%) leflunomide (LFN) and 30/321 (9.3\%) other sDMARDs] 298 (57.7\%) patients used NSAIDs. Concerning biological therapy 204 (39,5\%) received biological DMARDs (bDMARDs) [68 infliximab (IFX), 59 adalimumab (ADA), 35 etanercept (ETA), 6 golimumab (GOL), 2 certolizumab pegol (CTZ), 23 secukinumab (SEC), 10 ustekinumab (UST), 1 rituximab (RTX)]. AS patients 43/52 (82.7\%) are HLA-B27 positive; 152/195 (77.9\%) received NSAIDs; 95/195 (48.7\%) used sDMARDs (23.1\% MTX and $72.6 \%$ SSZ) and 79/195 (40.5\%) used bDMARDs (31 INF, 22 ADA, 19 ETA, 3 GOL, 3 SEC, 1 UST). Among the 198 PsA patients 148/198 (74.7\%) have solely peripheral involvement; 146/198 (73.7\%) were under sDMARDs (78.8\% MTX and $26.7 \%$ LNF) and 77/ 198 38.9\% were receiving bDMARDs (24 IFX, 18 ADA, 10 ETA, 19 SEC, 6 UST, 1 RTX). Concerning disease activity, 25/125 (20\%) of AS patients had ASDAS $\geq 2.1$ and 42/198 (21\%) of PsA patients had active arthritis in the last visit.

Conclusions: The description of epidemiological and clinical data of this cohort reinforces high prevalence of peripheral disease in Brazilian SpA patients. This fact could explain the wide use of sDMARDs in these patients. The frequency use of bDMARDs is in parallel with literature data including non-antiTNF drugs as SEC and UST.

\section{P016}

\section{ACUTE SACROILIITIS SECONDARY TO ISOTRETINOIN}

Guilherme Leví Tres, Ana Laura Didonet Moro, Luiza Rossi, Bruna de Lima Porto, Micheline Sulzbacher Batista, Afonso Papke, Fernando Schmidt Fernandes, Vanessa Hax HOSPITAL DE CLÍNICAS DE PORTO ALEGRE, PORTO ALEGRE, RS, Brasil Advances in Rheumatology 2018, 58(Suppl 1):P016

Background: Isotretinoin is a synthetic vitamin A derivative regarded as the most effective agent in the treatment of acne. Despite this, the rheumatic side-effects of this drug are still unknown for many rheumatologists. Our aim is to report a case of isotretinoin mimetizing a rheumatologic disease and its evolution.

Case report: A 16 year old male patient presented at the rheumatologic clinic complaining of severe progressive back pain of sudden onset with inability to walk. Over the last 2 weeks, he had already received 2 doses of intramuscular corticoid with no relief. He was previously healthy, except for moderate acne, which he was treating with isotretinoin. The back pain started 20 days after the beginning of isotretinoin and the drug was discontinued after 6 weeks due persistent pain. At his first rheumatologic visit, he was already 30 days without the medication. Examination revealed limitation of back movement in all planes and no peripheral arthritis or enthesitis. The patient had no other findings for SAPHO (synovitis, acne, pustulosis, hyperostosis, and osteitis syndrome) and had no history or signs of active infection or tuberculosis. Magnetic resonance imaging (MRI) showed evidence of bilateral sacroiliitis and osteitis pubis. Full therapeutic dose of nonsteroidal anti-inflammatory drug (NSAID) was prescribed. On the investigation, HLA-B27 antigen was absent, viral serologies were negative, erythrocyte sedimentation rate (ESR) was $68 \mathrm{~mm}$ and C-reactive protein was $0.7 \mathrm{mg} / \mathrm{dL}$. After 2 weeks of NSAID, the pain reduced, and patient ability to walk was completely restored after one month of continuous NSAID. Laboratory control showed normalization of inflammatory markers and the patient no longer had to take NSAIDs. Conclusion: In our case, acute sacroiliitis could be an adverse effect of isotretinoin that improved with its discontinuation and with NSAID. In another case report, patient had to use biological therapy for a short period of time, remaining asymptomatic after drug cessation. Therefore, the rheumatologist should be aware of the possibility of sacroiliitis caused by isotretinoin before establishing a definitive diagnosis of chronic rheumatologic disease.

\section{Consent for publication}

The authors declare that they have obtained informed written consent from the patient's tutors for publication

\section{P018}

Adherence to yellow fever vaccine (YFV) in patients with rheumatic diseases in Brazil: A Real-World Data

Gecilmara S. Pileggi', Priscila S. Torres², Ana Lúcia S. M. Paduelo", Ana Geórgia S. de Almeida ${ }^{4}$, Célia Maria Silva ${ }^{5}$, Eni Maria Silva ${ }^{6}$, Carlos Eduardo D. Tenório ${ }^{7}$, Izabel Teresinha S. Oliveira', Marta Maria S. Azevedo ${ }^{9}$, Samuel Oliveira ${ }^{10}$, Selma C. S. Merenlender ${ }^{11}$, Charlles Heldan M. Castro ${ }^{12}$, Valéria Valim Cristo ${ }^{13}$, Blanca Elena R. G. Bica ${ }^{14}$

Rodrigo Poubel V. Rezende ${ }^{15}$, Lícia Maria H. Mota ${ }^{16}$

${ }^{1}$ HOSPITAL DE MEDICINA DE RIBEIRÃO PRETO - FMUSP-RP, Brasil;

${ }^{2}$ GRUPO ENCONTRAR, Brasil; ${ }^{3}$ GRUPO DE APOIO AO PACIENTE REUMÁTICO DE RIBEIRÃO PRETO E REGIÃO - GRUPAR-RP, Brasil; ${ }^{4} A$ MENINA E O LÚPUS, BraSil; ${ }^{5}$ ASSOCIAÇÃO DE PACIENTES COM DOENÇAS REUMÁTICAS DO ESTADO DO RIO DE JANEIRO RECOMECAR-RJ, Brasil;

${ }^{6}$ GRUPO DE APOIO AO PACIENTE ARTRÍTICO DE SÃO PAULO - GRUPASP, Brasil; ; ${ }^{7}$ ASSOCIAÇÃO BRASILEIRA SUPERANDO O LÚPUS, Brasil;

${ }^{8}$ INSTITUIÇÃO ALUREL SINOS, Brasil; ${ }^{9}$ GRUPO DE APOIO AO PACIENTE REUMÁTICO DO CEARÁ - GARCE, Brasil; ${ }^{10}$ ESPONDILITE BRASIL, Brasil;

${ }^{11}$ SOCIEDADE DE REUMATOLOGIA DO RIO DE JANEIRO, Brasil;

${ }^{12}$ UNIVERSIDADE FEDERAL DE SÃO PAULO - UNIFESP, Brasil;

${ }^{13}$ UNIVERSIDADE FEDERAL DO ESPIRITO SANTO - UBES/ES, Brasil;

${ }^{14}$ UNIVERSIDADE FEDERAL DO RIO DE JANEIRO - UFRJ, Brasil;

${ }^{15}$ UNIVERSIDADE FEDERAL FLUMINENSE, Brasil; ${ }^{16}$ UNIVERSIDADE DE

BRASÍLIA - UNB, Brasil

Advances in Rheumatology 2018, 58(Suppl 1):P018

Since December 2016, Brazil is experiencing an upsurge of yellow fever virus activity. In January 2018, an informative Disease Outbreak News from the World Health Organization (WHO), states the need for control the yellow fever through the Vaccination, since this is the single most important measure for preventing this endemic disease with high mortality rates $(40-50 \%)$.

However, one of the main contraindications for this YFV is immunosuppression, a common condition among rheumatic patients, what raising many questions and hesitations regarding the safety on application of the YFV for this special group. Both, patients and health professionals had been faced with the dilemma, vaccinated or not?

To Understand the real scenario of adherence to the YFV during the mass vaccination campaign, according to the rheumatic patient's perspective.

A qualitative research, conducted through an electronic survey, between February 25 and April 17, 2018, published by social networks 
of associations to support rheumatic patients: Alurel Sinos, Encontrar, Garce, Grupar, Grupasp, Superando o Lúpus, RecomeçAR-RJ and bloggers, Blog Artrite Reumatoide, A Menina e o Lúpus e Espondilite Brasil. All the participants were volunteer, which have given their consent by mail and guarantee back to keep the information anonymous. The survey would reflect the real setting of the patients have or not receiving the YFV shot during the campaign.

We have assessed 675 answers from patients from all over Brazil, with a higher prevalence of urban residents (96.5\%), such as São Paulo (295), Rio de Janeiro (83), Minas Gerais (70) and Distrito Federal (37). The majority of them were patients with rheumatoid arthritis (RA) (318) and systemic lupus erythematosus (169). A total of 240 patients (35,5\%) had already been vaccinated, among them 129 (19\%) were vaccinated prior to diagnosis and 111 after it, and 10\% had received the fractionated dose. Important to note, 68 patients $(58 \%)$ reported the decision to take the vaccine was without consulting their rheumatologist and $42 \%$ (43) (performed the scheduled vaccine after a decision shared with their doctor, planning the medication withdraw. Thus, 77 (66\%) patients had vaccine during immunosuppressive therapy.

Of the 435/675 patients were not vaccinated, 264 (62\%) reported having received this guidance from their doctor, $124(29 \%)$ admitted were afraid to take the vaccine and $36(8 \%)$ patients had the vaccination denied at the health unit. Access to rheumatologic treatment comes from private resources for 201 (29.8\%), 254 (38\%) are cared for by the health plan and 220 (33\%) have access through the public health system.

We are presented the biggest survey involving a real patient's perspective on the YFV facing the actual epidemics settings, where a significant number of patients with rheumatic diseases, living in endemic areas, even at the risk of contracting this fatal disease, remain susceptible and are conflicting on the decision to vaccinate or not. In addition, the number of patients who were vaccinated without consulting the rheumatologist was higher than the number of patients vaccinated with medical planning advising. Highlighting the imminent need of a information regarding a management of vaccination with different approach's: to health professional of caring patients with rheumatic diseases under immunosuppressive therapy, as well to families and patient's education about immunization adherence as a protective measure for their health.

\section{P021}

\section{ADULT-ONSET STILL DISEASE ASSOCIATED WITH} MYOPERICARDITIS AND CARDIAC TAMPONADE -FIRST CASE

\section{REPORT IN BRAZIL}

Othon Moura Pereira Da Silva', Eduardo Thadeu De Oliveira Correia², Leticia Mara Dos Santos Barbetta ${ }^{2}$, Ronaldo Altenburg Odebrecht Curi Gismondi ${ }^{2}$, Luis Otávio Cardoso Mocarzel ${ }^{2}$, Adolpho Xavier De Carvalho Filho $^{2}$, Evandro Tinoco Mesquita ${ }^{2}$

'UNIVERSIDADE FEDERAL FLUMINENSE-UFF, NITÉROI, RJ, Brasil;

${ }^{2}$ UNIVERSIDADE FEDERAL FLUMINENSE, NITERÓl, RIO DE JANEIRO, Brasil Advances in Rheumatology 2018, 58(Suppl 1):P021

Introduction: Autoimmune diseases can affect the cardiovascular system causing pericarditis and/or acute myocarditis. Adult-Onset Still Disease (AOSD) has an unknown etiology, whose clinical findings are non-pathognomonic. This diagnostic is considered after exclusion of disorders such as neoplasias and seronegative autoimmune diseases, with the help of Yamaguchi et al criteria.

Case report: M.P., male, 40 years old with febrile prodrome, sore throat, chest pain, evolving within 48 hours to symmetrical impairment of the knees and ankles, followed by dyspnea. Admitted with electrocardiogram with signs of acute pericarditis, developing cardiac tamponade. X-ray of the thorax showed inflammatory infiltrate, being treated with antibiotic therapy. A pericardial window drainage was performed, withdrawing $350 \mathrm{ml}$ of pericardial fluid, serosanguinolent, with exudative and negative cytology. Extensive evaluation including blood counts, bacterial and viral studies and screening for rheumatoid and nuclear factor were performed, all resulting negative. Symptoms were ceased with Prednisone and Colchicine. After 3 weeks, the patient was rehospitalized with pleuritic chest pain, fever and odynophagia, with leukocytosis $\left(12800 / \mathrm{mm}^{3}\right)$ and neutrophilia, Creactive protein $16 \mathrm{mg} / \mathrm{dL}$ and normal troponin I. Magnetic resonance imaging (late-enhancement) showed evidence of myocarditis. At this internation ferritin values were dosed, which were increased (> 1,500 ng/mL). After one month, with attempt to reduce steroid therapy, the patient presented recurrence of fever and odynophagia. Corticoid dose was increased to $40 \mathrm{mg}$ and methotrexate and hydroxychloroquine were introduced.

Conclusion: The AOSD is based on clinical criterias and negative serological markers. In that way, an approach between cardiologists and internists/rheumatologists is fundamental for considering the AOSD as a differential diagnosis for miopericarditis and for choosing the best treatment options, specially with the emergence of new treatments such as the immunobiologics.

\section{Consent for publication}

The authors declare that they have obtained informed written consent from the patient's tutors for publication

\section{P022}

ADULT-ONSET STILL'S DISEASE ASSOCIATED WITH MACROPHAGE ACTIVATION SYNDROME WITH GREAT THERAPEUTIC RESPONSE IN THE COMBINATION CYCLOSPORINE AND CORTICOSTEROID: CASE REPORT

Eduarda Maria Schroeder, Jalyson de Freitas Souza, Marina Martinichen Furlaneto, Nycolle Louise Klein Ottoni Guedes, Ana Paula Adame,

Marcio Augusto Nogueira

UNIVERSIDADE ESTADUAL DO OESTE DO PARANÁ, CASCA, PARA, Brasil Advances in Rheumatology 2018, 58(Suppl 1):P022

Adult-onset Still's disease is characterized by severe polyarthritis, intermittent nocturnal fever peaks lasting more than 2 weeks, and extraarticular findings such as non-pruritic maculopapular erythematous rash, myalgia, odynophagia, hepatomegaly, splenomegaly, lymphadenopathy, serositis and hyperferritinemia. The manifestation of Adult-onset Still's Disease (AOSD) is extremely rare, with an incidence of $0.16 / 100,000$ individuals per year. This disease results from hyperactivation of innate immunity cells, with hypersecretion of pro-inflammatory cytokines and phagocytic proteins, such as S-100 protein. The AOSD may present with complications - an example is the Macrophage Activation Syndrome (MAS) - which is characterized by spontaneous hemophagocytosis, which results in severe pancytopenia, manifested by fatigue, infections and bleeding due to anemia, leukopenia and thrombocytopenia, respectively. It may cause acute liver dysfunction with elevated AST/ALT. Hemophagocytosis also accelerates the catabolism of hemoglobin, aggravating hyperferritinemia. In addition, it is responsible for the destruction of circulating fibrinogen, raising fibrin degradation products (FDPs), extending Prothrombin Time (PT) and Activated Partial Thromboplastin Time (APTT), and decreasing erythrocyte sedimentation rate (ESR). The decrease in ESR is important to distinguish MAS from an exacerbation of ASD. We describe the case of a patient previously diagnosed with polyarticular JIA in remission for years, who evolved to Adult-onset Still's Disease associated with Macrophage Activation Syndrome, and obtained a great therapeutic response with the proposed treatment. Initially, corticosteroid pulse therapy was prescribed for 3 days, and after that period oral corticosteroid $60 \mathrm{mg}$ per day associated with cyclosporine $6 \mathrm{mg} / \mathrm{kg}$ per day, both with a progressive reduction scheme, which lasted 14 months. The relevance of the case is due to the rarity and high morbimortality of AOSD complicated by MAS, and, also to the good response of the patient to the proposed immunosuppression. It may, therefore, be a useful aid in the conduct of similar cases.

\section{P025}

ALTERATION OF THE INFLAMMATORY PROFILE IN THE GINGIVAL FLUID OF PATIENTS WITH JUVENILE SYSTEMIC LUPUS

\section{ERYTHEMATOSUS}

Hellen De Souza Nascimento, Loreley Carlos Agostinho Bragard, Manuela Rubim Camara Sete, Flávio Roberto Sztajnbok, Marcelo da Silva Figueredo UERJ, RIO DE JANEIRO, RJ, Brasil

Advances in Rheumatology 2018, 58(Suppl 1):P025 
The objective of this study was to evaluate the expression of cytokines in the gingival crevicular fluid (GCF) of patients with juvenile systemic lupus erythematosus (SLE) and to compare them with healthy individuals. Thirty patients with SLE (mean age: $16.2 \pm 1.5$ years) and 29 without systemic disease (mean age $15.5 \pm 2.3$ years), both groups with gingivitis, participated in the study. Periodontal and gingival fluid data were collected. For cytokine analysis, fluid samples were collected from three to four sites per patient, and the most inflamed sites were selected. Cytokines were analyzed by the multiplex assay with the Bio-plex Pro TM Th17 panel assay kit. MannWhitney $\mathrm{U}$ test was used to evaluate numerical variables. The levels of probing depth, clinical insertion level, plaque index and gingival bleeding were significantly higher in the test group when compared to the control group. Immunological analysis revealed that IL-1 $\beta, \mathrm{IL}-7$, IL-8, IL-13, G-CSF, IFN- $\gamma$ and MCP-1 were significantly higher in the test group than in the control group. Cytokines IL-4, IL-12 (p70) and GM-CSF were significantly lower in the test group. It can be concluded that patients with SLE presented worse periodontal conditions and increased IL-1 $\beta, \mathrm{IL}-7, \mathrm{IL}-8, \mathrm{IL}-13, \mathrm{G}-\mathrm{CSF}$, IFN- $\gamma$ and MCP-1 cytokines when compared to systemic patients suggesting a more aggressive inflammatory profile in the FG of patients with SLE.

\section{P028}

AN UNUSUAL CASE OF OVERLAP INVOLVING SCLERODERMA, RHEUMATOID ARTHRITIS AND ANTIPHOSPHOLIPID SYNDROME

Julia Permegiani Vilarinho, Sandra Regina Miyoshi Lopes, Dênis Azuma, Larissa Alves da Silveira, Marcela Paganelli

FACULDADE DE MEDICINA DE CATANDUVA, CATANDUVA, SP, Brasil

Advances in Rheumatology 2018, 58(Suppl 1):P028

Overlap Syndrome is characterized by two or more autoimmune diseases in a patient. We report an overlap of Scleroderma, Rheumatoid Arthritis (RA) and Antiphospholipid Syndrome (APS).

Case report: Woman, 51 years old, with complaint of two Transient Ischemic Attacks 15 days ago. It reports two episodes of Ischemic Stroke being the first six years ago and the last, two years ago. History of arthritis in wrists, PIP of hands, elbows, knees and toes; Raynaud's phenomenon on hands and feet, diagnosing RA and scleroderma for six years ago. Since then, using methotrexate $15 \mathrm{mg} /$ week.

Report recurrent pneumonia and dyspnea on moderate exertion. Pregnancies whithout abnormalities. She denied smoking, alcoholism, liver disease and family history. Physical examination: arthritis in 2nd, 3rd phalangean metacarpal, right wrist and elbow; 2nd, 3rd, 4th phalangean metacarpal, left wrist; vesicular murmur positive with subcrepitant rales in 1/3 lower bilateral, respiratory frequency: 13 . (2018): Antinuclear Factor: nucleolar dotted 1: 160, antiSCL70: 35, antiCCP: 60, Waaler-rose: 40, CRP: 15, ESR: 62, lupus anticoagulant: 6, acid alpha1-glycoprotein: 150; anticardiolipin, serologies and cryoglobulins negative. CT thorax: reticulated pulmonary parenchyma, traction bronchiectasis, architectural distortion and bilateral sparse mosaic. MRI of the skull: chronic ischemic vascular injury in the left posterior artery. Echocardiogram: PSVD $31 \mathrm{mmHg}$. UGIE: hiatal hernia. Abdominal Ultrasonography: mild hepatic steatosis. MRI: chronic inflammatory arthropathy of phalangean metacarpal and degenerative of proximal and distal interphalangeals. Mammography and spirometry: normal.

Discussion: Autoimmune rheumatic diseases can coexist in the same patient, being able to evolve sequentially or appear simultaneously, configuring Overlap Syndrome. Association of manifestations equivalent to APS, Scleroderma and RA, as reported, is extremely uncommon, and only associations that contemplate two of these pathologies are described. Cases covering all these are not observed in the literature. Most of the reports are of cases of Scleroderma that throughout their evolution have overlapped with Polymyositis, Dermatomyositis, SLE, RA or Sjögren's Syndrome.

This syndrome stands out due to its clinical diversity, order of manifestation of the diseases and their consequences. This is believed to be due to multifactorial diseases.

Valuing history and physical examination, with the ability to interrelate clinical manifestations, is the best way to deal with rare and varied clinical courses.
Consent for publication

The authors declare that they have obtained informed written consent from the patient's tutors for publication

\section{P029}

ANALYSIS OF DRUG INTERACTIONS IN HOSPITALIZED RHEUMATOLOGY PATIENTS

Larissa Cristiane De Oliveira Souza, Marilma Galvão dos Santos Gomes, Francisco Alves Bezerra Neto

UFRN, NATAL, RN, Brasil

Advances in Rheumatology 2018, 58(Suppl 1):P029

Background: Medical prescriptions must be analyzed according to a variety of factors. Drug interaction is one of those factors. When harmful, they may extend hospital stay and heighten treatment costs.

The objective of the present investigation is to present the main drug interactions in admitted patients to a rheumatology infirmary as well as the drugs involved in such events.

Materials and methods: Forty three medical prescriptions from the rheumatology infirmary were analyzed between January and October of 2017. Subsequently, evidence-based medical information databases Micromedex and Uptodate were used for analysing of the possible interactions between the most prevailing drugs. Drug interactions deemed as contraindicated and of major severity were the ones considered for analysis.

Results: 54 pharmacological groups were identified along with 111 varieties of drugs. The mean amount of prescribed drugs per patient was 13. Drug interactions considered contraindicated were not found in prescriptions. However, interactions considered of major severity were found in all 43 analysed prescriptions. 6 prevalent drugs were found in rheumatology prescriptions and its respective therapeutic category (see Table 1). The Glucocorticoid class was found to be the most involved in drug interactions. The most prevailing drug-drug interactions were analysed (see Table 2).

Conclusions: The various comorbidities in rheumatology patients frequently result in polypharmacy and consequently in drug interactions. There is a growing need for the interaction of medical staff with other areas in health care. Drug interaction analysis in our institution is the result of our multidisciplinary staff's efficacy, which is composed by medical, clinical pharmacology, psychology, occupational therapy and nutrition professionals.

Thus, the study of prescriptions, and more specifically of the drugdrug interactions by the pharmacology staff contributes to action strategies which have as consequence, the reduction of possible harm inflicted to patients, as well as a reduction of healthcare costs due to the reduction of hospital stay.

Table 1 (abstract P029). See text for description

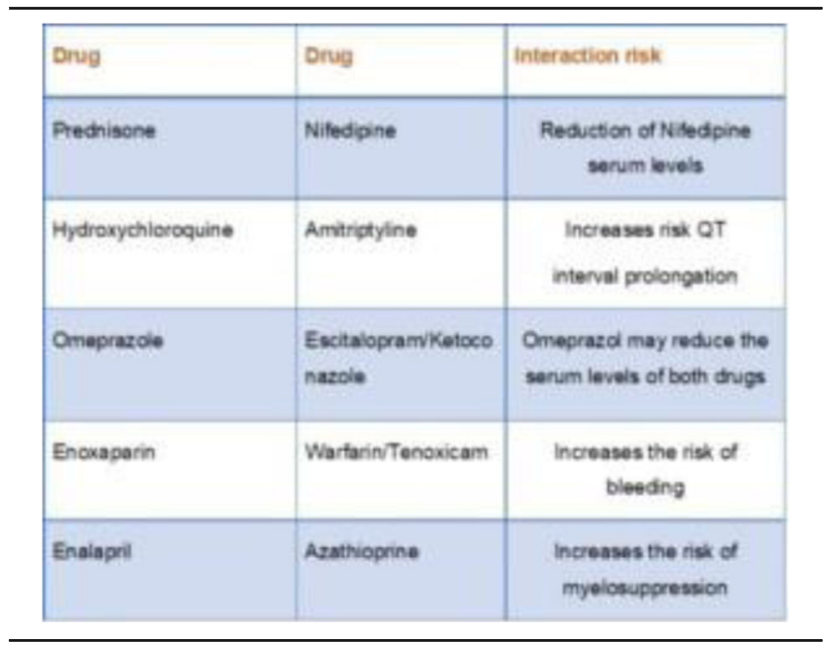


Table 2 (abstract P029). See text for description

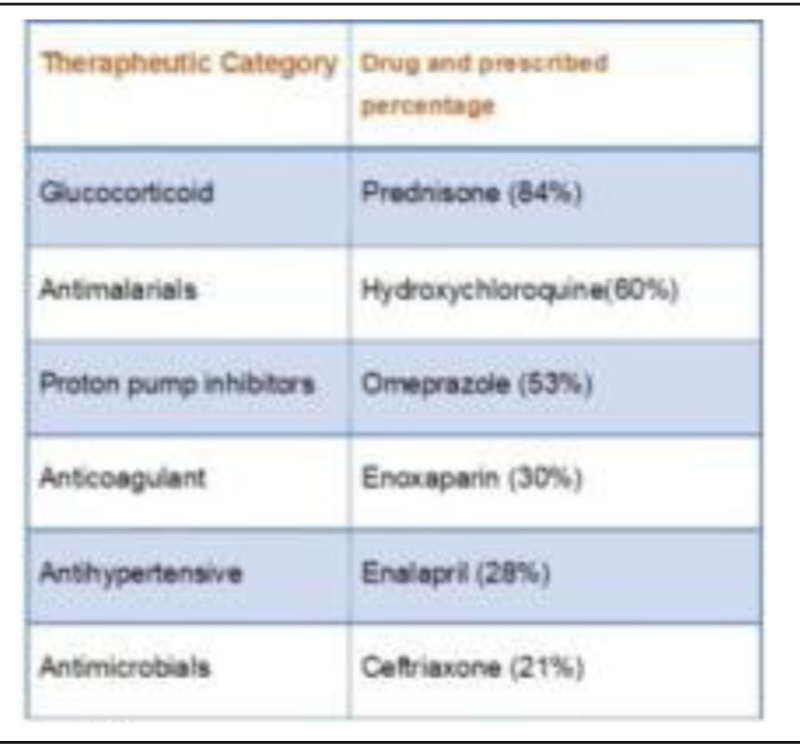

P030

ANALYSIS OF SURVIVAL, DEATHS AND ASSOCIATED FACTORS IN A COHORT OF SCLERODERMA

Vinicius Costa Vieira, Luiz Felipe Dipe Prates Miranda, Adib Chicre

Mansur Neto, Guilherme Salles de Escobar Gonçalves, Mauro Goldfarb,

Leonardo Domingues Romeiro

HOSPITAL FEDERAL DOS SERVIDORES DO ESTADO RJ, RIO DE JANEIRO,

RJ, Brasil

Advances in Rheumatology 2018, 58(Suppl 1):P030

Introduction: Systemic sclerosis (SS) represents an impact on morbidity and mortality rates and determines lower survival in relation to the general population. Cohort studies published worldwide reaffirm the importance of the cardiopulmonary involvement as the main cause of death in this disease.

Objective: Evaluate the survival, causes of deaths and prognostic factors in a cohort of patients with systemic sclerosis of a tertiary hospital. Methods: a retrospective cohort study of 97 patients followed from 2010 to 2018 with SS. The analysis was by review of medical records and telephone contact between February to April 2018. It was used an excel spreadsheet to the database. The deaths were obtained and analyzed the survival, the causes of deaths and related factors. It was performed a simple analysis of frequencies by EXCEL and also OPENEPI (openepi.com) bivariate analysis by the program.

Results: The average survival rate was $86.7 \%$ in the period. 13 deaths occurred: 6 by cardiopulmonary complications, 2 by neoplasms (pulmonar and large B-cell lymphoma), 3 of infectious complications of skin ulcerations and 2 without a defined cause. Associated with deaths, 10 (76.92\%) presented with pulmonary arterial hypertension (PAH), 6 (46.15\%), interstitial lung disease, $5(38.46 \%)$, anemia and $1(7.69 \%)$ alteration in renal function. The average age at death was 60.46 years. There was no occurrence of scleroderma renal crisis. In bivariate analysis, PAH was variable associated to death with $p$-value $<0.001$ calculated by Fisher's exact test (calculated on OpenEPI), but the other variables analyzed (anemia, serum creatinine and lung disease) were not statistically significant.

\section{P031}

\section{ANAPLASTIC NON-HODGKIN'S LYMPHOMA SIMULATING BEHÇET'S} DISEASE

Fernanda Tavares, Francisco Fellipe Claudino Formiga, Bruna Laiza Fontes Almeida, Ana Karla Guedes de Melo, Maria Roberta Melo Gomes Pereira, Danielle C. S. Egypto de Brito, Alessandra de Sousa Braz, Eutilia Andrade Medeiros Freire

UNIVERSIDADE FEDERAL DA PARAÍBA, JOÃO PESSOA, PB, Brasil

Advances in Rheumatology 2018, 58(Suppl 1):P031
Background: Behçet's disease (BD) is a systemic vasculitis characterized by recurrent oral and genital ulcers, uveitis and cutaneous lesions, and may develop with arthritis, gastrointestinal, neurological, vascular symptoms and orchiepididymitis. Pathergy test can be positive in $20 \%$ to $30 \%$ of cases and HLA-B51 is described in $20 \%$ of patients. The differential diagnosis of BD includes recurrent oral aphthosis, Crohn's disease, sarcoidosis, pemphigus, pyoderma gangrenosum, erythema nodosum leprosum, myelodysplastic syndrome, among others. Anaplastic T-cell non-Hodgkin's lymphoma (NHL) is a rare type of NHL that may have nodal and extranodal involvement (skin). On the skin, clinical signs range from erythematous plaques to pruritus, eczema, hypochromia, ulcers and tumors, and may cause disagreement in the characteristics of the lesions.

Case report: Male patient, 35 years, white, admitted on 07/03/18 with ulcers in the lower extremities and testicular region for 15 days. According to the report, on november 2017, he started cutaneous lesions with erythematous-brownish ascending non-nodular macules associated with daily fever. On December of the same year, he presented melena, anemia and two gastric ulcers were diagnosed. He was treated and released for outpatient follow-up. On march 2018, there was persistance of fever and the spots developed into painful ulcers, some with necrotic crusts, when he was admitted to our service. Examination at admission: cardiorespiratory, neuropsychiatric and abdominal devices without alterations. Skin and appendages: macules and erythematous-brown nodules and necrotic ulcers in lower limbs. Personal background: recurrent sores since the age of 14 years and partial bilateral hearing loss (recurrent otitis media). Initially, diagnostic hypotheses were suggested: BD, Crohn's disease, leprosy, Cogan's syndrome and necrotizing pyoderma gangrenosum. Blood count, renal and hepatic function, urinalysis, proteinuria $24 \mathrm{~h}$ ANCAs, fecal calprotectin, colonoscopy, chest $x$-ray, transthoracic echocardiogram, temporal bones computed tomography and lymph baciloscopy were investigated: unchanged. Testicular ultrasonography: orchitis. Upper digestive endoscopy showing the same two gastric ulcers (Grade 2 Los Angeles). Initiated prednisone $(40 \mathrm{mg} /$ day) and colchicine ( $1 \mathrm{mg} /$ day), with no response. Acute phase reactants, slightly elevated initially, got worse. The biopsy of a nodular lesion suggested $\mathrm{NHL}$, classified at immunohistochemistry as anaplastic NHL T cell.

Conclusion: the diagnosis of $\mathrm{BD}$ is made through clinical criteria and the exclusion of other diseases. The authors call attention to the occurrence of neoplasias with cutaneous manifestations in the differential diagnosis of $\mathrm{BD}$, especially in cases with atypical clinical evolution and with no response to recommended therapy.

Consent for publication

The authors declare that they have obtained informed written consent from the patient's tutors for publication

\section{P033}

ANTINUCLEAR ANTIBODIES DENSE FINE SPECKLED AND ASSOCIATION WITH AUTOIMMUNE DISEASES AT A TERTIARY HOSPITAL IN SAO PAULO

Fernanda Pulcheri Ramos, Matheus Xavier Guimarães, Andrey Tonetto Barbosa, Izaura Tereza Silva Guedes, Lisa Mielke de Oliveira, Bernardo Patrício Sequeira Dultra, Rina Dalva Neubarth Giorgi, Renata Ferreira Rosa, Nafice Costa Araujo

HOSPITAL DO SERVIDOR PUBLICO ESTADUAL DE SÃO PAULO, SÃO PAULO, SÃO PAULO, Brasil

Advances in Rheumatology 2018, 58(Suppl 1):P033

Background: The detection of antinuclear antibodies (ANAs) is a hallmark of systemic rheumatic diseases. Therefore, understanding the clinical relevance of various autoantibodies showing different staining patterns is crucial for the accurate diagnosis of ANA associated rheumatic disease. The dese fine speckled pattern (DFS-ANA) is one of the most commonly seen in routine diagnostic laboratories. Some authors point out that the DFS-ANA may be an exclusionary marker for ANA-associated rheumatic disease. OBJECTIVES: The objective of the present study is to evaluate the association of DFS pattern with SLE and other diseases. 
Methods: This is a longitudinal retrospective study carried out in 2018 in a tertiary hospital in São Paulo, in which all the patients with FANPFD were included in the year 2013. The laboratory evaluation was performed by means of indirect immunofluorescence with Hep cell substrate -2 . The medical records were analyzed to evaluate the presence or development of autoimmune disease until 2018. For statistical analysis, mean, standard deviation, frequency (\%), fisher test, chi-square tests were used. $\mathrm{P}<0.05$ was considered statistically significant.

Results: A total of 283 patients with DFS-ANA ins 2013 were selected. After excluding incomplete data and patients without follow-up, 136 patients were included for analysis, out of $90.4 \%$ were women. 77 patients $(56.6 \%)$ had clinical association, which corresponds to at least $27.2 \%$ of all DFS-ANA. Neoplasia was present in $6.6 \%$ of the patients, in which liver and breast were the most affected $(\mathrm{N}=4$ and $\mathrm{N}=2$, respectively). $5.9 \%$ presented hepatitis $\mathrm{B}$ or $\mathrm{C}(\mathrm{N}=1$ and $\mathrm{N}=4$, respectively) or autoimmune $(\mathrm{N}=3)$, while hyperthyroidism was the sixth most frequent association (3.7\%). $33.8 \%(\mathrm{~N}=46)$ presented a diagnosis of SARD, the most common being SLE (14\%), RA (9.6\%) and ES (4.4\%). Other SARDs included Sjogren's Syndrome, Gout, Cutaneous Vasculitis, LED, JIA and Reactive Arthritis (6.6\%). Patients with SARD presented DFS-ANA in higher titers than the others $(p=0.047$ for titles greater than 320 and $p=0.039$ for titles greater than 640 ). Conclusions: This paper does not corroborate data that relate DFS-ANA with absence of SARD. In this sample, DFS-ANA was associated with several clinical conditions, including a significant relationship between higher titers and the presence of autoimmune rheumatology disease.

\section{P034}

ANTINUCLEAR FACTOR REQUEST BY MEDICAL SPECIALTIES AT A TERTIARY HOSPITAL IN SAO PAULO

Fernanda Pulcheri Ramos, Andrey Tonetto Barbosa, Matheus Xavier Guimarães, Denise Moraes Horiy, Rina Dalva Neubarth Giorgi, Renata Ferreira Rosa, Nafice Costa Araujo HOSPITAL DO SERVIDOR PUBLICO ESTADUAL DE SÃO PAULO, SÃO PAULO, SÃO PAULO, Brasil

Advances in Rheumatology 2018, 58(Suppl 1):P034

Background: Laboratory tests have the objective of complementing and/or confirming the clinical suspicion generated from anamnesis and physical examination. The indiscriminate request for complementary tests leads to a greater financial cost, laboratories overload and, mainly, a greater risk of erroneous diagnosis and greater anxiety by patients on a positive result. The antinuclear factor (ANA) test is considered a screening method for various autoimmune diseases. It is often found in patients with a wide variety of rheumatic diseases, including systemic lupus erythematosus, systemic sclerosis, Sjogren's Syndrome, and myositis. However, despite high sensitivity, it does not characterize sufficient criteria for diagnosis and may even be present in healthy individuals.

Objectives: This study aims to correlate the origin of the FAN request with its positivity or negativity.

Materials and Methods: This is an analytical study conducted in 2018 at a tertiary hospital in São Paulo that included 16,181 FAN exams. The collections were carried out between 2010 and 2013 by means of indirect immunofluorescence with Hep-2 cells substrate. For statistical analysis, mean, standard deviation, frequency (\%), t-student and Chi-square tests were used. $\mathrm{P}<0.05$ was considered statistically significant.

Results: Out of the 16.181 exams, 11.354 (70\%) were nonreactive and 4.827 were reagents, 12.808 were collected in women (79\%). The internal medicine clinic was responsible for the largest number of requests $(\mathrm{N}=3.469,21.4 \%)$, followed by Rheumatology ( $\mathrm{N}=2.393,14.7 \%)$ and Gastroenterology ( $\mathrm{N}=1.849,11.4 \%)$. Among the tests requested by Internal medicine clinic and Gastroenterology, $73.9 \%$ and $74.8 \%$ were non-reactive, respectively. On the other hand, Rheumatology had $46.3 \%$ of negativity in their requests $(p=0.0001)$. The most common pattern was dense fine speckled (DFS) $(\mathrm{N}=1.754,36.3 \%)$, followed by fine speckled ( $N=1.117,23.1 \%)$, centromeric $(N=201,4.2 \%)$ and homogeneous ( $\mathrm{N}=195,4 \%)$. The prevalence of DFS pattern was similar among the three clinics (34.3\%, 36.4\% and $32.5 \%$, respectively). Conclusions: This high index of non-reagent ANA testing allows us to question the correct indication of this exam. Of course, rheumatology presents a higher clinical pre-test probability than other clinics and, therefore, more reagent results. In this way, continuing education in Rheumatology, specially focused on the major clinical specialties, is necessary to increase the value of the test and reduce indiscriminate requests.

\section{P035}

ANTIPHOSPHOLIPID SYNDROME AND THE IMPORTANCE OF DIFFERENTIATING ITS NEUROLOGICAL DISORDERS WITH THOSE FROM MULTIPLE SCLEROSIS: A CASE REPORT

Alana Micaela Araújo Lemos, Jonathan dos Anjos Rangel, Letícia Queiroga de Figueiredo, Evânia Claudino Queiroga de Figueiredo, Marcus Ivanovith Fernandes UNIVERSIDADE FEDERAL DE CAMPINA GRANDE, CAMPINA GRANDE, PARAÍBA, Brasil Advances in Rheumatology 2018, 58(Suppl 1):P035

The antiphospholipid syndrome is characterized by thromboembolism, repetitive spontaneous abortion and the antibodies presence. Although the antiphospholipid syndrome diagnostic criteria are different from multiple sclerosis, the white matter subcortical lesions might share the two diseases characteristics. Thus, the present report shows a patient with antiphospholipid syndrome, initially diagnosed with multiple sclerosis. Male patient, 42 years old, divorced and camera operator, presented two compatible episodes with cerebral ischemia occurred within a five-year interval, diagnosed with multiple sclerosis and prescribed interferon beta-1a, with symptomatic impairment. In the second ischemic event, the patient was reevaluated and hyperintense focus were observed in T2/Flair subcortical and deep in the supratentorial white matter, from non-specific nature that possibly indicated ischemic gaps, evidenced in Fig. 1, in addition to other complementary exams. The antiphospholipid syndrome diagnosis was made and the anticoagulation, oxcarbamazepine and ciprofibrate were initiated. The risk of treating antiphospholipid syndrome with interferon-beta is uncertain. The patient improvement with the use of this medication makes relevant the description of this case report and justifies the studies accomplishment that evaluate its interference in this syndrome. The differentiation among ischemic and demyelinating brain lesions is important in order to perform accurate diagnosis and safe treatment.

\section{Consent for publication}

The authors declare that they have obtained informed written consent from the patient's tutors for publication

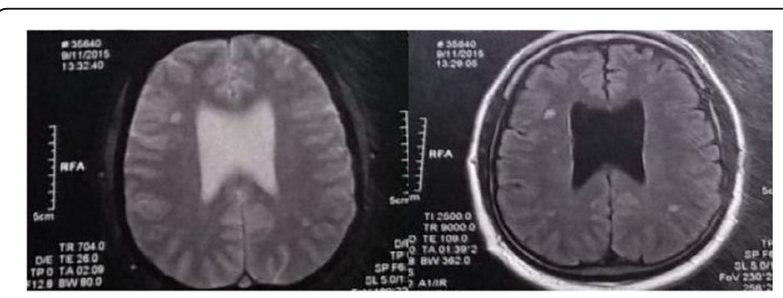

Fig. 1 (abstract P035). Left image indicating subcortical hyperintense focus and deep in the supratentorial white matter, from non-specific nature in T2. Right image indicating the same lesions, on Flair 


\section{P039}

APPETITE IN RHEUMATOID ARTHRITIS AND CLINICAL

\section{ASSOCIATIONS}

Hugo Deleon De Lima', Pedro Arturo Bismara Carneiro Santos ${ }^{2}$

Rafael da Rocha Caminha², Bruna Ferraz Gutierrez Piola²

Flávia Jatobá de Barros ${ }^{2}$, Laurindo Ferreira da Rocha Junior ${ }^{3}$

'INSTITUTO DE MEDICINA INTEGRAL PROF. FERNANDO FIGUEIRA, RECIFE,

PE, Brasil; ${ }^{2} F A C U L D A D E$ PERNAMBUCANA DE SAÚDE - FPS, RECIFE,

PERNAMBUCO, Brasil; ${ }^{3}$ INSTITUTO DE MEDICINA INTEGRAL PROF.

FERNANDO FIGUEIRA - IMIP, RECIFE, PERNAMBUCO, Brasil

Advances in Rheumatology 2018, 58(Suppl 1):P039

Background: Rheumatoid arthritis (RA) is a chronic systemic disease of autoimmune nature that affects mainly the synovial joints and leads to disability. Little is known about the relationship between RA and appetite. The objective of this study was to evaluate the relation of rheumatoid arthritis clinical characteristics and changes in appetite.

Materials And Methods: Cross-sectional study. Data collection of RA patients was performed through clinical evaluation, interview and review of medical records. To evaluate the occurrence of change in appetite and the intensity of the change we used pattern change scale of appetite of Beck Depression Inventory II (BDI-II). The question to evaluate changes in appetite patterns has seven affirmations with values of 0,1 , 2 and 3 indicating no change, mild, moderate and severe changes for decreased or increased appetite. Clinical Disease Activity Index (CDAl) and Disease Activity Score in 28 joints (DAS28) were calculated to measure the disease activity. Functional capacity was analyzed by the Health Assessment Questionnaire (HAQ). Statistical analysis was performed using Graph Pad Prism 6.01 software.

Results And Conclusions: Ninety-six included patients, thirty (30.92\%) presented loss of appetite, twenty-four (24.74\%) increased appetite and forty-three $(44.34 \%)$ had no change. When comparing patients with and without appetite change, there were difference in number of painful joints ( $p$ 0.414), visual analogue scale (VAS) for physician global assessment of disease ( $p$ 0.0349) and HAQ (0.0004). The dicreased appetite was associated with worse functional capacity median HAO 2.125 (IOR $1.594-2.406$ ) when compared to patients who did not have change in appetite and those who had increased appetite median HAQ 1.5 (IQR 0.75-22.25) (p 0.0112). On the other hand, increased appetite had association with swollen joints ( $p$ 0.0475). Additionally, the the intensity of increased appetite also correlated with swollen joints ( $p$ 0.0367; $r$ 0.2558) and with worse functional capacity ( $p 0.017 ; r$ 0.2906). There was a tendency for significance in the correlations of this group with VAS for physician global assessment of disease ( $p 0.0751 ; r$ 0.2189) and CDAI ( $p$ 0.0656; $r$ 0.2263 ). Dicreased appetite correlated with functional capacity ( $p$ $0.0006, r 0.391)$ and CDAI ( $p 0.043, r 0.2376)$. The findings suggest that appetite disorders may influence the inflammatory picture of RA. Understanding the role of these disorders in their relation to the etiology and clinical manifestations of RA can point to new therapeutic approaches.

\section{P045}

ASSESSMENTS OF CENTRAL PAIN MODULATORS IN ACTIVE AND INACTIVE RHEUMATOID ARTHRITIS PATIENTS

Luis Henrique Savoi De Almeida ${ }^{1,3}$, Juline do Prado Paes ${ }^{2}$, Maria Carolina Coelho Gozzano ${ }^{3}$, Isabella Guadanhim Guerra ${ }^{3}$, Melissa Nobrega Vasques de Freitas ${ }^{3}$, Walace Dorneles Gomes ${ }^{3}$, Gilberto Santos Novaes ${ }^{3}$

${ }^{1}$ PONTIFÍCIA UNIVERSIDADE CATÓLICA DE SÃO PAULO, SOROCABA, SP, Brasil; ${ }^{2} P U C$ SP, SOROCABA, SAO PAULO, Brasil; ; ${ }^{3}$ UUC SP (SOROCABA),

SOROCABA, SAO PAULO, Brasil

Advances in Rheumatology 2018, 58(Suppl 1):P045

Background: Rheumatoid Arthritis (RA) is a chronic, inflammatory autoimmune disease that leads to deformity and destruction of the joints. Although the inflammatory process of RA activity is responsible for the initiation of pain, studies indicate that its intensity may be disproportionate to the severity of the inflammation. It is believed that this is due to the amplification of pain by the central nervous system, mainly due to the decrease in the modulation of conditioned pain.
Thereby this study has the objective of evaluating the characteristics of the main modulators of central pain, as fatigue, sleep and pain intensity, in RA patients with or without active disease.

Methods: The research was developed from the evaluation of 51 patients with RA according to the criteria of the ACR 2011. Simplified Disease Activity Index (SDAl) was used to evaluate disease activity. Pain was assessed according to the SF-36 body pain item. Fatigue severity scale was used in the fatigue assessment and sleep was evaluated by the Pittsburgh Sleep Quality Index (PSQI).

Statistical analysis was performed using Chi-square test, which was considered significant $p<0.05$.

Results: For the study 41 women (80.4\%) and 10 men (19.6\%) were selected.

There was a higher prevalence of pain symptom in the group undergoing active disease $(50.98 \%)$ than in the patient group in clinical remission (19.61\%), $\mathrm{p}=0.003$.

Likewise, a higher prevalence of fatigue symptoms were observed in the active disease group (39.22\%), when compared to the group of patients in remission (19.61\%), $p=0.17$.

There was a higher prevalence of poor sleep quality in the active disease group (50.98\%) than in the remission group (7.84\%), $p=0.01$.

The 3 parameters were found in $9.52 \%$ of the patients in remission and in $66.67 \%$ of the patients with the active disease, $p<0.05$. At least two parameters in $28.57 \%$ of the patients in remission and $83.33 \%$ of the active patients, $p<0.05$. An isolated parameter was found in $52.38 \%$ of patients in remission and $96.66 \%$ in patients with active disease, $\mathrm{p}<0.05$.

Conclusions: Analysis of the results showed an association between the three parameters evaluated and the active RA. None of these parameters were able to discriminate central symptoms, sleep, fatigue and pain from active disease suggesting that central pain sensitization modulators do not apply to RA.

\section{P046}

ASSOCIACÃO ENTRE DENSIDADE MINERAL ÓSSEA E SÍNDROME METABÓLICA EM HOMENS IDOSOS DA COMUNIDADE: SÃO PAULO AGEING \& HEALTH STUDY (SPAH)

João De Mendonça Alho Teixeira', Diogo S Domiciano², Luana G Machado², Jaqueline B Lopes ${ }^{2}$, Camille P Figueiredo², Valeria F Caparbo ${ }^{2}$, Liliam

Takayama², Rosa M R Pereira²

${ }^{1}$ HC FMUSP, SÃO PAULO, SP, Brasil; ${ }^{2}$ HOSPITAL DAS CLÍNICAS FMUSP, SÃO PAULO, SP, Brasil

Advances in Rheumatology 2018, 58(Suppl 1):P046

Background: Recent studies have shown a link between metabolic syndrome (MS) and bone mass. These results are uncertain about the effect of the components of MS on bone mineral density (BMD) and risk of fragility fractures. Furthermore, the higher prevalence of MS among subjects with higher body mass index (BMI) is a confounding factor, since previous findings have demonstrated that obesity could be a protective factor against bone loss. The aim of this study was to evaluate the prevalence of MS in a community-dwelling older men with high frequency of overweight/obesity and its association with bone parameters. Methods: 258 community-dwelling older men were evaluated by specific questionnaire. Lumbar spine, femoral neck and total hip BMD were evaluated by DXA. Laboratory tests, including calcium, phosphorus, creatinine, lipid profile and glucose were performed. Xrays were assessed to identify vertebral fractures. National Cholesterol Education Program-Adult Treatment Panel III (NCEP-ATPIII) criteria were used to define MS.

Results: Prevalence of MS was high (41.5\%). Those with MS had higher BMI $(28.0 \pm 3.9$ vs. $25.4 \pm 3.4 \mathrm{~kg} / \mathrm{m} 2, P<0.001)$, total body fat $(19.1 \pm 6.4$ vs. $15.1 \pm 4.6 \mathrm{~kg}, P<0.001)$, body fat percentage $(25.5 \pm 4.9$ vs. $22.4 \pm 4.6 \%$, $P<0.001)$, serum levels of creatinine $(1.2 \pm 0.3$ vs. $1.1 \pm 0.3 \mathrm{mg} / \mathrm{dl}, P=0.04)$, lumbar spine BMD $(1.082 \pm 0.202$ vs. $1.000 \pm 0.190 \mathrm{~g} / \mathrm{cm} 2, P=0.001)$ and total hip BMD $(0.947 \pm 0.137$ vs. $0.876 \pm 0.319 \mathrm{~g} / \mathrm{cm} 2, P=0.031)$, but had lower frequency of use of calcium ( 24.2 vs. $75.8 \%, P=0.034$ ) and vitamin D supplements ( 12.5 vs. $87.5 \%, \mathrm{P}=0.015$ ). After adjustments for $\mathrm{BMl}$, hip BMD (OR:1.42 95\% Cl:1.04-1.93, P=0.029), creatinine (OR:2.79 95\% $\mathrm{Cl} 1.17-6.61, \mathrm{P}=0.02)$ and total body fat (OR:1.12 95\% Cl:1.06-1.19, $\mathrm{P}=0.001$ ) remained as independent factors associated with MS. No 
significant difference concerning the prevalence of fractures was observed between the men with and without MS.

Conclusion: A positive association between total hip BMD and MS was found in our community-dwelling older men. Nevertheless, the frequency of fractures was similar in both groups. It suggests that higher BMI does not explain the positive association between higher BMD and MS, and does not protect against fractures. Further studies are necessary to elucidate the effect of MS on bone mass and fracture risk, possibly related to bone quality.

\section{P047}

ASSOCIATION BETWEEN ANTINUCLEAR ANTIBODIES CENTROMERE PATTERN AND DIFFERENTS AUTOIMMUNE DISEASES AFTER FIVE YEARS IN BRAZILIAN PATIENTS

Matheus Xavier Guimarães ${ }^{1}$, Andrey Tonetto Barbosa ${ }^{2}$, Fernanda Pulcheri Ramos $^{2}$, Raissa Barbosa De Souza2, Lisa Mielke De Oliveira², Kioko Takei², Daniela Crema ${ }^{2}$, Rina Dalva Neubarth Giorgi ${ }^{2}$, Renata Ferreira Rosa ${ }^{2}$, Nafice Costa Araujo ${ }^{2}$

${ }^{1}$ HOSPITAL DO SERVIDOR PUBLICO ESTADUAL, Brasil; ${ }^{2}$ HOSPITAL DO SERVIDOR PUBLICO ESTADUAL DE SÃO PAULO, SÃO PAULO, SÃO PAULO, Brasil

Advances in Rheumatology 2018, 58(Suppl 1):P047

Introduction: Antinuclear antibodies (ANA) are the first-line autoantibodies in suspicion of mostly autoimmune diseases. ANA are immunoglobulins that recognize a broad spectrum of not only nuclear, but also cytoplasmic components. They occur frequently in the sera of patients with a wide variety of rheumatic diseases, including systemic lupus erythematosus (SLE) and Sistemic Esclerosis (SE). According to the ANA consensus, as well as other studies, the centromeric parttern (CP) is on of the least associated with findings in healthy individuals. Objectives: To determine the frequency of current and new autoimmune manifestations or autoimmune disease five years after detection of antinuclear antibodies centromeric pattern

Methods: This is a retrospective study, conducted in 2018, included 132 patients with ANA CP between 2010 and 2013. Only 101 patients followed at Rheumatology clinic or other in a tertiary hospital. Were excluded 31 patients who had no follow-up. The 101 patients were divided into two groups according to ANA titles; greater than or equal to $1 / 640(N=81$, group 1$)$ and less than or equal to $1 / 320$ $(\mathrm{N}=20$, group 2). In both groups we evaluated the presence of autoimmune diseases or autoimmune manifestations. For ANA detection, indirect immunofluorescence is performed using Hep-2 cells as a substrate. For statistical analysis: mean, standard deviation, frequency (\%), t-student, Mann-Whitney and Chi-square tests were used. $\mathrm{P}<0,05$ was considered statistically significant.

Results: Of the group 1 patients, the mean age was 63,62 $\pm 12,65$ years, without statistical difference with group $2(p=0,153)$. Forty out of 81 patients in group 1 had already diagnosed some autoimmune disease in the ANA achievement $(p=0,0017)$, of which 15 had SE $(p=0,0374), 9$ Sjogren, 5 Indifferentiated Connective Tissue Disease and 3 Primary Biliar Cirrosis, SLE and reumatoid arthrits. In both groups there was a predominance of females $(N=97,96,03 \%)$. After, at least, five years, the most common symptom related were Raynaud phenomenon (FRY) $(N=22, p=0,0056)$, sclerodactyly $(N=15$, $\mathrm{p}=0,0374)$ and esophagophathy $(\mathrm{N}=15, \mathrm{p}=0,0374)$. Sixteen patients in group 1 developed some autoimmune disease after five years, of which 14 were SSc $(p=0,036)$.

Conclusion: ANA is more associated with females and titers greater or equal to $1 / 640$ are positively associated with autoimmune diseases, mainly SE and its related manifestations. We propose active investigation of all patients who present this standard of ANA in very high titers, considering the incidence of evolution to autoimmune disease after 5 years of follow-up.
P048

\section{ASSOCIATION BETWEEN ERECTILE DYSFUNCTION AND GOUT IN}

BRAZILIAN PATIENTS

Andrey Tonetto Barbosa, Fernanda Pulcheri Ramos, Raissa Barbosa De

Souza, Matheus Xavier Guimaraes, Lisa Mielke De Oliveira, Rina Dalva

Neubarth Giorgi, Renata Ferreira Rosa

HOSPITAL DO SERVIDOR PUBLICO ESTADUAL DE SÃO PAULO, SÃO

PAULO, SÃO PAULO, Brasil

Advances in Rheumatology 2018, 58(Suppl 1):P048

Introduction: Erectile dysfunction (ED) is defined as the inability to achieve or maintain an erection appropriate for sexual satisfaction and may have multiple causes, such as psychological, vascular, neurological and/or endocrine. Clinical studies have shown that ED shares several risk factors with coronary heart disease, suggesting that ED may be a clinical marker of cardiovascular disease (CVD). Gout correlates with various metabolic comorbidities, including arterial hypertension, insulin resistance, dyslipidemia and obesity. New evidences show that hyperuricemia and inflammation may be independent risk factors for ED in addition to those already established.

Objectives: To determine the frequency of ED in patients with and without gout and define their demographic and laboratory features. Methods: This cross-sectional study, conducted in 2017, included 66 men aged between 40 and 65 years with gout according ACR/EULAR 2015 criteria followed at Rheumatology clinic in a tertiary hospital. As a control group, 37 healthy volunteers were recruited, matched for age. ED was determined by means of questionnaire (Sexual Health Inventory in Men - SHIM), with scores varying from 1 to 25 , and classified ED into one of five categories: absent (22-25), mild (17-21), mild to moderate (12-16), moderate (8-11) and severe (1-7). Patients with pre-existing cardiovascular disease and diabetes mellitus were excluded. For statistical analysis: mean, standard deviation, frequency (\%), t-student, Mann-Whitney and Chi-square tests were used. $\mathrm{P}<0,05$ was considered statistically significant.

Results: Of the 66 gout patients, the mean age was $63,20 \pm 8,36$ years and the most common comorbidities were arterial hypertension $(57,6 \%)$, dyslipidemia $(39,4 \%)$ and smoking $(34,9 \%)$. Fourteen out of 17 patients with tophi had ED $(82,8 \%)$. In gout group, 50 patients $(75,8 \%)$ presented ED, while $19(51,4 \%)$ in control group $(p=0,0162)$. The mean SHIM score of patients with gout was significantly lower than in control group $(15,85 \pm 6,61$ versus $20,03 \pm$ $3,37 ; p<0,001)$. A significantly greater proportion of gout patients $(29,5 \%)$ had moderate to severe ED compared with control subjects $(p<0,001)$.

Conclusion: $E D$ is present in most men with gout and is frequently moderate to severe. Increasing awareness of the presence of ED in gout patients may lead to earlier medical attention and treatment as well as evaluation of possible CVD. We propose active investigation of ED in patients with gout in order to anticipate unwanted cardiovascular events

\section{P051}

ASSOCIATION OF EPSTEIN BARR VIRUS AND

CITOMEGALOVIRUS WITH RHEUMATOID ARTHRITIS AND SJÖGREN'S SYNDROME

Camila Nunes Carvalho Sorgato', Angela Luzia B', Tatiany Romão Pompílio De Melo', Jair Carneiro Leão', Aysa Cesar Pinheiro', Paula Regina Toche Dos Santos', Luiz Alcino Monteiro Gueiros ${ }^{2}$

${ }^{1}$ UFPE, RECIFE, PE, Brasil; ${ }^{2} \mathrm{RE}, \mathrm{PE}$, Brasil

Advances in Rheumatology 2018, 58(Suppl 1):P051

Background: The role of viral infections in the pathogenesis of autoimmune diseases has long been suggested, but available evidence is 
still available. The objective of this study was to evaluate an association between Epstein Barr virus (EBV) and Citomegalovirus (CMV) and the presence of Rheumatoid Arthritis (RA) and its association with Sjögren's Syndrome (SS).

Material and Method: A sample composed of 256 patients of both gender and older than 18 years, divided into three groups: rheumatoid arthritis (RA, $n=108)$, Sjögren's syndrome associated with rheumatoid arthritis (RA/SS, $\mathrm{n}=20)$ ) and healthy controls $(C, \mathrm{n}=$ 128). Patients were submitted to resting sialometry, Schirmer's test and minor salivary gland biopsy in case of suspected SS. Blood samples were collected for detection and quantification of viral loads of CMV and EBV and glandular tissue samples for the detection of these viruses by in situ hybridization (EBV) and immunohistochemistry (CMV).

Results and Conclusions: EBV was more frequent in patients with RA and SS than in healthy controls ( $p<0.000007$ ), but no correlation with clinical markers ( $p>0.05$ ) and no difference between RA and AR/ SS ( $p>0.05)$. A higher number of EBV/DNA copies was found in RA

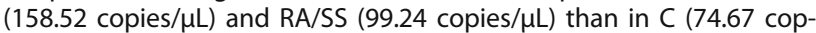
ies $/ \mu \mathrm{L}, \mathrm{p}=0.739$ ). A statistically significant association was found between the Schirmer test and an EBV/DNA presence $(p=0.0231)$. CMV was only found in one patient of RA group. The two viruses were not detected in biopsies of minor salivary glands. The presence of EBV/ DNA in peripheral blood but not its viral load is associated with RA but not with SS.

\section{P052}

\section{ASSOCIATION OF HLA-B27 POSITIVITY WITH CLINICAL} MANIFESTATIONS OF SPONDYLOARTHRITIS

Jhésyca Flávia Sousa Castaman Stédile', Betânia Longo², Bárbara Stadler Kahlow², Juliana Simioni², Ana Paula Beckhauser de Campos², Thelma Larocca Skare ${ }^{2}$

'HOSPITAL UNIVERSITÁRIO EVANGÉLICO DE CURITIBA (PR), CURITIBA, PR, Brasil; ${ }^{2}$ HOSPITAL UNIVERSITÁRIO EVANGÉLICO DE CURITIBA, CURITIBA, PR, Brasil

Advances in Rheumatology 2018, 58(Suppl 1):P052

Background: HLA-B27 is one of the most well studied genetic factors within spondyloarthritis (SpA). Although ankylosing spondylitis (AS) is the disease most associated with this HLA, its association is also verified, to a lesser extent, in other forms of $\mathrm{SpA}$ and its presence can modulate the patient's phenotype. In this study we sought to verify the clinical variability associated with the presence of HLA B27 in a group of patients with $\mathrm{SpA}$.

Methods: This is a retrospective study of 119 medical records of patients of both sexes, followed in a single university hospital with a diagnosis of SpA. For inclusion, patients should meet the ASAS criteria for SpA and have HLA B27 screening performed.

Results: In this sample, $56.3 \%$ had AS, $15.9 \%$ had psoriatic arthritis; $10.9 \%$ had undifferentiated arthritis, $6.7 \%$ had non radiological SpA and $3.3 \%$ had reactive arthritis, juvenile $\mathrm{SpA}$ and $\mathrm{SpA}$ associated with inflammatory bowel disease each. The prevalence of HLA-B27 positivity was $62.1 \%$ (74/119). Comparison of HLA-B27 positive and negative individuals can be seen on Table 1 .

Conclusion: The studied sample showed association of HLA-B27 with axial involvement of SpA, with AS being the most related phenotype. It was also observed a negative association of HLA-B27 with skin lesions and inflammatory bowel disease.

\section{References}

1. Lin $H$. et al. Association of HLA-B27 with ankylosing spondylitis and clinical features of the HLA-B27-associated ankylosing spondylitis: a meta-analysis. Rheumatol. Intern. 2017; 37:1267-80.

2. Yu DT et al. Overview of the clinical manifestations and classification of spondyloarthritis. UpToDate. 2017. Disponível em: . Acesso em: 11/ $01 / 2018$
Table 1 (abstract P052). COMPARISON OF SPONDYLOARTHRITIS (SPA) PATIENTS WITH AND WITHOUT HLA-B27

\begin{tabular}{|c|c|c|c|}
\hline & $\begin{array}{l}\text { RLA-B27 Positive } \\
\qquad \begin{array}{l}N=74 \\
\text { a }\end{array}\end{array}$ & 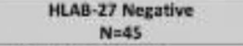 & $\mathbf{p}$ \\
\hline \multirow[t]{2}{*}{ Gender } & Females= 25/74 [33.7W] & Females=18/45 (40X) & 0.49 \\
\hline & Males $=49 / 74\left[66.2 \%_{1}\right]$ & Males $=27 / 45$ [60s; & \\
\hline \multirow[t]{2}{*}{ Age (years) } & 21-BO & 22.87 & 0.0003 \\
\hline & Mean=64.9n11.8 & Meann $53.3+12.4$ & \\
\hline Ethnit & Caucasian-49/51 (80 -3\%) & Coucasian, $18 / 25(69.2 \mathrm{~N})$ & \\
\hline background & Afro-descendents-12/61 (19.65) & Afro-descendants- $8 / 26(39.7 \%)$ & 0.26 \\
\hline \multirow{3}{*}{ Spa dagnosis } & AS $-48 / 74$ (62.836) & AS $19 / 45(42.226)$ & 0.001 \\
\hline & $P_{50 A-5 / 74}(5.7 \%)$ & PSOA-19/445 (31.18) & \\
\hline & Others-21/74 [28. $3 \times$ ] & Othess-12/45 (26.65) & \\
\hline & $1 / 74(13 \times 6)$ & \multicolumn{2}{|c|}{ bowell disease } \\
\hline Psoriasis & $5 / 74(6.7 \%)$ & $12 / 45(32.156)$ & 0.0004 \\
\hline Uveltis & $29 / 74$ [39.1\%] & $12 / 45$ (26.5\%) & 0.15 \\
\hline Dactylits & $5 / 74(6.7 \%)$ & $6 / 45(13.3 \times)$ & 0.22 \\
\hline Artheitis & $35 / 74(47.266)$ & $27 / 45(5096)$ & 0.17 \\
\hline Entesitis. & $42 / 74(56.7 \%)$ & $19 / 45(42.26)$ & 0.12 \\
\hline Low back pain & $67 / 74(90.5 \%)$ & $37 / 45(82.26)$ & 0.18 \\
\hline \multirow[t]{3}{*}{ Cinical form } & Axial-36/74 (4R.6\%) & Axial-15/45 (33.33) & 0.22 \\
\hline & Peripheral. $8 / 74$ (10.8\%) & Peripheral $\mathrm{B} / 45(17.7 \mathrm{~W})$ & \\
\hline & Mixed $-30 / 7$ t $\{40554$ ] & Mixed -22/45 (4ts 8x) & \\
\hline
\end{tabular}

P057

ATYPICAL FEMORAL FRACTURES - CLINICAL-EPIDEMIOLOGICAL PROFILE OF PATIENTS IN A TERTIARY HOSPITAL

Natália Carneiro Dos Santos, Ana Clara Ribeiro, André Ozela Augusto, Dayrana Alves Lucena, Lais Farrapo De Barros Leite, Marcelo Nora

Resende, Elaine De Azevedo, Rina Dalva Neubarth Giorgi HOSPITAL SERVIDOR PÚBLICO ESTADUAL - SP, SÃO PAULO, SÃO PAULO, Brasil

Advances in Rheumatology 2018, 58(Suppl 1):P057

Introduction: Atypical femoral fractures are a rare complication of chronic therapy with bisphosphonate (BF). The American Society for Bone and Mineral Research (ASBMR) generically estimates a cumulative incidence of 0.9 atypical fractures per 100,000 people per year. They differ from classical osteoporotic fractures in several aspects, but mainly on location, since it involves the strongest regions of the femur, subtrochanteric and diaphyseal, differently to osteoporotic fracture, which commonly occurs in the femoral neck. BF are among the main prescribeds medications worldwide for the treatment of osteoporosis (OP) and its role in reducing the incidence of vertebral and non-vertebral fractures when used in the treatment of senile and postmenopausal OP is already established.

Methods: A cross-sectional observational study, including patients following up at an OP service of a tertiary hospital in the state of São Paulo, from 2008 to 2018, who presented atraumatic, subtrochanteric or diaphyseal femoral fractures, in the use of bisphosphonate, and who did not present other conditions associated with the alteration of bone integrity $(\mathrm{N}=10)$.

Results: It was observed that $100 \%$ of the studied population were female, with a mean age of 76.2 years old (65-90). At the time of diagnosis of OP, $50 \%(n=5)$ were younger than 60 years old and only one patient was older than 70 years. Eight patients were taking oral BF while only two were taking zoledronic acid. The average time using BF before fracture was 10.2 years $(5-20)$, and $30 \%(n=3)$ of the patients were in use for just 5 years. The type of atypical fracture most observed was the diaphyseal, in $40 \%$ of the cases $(n=4)$. No patient presented a new atypical fracture after discontinuation of BF, but $70 \%(n=7)$ of them presented fragility fractures, the most prevalent was the vertebral fracture $(n=2)$.

Conclusion: Patients with a history of prolonged treatment ( $>5$ years) with BF for OP, in general, with a longer diagnosis time, are more likely to evolve with atypical fractures. Even with the small number of cases, there was an expressive increase of fragility fractures after the suspension of BF. It is inferred, therefore, as already established in the literature, that the risk for atypical fractures is small compared to the benefit of the protection conferred by antireabsorption treatment. 


\section{P058}

ATYPICAL FRACTURE IN PATHOLOGICAL BONE OF A PATIENT WITH IMPERFECT OSTEOGENESIS AFTER PROLONGED USE OF PAMIDRONATE: CASE REPORT

Beatriz Barbosa Teixeira, Thais Lavareda Nascimento, Igor dos Santos Costa, Matheus Lucas da Silva Santana, Camila Alves do Amaral Silva UNIVERSIDADE FEDERAL DE RORAIMA, BOA VISTA, RR, Brasil

Advances in Rheumatology 2018, 58(Suppl 1):P058

Imperfect Osteogenesis $(\mathrm{IO})$ is a genetic disease of the connective tissue caused by the synthesis of type 1 collagen of poor quality or in reduced quantity. Consequently, hydroxyapatite crystals do not properly penetrate the gaps of the collagen to mineralize it and the connective tissue becomes brittle. Therefore, the bone will have impaired quality and mineralization, with a high risk of fragility, especially in long bones. In addition, other manifestations of 10 include imperfect dentinogenesis, bluish sclera, short stature, triangular face and scoliosis. Diagnosis is usually based on clinical criteria (history of repetitive fractures in children), family history and radiological findings. A case of a 24-year-old patient with 10 of a hybrid form is reported, and does not matching types determined by the Classification of Imperfect Osteogenesis proposed by Sillence, it made prolonged use of Pamidronate at a dosege of $90 \mathrm{mg} /$ day for 3 days every 6 months for 10 years. In the tenth year of treatment, there was an atypical fracture in the right femur. The event caused the insertion of a plaque,

10 screws, grafting and realignment in the bone affected 6 months ago.

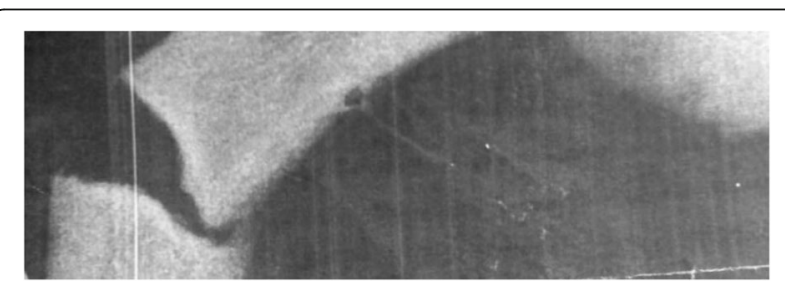

Fig. 1 (abstract P058). Fracture in the right femur

\section{P059}

ATYPICAL INITIAL MANIFESTATION OF SYSTEMIC LUPUS ERYTHEMATOSUS WITH MYOCARDITIS IN A 62-YEAR-OLD WOMAN

Lucas José Sá Da Fonseca, Lerika Moreira Rêgo, Lais Quintiliano Pedroza, Thiago Sotero Fragoso, Vanessa Miranda Pereira Fausto, Sasha Rodrigues de Vasconcellos Costa

UNIVERSIDADE FEDERAL DE ALAGOAS, MACEIÓ, AL, Brasil

Advances in Rheumatology 2018, 58(Suppl 1):P059

Background: Systemic Lupus Erythematosus is a systemic autoimmune disease virtually capable of compromising all organic systems. More commonly affecting women between 16 and 55 years old, its onset in older persons is much less frequent (about 15 percent of the cases) and usually milder. Furthermore, the women: men ratio tend to be reduced with the advance of age.

Case report: a 62-year-old previously healthy woman presented to the Rheumatology department complaining of dyspnea, alopecia and polyarthralgia initiated in the preceding twelve months. The patient was already under treatment for heart failure prescribed by a cardiologist, without significant symptomatic improvement. Clinical history revealed unintentional weight loss of 48 percent of habitual body weight in the preceding year, recurrent fever without evident infection, and previous full alopecia. Physical examination showed crackles in both lung bases, painful hepatomegaly and notable lower limbs edema, with the laboratorial findings of anemia, leucopenia, lymphopenia, elevated erythrocyte sedimentation rate, renal dysfunction (creatinine levels of $1.36 \mathrm{mg} / \mathrm{dL}$, with clearance of $31 \mathrm{ml} / \mathrm{min} /$ $1.73 \mathrm{~m} 2$; previous creatinine of $0.77 \mathrm{mg} / \mathrm{dL}$ ) and positive antinuclear antibody (ANA; nuclear homogeneous pattern at a titer superior to 1:640). As her echocardiogram showed ejection fraction of 45 percent (while being pharmacologically treated for heart failure), with diffuse hypokinesia of both ventricles, the diagnosis of systemic lupus erythematosus with myocarditis was established, with the prescription of prednisone $0.5 \mathrm{mg} / \mathrm{kg} /$ day (higher doses were avoided at first, because of the advanced age and uncontrolled systemic arterial hypertension at that time). Two weeks later, the patient returned for clinical reassessment, without peripheral edema or dyspnea, and with normal breath sounds and abdominal examination. New laboratory findings demonstrated reduced complement levels, as well as high titers of double-strand anti-DNA and anti-Ro antibodies, when corticoid dose was then increased to $1 \mathrm{mg} / \mathrm{kg} /$ day. Once 24 -h proteinuria fulfilled criterion for nephritis (792 mg; renal biopsy showing class IV lupus nephritis), treatment with monthly intravenous cyclophosphamide for six months was initiated. After a five-dose course of cyclophosphamide, the general clinical status dramatically improved, along with normalization of the cardiac ejection fraction (from 45 percent to 74 percent) and reduction in 24 -h proteinuria (which was shown to be $406 \mathrm{mg}$ ).

Conclusion: the case presented emphasizes the importance of considering systemic autoimmune diseases in the differential diagnosis of cardiac compromise in older women with heart failure of apparently cryptogenic ethiology.

\section{P065}

AUTOINFLAMMATORY DISEASES: CLINICAL, LABORATORY, GENETIC FEATURES ANDRESPONSE TO TREATMENT OF A REFERENCE CLINICAL PRACTICE IN RHEUMATOLOGICPEDIATRIC POPULATION

Erica Naomi Naka Matos, Milena Foizer Leite, Carolina Yume Arazawa, Laura Loureiro De Souza Rosa

UNIVERSIDADE FEDERAL DE MATO GROSSO DO SUL, CAMPO GRANDE, SSP/MS, Brasil

Advances in Rheumatology 2018, 58(Suppl 1):P065

BACKGROUND: Autoinflammatory Syndromes (AIS) are a group of rare diseases with genetic alterations characterized by the presence of recurrent or continuous episodes of fever, associated with systemic or localized inflammatory signs that affect mainly the musculoskeletal, cutaneous, gastrointestinal, ocular and neurological systems. The objective of this study was to investigate epidemiological, clinical and laboratory features of patients with AIS followed in our clinical practice and evaluate the difficulties in diagnosis and treatment. MATERIALS AND METHODS: An observational, descriptive, retrospective study was perfomed. Were analysed data of patients with AIS followed between 2003 and 2017, collected through a form (ethnic group, gender, age of onset symptoms and diagnosis, family history, clinical, laboratory and genetic features, therapeutic response).

RESULTS AND CONCLUSIONS: There was six patients whit AIS, 5:1 males-female ratio. The age in the moment of diagnosis ranged from three to ten yearsold. The averaged time passed since the symptoms onset was 32 months. Two patients had mediterranean progeny. Diagnoses were: Family Mediterranean Fever - FMF (4), Neonatal Onset Multisystem Inflammatory Disease - NOMID (1) and Periodic Syndrome Associated with the Receiver of Tumor Necrosis Factor TRAPS (1). Fever and abdominal pain were present in $100 \%$ and all patients presented arthritis and arthralgia in some time of the diseases evolution. One patient presented delayed neuropsychomotor development (NOMID). Regarding the laboratory tests, were found: anemia (4), leukocytosis (3) and raise in platelets (2), inflammatory tests (6) and ferritin (3). The patient with NOMIDhad radiographic changes (bone rarefaction, enlarged metaphyses and epiphyses, patellar fragmentation and hypertrophy). Three patients had access to the molecular study: one had a mutation in the CIAS1 gene, one had a variant in the NOD2 gene (NM_022162) that is considered a risk modifier, but non-specific and another had negative result. Colchicine was the initial empirical choice therapy, being the first choice for Family Mediterranean Fever. Although they did not gain significant symptom control, nonsteroidal anti-inflammatory drugs and corticosteroids were the most widely used drugs in the different 
diseases. Immunobiological therapies were reserved for refractory and clinically more exacerbated casas as in NOMID and TRAPS. Therefore, AIS remains a challenge thatshould be recognized by pediatricians treating children with recurrent fever, to be early referred to a rheumatologist for treatment and avoid the perpetuation of the inflammation that affects the patient's well-being.

\section{Consent for publication}

The authors declare that they have obtained informed written consent from the patient's tutors for publication

\section{P066}

\section{AXIAL SPONDILOARTHRITIS FEATURES IN FIBROMYALGIA} PATIENTS

Fernada Del Castanhel, Mariana Sanchez Malagutti, Valderílio Feijó Azevedo, Eduardo Dos Santos Paiva

UFPR, CURITIBA, PR, Brasil

Advances in Rheumatology 2018, 58(Suppl 1):P066

Background: Fibromyalgia (FM) is a syndrome that can mask other rheumatological diseases, such as axial spondyloarthritis (axSpA). Widespread pain, fatigue and stiffness are common symptoms. The aim of this study was to evaluate the prevalence of clinical features of axSpA in patients suffering from FM and chronic back pain, seeking for indicators that could suggest the presence of this underlying condition. Materials and methods: One hundred FM patients between 18 and 65 years old were assessed. This evaluation consisted of a questionnaire based on the clinical arm (clinical features of axSpA, without HLA-B27 testing) of the ASAS ("Assessment of Spondyloarthritis International Society") axSpA criteria set and a questionnaire on the impact of FM (Revised Fibromyalgia Impact Questionnaire - FIQR) Results and conclusion: We found that $80 \%$ of the patients met clinical criteria for the diagnostic of axSpA. Inflammatory back pain, good response to non-steroidal anti-inflammatory drugs (NSAIDs), arthritis and enthesitis were significantly more common in these patients than in the ones who did not meet the criteria (Table 1). Patients with FM and positive axSpA criteria also presented with higher CRP ( $C$ - reactive protein) levels $(p=0.00035)$ as illustrated in Table 1. FIQR mean value was 63.6 and did not show significant difference between patients that met and did not meet axSpA criteria. The investigation of axSpA in patients suffering from FM and chronic back pain should be pursued, in particular if there is associated enthesitis, arthritis, good response to NSAIDs, inflammatory pain and elevated CRP.

Table 1 (abstract P066). CLINICAL FEATURES AMONG PATIENTS IN THE STUDY

\begin{tabular}{|c|c|c|c|}
\hline Characteristic & Criteria axSpAt & Criteria axEpA- & P value \\
\hline $\begin{array}{l}\text { Inflammatory back } \\
\text { pain }\end{array}$ & $59(59 \%)$ & $7(7 \%)$ & 0,0026 \\
\hline $\begin{array}{l}\text { Good response to } \\
\text { NSAIDs }\end{array}$ & $59(59 \%)$ & $4(4 \%)$ & $<0,001$ \\
\hline Arthritis & $29(29 \%)$ & $2(2 \%)$ & 0,029 \\
\hline Enthesitis & $30(30 \%)$ & $1(1 \%)$ & 0.0055 \\
\hline Uveitis & $1(1 \%)$ & 0 & 1.00 \\
\hline Dactylitis & $12(12 \%)$ & 0 & 0.1173 \\
\hline Psoriasis & $4(4 \%)$ & 0 & 0,5809 \\
\hline Crohn's/colitis & $2\{2 \%)$ & 0 & 1.00 \\
\hline $\begin{array}{l}\text { Family history of } \\
\text { SpA }\end{array}$ & $1(1 \%)$ & 0 & 1,00 \\
\hline
\end{tabular}

P067

AXONAL MOTOR AND SENSORY ACUTE NEUROPATHY IN SYSTEMIC LUPUS ERYTHEMATOSUS: RARE VARIANT OF GUILLAIN-BARRÉ SYNDROME: CASE REPORT

Andressa Alexandre de Araujo, Débora Rocha de Moura Rodrigues de Aguiar, Julia Yoneshigue Laranja de Oliveira, Paula Guedes Ferreira da Silva, Elba Sophia Theodoro Santos de Oliveira, Luciana de Abreu e Lima Pamplona, Luiz Felipe Dipe Prates Miranda, Carlos Baptista de Figueiredo HOSPITAL FEDERAL DE BONSUCESSO, RIO DE JANEIRO, RJ, Brasil

Advances in Rheumatology 2018, 58(Suppl 1):P067

Background: Guillain-Barré syndrome (GBS) is an immune-mediated inflammatory polyradiculoneuropathy usually occurring after infection and is the main cause of acute neuromuscular paralysis. Systemic lupus erythematosus (SLE) has neuropsychiatric manifestations, including GBS, occurring in $0.1 \%$ of cases.

Case report: A 19-year-old female patient diagnosed in January 2016 with cutaneous-articular SLE, using hydroxychloroquine $400 \mathrm{mg} /$ day and prednisone $10 \mathrm{mg} / \mathrm{day}$, immunology with ANA $1 / 640$ nuclear fine dotted dense, consumed complement, positive anti-DNA. Anti-RNP, anti-Sm, anti-Ro, anti-LA and anticardiolipins was negative. Presented in our hospital with nausea and intense myalgia which had started 3 days before admission, including face paresthesia, bilateral conjunctival hyperemia. She denied fever. During hospitalization, she developed urinary retention, intestinal constipation paralytic mydriasis of the left eye, descending paresthesia and paresis, with sensitive level. CSF with only a mild protein increase. Methylprednisolone $1 \mathrm{~g}$ for 5 days was initiated, however that was a rapid progression to tetraplegia, aphasia, arreflexia and anesthesia in the four limbs.

With this evolution, we started immunoglobulin intravenously $400 \mathrm{mg} / \mathrm{kg} /$ day for 5 days. She responded well with improvement of paresthesia and partial recovery of strength. However, the symptoms of dysautonomia remain.

Magnetic resonance of the brain and electroencephalogram showed no abnormalities. Otherwise, electroneuromyography showed alteration of the axonal sensory-motor type. These findings lead us to the diagnosis of Acute Motor Sensitive Axonal Neuropathy (AMSAN), a rare variant of SGB. Although intravenous immunoglobulin led to improved strength and sensory changes, there was no response to dysautonomic symptoms. Then, it was started pulse therapy with methylprednisolone $1 \mathrm{~g}$ for 5 days, cyclophosphamide $500 \mathrm{mg} / \mathrm{m} 2$ single dose and $1 \mathrm{mg} / \mathrm{kg} /$ day prednisone regular, obtaining a better control of nausea and emesis. The patient is followed up in our outpatient clinic, with cyclophosphamide $500 \mathrm{mg} / \mathrm{m} 2$ and immunoglobulin $400 \mathrm{mg} / \mathrm{kg} /$ day for 5 days, monthly. Comments: Most SLE patients have atypical GBS symptoms. Few studies related AMSAN variant to SLE. Another differential of our case is that the paralysis was descending, which can occur in the variants of GBS. Urinary and intestinal retention, which made the differential diagnosis more difficult, are due to dysautonomia, and may be associated with GBS. AMSAN variant consists in rapid evolution and poor prognosis. In our case, although dysautonomic symptoms remained, we observed a good response to immunoglobulin in strength and sensations.

Consent for publication

The authors declare that written informed consent was obtained from patient's mother.

P073

BELIMUMAB IN JUVENILE SYSTEMIC LUPUS ERYTHEMATOSUS (JSLE)

Marcia Bandeira, Christina Feitosa Pelajo, Ingrid Herta R Grein, Rafaela Wagner, Luana Ribeiro Carlos, Loris Lady Janz Junior

HOSPITAL PEQUENO PRINCIPE, CURITIBA, PR, Brasil

Advances in Rheumatology 2018, 58(Suppl 1):P073 
Background: Systemic lupus erythematosus (SLE) is a chronic multisystem autoimmune disorder in which $20 \%$ of patients are diagnosed in childhood. Childhood-onset SLE is associated with higher morbidity and mortality than adult-onset SLE. Most pediatric patients will require glucocorticoids and often immunosuppressive drugs, with the aim of reducing disease activity while also preventing longterm toxicities from medications.

Belimumab is a human monoclonal antibody that inhibits soluble Blymphocyte stimulator with small data in pediatric patients.

Methods: Observational study of patients with JSLE diagnosed before their 16th birthday, and treated with belimumab, from a single center in Brazil. Data were collected on demographic and disease characteristics, clinical manifestations requiring treatment, concomitant medications, disease course, and treatment outcomes. The outcome was defined as the physician's impression of improvement in the initial manifestation (s) being treated without worsening in other organ systems.

Results: 5 children treated with belimumab in addition to standard of care therapy had a follow up of at least 9 months. The median age at diagnosis was 11.3 years old (range 9-14 years old); the median disease duration at initiation of belimumab was 4 years (range 2-6 years). All patients were taking other background medications prior to initiation of belimumab (hydroxychloroquine, prednisone, cysclophosphamide, mycophenolate mofetil and azathioprine). The most common indications for initiation of therapy were inability to taper steroids, arthritis, rash and persistent or worsening serologic activity (increasing anti-dsDNA and hypocomplementemia). All patients had a significant decrease in disease activity as measured by SLEDAI, laboratory improvement and decreased autoantibody levels were noted 4 months after starting treatment. And a significant decrease in the amount of corticosteroid was allowed in $80 \%$ of the sample. Conclusion: With improvement of the survival in JSLE the prevent of accrue organ damage secondary to the disease process itself and to its therapy has become a major goal in the management of pediatric lupus in our era. This serie suggest a important role for belimumab in pediatric-onset SLE in tapering steroids and minimizing damage associated with disease flares.

\section{P074}

\section{BILATERAL ANEURYSM OF CAROTID ARTERIES AND} INTRACAVITARY THROMBUS IN THE RIGHT VENTRICLE AS RARE VASCULAR INVOLVEMENT IN A PATIENT WITH BEHÇET'S DISEASE -

\section{CASE REPORT}

Pedro Paulo De Alcantara Pedro ${ }^{12}$, Priscila Dias Cardoso Ribeiro², Rywka Tenenbaum Medeiros Golebioviski ${ }^{2}$, Dennise de Oliveira Nogueira Farias $^{2}$, Flávia Maria Matos Melo Campos Peixoto², João Victor Campos de Oliveira ${ }^{2}$, Pedro Matos ${ }^{2}$, Edgard Torres dos Reis Neto ${ }^{2}$

${ }^{1}$ UNIVERSIDADE FEDERAL DE SAO PAULO, SÃO PAULO, SP, Brasil; ${ }^{2}$ UNIVERSIDADE FEDERAL DE SÃO PAULO, SÃO PAULO, SÃO PAULO, Brasil

Advances in Rheumatology 2018, 58(Suppl 1):P074

Background: Behçet's disease (BD) is a systemic disorder characterized by recurrent bipolar ulcers, cutaneous lesions, ocular, neurological and vascular involvement. Arterial involvement may affect any vessel conferring greater morbidity and mortality and the need for aggressive immunosuppressive treatment due to the high risk of complications, such as rupture of aneurysms.

Case Report: A 25-year-old male patient with history of recurrent oral and genital ulcers during the last 10 years associated with pustular lesions on buttocks was admitted with hemoptysis and dyspnea in the last two weeks. Lung tomography confirmed the diagnosis of pulmonary thromboembolism with multiple acute and chronic thrombosed arterial branches, without pulmonary artery aneurysms. Patient also had previous history of bilateral lower limbs venous thrombosis four years ago and one month before admission he was submitted in another hospital to embolization of left internal carotid artery aneurysm after the diagnosis of ipsilateral abducent nerve palsy. Pathergy test, antinuclear antibody and antineutrophil cytoplasmic antibody were negative. Angiotomography of the aorta and its main branches demonstrated saccular aneurysm in the right common carotid artery and left common carotid thrombosis. Transthoracic echocardiography revealed an intracavitary thrombus in the right ventricle with $5.4 \mathrm{~cm}$ of diameter. A diagnosis of $\mathrm{BD}$ was performed with severe arterial involvement with multiple thrombosis and aneurysms. Immunosuppressive treatment was initiated with methylprednisolone pulse therapy $1 \mathrm{~g} /$ day for three consecutive days (followed by oral prednisone $1 \mathrm{mg} / \mathrm{kg} /$ day) associated with cyclophosphamide $0.5 \mathrm{~g} / \mathrm{m}^{2}$ and anticoagulation. Despite this treatment, patient evolved with right carotidinia and expansion of the aneurysm. He was submitted to surgical aneurysm repair (endarterectomy) without intercurrences and symptom improvement. Vessel biopsy through surgery confirmed the etiology of vasculitis of the aneurysm. There was also a reduction in the right ventricular thrombus size. Patient was referred to the outpatient clinic without complaints, using prednisone $1 \mathrm{mg} / \mathrm{kg} /$ day and monthly intravenous cyclophosphamide.

Conclusion: Vascular involvement in BD can occur in any vessel, arterial or venous. Arterial involvement of large vessels is a severe manifestation of the disease due to the risk of aneurysms, stenosis or thrombosis. In these cases, immunosuppression is the main therapy with high corticosteroids dose associated with immunosuppressants, such as cyclophosphamide or anti-TNFa. In aneurysms with risk of rupture, surgical treatment should be performed.

\section{Consent for publication}

The authors declare that they have obtained informed written consent from the patient's tutors for publication

\section{P076}

BISPHOSPHONATE-ASSOCIATED OSTEONECROSIS OF THE JAW: CASE SERIES

Caroline Almeida Oliveira', Lisa Mielke de Oliveira², Laís Farrapo de Barros Leite ${ }^{2}$, Matheus Xavier Guimarães ${ }^{2}$, Denise Moraes Horiy ${ }^{2}$, Bernardo Patrício Sequeira Dultra², Elaine de Azevedo², Rina Dalva Neubarth Giorgi ${ }^{2}$

${ }^{1}$ HOSPITAL DO SERVIDOR PÚBLICO ESTADUAL, SÃO PAULO, SP, Brasil; ${ }^{2}$ HOSPITAL DO SERVIDOR PÚBLICO ESTADUAL DE SÃO PAULO, SÃO

PAULO, SÃO PAULO, Brasil

Advances in Rheumatology 2018, 58(Suppl 1):P076

Introduction: Bisphosphonates (BF) are effective drugs in the treatment and control of conditions related to the loss of bone mineral density. The first description of bisphosphonate-induced osteonecrosis of the jaw (ONJ) occurred in 2003. Recently, it has also been identified in patients who used denosumab (Dmabe), without previous use of BF. ONJ has an incidence between $1 / 10.000$ and 1/100.000, being slightly higher than the general population. Although it may happen spontaneously, parenteral use remains the main risk factor for this event, most commonly in the jaw. In addition, it is necessary to exclude other predisposing factors, such as periodontal diseases, poor oral hygiene, smoking, diabetes, presence of malignant neoplasia, type of chemotherapy, chronic use of steroids and advanced age. Case Study: In the outpatient clinic of osteometabolic diseases of a public tertiary hospital in the city of São Paulo, seven female patients with a previous diagnosis of osteoporosis presented osteonecrosis of the jaw during follow-up in the last six years. The mean age during the event was 69 years, all with more than three years of antireabsorption therapy. Of these, three were using intravenous bisphosphonate (Zoledronic Acid) and four used the oral presentation (Alendronate or Ibandronate). Five patients had other associated risk factors for ONJ: two in prolonged use of steroids, two in diabetic patients and the other in patients with periodontal disease as the initial lesion.

Conclusion: The prevalence of ONJ in patients taking antireabsorbents is close to $0.10 \%$. Even though it is a serious adverse effect, the recommendation is to maintain the use of the medication in cases of high risk for fragility fractures. Attention should be given in cases where, in addition to prolonged use of BF, risk factors such as corticotherapy, dental manipulation with inadequate technique, periodontal disease and diabetes are associated. As seen in our series, these overlapping factors seem to increase the chance of ONJ. 
P077

BONE MINERAL DENSITY AS A PREDITOR OF OSTEOPOROTIC FRACTURES IN GROUP OF BRAZILIAN VERY ELDERLY PATIENTS Lisa Mielke de Oliveira', Raissa Barbosa De Souza ${ }^{2}$, Andrey Tonetto Barbosa ${ }^{2}$, Fernanda Pulcheri Ramos ${ }^{2}$, Denise Moraes Horiy ${ }^{2}$, Rina Dalva Neubarth Giorgi ${ }^{2}$, Elaine De Azevedo ${ }^{1}$

${ }^{1}$ HOSPITAL DO SERVIDOR ESTADUAL DE SÃO PAULO, SÃO PAULO, SÃO PAULO, Brasil; ${ }^{2}$ HOSPITAL DO SERVIDOR PUBLICO ESTADUAL DE SÃO PAULO, SÃO PAULO, SÃO PAULO, Brasil

Advances in Rheumatology 2018, 58(Suppl 1):P077

Introduction: Osteoporosis is a systemic skeletal disease characterized by low bone mass and microarchitectural deterioration of bone. The incidence of osteoporotic fractures (OF) increases with age and according to FRAX Brazil, the central risks for OF are: family history of fracture, female, smoking, alcoholism, rheumatoid arthritis and chronic use of glucocorticoids. The global prevalence of OF is growing with the aging of the population, which increases substantially the morbidity, mortality and economic cost. It should be considered that at very elderly (VE) there is a main prevalence of multiple diseases and consequently polypharmacy.

Objectives: To compare risk factors for OF and bone mineral density (BMD) in VE patients with primary osteoporosis and relate to the presence of OF.

Methods: This retrospective study, conducted in 2018 included 91 patients with the diagnosis of osteoporosis aged above 85 years followed at Rheumatology clinic in a tertiary hospital. The subjects were divided in two groups, with and without OF (group 1, N=72, group 2, $\mathrm{N}=19$, respectively). For statistical analysis: mean, standard deviation, frequency (\%), t-student, Mann-Whitney and Chi-square tests were used. $P<0,05$ was considered statistically significant.

Results: Of the 91 VE patients, $89,01 \%$ were females $(\mathrm{N}=81)$ and the mean age was $87,87 \pm 3,43$ years. The most prevalent diseases in both groups were hypertension $(\mathrm{N}=69,75,82 \%)$, osteoarthritis $(\mathrm{N}=58$, $63,74 \%)$, chronic kidney disease $(\mathrm{N}=49,53,84 \%)$ and gastroesophageal reflux $(\mathrm{N}=39,42,85 \%)$. The main prescribed medicines were zoledronic acid $(\mathrm{N}=42,46,15 \%)$, denosumab $(\mathrm{N}=32,35,16 \%)$ and teriparatide $(\mathrm{N}=5,5,49 \%)$. In group 1 the most commons OF were vertebral $(\mathrm{N}=34,47,22 \%)$, wrist $(\mathrm{N}=17,18,06 \%)$, hip $(\mathrm{N}=15,16,67 \%)$ and others $(\mathrm{N}=18,25 \%)$, however $33(45,83 \%)$ patients had two or more OF. The menopause age of the group 1 was earlier $(p=0,045)$ being the only clinical risk factor with significance for OF. When compared BMD of the total femur, was observed lower BMD of the densitometry in group $1(0,668 \mathrm{~g} / \mathrm{cm} 3)$ versus group $2(0,755 \mathrm{~g} / \mathrm{cm} 3)$ $(p=0,0071)$. According to ROC curve analysis, the best cutoff value for BMD was $0,768 \mathrm{~g} / \mathrm{cm} 3$, yielding sensitivity and specificity of $50 \%$ and $86,96 \%$, respectively, for predicting OF events in this study. Conclusion: In the present study, it was found that both, comorbidities and clinical risk factors, were not sufficient to determine an increased risk for OF. The complementary exam, bone densitometry, was good predictor for risk of OF after 85 years old. Therefore, we suggest that this test be performed at least once in VE patients.

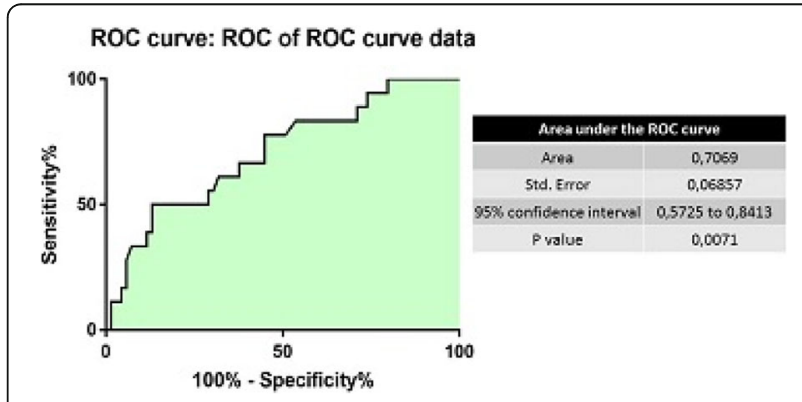

Fig. 1 (abstract P077). ROC curve analysis
P080

CANNABIS ARTERITIS: A CASE WITH A STRONG ASSOCIATION

Luiz Felipe Dipe Prates Miranda ${ }^{2}$, Gabriela Araújo Campos ${ }^{2}$, Filipe Kruger $^{2}$, Larissa Franco Motta de Souza', Guilherme Nogueira d'Utra', Débora Rocha de Moura Rodrigues de Aguiar², Carlos Baptista de Figueiredo ${ }^{2}$

${ }^{1}$ HOSPITAL MUNICIPAL MIGUEL COUTO, RIO DE JANEIRO, RJ, Brasil;

${ }^{2}$ HOSPITAL FEDERAL DE BONSUCESSO, RIO DE JANEIRO, RJ, Brasil

Advances in Rheumatology 2018, 58(Suppl 1):P080

Introduction: Cannabis is the psychoactive substance most consumed by young people. The thromboangiitis obliterans is an inflammatory, segmental and non-atherosclerotic disease, which most commonly affects the arteries and veins of small and medium calibers of the extremities. It is most frequent in men and is strongly associated with tobacco use. The cessation of smoking is important to decrease the risk of amputation. It has also been reported in marijuana users (Cannabis Arteritis).

Case report: Woman, 33 years old, former smoker of 20 packs-year and a regular marijuana user. Deny use of other illicit drugs. She presents ischemic lesions of 10 years evolution in extremities, with a previous history of amputation of her second left pododactyl. In another hospitalization she had negative research for thrombophilia and she stopped smoking because of the thromboangiitis obliterans hypothesis, however the lesions continued to progress. She was admitted for investigation of the lesions in September 2017, when she was submitted to an angio tomography and arterial Doppler of the limbs, without alterations. ANA, ANCA, VDRL, RF and serologies for HIV and hepatitis $B$ and $C$ were negative and there was no monoclonal peak into electrophoresis of serum proteins. Arteriography of the lower limbs showed obstruction of the left anterior tibial artery in the middle and distal thirds and of the microcirculation of the ipsilateral foot. Because of the evolution of the lesions, treatment with alprostadil, acetylsalicylic acid, cilostazol and calcium channel blocker was initiated. During the hospitalization period, the lesions showed improvement, with complete healing. After 3 months of discharge, the patient returned to a routine visit with signs of digital ischemia and with the reappearance of the ulcers, when she admitted having returned the use of marijuana and noticed its correlation with the lesions. We kept the same treatment of the first hospitalization and referred to psychology and psychiatry services for assistance in the treatment of drug withdrawal. The lesions regressed again, reinforcing the suspicion of association with the use of Cannabis.

Comments: Despite currently available reviews do not suggest the use of Cannabis as a cause of arteritis due to the concomitant use of tobacco in most patients, our patient presented worsening of the lesions after cessation of smoking and important improvement after the suspension of marijuana, strengthening the hypothesis of this association.

Consent for publication

The authors declare that they have obtained written informed consent from the patient for publication.

Consent for publication

The authors declare that they have obtained informed written consent from the patient's tutors for publication

P083

CARDIAC VALVAR INVOLVEMENT IN SLE REQUIRING SURGERY: REPORT OF FIVE CASES

Jhésyca Flávia Castaman Stédile', Aiessa Z. Fedrigo ${ }^{2}$, Betânia Longo ${ }^{2}$ Carla Luchese de Almeida ${ }^{2}$, Juliana Delfino ${ }^{2}$, Deborah Cristyne Colombo ${ }^{2}$, Thelma Larocca Skare ${ }^{2}$

${ }^{1}$ HOSPITAL UNIVERSITÁRIO EVANGÉLICO DE CURITIBA (PR), CURITIBA, PR, Brasil; ${ }^{2}$ HOSPITAL UNIVERSITÁRIO EVANGÉLICO DE CURITIBA, CURITIBA, $\mathrm{PR}$, Brasil

Advances in Rheumatology 2018, 58(Suppl 1):P083

Background: Systemic lupus erythematosus (SLE) is a chronic, multisystemic and autoimmune disease that may affect all structures of the heart. Valvar involvement, when studied by echocardiography, 
occurs in almost $60 \%$ of the patients. They are usually described as diffuse valvar thickness of mitral or aortic valves. Severe hemodynamic changes requiring cardiac valve replacement are rare. Here, we describe five cases of SLE patients needing cardiac valve substitution.

Cases Report: All five patients filled the ACR classification for SLE diagnosis. In $80 \%(4 / 5)$ of the cases, it was found double mitral lesion (stenosis and insufficiency); only one (1/5) had isolated aortic lesion (stenosis). All patients were female with mean age of 35 years at valvar lesion diagnosis and all were negative for antiphospholipid antibody. In all, but one case, the diagnosis of valvar lesion was done after the lupus diagnosis. In addition, in all patients, metallic prosthesis was used for substitution. In this series, pericarditis was seen in $3 / 5$ cases and $1 / 5$ had myocarditis. Glomerulonephritis was the most commonly associated extra cardiac manifestation.

Discussion: Usually cardiac valvar lesions in SLE are associated with SAA, but this could not be verified in our cases. Interestingly, Gabrieli et al, studying 39 lupus and 20 primary SAA patients concluded that valvular involvement is frequent in SLE but it is apparently unrelated to antiphospholipid autoimmunization. In our series mitral is the most affected valve and the pericardium was the second cardiac structure more involved. Glomerulonephritis was also commonly seen in this series.

\section{References}

1. CORDEIRO A et al. Envolvimento valvular cardíaco em doentes com lúpus eritematoso sistêmico. Correlação com presença de anticorpos anticardiolipina. Acta Reum Port. 2004;29: 97-103.

2. GABRIELLI F et al. Cardiac valve involvement in systemic lupus erythematosus and primary antiphospholipid syndrome: lack of correlation with antiphospholipid antibodies. Int J Cardiol. 1995;51:117-26.

Table 1 (abstract P083). MAIN CHARCATERISTICS OF 5 SYSTEMIC LUPUS ERYTHEMATOSUS PATIENTS SUBMITTED TO CARDIAC VALVE REPLACEMENT

\begin{tabular}{lllllccl}
\hline & $\begin{array}{c}\text { Ethnic } \\
\text { background }\end{array}$ & $\begin{array}{c}\text { Age } \\
\text { (Years) }\end{array}$ & Gender & Valve & $\begin{array}{c}\text { Anti- } \\
\text { Ro }\end{array}$ & $\begin{array}{c}\text { Anti-ds } \\
\text { DNA }\end{array}$ & Other characteristics \\
\hline 1 & Afrodescendant & 53 & Female & Mitral & + & + & Glomerulonephritis \\
2 & Afrodescendant & 44 & Female & Mitral & - & + & Myocarditis, Pericarditis \\
3 & Caucasian & 52 & Female & Aortic & - & - & Glomerulonephritis \\
4 & Caucasian & 26 & Female & Mitral & - & + & Glomerulonephritis, Pericarditis \\
5 & Afrodescendant & 31 & Female & Mitral & + &. & Glomerulonephritis, Pericarditis \\
\hline
\end{tabular}

\section{P090}

CAUSES OF INTERNATION AND CLINICAL EVOLUTION OF RHEUMATOLOGICAL PATIENTS IN A INTENSIVE THERAPY UNIT OF THE REGIONAL HOSPITAL OF MATO GROSSO DO SUL

Solino de Matos Neto', Gláucia Moreira Souza de Queiroz', Ana Elisa Dantas Modesto', Laize Guerreiro de Jesus', Alex Magno Coelho Horimoto ${ }^{1}$, Patricia Rubini ${ }^{2}$, Claudnei Menezes de Rezende ${ }^{2}$ 'UNIVERSIDADE FEDERAL DE MATO GROSSO DO SUL, CAMPO GRANDE, MS, Brasil; ${ }^{2}$ HOSPITAL REGIONAL DE MATO GROSSO DO SUL, CAMPO GRANDE, MATO GROSSO DO SUL, Brasil

Advances in Rheumatology 2018, 58(Suppl 1):P090

Introduction: Patients with systemic autoimmune rheumatic diseases (SARD) may require admission to an intensive care unit (ICU) due to underlying disease, associated infections, complications or new manifestations. Usually these patients present significant morbidity and mortality, with death rates ranging from $16.7 \%$ to $64.3 \%$. Patients with disseminated intravascular coagulation or infection have the highest mortality rates. Coma, renal failure and acute abdomen are also predictors of worse prognosis. Mortality rates in patients with SARD exceed those predicted by the APACHE II score, and are higher than those in non-rheumatologic patients admitted to the ICU.

Objectives: To identify the main causes of hospitalizations of rheumatologic patients in the ICU, to describe the epidemiological profile of the patients assisted under this regimen and to determine the rate and cause of mortality of the patients during the period of 56 weeks.
Methods: An observational, analytical and cross - sectional study. All patients admitted to the Intensive Care Unit of the Regional Hospital of Mato Grosso do Sul from July 2015 to June 2016 were prospectively identified and monitored.

Results and Conclusions: A sample of 12 patients was obtained in the period, which had a mean age of $41.33 \pm 17.35$ years; with a predominance of females (58.3\%). Systemic lupus erythematosus (SLE) was the most common rheumatic disease $(41.7 \%)$ in patients admitted to the ICU, followed by systemic vasculitis (granulomatosis with polyangiitis and microscopic polyangiitis) in $25 \%$ of hospitalizations. The main reasons for the hospitalization of these patients were infections, followed by exacerbations of rheumatologic diseases. Among the patients evaluated, $41 \%$ had the first diagnosis of SARD in the ICU and found a mortality rate of $25 \%$ in the sample, with alveolar hemorrhage in the context of vasculitis being the main cause of mortality. Although the use of the APACHE II score is contradictory in predicting the outcomes of patients admitted to the ICU, it has shown a significant relationship with evolution for renal failure in our study, considering the importance of this tool in the evaluation of rheumatic patients in the ICU.

P091

CAUSES OF MORTALITY IN JUVENILE SYSTEMIC LUPUS ERYTHEMATOSUS - EXPERIENCE OF A TERTIARY HOSPITAL IN RIO DE JANEIRO

Maurício Petroli, Ana Carolina Lopes Santiago, Jéssica Costa Farias, José Paulo Amoedo Bueno Brandão, Daniely Das Graças Roberto,

Blanca Elena Rios Gomes Bica

UFRJ, RIO DE JANEIRO, RJ, Brasil

Advances in Rheumatology 2018, 58(Suppl 1):P091

Background: Juvenile Systemic Lupus Erythematosus (JSLE) is an autoimmune chronic inflammatory disease that affects more girls than boys, with median age of onset at 12 to 13 years old. Its etiology is multifactorial. JSLE generates systemic repercussions that impacts especially pediatric range because of the disease's and its immunosuppressive therapy's effects on growth and development. Methods: Cross-sectional study based on review of 18 dead patients' medical records within a group of 120 patients diagnosed with JSLE between 1989 and 2016 and followed up in a Rheumatology service of a university hospital in Rio de Janeiro between 2000 and 2017. A retrospective analysis of medical records allowed collection of various data, whose subsequent analysis focused on clinical conditions at time of death and causes of mortality.

Results And Conclusions: Among the $18(15 \%)$ patients who died during follow-up, the ratio between female $(15,83.3 \%)$ and male $(3,16.7 \%)$ was 5:1. The mean time of disease until death was 101.5 months. The age at death ranged from 195 to 348 months. The mean age was 261.5 months, corresponding to 21.8 years old. $11(61.1 \%)$ patients died of infectious causes. Within infectious causes of death, the primary site of infection was: pneumonia $(2,16.7 \%)$, urinary tract infection $(1,8.3 \%)$, cutaneous infection $(2,16.7 \%)$, central nervous system infection (1, $8.3 \%)$, and acute gastroenteritis $(1,8.3 \%)$. In addition, 1 case was described as fungal infection (8.3\%) and 4 cases had no specific infectious focus (33.3\%). Another causes of mortality included: chronic kidney disease (1,5.5\%); refractory hemolytic anemia (1,5.5\%); macrophage activation syndrome (2, 11.1\%); transplant-associated graft-versus-host disease $(1,5.5 \%) .1$ (5.5\%) patient had both septic shock and macrophage activation syndrome. Cause of death remained unknown in 2 $(11.1 \%)$ cases. Among reviewed patients, the major death cause was infectious diseases, highlighting septic shock. The immunosuppressive therapy used to control the disease had difficult adherence in pediatric range, besides contributing with undesirable clinical outcomes, especially higher risk of infections. JSLE is an important disease in pediatric context due to its diagnostic difficulty, complex management and impairment in growth and development phase. Early diagnosis, close control and encouragement to therapeutic adherence contribute to the reduction of damage and the control of the disease. Early diagnosis of infections and its prompt treatment can minimize mortality rates secondary to infections in immunosuppressed patients. 


\section{P093}

CELIAC SYMPTOMS IN BRAZILIAN PATIENTS WITH FIBROMYALGIA FROM A TERTIARY HOSPITAL

Caio Bosquiero Zanetti, Diogo Souza Domiciano

HC-FMUSP, AMERICANA, SÃO PAULO, Brasil

Advances in Rheumatology 2018, 58(Suppl 1):P093

Background: Fibromyalgia (FM) is a chronic syndrome characterized by diffuse musculoskeletal pain and multiple somatic symptoms including functional gastrointestinal disorders. Celiac disease and nonceliac gluten sensitivity (NCGS) frequently evolve in adults with symptoms similar to those found among FM patients. However, prevalence of $C D / N C G S$ vary according to genetic background from different populations. There is no consistent data on frequency of gastrointestinal symptoms as well as no study performed duodenal biopsies to investigate CD/NCGS in Brazilian FM patients.

Objective: to determine the prevalence of CD/NCGS in patients with FM from a tertiary hospital and to verify the association between gastrointestinal manifestations and classical symptoms of FM.

Methods: 62 women (age 54.6 \pm 12.5 years) with FM (ACR 2010) were consecutively recruted from FM Outpatient clinics of a tertiary hospital. Patients were excluded if they had other disease than osteoarthritis. Clinical evaluation included Widespread Pain Index (O-19), Severity Symptom Scale (0-12), fibromyalgia survey score ("fibromyalgia-ness") (0-31) and revised Fibromyalgia Impact Questionnaire, besides presence of gastrointestinal manifestations during at least 3 months in the last year. Subjects were screened for the presence of serum IgA antiendomysial antibodies and IgA tissue transglutaminase antibodies. An upper gastrointestinal endoscopy with duodenal biopsies was performed and samples were studied by two expert pathologists according to classification for CD (Marsh-Oberhüber). Potential statistical associations between gastrintestinal manifestations and FM symptoms were analyzed by chi-square, likelihood ratio, Student's t or MannWhitney's tests. Significance was set at $\mathrm{P}<0.05$. Results: 46 (74.2\%) women reported at least one digestive symptom: 34 (54.8\%) constipation, $35(56.5 \%)$ abdominal distension, 21 (33.9\%) loss of weight or inappetence, and 21 (33.9\%) nausea or vomiting. The average fibromyalgia survey score and FIQR were 16.9 points and 56.5 (moderate impact on quality of life), respectively. There was an association between presence of any digestive symptom and scores Widespread Pain Index and Severity Symptom Scale (fatigue, waking up tired and cognition). $14(31.8 \%)$ individuals presented macroscopic duodenitis and 2(4.5\%) had lymphocytic infiltrates on duodenal tissue, but none met CD criteria. In 1 (2.3\%) patient the NCGS was confirmed.

Conclusion: In this sample of FM women from a tertiary hospital, we did not find any case of CD and only subject had NCGS. There was a high prevalence of gastrointestinal symptoms (more than half referring constipation or abdominal distension). A positive association between these manifestations and greater degree of central pain amplification was observed. Nevertheless, no difference was found in the impact of FM on quality of life (FIQR) in patients with and without gastrointestinal symptoms.

\section{Consent for publication}

The authors declare that they have obtained informed written consent from the patient's tutors for publication

\section{P095}

CEREBRAL PSEUDOTUMOR IN CHILDHOOD AND ADOLESCENCE: DATA FROM A SPECIALIZED SERVICE

Gabriela Guimarães Moreira Balbi, Iggor Oliveira de Sousa, Jade Dib Fernandez, Annelyse de Araújo Pereira, Melissa Marati Fraga, Claudio Arnaldo Len, Sandro Luiz de Andrade Matas, Maria Teresa Terreri UNIVERSIDADE FEDERAL DE SÃO PAULO, SÃO PAULO, SP, Brasil Advances in Rheumatology 2018, 58(Suppl 1):P095

Background: Cerebral pseudotumor or benign intracranial hypertension $(\mathrm{BIH})$ is a syndrome that presents with clinical features of elevated intracranial pressure without radiological evidence of intracranial mass, infection, vascular abnormality, hydrocephalus or changes in level of consciousness. It is rare in childhood. The incidence increases between the ages of 12 to 15 years, and $60 \%$ of children who develop the syndrome are over 10 years of age. Several conditions are associated with pseudotumor (secondary pseudotumor), such as systemic diseases and drug exposure. The term idiopathic intracranial hypertension is used when the cause of this condition is not found. There are few studies in the literature, most of them case reports, describing secondary pseudotumor syndrome in pediatrics. Our objective was to report all cases of children and adolescents diagnosed with cerebral pseudotumor, with or without rheumatic disease, who were followed by the pediatric rheumatologists and neurologists of our hospital.

Method: Retrospective study based on medical reports, 29 patients aged up to 18 years and diagnosed with cerebral pseudotumor, registered until December 2016 and followed up in pediatric rheumatology and neurology outpatient clinics of a tertiary hospital.

Results and conclusions: Among the 29 patients diagnosed with cerebral pseudotumor, $51.7 \%$ were girls and the mean age at disease onset was 12.3 years. Etiology was found in 18 patients (62\%) associated with a rheumatic disease in 4 cases. Among these, two of them had juvenile dermatomyositis undergoing oral glucocorticoid withdrawal. One patient had Henoch-Schönlein purpura also receiving glucocorticoid. The fourth patient presented anti-phospholipid antibody syndrome. The most common symptom was headache $(69 \%)$ and acetazolamide was the most used medication (69\%). Two patients developed blindness and 10 are still in follow-up. Although rare, pseudotumor cerebri should be considered in children with headaches, especially in patients with rheumatic disease.

\section{P097}

\section{CHANGES IN CYTOKINE EXPRESSION IN PATIENTS WITH JUVENILE} SYSTEMIC LUPUS ERYTHEMATOSUS SUBMITTED TO GINGIVAL INFLAMMATION CONTROL

Loreley Carlos Agostinho Bragard $^{3}$, Manuerla Rubim Camara ${ }^{1}$

Carlos Marcelo da Silva Figueredó, Flavio Roberto Sztajnbok ${ }^{3}$

${ }^{1}$ UNVERSIDADE DO ESTADO DO RIO DE JANEIRO, RIO DE JANEIRO, RIO

DE JANEIRO, Brasil; ${ }^{2}$ UNVIERSIDADE DO ESTADO DO RIO DE JANEIRO,

RIO DE JANEIRO, RIO DE JANEIRO, Brasil; ${ }^{3}$ UNIVERSIDADE DO ESTADO

DO RIO DE JANEIRO, RIO DE JANEIRO, RIO DE JANEIRO, Brasil

Advances in Rheumatology 2018, 58(Suppl 1):P097

The aim of this study was to evaluate the impact of treatment of gingival inflammation on the expression of cytokines in serum and gingival crevicular fluid (GCF) in patients with Juvenile Systemic Lupus Erythematosus (SLE). Twenty-four patients with SLE (mean age: $16.1 \pm$ 1.6 years) who had gingivitis participated in the study. Periodontal, rheumatologic, blood and GCF data were collected. Cytokines were analyzed by the multiplex assay with the Bio-plex Pro TM Th17 panel assay kit. The McNemar test was used for qualitative data and the Wilcoxon test was used for numerical data. After treatment of gingival inflammation, there was a decrease in SLEDAI $(p=0.039)$, IL-4 and IL-5 ( $p$ $=0.023$ and 0.026 ) in serum. In the gingival fluid, there was a significant decrease in IL-1 $\beta$, IL-10 and MCP-1 ( $p=0.039 ; 0.010 ; 0.025$; respectively) and a significant increase in IL-4, IL-12 (p70), IL-17, GM -CSF and INF-V $(p=0.003 ; 0.025 ; 0.001 ; 0.049 ; 0.004$; respectively). After the treatment of gingival inflammation, it was concluded there was no significant difference in SLICC (Systemic Lupus International Collaborating Clinics) values, but there was a significant reduction of absolute SLEDAI and serum IL-4 and IL-5 levels, probably in due to the time of treatment of SLE. The increase of the IL-12, IL-17 and GM-CSF cytokines in the GF analysis demonstrated a possibly unfavorable prognosis, since they are associated with local bone and tissue loss.

\section{P098}

CHARACTERISTICS OF CARE GIVEN TO HOSPITALIZED PATIENTS WITH OSTEOARTHRITIS IN THE LAST 9 YEARS IN SERGIPE

Luíza Brito Nogueira, João Victor de Andrade Carvalho, Bruno José

Santos Lima, Angela Santos Lima, Henrique Gouveia Borba e Souza,

Larissa Sá dos Santos, Juliana Monroy Leite, Nicole Santiago Leite,

Meyling Belchior de Sá Menezes, Yasmin Oliveira Santos

UNIVERSIDADE TIRADENTES, ARACAJU-SE, SE, Brasil

Advances in Rheumatology 2018, 58(Suppl 1):P098 
Background: The arthrosis is an painful infection of the joints caused by the unbalance between the formation and destruction of cartilage. It has already been classified as the most prevalent rheumatic disease among people over 65 years of age and, for being a chronic multifactorial disease, leading to progressive functional disability, patients with arthrosis present different demands of care - elective or urgent. Then, as the treatment of the condition varies from pharmacological to surgical, the hospitalization profile seems to be directly related to such demands.

Methods: This is an exploratory documentary study with secondary data obtained by DataSUS with the Sistema de Informações Hospitalares $(\mathrm{SIH})$ about the influence of elective or urgent care in patients hospitalized for arthrosis in Sergipe in the interval from October 2008 to October 2017.

Results And Conclusions: In the past years, about $67,6 \%$ of the patients admitted with arthrosis were assisted in an elective way. This predominance kept on mostly each year, witch its smallest number was in 2010 with $71,2 \%$ for the electives and the biggest, in 2008, reached 100\%. However, in the years of 2013 and 2014, not only there was an abrupt variation of this average, it was predominant the urgent stamp of the medical assistance with in-patients. Respectively, the numbers reached only $36,6 \%$ and $44,6 \%$ for the elective support. In general, from 2008 to 2017, there was an alarming 99,4\% increase in the hospitalization by arthrosis and, besides mostly of these have been assisted in an elective way, the urgent stamp of the medical support grew $12,7 \%$. Figure 1 shows this variation over the years. It is concluded that the relationship between the stamp of medical assistance and hospitalization of patients with arthrosis in Sergipe not only exists, but also it does not happen in a regular way throughout the years. The reason remains unknown, but the relationship can be predominant of the in-patients that were electively assisted because of the variety of treatments for arthrosis, like physiotherapy, the non-pharmacological, the pharmacological and the surgical, and their different levels of evolution.

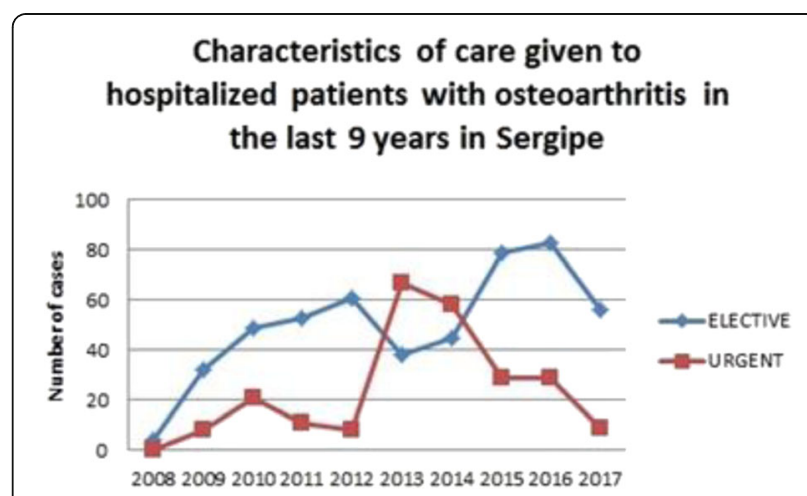

Fig. 1 (abstract P098). Characteristics of care given to hospitalized patients with osteoarthritis in the last 9 years in Sergipe

\section{P099}

CHARACTERIZATION OF CLINICAL BENEFITS IN SUBJECTS CLASSIFIED AS ACR20 NON-RESPONDERS AT WEEK 104 OF APREMILAST TREATMENT: SUBANALYSIS OF 3 LONG-TERM, PHASE III TRIALS

Priscila Magalhaes Reis Nakasato

CELGENE, Estados Unidos

Advances in Rheumatology 2018, 58(Suppl 1):P099

Background: The PALACE 1, 2, and 3 studies evaluated apremilast (APR) efficacy and safety in subjects with active psoriatic arthritis (PsA) despite prior conventional DMARDs and/or biologics. This analysis further characterizes the clinical benefits associated with longterm APR exposure in subjects failing to achieve an ACR20 response at Week 104.
Material and Methods: Subjects were randomized (1:1:1) at baseline to placebo (PBO), APR $30 \mathrm{mg}$ twice daily (APR30), or APR $20 \mathrm{mg}$ twice daily. Subjects randomized to APR30 at baseline and classified as ACR20 non-responders (ACR20NRs) at Week 104 were considered for this analysis. At Weeks 24, 52, and 104, ACR core components were examined as well as the proportions of subjects achieving PASI$75 /$ PASI- 50 among those with psoriasis involvement $\geq 3 \%$ of the body surface area (BSA) at baseline and dactylitis count and Maastricht Ankylosing Spondylitis Enthesitis Score (MASES) of 0 among those with dactylitis or enthesitis, respectively, at baseline. Safety is described for the overall PALACE 1-3 population.

Results: A total of 109 subjects randomized to APR30 at baseline were ACR20NRs at Week 104. Baseline ACR core components were similar for ACR20NRs and ACR20 responders at Week 104. Among these ACR20NRs, several core components of ACR response showed sustained improvements from baseline through Week 104 with continued treatment: swollen/tender joint counts (mean \% change, $-58.0 \% /-41.7 \%)$ and Physician's Global Assessment of Disease Activity (visual analog scale) scores (mean \% change, $-44.3 \%$ ). Importantly, of those with psoriasis BSA involvement $\geq 3 \%$ at baseline, $50.0 \%$ achieved a PASI-50 response and $36.0 \%$ achieved a PASI- 75 response after continued treatment with APR30 through Week 104. Among ACR20NRs with baseline dactylitis $(n=44)$ or enthesitis $(n=74)$, $68.2 \%$ achieved a dactylitis count of 0 and $33.8 \%$ achieved a MASES of 0 at Week 104, respectively. More limited improvements in Subject's Global Assessment of Disease Activity, Subject's Assessment of Pain, HAQ-DI, and CRP outcomes most commonly had an impact on subjects' ability to achieve an ACR20 response. In the overall subject population, no new safety concerns were identified through 104 weeks.

Conclusions: ACR20NRs receiving APR30 demonstrated significant improvements in core PsA domains. The data may explain why subjects who failed to achieve an ACR20 response remained on long-term APR treatment. These findings suggest some subjects with PsA may experience meaningful clinical improvements not completely captured by assessment of ACR20 response criteria. Outcome measures specifically designed for PsA subjects may be more suitable to evaluate treatment response in PsA subjects.

\section{P100}

CHARACTERIZATION OF PSORIATIC ARTHRITIS MUTILANS PATIENTS FROM A SINGLE TERTIARY CENTER

Jobson Lopes De Oliveira ${ }^{2}$, Rafael Alves Cordeiro ${ }^{2}$, Solange Carrasco', Célio Roberto Gonçalves², Julio César Bertacini De Moraes²,

Carla Gonçalves Schahin Saad², Percival Degrava Sampaio-Barros², Claudia Goldenstein-Schainberg

${ }^{1}$ FACULDADE DE MEDICINA DA UNIVERSIDADE DE SÃO PAULO, SAO

PAULO, SP, Brasil; ${ }^{2}$ DIVISAO DE REUMATOLOGIA, HOSPITAL DAS

CLINICAS HCFMUSP, FACULDADE DE MEDICINA, UNIVERSIDADE DE SAO

PAULO, SAO PAULO, SP, Brasil; ${ }^{3}$ HOSPITAL DAS CLÍNICAS DA

FACULDADE DE MEDICINA DA UNIVERSIDADE DE SÃO PAULO,

SÃO PAULO, SÃO PAULO, Brasil

Advances in Rheumatology 2018, 58(Suppl 1):P100

Background: Psoriatic arthritis mutilans (PsAM) occurs in about 5\% patients representing the most serious and uncommon form of psoriatic arthritis (PsA). Data concerning PsAM subtype are scarce. It is characterized by shortening of fingers and/or toes, intense osteolysis of those peripheral joints and digital telescoping. In this study we evaluated the prevalence and clinical characteristics of PsAM patients from our tertiary rheumatology center

Methods: All PsA patients $\geq 18$ years according to CASPAR criteria followed at our rheumatology division between 2002 and 2017 were actively searched for PsAM deformity of hands and feet. PsAM was considered in the presence of shortened fingers, digital telescoping, and anteroposterior radiography showing at least one joint with severe erosive arthritis (for example pencil-in-cup or gross osteolysis of the bones) without osteophytes. Demographic, clinical, laboratory and radiographic data were searched.

Results: PsAM was found in $8.2 \%$ PsA patients (17/207), 12 males and 5 females with mean age $=59.0 \pm 9.9$ years and mean age at arthritis 
onset of $34 \pm 12.6$ years. According to co morbidities, hypertension occurred in 13 patients (76\%), dyslipidemia in $10(59 \%)$, metabolic syndrome in $9(53 \%)$, osteoporosis in $6(35 \%)$, psychiatric disorders in $4(24 \%)$, diabetes in $2(12 \%)$. Of note, $3(18 \%)$ patients had cancer $(1$ colon, 1 breast, 1 multiple myeloma). Syndesmophytes were evident in $9 / 16(56 \%)$ patients and radiographic sacroiliitis in 7/16 (44\%). Surprisingly 9 of $13(69 \%)$ patients tested for HLA-B27 were positive. Regarding therapy, 9/17 PsAM patients (53\%) were on biologic agents ( 2 adalimumab, 4 infliximab, 2 secukinumab, 1 etanercept).

Conclusion: We have shown a $8.2 \%$ prevalence of PsAM in our PsA patients, slightly higher than previously reported. The enhanced positivity of HLA-B27 and radiographic axial involvement in PSAM demonstrated for the first time is intriguing. Furthermore, biologic therapy use in more than half PsAM patients reinforces the severe nature of PsA mutilans involvement.

\section{P101}

\section{CHARACTERIZATION OF THE EPIDEMIOLOGICAL PROFILE OF} RHEUMATIC HEART DISEASE IN BRAZIL IN THE LAST 10 YEARS

Mariana Bomfim De Menezes, Érica Nascimento, Andressa Borelli, Debora Montarroyos

UNIT, ARACAJU, SE, Brasil

Advances in Rheumatology 2018, 58(Suppl 1):P101

Introduction: Rheumatic heart disease represents a high cost in health, not only in hospitalization, but also in post-discharge care. Knowing its epidemiology allows the adoption of preventive measures and the choice of the most appropriate therapy. The authors present the epidemiological characterization of the pathology over 10 years in Brazil.

Methods: Retrospective descriptive study that shows all Rheumatic heart disease between 2008 and 2018. Epidemiological data were collected database of System of Hospital Information of the Unified Health System (SIH-SUS).

Results: The present study demonstrated 6,383 cases in Brazil. It is also observed a heterogeneous distribution of the number of cases related to the southeastern region in the leadership with 2,853 reported cases. Infections in women were higher than in men during the period.

Conclusions: Through the data collected it is possible to observe the high index of cases of rheumatic heart disease. The fact that the southeastern region represents the second place for cases of rheumatic fever in the country, but leadership in this pathology demonstrates the local need for better prevention.

\section{P102}

\section{CHARCOT NEUROPATHIC ARTHROPATHY SECONDARY TO} SYRINGOMYELIA: A CASE REPORT

Natália Jardim Martins da Silva Brasil ${ }^{1,2}$, Ruy Sampaio de Siqueira Neto ${ }^{2}$, Leila Patrícia Fonseca Andrad Oliveira², Vitor Silva Souza², Fernanda Nogueira Holanda Ferreira Braga ${ }^{2}$

${ }^{1}$ JANSSEN BRASIL, FORTALEZA, CE, Brasil; ${ }^{2}$ HOSPITAL UNIVERSITÁRIO WALTER CANTÍDIO/UFC, FORTALEZA, CE, Brasil

Advances in Rheumatology 2018, 58(Suppl 1):P102

Introduction: Neuropathic arthropathy, also known as Charcot arthropathy, is a chronic, progressive degeneration of a joint associated with an underlying neurological disorder. It is characterized by pathological fractures, destruction, which eventually leads to debilitating deformities and loss of function. ' We report a case of Charcot neuroarthropathy, secondary to syringomyelia.

Case Report: A 57-year-old woman with complain of arthralgia on the left shoulder about eight years, evolving with decreased strength, tenderness and left upper limb atrophy. The physical exam showed significant left shoulder atrophy with functional limitation and mild movement pain, decreased strength (Grade 3), hypoesthesia of all limbs with deep reflexes absent. The left shoulder X-ray showed head and proximalhumeral metaphysis with multiple intra-articular bone fragments due to the joint destruction. On this, it was hypothesized of neoplasic or neuropathic arthropathy, and a cervical spine and left shoulder magnetic resonance imaging (MRI) were requested.
MRI of the left shoulder confirmed the extensive joint destruction; Cervical spine MRI suggested syringomyelia in cervical and dorsal spine. MRI of the thoracic and lumbar spine was performed to estimate the extent of lesion, however was evidenced only in the thoracic spine. With Charcot's neuropathic arthropathy secondary to syringomyelia hypothesis, the patient was referred to the neurosurgery and orthopedics to analyze the possibility of surgical intervention.

Discussion: Charcot arthropathy is a rare cause of joint destruction in individuals with various forms of neuropathy. The most common causes include: syringomyelia, diabetes mellitus, peripheral neuropathy and tabes dorsalis. The shoulder is involved in less than $5 \%$ of patients, and cervical syringomyelia accounts for $75 \%$ of cases. $2{ }^{3}$ There are two theories currently accepted about its pathogenesis. The neurotraumatic theory involves repeated trauma by an insensitive joint and neurovascular theory with osteoclastic activation leading to joint inflammation and bone resorption. 4 Treatment goals are to reduce further articular damage while retaining a functional joint. Initially conservative measures are adopted, including symptomatic treatment, anti-inflammatory, orthotics, weight reduction and patient education to minimize mechanical trauma. Recently, several studies have demonstrated a beneficial effect of bisphosphonates. Failure of conservative measures is an indication for surgical intervention.

\section{Consent for publication}

The authors declare that they have obtained informed written consent from the patient's tutors for publication

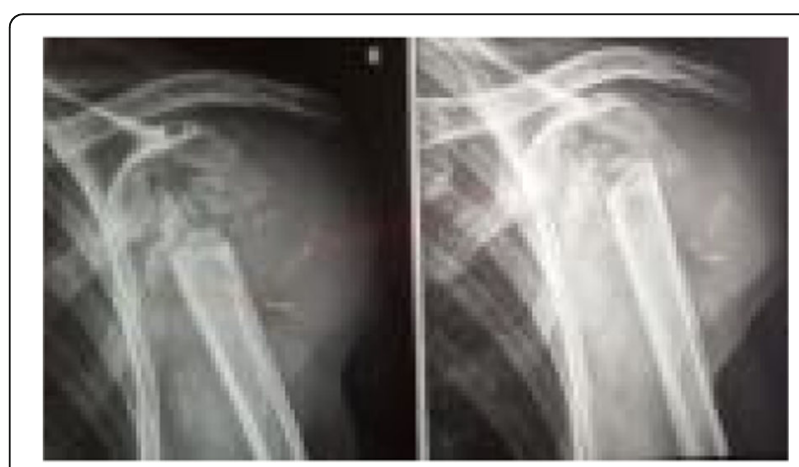

Fig. 1 (abstract P102). Radiograph of the left shoulder showing joint destruction of the head and proximal humeral metaphysis with multiple intra-articular bone fragments

\section{P107}

CHILBLAINS IN PEDIATRIC: A SERIES OF A CONFOUNDER FOR ARTHRITIS

Rafael Coradin, Thiago Willers, Maria Odete Esteve Hilario, Tatiana Freitas Tourinho, Maria Lúcia Lemos Lopes, Eduardo Souza da Rosa,

Elisa Pacheco Estima Correa

UNIVERSIDADE FEDERAL DE CIÊNCIAS DA SAÚDE DE PORTO ALEGRE,

PORTO ALEGRE, RS, Brasil

Advances in Rheumatology 2018, 58(Suppl 1):P107

Introduction: Chilblains (CB) is a disease with a eritematous localized purple cutaneous lesion associated with pain or itch. It occurs with after 12 to 24 hours after a cold exposure. It is usually self limited. In the south of Brazil it can appear with a relative frequency.

The objective of this article is to demonstrate our experience with $C B$ in pediatric patients in this case report.

Case reports: From 2014 to 2018 there was 9 cases. 88,8\% were female with an average of 15 -years-old. The hands (44\%) and the fingers $(77 \%)$ were the most affected, and the symptoms remains for about 4,5 weeks, being the itch and erythema the most common. In the laboratory tests, the Antinucleous Antibody (ANA) were present 
in $44 \%$ of the cases, with Nuclear Fine Dotted pattern in $75 \%$ of them. Two patients used Nifedipin and other two used naproxen as treatment, all others didn't needed any kind of medicine. One patient had a family story of $C B$ and other of Lupus.

Discussion/Conclusion: The $\mathrm{CB}$ is a benign disease. The understanding of this pathology is important to rheumatologists, because it can be a differential diagnosis of arthritis. The CB can occur secondary to Lupus, Behçet, malignancy and immunoglobulinopathies, enhancing its relevance. The literature in pediatric cases is scarce, with only few case reports. Differently of them, our children were more affected in the upper limbs (88\%). We had a predominance of female adolescents, as seen in previous articles. We had no cases with associated disorders, although almost half of the children had a positive ANA We did not perform skin biopsy because the cases were mild and self-limiting. Due to the possibility of chronicity, as well the risk of associated diseases, we should be aware of CB in childhood.

\section{P109}

CHOREA AS INITIAL MANIFESTATION OF SYSTEMIC LUPUS ERYTHEMATOSUS IN A YOUNG MAN: CASE REPORT

Larissa Cristiane De Oliveira Souza, Rodrigo Alencar e Silva, Juliana Maia Marinho, Saulo de França Oliveira, Olivia de Fátima Costa Barbosa,

Francisco Alves Bezerra Neto

UFRN, NATAL, RN, Brasil

Advances in Rheumatology 2018, 58(Suppl 1):P109

Background: Movement disorders are rare in patients with systemic lupus erythematosus (SLE). With a prevalence rate of $1.3 \%$, chorea is the most common amongst them. It usually occurs within the first years after the onset of SLE.

Case report: Male, 16 years of age, otherwise healthy, presented with migratory arthritis, anasarca and oral ulcers. Approximately 30 days after the onset of such symptoms, the subject experienced choreic movements, dysarthria and mental confusion. The patient denied recent history of seizures, fever or cutaneous lesions. Upon admission, he was alert, feverless, displaying equal and fotorreactive pupils, affective lability, generalized choreic movements (picture 1), appendicular hypotonia, normal deep tendon reflexes, bilateral flexor plantar reflexes and no meningeal signs. Tongue ulcerations on the inferior portion of the tongue were also observed. Left elbow and right metacarpophalangeal joint arthritis were also present. Lab workup revealed positive ANA 1/ 1280 (homogeneous nuclear and chromosomal metaphase plate pattern), positive Anti-DNA, leukopenia (3000), lymphopenia (1032) and hypocomplementemia. ESR was 28, 61.3 CRP, positive rheumatoid factor, negative P-ANCA and C-ANCA, negative ASO, hepatitis-related serology, VDRL and HIV. Skull CT scan and cerebrospinal fluid showed no abnormalities. The patient was given a diagnosis of SLE with movement disorder (chorea). He was given $1 \mathrm{~g}$ methylprednisolone daily for three days, and then started on prednisone $1 \mathrm{mg} / \mathrm{Kg}$, hydroxychloroquine $400 \mathrm{mg}$ and azathioprine $50 \mathrm{mg}$ daily, achieving full symptom remission. The patient is currently asymptomatic under hydroxychloroquine and azathioprine.

Conclusion: Chorea, ataxia, choreoathetosis, dystonia and hemiballismus figure among SLE movement disorders. Out of all SLE patients who develop chorea, $50 \%$ will do so after after being diagnosed with SLE, $25 \%$ will develop it before and $25 \%$ at the time of SLE diagnosis. It is paramount to bear in mind the existing association between Chorea and SLE as well as its differential diagnoses: rheumatic fever, infectious diseases, metabolic disorders. drug-induced and occasionally, other autoimmune disease. P.S.: Publication with consent of the patient.

\section{P111}

CHRONIC DIARRHEA AS INITIAL MANIFESTATION OF SYSTEMIC LUPUS ERYTHEMATOSUS

Mario Sergio Ferreira Santos ${ }^{1,2}$, Francisco Leonardo De Oliveira Leal ${ }^{2}$, Francisco Ewardo Rodrigues Da Silva ${ }^{2}$, Joyce Reis Costa ${ }^{2}$, Yane Chaves Martins Resende ${ }^{2}$, Jozelda Lemos Duarte ${ }^{2}$, Joelma Moreira De Noroes Ramos $^{2}$, Ingrid Mayra Pereira De Oliveira², Gabriela Grabowski Amorim² Therezinha Dantas Nobre Neta², Vinicius Leal Veloso ${ }^{2}$

${ }^{1}$ TERESINA, PI, Brasil; ${ }^{2}$ UNIVERSIDADE ESTADUAL DO PIAUÍ, TERESINA, PI, Brasil Advances in Rheumatology 2018, 58(Suppl 1):P111
Background: Systemic lupus erythematosus (SLE) is a chronic, autoimmune inflammatory disease of unknown cause, and diverse symptomatology. The gastrointestinal tract involvement is common, but chronic diarrhea as the initial manifestation of the disease is rare. We present a patient who started the clinical picture with chronic diarrhea, making the diagnostic suspicion difficult.

Case Report: 38-year-old patient, female. Five months ago, she started with diarrhea, abdominal pain and vomiting. Two months later the diarrhea remained about 5 times a day, without blood or pus. A month ago, she was treated at the Emergency Unit. The medical record of the occasion reports a picture of acute respiratory failure, attributed to acute pulmonary edema. Once the clinical emergency was resolved, she was sent to a main hospital (Getulio Vargas Hospital in the city of Teresina-PI). The patient reported that in the last month she presented skin rash with photosensitivity and wrists and hands edema. In the evaluation, generalized edema was identified, besides increased volume and wrists and hands joints sensitivity. Complementary tests: Hemogram with anemia, leukopenia and thrombocytopenia. Urine summary with proteinuria and granular cylinders ( $2 \mathrm{~g}$ in $24 \mathrm{~h}$ proteinuria). FAN 1/320, homogeneous nuclear pattern, C3 and C4 downgraded, anti-DNA and anti-SM, and nonreagent antiphospholipids, antitransglutaminase, antiendomysium and antigliadin. Negative results for gastrointestinal parasites and coproculture. Subsequent investigation for gastrointestinal disease, including endoscopic examination with biopsy and MRI enterography, revealed diffuse intestinal and colonic loops distension, diffuse thickening of this last one, in addition to mild enanthematous gastritis and severe erosive esophagitis. After SLE diagnosis, therapy with $500 \mathrm{mg}$ of methylprednisolone began once a day for 3 days, followed by predinisone $1 \mathrm{mg} / \mathrm{kg} /$ day, associated with hydroxychloroquine $400 \mathrm{mg} /$ day, plus general clinical support measures such as diuretics and hydroelectrolytic care, plus albendazole $400 \mathrm{mg} /$ day for 5 days. In the patient's evolution, a normalization of clinical and laboratory parameters was observed.

Conclusion: Chronic diarrhea as a manifestation of SLE can be explained by enteritis, either by mesenteric vasculitis and/or by intestinal mucosa inflammation. The possibility of vasculitis was reinforced MRI enterography findings. Since protein loss can be identified by proteinuria, the possibility of protein-losing enteropathy was excluded.

\section{Consent for publication}

The authors declare that they have obtained informed written consent from the patient's tutors for publication

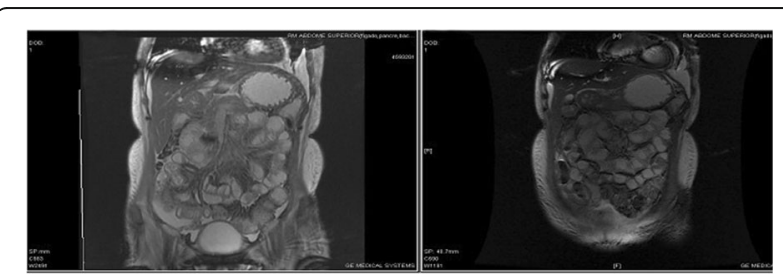

Fig. 1 (abstract P111). MRl enterography showing diffuse intestinal and colonic loops distension, diffuse thickening of this last one, in addition to mild enanthematous gastritis and severe erosive esophagitis

P112

CHRONIC DISCOID LUPUS ERYTHEMATOSUS (CDLE), WITH ANTIPHOSPHOLIPID SYNDROME (APS) AND CUTANEOUS ULCER Mario Sergio Ferreira Santos ${ }^{1}$, Mateus Dutra Batalha Costa ${ }^{3}$, Therezinha Dantas Nobre Neta ${ }^{3}$, Ana Luiza Gonçalves Santos², Késsia Pachêco Leal ${ }^{3}$, Francisca Daline dos Santos Silva ${ }^{3}$, Sérgio Augusto de Souza Cavalcante ${ }^{3}$ Gabriel Lima Jurema ${ }^{3}$, Lucas Moura Santana ${ }^{3}$, Beatriz Saraiva Araujo ${ }^{3}$ ${ }^{1}$ UNIVERSIDADE ESTADUAL DO PIAÚ, TERESINA, PI, Brasil; ${ }^{2}$ PPEMED, SÃO PAULO, SÃO PAULO, Brasil; ${ }^{3}$ UNIVERSIDADE ESTADUAL DO PIAUÍ, TERESINA, PIAUÍ, Brasil

Advances in Rheumatology 2018, 58(Suppl 1):P112 
Background: CDLE may exist as an independent clinical entity or in association with Systemic Lupus Erythematosus (SLE). Antiphospholipid antibodies (aPL) may appear in the course of different clinical disorders, particularly in SLE. However, the presence of these antibodies in the course of CDLE is rare. Moreover, when associated with thrombotic events and cutaneous ulcer. This is what we present in this case report. Case Report: A 62-year-old female patient on CDLE treatment for face and scalp in the last 5 years. Interrupted the use of hydroxychloroquine (HCQ) 2 months ago. In the last month, constitutional symptoms appeared with worsening of cutaneous lesions, in the form of deep and painful ulcers, on the face and scalp with secondary infection (Klebsiella and Pseudomona). Hb $10 \mathrm{~g} / \mathrm{dl}$; PT, platelets, leukocytes, kidney and hepatic functions tests were normal; ESR: $140 \mathrm{~mm}$ hr; ANA, anti-SM, anti-native DNA and anti-Ro unreacted; Anticardiolipin IgG: 9U GPL and IgM: 40U MPL; lupus anticoagulant was present; anti-Beta-2-Glycoprotein I: 22 and $6.1 \mathrm{U} / \mathrm{mL}$, IgG and IgM respectively. Wide antibiotic therapy, warfarin, treatment of skin ulcers with alginate, silver and calcium, reintroduction of $\mathrm{HCQ}$, plus prednisone at a low dose were instituted. During hospitalization, the patient presented thrombosis of the axillary and left basal veins, requiring full anticoagulation. aPLs remained positive in moderate to high titers, confirming the presence of APS. The patient received Rituximab, $1 \mathrm{~g}$ $\mathrm{EV}$, reinfused 15 days later. In the evolution, it was observed a regression of cutaneous ulcers, elevation of hemogobin levels and normalization of inflammatory activity tests.

Conclusion: This is one of the few CDLE records, presenting with APS and cutaneous ulcer (CU). CU is accepted as a non-APS criterion. Rituximab appears as an alternative for cases of APS, with skin ulcer difficult to control.

\section{Consent for publication}

The authors declare that they have obtained informed written consent from the patient's tutors for publication

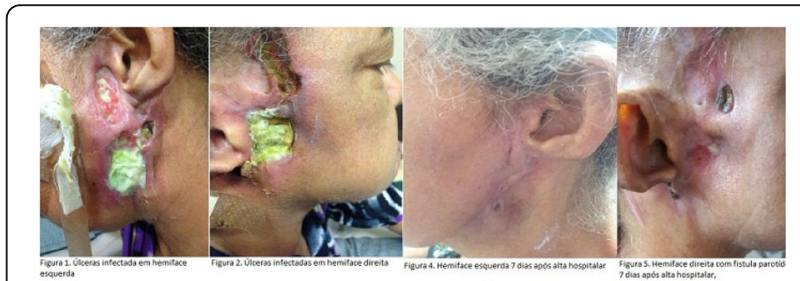

Fig. 1 (abstract P112). Ulcera cutânea numa paciente com Lupus Discoide associado a SAF

\section{P113}

\section{CHRONIC MUSCULOSKELETAL PAIN IN RHEUMATIC} OUTPATIENTS ATTENDING PHYSICAL THERAPY AT A UNIVERSITY

\section{HOSPITAL}

Mariana Alonso Monteiro Bezerra, Victor Emmanuel Cavalcanti Zamora, Geraldo da Rocha Castelar Pinheiro

UNIVERSIDADE DO ESTADO DO RIO DE JANEIRO, RIO DE JANEIRO, RJ, Brasil Advances in Rheumatology 2018, 58(Suppl 1):P113

Background: Chronic pain is considered a conscious experience modulated by neurobiological, environmental and cognitive factors. Mechanisms-based classification of chronic musculoskeletal pain is a useful tool in clinical practice, as it combines self-reported pain characteristics and a physical examination, pointing symptoms and signs and leading professionals in the decision making process $[1,2]$. The purpose of this study was to provide a musculoskeletal pain profile of rheumatic patients assisted by a physiotherapist from a university hospital in Rio de Janeiro.

Methods: A retrospective descriptive study was conducted between January 2016 and September 2017. Data was collected at the first physiotherapy evaluation session. Chronic musculoskeletal pain was classified as predominantly mechanical or inflammatory "nociceptive" pain, "peripheral neuropathic" pain, or pain related to "central sensitization" based on clinical classification previously described [2]. Sociodemographic, economic and clinical factors were also assessed. Results and conclusions: Of 176 patients evaluated for physiotherapy treatment, $83 \%$ were female, $36 \%$ were middle-aged adults, $61 \%$ had low education, $60 \%$ had no current occupational activity, and about $79 \%$ were sedentary. The most prevalent clinical diagnosis was osteoarthritis $(25 \%)$, followed by rheumatoid arthritis $(22.1 \%)$, spondyloarthritis (11.4\%), soft tissues lesions (10.8\%), gout (5.7\%), systemic lupus erythematosus (4\%), osteoporosis (3.4\%), and others (17.6\%). Regarding the characteristics of the chronic musculoskeletal pain, $60 \%$ reported their pain as intense (VAS $>7$ ), $70 \%$ were classified as nociceptive pain, $18 \%$ as chronic widespread pain associated with central sensitization and $12 \%$ as peripheral neuropathic pain. The most affected anatomic areas were the knee (65\%) and the lumbar spine $(44 \%)$. Considering the number of body sites, $28.4 \%$ of patients referred pain in only one site, $28.4 \%$ referred pain in two sites, $18.8 \%$ referred pain in three sites and $24 \%$ referred pain in four or more sites of the body. Even with a small number of participants, we found a high occurrence of nociceptive pain and this information is useful to guide the dynamics of our service in terms of human resources and therapeutic approach to rehabilitation of patients with chronic musculoskeletal pain.

\section{References}

1. Smart KM, Blake C, Staines A, Doody C. Clinical indicators of "nociceptive", "peripheral neuropathic" and "central" mechanisms of musculoskeletal pain. A Delphi survey of expert clinicians. Manual Therapy. 2010;15(1):80-7.

2. Smart KM, Blake C, Staines A, Doody C. The Discriminative Validity of "Nociceptive", "Peripheral Neuropathic", and "Central Sensitization" as Mechanisms-based Classifications of Musculoskeletal Pain. The Clinical Journal of Pain. 2011;27(8):655-63.

\section{P115}

\section{CHURG-STRAUSS SYNDROME IN A YOUNG MAN}

Marcio Ximendes Espirito Santo

UNIMED COSTA DO SOL, MACAE, RJ, Brasil

Advances in Rheumatology 2018, 58(Suppl 1):P115

Background: Churg-Strauss syndrome, also known as eosinophilic granulomatosis with polyangiitis, is a necrotizing vasculitis that affects small-sized vessels and is associated with severe asthma, blood and tissue eosinophilia and a positive antineutrophil cytoplasmic antibody. People around the age of 50 years are most affected, but can occur in all ages.

Case Report: A male aged 28 years was admitted to the intensive care unit with accute breathing insufficiency, hemoptoic sputum and fever. He complained about weight loss, arthralgia and worsening asthma. Before admission, he has received antiobiotics for pneumonia treatment without any result. On examination he had proximal interphalangeal arthritis, small violaceous nodules in hands and foot and distal asymmetric sensory neuropathy. On investigation the computed tomography showed bilateral pulmonary infiltrate in appearance of budding tree and hilar linfadenopathy, he had eosinophilia in blood test and P- antineutrophil cytoplasmic antibody was positive. At first, he received corticoid pulse with great clinical improvement, but with maintenance of the pulmonary infiltrate. After that, he was submitted to lung biopsy that confirmed small vessel vasculitis with eosinophilic infiltrate compatible to the ChurgStrauss syndrome. Finaly, he was treated with cyclofosfamide and was discharged.

Conclusion: We concluded that Churg-Strauss syndrome is a rare disease that can affect any age, depends on early aggressive immunosuppressive therapy and needs to be searched in pacients that have history of nasal allergies, chronic sinusitis or asthma with hard control.

\section{Consent for publication}

The authors declare that they have obtained informed written consent from the patient's tutors for publication 
P116

\section{CIITA RS3087456 PROTECTIVE ASSOCIATION TO}

PHOTOSENSITIVITY IN LUPUS ERYTHEMATOSUS SYSTEMIC

Denise de Queiroga Nascimento ${ }^{3}$, Suelen Cristina de Lima ${ }^{3}$, Isaura

Isabelle Fonseca Gomes da Silva ${ }^{3}$, Nadja Maria Jorge Asano ${ }^{1}$, Gisele

Vajgel Fernandes ${ }^{2}$, Lucila Maria Valente ${ }^{2}$, Paula Sandrin Garcia ${ }^{3}$

${ }^{1}$ HOSPITAL CLÍNICAS DE PERNAMBUCO, RECIFE, PERNAMBUCO, Brasil;

${ }^{2}$ HOSPITAL DAS CLÍNICAS DE PERNAMBUCO, RECIFE, PERNAMBUCO,

Brasil; ${ }^{3}$ UNIVERSIDADE FEDERAL DE PERNAMBUCO, RECIFE,

PERNAMBUCO, Brasil

Advances in Rheumatology 2018, 58(Suppl 1):P116

Background: Systemic lupus erythematosus (SLE) is a chronic autoimmune disease and genetic, environmental and immunological factors are involved in this pathogenesis. Patients with SLE present photosensitivity to UV radiation, presenting an abnormal cutaneous response. A large number of genes contribute to SLE pathogenesis. Studies have shown there are considerable evidence that single MHC genes contribute to the SLE development. One of them is the Class II Histocompatibility Complex Transformer (CIITA). It is able to alter the level of transcription of the Class II Major Histocompatibility Complex (MHC II), an important regulator of the adaptive immune response. The polymorphism rs3087456 at position -168 (A/G) in the CITTA gene may be responsible for this change. Only one association study was performed between this polymorphism and SLE. However, to date, no association study was performed between this polymorphism and SLE clinical features. Photosensitivity is a clinical feature that affects about $70 \%$ of SLE individuals, and this number increases in patients living in places with high UV radiation incidence, as is the case of the Brazilian Northeast population. Thus, our study aims to evaluate the binding of rs3087456 to photosensitivity in SLE in Northeast Brazilian population.

Methods: We collected 74 individuals with SLE in Recife, from the Rheumatology Outpatient Clinic of the UFPE Hospital das Clínicas. The control group consisted of 180 healthy individuals from the analyzed region. All subjects were genotyped using the TaqMan genotyping assay by the ABI 7500 Real Time PCR platform. Statistical analyzes were performed using Fisher's exact test by program $R$. Results: The chi-square test did not indicate significant differences when comparing patients and controls. Using the codominant model, the G/G genotype showed associated to high susceptibility to photosensitivity in SLE patients $(p<0.05)$. Other clinical features analyzed in the present study did not present significant statistical differences between the allelic and genotype frequencies.

Conclusion: Our findings suggests that the CIITA SNP rs3087456 is associated to high susceptibility to photosensitivity in SLE patients from Northeast Brazilian population.

\section{P117}

CLASSIFICATION OF MUCOCUTANEOUS MANIFESTATIONS IN SYSTEMIC ERITEMATOSUS LUPUS (SLE): DE GILLIAM JN \& SONTHEIMER RD A RIBERO S \& LIPSKER D

Luciano Junqueira Guimarães, Ana Paula Monteiro Gomides, Izelda Maria

Costa Carvalho, Leopoldo Luiz Dos Santos Neto

HOSPITAL UNIVERSITÁRIO DE BRASÍLIA, BRASÍLIA, DF, Brasil

Advances in Rheumatology 2018, 58(Suppl 1):P117

Background: The spectrum of mucocutaneous manifestations that occur in the course of SLE is broad and the classification is heterogeneous, such manifestations are among the most prevalent in SLE, the most common being malar rash and photosensitivity.

Methods: Bibliographic review.

Results and conclusions: In 1981 James N. Gilliam and Richard D. Sontheimer proposed the classification of lesions in specific and nonspecific lupus erythematosus (LE) based on the histopathological findings of skin biopsies. They defined as specific lesions those presenting dermatitis interface to histopathology. Subsequently, the following findings were added: presence of necrosis and vacuolization of the basal layer of keratinocytes, thickening of the basement membrane, incontinence of pigmentation and lymphocyte infiltration at the epidermal dermal junction. Acute LE (erythema malar, maculopapular and bullous erythema), subacute LE (psoriasiform and polycyclic) and chronic cutaneous LE (discoid, verrucous, tumoid, lupus panniculitis, pernium, discoid/lichen planus and mucosal) are included. Manifestations such as periungual telangectasia, livedo reticularis, occlusive vasculopathy, Raynaud's phenomenon, leukocytoclastic vasculitis and thrombophlebitis are the most frequent non-specific lesions in SLE, but may occur in other diseases. In 2010 Dan Lipsker suggested a new classification for cutaneous lesions, based on clinical signs and findings under microscopy. The lesions were classified according to the level of the cellular infiltrate and the tissue damage in the epidermis, dermis and or subcutaneous. In 2016 Simone Ribero et al, separated the nonspecific lesions between those that indicated the presence of thrombotic vasculopathy and those that presented a high standard neutrophil reaction. The specific lesions, most common in SLE according to the latter classification are: epidermal dermis (acute, subacute, chronic/discoid and bullous LE specific disease), dermal (tummy, papulonodular mucinose, erythematous reticular mucinous syndrome and lymphocytic skin infiltrate of Jessner-Kanof) and hypodermic (lupus panniculitis and lupus). The non-specific LE are indicative of thrombotic vasculopathy (livedo reticular, purpura, cutaneous necrosis and thrombophlebitis), neutrophil cutaneous LE (bullous LE, neutrophilic LE dermatosis, neutrophilic urticaria, urticaria LE vasculitis and pyoderma gangrenosum).

Conclusion: Mucocutaneous manifestations constitute 4 of the $17 \mathrm{cri}-$ teria established by Systemic Lupus International Collaborating Clinics (SLICC) in 2012 for the classification of SLE, denoting the importance of such manifestations in the course of the disease. A broad knowledge of these manifestations is fundamental for the diagnosis and management of SLE.

P118

CLASSIFICATION OF PHYSICAL ACTIVITY LEVEL OF OSTEOPOROSIS PATIENTS ACCOMPANIED AT A RHEUMATOLOGY SERVICE OF SÃO PAULO IN 2018

André Ozela Augusto, Dayrana Alves Lucena, Natália Carneiro dos Santos, Matheus Xavier Guimarães, Lisa Mielke de Oliveira, Bernardo Patrício Sequeira Dultra, Rina Dalva Neubarth Giorgi, Elaine de Azevedo HOSPITAL SERVIDOR PÚBLICO ESTADUAL-SP, SÃO PAULO, SÃO PAULO, Brasil

Advances in Rheumatology 2018, 58(Suppl 1):P118

Introduction: For the treatment of Osteoporosis, a comprehensive plan should be proposed, including pharmacological therapy associated with practice of physical activity, a fundamental item as it increases the resistance of the bones and improves muscle performance by reducing the number of falls.

Objective: To identifying the physical activity level among the population served, so that one can try to act in order to generate positive impact on this population's quality of life.

Method: Data were collected from 66 female patients, accompanied in the osteoporosis ambulatory in a rheumatology service. The nationally validated questionnaire "Physical Activity Level Rank (Short Version) IPAQ" was used, using the classification suggested by it. The data were computed and analysed using the Excel 2013 software.

Results and discussion: The mean age found among the study population was 71 years, with a minimum of 35 and maximum of 94 years, mode of 62 and median of 71 , obtaining the following distribution according to the classification proposed by IPAQ: very active $15.2 \%$ (10); active $42.4 \%$ (28); irregularly active A $16.7 \%(11)$; irregularly active B $13.6 \%$ (9) and sedentary $12.1 \%$ (8). Comparing the obtained results with the São Paulo's female population profile in 2011, elaborated by Matsuda et al., which evaluated 1048 women with no age or comorbidities restrictions, a higher proportion of very active women $(4.1 \%)$ were found, maintaining the same percentage of sedentary patients (8\%).

Conclusion: This comparison suggests that the perception of the benefits of physical activity in the Osteoporosis treatment reached positively the groups that were previously active, leading to an increase in the level of physical activity. Unfortunately, the greater understanding about the need to adopt more active habits was not sufficient to reduce the number of sedentary patients (which was unchanged in relation to the general population 
P120

COEXISTENCE OF PSORIATIC ARTHRITIS AND SYSTEMIC LUPUS ERYTHEMATOSUS: A CASE REPORT

Ítalo José Araújo Silveira De Sá1, Fernanda Tavares De Melo Cavalcanti Ana Valeska Lisboa Carvalho², Karla Valéria Miranda De Campos², Esther Bastos Palitot ${ }^{2}$, Maria Roberta Melo Pereira Soares², Alessandra Sousa Braz $^{2}$, Eutília Andrade Medeiros Freire ${ }^{2}$

${ }^{1}$ HOSPITAL UNIVERSITÁRIO LAURO WANDERLEY, JOÃO PESSOA, PB, Brasil; ${ }^{2} \mathrm{HULW}$, JOÃO PESSOA, PB, Brasil

Advances in Rheumatology 2018, 58(Suppl 1):P120

Systemic Lupus Erythematosus (SLE) is an autoimmune systemic inflammatory disease of multifactorial etiology affecting multiple systems. Although it has been described in association with various autoimmune diseases, there is only minimal data available regarding coexistence of Psoriatic Arthritis (PSA) and SLE. We report the first case of a brazilian patient suffering from both PsA and SLE.

A 53 years old woman presented to a tertiary hospital in 2016 with a 5-year history of polyarthralgia and well-demarcated erythematous and scaly plaques on her scalp (Fig. 1) and hands, that were misdiagnosed and treated as leprosy in 2014 by a infectologist, without any improvement. Physical examination reaveled nail dystrophy and symmetric polyarthritis, which affected several proximal interphalangeal (PIP) joints, wrists, shoulders, knees and ankles. A skin biopsy was performed in July 2016 and histology corroborated the diagnosis of psoriasis. Methotrexate was prescribed orally in a weekly dose of 15 $\mathrm{mg}$ with supplementation of folic acid $5 \mathrm{mg} /$ week. Three weeks later she developed bone marrow supression and acute kidney failure requiring dialitic therapy, with complete recovery after discontinuation of methotrexate. In 2017 she presented marked photosensivity and nonscarring hair loss. Laboratory tests included: sedimentation rate of $60 \mathrm{~mm}$ in the first hour; negative serology test for rheumatoid factor; presence of Direct Coomb's test in the absence of hemolytic anemia; serum ANA positive (1:640 - homogeneous staining pattern), anti-dsDNA positive (1/40), Anti-Sm positive $(36,9 \mathrm{U} / \mathrm{ml})$, and serology test for anti-Ro positive $(107,3 \mathrm{U} / \mathrm{ml})$. Serum C3, C4 and CH50 complement components were normal. A subsequent $\mathrm{X}$-ray revealed irregular periosteal bony proliferation resulting in periostitis at the 3rd and 4th PIP joints of the left hand. Those clinical, radiographic and laboratorial findings allowed the diagnosis of SLE (based on the Systemic Lupus International Collaborating Clinics classification criteria) and PsA (based on the Classification of Psoriatic ArthritisCASPAR - criteria), and the patient improved after therapy with corticosteroids, hydroxychloroquine and leflunomide.

The coexistence of psoriasis and SLE is uncommon, while the association of SLE and PSA has rarely been described in medical literalute. To the best of our knowledge, this is the first brazilian pacient reported as suffering from both conditions.

\section{Consent for publication}

The authors declare that they have obtained informed written consent from the patient's tutors for publication

\section{P122}

COGNITIVE IMPAIRMENT IN RHEUMATOID ARTHRITIS PATIENTS IS INDEPENDENT OF GLUCOCORTICOID USE

Tatiane Bellafronte Betoni, Fernanda de Almeida Said, Silvio Almeida de Lima, Thiago Alberto Fernandes Gomes dos Santos, Barbara Stadler Kahlow, Marilia Barreto Gameiro e Silva, Thelma Laroca Skare HOSPITAL UNIVERITÁRIO EVANGÉLICO DE CURITIBA, CURITIBA, PARANÁ, Brasil

Advances in Rheumatology 2018, 58(Suppl 1):P122

Background: Cognitive impairment has been observed in rheumatoid arthritis (RA) patients. Chronic pain, depression and glucocorticoid use has been implicated in its etiology. Animal studies have shown that glucocorticoids reduce the plasticity of astrocytesand the volume of the hippocampus, inducing cerebral atrophy. Herein we studied a sample of RA patients for cognitive impairment to see if it was associated with cumulative doses of glucocorticoid.

Methods:The sample had 60 RA patients and 64 controls paired for gender $(p=0.9)$, age $(p=0.12)$ and educational level $(p=0.12)$. Epidemiological data were collected and all were invited to answer the mini-mental and the CES-D (Center for Epidemiological Scale-Depression) questionnaires, and a VAS (from zero to 10) of pain. The charts of RA patients were reviewed for extra-articular manifestations, antibody profile, ESR, CPR, DAS-28-ESR and used medications including cumulative dose of glucocorticoids. Patients with neurological and psychiatric problems and using drugs with SNC action were excluded.

Results: RA patients were more depressed than controls $(p=0.03)$ and had worse mini-mental score $(p=0.002)$. RA patients with altered mini-mental were compared with those with normal mini-mental. The results are on Table 1.

Conclusion: Our results showed that RA patients had more cognitive impairment than controls and that the glucocorticoid cumulative dose did not influence in this result.

References

1. Zhang $\mathrm{H}$ et al. Chronic corticosterone exposure reduces hippocampal astrocyte structural plasticity and induces hippocampal atrophy in mice. Neurosci Lett. 2015; 592:76-81

2. Joaquim AF et al. Neuropsychiatric manifestations in rheumatoid arthritis. Autoimmun Rev. 2015;14(12):1116-22.

Table 1 (abstract P122). COMPARISON OF RA PATIENTS WITH NORMAL AND ALTERED MINI-METNAL (SCORES CORRECTED FOR EDUCATIONAL LEVEL)

\begin{tabular}{|c|c|c|c|}
\hline & $\begin{array}{l}\text { Altered } \\
\text { mini-mental } \\
N=31\end{array}$ & $\begin{array}{c}\text { Normal mini- } \\
\text { mental } \\
N=29\end{array}$ & P \\
\hline Male/female & $3 / 28$ & $2 / 27$ & 1.00 \\
\hline Mean age (years) & $55.5+9.7$ & $56.5 \pm 9.9$ & 0.68 \\
\hline Smokers(n) & $16 / 31(51.1 \%)$ & $16 / 29(55.1 \%)$ & 0.78 \\
\hline Disease duration (vears) - mean(SD) & $41.3+10.4$ & $45.7 \pm 12.2$ & 0.15 \\
\hline Nodules (n) & $2 / 25(8 \%)$ & $2 / 22(9 \%)$ & 1.00 \\
\hline Dositive rheumatoid fator(n) & $18 / 29(62 \%)$ & $14 / 28(50 \%)$ & 0.35 \\
\hline Antinudearfator(n) & $9 / 26(34.6 \%)$ & $8 / 25(32 \%)$ & 0.84 \\
\hline CES D-mean(SD) & $25.8 \pm 10.8$ & $27.2 \pm 10.1$ & 0.60 \\
\hline Das-28-ESR-median (IOR) & $3.25(2.52-4.95)$ & $4.08(3.21-5.59)$ & 0.15 \\
\hline ESR & $38.0(18.0-63.0)$ & $35.0(20.5-57.0)$ & 0.89 \\
\hline Creactive protein-median(ICR) & $4.0(1.1-6.0)$ & $6.0(4.35-12.5)$ & 0.01 \\
\hline Pain-Visual analogic scale-mean(SD) & $5.12+3.06$ & $4.65+3.10$ & 0.55 \\
\hline Glucocorticoid cumulative dose fimg & 16,385 & 10,960 & 0.82 \\
\hline prednisone)-median(IQR) & $(1.850 \cdot 26,350)$ & $(709-20,970)$ & \\
\hline
\end{tabular}

P123

COGNITIVE IMPAIRMENT IN RHEUMATOID ARTHRITIS PATIENTS: A CASE-CONTROL STUDY

Bruno Kusznir Vitturi, Beatriz Rizkallah Alves, Bruno Azeredo Coutinho Nascimento, Felipe Sobolewski Carneiro de Campos, Dawton Yukito Torigoe

FACULDADE DE CIÊNCIAS MÉDICAS DA SANTA CASA DE SÃO PAULO, SÃO PAULO, SP, Brasil

Advances in Rheumatology 2018, 58(Suppl 1):P123

Background: Rheumatoid arthritis (RA) is the most common autoimmune inflammatory arthritis in adults. Extra-articular manifestations of RA can occur in about $40 \%$ of patients, either in the beginning or during the course of their disease. Recent studies have suggested that RA may have an important relation in the development of cognitive and neurological dysfunction. However, the bond between RA and the brain is still uncertain. 
Objective: The purpose of this study was to assess the frequency and the clinical predictors of cognitive impairment in rheumatoid arthritis (RA) patients.

Methods: A cross-sectional and case-control study was performed including consecutive RA patients seen in a rheumatology outpatient clinic of referral tertiary hospital. The control group included 100 healthy subjects. We registered clinical and demographic data including age, sex, level of education, time of disease, time of diagnosis, drugs in use, cardiovascular risk factors and other comorbidities. Functional capacity was assessed using the Health Assessment Questionnaire (HAQ). Neurological appraisal was made with standardized questionnaires: Mini-Mental State Examination (MMSE), Montreal Cognitive Assessment (MoCA) and the Hospital Anxiety and Depression (HAD). Analysis of the data was performed using qui-square and t-tests and a multivariate analysis (SPSS 22.0). Significance level was set as $<0.05$.

Results: We included 200 patients (166 were female) with a mean age of $57.4( \pm 11.4)$ years; Among these patients, $163(81.5 \%)$ used corticosteroids, $148(74 \%)$ methotrexate, $16(8 \%)$ sulfasalazine, 91 (45.5\%) leflunomide, 35 (17.5\%) chloroquine and 57 (28.5\%) biologic therapy. The mean age of RA diagnosis was 43.0 ( \pm 13.6$)$ years. In both univariate and multivariate analysis, compared to the control group, patients with RA presented significant lower MMSE (21.9 $\pm 3.9)$ and MoCA $(17.0 \pm 4.4)$ scores $(p<0.05)$. Adjusting for level of education, just 61 and 50 patients presented normal MMSE and MoCA scores $(p<0.01)$, respectively. Cognitive decline was associated with higher HAQ scores (functional outcome due to RA) and prolonged time of disease $(p<0.05)$. No correlation was found between sex, disease-modifying antirheumatic drugs, rheumatoid factor, C-reactive protein levels and the neurological impairment. The mean HAD score was $17.7( \pm 7.7)$ and anxiety and depression were more prevalent in RA patients than in control group $(p<0.01)$.

Conclusions: Patients with rheumatoid arthritis may present with cognitive decline and dementia as extra-articular manifestations of the disease. Neurological impairment is usually disregarded and might be under-diagnosed in RA patients. Future studies are necessary in order to better understand the relationship between RA and the brain.

\section{P124}

COHORT COMPOSED OF 100 PATIENTS WITH HENOCH-SCHÖNLEIN PURPURA FROM 2010 TO 2017

Iloite Maria Scheibel ${ }^{1}$, Heloisa Pittoli Silva², Alvares Alves Ferreira Filho²,

Paula Gozzi ${ }^{2}$

${ }^{1}$ HOSPITAL CRIANCA CONCEICÃO DO GRUPO CONCEICÃO, PORTO ALEGRE, RS, Brasil; ${ }^{2}$ HOSPITAL CRIANÇA CONCEIÇÃO, PORTO ALEGRE, RS, Brasil Advances in Rheumatology 2018, 58(Suppl 1):P124

The Henoch-Schönlein purpura (HSP) is the most common vasculitis in children, but there's still questions regarding the long-term renal vascular performance in those patients. The goal of this study is to describe the demographic profile and the clinical evolution of 100 children who presented HSP from 2010 to 2017. Methodology: anterograde retrospective cohort study evaluating 100 patients that were followed up from 2010 to 2017 in a pediatric rheumatology service of a tertiary hospital at southern Brazil. Results: the average age was 5,42 years; divided in two age groups, equal to or under 5 years old and over 5 years old thru 16, the number of relapses were not statistically different. $25 \%$ of the patients of 5 years old or under relapsed at least once in the following year, whilst the relapse rate in patients over 5 years old was $18,8 \%$. Sixty four percent presented with arthritis, 58\% had extra-articular edema, $40 \%$ had abdominal pain, $6 \%$ had gastrointestinal bleeding and $3 \%$ presented with macroscopic hematuria. Of those symptoms, only extra-articular edema was more frequent among the patients of 5 years old and under $(68,8 \%$ versus $51,7 \%)$. Corticosteroid use was more prevalent among those with abdominal pain $(\mathrm{p} 0,004)$ and arthritis (p 0,04). Forty five percent presented renal impairment, $18 \%$ presented with proteinuria (one of them reaching nephrotic range), 30\% with microscopic hematuria. Renal biopsy was performed in 2 patients, one of them with nephrotic syndrome and the other with persistent and abundant hematuria. Only one patient, that had both HSP and post-streptococcic glomerulonephritis, presented altered blood pressure. Of those who started follow-up at the hospital, $71 \%$ kept coming to appointments for at least 6 months, $51 \%$ for a year and $26 \%$ for over 2 years. Conclusion: the demographic data corroborated those found on the literature. Most patients presented with edema and arthritis, but abdominal pain appears to be the most motivating isolated symptom for corticosteroid prescription, along with severe acute complications (Gl bleeding). Initial corticosteroid use didn't seem to have protective effect against relapses. There was no hypertension in this group of patients, but $29 \%$ were followed for less than 6 months, being necessary the search of those patients to complement their data.

\section{P125}

\section{COMORBIDITIES ON RHEUMATOID ARTHRITIS: ANALYSIS OF A}

\section{BRAZILIAN COHORT}

Ana Paula Monteiro Gomides ${ }^{1}$, Natália Mariana Diógenes Silva de Albuquerque $^{2}$, Jeniffer Yumie Sonobe Hable ${ }^{2}$, Brenda Magalhães Rocha ${ }^{2}$, Lorenna Alves Bezerra², Licia Maria Henrique da Mota ${ }^{3}$, Luciana Feitosa Muniz ${ }^{3}$, Talita Yokoy de Souza ${ }^{3}$, Viviane Cristina Uliana Peterle ${ }^{2}$, Luciano Junqueira Guimarães ${ }^{3}$, Regina Alice Fontes Von Kirchenheim ${ }^{4}$, Andressa Junqueira Osório ${ }^{3}$, Luciana Teófilo Lourençoni ${ }^{3}$, Isabela de Sousa Russo ${ }^{3}$, Tassiane Raquel Cunha Martins de Moraes ${ }^{3}$

${ }^{1}$ UNB/UNICEUB, BRASILIA, DF, Brasil:; ${ }^{2} U N I C E U B$, BRASÍLIA, DF, Brasil; ${ }^{3} U N B$, BRASÍLIA, DF, Brasil; ${ }^{4}$ SESDF, BRASÍLIA, DF, Brasil

Advances in Rheumatology 2018, 58(Suppl 1):P125

Introduction: Rheumatoid arthritis (RA) is a chronic condition, with potential irreversible bone and cartilage damage. In addition to musculoskeletal impairment, patients with RA can manifest other diseases of autoimmune or non-autoimmune etiology, making it difficult to manage patients and aggravating the condition. The association of different comorbidities in individuals with RA causes a marked decline in the quality of life of these individuals, and it is essential that the diagnosis and appropriate treatment be instituted as early as possible.

Methods: Patients with rheumatoid arthritis were analyzed in a University Hospital, participants of an initial RA cohort. For the present study, a cross-sectional study was performed and the patients' data were analyzed in the year 2018. Participants underwent a thorough clinical evaluation and medical records analysis. The work was approved by the Ethics Committee and the patients signed the consent form.

Results And Conclusions: We evaluated 107 patients with RA with a mean age of 54.5 years. The female gender (95.3\%) and the brown ethnic group (47.7\%) predominated. The mean duration of disease was 12.8 years and $75.5 \%$ had a positive rheumatoid factor. 12 patients (11.3\%) had documented erosive disease. The mean HAQ at the time of the evaluation was 0.6 (median of 0.5 ). The comorbidities found in this population can be seen in Table 1. Based on the data presented, a high association of comorbidities in the course of RA is observed, with prominence for arterial hypertension, dyslipidemia and fibromyalgia. Thus, the importance of exploring this relationship and its respective prevalences is emphasized due to the potential increase in morbidity and mortality, as well as the loss of functional capacity of the patients. 
Table 1 (abstract P125). Comorbidities in patients with RA

\begin{tabular}{lll}
\hline COMORBITIES & $\mathrm{N}$ & $\%$ \\
\hline Cerebrovascular disease & 1 & 0.9 \\
Arterial hypertension & 70 & 65.4 \\
Myocardial infarction & 5 & 4.7 \\
Other heart diseases & 4 & 3.7 \\
Asthma & 2 & 1.9 \\
Chronic obstructive pulmonary diseases & 2 & 1.9 \\
Other chronic lung diseases & 2 & 1.9 \\
Moderate or severe liver diseases & 1 & 0.9 \\
Diabetes without damage to vital organ & 15 & 14 \\
Diabetes with damage to vital organs & 1 & 0.9 \\
Dyslipidemia & 32 & 30 \\
Osteoporosis by bone densitometry & 15 & 14 \\
Psychiatric illness & 6 & 5.6 \\
Metastases without tumor & 3 & 2.8 \\
Connective tissue disease & 2 & 1.9 \\
Fibromyalgia & 31 & 29 \\
Chronic low back pain & 3 & 2.8 \\
Osteoarthritis & 10 & 9.3 \\
Cataract & 3 & 2.8 \\
Thyroid disease & 11 & 10.3 \\
\hline
\end{tabular}

\section{P128}

CORRELATION BETWEEN CAPILLAROSCOPY PATTERNS AND AUTOANTIBODY PROFILES IN SYSTEMIC SCLEROSIS PATIENTS Laíssa Cristina Alves Alvino ${ }^{1}$, Gabriela Santiago Brum Marques ${ }^{2}$ Camilla de Castro Silva ${ }^{2}$, Verônica Silva Vilela², Roger Abramino Levy², Cláudia Henrique da Costa², Rogério Lopes Rufino

${ }^{1}$ UNIVERSIDADE ESTADUAL DO RIO DE JANEIRO, RIO DE JANEIRO, RJ, Brasil; ${ }^{2}$ UNIVERSIDADE DO ESTADO DO RIO DE JANEIRO, RIO DE JANEIRO, RIO DE JANEIRO, Brasil

Advances in Rheumatology 2018, 58(Suppl 1):P128

Background: Specific autoantibodies were reported to correlate with nailfoldcapillaroscopy morphologic alterations in systemic sclerosis (SSc). This study was performed with the objective to evaluate if specific autoantibodies were associated with corresponding nailfoldcapillaroscopy patterns and morphological alterations in a Brazilian population of patients with SSC.

Methods: We evaluated 61 SSc patients that had videocapillarocopy performed and antinuclear antibodies (ANA) and specific autoantibodies registered. Videocapillaroscopy were graded by the following qualitative method as: (1) normal, with more than 9 capillaries per millimiter, no morphological abnormalities and less than 3 microhemorrages per 8 digits; (2) "Early", few enlarged/giant capillaries, few capillary haemorrhages, relatively well-preserved capillary distribution, no evident loss of capillaries; (3) "Active", frequent giant capillaries, frequent capillary haemorrhages, moderate loss of capillaries, mild disorganisation of the capillary architecture, absent or mild ramifi ed capillaries; (4) "Late", irregular enlargement of the capillaries, few or absent giant capillaries and haemorrhages, severe loss of capillaries with extensive avascular areas, disorganisation of the normal capillary array, ramified capillaries; (5) ES like. ANA were performed by IF and national consensus patterns were reported. Specific SSc autoantibodies measured were $\mathrm{Scl}-70$ and centromere. The nonspecific RNP were also measured and correlated.

Results and conclusions: In this present study we found that the anticentromere was related with $43,75 \%$ of the pattern active scleroderma; $31,25 \%$ of early scleroderma; $6,24 \%$ of late escleroderm and ES like; and $12 \%$ of normal pattern. The anti Scl-70 was associated with $38,09 \%$ of the pattern active scleroderma; $4,16 \%$ with early scleroderma; $57,14 \%$ of late escleroderm and did not show relation with the ES like and normal patterns. The anti-RNP was related with $50 \%$ of the pattern active scleroderma; $12,5 \%$ of early scleroderma and late escleroderm; $25 \%$ of ES like; and didn't show correlation with the normal pattern. Furthermore, the patients without autoantibodies showed relation with $25 \%$ of the active, early and late escleroderm patterns; $18,75 \%$ of the ES-like pattern and $6,25 \%$ of the normal pattern. Therefore, the union of the analysis with nail fold videocapillaroscopy in the association with different phenotypes described by each antibody may be use for suggest about the diagnosis and with the intensity of activity of the disease.

P129

CORRELATION BETWEEN SLICC/ACR-SDI CUMULATIVE DAMAGE INDEX AND MATERNAL-FETAL OUTCOME IN WOMEN WITH SYSTEMIC LUPUS ERYTHEMATOSUS (SLE)

Evandro Mendes Klumb ${ }^{1,2}$, Bruna Costa Rodrigues ${ }^{2}$, Marcela Ignacchiti Lacerda², Camilla de Castro e Silva², Camila Pitasi Argueles²,

Guilherme Ribeiro Ramires de Jesús², Roger Abramino Levy²,

Nilson Ramires de Jesús ${ }^{2}$

'UNIVERSIDADE DO ESTADO DO RIO DE JANEIRO, RIO DE JANEIRO, RJ, Brasili: ${ }^{2}$ UERJ, RJ, RJ, Brasil

Advances in Rheumatology 2018, 58(Suppl 1):P129

Background: The presence of at least one point in the SLICC/ACR-SDI cumulative damage index in patients with systemic lupus erythematosus (SLE) is associated with a higher risk of future cumulative damage and increases the mortality was 1.46 times in this group when compared to patients with a score equal to zero. Recent studies have shown that gestation does not contribute to the increase in the SDI index. However, the impact of the presence of cumulative damage on gestational, maternal and fetal outcomes has not been investigated. The objective of this study was to analyze the impact of the presence of permanent damage (SDI) on the maternal-fetal outcome in pregnancies of women with SLE.

Methods: Analysis of the evolution of 152 single pregnancies in SLE patients ( $\geq 4$ ACR criteria) with delivery after 22 weeks in the period of 2011 and 2016. Statistical analysis included the Student t test and chi-square test $(x 2)$ as indicated with a significance level of $5 \%$ $(\mathrm{p} \leq 0.05)$.

Results and Conclusions: Patients with SDI $>1$ presented more frequently nephritis $(p=0,006)$, neurologic manifestations $(p=0,007)$, anti DNA $(P=0,01)$ and less frequently positive $\mathrm{aPL}(p=0,02)$. In reference to fetal outcomes patients with $\mathrm{SDI}>1$ presented more frequently peripartum hemorrhage $(p=0,01)$, any infection $(p=0,0001)$ and hospital admissions $(p=0,02)$.

\section{Consent for publication}

The authors declare that they have obtained informed written consent from the patient's tutors for publication

P130

CORRELATION BETWEEN SLICC/ACR-SDI CUMULATIVE DAMAGE INDEX AND MATERNAL-FETAL OUTCOME IN WOMEN WITH SYSTEMIC LUPUS ERYTHEMATOSUS (SLE)

Camilla De Castro E Silva ${ }^{1,2}$, Bruna Costa Rodrigues ${ }^{2}$, Marcela Ignacchiti Lacerda², Flavia Cunha dos Santos², Guilherme Ribeiro Ramires de Jesús ${ }^{2}$, Roger Abramino Levy ${ }^{2}$ Evandro Mendes Klumb ${ }^{2}$

${ }^{1}$ HOSPITAL UNIVERSITÁRIO PEDRO ERNESTO -, Brasil; ${ }^{2}$ HOSPITAL

UNIVERSITÁRIO PEDRO ERNESTO - UERJ, Brasil

Advances in Rheumatology 2018, 58(Suppl 1):P130

Background: The presence of at least one point in the SLICC/ACR-SDI cumulative damage index in patients with systemic lupus erythematosus (SLE) is associated with a higher risk of future cumulative damage and increases the mortality was 1.46 times in this group when compared to patients with a score equal to zero. Recent studies have shown that gestation does not contribute to the increase in the SDI 
index. However, the impact of the presence of cumulative damage on gestational, maternal and fetal outcomes has not been investigated. The objective of this study was to analyze the impact of the presence of permanent damage (SDI) on the maternal-fetal outcome in pregnancies of women with SLE.

Methods: Analysis of the evolution of 152 single pregnancies in SLE patients ( $\geq 4$ ACR criteria) with delivery after 22 weeks in the period of 2011 and 2016. Statistical analysis included the Student $t$ test and chi-square test $(x 2)$ as indicated with a significance level of $5 \%(p \leq 0.05)$.

Results and Conclusions: Patients with SDI $>1$ presented more frequently nephritis $(p=0,006)$, neurologic manifestations $(p=0,007)$, anti DNA $(P=0,01)$ and less frequently positive $\mathrm{aPL}(\mathrm{p}=0,02)$. In reference to fetal outcomes patients with SDI $>1$ presented more frequently peripartum hemorrhage $(p=0,01)$, any infection $(p=0,0001)$ and hospital admissions $(p=0,02)$.

\section{P131}

CORRELATION OF THE PSORIATIC ARTHRITIS IMPACT OF DISEASE SCORE (PSAID) WITH THE MINIMAL DISEASE ACTIVITY (MDA) STATUS: A MULTICENTER STUDY

Elziane Da Cruz Ribeiro E Souza' Sueli Coelho Da Silva Carneiro² Michel Alexandre Yazbek ${ }^{3}$, Rita De Cassia Menin ${ }^{4}$, Cristiano Barbosa Campanholo ${ }^{5}$, Jamille Nascimento Carneiro ${ }^{6}$, Carlos Henrique Martins Da Silva ${ }^{7}$, Roberto Ranza

'UNIVERSIDADE FEDERAL DE UBERLANDIA, UBERLÂNDIA, MG, Brasil; UNIVERSIDADE DO ESTADO DO RIO DE JANEIRO, RIO DE JANEIRO, RIO DE JANEIRO, Brasil; ${ }^{3}$ UNIVERSIDADE ESTADUAL DE CAMPINAS,

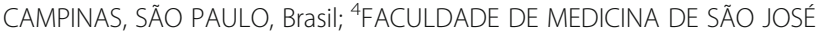
DO RIO PRETO, SÃO JOSÉ DO RIO PRETO, SÃO PAULO, Brasil; ${ }^{5}$ FACULDADE DE CIÊNCIAS MÉDICAS DA SANTA CASA DE SÃO PAULO, SÃO PAULO, SÃO PAULO, Brasil; ${ }^{6}$ HOSPITAL DE BASE DO DISTRITO FEDERAL, BRASILIA, DISTRITO FEDERAL, Brasil; ${ }^{7}$ UNIVERSIDADE FEDERAL DE UBERLÂNDIA, UBERLÂNDIA, MINAS GERAIS, Brasil Advances in Rheumatology 2018, 58(Suppl 1):P131

Background: Psoriatic arthritis (PsA) is a complex and heterogeneous disease, which causes functional impairment and reduction of health-related quality of life. These characteristics represent a real challenge in the evaluation of patients with PsA, and it is essential to add to the medical evaluation the patient's impression of their state of health in order to assess the real impact of the disease and define the target of treatment. The PSAID is a validated patient-reported questionnaire of disease impact in PsA.

Objective: To study the correlation between the PsAID score and the minimal disease activity (MDA) and very low disease activity (VLDA) status.

Methods: 150 patients who fulfilled the Classification for Psoriatic Arthritis (CASPAR) criteria were recruited in a cross-sectional study by 6 Brazilian rheumatology centers. Patients were grouped according to MDA status: MDA (if they met at least 5/7 of the MDA criteria), in VLDA (if they met 7/7 of the MDA criteria) and non-MDA. To compare the domains of PSAID among the MDA groups, the KruskalWallis tests and the Dunn test were applied. The level of significance was set at $5 \%(p<0.05)$.

Results: Of the 150 included patients, 76 were men and 74 women, with a mean (SD) age of 54.4 (11.3) years. The median of PsA duration (P25-P75) was 9 years (6-14) and 103 (68.7\%) patients were on biological treatment. Overall, $16.7 \%$ achieved MDA and $11.3 \%$ VLDA vs $72 \%$ non-MDA. Interestingly, the MDA criteria less frequently met were patient pain visual analog scale and patient global disease activity. Out of 103 patients on biologic, only $26.2 \%$ were MDA status. Analyzing the median (P25-P75) of the PsAID total score between the groups, patients in MDA and VLDA had a significantly lower scores [MDA 1.7 (1-3.6), VLDA 0.6 (0.2-0.9) vs non-MDA 4.6 (2.9-6.8)], $\mathrm{p}<0.001$. Accordingly, statistically significant differences were observed between MDA/VLDA groups and non-MDA patients for all PsAID domains $(p<0.001)$. PsAID score $<4$ was expressed by $84 \%$ of patients in MDA and $100 \%$ of patients in VLDA vs $42.6 \%$ of non-MDA $(p<0.0001)$. The presence of fibromyalgia did not significantly interfere in the total PsAID score, showing a statistical correlation only with the depression domain.

Conclusions: In our experience, the PsAID score correlated with the MDA status: patients in MDA/VLDA considered they have a significantly lower impact of the disease as measured by PsAID.

\section{P134}

\section{CROSS CULTURALLY ADAPTATION AND VALIDATION OF THE} BRAZILIAN-PORTUGUESE VERSION OF HAMIS

Pedro Ming Azevedo ${ }^{1}$, Patricia Martin², Estefania Sartorato Sanson², Thiago Alberto Fernandes Gomes dos Santos², Thelma Laroca Skare ${ }^{2}$ ${ }^{1}$ HOSPITAL UNIVERITÁRIO EVANGÉLICO DE CURITIBA, CURITIBA, PR, Brasil; ${ }^{2}$ HOSPITAL UNIVERSITÁRIO EVANGÉLICO DE CURITIBA, CURITIBA, PR, Brasil

Advances in Rheumatology 2018, 58(Suppl 1):P134

Background: Hand involvement secondary to systemic sclerosis causes disability that can be measured by the Hand Mobility in Scleroderma (HAMIS) questionnaire. The aim of this study is to validate the Brazilian version of HAMIS.

Methods: Translation from English to Brazilian-Portuguese was done by two of the authors, and the consensus was back-translated to English by a native English speaker fluent in Brazilian-Portuguese with no prior knowledge of the questionnaire. After verification of coherence between the back-translated and original English version, the consensus Brazilian-Portuguese version was applied to 10 scleroderma patients, to check their understanding. The final version was applied to 31 stable scleroderma patients. One examiner performed the test on day 1 and day 15. A second examiner applied the test on day 15. Intra and inter reliability for each individual question were evaluated by intraclass correlation coefficient (ICC). Intra and inter reliability between final scores were evaluated by Spearman's coeficient. Internal consistency was evaluated by Kronbach's alpha coefficient. Factorial analysis was performed to assess the factorial structure of the questionnaire.

Results: The Brazilian version of HAMIS showed a good intra and inter-reliability for all questions (Table 1). Although the examiners disagreed about the results of question 8 in only two patients, the statistical method was not able to detect inter-observer agreement for the question, because responses were limited to first and second domains. The correlation coefficient for the final score was 0.99 $(p<0.0001)$ for intra observer and $0.97(p<0.0001)$ for inter observer evaluation, revealing excellent correlation. The alpha's Cronbach coefficient was 0.92 showing a good internal consistency. The factorial analysis extracted 3 factors that could be clinically characterized. The first factor aggregated questions 2/5/7/9, second factor aggregated questions $1 / 3 / 4$ and question 8 stood alone as a factor. All movements tested by questions of the first factor are affected by the extension of the fingers and, similarly, second factor is affected by flexion of the fingers. For instance, supination can be misinterpreted if fingers don't touch the table because of reduced extension. Therefore, examiners must pay attention to the movement being tested. Conclusion: The translation and cultural adaptation of HAMIS to Brazilian-Portuguese language is supported by our study.

Table 1 (abstract P134). ICC for inter and intra-observer reliability

\begin{tabular}{|c|c|c|}
\hline & Reliability intra-observer(ICC) & Reliability inter-observer(ICC) \\
\hline Q1 & 0.90 & 0.80 \\
\hline Q2 & 1 & 1 \\
\hline Q3 & 1 & 0.86 \\
\hline Q4 & 0.93 & 0.77 \\
\hline Q5 & 0.92 & 1 \\
\hline Q6 & 0.78 & 0.81 \\
\hline Q7 & 0.90 & 0.91 \\
\hline Q8 & 1 & - \\
\hline Q9 & 1 & 0.93 \\
\hline
\end{tabular}




\section{P135}

CROWNED DENS SYNDROME: A CHALLENGING DIAGNOSIS

Eduardo S. Paiva, Salun Coelho Aragao, Maiara Bastiani Bisogni, Luiza Bueno Zeni, Luan Luckmann, Keoma Azevedo Sabiao, Chayanne Natielle Rossetto, Isadora Welter Pioresan, Ana Beatriz Artigas Guimaraes UFPR, CURITIBA, PR, Brasil

Advances in Rheumatology 2018, 58(Suppl 1):P135

Background: The crowned dens syndrome is characterized by acute cervicalgia associated with a decreased cervical range of motion, mainly rotation, caused by deposition of calcium pyrophosphate or hydroxyapatite crystals around the axis odontoid process. As it may be associated with systemic symptoms, such as fever, it can mimic diseases like infectious meningitis. The radiological diagnosis is made by computed tomography (CT), which is superior to simple radiography and magnetic resonance imaging. We herein present a case of crowned dens syndrome in which there was a dramatic response to the use of corticosteroids.

Case report: Female patient, 47 years old, came to emergency department with acute neck pain, with progressive symptoms in the last few days. Since the day before, she was unable to perform any kind of cervical movement. She also reported low-grade fever and headache. Plain radiographs and magnetic resonance of the cervical spine were performed, with no diagnostic definition. She was initially treated with NSAIDs and muscle relaxants, without any improvement. Blocking of the greater occipital nerve was performed, again with no improvement. A spinal tap was negative. Since there was a suspicion of crowned dens syndrome, a cervical spine CT was performed, which showed calcification around the odontoid process of the axis. She underwent pulse therapy with methylprednisolone $500 \mathrm{mg} /$ day for a total of 3 days, along with a full-dose NSAIDs, with improvement of symptoms during the following week. The maintenance treatment was done with colchicine, and she remains symptoms-free for the last few months.

Conclusion: Crowned dens syndrome is a difficult diagnosis that should be thought every time there is a case of acute cervical spine pain. The diagnosis is not always easy since the main findings are common to other diseases. The main imaging modality is computed tomography, since the deposits may be difficult to visualize with plain radiographies and magnetic resonance.

\section{Consent for publication}

The authors declare that they have obtained informed written consent from the patient's tutors for publication

\section{P137}

CRYPTOCOCOSIS: A DIFFERENTIAL DIAGNOSIS FOR EXACERBATION OF PULMONARY INVOLVEMENT BY GRANULOMATOSIS WITH POLYANGIITIS

Mateus De Miranda Moura Cortês ${ }^{1,2}$, Jacqueline Foelkel Pignatari ${ }^{2}$ Pamella de Paula Bellini², Gabriela Miyuki Teodoro Ogawa ${ }^{2}$, Lara Ribeiro Teixeira Bonfim², Samuel de Oliveira Andrade ${ }^{2}$, Alisson Aliel Vigano Pugliesi $i^{2}$, Zoraida Sachetto ${ }^{2}$

${ }^{1}$ UNIVERSIDA ESTADUAL DE CAMPINAS - UNICAMP, CAMPINAS, SP, Brasil; ${ }^{2}$ UNIVERSIDADE ESTADUAL DE CAMPINAS - UNICAMP, CAMPINAS, SP, Brasil

Advances in Rheumatology 2018, 58(Suppl 1):P137

Background: Cryptococcus gattii and Cryptococcus neoformans are encapsulated fungi responsible for the greater part of the cryptococcosis, which manifest most frequently as pneumonia and/or meningoencephalitis. Pulmonary nodules, or cryptococomas, are the manifestation of pulmonary involvement and lead to differential diagnosis with other granulomatous diseases. We report a case of granulomatosis with polyangiitis (GPA) with previous lung involvement, under irregular immunosuppressive treatment, in which pulmonary cryptococcosis developed as an opportunistic infection.

Case Report: A 61-year-old male patient, diagnosed with GPA in 2016 (c-ANCA+, rhino-sinusopathy, rapidly progressive glomerulonephritis pauci-immune to renal biopsy, alveolar hemorrhage, pulmonary nodules). Because of illness severity, he underwent induction treatment with oral prednisone and monthly intravenous cyclophosphamide
(CYC), but at suboptimal doses and with irregular frequency due to repetitive infectious complications. In 2017, days after the fifth infusion of CYC, the patient was hospitalized for investigation of dry cough and fever. He presented negative initial infectious screening, absence of leukocytosis or increased inflammatory markers, but with bibasilar crackles on pulmonary auscultation and chest X-rays showing diffuse patchy infiltrates. Tomography of the chest revealed multiple nodules and solid masses distributed diffusely across both lung fields, absent in a previous study (Fig. 1). The patient was treated empirically with broad-spectrum antibiotic therapy for five days, without improvement of the febrile curve, until the end of the infectious investigation with bronchoscopy with bronchoalveolar lavage, whose culture was positive for C. gattii. Serum antigenemia for cryptococcosis was positive (1/128), with screening for fungus at other sites proving negative, including cerebrospinal fluid. Pulmonary cryptococcosis was treated with oral fluconazole $400 \mathrm{mg} /$ day, with fever resolution and cough reduction, the patient being discharged for continuity with antifungal for 12 months. Conclusion: The clinical manifestations of pulmonary cryptococcosis include nodular lesions with or without cavitations, segmental pneumonic infiltrates, segmental interstitial or alveolar infiltrates, pleural effusions, hilar masses and thoracic lymphadenopathy. These findings, however, require a differential diagnostic approach, since they are part of the clinical spectrum of other diseases, such as GPA. In our case, the pulmonary manifestation appeared to be an exacerbation of the basal vasculitis, requiring immunosuppressive treatment, but it was actually a fungal infection, for which more immunosuppression could have had an aggravating effect.

\section{Consent for publication}

The authors declare that they have obtained informed written consent from the patient's tutors for publication

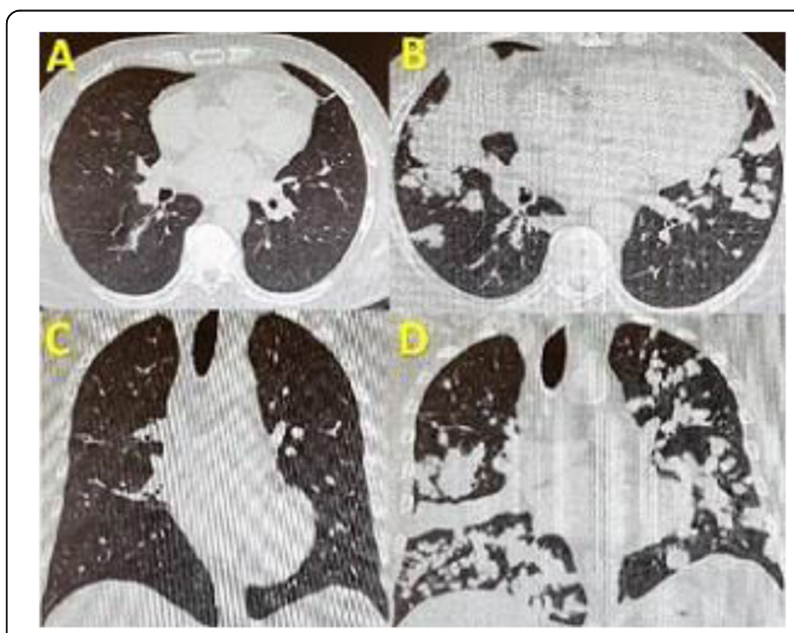

Fig. 1 (abstract P137). Tomography of the chest. Solid, confluent and poorly defined nodules and masses, some with permeating air bronchograms, distributed diffusely across both lung fields (b and d), absent in a previous study (a and $\mathbf{c}$ )

\section{P140}

CYTOMEGALOVIRUS INFECTION IN A SYSTEMIC LUPUS ERYTHEMATOSUS PATIENT TREATED WITH BELIMUMAB: A CASE REPORT

Andréa Tavares Dantas', Vanessa Fonseca De Jesus'², Henrique De Ataíde Mariz', Rafaela Silva Guimarães Gonçalves², Claudia Diniz Lopes Marques², Angela Luzia Branco Pinto Duarte ${ }^{2}$

${ }^{1}$ UNIVERSIDADE FEDERAL DE PERNAMBUCO, RECIFE, PE, Brasil;

${ }^{2}$ HOSPITAL DAS CLÍNICAS - UNIVERSIDADE FEDERAL DE PERNAMBUCO, RECIFE, PERNAMBUCO, Brasil

Advances in Rheumatology 2018, 58(Suppl 1):P140 
Background: Treatment of systemic lupus erythematosus (SLE) with belimumab has been associated with a low risk of infection. Cytomegalovirus (CMV) infection in SLE patients may represent a diagnostic challenge because of the difficulty of differentiation with disease flare and the potential to evolve with severe complications. The aim of this case was to report CMV infection in a SLE patient after initiation of therapy with belimumab.

Case report: A 31-year-old female patient diagnosed with SLE for 5 years with a history of arthritis, lymphopenia, thrombocytopenia, nephritis, complement consumption, antinuclear antibodies (ANA) and anti-DNA positive. She demonstrated good control of nephritis after treatment with corticosteroids and mycophenolate mofetil, which was later switched to azathioprine due to intolerance. Because the difficulty of weaning from the corticosteroid due to the recurrence of joint complaints, treatment with belimumab was chosen in January 2017. At the 5th month of treatment, fever, retroorbital pain, odynophagia, headache and erythematous skin rash began to occur, as well as intense fatigue. Laboratory tests revealed anemia $(\mathrm{Hb}=9.8$ $\mathrm{mg} / \mathrm{dl}$ ), leukopenia and lymphopenia (later with lymphocytic atypia), decreased complement, anti-DNA 1:20, positive direct Coombs test, hematuria, and increased LDH, transaminases, erythrocyte sedimentation rate (ESR) and $C$ reactive protein (CRP). In this moment, it was decided to increase dose of prednisone to $0.5 \mathrm{mg} / \mathrm{kg} / \mathrm{day}$ and to suspend infusion of belimumab. Serologies were negative for dengue, rubella, zika, parvovirus B19, syphilis, hepatitis A, B and C, but with IgM and IgG positive for Cytomegalovirus. DNA PCR for CMV showed 1660 copies $/ \mathrm{mL}$. The patient presented with clinical improvement without the need for ganciclovir therapy. The viral load was negative after 2 months and she resumed the treatment with belimumab 5 months after its suspension.

Conclusion: Although only two cases of CMV infection have been reported in pivotal clinical trials of belimumab, one of which was fatal, this is a common infection in immunosuppressed patients and should be considered in the differential diagnosis of SLE flare. Milder cases may be managed symptomatically with the temporary suspension of the immunobiological therapy.

\section{Consent for publication}

The authors declare that they have obtained informed written consent from the patient's tutors for publication

\section{P142 \\ DATA ON MORBITY AND MORTALITY DUE TO OSTEOPOROTIC FEMORAL FRACTURE AND EVIDENCE ON PRIMARY AND SECONDARY PREVENTION \\ Dayrana Alves Lucena ${ }^{2}$, Caroline de Almeida Oliveira ${ }^{2}$, Matheus Xavier Guimarães ${ }^{1}$, Fernanda Pulcheri Ramos ${ }^{2}$, Laís Farrapo de Barros Leite², Marcelo Nora Resende ${ }^{2}$, Natália Carneiro dos Santos², Rina Dalva Neubarth Giorgi ${ }^{2}$, Elaine de Azevedo ${ }^{2}$ \\ ${ }^{1}$ HOSPITAL DO SERVIDOR PUBLICO ESTADUAL DE SÃO PAULO, SÃO PAULO, SÃO PAULO, Brasil; ${ }^{2}$ HOSPITAL DO SERVIDOR PÚBLICO ESTADUAL - SÃO PAULO, SÃO PAULO, SÃO PAULO, Brasil Advances in Rheumatology 2018, 58(Suppl 1):P142}

Background: Osteoporosis (OP) is characterized by bone fragility and changes in its microarray, with the most important clinical outcome being the occurrence of low impact fractures and affecting more than 200 million people worldwide. In Brazil, OP affects around 10 million people. It is estimated that $50 \%$ of women and $20 \%$ of men aged 50 years or older will suffer a lifetime osteoporotic fracture. Approximately $5 \%$ of individuals with hip fractures die during hospital stay, $12 \%$ die within the subsequent 3 months, and $20 \%$ die within the first year following fracture.

Objective: To evaluate the evolution after a minimum of 24 months of 66 patients who presented a femoral neck fracture in the year 2015 , with emphasis on functional capacity.

Methods: Sixty-six patients attended at a tertiary hospital during the year 2015 after hip fracture were selected. Of these 66, 41 were submitted to an inquiry via telephone contact. Epidemiological data such as: identification, age, sex, date of fracture and its mechanism (excluding cases of traumatic fractures), length of hospital stay, information on OP (knowledge about diagnosis and/or previous previous treatment, specific treatment for OP); and functional capacity after two years of fracture.

Results: Of the 41 patients evaluated, $73.1 \%$ were female, with a mean age of 71.1 years. Regarding the cause of the fracture, the fall of the height itself occurred in 38 patients. Surgical treatment was performed in $92.6 \%$ of the cases with a mean hospital stay of 11.3 days. The majority of the patients $(92.6 \%)$ were unaware of having OP prior to fracture and $75.6 \%$ had never had treatment for the disease. After one year of the event, the number of deaths was $21.9 \%$, 14 patients were completely restored $(100 \%$ of post-fracture functional capacity), and $43.9 \%$ evolved with some degree of functional disability. Only $24.39 \%$ of the cases were referred for secondary cause assessment and treatment for PB.

Conclusion: The population studied presented similar morbidity and mortality described in the literature. A special relevance should be given to the absence of diagnosis and treatment for OP prior to fracture and inefficiency in referring patients for secondary prevention. It is imperative to adopt educational, preventive, diagnostic and early treatment measures in order to minimize the complications of this disease.

\section{P143}

DEMOGRAPHIC AND EPIDEMIOLOGICAL PROFILE OF PATIENTS WITH RHEUMATIC DISEASES SERVED AT SUS HEALTH UNIT

Maria Carolina Vasconcelos Fecury', Regina Alice Fontes Von Kirchenheim², Ana Paula Monteiro Gomides ${ }^{3}$, Viviane Cristina Uliana Peterle ${ }^{1}$, Luciano Junqueira Guimarães ${ }^{3}$

${ }^{1}$ HOSPITAL DA REGIÃO LESTE - HRL, BRASÍLIA, DF, Brasil; ${ }^{2} \mathrm{HOSPITAL}$ REGIONAL DA ASA NORTE - HRAN, BRASÍLIA, DF, Brasil; ${ }^{3}$ CENTRO UNIVERSITÁRIO DE BRASÍLIA - UNICEUB, BRASÍLIA, DF, Brasil Advances in Rheumatology 2018, 58(Suppl 1):P143

Rheumatology is characterized by a wide variety of diseases. More than 100 different conditions are labeled as rheumatic diseases including rheumatoid arthritis, osteoarthritis, autoimmune diseases such as systemic lupus erythematosus (SLE), scleroderma, osteoporosis, back pain, gout, fibromyalgia and tendinitis. In Brazil, there is a lack of data on the population affected by rheumatic diseases, morbidity, mortality and costs. The present research is a cross-sectional, retrospective, descriptive study with secondary data analysis. It was performed with an electronic records analysis of patients over 18 years-old with a diagnosis of rheumatic diseases treated at a SUS rheumatology outpatient clinic from 2012 to 2017. The variables collected were: age, sex, where they were living, pathologies served by the international classification of diseases (ICD 10). The statistical analysis was performed using Excel, and the results were quantitatively descriptive and presented as percentage. The electronic medical records of 80 patients were analyzed, being 90\% ( $n=72)$ females and $10 \%(n=8)$ males. The mean age among the female patients was 55.3 years and the male sex was 66.8 years. Of the total number of patients, $96.25 \%(n=77)$ were from the Federal District and $3.75 \%(n=3)$ were from Goiás. The main rheumatic diseases found in the sample were fibromyalgia, 52.5\% ( $n=42)$, Osteoarthritis $37.5 \%(n=30)$, Osteoporosis 31.25\% $(n=25)$, Rheumatoid Arthritis 17.5\% ( $\mathrm{N}=3$ ), Ankylosing Spondylitis 2.5\% ( $=2$ ) and Sclerosis $(n=3)$, Lupus $11.25 \%(n=9)$, Psoriatic Arthritis $3.75 \%$ Systemic $1.25 \%(n=1)$. SENNA et al. estimated the prevalence of rheumatic diseases in residents of city of Montes Claros, Brazil. Fibromyalgia in $34.7 \%(n=76)$, Rheumatoid Arthritis in $6.4 \%$ $(n=14)$ and Lupus in $1.4 \%(n=3)$ were observed in $57.5 \%(n=$ 126). WONG et al. stated that the prevalence of Ankylosing Spondylitis varies from 0.1 to $0.5 \%$ worldwide, Gout in Canada affects $3 \%$ of adults and other diseases such as Psoriatic Arthritis, Lupus and Scleroderma present a prevalence ranging from 0.1 to $0.5 \%$. There is a great need for epidemiological studies that serve as a basis for strategies and actions for the development of a health policy aimed at diagnosis and early treatment. In addition, the results of the research are corroborating with literature data found in Brazil and Internationally. 


\section{P145}

DENGUE ASSOCIATED TO SEPTIC ARTHRITIS OF THE HIP A CASE REPORT

Isadora Carvalho Medeiros Francescantonio, Jéssica Ferreira Souza,

Ana Márcia Guimarães Alves, Ana Paula Vecchi

HOSPITAL MATERNO INFANTIL, GOIANIA, GO, Brasil

Advances in Rheumatology 2018, 58(Suppl 1):P145

Background: The painful hip syndrome in children can have many causes, including infectious and inflammatory $(1,2)$. The viral infections can be part of this group and lead to acute arthritis (3). How ever the diagnosis can be hard, that's why is necessary the association of other signs and symptoms and serologies (3). The arboviruses can be related to articular symptoms, being this more prevalent in the cases of chikungunya and rare in dengue (3). The septic arthritis in children is usually caused by hematogenous dissemination of Staphylococcus aureus (SA). It frequently needs early surgical treatment to reduce complications (4). It is described a case of a patient with dengue associated to septic arthritis of the hip.

Case Report: D.P.M, male, 8 years old, with high fever $\left(39^{\circ} \mathrm{C}\right)$ for 8 days and pain in left hip, which was mild at the beginning and was increasing in intensity until leads to movement limitation. He also complains about abdominal pain, vomit and dyspnea. The parents brought some exams: computed tomography showing right pleural effusion, massive ascites and left synovitis of the hip; hemoglobin $19 \mathrm{~g} / \mathrm{dL}$, hematocrit $52 \%$, leukocytes 20.000 , platelets 107.000 . On admission he received oxacilin and ceftriaxone and was submitted to a hip joint puncture. In the puncture was drained a big quantity of pus, which have positive culture for SA. During the hospitalization occurs improvement of the hemoconcentration with venous hydration. Also in the recovery period, he presented some episodes of orthostatic hypotension. Because of these symptoms dengue serologies was requested, and have a positive IgM result. The children presented improvement of the articular symptoms and recovered the movements completely.

Conclusion: Dengue can be presented by a variety of symptoms (2). The joint involvement is unusual and frequently transitional with spontaneous resolution (2). Chikungunya causes tissue damage and leads to an inflammatory process. Is possible that the inflammation causes a deregulation of inflammatory process control mechanisms because of the persistence of viral RNA in macrophages. Therefore is also possible that the dengue virus, could cause a similar inflammatory process, and because of that leads to a secondary bacterial infection, which can progress to septic arthritis of the hip. A case with joint involvement associated to serosite can be related to autoimmune diseases and the diagnosis of infectious diseases may be neglected. Therefore it's fundamental to make a global analyses of the patient taking into account its epidemiological characteristics to make a early diagnoses and treatment.

\section{Consent for publication}

The authors declare that they have obtained informed written consent from the patient's tutors for publication

\section{P146 \\ DEPRESSION AND ANTI-TNF USE: AN UNCOMMON ADVERSE EVENT \\ Caroline Almeida Oliveira', Matheus Xavier Guimarães ${ }^{2}$, Lisa Mielke de Oliveira ${ }^{2}$, André Ozela Augusto ${ }^{2}$, Denise Moraes Horiy², Fernanda Pulcheri Ramos², Sônia Maria Alvarenga Anti Loduca Lima², Rina Dalva Neubarth Giorgi ${ }^{2}$ \\ ${ }^{1}$ HOSPITAL DO SERVIDOR PÚBLICO ESTADUAL, SÃO PAULO, SP, Brasil; ${ }^{2}$ HOSPITAL DO SERVIDOR PÚBLICO ESTADUAL DE SÃO PAULO, SÃO PAULO, SÃO PAULO, Brasil \\ Advances in Rheumatology 2018, 58(Suppl 1):P146}

Introduction: Major depression is a mental health problem that affects approximately $8 \%$ of men and $15 \%$ of women in the world population. Some evidence shows that this disease is mediated by an inflammatory disorder caused by the activation of cytokines, such as interleukins (IL-2, IL-6, IL-12) and tumor necrosis factor (TNF-alpha) at high levels in depressed patients. However, we report four cases of patients who progressed with depression after using anti-TNF.

Case Study: Four female patients, aged 35 to 58 years, in regular follow-up at the Rheumatoid Arthritis outpatient clinic of a tertiary hospital in the city of São Paulo, reported a depressive picture or worsening of mood during the use of Infliximab or Etanercept to control articular disease activity. Complaints such as discouragement, fatigue, easy crying, marked depression were reported spontaneously during routine care related to the medication. The mean time of disease was 14 years, with inflammatory polyarticular involvement, which required therapeutic changes during evolution. All of them noticed improvement in mood after anti-TNF suspension, maintaining a good clinical and laboratory response from the joint point of view.

Conclusion: Patients with RA have a prevalence of above-average mood disorders, varying from $13 \%$ to $47 \%$, possibly because it is a disabling disease or because of chronic inflammatory activity. Two analyzes of depressed patients using Infliximab concluded that there was no generalized efficacy when compared to antidepressants, but there was less need for prescription in this population. Uguz et al., In 2009, evaluated the impact of Etanercept or Infliximab on the severity of depression and anxiety symptoms in 83 outpatients with $\mathrm{RA}_{\text {i }}$ those on anti-TNF agents had a lower prevalence of mood symptoms compared to those who were not on treatment. To date, there is no evidence of efficacy of IL- 6 blockade in the treatment of major depression. The most likely hypotheses for the unexpected effect reported by the four RA patients would be the fact that only a very limited number of proinflammatory cytokines could be inactivated by the currently available blockers, or the possibility that cytokines involved in the pathophysiology of RA depression may be different from those involved in the more typical autoimmune disorders.

\section{P147}

DEPRESSION, INTENSITY OF DEPRESSIVE SYMPTOMS AND CORRELATION WITH CLINICAL PARAMETERS IN PATIENTS WITH RHEUMATOID ARTHRITIS

Paulo Gabriel de Oliveira Cerquinho', Rafael da Rocha Caminha², Bruna Ferraz Gutierrez Piola², Flávia Jatobá de Barros², Pedro Arturo

Bismara Carneiro Santos ${ }^{2}$, Hugo Deleon de Lima ${ }^{3}$, Mariana Souza Pessoa de Luna ${ }^{3}$, Marina de Oliveira Stojanovic Gomes ${ }^{3}$, Leia Teixeira de Andrade ${ }^{3}$ Dennys Lapenda Fagundes ${ }^{3}$, Laurindo Ferreira da Rocha Junior ${ }^{3}$

${ }^{1}$ FACULDADE PERNAMBUCANA DE SAÚDE, RECIFE, PE, Brasil;

${ }^{2}$ FACULDADE PERNAMBUCANA DE SAÚDE -FPS, RECIFE, PERNAMBUCO, Brasil; ${ }^{3}$ INSTITUTO DE MEDICINA INTEGRAL PROF. FERNANDO FIGUEIRA IMIP, RECIFE, PERNAMBUCO, Brasil

Advances in Rheumatology 2018, 58(Suppl 1):P147

Background: Rheumatoid arthritis (RA) is a chronic inflammatory autoimmune disease that primarily affects the joints. It is well known that RA has a profound impact on the social, economic and psychological aspects of the patients' lives. The objective of this study is to evaluate the presence of depression and the intensity of depressive symptons in RA patients as well as to correlate these symptons with clinical parameters.

Methods: The data collection was done by interview, clinical evaluation and review of medical records of patients. Evaluation of functional capacity was performed wiht Health Assessment Questionnaire (HAQ). For the clinical diagnosis of depression, the DSM-5 (Diagnostic and Statistical Manual of Mental Disorders - 5th edition) was used, while the BDI-II (Beck Depression Inventory II) was applied to evaluate the intensity of depression symptoms. Thirty-one age and sexmatched healthy individuals were enrolled as controls. Statistical association and correlation were performed using software Graphpad prism 6.0. In the associations, the methods of Mann-whitney and $t$ student were used. Pearson and Spearman tests were utilized in the correlations. Fisher exact test was used for categorical variables.

Results And Conclusions: A total of 98 patients participated in this study of which 95 were women with median age of 55 years (IQR 46.25-62.5). Forty patients (40.82\%) and $2(6.5 \%)$ healthy controls were clinically confirmed to have depressive disorder after responding to DSM-5 $(p=0.0003)$. Patients with depression compared to patients with no depression had higher median visual analogue scale 
(VAS) score for stiffness $(55 \mathrm{~mm}$ [IQR $21.75-85.25]$ vs. $12 \mathrm{~mm}$ [IQR 052.5], $\mathrm{P}=0.0005)$, for fatigue $(63.5 \mathrm{~mm}$ IQR [21.75-89.5] vs. $32 \mathrm{~mm}$ [IQR 3-69], $p=0.0106)$, for patient global assessment $(82.5 \mathrm{~mm}$ [IQR 61.25$96.5]$ vs. $52 \mathrm{~mm}$ [IQR 8-84.5], $\mathrm{p}=0.0029)$ and for Physician Global assessment (45mm [IQR 23.25-66] vs. $22 \mathrm{~mm}$ [IQR 7.5-50], $\mathrm{p}=0.0095)$. Additionally, greater number of painful $(p=0.0259)$ and swollen joints $(p=0.0199)$, greater duration of morning stiffness $(p=0.0306)$, worse disease activity indexes (CDAl with $p=0.0036$ and DAS28 $p=0.0099$ ) and worse functional capacity $(p=0.0078)$ was also associated with depression. Patients had higher BDI scores compared to controls (median 13.5 [IQR 5-21] vs. 6 [IQR 2-9], p=0.0007). The intensity of depressive symptons correlated positively with weight $(r=0.2209$; $p=0.0315)$, mean arterial pressure $(r=0.2681 ; p=0.0098)$ and functional capacity $(r=0.3883 ; p<0.0001)$. These results suggest that patients with depression have more severe clinical features of RA. Patients with RA had more intense depressive symponts than healthy subjects.

\section{P153}

DORSALGIA IN A GOUT PATIENT: THE ROLE OF DUAL ENERGY

\section{TOMOGRAPHY}

Eduardo S. Paiva, Ana Beatriz Artigas Guimaraes, Salun Coelho Aragao,

Maiara Bastiani Bisogni, Luiza Bueno Zeni, Luan Luckmann, Keoma Azevedo Sabiao, Chayanne Natielle Rossetto, Isadora Welter Pioresan

UFPR, CURITIBA, PR, Brasil

Advances in Rheumatology 2018, 58(Suppl 1):P153

Background: Gout is a metabolic disease caused by monosodium urate crystals deposits leading to a classic picture of acute arthritis and periarthritis that occurs asymmetrically and episodically. The main diagnostic procedure is to proceed with an arthrocentesis of the affected joint. Although described in the literature, the involvement of the spine by the disease is not easy to prove, given the difficulty of performing any kind of puncture at that site. Dual-energy tomography has emerged in recent years as a method for the detection of joint and periarticular deposits of urate, and also serving as a way to differentiate between these kinds of deposits from those of calcium pyrophosphate. Due to its clinical application, it can be a valuable tool for the rheumatologist, helping him differentiate among the causes of episodic arthropathies. We herein describe a case in which the dual energy tomography was useful for the diagnosis of the spinal cord involvement by gout.

Case report: A 57-year-old male patient had a 15-year history of gout. His last crisis occurred one year ago and he was being treated with an inadequate dose of allopurinol. He presented to the emergency department with a recent history of acute dorsal pain followed by arthritis in his left knee and foot, with no response to low-dose overthe-counter NSAIDs. Because there was a strong suspicion of thoracic spine gout, a double energy tomography was requested, which confirmed the diagnosis. There were deposits in the left T8/T9 facet joint and in the T1 vertebral body. He was treated with a combination of a prescription NSAID and moderate-dose prednisone. There was a quick resolution of the spine pain after 2 days of treatment an in the following days he had complete resolution of the foot and knee arthritis.

Conclusion: Dual energy tomography may be useful in the diagnosis of gout patients, especially when there is any kind of diagnostic doubt, or when there is involvement of sites that are hard to access.

\section{Consent for publication}

The authors declare that they have obtained informed written consent from the patient's tutors for publication
P154

DRUG TREATMENT ADHERENCE IN SYSTEMIC LUPUS

ERYTHEMATOSUS (SLE): A LONG TERM ANALYSIS OF ADHERENCE

AND A POST-HOC ANALYSIS OF MORISKY MEDICATION

ADHERENCE SCALE MODIFIED BY DELGADO AND LIMA

Karen Sanae Takehara Vieira', Gisele Veiga Guimarães ${ }^{1}$, Felipe Silva

Medeiros ${ }^{1}$, Pedro Felipe De Almeida Vianna², Laíssa Cristina Alves

Alvino $^{2}$, Elisa Martins Das Neves De Albuquerque ${ }^{2}$, Marise Oliveira Dos

Santos ${ }^{2}$, Evandro Mendes Klumb ${ }^{2}$

${ }^{1}$ UNIVERSIDADE DO ESTADO DO RIO DE JANEIRO, RIO DE JANEIRO, RJ, Brasil; ${ }^{2}$ HOSPITAL UNIVERSITÁRIO PEDRO ERNESTO - UERJ, RIO DE

JANEIRO, RJ, Brasil

Advances in Rheumatology 2018, 58(Suppl 1):P154

Background: SLE is an autoimmune disease with a variable clinical course and severity. Drug treatment adherence (DTA) is frequently low, especially in undeveloped countries, as in other chronic diseases. However DTA may be influenced by the method employed for its measurement, the reason why this study was developed.

Materials and methods: This study included 246 lupus patients followed at a university hospital in Rio de Janeiro and was designed to investigate DTA in 2009. Adherence was primarily evaluated using the Morisky Medication Adherence Scale modified by Delgado and Lima (MMAS), which was set as a dichotomous variable (adherent or not). Patients were considered adherent whenever they answered "rarely" or "never" to the four questions of MMAS. However, possibly due to cultural characteristics of Brazilian population, the term "sometimes" is commonly used to specify an event that occurs with similar frequency of "rarely" which justified its inclusion as adherence. For this purpose, the 246 questionnaires were reviewed and those with "sometimes", "rarely" or "never" for the 4 questions were considered adherents (MMAS remodified). A second interview was performed for 123 among the 246 patients previously selected in 2017 and data was analyzed using MMAS and MMAS remodified.

Results: The DTA for the 246 patients was of $31.7 \%$ (MMAS), which turned out to be $67.1 \%$ when adherence scale included "sometimes" as a positive adherence answer (MMAS remodified). For the 123 patients interviewed in 2017, MMAS showed DTA of 30.1\% (2009) and $51.2 \%$ (2017). With the employ of MMAS remodified, DTA was $61.7 \%$ in 2009 and $79.7 \%$ in 2017. The intensity of concordance for DTA between the tests performed (MMAS and MMAS remodified) was moderate (kappa value: 0.37 - for 246 cases), or strong (kappa value: 0.42 - for 123 cases) which indicates a high variability of adherence percentage depending on the scale employed and patient interpretation of terms used.

Discussion and Conclusion: The present study showed that the amount of DTA percentage depends on the method employed. The DTA determination remains a complex issue and its variability is influenced not only by patient compliance but also by methodology employed to analyze data. More accurate methods for DTA determination are needed and they should ideally include patient subjectiveness.

\section{P155}

EARLY PULMONARY HYPERTENSION IN YOUNG PATIENT WITH MIXED CONNECTIVE TISSUE DISEASE

Igor André Telles Da Cunha', Fernando César Duarte', Leandro Bonecker Lora', João Renato Cardoso Mourão', Priscilla Souza da Cruz², Leonardo Motta Ramos ${ }^{1}$, Paula Sampaio Azevedo ${ }^{1}$, Laura Pessanha Nunes', Bruna Sande Miguel' ${ }^{1}$, Camilla Meira Riccioppo ${ }^{1}$, Alessandra Cardoso Pereira 'UNIGRANRIO, RIO DE JANEIRO, RJ, Brasil; ' ${ }^{1} E S T A ́ C I O$, RIO DE JANEIRO, RIO DE JANEIRO, Brasil

Advances in Rheumatology 2018, 58(Suppl 1):P155 
Mixed Connective Tissue Disease (MCTD) is an overlapping syndrome associated with the presence of anti-U1 RNP antibodies, which incorporates clinical manifestations of systemic lupus erythematosus, sclerodemia and poliomyositis. Definitive diagnosis of MCTD is often complicated by the fact that overlapping features tend to occur sequentially. This confusion arises because of the concomitance of various diffuse connective tissue diseases, as well as changes in the underlying disease pathology. Pulmonary hypertension appears as a late manifestation and appears to be the leading cause of death in MCTD.

We report the case of a female patient, 33 years old, black, who initially had an anastarch, chest pain, dyspnea on minor exertion. She was admitted to a referral hospital in cardiology, with a suspected diagnosis of pulmonary thromboembolism. A chest CT angiography showed no abnormalities, and transthoracic echocardiography revealed significant pulmonary hypertension (mPAP $73 \mathrm{mmHg}$ ) associated with dilatation and right ventricular systolic dysfunction. He was discharged with prescribed anticoagulant medication. She reported a return of symptoms after three days and a new search for hospital care, and was then referred to the Rheumatology outpatient clinic. In the consultations that occurred between February 2017 and April 2018, alopecia, painful subcutaneous nodules in the lower limbs, visual turbidity, xerostomia, oral ulcers, thoracic pain, cramp, abdominal pain, Raynaud's phenomenon, arthralgia of the metacarpophalangeal joints of both hands, finger edema and sclerodactyly, as well as an episode of pericardial effusion. Laboratory tests revealed thrombocytopenia, elevated CPK (1.482 U /L), anti-Ro and antiRNP (greater than $240 \mathrm{Elia} \mathrm{U} / \mathrm{ml}$ ), anti-LA transiently positive. Before the findings, the diagnosis of MCTD was made.

The relevance of this case is the atypical clinical evolution of the young patient, who presented pulmonary hypertension in an early form of her disease. This fact is unusual because this complication is related to a longer time of illness and a more advanced age. It is worth mentioning also that anti-Ro positive in the MCTD happens in only $25 \%$ of the cases.

\section{Consent for publication}

The authors declare that they have obtained informed written consent from the patient's tutors for publication

\section{P156}

\section{EARLY RESPIRATORY DISEASE ACTIVITY IS RISK FOR DAMAGE IN} PRIMARY SJÖGREN'S SYNDROME

Leticia Fonseca Favarato, Wildner Mardegan Sardenberg, Maria Carmen Lopes Ferreia Silva Santos, Larissa Carvalho Caser, Letícia Fonseca

Favarato, Sabrina Zanardi Machado, Valéria Valim

UNIVERSIDADE FEDERAL DO ESPÍRITO SANTO, VITÓRIA, ESPÍRITO SANTO,

Brasil

Advances in Rheumatology 2018, 58(Suppl 1):P156

Background: We studied clinical and epidemiological variables that could be related to greater severity and damage in primary Sjögren's syndrome (pSS).

Methods: Cross-Sectional study including patients with pSS (AECG 2002 or ACR/EULAR 2017). EULAR Sjögren's syndrome Disease Index (ESSDAl) was calculated at diagnosis and currently. Patients have performed EULAR Sjögren's syndrome Patient Report Index (ESSPRI) and Sjögren's syndrome Disease Damage Index (SSDDI). Non-parametric statistics and logistic regression were applied with $p$-value of 0.05 . Results: 104 patients with pSS were included, $51.49 \pm 12.13$ years, $95,2 \%(n=99 / 104)$ were women. Disease duration was $65,44 \pm 41.89$ months. Anti-Ro was positive in $68 \%(n=70 / 103)$, FAN in $82 \%(n=82 /$ $100), F R$ in $37.4 \%(n=37 / 99)$ and anti-La in $30.3 \%(n=30 / 99)$, focal lymphocytic sialoadenitis in $73 \%$ (71/91). The ESSPRI was $5.62 \pm 2.56$ and the ESSDAI $3.63 \pm 5.12 .53 .8 \%(n=56 / 104)$ was using hydroxychloroquine and $51 \% \quad(n=53 / 104)$ immunosuppressant. The higher damage score was associated with disease duration $(0.270 ; \mathrm{p}=0.007)$, high disease activity at the onset of the disease (initial ESSDAl)
$(0.226, p=0.021)$, respiratory evolvement at the onset of the disease $(0.283, p=0.004)$ and impairment of the peripheral nervous system $(0,273, p=0,005)$, at some point. After multiple regression analysis, the initial involvement of the respiratory system was confirmed to be a risk factor for damage (SSDDI > 3) independent of disease duration and current disease activity (ESSDAl) (OR=8.94, 1.44-55.64C195\%; $p<0.05)$. The respiratory domain was also associated with higher mortality $(66.7 \%$ vs. $5 \%, p=0.011)$.

Conclusion: Early respiratory disease activity is associated with death and predicts damage in primary Sjögren's syndrome

P157

EARLY-ONSET HELLP SYNDROME AND HEPATIC INFARCTIONS RELATED TO PRIMARY ANTIPHOSPHOLIPID SYNDROME (APS)

Rafael Alves Cordeiro, Jobson Lopes De Oliveira, Camila Nobre Bulhões, Steeven Shu Kai Yeh, Danieli Castro Oliveira De Andrade DIVISAO DE REUMATOLOGIA, HOSPITAL DAS CLINICAS HCFMUSP, FACULDADE DE MEDICINA, UNIVERSIDADE DE SAO PAULO, SAO PAULO, SP, Brasil

Advances in Rheumatology 2018, 58(Suppl 1):P157

Background: Antiphospholipid syndrome (APS) in pregnancy has a serious impact on maternal and fetal morbidity. We report a case of a pregnant woman with previously known primary APS who developed early onset hemolysis, elevated liver enzymes, low platelets syndrome (HELLP) and multiple liver infarctions during the 18th week of gestation.

Case Report: A 25-year-old female patient has had a diagnosis of primary APS for 8 years based on the presence of deep venous thrombosis associated with anticardiolipin and lupus anticoagulant positivity. At the time of the diagnosis (another service), she was prescribed enoxaparin, warfarin and aspirin. However, she presented retroperitoneal hemorrhage which led to suspension of the anticoagulation. She remained without anticoagulant treatment until starting follow-up in a high-risk prenatal service in 2017, where she was introduced enoxaparin at a prophylactic dose $(40 \mathrm{mg} \mathrm{SC} /$ day $)$ and ASA $100 \mathrm{mg} /$ day. At the eighteenth week of gestation, she went to emergency department with abdominal pain in the right quadrant pain. She denied arthralgia, photosensitivity, alopecia, cutaneous lesions, oral or nasal ulcers, dyspnea, chest pain, hematuria and fever. Physical exam: arterial hypertension $(140 / 100 \mathrm{mmHg})$ and livedo reticularis in the lower limbs. Laboratory evaluation: anemia $(9.9 \mathrm{~g} / \mathrm{dL})$, elevation of LDH, elevated transaminases (AST: 814 and ALT: 678), thrombocytopenia $(98000 / \mathrm{mm} 3)$ and proteinuria $(2.34 \mathrm{~g} / \mathrm{L})$. Immunological profile: ANA 1/320 (homogeneous nuclear pattern) with antidsDNA negative; absence of complement consumption (C3: 89 and C4: 16); anti-cardiolipin lgG (112.9 GLP) and lupus anticoagulant positive. Obstetric USG: increased resistance of the umbilical and uterine arteries. Abdominal tomography: enlarged liver, multiple hypovascularized and poorly delimited areas in all hepatic segments, compatible with ischemia and tissue necrosis. Hypertension was initially controlled with amlodipine, but fetal death was observed at 19 weeks and 5 days. After pregnancy termination, the patient progressed with resolution of laboratory abnormalities and with no need for antihypertensive drugs. Due to clinical and laboratory evolution (lupus and sepsis were excluded), the diagnosis of early-onset HELLP syndrome and hepatic infarctions associated with the antiphospholipid syndrome was confirmed.

Conclusion: HELLP syndrome is a relatively rare pregnancy-related thrombotic microangiopathic disorder, usually observed during the third trimester. The occurrence of HELLP syndrome before 20 weeks of gestation is extremely rare. However, clinicians should be aware to the diagnosis of early-onset HELLP syndrome and hepatic infarctions associated with APS.

\section{Consent for publication}

The authors declare that they have obtained informed written consent from the patient's tutors for publication 


\section{P159}

EFFECTIVENESS OF NIGHT-TIME ORTHOSES IN THE PAIN FOR WOMEN WITH HAND OSTEOARTHRITIS: RANDOMIZED AND CONTROLLED TRIAL

Paula Gabriel Silva, Fabiana de Carvalho Silva, Artur da Rocha Correa Fernandes, Jamil Natour

UNIFESP, SÃO PAULO, SÃO PAULO, Brasil

Advances in Rheumatology 2018, 58(Suppl 1):P159

Objective: To evaluate the effectiveness of night-time orthoses in women with symptomatic osteoarthritis (OA) diagnosis in the second or third fingers of the dominant hand.

Methods: Design: A randomized controlled trial, masked assessor and intention to treat analysis. Setting: the outpatient clinic of the rheumatology department, referral center. Financial support: Support in the grant\#13/14460-3 process and grant\#13/221591, São Paulo Research Foundation (FAPESP). Participants: Seventy-seven patients with a diagnosis of hand OA were contacted, fifty-two were eligible and allocated randomly to 2 groups. Interventions: The intervention group (IG) N26 used a night-time orthosis starting at baseline and the control group (CG) N26 did not. However, both groups participated in educational group discussions about hand OA. Participants were evaluated at baseline and at 45,90 , and 180 days after inclusion in the study. Main Outcome Measure: Numerical Rating Scale for pain; the Jamar dynamometer for grip strength; a pinch gauge for pinch strength; Cochin and AUSCAN questionnaires for function, and the Moberg pick-up test for manual performance.

Results: The IG showed a statistically significant improvement versus the CG in relation to pain and function; the IG had decreased pain resting at $2.1 \mathrm{~cm}(p=0.002)$ and had decreased pain during activity at $4.3 \mathrm{~cm}(\mathrm{p}<0.001)$. The improvement in pain had a correlation with the Cochin questionnaire and absence of Bouchard's node in the third finger, as well as a thin pinch performed without difficulty; these are predictors of the best prognosis for treatment with the night-time orthoses.

Conclusion: The use of night-time orthoses reduces pain and leads to improvement in hand function in women with hand OA.

\section{P160}

\section{EFFECTIVENESS OF SECUKINUMAB FOR HIGHLY RESISTANT}

JUVENILE PSORIATIC ARTHRITIS: CASE REPORT

Cleandro Pires Albuquerque, Ana Paula Faria Carvalho, Luciana Teófilo Lourençoni, Andressa Junqueira Osorio, Isabela de Sousa Russo, Tassiane Raquel Cunha Martins de Moraes, Talita Yokoy de Souza, Ana Carolina Emy Vicente Hidaka, Sandra Maximiano de Oliveira HOSPITAL UNIVERSITÁRIO DE BRASÍLIA, BRASÍLIA, DF, Brasil Advances in Rheumatology 2018, 58(Suppl 1):P160

We describe an atypical case of aggressive psoriatic arthritis, refractory to nearly every attempt of treatment, including multiple biologics, but then achieving an excellent response after initiating secukinumab. Female, 26 years old, diagnosed with axial and peripheral psoriatic arthritis at the age of four. Psoriasis had started only months after birth. The disease followed an aggressive and relentless course through childhood and adolescence, despite every attempt of treatment with DMARDs. At the age of 15 , she was submitted to hip arthroplasty. By that time, she had some deformities in hands and feet. She also developed asthma and corticosteroid-induced osteoporosis, with a pelvic fracture.

Throughout the course of the disease, she maintained cutaneous and articular inflammatory activity, refractory to methotrexate, sulfasalazine, leflunomide, cyclosporine, adalimumab, etanercept, infliximab, and ustekinumab. Biologics were always used in combination with conventional DMARDs. She had an anaphylatic shock with infliximab, which was discontinued. For a while, she achieved a good response with the combination of adalimumab and cyclosporine, but eventually this also had to be discontinued due to acute kidney injury. At last, when she was 24 years old, we initiated secukinumab in combination with methotrexate.
Soon after induction therapy, she developed a community acquired pneumonia, requiring hospitalization, and demanding suspension of immunosuppressants. By the time of recovery from the infection, the cutaneous and articular disease was already inactive, and so persisted even after six months of discharge. For that reason, methotrexate was not reinstituted, and she stayed with secukinumab monotherapy.

To this date, two years after starting secukinumab, the patient maintains remission from articular and cutaneous symptoms. BASDAI 0.4, ASDAS-ESR 0.69, ASDAS-CRP 1.1, PASI 0.4.

This case suggests the potential usefulness of secukinumab for highly refractory psoriatic arthritis, even within a monotherapy scenario. Nevertheless, it also points to the possibility of severe infectious complications, associated with the treatment, requiring careful monitoring for prompt intervention throughout.

\section{Consent for publication}

The authors declare that they have obtained informed written consent from the patient's tutors for publication

\section{P161}

EFFECTS OF EXERCISE TRAINING PROGRAM ON ENDOTHELIAL FUNCTION AND STRUCTURAL PROPERTIES OF LARGE ARTERIES OF SYSTEMIC AUTOIMMUNE MYOPATHY

Rafael Giovane Missé2, Diego Sales De Oliveira², Alexandre Moura Santos², Jean Marcos Souza², Bruno Gualano, Ana Lúcia De Sá Pinto²

Valéria Aparecida Costa Hong ${ }^{3}$, Luiz Aparecido Bortolotto3,

Samuel Katsuyuki Shinjo ${ }^{2}$

${ }^{1}$ ESCOLA DE EDUCAÇÃO FÍSICA E ESPORTES DA UNIVERSIDADE DE SÃO PAULO, SÃO PAULO, SÃO PAULO, Brasil; 'FACULDADE DE MEDICINA DA UNIVERSIDADE DE SÃO PAULO, SÃO PAULO, SÃO PAULO, Brasil;

${ }^{3}$ INSTITUTO DO CORAÇÃO DO HOSPITAL DAS CLÍNICAS DA FACULDADE DE MEDICINA DA UNIVERSIDADE DE SÃO PAULO, SÃO PAULO, SÃO

PAULO, Brasil

Advances in Rheumatology 2018, 58(Suppl 1):P161

Background: Significant impairment in vascular structure and function has been described in several rheumatic diseases, including in a unique cross-sectional study with systemic autoimmune myopathies (SAM). These vascular abnormalities closely linked to cardiovascular diseases and their risk factors. Exercise training has useful tool for promotes reduction and control in these risks. In this context, the purpose of this study was to evaluate the effect of chronic exercise training in vascular structure and function in patients with SAM.

Methods: This quasi-prospective study included 5 adult female with SAM (2017 EULAR/ACR classification criteria: 3 dermatomyositis and 1 polymiositis). All patients underwent to carotid-femoral pulse wave velocity (PWV) and flow-mediated vasodilation (FMD) analysis achieved from brachial arterial above the antecubital fossa. Disease status was assessed with International Myositis Assessment \& Clinical Studies Group (IMACS) core set measures. These exams were applied at baseline and after 12-week, twice weekly, exercise training program that was composed of 60 minutes of aerobic training and strength training composed of 7 exercises (leg press, bench press, leg extension, pulley, leg flexion, seated cable rows and abdominal exercises).

Results: Mean age of the patients was 48 years, whereas median disease duration was 8 years. All patients were clinically and laboratory stable, according to IMACS core set measures, using different immunosuppressive drugs and prednisone dose $<10 \mathrm{mg} /$ day. After exercise training, there was an increase in aerobic capacity and muscle strength $(\mathrm{P}<0.05)$. In vascular parameters, a reduction tendency with a high effect size was observed in endothelial function parameters $(P=0.057$; ES: 0.90$)$, but not in arterial stiffness parameters $(P=0.438$; ES: 0.40$)$.

Conclusion: In patients with clinical and laboratory stable SAM, exercise training promoted a reduction tendency in endothelial function, but not sufficient in arterial stiffness. Further studies with large sample are necessary to confirm these findings.

Support by: FAPESP \#2016/19771-5 (DSO), \#2016/23574-0 (RGM), \#2017/13109-1 (SKS) 


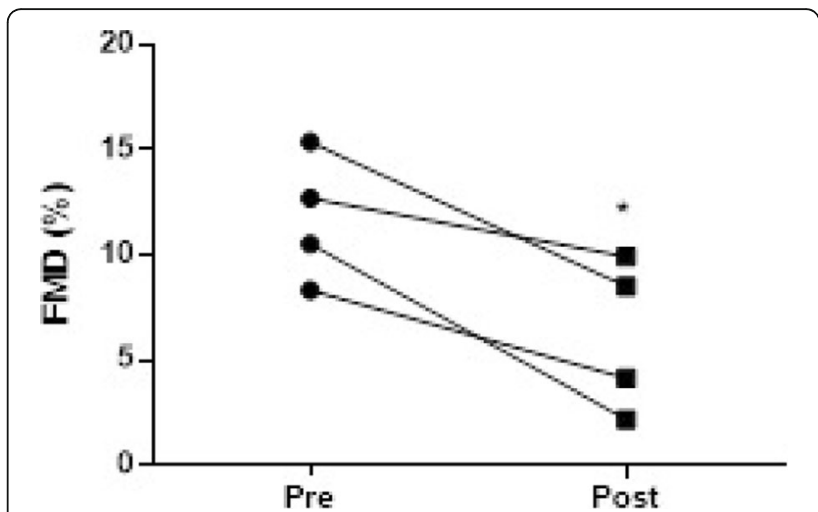

Fig. 1 (abstract P161). Effect of exercise training (pre and post 12weeks) in endothelial function measured by flow-mediated vasodilation (FMD) in patients with systemic autoimmune myopathies

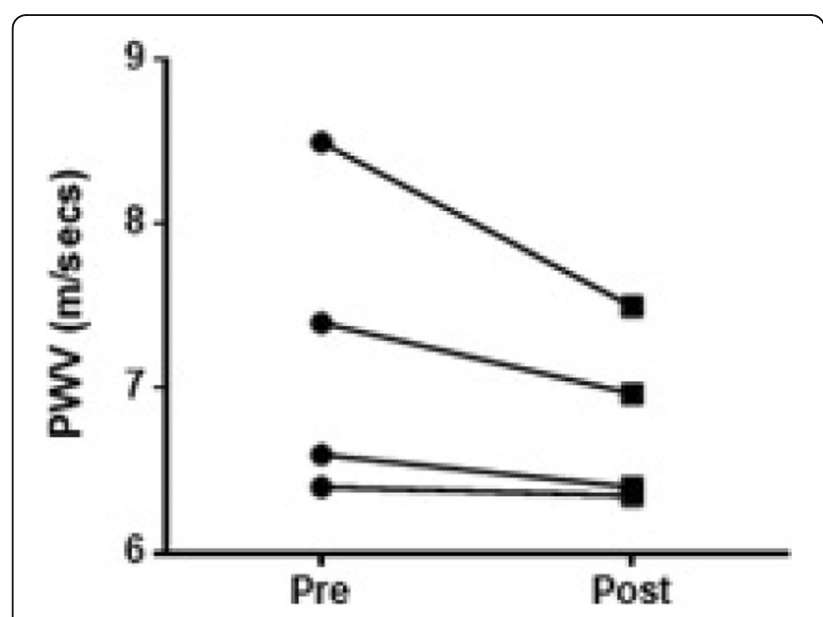

Fig. 2 (abstract P161). Effect of exercise training (pre and post 12weeks) in arterial

\section{P165}

EOSINOPHILIC FASCIITIS: DIAGNOSTIC USEFULNESS OF MAGNETIC RESONANCE IMAGING IN A SCLERODERMA-LIKE DISORDER

Bruna Giusto Bunjes, Marília Ambiel Dagostin, Douglas Amaral Moreira, Ana Paula Luppino Assad, Henrique Carriço da Silva, Percival Degrava Sampaio Barros

FACULDADE DE MEDICINA DA UNIVERSIDADE DE SÃO PAULO FMUSP, SÃO PAULO, SÃO PAULO, Brasil

Advances in Rheumatology 2018, 58(Suppl 1):P165

Background: Eosinophilic fasciitis (EF) is a rare disease characterized by symmetrical induration of the skin and inflammation of deep muscle fascia. The onset is typically acute and the clinical findings include erythema, swelling and thickening of the extremities, usually accompanied by peripheral blood eosinophilia, hypergammaglobulinemia and elevated erythrocyte sedimentation rate. Although the affected skin is somewhat similar to that observed in other scleroderma spectrum disorders, the skin of the hands and feet is generally spared.

Case Report: The authors describe the case of a 45-year-old woman who was referred to the Rheumatology Division of our institution due to symmetrical and progressive stiffness, induration and swelling of forearms and legs. As the patient also presented concomitant inflammatory arthralgia and dyspnea on moderate exertion, the diagnosis of systemic sclerosis (SSc) was initially considered. Laboratory tests presented peripheral eosinophilia, positive antinuclear antibodies (ANA), with negative antitopoisomerase I (antiScl70) and anticentromere antibodies. Deep skin biopsy showed a lymphomononuclear infiltrate associated with moderate thickening in fascia, compatible with EF. Magnetic resonance imaging (MRI) of lower and upper limbs showed diffuse thickening with hyperintense signal on $\mathrm{T} 2$ fascia, involving all muscle groups, sparing muscle belly. The initial treatment was with oral glucocorticoids with significant improvement. Conclusion: EF should be considered as a differential diagnosis in fibrosing disorders. The early suspicion is important because, different from others scleroderma-like disorders, EF presents a good response to glucocorticoids. In our patient, MRI showed a good correspondence with anatomopathological data. Therefore, in cases with typical clinical findings, we can presume that MRI findings could possibly substitute deep skin biopsy.

Consent for publication

The authors declare that they have obtained informed written consent from the patient's tutors for publication

P166

EPIDEMIOLOGICAL CLINICAL PROFILE OF PATIENTS WITH

SPONDYLOARTHRITIS IN THE CENTER-WEST REGION OF BRAZIL

Fabiana Pompeo De Pina, Lucas Carvalho Velloso De Oliveira,

Lucas Alves Oliveira, Kleverton Augusto de Castro Siqueira

UNIVERSIDADE FEDERAL DE GOIÁS, GOIÂNIA, GO, Brasil

Advances in Rheumatology 2018, 58(Suppl 1):P166

Background: The epidemiological, demographic and clinical characteristics of spondyloarthritis are variable according to the affected population. There are few studies conducted in the brazilian population. This study carries out a survey of the epidemiological clinical profile of patients diagnosed with spondyloarthritis in a university hospital in the center-west region of Brazil.

Methods: A retrospective analytical-descriptive study was carried out with a cross-sectional design, in which data in medical records from patients with established diagnosis of spondyloarthritis of the spondyloarthritis ambulatory of the Rheumatology Service of a University Institution in central-western region of Brazil were analyzed. The analyzed data were: gender, age, color, occupational disability, signs and symptoms of spondyloarthritis, extra-articular disorders, use of drugs to treat the disease, physical characteristics and metric (Schober, Occiput-to-wall), HLA-B27, evidence of ESR and CRP, functional assessment (BASFI) and disease activity (BASDAI), type of diagnosis and clinical form.

Results and Conclusions: Of the 49 medical records analyzed, $61.22 \%$ presented the diagnosis of ankylosing spondylitis, followed by $36.73 \%$ of cases with Psoriatic Arthritis and 2.04\% with Enteropathic Arthritis. Of the total, $69.39 \%$ were male and $30.61 \%$ female. Mean age was 49.73 years. The patients most affected by some spondyloarthritis were the self-reported white with $46.81 \%$, followed by the browns with $42.55 \%$ and blacks with $10.64 \%$. Of these, $38.78 \%$ presented exclusively axial involvement; $34.69 \%$ axial and peripheral involvement; and $26.53 \%$ were exclusively peripheral. $38.78 \%$ of the analyzed patients reported work disability due to the disease. The Schober Test average for the total group analyzed was $13.84 \mathrm{~cm}$; those of occiput-wall distance $24.33 \mathrm{~cm}$; the number of inflamed joints had an average of 1.79; the number of inflamed entheses had an average of 1.05 . Of the 42 patients in whom the functionality was assessed by BASFI, the mean was 4.83 . Of the 41 patients in whom the disease activity was assessed by BASDAl, the mean was 4.83 .26 patients underwent HLA-B27, $57.69 \%$ of whom were positive for the test. From the analysis of the data of these medical records, a greater prevalence of ankylosing spondylitis is observed than of the other forms. There is a slight prevalence of axial involvement. The male was the most affected, as were the white group. The important functional impact was also observed in these patients. This is an unpublished study in this federative unit of the center-west region of Brazil. The original data obtained, justify the importance of its accomplishment. 


\section{P167}

EPIDEMIOLOGICAL PROFILE OF LEPROSY IN BRAZIL FROM 2010 TO

2017

Érica Alves Nascimento, Andressa Borelli Sandos, Mariana Bomfim

Menezes, Débora Larissa Montarroyos Leite

UNIVERSIDADE TIRADENTES, ARACAJU-SE, SE, Brasil

Advances in Rheumatology 2018, 58(Suppl 1):P167

Introduction: Leprosy is a chronic and infectious disease caused by the Mycobacterium leprae, which has a high infective power but low pathogenicity and affects the skin and regions of the peripheral nervous system. It is transmitted directly from an infected person to another through the upper airways. In Brazil, it is an endemic disease and has compulsory notification. It is also classified as paucibacillary, when the patient has 1 to 5 lesions, showing a lower bacilli load and in multibacillary, when the patient has more than 5 lesions, showing a greater bacilli load.

Objective: To analyze the epidemiological profile of patients infected with Mycobacterium leprae between 2010 and 2017 in Brazil.

Methodology: Retrospective descriptive study that shows all leprosy cases presents in Brazil between 2010 and 2017. Epidemiological data were collected through the database of the Notification of Injury Information System (SINAN).

Results: There was a decrease in the number of Mycobacterium leprae infections between 2010 and 2015. From 2010 to 2015, the number of cases dropped from 42,433 to 35,131 . In 2014, there was a small increase to 38,562 cases. In 2016, the number increased to 64,545 , decreasing again in 2017 , with 22,495 registered cases. Infections in men were higher than in women in all regions during the period analyzed. It is also observed a heterogeneous distribution of the number of cases recorded in the different Brazilian regions, which expose the socioeconomic differences of the country. Of the 323,182 cases registered, approximately $42 \%$ are in the Northeast region. The region with the lowest number of registered cases was the South, representing only $3.5 \%$ of the total. Infected patients, aged 20-64 years (economically active age group), represent the majority of the cases occurring between 2010 and 2017.

Conclusions: The study shows that there was a decrease in the number of leprosy cases registered in Brazil, however, this disease should not be neglected, since it affects mainly the economically active population. It is a disease, which, although incapacitating, is easily diagnosed and treated, as long as it occurs early. Because of this, the active search and compulsory notification by family health teams of suspected cases is so important.

\section{P168}

EPIDEMIOLOGY, CLINICAL FEATURES AND OUTCOME OF UVEITIS IN JUVENILE IDIOPATHIC ARTHRITIS

Renata Lopes Francisco De Andrade, Aline Maria de Oliveira Rocha, Liana Soido Teixeira e Silva, Vânia Schinzel, Yuslay Fernández Zamora, Cristina Muccioli, Maria Teresa de Sande e Lemos Ramos Ascensão Terreri UNIFESP, SÃO PAULO, SP, Brasil

Advances in Rheumatology 2018, 58(Suppl 1):P168

Background: Juvenile Idiopathic Arthritis (JIA) is the most prevalent chronic arthropathy in childhood. Among the extra-articular manifestations, the most frequent is chronic anterior uveitis. We aim to describe the cases of chronic anterior uveitis associated with JIA and the epidemiological characteristics, presence of autoantibodies, follow-up, complications and treatment.

Materials and methods: Retrospective analysis based on chart reviews of $329 \mathrm{JIA}$ patients at the Pediatric Rheumatology outpatient clinic. The ophthalmologic evaluation was performed using a slit lamp with biomicroscopy by a specialized ophtalmologist.

Results and Conclusions: Among the analyzed patients, 32 (9.7\%) presented uveitis. Of these, $26(81.3 \%)$ were female, mean age at symptoms onset was 5.4 years (ranged from 8 months to 15 years), mean age at the diagnosis was 6.6 years (ranged from 2 to 15 years) and the mean time of disease follow-up 9.2 years. The mean time between the diagnosis of JIA and uveitis was 8.3 years (between 4 years before and 3.7 years after). FAN Hep2 positive was identified in 21
(65.6\%) patients. No patient presented positive rheumatoid factor. According to the ILAR criteria, $20(62.5 \%)$ patients belonged to the oligoarticular group and $12(37.5 \%)$ to the rheumatoid factor negative polyarthritis. All patients received methotrexate as their initial DMARD, 16 oral prednisone, 8 cyclosporine, and 9 leflunomide. Fourteen patients $(43.8 \%)$ were treatment refractory and needed biological drugs: 4 etanercept, 3 infliximab, 11 adalimumab and 1 had abatacept. Four of the 14 patients $(28.6 \%)$ took more than one biological drug. In the follow-up, recurrent uveitis occurred in 23 patients $(71.9 \%)$; of these, 11 presented only 1 recurrence, while the other 12 recurred between 2 and 9 times. Five (15.6\%) patients had sequelae lesions, being 4 cataract (one with partial loss of vision), and one corneal opacity. FAN positivity, early age at diagnosis and female sex were related to uveitis development, although not predictors of severity. We concluded that our ocurrence of uveitis was similar to that found in the international literature and treatment refractoriness and recurrence were frequent.

\section{P174 \\ EVALUATION OF BODY COMPOSITION THROUGH BONE DENSITOMETRY IN PATIENTS SUBMITTED TO IMMUNOBIOLOGICAL THERAPY WITH RHEUMATOID ARTHRITIS AND SPONDYLOARTHRITIS \\ Manuela Amoedo Cox ${ }^{1}$, Rafaela Amoedo Cox ${ }^{1}$, Ana Teresa Amoedo ${ }^{2}$ ${ }^{1}$ UNIFACS, SALVADOR, BA, Brasil; ${ }^{2}$ UEFS, FEIRA DE SANTANA, BAHIA, \\ Brasil \\ Advances in Rheumatology 2018, 58(Suppl 1):P174}

Background: The present study aims to establish a body mass analysis through double emission bone densitometry (BMD) in patients with rheumatoid arthritis (RA) and spondylarteritis (SPS) using assisted immunobiological therapy.

Materials And Methods: For this, the method of collecting data from the medical records of these patients was used in a private clinic in Feira de Santana. Through the study of scientific articles on the subject and the data collection, it is expected to better understand the relationship between body composition (BC) and these patients. These data have relevance, since it allows to know the health of the patients with RA and SPS in use of this therapy. All patients with RA and SPS, who uses the immunobiological therapy from January 2018 to March 2018, were included in this study.

Results And Conclusion: Twenty patients with RA and SPS, equally distributed, were evaluated, being 17 women and 3 men. The study of BC demonstrated normal bone mass (NBM) in the majority of patients, $85 \%$ in both pathology groups. $65 \%$ of the patients shown high fat percentage, $45 \%$ with overweight, $40 \%$ with normal BMI and only $5 \%$ of obesity. Concluding that in the present study, there was no change in BMD in the majority of patients in both groups; although AR and SPS are diseases with potential risk factors for bone loss, it can be explained by many other factors like drugs use or sedentary lifestyle. Other studies are needed to establish cause-effect change in the $B C$ in patients using immunobiological therapy.

\section{P175}

EVALUATION OF BONE MASS THROUGH BONE

DENSITOMETRY IN PATIENTS SUBMITTED TO IMMUNOBIOLOGICAL THERAPY WITH RHEUMATOID ARTHRITIS AND

\section{SPONDYLOARTHRITIS}

Rafaela Amoedo Cox', Manuela Amoedo Cox ${ }^{1}$, Ana Teresa Amoedo ${ }^{2}$ ${ }^{1}$ UNIFACS, SALVADOR, BAHIA, Brasil; ${ }^{2}$ UEFS, FEIRA DE SANTANA, BAHIA, Brasil

Advances in Rheumatology 2018, 58(Suppl 1):P175

Background: The present study aims to establish a bone mass analysis through double emission bone densitometry (BMD) in patients with rheumatoid arthritis (RA) and spondylarteritis (SPS) using assisted immunobiological therapy. These data have relevance, since it allows to know the bone health of the patients with RA and SPS in use of this therapy.

Methods: This is a cross-sectional study, based on the collection of data from patients' medical records with RA and SPS, submitted to 
immunobiological therapy at a private clinic in Feira de Santana-BA in the year 2018. Through the study of scientific articles on the subject and the data collection, it is expected to better understand the relationship between bone mass and these patients. All patients with RA and SPS, who uses the immunobiological therapy from January 2018 to March 2018, were included in this study.

Results And Conclusion: Twenty patients with RA and SPS, equally distributed, were evaluated, being 17 women and 3 men. The study of $\mathrm{BMD}$ in the lumbar spine and femur (total femoral neck) demonstrated normal bone mass (NBM) in the majority of patients, with $60 \%, 80 \%$ and $85 \%$, respectively. The radio evaluation of $33 \%$ showed bone loss in $60 \%$ of the patients, with a predominance in the RA group. Concluding that in the present study, there was no change in BMD in the majority of patients in both groups; although they are potential risk factors for bone loss. Radio 33\% demonstrated a greater alteration of BMD in patients with RA, which may be justified by the high frequency of wrist involvement in this pathology. Other studies are needed to establish cause-effect change in bone mass in patients using immunobiological therapy.

\section{P176}

\section{EVALUATION OF COMORBIDITY IN PATIENTS WITH MULTIPLE} SCLEROSIS AND AUTOIMMUNE DISEASE

Francielle Santana Santos, Jussara Mathias Netto Khouri,

Greice Nascimento Pires

UNIVERSIDADE FEDERAL DO RIO DE JANEIRO, MACAE, RJ - RIO DE JANEIRO, Brasil

Advances in Rheumatology 2018, 58(Suppl 1):P176

Multiple sclerosis (MS), as well as classical autoimmune diseases, predominate in women and there is evidence that heredity may influence the occurrence of these diseases. They also have in common the facts that sometimes the diagnosis of these conditions is difficult, mainly due to the variety of symptoms, involving one or multiple organs, demanding clinical criteria and specific complementary tests. One of the severity parameters of an autoimmune disease is determined by impairment of the central nervous system. Neurological manifestations in autoimmune diseases require investigating the existence of comorbidities with multiple sclerosis. Patient AF, 38 years old, female, Caucasian, coming from Aracaju. Multiple sclerosis diagnosed at age 32 and psoriasis. The treatment offered to this patient was made with Dimethyl Fumarate, derived from fumaric acid. The immunomodulatory properties of dimethyl fumarate in MS were extrapolated from the fumaric acid results in psoriasis. Dimethyl fumarate (DMF), the methyl ester of fumaric acid, is a new agent that was recently (2013) approved by Food and Drug Administration (FDA) and European Medicine Agency (EMA) for the management of relapsing forms of multiple sclerosis. Being an orally available agent with a favourable safety profi le, DMF has become one of the most commonly prescribed disease-modifying agents in the USA and Europe. DMF induces apoptosis of T cells. It was greater for CD8+ vs CD4+, as well as for memory vs naive, and conventional vs regulatory T-cell subsets. In addition, DMF infl uenced dendritic cells by a reduction in their IL-12 and IL-23 synthesis. This attenuation of myeloid antigenpresenting cells in turn reduced the differentiation of CD4+ Th cells into proinfl ammatory Th1 and Th17 cells, and increased the expression of the Th2 cytokine IL-4. A potential neuroprotective role of DMF has been postulated and is currently thought to be mediated by its action on nuclear factor NRF-2 in astrocytes, oligodendrocytes, and neurons. The binding of NRF-2 to its target genes leads to a transcription of ROS (reactive oxygen species) protective genes. At the moment there is still no consensus in the literature regarding the ideal treatment for patients with psoriasis, so it is important to individualize the treatment based on the clinical picture. There is a need for studies/research associating these diseases.

\section{Consent for publication}

The authors declare that they have obtained informed written consent from the patient's tutors for publication

\section{P178}

EVALUATION OF FUNCTIONAL DISABILITY IN PATIENTS WITH SYSTEMIC SCLEROSIS

Lettícia Cristina Santos Cardozo Roque ${ }^{1}$, Andréa Tavares Dantas²,

Victória Pimentel Jatobá ${ }^{3}$, Rafaela Silva Guimarães Gonçalves ${ }^{2}$,

Angela Luzia Branco Pinto Duarte

${ }^{1}$ DEPARTAMENTO DE FISIOTERAPIA - UFPE, RECIFE, PE, Brasil; ${ }^{2}$ SERVICO

DE REUMATOLOGIA - HC/UFPE, RECIFE, PE, Brasil; ${ }^{3}$ UNIVERSIDADE

FEDERAL DE PERNAMBUCO, RECIFE, PE, Brasil

Advances in Rheumatology 2018, 58(Suppl 1):P178

Background: Systemic sclerosis (SSc) is a chronic and severe disease characterized by cutaneous and visceral involvement, presenting a high morbidity and mortality rate. The repercussions of the disease in the various systems are responsible for important functional impact and quality of life impairment. The objective of this study was to evaluate the functional disability in individuals with SSc and their association with clinical manifestations of the disease.

Methods: Descriptive and transversal study, with evaluation of 73 patients with SSC diagnosis. The data were obtained from the interview and review of the medical records. The disability was assessed using the Scleroderma Health Assessment Questionnaire (SHAQ), which contains questions about activities of daily life, as well as five additional domains assessing clinical manifestations of the disease through visual analog scale (VAS). Results were categorized into mild to moderate difficulty (score of 0 to $<1$ ), moderate to severe disability (score of 1 to $<2$ ) and severe to very severe disability (score of 2-3).

Results and Conclusions: Of the patients evaluated, $95.9 \%$ were female, at the average age of 46.0 ( \pm 13.2 ) years and a median time of diagnosis of 60.5 months. Most of the patients had a limited cutaneous form $(54.8 \%)$. The median of HAQ and SHAQ scores were 1.38 $(0.25-1.88)$ and $1.13(0.52-1.52)$ respectively, indicating moderate to severe disability. Regarding the clinical manifestations, the median values of VAS were: $5.0 \mathrm{~cm}$ for Raynaud's phenomenon, gastrointestinal complaints and digital ulcers, $3.0 \mathrm{~cm}$ for pulmonary symptoms and $6.0 \mathrm{~cm}$ for the general disease. About $43.8 \%$ of the patients were in the mild to moderate difficulty SHAQ category, $48.0 \%$ in the moderate to severe category and $8.2 \%$ in the severe to very severe category. Patients with the diffuse cutaneous form of the disease had a worse SHAQ score when compared to the limited form (1.38 and 0.91 , respectively, $p=0.02$ ). Patients with microstomia, arthralgia, myalgia, dysphagia, regurgitation, dyspepsia and dyspnea presented higher SHAQ scores compared to those without these clinical manifestations. In conclusion, patients with SSc have a significant impairment of functional capacity, especially those with diffuse cutaneous form, microstomia, musculoskeletal and gastrointestinal complaints. Measures to improve disability must be emphasized in SSc treatment protocols.

\section{P179}

EVALUATION OF HEALTH-RELATED QUALITY OF LIFE, SELF-ESTEEM, ANXIETY AND DEPRESSION IN WOMEN WITH SYSTEMIC LUPUS ERYTHEMATOSUS PRESENTING SCARS OF CUTANEOUS LUPUS

Rosa Weiss Telles ${ }^{1}$, Fernando Afrânio Palmeira de Oliveira ${ }^{2}$, Fabiana de Miranda Moura Santos ${ }^{2}$, Maria Veloso Rocha Mameluque ${ }^{2}$, Thiago Loredo e Silva², Maria Isabel Menezes Guedes², Maria Carolina Padovani Guerra $^{2}$, Vinicius Ornela Bicalho², Claudia Santoro Neiva ${ }^{3}$, Ana Flavia Madureira de Pádua Dias ${ }^{3}$, Rosa Weiss Telles ${ }^{2}$, Cristina Costa Duarte Lanna ${ }^{2}$

${ }^{1}$ FACULDADE DE MEDICINA DA UFMG, BELO HORIZONTE, MG, Brasil;

${ }^{2}$ FACULDADE DE MEDICINA - UFMG, BELO HORIZONTE, MG, Brasil; ${ }^{3}$ SANTA CASA DE MISERICÓRDIA, BELO HORIZONTE, MG, Brasil Advances in Rheumatology 2018, 58(Suppl 1):P179

Background: Few studies have analyzed health-related quality of life (HRQoL) in patients with systemic lupus erythematosus (SLE) and cutaneous manifestations in the Brazilian population. Although it is known that active skin disease is related to poor HRQoL, it is not well defined if the indexes are maintained in inactive patients. The aim of 
this study was to describe HRQoL, self-esteem, anxiety and depression in inactive women with SLE and cutaneous scars on the face. Methods: This is a cross-sectional, descriptive study with SLE women patients according to the ACR/1997 and/or SLICC/2012 criteria, included from September/2015 to March/2018. Patients were 18 years or older and had scars of cutaneous lupus on the face. Patients were excluded if they had moderate to severe systemic activity (SLEDAI $2 \mathrm{k}$-modified $>4$ ), did not understand the questionnaires or had started psychological and/or psychiatric treatment within four weeks prior to inclusion. The following validated Brazilian-Portuguese questionnaires were applied: SLE-Quality of Life (SLEQOL), with scores varying from 40 to 280 , and Dermatology Life Quality Index (DLQI), with scores varying from 0 to 30, of which higher values identify higher effect on HRQoL for both questionnaires; the Rosenberg Self-esteem Scale with scores varying from 10 to 40 , of which higher scores identify higher selfesteem; and Hospital Anxiety and Depression scale (HADs) with scores varying from 0 to 21 for each disorder (scores above seven identify patients with signs of anxiety and/or depression separately).

Results and Conclusions: The mean (SD) age of the 56 outpatient women was 46.3 (11.6) years and the median (IQR) of modified SLEDAl$2 \mathrm{k}$ was 2 (2). The SLEQOL scores ranged from 53 to 216 with a median (IQR) of 111 (68.5), the DLQI scores ranged from 0 to 28 with a median (IQR) of 7 (11). The Rosenberg scale scores ranged from 22 to 40 with a median (IQR) of 29 (4). The HADs scores were higher than seven for depression in $48.3 \%$ of the patients and for anxiety in $60.7 \%$, identifying cases of doubt and/or presence of signs of depression/anxiety. The study has shown in this group of SLE women presenting scars of cutaneous manifestations and low systemic disease activity an impairment in the HRQoL, in the self-esteem and in the mood/anxiety. This preliminary analysis indicates the need of strategies that could interfere positively in lupus patients' quality of life.

\section{P180}

EVALUATION OF SENSITIVITY AND SPECIFICITY OF BECK DEPRESSION INVENTORY (BDI-II) TO DIAGNOSIS DEPRESSION IN HEALTHY INDIVIDUALS AND PATIENTS WITH RHEUMATOID ARTHRITIS

Rafael da Rocha Caminha², Paulo Gabriel de Oliveira Cerquinho², Flávia Jatobá de Barros'², Bruna Ferraz Gutierrez Piola², Hugo Deleon de Lima ${ }^{1}$ Pedro Arturo Bismara Carneiro Santos ${ }^{1}$, Amanda Fernandes Vieira Mendes da Silva², Leia Teixeira de Andrade', Dennys Lapenda Fagundes $^{1}$, Laurindo Ferreira da Rocha Junior ${ }^{1}$

'INSTITUTO DE MEDICINA INTEGRAL PROFESSOR FERNANDO FIGUEIRA IMIP, RECIFE, PERNAMBUCO, Brasil; ${ }^{2}$ FACULDADE PERNAMBUCANA DE

SAÚDE - FPS, RECIFE, PERNAMBUCO, Brasil

Advances in Rheumatology 2018, 58(Suppl 1):P180

Background: The Beck Depression Inventory (BDI-II) is an instrument used worldwide to measure the severity of depressive episodes in clinical and non-clinical populations. The aim of this study is to evaluate the ability of BDI-II to diagnosis depression and calculate sensitivity and specificity of this instrument in identifying depression among healthy individuals and patients with Rheumatoid Arthritis (RA).

Methods: BDI-II was applied in two populations: RA patients and healthy individuals. For the calculation of associations of BDI-II values between the groups the Mann-Whitney test was used (software Graphpad prism 6.0). Optimum cut-off values were calculated to optimize sensitivity and specificity (i.e. the Young Index). Receiveroperating characteristic (ROC) were plotted (sensitivity against 100specificity) and the areas under the ROC curves were calculated to assess the performance of BDI-II to distinguish individuals with depression from individuals with no depression. The gold standard method used for diagnosis of depression was the diagnosis criteria of the fifth edition of the Diagnostic and Statistical Manual of Mental Disorders (DSM-5).

Results And Conclusions: Ninety-eight patients with RA were included, of whom $40(40.82 \%)$ were considered with depression according to DSM-5. Out Of the 31 healthy subjects who participated in the study, 2 (6.45\%) were diagnosed with depression. We Stratified the participants in three groups: 1) RA patients 2) healthy subjects 3 ) RA patients and healthy subjects. The optimal cut-off value for BDI-II to distinguish subjects with depression was $>13$ for all the three groups analyzed. With this optimal cut-off the yielded sensitivity of BDI-II to diagnosis depression was $100 \%(95 \% \mathrm{Cl} 92.6-100 \%)$ and specificity was $100 \%$ (95\% Cl $92.9-100 \%)$ in RA patients. For healthy controls the sensitivity was $100 \%(95 \% \mathrm{Cl} 39.8-100 \%)$ and specificity was $100 \%(95 \% \mathrm{Cl} 87.2-100 \%)$. For all the participants the yielded sensitivity was $100 \%(95 \% \mathrm{Cl} 93.2-100 \%)$ and specificity was $100 \%$ (95\% Cl 95.3-100\%). The ability of BDI-II to distinguish depression from all subjects in the study demonstrated an AUC of ROC analysis of $1,000(95 \% \mathrm{Cl} 0.972-1000)$ (Fig. 1). Numerical values of BDI-II may have the potential to be used as diagnostic markers in depression. Studies with a greater number of RA patients (with and without depression) and healthy controls are needed to better understand the role of BDI-II as a diagnostic tool for this purpose.

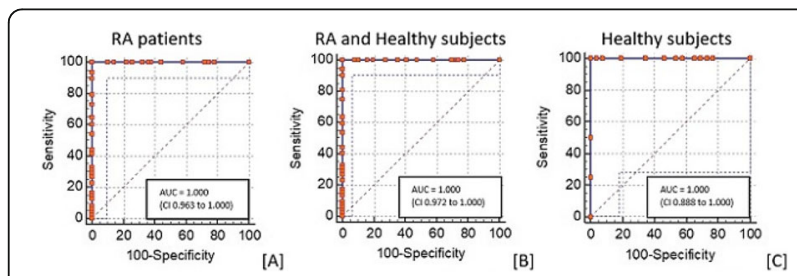

Fig. 1 (abstract P180). Receiver Operating Characteristic (ROC) curve for BDI-II to diagnosis depression in RA (Rheumatoid Arthritis) patients and healthy controls. Patients with RA [A]; patients with RA and Healthy subjects [B]; Healthy subjects [C]

\section{P181}

EVALUATION OF THE CLINICAL AND DEMOGRAPHIC PROFILE OF PATIENTS WITH TERIPARATIDE THERAPY IN A TERTIARY HOSPITAL IN SÃO PAULO

Bernardo Patricio Sequeira Dultra, Denise Moraes Horiy, Andre Ozela Augusto, Dayrana Alves Lucena, Laís Farrapo de Barros Leite, Marcelo Nora Resende, Elaine de Azevedo, Rina Dalva Neubarth Giorgi HOSPITAL SERVIDOR PÚBLICO ESTADUAL - SP, SÃO PAULO, SÃO PAULO, Brasil

Advances in Rheumatology 2018, 58(Suppl 1):P181

Introduction: Teriparatide ((PTH[1-34]rh), fraction 1-34 of human recombinant parathyroid hormone, directly stimulates osteoblastic activity, restoring bone microarchitecture and promoting bone mass gain in the lumbar spine and femoral neck, with a consequent reduction in the risk of vertebral fractures in patients with osteoporosis, both men and women, whether or not glucocorticoid users. Objectives: Describe the demographic and clinical profile of patients with osteoporosis in current or previous treatment with PTH[1-34]rh in a tertiary hospital in the State of São Paulo.

Methods: An analytical-descriptive study, conducted in 2018, which included 42 patients with Osteoporosis in current or previous use of PTH[134]rh. Results: Among those included, $95.2 \%(\mathrm{~N}=40)$ were women, with a mean age of $70.2 \pm 10.8$ years. The indication of the use of PTH[1-34]rh due to therapeutic failure with antireabsorbents (oral bisphosphonate, parenteral or Odanacatib) occurred in $59.5 \%(\mathrm{~N}=25)$ of the patients; In $22.2 \%(\mathrm{~N}=10)$ it was indicated because of Corticosteroid-induced osteoporosis; in $12.8 \%(\mathrm{~N}=5)$ the exchange was performed after complication of the prolonged use of Bisphosphonate (Osteonecrosis of Jaw) and in another $4.8 \%(\mathrm{~N}=2)$ was already used as first line therapy. Regarding the identified fractures, $90.5 \%(\mathrm{~N}=38)$ of the patients had a history of at least one fragility fracture. The most prevalent fractures were vertebral $35.7 \%$ $(\mathrm{N}=15)$ and femoral neck $31 \%(\mathrm{~N}=13)$. About $1 / 3$ of the cases $(28.6 \%$, $\mathrm{N}=12$ ) had a history of more than one fracture. The majority of cases, $76.2 \%(\mathrm{~N}=32)$ are in current use of PTH[1-34]rh, while $23.8 \%(\mathrm{~N}=10)$ already receive sequential post anabolic treatment: $14.3 \%(\mathrm{~N}=6)$ with Denosumab and $9.5 \%(\mathrm{~N}=4)$ with Zoledronic Acid. No cases of osteosarcoma were identified. 
Conclusion: The profile of patients for whom anabolic therapy was indicated is compatible with the recommendations in the literature. It was observed that the most severe cases and those at high risk for fracture (patients already fractured, at high risk of falls or with very low bone mineral density), as well as who met criteria for therapeutic failure and those with adverse events to previous therapy, are the cases with most benefit from this type of treatment

\section{P183}

EVALUATION OF THE SERUM 25-HYDROXYVITAMIN D LEVEL IN DIFFERENT AGE GROUPS WITH EMPHASIS ON INFANTS AND

\section{ELDERLY OVER 60 YEARS}

Fernanda Pulcheri Ramos, Matheus Xavier Guimarães, Andrey Tonetto Barbosa, Bernardo Patrício Sequeira Dultra, Kioko Takei, Daniela Crema, Rina Dalva Neubarth Giorgi, Elaine de Azevedo

HOSPITAL DO SERVIDOR PÚBLICO ESTADUAL - SP, SÃO PAULO, SP, Brasil Advances in Rheumatology 2018, 58(Suppl 1):P183

Background: Vitamin D plays an important role in bone formation and reabsortion. The serum concentration of 25-hydroxyvitamin D (25OHD) is used to monitor. Among the risk groups for hypovitaminosis $D$, the following stand out: elderly (over 60 years), infants, pregnant women, individuals with osteoporosis and other osteometabolic diseases, chronic kidney disease, and disabsorptive syndrome. The recommended level in these groups should be between 30 and $60 \mathrm{ng} / \mathrm{dL}$, while for healthy people under 60 years levels above $20 \mathrm{ng} /$ $\mathrm{dL}$ are recommended. Adequate monitoring and supplementation of $25 \mathrm{OHD}$ should be performed in risk groups, since its deficiency is related to certain conditions such as rickets, osteomalacia and secondary hyperparathyroidism, thus increasing the risk of fractures. The objective of this study was to evaluate vitamin D levels in patients with different ages attended in a public tertiary hospital in São Paulo, focusing on two age groups at greatest risk for disability: infants $(<2$ years) and the elderly over 60 years.

Methods: This is a cross-sectional and analytical study where the results of 32.535 patients residing in the state of São Paulo, Brazil, who underwent 25OHD dosing between January and December 2016, regardless of socio-cultural habits, supplementation, solar exposure or diseases. Laboratory tests were performed on the Architect with Abbot $25-\mathrm{OH}$-VitaminaD kit $5 \mathrm{P} 02$ by the competitive immunoassay technique. The reference values for normality were based on the official Positioning of the Brazilian Society of Clinical Pathology/Laboratory Medicine and the Brazilian Society of Endocrinology and Metabology published in December 2017.

Results: The overall calculated mean of all patients selected in the study, regardless of age, was $27.6 \pm 9.0 \mathrm{ng} / \mathrm{dL}$. In infants $(\mathrm{N}=92)$, the mean was $34.1 \pm 12.7 \mathrm{ng} / \mathrm{dL}$, while in subjects older than 60 years $(\mathrm{N}=16,667)$ it was $28.5 \pm 9.5 \mathrm{ng} / \mathrm{dL}$. In this group over 60 years, 38.7\% had adequate levels above $30 \mathrm{ng} / \mathrm{dL}$, most of them with levels between 21-30ng / dL (40.9\%). Most infants had vitamin D levels above $30 \mathrm{ng} / \mathrm{dL}$ (57.6\%).

Conclusions: The data presented in the present study can conclude that in this population, unlike that described in the literature, infants had adequate levels of vitamin D, while the elderly, although not fully complying with the recommendation of the new guideline, are close to ideal, perhaps reflecting greater disclosure in recent years of the role of vitamin $\mathrm{D}$ among health professionals and the general public.

Table 1 (abstract P183). See text for description

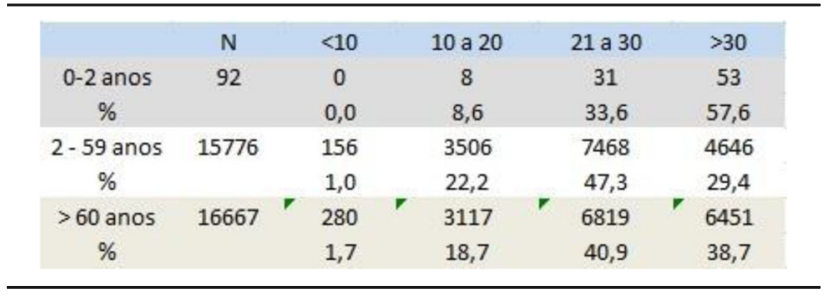

P185

EXERCISE TRAINING ATTENUATES INSULIN RESISTANCE IN PATIENTS WITH SYSTEMIC AUTOIMMUNE MYOPATHIES

Diego Sales De Oliveira, Jean Marcos de Souza, Rafael Giovani Misse, Isabela Bruna Pires Borges, Alexandre Moura dos Santos,

Marilda Guimarães Silva, Samuel Katsuyuki Shinjo

UNIVERSIDADE DE SÃO PAULO, CAMPO LIMPO PAULISTA, SP, Brasil

Advances in Rheumatology 2018, 58(Suppl 1):P185

Background. Recently studies have demonstrated increase metabolic syndrome and insulin resistance in patients with systemic autoimmune myopathies (SAM). Exercise training has been reported as a coadjuvant in improve metabolic parameters in several autoimmune diseases, but not specifically in SAM. Therefore, the aim of the present study was to evaluate the effects of exercise training in insulin resistance in SAM.

Materials and Methods. This prospective cohort study engaged 7 patients ( 6 female and 1 male gender) with definite SAM ( 4 dermatomyositis, 2 antisynthetase syndrome and 1 polymyositis) to a 12 weeks, twice-a-week, exercise training program. All sessions started with strength training that was composed by 6 exercises using machines or assisting devices (horizontal leg press, horizontal bench press, let pull down, narrow-grip seated rows, weighted knee extensions and seated hamstring curl). After strength training, the patients performed aerobic exercises on a treadmill in a 30-50 min of moderate-intensity walk/running period. Baseline glucose, insulin and c-peptide were evaluated through blood sample. To evaluate insulin resistance, the oral glucose tolerance test (OGTT) was used before and after exercise training. The disease status was evaluated by International Myositis Assessment and Clinical Studies Group (IMACS) core set measures.

Results. Mean age of the patients was 45.6 years, with mean disease duration of 7.3 years. All patients have a stable disease, according to IMACS core set measures. At least one immunosuppressive drugs was used by all patients and glucocorticoids drugs in low dosage $(<5 \mathrm{mg})$ was used by just one patient. After exercise training program, there was no disease relapsing or auto-related events. In addition, there was an important increase in aerobic capacity, muscle strength and function in the patients $(P<0.05)$. The area under curve (AUC) for glucose and baseline glucose has no change $(P>0.05)$, while baseline insulin and baseline c-peptide has a decrease (insulin: $18.7 \pm 7.6$ vs. $13.5 \pm 4.8 \mathrm{uU} / \mathrm{mL}, \mathrm{P}=0.104$, effect size: 0.82 ; C-peptide: $4.0 \pm 1.0$ vs. $3.0 \pm 0.4 \mathrm{ng} / \mathrm{mL}, \mathrm{P}=0.043$, effect size: 1.31 ) after exercise training program. AUC for insulin and c-peptide has an important decrease after exercise training $(P=0.025$ and $P=0.020$, respectively) in the OGTT.

Conclusions. Exercise training was effective in attenuates insulin resistance and improve b-cell function in patients with SAM. This findings contributes to attenuate metabolic impairment and cardiovascular risks in these diseases.

Support by FAPESP \#2016/19771-5 (DSO), \#2016/23574-0 (RGM), \#2016/20371-1 (IBPB), \#2017/13109-1 (SKS), CAPES (MGS)

\section{P186 \\ EXPERIENCE OF A PATIENT WITH ANTIPHOSPHOLIPID SYNDROME (APS) \\ Karolyne Nogueira De Medeiros, Roberta Laís de Souza Bezerra, Ádala Nayana de Sousa Mata, Rafael BArros Gomes da Câmara UNIVERSIDADE FEDERAL DO RIO GRANDE DO NORTE, CAICÓ, RIO GRANDE DO NORTE, Brasil \\ Advances in Rheumatology 2018, 58(Suppl 1):P186}

Background: APS is a chronic disease characterized by thrombotic events, which can cause recurrent abortions. Being a chronic disease, there may be some impact on the lives of its patients. Thus, this study sought to evaluate the experience of a patient with APS, regarding their experience with the disease and its biological/psychological/social repercussions.

Methods: The research was developed through an interview with a SAF carrier. The interview method chosen was the McGill Illness 
Narrative Interview, and was done after approval of the Ethics Committee and signing of the TCLE.

Results And Conclusions: The difficulty of the diagnostic/therapeutic itinerary for rare diseases generates feelings of anguish, fear and doubt: "When I was hospitalized, I knew very little about my problem ... I felt worried ... I was afraid that was something recurring ... The doctor even told me not to ask too many questions because even he did not know how to answer everything. Many do not even know which remedies I can take." In addition to the better knowledge about APS, it is also important to take into account the patient's longings: "He wrote the APS acronym on a paper, and said that I had it. He said that I would do the treatment for the rest of my life, which would have a normal life, but with restrictions ... just told me that. My restrictions! I believe the treatment works, but I think the doctor should be something more present in my life." According to data, chronic diseases have an impact on both subjectivity and their way of life. These repercussions can be alleviated in the face of social support, in the sense of knowing other people with the same health problem, and help in a greater coping capacity: "I would like to have more contact with people who have APS as a group, to share things." It is observed as resources of support/confrontation cited by the patient, references to the family and spiritual support. The concept of health/illness is produced from the subjects' experiences in knowledge and ways of thinking, which incorporate aspects related to pain, suffering, life and death. Thus, interpersonal relationships, such as the support of friends, family, health team, and faith, are important coping resources.

\section{Consent for publication}

The authors declare that they have obtained informed written consent from the patient's tutors for publication

\section{P190}

\section{FAMILY HISTORY OF AUTOIMMUNE AND NON-AUTOIMMUNE} DISEASES FROM A BRAZILIAN COHORT OF INITIAL RHEUMATOID ARTHRITS

Ana Paula Monteiro Gomides', Ana Paula Faria de Carvalho², Licia Maria Henrique da Mota², Luciana Feitosa Muniz'2, Talita Yokoy de Souza² Andressa Junqueira Osório ${ }^{2}$, Luciana Teófilo Lourençoni ${ }^{2}$, Isabela de Sousa Russo' ${ }^{2}$, Tassiane Raquel Cunha Martins de Moraes ${ }^{2}$ ${ }^{1}$ UNB/UNICEUB, BRASILIA, DF, Brasil; ${ }^{2} U N B$, BRASÍLIA, DF, Brasil Advances in Rheumatology 2018, 58(Suppl 1):P190

lintroduction: Rheumatoid arthritis (RA) is a chronic and systemic disease in which genetic and environmental factors are involved in its etiology. In the literature we find epidemiological and genetic studies that suggest the familial aggregation of autoimmune diseases. In addition, the comorbidities commonly found in RA, such as arterial hypertension for example, may be common in first-degree relatives. The aim of this study was to evaluate family history in first-degree relatives of patients with RA.

Methods: Patients from an initial RA cohort were analyzed in a Brazilian university hospital. For the present study, a crosssectional study was performed and the patients' charts were analyzed from December 2016 to December 2017. Participants underwent routine clinical evaluation. The work was approved by the local ethics committee.

Results And Conclusions: We included 107 RA patients, 101 women and 5 men. The mean age was 62 years and $43 \%$ of the white race. The median time to illness was 12 years.

When we analyzed the first-degree relatives of the patients we verified that the non-autoimmune disease most common was hypertension $(n=56,52,33 \%)$, that was more prevalent in the mothers of patients with RA ( $n=31,28,97 \%)$. The second most found non-autoimmune disease was diabetes mellitus $(n=22$,
$20,56 \%)$, was also more common in the mothers of patients with RA $(n=13,12,14 \%)$.

A personal family history of rheumatoid arthritis $(n=17,15,88 \%)$ was the most prevalent data in this cohort for an autoimmune disease. It was more present in sisters of patients with RA.

The full evaluation can be seen in Table 1

This result, reasures the relevance of inquiring about RA family history, specially at the suspected diagnosis of RA. Environmental factors might contribute toward increased familial aggregation. The results for non-autoimmune diseases prevalences in family history, sugests the need for cardiovascular risk acessment in the RA patients.

\section{P191}

FEVER OF OBSCURE ORIGIN LEADING BEHÇET'S DISEASE WITH SEVERE VASCULAR MANIFESTATIONS

Vívian Garcia Moreira, Rayla Felizardo Oliveira Marques, Fernando de Almeida Marques, Nayara Ramos Antelo, Caroline Kauscher Santa Rita, Fernando Henrique Souza, Flavio Ribeiro Pereira, Isabella Matias Ribeiro SEMUSA, MACAÉ, RJ, Brasil

Advances in Rheumatology 2018, 58(Suppl 1):P191

Background: Behçet's disease (DB) is a multisystemic condition characterized by vasculitic lesions, affecting venous and arterial vessels of varying caliber. It may be associated with genetic factors with the presence of HLA-B51. It is manifested by recurrent oral and genital ulcers, cutaneous lesions, arthritis, uveitis, thrombophlebitis, and gastrointestinal and central nervous system implication. Vascular lesions may be associated with high morbidity and mortality, especially when associated with the existence of thrombi and aneurysms.

Case Report: MP, 55 years old, female, presenting weight loss, left knee arthritis, dislocating lumbosacral pain in the right knee, painful oral ulcer, prolonged fever, cicatricial lesions on the legs suggestive of folliculitis, in addition to normocytic and normochromic anemia and increased HSV. During the investigation, we discarded differential diagnoses such as chronic infections, neoplasms and other chronic inflammatory diseases, such as diffuse collagen diseases and inflammatory bowel disease. Patient evolved deep venous thrombosis in right lower limb and single genital ulcer. She was submitted to High Digestive Endoscopy which revealed multiple ulcers in gastric antrum. The paternal test was positive. Thus, Behçet's disease was the main diagnostic hypothesis. Anticoagulation with rivaroxaban was initiated and immunomodulation with steroid and colchicine. She then evolved bilateral DVT associated with a $7 \mathrm{~cm}$ right femoral artery saccular aneurysm (Fig. 1), and then was submitted to immunosuppression with cyclophosphamide pulses. EcoDoppler and Cineangiocoronariography were performed and discarded evidence of aneurysms or thrombi in pulmonary artery and/or coronary arteries. Then she was submitted to surgical procedure, femoral bypass femoral vein with reversed major saphenous vein technique, evidencing the presence of ruptured aneurysm, with a satisfactory evolution on the postoperative period.

Conclusion: DB is a rare condition, with chronic evolution, acute outbreaks, an essentially clinical diagnosis, and no predilection for gender. It is important to point out that there is no evidence of the benefit of using anticoagulants for the management of venous or arterial thrombotic events in patients with this disease, since thrombi adhered to the vessel hardly result in embolic events. Pulmonary embolism is rare despite the high prevalence of DVT. Another reason for not recommending such drugs would be the high risk of fatal bleeding, given the high rate of pulmonary artery aneurysm; besides not reducing the recurrence of new thrombotic events. Thus, the best strategy for disease control is immunosuppression.

\section{Consent for publication}

The authors declare that they have obtained informed written consent from the patient's tutors for publication 


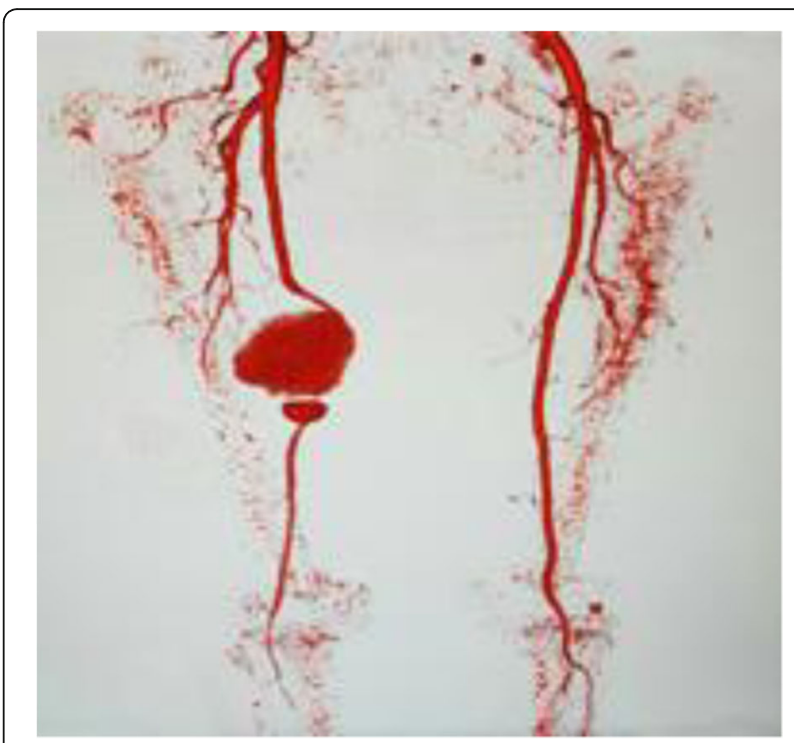

Fig. 1 (abstract P191). Aneurisma de Artéria femoral

P192

FIRST CASE OF BAGGIO-YOSHINARI SYNDROME IN THE STATE OF MATO GROSSO

Maira Sant Anna Genaro, Matheus Yung Perin

UNIVERSIDADE DE CUIABÁ, CUIABÁ, MT, Brasil

Advances in Rheumatology 2018, 58(Suppl 1):P192

Background: Baggio-Yoshinari syndrome, also called Lyme-like disease (DL-S), is an infectious disease caused by spirochetes of the Borrelia burgdorferi complex and transmitted by ticks of the genera Amblyomma and Rhipicephalus. It is considered an emerging zoonosis in Brazil that shares clinical similarities with Lyme Disease (DL) found in North America and Eurasia. In Brazil, the first case of DLsimile was described in the 1990s and to date it has not been possible to isolate the spirochete from atypical morphology, even with the use of enriched culture media.

Case Report: A 29-year-old woman, previously living in Cuiabá, Mato Grosso, Brazil, with a history of pruritic cutaneous lesion of centrifugal growth on the left thigh, fatigue, and non cicatricial alopecia for more than fifteen days. At the afebrile physical examination, macular lesion with erythematous borders and light center, on the lateral side of the left thigh. The complementary tests showed antibodies against Borrelia burgdorfer IgM 3,1 and IgG 95, positive according to the reference. The patient presented complete response to systemic therapy after 12 weeks of the treatment, without reporting constitutional symptoms in this period.

Conclusion: Characterization according to LIM-17 HCMUSP criteria are higher criteria: clinical manifestations (erythema migrans or systemic symptoms), epidemiological evidence (tick bite, endemic region, contact with animals or rural area) and positive serology; the minor criteria are: recurrent episodes, fatigue, arthralgia, myalgia, paresthesia and cognitive disorders, and identification of the spirochete in the dark field. For diagnosis it is considered 3 major criteria or 2 major and 2 minor.

The patient presented, according to LIM-17 protocol, three major criteria: reactive serology, migratory erythema and epidemiological data.

Thus, the first case of Boggio-Yoshinari syndrome occurred in the State of Mato Grosso and the sequence of clinical reasoning necessary for the diagnostic elucidation was presented.
Consent for publication

The authors declare that they have obtained informed written consent from the patient's tutors for publication

\section{P194}

FOLATE SUPPLEMENTATION ASSOCIATED WITH METHOTREXATE IN JUVENILE IDIOPATHIC ARTHRITIS: SYSTEMATIC REVIEW

Debora P Souza, Ana Carolina Londe, Antonio de Azevedo Barros Filho, Simone Appenzeller

UNICAMP, SP, SP, Brasil

Advances in Rheumatology 2018, 58(Suppl 1):P194

Background: Juvenile Idiopathic Arthritis (JIA) is the most common rheumatic disease in childhood, with a prevalence ranging from 7 to $150 / 100,000$ children. It is defined by onset of disease before age 16 and persistent arthritis for more than six weeks after exclusion of other causes. The main characteristic of the disease is chronic arthritis, and may also present systemic involvement and lead to permanent functional disability outcomes. Patients with JIA commonly use Anti-Rheumatic Disease Modifying Drugs (DMARDs), capable of attenuating clinical evolution and leading to remission of the disease. Methotrexate is a DMARD, analogue of folic acid. Folate supplementation is indicated to decrease the gastrointestinal and hepatotoxic adverse effects of methotrexate, but its efficacy in JIA is not well defined. The aim of this study was to perform a systematic literature review whether supplementation of folic or folinic acid may decrease the efficacy of MTX and whether it is able to reduce or ameliorate the adverse effects caused by MTX in JIA.

Methods: We conducted the systematic review in the literature, through the sensitive search strategy, in the Pubmed, BVS, Cochrane and Embase databases, from September to October 2017, searching for published studies in the last 22 years, including MESH terms: folic acid; arthritis, juvenile; methotrexate and their synonyms.

Results and conclusions: We identified 108 studies, after removal of the duplicates, 90 were sorted through the abstract, 87 were excluded because they did not meet the objective of the study, and 3 were considered eligible. The Hunt et al (1997) was a randomized double blind study conducted in a short period of 13 weeks including only 18 patients, being considered a moderate quality study due to unknown selection and allocation bias; the study of Ravelli et al (1999), uncontrolled retrospective included 43 patients and was not controlled, featuring a study with high risk of bias; and the study of Modesto et al (1996), case report with 3 patients, considered a low level of scientific evidence. It was concluded that despite the widespread use of folate supplementation to prevent adverse effects related to the use of MTX in JIA, there is a lack of evidence to support this practice, so it is necessary to conduct a well-designed clinical trial to support a regimen of supplementation.

\section{P195}

FOLLOW UP OF A PATIENT WITH ANTIPHOSPHOLIPID SYNDROME

\section{(APS): A CASE REPORT}

Roberta Lais De Souza Bezerra, Karolyne Nogueira de Medeiros

UNIVERSIDADE FEDERAL DO RIO GRANDE DO NORTE, CAICO, RN, Brasil Advances in Rheumatology 2018, 58(Suppl 1):P195

Background: APS corresponds to the association of thrombotic events with repetitive abortions and/or thrombocytopenia, in the presence of specific antibodies. The pathogenesis of APS has not been fully elucidated yet, however, it is known that autoantibodies when interacting with negatively charged phospholipids from membranes generate immune complexes that are deposited in vessels and predispose to formation of clots, generating a prothrombotic condition. The diagnosis is given through the association of clinical and laboratory criteria, but there are cases that not have clinical or laboratory evidence, or associated with other autoimmune diseases. Even today, there is a lack of information about APS, both in the literature and in clinical practice, causing their diagnosis to be postponed and favoring the development of complications in the 
patient. In this way, the search for a deeper understanding of the disease contributes to its early diagnosis and better management of the patients. Thus, this work sought to make a case report of a patient with APS, highlighting the main events from the onset of symptoms to the implementation of appropriate treatment.

Case Report: R.F.S, 35-year-old female, from Currais Novos-RN, started with epigastric pain, 10/10 intensity associated with dyspnea, with irradiation to the cervical region, investigated with endoscopy. It was evidenced gastritis, which had its treatment initiated. Subsequently, with the maintenance and progression of the condition, an abdominal ultrasonography was performed which showed an echogenic image in the splenic vein, suggestive of thrombus, leading to the patient's hospitalization for treatment and better investigation by computed tomography (CT). CT scan (Fig. 1) revealed tomographic signs of thrombosis of the splenic vein and the intrahepatic portion of the portal vein, as well as of its main and peripheral branches. In addition, serology was positive for IgM-ACL on two occasions, confirming the diagnosis of APS, and treatment with warfarin, food care and indication of physical exercise.

Conclusion: Although the incidence of APS has grown since its description, its diagnosis is still difficult to achieve. Thus, the more detailed knowledge about its clinical manifestations and pathogenesis end up favoring the diagnosis, treatment and quality of life of the affected patients.

\section{Consent for publication}

The authors declare that they have obtained informed written consent from the patient's tutors for publication

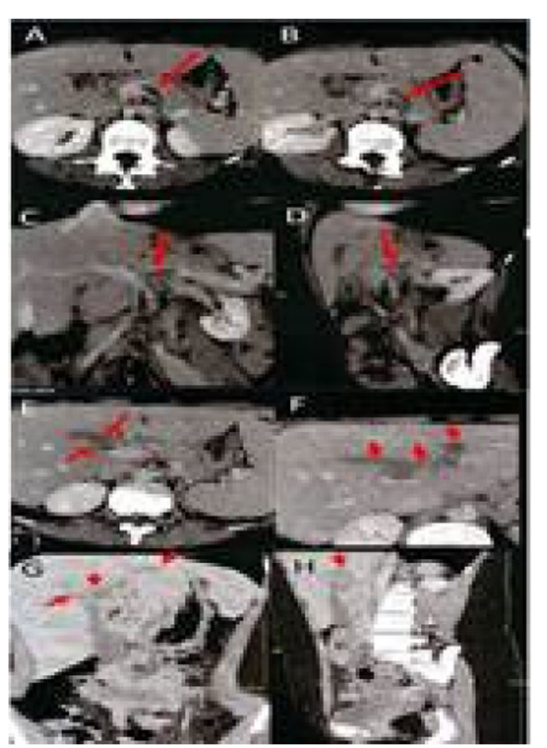

Fig. 1 (abstract P195). Computed tomography images of total abdomen with contrast application showing thrombi in the splenic vein ( $A$ and $B$ - axial sections, $C$ - coronal section and D - sagittal section) and in the intrahepatic portion of the portal vein (E and $F-a x i)$

\section{P196}

GIANT CELL ARTERITIS ASSOCIATED WITH MYOPERICARDITIS AS A PARANEOPLASIC MANIFESTATION OF COLON

\section{ADENOCARCINOMA}

Jeane Oliveira Da Silva, Igor da Fonseca Rangel, Lionete Gall Acosta Filha, Marília Daniela Silveira da Silva, Beatriz Gonçalves Batista Lamounier, Belisia Vasconcelos de Barros, Maria Isabel Dutra Souto, Laura Hoffmann de Segadas Soares, Afonso Celso de Oliveira Senos UNIVERSIDADE FEDERAL DO RIO DE JANEIRO, RIO DE JANEIRO, RJ, Brasil Advances in Rheumatology 2018, 58(Suppl 1):P196
Backgroud: Giant cell arteritis (GCA) is a chronic inflammation vasculitis of large and medium arteries wall, mainly affecting extracranial branches of the carotid artery. It is the most common vasculitis in people over 50 years, with a peak incidence in the 70-80 year age group. A few case reports have described rare myopericarditis manifestations in temporal arteritis disease. In addiction, rheumatic diseases can be associated with malignant diseases characterizing a paraneoplastic syndrome and concurrent malignancy in GCA is observed in up to $7,4 \%$ of cases. The objective of this case report is to describe an illustrative rare case about an elderly man with temporal arteritis evolving with myopericarditis and associated with colon tubular adenocarcinoma.

Case Report: A 79-year-old black man with multiple comorbidities presented with anemia, hematochezia and bilateral temporal headache was admitted for investigation of the cause of anemia. Bilateral temporal headache in the last 6 months, palpable left temporal artery, high erythrocyte sedimentation rate a led to clinical suspicion of temporal arteritis, following the criteria classification of GCA by the American College of Rheumatology. As a result, prednisone (40mg/ day) and aspirin (100mg/day) were initiated. Temporal artery biopsy confirmed the diagnosis of giant cell arteritis. Colonoscopy and upper digestive endoscopy were performed and showed gastric, transverse and ascending colon polyps, whose biopses confirmed the diagnosis of colon tubular adenocarcinoma. The patient was discharged with a prescription for prednisone. He was subsequently admitted with moderate pericardial effusion and a severe ventricular dysfunction of recent onset. Coronary angiography showed normal coronary arteries. Myopericarditis associated to giant cell arteritis was suspected. Due the severity of the case, a methylprednisolone $(250 \mathrm{mg} /$ day) pulse therapy was initiated and maintained for 3 days, with improvement of the ventricular dysfunction (ejection fraction: $33 \%$ to $43 \%$ ). The patient's history and physical examination led to suspicion of GCA as a paraneoplastic syndrome.

Conclusion: This case report contributes to emphasize the few number of publications which describe the association of GCA and myopericarditis in the elderly. Furthermore, this is possibly one of the first cases reporting a relationship between colon adenocarcinoma and myopericarditis-associated GCA. Therefore, physicians should be aware of the rarer complications of GCA and temporal arteritis symptoms as one of the first manifestations of malignant diseases.

Consent for publication

The authors declare that they have obtained informed written consent from the patient's tutors for publication

P199

GOOD EFFICACY OF ANTITUMOUR NECROSIS FACTOR ALPHA

TREATMENT IN RHUPUS: ONE CASE REPORT

Leticia Resende Brandao, Elizandra Tomazela Laurenti Polito

HOSPITAL ESTADUAL DORIO SILVA, VITÓRIA, ES, Brasil

Advances in Rheumatology 2018, 58(Suppl 1):P199

Background: The efficacy of antitumour necrosis factor alpha (antiTNF-a) treatment is well recognised in rheumatoid arthritis (RA) but remains controversial in systemic lupus erythematosus (SLE). Therefore, the role of anti-TNF-a treatment in 'Rhupus', a disease sharing features of RA and SLE, is still debated.

Case Report: J.L.C, male, 67 years old, ex smoker. In November 2016, presented with small and large polyarthritis joints, including hands (MCFs, IFPs), bilaterally and symmetrically, with intense morning stiffness. He was walking with difficulty due to arthritis on the knees. It had areas of hyperemia in the trunk and proximal thigh (itchy rashes). Denied infectious complaints or fever.

Laboratory tests: FAN 1:320 Nuclear Homogeneous, reagent metaphase plate, rheumatoid factor 32, Anti-CCP $>340$, VHS 83 and negative PCR, hemoglobin 14, leukocytes 4810, lymphocytes 1029, platelets 149000 . Serologies for Hepatitis B, C and HIV negative.

Chest computed tomography: fibrotic-like cortical foci, panlobular emphysema.

Initiated hydroxychloroquine and methotrexate, but presented gastric intolerance. Replaced by leflunomide, but progressed with 
worsening of gastric intolerance, alopecia, leucopenia and lymphopenia. (hemoglobin 10.4, leukocytes 2960, lymphocytes 592, platelets 168000 , VHS 98, PCR 9, rheumatoid factor positive, Anti-Sm positive, Anti-La/SSB 13, Anti-dsDNA negative and Anti-Ro/SSA negative).

He maintained with polyarthritis, and began to present Raynaud and calcinosis in hands and periungual vasculitis.

Diagnosis hytothesis: RHUPUS.

We tried Methotrexate SC and corticotherapia with $60 \mathrm{mg} / \mathrm{d}$ of prednisone, in addition to Ca and vit D. In October 2017 he was hospitalized for septic arthritis of the right hand, with improvement after antibiotic therapy (oxacillin and Rocefin intrahospital and high with ciprofloxacim and clindamycin). In December 2017 started Infliximabe and in January with an important improvement of polyarthritis, and corticoid dose reduction scheme. In February 2017 without arthritis, maintaining Infliximab, methotrexate subcutaneous, and corticoid $10 \mathrm{mg} /$ day, in decreasing plans.

Conclusion: Anti-TNF-a treatments could be an alternative strategy to rituximab in rhupus cases in which arthritis is the most prominent component. Further long-term evaluation and comparative studies are still needed to confirm the favourable benefit/risk ratio of antiTNF-a therapy in rhupus.

\section{Consent for publication}

The authors declare that they have obtained informed written consent from the patient's tutors for publication

\section{P202}

GRANULOMATOSIS WITH POLYGIENE (GPA) - OTOLOGICAL, TRACHAL AND RENAL ACHIEVEMENT: CASE REPORT

Ruy Sampaio de Siqueira Neto, Natália Jardim Martins da Silva Brasil, Leila Patrícia Fonseca Andrade Oliveira, Vitor Silva Souza, Leonardo Ribeiro Sampaio

HOSPITAL UNIVERSITÁRIO WALTER CANTÍDIO, FORTALEZA, CE, Brasil Advances in Rheumatology 2018, 58(Suppl 1):P202

Introduction: Granulomatosis with polyangiitis (GPA), Wegener's granulomatosis, is a rare and idiopathic vasculitis associated with antineutrophil cytoplasmic antibodies (ANCAs), characterized by the involvement of small and medium-sized bood vessels. The disease mostly affects the upper and lower airways and kidneys, leading to the formation of granulomas and necrosis of these organs. We report a case of Granulomatosis with Poliangiitis with respiratory and renal involvement.

Case Report: A 23 years old female patient with complain of epistaxis, retro-orbital pain and dyspnea with no triggering factors with spontaneous resolution since childhood. During pregnancy, 1 year and 8 months ago, intensified the dyspnea with deafness and polyarthritis associated. She underwent examinations showing subglottic narrowing. Computadorizer Tomography (CT) scan of the cervical region showed a significant tracheal lumen restriction with a $10 \mathrm{~mm}$ thickening. She was submitted to a emergency tracheostomy. PR3 and C-ANCA (1:40) were reagents. Videolaryngoscopy showing subglottic narrowing with $95 \%$ reduction in the sizer of lumen. Proteinuria: $987,5 \mathrm{mg} / 24 \mathrm{H}(1250 \mathrm{ml})$ and renal biopsy (16 glomeruli) showed: 01 with focal and segmental proliferation with fibrinoid necrosis associated, 01 with intense global - epithelial cell growing proliferation, 01 with discrete segmental epithelial proliferation with adhesion between the epithelial leaflets, 02 globally sclerosed. The others (11) with basal membrane thickening by fibrinoid epithelium with a slight increase of mesangial matrix and rare leukocytes. Immunofluorescence (IF): positive for $\mathrm{C} 3 \mathrm{c}$ and fibrinogen. The GPA diagnostic was confirmed and methylprednisone and cyclophosphamide treatment was started.

Discussion: GPA is a granulomatous vasculitis of idiopatic cause, occurs more frequently in the fifth decade of life, onset may be insidious, acute, or even fulminant. About $90 \%$ are associated with ANCAs. Regarding the immunofluorescence, 3 patterns are known: c-ANCA,
p-ANCA and atypcal x-ANCA. C-ANCA is associated to the presence of anti-PR3 antibodies and p-ANCA is typically related to Anti-MPO antibodies. Tracheal stenosis is a less common impairment. Nasal, sinus, oral, laryngopharyngeal, tracheal and/or ear signals and symptoms are observed in more than $90 \%$ of GPA cases. When hearing loss occurs it can be conductive, sensorineural or mixed. Subglottic stenosis results from tracheal scars and inflammation and is considered a serious complication of GPA which can eventually cause tracheal stenosis. Almost $80 \%$ of patients with GPA develop kidney disease and without a proper therapy can lead to irreversible kidney function loss. The histological differentiation between GPA, PAM and GEPA can be a challenge. Treatment includes, corticoid, and immunosuppression.

\section{Consent for publication}

The authors declare that they have obtained informed written consent from the patient's tutors for publication

\section{P212}

\section{HEERFORDT-WALDENSTRÖM SYNDROME: CASE REPORT}

Pamella de Paula Bellini ${ }^{1}$, Jacqueline Foelkel Pignatari ${ }^{2}$, Mateus de Miranda Moura Cortes², Gabriela Miyuki Teodoro Ogawa², Lara Ribeiro Teixeira Bonfim², Samuel de Oliveira Andrade², Alisson Aliel Vigano Pugliesi ${ }^{2}$, Zoraida Sachetto ${ }^{2}$

1 UNIVERSIDA ESTADUAL DE CAMPINAS - UNICAMP, CAMPINAS, SP, Brasil; ${ }^{2}$ UNIVERSIDADE ESTADUAL DE CAMPINAS (UNICAMP), CAMPINAS, SP, Brasil

Advances in Rheumatology 2018, 58(Suppl 1):P212

Introduction: Heerfordt-Waldenström syndrome (HWS) is a rare variant of sarcoidosis first described in 1909 by Heerfordt and Waldenström, being associated with sarcoidosis in 1937. HWS is characterized by the triad: parotid gland edema, facial nerve paralysis and anterior uveitis. The disease is considered complete when the three symptoms are present and incomplete in the presence of two. Case description: A 34-year-old male patient presenting headache and fever progressing to left facial paralysis and bilateral parotitisis hospitalized for investigation. He did not present uveitis. During hospitalization, a computed tomography of the chest was performed, identifying hilar and mediastinal lymph node enlargement, as well as pulmonary nodules.

A lymph node biopsy was performed, which revealed sarcoid granuloma, a finding compatible with sarcoidosis. Treatment with corticosteroids in high doses was initiated, with no adequate response, and a progression to cyclophosphamide was required, when total remission of symptoms was achieved. Currently, the patient is still on azathioprine.

Discussion: The case report aimed to remind the rheumatologist of this variant of sarcoidosis when encountering patients with associated facial paralysis, uveitis and parotitis. As with sarcoidosis, corticosteroids are first-line therapy, and other immunosuppressants are used in cases of refractoriness.

\section{Consent for publication}

The authors declare that they have obtained informed written consent from the patient's tutors for publication

\section{P216}

HIPOCOMPLEMENTEMIC URTICARIAL VASCULITIS - A SERIES OF SEVEN CASES

Lais Gomes², Carolina Oliveira², I Thien Chan², Liliana C C Castro², Chiara Borges' ${ }^{2}$, Camila Souto ${ }^{2}$, Manuella Gomes Ochtrop², Evandro Mendes Klumb²

'UNIVERSIDADE DO ESTADO DO RIO DE JANEIRO, RIO DE JANEIRO, RJ, Brasil; ${ }^{2}$ UERJ, RJ, RJ, Brasil

Advances in Rheumatology 2018, 58(Suppl 1):P216 
Background. The hipocomplementemic urticarial vasculitis (HUV) or anti-C1q vasculitis is a rare disease that may present isolated or in association with other autoimmune diseases (AID) and has its main characteristic as hives that last for more than 24 hours and hurts instead of itching. HUV is an immune complex vasculitis according to the Chappel-Hill classification and presents small vessel leukocytoclastic lesions. At the same time, severe internal organ involvement may occur with articular, ocular, lung, renal and digestive tract manifestations which may simulate other AID as SLE. In the present study we report clinical features and treatment of seven patients (6 women, mean age of 39,7 years) followed at a university hospital.

Discussion and conclusion: Four patients also had SLE, one, Sjögren syndrome and one APS. Treatment included prednisone (7/7), hydroxichloroquine (5/7), dapsone (2/7), azathioprine (6/7), methotrexate (1/7), mycophenolate (2/7) and cyclophosphamide (1/7). All patients reported oligo or polyarthritis, four had proliferative glomerulonephritis (2 with concomitant SLE), Episcleritis was found in 2, escleritis in 2 , uveitis in 2, lung nodules in one and intersticial pneumonitis in one. Two out of the seven cases reported had the isolated form of HUV (IHUV) and interestingly were the two more severe patients despite the absence of another AID. These two IHUV patients developed glomerulonephritis (one with hemodyalisis), which is described in $50 \%$ of HUV, pulmonary nodules (wicth required biopsy and immunesuppressive treatment) (1 case) and retinal vasculitis with amaurosis (1 case). We did not had any patient with COPD that is described in $20 \%$, except in one case with a high smoking load. All cases including those with associated AID had strong steroid dependence and required immunesuppressive agents due to cutaneous and systemic manifestations. All patients presented low serun complement levels along follow up, a common feature in HUV. The antiC1q antibody that is present in 50\% of patients, was negative in the only one studied. The main objetive of the present report is to bring to light this rare disease that may simulate other AID especially SLE and also be associated with them. We herein also describe two cases with isolated HUV and severe clinical manifestations as glomerulonephritis and renal insuficiency, pulmonary nodules, uveitis and amaurosis, including a death due to sepsis.

\section{P218}

HOSPITAL INTERVENTIONS CAUSED BY RHEUMATOID ARTHRITIS AND OTHER INFLAMMATORY POLYARTHROPATHIES IN THE NORTH REGION AND ITS PERCENT VARIATIONS IN THE LAST 8 YEARS

Amanda Domingues Barreto, Gislanne Dos Santos Aguiar, Bráulio Érison França Dos Santos

UNIVERSIDADE FEDERAL DO AMAPÁ, MACAPÁ, AP, Brasil

Advances in Rheumatology 2018, 58(Suppl 1):P218

Background: Rheumatoid arthritis (RA) is an autoimmune, inflammatory, systemic and chronic disease, characterized by synovitis and various extra-articular manifestations. The AR does not yet have its well-known etiopathogenesis, however, the human leukocyte antigen (HLA), considered the main genetic factor related to the disease, has been identified. It is estimated that about $1 \%$ of the world's population is affected, especially between 40 and 60 years. This pathology is related to decreased functional capacity and reduced quality of life. The objective of this study was to compare the numbers of hospital admissions caused by Rheumatoid Arthritis and Other Inflammatory Polyarthropathies (OPI), from 2010 to 2017, in the North Region. Methods: A quantitative approach was carried out using secondary data obtained from the Department of Informatics of the Brazilian Unified Health System (DATASUS), with access in April 2018.

Results And Conclusions: According to information provided by DATASUS, in 2017 there was a $42.9 \%$ decrease in the number of hospitalizations caused by RA and IPO in the North Region in comparison to 2010. Among the falls in the number of hospitalizations, the highlight is the year 2011, with $-21.2 \%$ of cases compared to the year 2010; and the year 2017, with $-15 \%$ of the cases compared to the year 2016 (Fig. 1). It is concluded that the fall in the numbers of hospitalizations in the last 8 years in the North Region suggests less seriousness in the cases, leading to estimate that the treatment is being performed in an adequate way, since it can be done in the basic network, without hospitalization when there are no complications, or that the early diagnosis of the initial RA has been performed as proposed by the Brazilian Society of Rheumatology in 2010, improving the prognosis when the diagnosis is early.

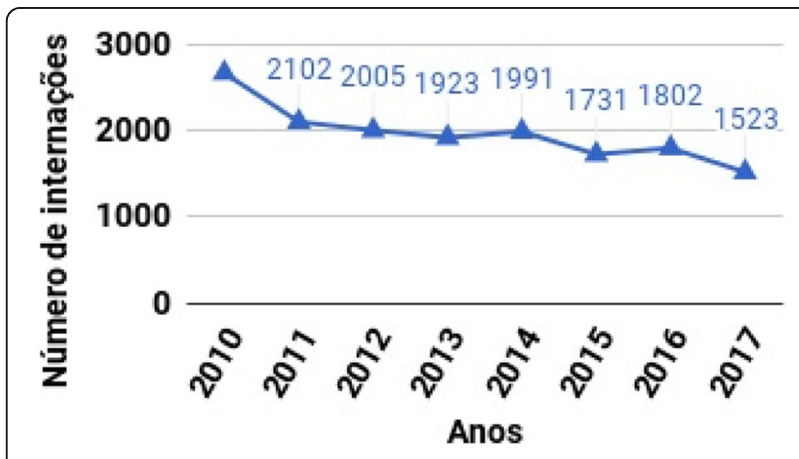

Fig. 1 (abstract P218). Number of hospital admissions caused by rheumatoid arthritis per year

P220

HUGHES-STOVIN SYNDROME: A CASE REPORT

Taíssa Cerqueira dos Santos, Luiz Felipe Dipe Prates Miranda, Paulo Cesar Meucci Pereira Nogueira, Lucas Balieiro Dias, William dos Santos

Gonçalves, Patrícia Lobo Nunes Moll, Débora Rocha de Moura Rodrigues de Aguiar, Carlos Baptista de Figueiredo

HOSPITAL FEDERAL DE BONSUCESSO, RIO DE JANEIRO, RJ, Brasil

Advances in Rheumatology 2018, 58(Suppl 1):P220

Background: The Hughes-Stovin Syndrome (HSS) is a rare condition, which preferentially affects men between 20 and 40 years. The typical presentation includes recurrent fever, venous thrombosis and hemoptysis due to pulmonary arteries aneurysms.

It may be considered as an incomplete form of Behcet's disease, since not all required criteria are presented, such as recurrent oral and genital ulcer and positive pathergy test.

Case report: A 23-year-old male was hospitalized with headache, chest pain, dyspnea, cough and jugular vein distention. The symptoms started four months earlier with daily fever, inappetence and lost of 20 kilograms.

Contrast computer tomography scans revealed an extensive thrombosis in inferior vena cava, common iliac veins, internal jugular veins and sagittal sinus. Echocardiography showed a large thrombus on right ventricle. He had normocytic and normochromic anemia, inversion of the albumin-globulin ratio, elevated CRP and several negative blood cultures. Infectious etiologies were discarded and autoimmune serologic markers were negative. The funduscopic examination showed papilledema and micro hemorrhages.

After discarding thrombophilia, Behçet's disease was suggested, but the patient didn't met the diagnostic criteria. The hypothesis of HSS was raise and an angio-MRI was requested. In the meantime the patient developed haemoptysis, so anticoagulant drug was suspended and immunosuppressive therapy was started with methylprednisolone pulse therapy and cyclophosphamide. He had good clinical outcome, interruption of fever and weight gain. However, the patient presented with deep vein thrombosis and had the anticoagulation therapy restarted.

The angio-MRI detected three pulmonary arteries aneurysms and the hypothesis of HSS was confirmed.

Conclusion: There are few cases described in the literature. The diagnosis is clinical and must be suspected when there are the concomitant presence of deep vein thrombosis and signs of inflammatory disease associated with aneurysms of the pulmonary arteries. Behçet's disease becomes the main differential diagnosis and must be excluded. 
HSS has a three stage course: thromboses, formation of pulmonary aneurysms and hemoptysis. The thrombophlebitis usually affects peripheral veins, being the vena cava thrombosis and the presence of thrombus in right ventricle rare conditions.

Immunosuppressive therapy with a combination of glucocorticoids and cyclophosphamide is the cornerstone of treatment. The use of anticoagulants remain controversial because of the risk of fatal hemoptysis.

\section{Consent for publication}

The written informed consent was obtained from the patient for publication.

Consent for publication

The authors declare that they have obtained informed written consent from the patient's tutors for publication

\section{P224}

HYPOTHYROIDISM AND HAND OSTEOARTHRITIS

Lila Morena Bueno Da Silva², Jobson Lopes de Oliveira², Cláudia Goldenstein Schainberg ${ }^{1}$, Ricardo Fuller ${ }^{2}$, Henrique Carriço da Silva ${ }^{2}$ ${ }^{1}$ HFMUSP, Brasil; ${ }^{2}$ HCFMUSP, Brasil

Advances in Rheumatology 2018, 58(Suppl 1):P224

Background: Recent studies indicate a possible participation of thyroid dysfunction in osteoarthritis, but the results are still controversial. The aim of this study is to verify if there is an association between hypothyroidism and hands osteoarthritis-HOA (erosive and non-erosive), both considered generalized and genetic forms of the disease.

Methods: 168 participants were included consecutively: 115 with HOA and 53 with knee osteoarthritis (KO) (no-hand osteoarthritis, or control group), according to ACR criteria. HOA was classified as erosive osteoarthritis (EO, $\mathrm{n}=71$ ) and non-erosive osteoarthritis (NEO, $\mathrm{n}=44$ ). EO were classified according to presence of at least one central interphalangeal joint erosions (Gull-wing sign and/or saw-tooth sign) on $x$ ray. Exclusion criteria: patients with hand pain in the KO group, inflammatory articular diseases, endocrine-metabolic diseases associated with joint manifestations (except thyroid disease) and history of trauma. Individuals were compared using logistic regression adjusted for age and body mass index (BMI), for the presence of hypothyroidism (classified as a TSH $>5 \mathrm{mU} / \mathrm{L}$ or previous diagnosis of hypothyroidism already receiving treatment). The statistical analysis considering statistically significant a $p$-value $<0.05$.

Results and conclusion: hypothyroidism prevalence was $39.4 \%, 18.2 \%$ and $11.3 \%$, respectively in the EO, NEO and KO groups $(p=0.001)$. Comparisons between the groups showed: EO vs NEO, $p=0.017$; EO vs KO, $p=0.001$ and NEO vs KO, $p=0,33)$. The differences were independent of the BMI and age ( $p$ 0.034). TPO, anti-thyroglobulin, TSH and $\mathrm{T} 4 \mathrm{I}$ showed no statistical significance.

Conclusion: we demonstrated a higher prevalence of hypothyroidism in patients with osteoarthritis of the hands, especially in erosive form compared to patients with no hand osteoarthritis.

\section{P228}

IMMUNE-MEDIATED NECROTIZING MYOPHATY ASSOCIATED WITH STATINS

José Marques Filho', João Aris Kouyoumdjian ${ }^{2}$

${ }^{1}$ FACULDADE DE MEDICINA UNISALESIANO ARACATUBA/CREMESP,

ARACATUBA, SP, Brasil; ${ }^{2}$ FACULDADE DE MEDICINA SÃO JOSÉ DO RIO

PRETO, SÃO JOSÉ DO RIO PRETO, SP, Brasil

Advances in Rheumatology 2018, 58(Suppl 1):P228

The idiopathic inflammatory myopathies (IIMs) comprise a group of autoimmune disorder that target skeletal muscle. They are characterized by typical laboratory and clinical features including muscle weakness, elevated muscle enzymes, characteristics histopathology of muscles biopsies, as well a electromyography abnormalities. The IIMs are divided into polymyositis, dermatomyositis, inclusions body myositis, nonspecific myositis and immune-mediated necrotizing myopathy (IMNM), characterized by absence of primary inflammation on muscle biopsy. One the most common complaints of people taking statins is muscle pain. It can feel like a soreness, tiredness or weakness in the proximal muscles. Withdrawal of the statin usually improves symptoms in a days or few weeks. We report a case of 70years-old female patient in treatment to rheumatoid arthritis (RA) about seven years taking leflunomide $20 \mathrm{mg}$ by day and deflazacort $6 \mathrm{mg}$ by day. She was in remission of RA by years. Four previous months she lost weight, generalized complaints such fatigue, proximal muscular pains and muscle weakness. She was in use of atorvastatine $10 \mathrm{mg}$ by day for about one year. The elevated creatine kinase (CK) and clinical symptoms were unchanged even after withdrawal of statin. Electromyography demonstrated typical myopathic pattern. Muscle biopsy was indicated and show myopathy necrotizing without significant inflammation. A diagnosis of immune-mediated necrotizing myopathy associated with statin was made and therapy with azathioprine $150 \mathrm{mg}$ by day was introduced with rapid clinical improvement and normalization of level of CK. Concluding, the lack of improvement following discontinuation of statin in patient with myalgia and elevated CK, the need for immunosuppressive therapy and frequent relapse when treatment was tapered suggest an immunemediated etiology for this rare, statin-associated necrotizing myopathy.

\section{P229}

IMMUNOGLOBULIN G4-RELATED HYPERTROPHIC

PACHYMENINGITIS (IGG4-RHP): CASE REPORT

Déborah Lobato Guimarães, Ihan Bruno Lopes Rabelo, Débora Cerqueira Calderaro, Caio Moreira

HOSPITAL DAS CLÍNICAS DA UFMG, BELO HORIZONTE, MG, Brasil

Advances in Rheumatology 2018, 58(Suppl 1):P229

Background: Immunoglobulin G4 (IgG4)-related disease is a newly recognized fibroinflammatory systemic condition characterized by tumefactive lesions, a dense lymphoplasmacytic infiltrate rich in IgG4-positive plasma cells, storiform fibrosis, and, often but not always, elevated serum IgG4 concentrations. Hypertrophic pachymeningitis is an infrequent disorder that produces focal or diffuse thickening of the dura mater that has recently been described as secondary to 'IgG4-related disease'.

Case report: A male 64 years-old patient from Lagoa Santa (MG), came to the Rheumatology facility complaining of important thoracic pain in the interescapular region not ventilatory dependent for the last six months. Magnetic ressonance showed dural thickening in cervical and thoracic spine (Fig. 1a). Serum lgG4 was $2450 \mathrm{mg} / \mathrm{dL}$ (increased). Dural biopsy microscopic examination revealed it was expanded by a patchy dense infiltrate of plasma cells and small lymphocytes. Immunohistochemistry presented $>60$ plasma cells/high power field and more than $60 \%$ lgG4 + plasma cells (Fig. 1b). IgG4RHP was diagnosed. Patient was treated with IV Methylprednisolone $1 \mathrm{~g} /$ day for 3 days, followed by oral prednisone $(60 \mathrm{mg} /$ day) and slowly glucocorticoid taper (currently, $10 \mathrm{mg} / \mathrm{day}$ ) and azathioprine (currently, $100 \mathrm{mg} /$ day). Thoracic pain resolved in two weeks. No neurological complications arised. He is a lawyer currently working normally.

Conclusion: Clinicians should become familiar with IgG4-RHP, because a prompt, specific therapeutic approach may avoid long-term neurological complications. There is currently no consensus about treatment for patients with lgG4-RHP. High-dose glucocorticoids (eg, $1 \mathrm{~g}$ of methylprednisolone a day for 3 days) followed by a long glucocorticoid taper are still the treatment of choice, followed by the addition of other immunosuppressive agents in the event of a recurrence. Methotrexate, azathioprine, mycophenolate mofetil and cyclophosphamide have been used as steroid-sparing agents. Rituximab is a promising therapeutic approach but experience with B-cell depletion strategies remains limited.

\section{Consent for publication}

The authors declare that they have obtained informed written consent from the patient's tutors for publication 


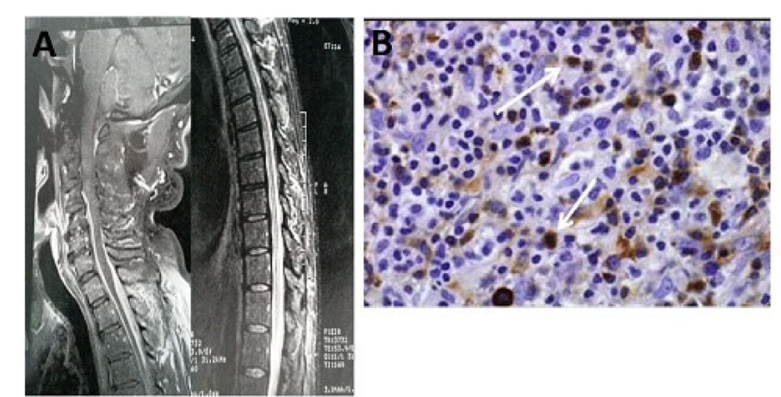

Fig. 1 (abstract P229). A: T1-weighted magnetic resonance (MR) imaging, showing hyperintense linear dural thickening in cervical and thoracic spine. B: Dural biopsy immunohistochemistry: Numerous IgG4+ plasma cells. In this field, the lgG4+ plasma cells constitute

\section{P231}

INDICATIONS AND EXPERIENCE WITH USE OF RITUXIMABE IN SYSTEMIC SCLEROSIS PATIENTS

Ana Elisa Dantas Modesto, Gláucia Moreira Souza de Queiroz,

Laize Guerreiro de Jesus, Solino de Matos Neto, Alex Magno Coelho Horimoto UNIVERSIDADE FEDERAL DE MATO GROSSO DO SUL, CAMPO GRANDE,

MS, Brasil

Advances in Rheumatology 2018, 58(Suppl 1):P231

Introduction: Systemic sclerosis (SSc) is an autoimmune disease, characterized by the triad of vascular injury, autoimmunity and tissue fibrosis. There is robust evidence to support B cell depletion in the treatment of SSc, since functional alterations of these cells have been demonstrated in both animal models and in SSc patients.

Objectives: To evaluate the effect of Rituximab (RTX) in patients with SSc in a population of the pulse therapy sector of Maria Aparecida Pedrossian University Hospital (HUMAP) in Campo Grande, Mato Grosso do Sul, Brazil.

Methods: This is an observational, retrospective study in which all patients using RTX in the pulse therapy sector of HUMAP in 2016 were evaluated. The indications for the use of RTX were evaluated. The analysis of pulmonary involvement was through computed tomography $(\mathrm{CT})$ of the chest and spirometry (FVC $<70 \%)$. The degree of cutaneous involvement was assessed through the modified Rodnan score and the joint evaluation by radiographic analysis of the hands and the composite index DAS 28.

Results and conclusion: Six patients were included in the use of RTX, with a mean age of $57.33 \pm 5.16$ years, all female $(100.0 \%-n=6)$. Joint involvement $83.3 \%(n=5)$ and pulmonary involvement $66.7 \%(n=4)$ were the indications for its use, and cyclophosphamide $(83.3 \%-n=5)$ was the medication most used previously to the drug studied. The total cutaneous score varied between 6 and 37 points $(16.33 \pm 11.57)$. Most of the patients presented joint involvement $(83.3 \%-n=5)$ with DAS28 of $2.63 \pm 1.30$, being observed in the radiograph: bone erosions, just-articular osteopenia and digital pulp reabsorption $(66,7 \%-n=4$, each change). Restrictive pattern was observed in $66.7 \%(n=4)$ of the patients and changes in chest tomography with bronchiectasis, traction, frosted glass and honeycombing (33.3\% $-n=2$, for each change). All patients are in the follow-up in the sector, with cutaneous, articular, pulmonary and disease stability, in use of rituximab, except for one patient with joint worsening, being indicated the exchange of immunosuppressive medication for tocilizumab with satisfactory response. This study, according to the literature, demonstrated good control and tolerability to the use of RTX in SSc. This fact reinforces the importance of this medication in patients with SSc because it presents a potential for improvement in the clinical picture of patients with cutaneous, articular and pulmonary involvement.
P233

INFLAMMATION INFLUENCE ON INTIMA-MEDIA THICKNESS (IMT) IN RHEUMATOID ARTHRITIS POPULATION: A SINGLE-CENTER POST-HOC ANALYSIS

Bruno Cesar Bacchiega ${ }^{1,2}$, Ana Beatriz Santos Bacchiega ${ }^{3}$, Manuella Lima Gomes Ochtrop ${ }^{3}$, Camila Souto Oliveira ${ }^{3}$, Ricardo Bedirian², Geraldo da Rocha Castelar Pinheiro ${ }^{3}$

${ }^{1}$ HOSPITAL DO CÂNCER DE BARRETOS, Brasil; ${ }^{2}$ HOSPITAL DO CÂNCER DE BARRETOS - BARRETOS - SP, Brasil; ${ }^{3}$ UNIVERSIDADE DO ESTADO DO RIO DE JANEIRO - RJ, Brasil

Advances in Rheumatology 2018, 58(Suppl 1):P233

Background: Patients with rheumatoid arthritis (RA) are at increased risk of mortality compared with the general population, mainly from cardiovascular causes. Intima-media thickness (IMT) has been described as a subclinical atherosclerosis screening tool. The main objective of this study was to evaluate the influence of inflammatory markers on IMT in RA patients.

Methods: We performed a post hoc analysis of an observational cohort data previously published and collected from December 2014 to November 2015. Thirty-nine RA patients according to the 1987 American College of Rheumatology criteria, older than 18 years-old, presented with moderate/high disease activity, starting on biologic or traditional disease modifying antirheumatic drugs (DMARDs) were enrolled. Variables assessed were clinical parameters, $C$ reactive protein (CRP), RA disease activity score 28 joints (DAS28_CRP), Health Assessment Questionnaire-Disability Index (HAQ-DI), lipid profile, subclinical atherosclerosis evaluated by intimal-media thickness (IMT) assessed by ultrasonography bilaterally in common carotid artery, internal carotid artery and in the bifurcation. We performed a linear regression, based on an inflammatory pathophysiology model (DAS28_CRP, CRP, LDL-c), to assess its influence over IMT.

The exclusion criteria were acute coronary syndrome in the last 3 months before enrollment and/or uncontrolled hypertension (SBP $\geq 160 \mathrm{mmHg}$ or $\mathrm{DBP} \geq 110 \mathrm{mmHg}$ ). The present investigation was approved by ethic review board. All participants signed a written informed consent form.

Results and conclusions: Demographic characteristics are shown in Tables 1 and 2. Four patients (10,5\%) had an IMT measurement beyond $0,90 \mathrm{~cm}$ (higher risk of cardiovascular events) and were not on statin therapy. Linear regression showed a positive correlation between IMT and CRP and LDL-c levels. These findings reinforce the influence of systemic inflammation on accelerated atherosclerosis and the awareness of RA patients as a high-risk cardiovascular population.

Funding

This work was supported by the Pedro Ernesto Rheumatology Study Center.

Table 1 (abstract P233). Demographic characteristics

\begin{tabular}{l|c|c|}
\hline Table 1 & Frequency & $(\%)$ \\
\hline Gender (female) & 33 & $84.65 \%$ \\
\hline Erosive disease & 32 & $82.05 \%$ \\
\hline RF+ & 30 & $76.92 \%$ \\
\hline ACPA+ & 27 & $69.23 \%$ \\
\hline Statin & 7 & $17.95 \%$ \\
\hline Tobacco & 4 & $10.25 \%$ \\
\hline Tobacco (former) & 12 & $30.75 \%$ \\
\hline
\end{tabular}


Table 2 (abstract P233). Demographic characteristics

\begin{tabular}{|l|c|c|}
\hline Table 2 & Median & IUK \\
\hline Age $i \mathrm{y})$ & 53 & $(45-61)$ \\
\hline BMI $\left(\mathrm{kg} / \mathrm{m}^{2}\right)$ & 26.99 & $(24.26-30.81)$ \\
\hline DIsease duration $(\mathrm{Y})$ & 9.5 & $(5-16)$ \\
\hline HAO & 1.75 & $(1.125-2.25)$ \\
\hline nAS $) 8 \mathrm{CRP}$ & 5.38 & $(4.51-6.35)$ \\
\hline CRP $(\mathrm{mg} / \mathrm{dL})$ & 1.36 & $(0.91-3.05)$ \\
\hline ESR $/ \mathrm{mm} / \mathrm{h})$ & 32 & $(14-57)$ \\
\hline Total $\mathrm{cholest}(\mathrm{mg} / \mathrm{dL})$ & 200 & $(168-2271$ \\
\hline IIDL-c $(\mathrm{mg} / \mathrm{dL})$ & 58 & $(4672)$ \\
\hline LDL-: $(\mathrm{mg} / \mathrm{dL})$ & 111.4 & $(91.4-142.6)$ \\
\hline Trig/vcerides $(\mathrm{mg} / \mathrm{dL})$ & 91 & $(73-132)$ \\
\hline IMT $(\mathrm{mm})$ & 0.696 & $(0.607-0.796)$ \\
\hline
\end{tabular}

\section{P235}

INFLUÊNCIA DA FIBROMIALGIA NA AVALIAÇÃO DO BASDAI NOS PACIENTES COM ESPONDILOARTRITE

Lara Francischetto Milleri, Renan de Souza Santana Soares, Larissa de

Barros Oliveira, Gabriela Stutz Francisco Moreira

HOSPITAL FEDERAL DOS SERVIDORES DO ESTADO, RIO DE JANEIRO, RIO

DE JANEIRO, Brasil

Advances in Rheumatology 2018, 58(Suppl 1):P235

Spondyloarthritis is a chronic inflammatory rheumatic disease characterized by entheses, axial and peripheral joints involvement, often associated with fatigue and intense pain, which are important symptoms in Fibromyalgia. These diseases can occur simultaneously, so the aim of this study was to measure the influence of Fibromyalgia on Bath Ankylosing Spondylitis Disease Activity Index (BASDAI) in the cohort of patients of the Hospital Federal dos Servidores do Estado do Rio de Janeiro with spondyloarthritis diagnosis in concomitance.

It's used as a BASDAI classification: classified $\geq 4$ and unclassified $<4$. Patients with arthropathic psoriasis were not included in the study.

The study included 149 patients, 37 (24.8\%) were diagnosed with Fibromyalgia. Of 112 patients without Fibromyalgia, 73.2\% had unclassified BASDAI. In contrast, of 37 pacients with Fibromyalgia, $48.6 \%$ had unclassified BASDAl and $51.4 \%$ had BASDAI classified When comparing patients with Spondylarthritis associated Fibromyalgia with those without Fibromyalgia, the odds ratio for classified BASDAl was 2.88 (95\% Cl, 1.33 to 6.22 ).

It's concluded that Fibromyalgia affects a significant proportion of patients with Spondylarthritis and may compromise the reliability of the evaluation of true inflammatory disease through BASDAl, since it is a subjective index of analysis.

\section{P239}

\section{INTRAVENOUS PAMIDRONATE FOR CALCINOSIS IN JUVENILE} DERMATOMYOSITIS

Clovis Artur Almeida Da Silva ${ }^{1,2}$, Verena Balbi ${ }^{2}$, Tamima Mohamad Abou Arabi $^{2}$, Vitor Cavalcanti da Trindade ${ }^{2}$, Ana Carolina Cortez Maia², Izabel Mantovani Buscatti², Flavia Sakamoto², Lucia Maria Arruda Campos², Adriana Maluf Elias Sallum²

${ }^{1}$ ICR-HC-FMUSP, SÃO PAULO, SP, Brasil; ${ }^{2}$ HOSPITAL DAS CLINICAS HCFMUSP, SAO PAULO, SP, Brasil

Advances in Rheumatology 2018, 58(Suppl 1):P239

Background: Calcinosis occurs in up to $47 \%$ juvenile dermatomyositis (JDM) patients, and generally is associated with disease activity. There is no standard treatment for this potentially limiting condition and a few reported cases have improved after intravenous pamidronate (IVP). Therefore, the objective of the present study was to evaluate efficacy and safety of IVP in real life situation.
Methods: A prospective study included all JDM patients (Bohan and Peter criteria) with refractory calcinosis to diltiazem, bisphosphonates and/or intravenous immunoglobulin selected from a population of 63 JDM patients followed-up between 2013 to 2018. Dystrophic calcification was classified as superficial plaques/nodules, calcinosis circumscripta, calcinosis universalis, exoskeleton-like deposits and mixed forms. Patients were systematically treated with IVP $1 \mathrm{mg} / \mathrm{kg} /$ month for at least three consecutive months. Disease activity (Disease Activity Scale-DAS), muscle strength tests (Childhood Myositis Assessment Scale-CMAS and Manual Muscle Text-MMT), muscle enzymes levels and inflammatory markers were prospectively assessed. Experienced pediatric radiologist blindly assessed radiographic exams before and between three to six months after drug infusion.

Results: Refractory calcinosis was treated with IVP in 8/63 (13\%) JDM patients. Of note, $3 / 8$ were excluded due to incomplete radiographic evaluation, and $1 / 8$ had just started the first dose of IVP. The median age at JDM onset was 6 years (range 3-7 years) and at calcinosis diagnosis was 7 years (range 4-11 years). No adverse events were observed 4 JDM patients. The median interval between calcinosis presentation and IVP treatment was 3 years (range 1-6 years). Importantly, a 6 years old patient, who received earlier IVP treatment (one-year interval), had a dramatically enhancement of exoskeletonlike calcinosis as well as CMAS, DAS and MMT scores, and muscle enzymes. The second patient, 13 years old, had no calcinosis changes in x-ray images, even though no new calcinosis was reported. A 12 years old patient had partial improvement of calcinosis on lower member and right upper member, although new calcinosis was shown in left arm. Finally, the fourth child, 12 years old, presented dystrophic calcification reduction on $\mathrm{x}$-ray, however she developed new lesions on the follow-up.

Conclusion: IVP seems to be a safe option by refractory calcinosis, however with limited efficacy. Further prospective and multicenter studies, with a large JDM population, are necessary to clarify which factors are associated with better outcomes using this medication.

\section{P245}

JUVENILE DERMATOMYOSITIS: CLINICAL, LABORATORIAL, HISTOLOGICAL, THERAPEUTICAL AND EVOLUTIVE PARAMETERS OF NINE CHILDREN

Erica Naomi Naka Matos, Cristiane Harumi Bazhuni Tsuge Neiva, Jamima Oliveira Santos, Milena Foizer Leite, Querolai Gomes Gadelha, Laize Guerreiro De Jesus, Solino De Matos Neto

UNIVERSIDADE FEDERAL DE MATO GROSSO DO SUL, CAMPO GRANDE, MS, Brasil

Advances in Rheumatology 2018, 58(Suppl 1):P245

Background: Juvenile Dermatomyositis (JDM) is a rare multisystem disease, whose etiology is unknown, characterized by a vasculitis that affects skin and muscles. The objective of this study was to describe clinical, laboratorial, therapeutical and outcome parameters of nine children with JDM followed between 2004 and 2017.

Methods: This study was based on a prospective and a retrospective analysis of nine children who met Bohan and Peter criteria for JDM diagnosis. A diagnosis of JDM requires the presence of the pathognomonic rash and three of the other criteria.

Results And Conclusions: The mean age of onset of the disease was 7 years and 6 months and the median age was 8 years, ranging from 3 years to 5 months to 10 years. The mean time until the diagnosis of JDM was between 15 days and 24 months. The distribution by gender showed a predominance of females (100\%). All patients met the criteria of Bohan and Peter for JDM, with characteristic cutaneous lesions (Gottron papules and/or heliotrope), as well as three other criteria. Muscle weakness was present in $100 \%$. The cutaneous and subcutaneous involvement was severe in two (22\%), presenting skin ulcer, while another three patients presented calcinosis. Gastrointestinal involvement was observed in four (44.4\%) patients, while three had pulmonary involvement (33.3\%) and none presented cardiac compromise. All patients had at least one of the altered muscle enzymes. Anti-nuclear antibody was positive in all patients and anti-Jo1 antibody was positive in two patients, one patient with pulmonary involvement. The erythrocyte sedimentation rate was positive in 
seven patients (77.7\%). All patients underwent electromyography and two were muscle biopsy, with compatible results for JDM. All patients received corticosteroids (prednisone or methylprednisolone) and methotrexate. The response was considered good in four patients, with improvement of muscle strength and cutaneous lesions. Partial response was observed in two patients and bad response in three, whom received additional associated therapy with cyclosporine (1), cyclophosphamide (2) and intravenous immunoglobulin (2). At study end-point, on April 2018, two patients (22\%) lost follow-up, two patients remain with cutaneous manifestations and laboratorial activity (22\%), five patients (55\%) were out of activity and in remission. This study aims to highlight the importance of establishing diagnostic criteria for JDM early, aiming diagnostic agility and the institution of treatment, which significantly alter the progression of the disease and its prognosis.

\section{P250}

\section{JUVENILE IDIOPATHIC ARTHTRITIS ASSOCIATED WITH}

\section{NUTCRACKER SYNDROME}

Anna Clara Dos Santos Da Costa ${ }^{1}$, Farley Carvalho Araújo ${ }^{1}$, Karen Santos

Lima', Vivian Louise Syrio Pessoa', Leandro Liberino da Silva ${ }^{2}$

'UNIVERSIDADE FEDERAL DOS VALES DO JEQUITINHONHA E MUCUR

(UFVJM), DIAMANTINA, MINAS GERAIS, Brasil; ${ }^{2}$ SERRA MEDICINA

DIAGNÓSTICA, DIAMANTINA, MINAS GERAIS, Brasil

Advances in Rheumatology 2018, 58(Suppl 1):P250

Background. Juvenile Idiopathic Arthritis (JIA) is the most common rheumatic condition in childhood, and has well known articular, ophthalmologic and hematologic manifestations. Nutcracker Syndrome (NCS) is a rare condition that refers to compression of the left renal vein, most commonly between abdominal aorta and superior mesenteric artery, with symptoms, more frequently hematuria and proteinuria, besides unilateral vascular ectasia. The combination of the two conditions has never been described in literature. Moreover, renal manifestations in JIA is possible. IgA nephropathy could happens in both.

Case report. ROA is a 4 years old girl, Brazilian, presenting inflammatory pain on left knee at least during last 5 months. She also had painful dilated collateral veins on left buttock, left side of the vulva and left thigh. At the clinical exam, the left knee was warm and had synovitis. Antinuclear antibodies (FAN) were positive (1:80), presenting fine dotted nuclear pattern. Rheumatoid factor was negative. Urine analysis presented without changes. Renal function was normal, either. Ultrasonography of left knee showed small amount of free suprapatellar liquid. Column, hips, hands and fists X-rays were normal. Abdominal dopplervelocimetry showed left renal vein entrapment at the aortic-mesenteric portion, with vascular angle narrowing. Patient was diagnosed with oligoarticular JIA (with positive FAN) and NCS. We prescribed ibuprofen $200 \mathrm{mg} /$ day, metotrexate $5 \mathrm{mg} /$ week and folic acid $5 \mathrm{mg} /$ week. Pain reduced, but the swelling maintained. Hexacetonide triamcinolone intra-articular infiltration was performed, resolving the remaining edema. We opted for conservative management of NCS.

Conclusion. NCS presenting pelvic congestion at this age and oligoarticular JIA have bad prognostics. Renal injury can be expected in both cases. Besides that, IgA nephropathy could arise in JIA and NCS. Therefore, renal manifestations, in this context, could represent a confusion factor and make difficult to define best treatment for the case. Aleatory cause seems be the most probable etiology of this association, although common pathologic ways could be identificated (IgA nephropathy could be a reflex of this). So, it is very important to recognize this association in clinical practice, beyond what more studies are necessary to define nature of that.

\section{Consent for publication}

The authors declare that they have obtained informed written consent from the patient's tutors for publication.

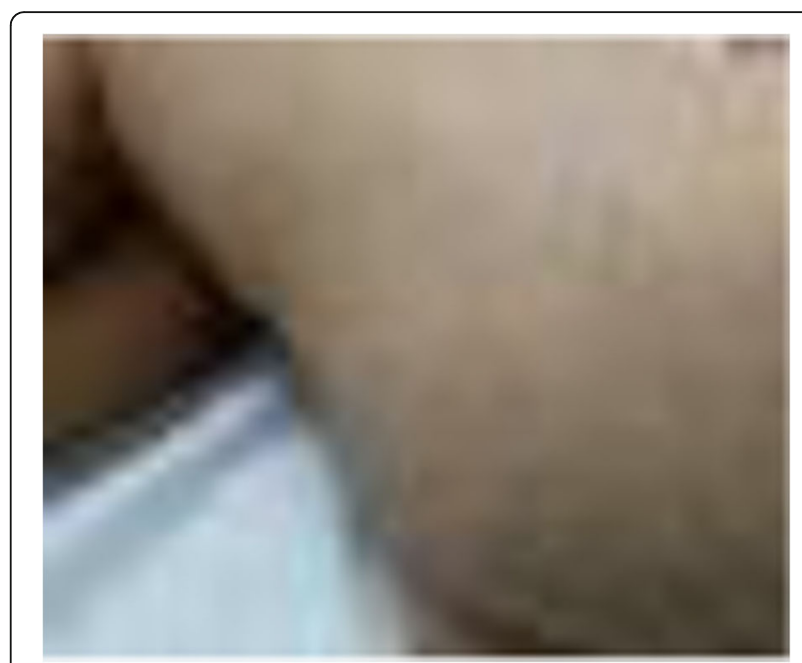

Fig. 1 (abstract P250). Dilated collateral veins on left buttock

P253

JUVENILE-ONSET SYSTEMIC LUPUS ERYTHEMATOSUS AND SYMPATHIC-REFLEX DYSTROPHY: A CASE REPORT

Marcela Paganelli, Sandra Regina Myioshi Lopes, Lígia Rocha Chaim, Dênis Azuma, Durval Ribas Neto, Julia Permegiani Vilarinho FACULDADE DE MEDICINA DE CATANDUVA, CATANDUVA, SP, Brasil Advances in Rheumatology 2018, 58(Suppl 1):P253

Introduction: Juvenile-onset Systemic Lupus Erythematosus (JSLE) is a rare multisystemic autoimmune disease.

There are several conditions associated with the development of sympathetic-reflex dystrophy (DSR). In adults there is a history of trauma in more than $60 \%$ of cases, already in childhood it is a rare and poorly diagnosed condition. There are no reports on the incidence and prevalence of this condition in the pediatric age group.

Case report: V, G, S, male, 13 years, with SLE for 10 months, treated with hydroxychloroquine, methotrexate and demand corticosteroid. Inhaled with pain and edema in the left upper limb and lower left limb. He showed no signs of infection at the scene, he denied trauma, he denied the use of medications without medical prescription. LLL edema up to knee height maintaining good peripheral perfusion and left hand edema. Severe pain limiting movements and keeping limbs in antalic position. Laboratory findings:

anti-native DNA $=1 / 80$, FAN positive homogenous nuclear standard, $\mathrm{C} 4=32, \mathrm{CH} 50=73.9$, IgM and IgG negative anticardiolipins, lupus anticoagulant negative, hepatitis $B$ and $C$ negative, anti HIV negative, urine type 1 and 24-hour proteinuria normals. Radiographic examinations without any signs of pathology. Remaining laboratory exams without changes. LLL and ULL US doppler with no evidence of thrombosis. MRI of the left ankle evidencing diffuse edema of the subcutaneous and deep myofacial planes in the ankle and lateral aspect of the forefoot, without significant atrophy of the soft tissues. Reflex sympathetic dystrophy?

Performed pulse therapy with methylprednisolone for 3 days, painkillers and neuromodulation with amitriptyline, gabapentin, paracetamol, ibuprofen and codeine. Physiotherapy started.

At the moment the patient is in reduction of edema concomitantly with the pain. Initiated azathioprine $1 \mathrm{mg} / \mathrm{kg} /$ day.

Clinical Discussion:

Expression in children and young adults with SLE may be different than in adults. The diagnosis of sympathetic-reflex dystrophy in patients with rheumatic diseases in childhood and adolescence should be considered as differential diagnosis in cases of neuropathic pain. Treatment is based on physiotherapy and pain relief. 
Conclusion: CRD is a chronic painful disease that can cause permanent or temporary functional incapacitation, it should have its diagnosis and early intervention.

\section{Consent for publication}

The authors declare that they have obtained informed written consent from the patient's tutors for publication

\section{P254}

\section{KAWASAKI DISEASE IN A NEW PEDIATRIC RHEUMATOLOGY}

\section{CENTER FROM BRAZIL}

Larissa Elias Pinho². Marina de Andrade Barbosa' Vitória Maria Batista Freire ${ }^{1}$ Thaís Sales Santana', Robério Crister Rabelo Lima Filho', Marco Felipe Castro da Silva², Carlos Nobre Rabelo Júnior ${ }^{2}$

'CENTRO UNIVERSITÁRIO CHRISTUS, FORTALEZA, CEARÁ, Brasil;

${ }^{2}$ HOSPITAL GERAL DE FORTALEZA, FORTALEZA, CEARÁ, Brasil

Advances in Rheumatology 2018, 58(Suppl 1):P254

Background: Kawasaki disease (KD) is one of the primary main vasculitis of childhood. It primarily affects individuals before 5 years, with risk of diagnostic delay and short- and long-term complications. The objective of the present study is describe the clinical and epidemiological profile as well as complications of the disease, especially coronary abnormalities.

Methods: This is a cross-sectional, observational and descriptive study that analyzed medical records of patients attending a tertiary service of pediatric rheumatology in Fortaleza, Ceará. Initially, 50 patients with a diagnosis of KD, younger than 18 years of age, were selected, and 16 cases were excluded due to incomplete data. The study was conducted in April 2018, including patients diagnosed between May 2008 and July 2017.

Results and Conclusion: Of 34 cases analyzed, 24 (70.5\%) were male. The mean age at diagnosis and current age were 44.4 (3-176) and 92 (13-210) months, respectively, and $73.5 \%$ were younger than 5 years. The majority of patients were diagnosed in the first semester of the year, corresponding to $64.7 \%(n=22)$. Other initial diagnoses were reported in 28 patients (82.3\%), especially bacterial tonsillitis, arbovirosis and scarlet fever. Of the total number of patients, $31(91.2 \%)$ received at least one IVIG dose, 4 (12.9\%) refractory to the first dose. The mean duration of fever and fever up to IVIG were 15.4 and 12.5 days, respectively. Atypical DK was found in 8 (23.5\%) patients. Acute complications were diagnosed in $13(38.2 \%)$ cases: coronary abnormalities (10), Kawasaki disease shock syndrome (3), macrophage activation syndrome (1). Patients with fever lasting more than 15 days had a higher incidence of coronary diseases $(p=0.0168)$. Out of the patients followed up after hospital discharge (32), 70\% of those with initial coronary disease evolved with clinical resolution. Loss of follow-up occurred in $50 \%$ of cases. It is important for the general pediatrician to suspect diagnosis for KD in patients with febrile infectious diseases of atypical course. The high rate of cardiac complications in the present study highlight the need for early diagnosis and treatment, especially in patients with prolonged fever.

\section{P258}

LATE-ONSET SYSTEMIC LUPUS ERYTHEMATOSUS: COMPARISON BETWEEN GROUPS WITH AND WITHOUT IMMUNOSUPPRESSION IN THE POPULATION OF A TERTIARY

\section{HOSPITAL}

Denise Moraes Horiy ${ }^{1}$, Andrey Tonetto Barbosa ${ }^{2}$, Fernanda Pulcheri Ramos ${ }^{2}$ Bernardo Patrício Sequeira Dultra ${ }^{2}$, Rina Dalva Neubarth Giorgi ${ }^{2}$, Renata Ferreira Rosa ${ }^{2}$, Nafice Costa Araújo ${ }^{2}$

${ }^{1}$ HOSPITAL DO SERVIDOR PÚBLICO - SP, SÃO PAULO, SP, Brasil; ${ }^{2}$ HOSPITAL DO SERVIDOR PÚBLICO ESTADUAL - SP, SÃO PAULO, SP, Brasil

Advances in Rheumatology 2018, 58(Suppl 1):P258

Introduction: Systemic lupus erythematosus (SLE) is a multisystem inflammatory autoimmune disease of unknown etiology with variable laboratory and clinical manifestations. It is most common in young women, with a peak age of 15 to 45 years, but may also be late onset. Elevated levels of female sex hormones are found in women with
SLE and the use of contraceptives and hormone replacement therapy after menopause were associated with a higher incidence of SLE. Objectives: To find determinants for the use of immunosuppressants in patients with SLE at baseline after 50 years of age and define their demographic and laboratory features.

Methods: The retrospective study included 32 patients accompanied in the rheumatology of the tertiary hospital. They were over 50 years old when they received the diagnosis of SLE according to the 1997 ACR criteria and were divided into two groups, according to an immunosuppression use in the group $1(\mathrm{~N}=11)$, that received azathioprine, mycophenolate mofetil, rituximab or cyclophosphamide and in the group $2(\mathrm{~N}=21)$ no history of immunosuppression. For the analysis, mean, standard deviation, frequency (\%), t-student, MannWhitney and Chi-square tests were used. $\mathrm{P}<0.05$ was considered statistically significant.

Results: The mean age was $69,91 \pm 10,71$ and $66,62 \pm 7,58$ years, for the group 1 and the group 2 , respectively $(p=0,3204)$, but the the mean diagnosis time was bigger in the group $1(12,36 \pm 8,85$ years, $p=0,05)$. Acute cutaneous injury was more common in the group $2(\mathrm{~N}=18, \mathrm{p}=0.035)$. Renal and neurological manifestations prevailed in the group $1(\mathrm{~N}=5, \mathrm{p}=0.011$ and $\mathrm{N}=3, \mathrm{p}=0.033$, respectively). Need of pulse with intravenous corticosteroid ( $N=6$, $p<0,001)$ and renal biopsy $(N=5, p=0,0023)$ were also significantly higher in the group 1. One hundred percent of the patients of the both groups used hydroxychloroquine and the most common antibodies were ANA, anti-DNA and anti-SM without statistically difference between the groups $(p=1,000)$. Secondary Sjögren syndrome were present in 6 patients in the group 2 and zero patients in the group $1(p=0,0711)$.

Conclusion: The study demonstrated a relationship between a longstanding disease and the need for immunosuppression in these patients. Renal and neurological involvements were verified pointing to a less favorable outcome. It is necessary to be attentive to this subgroup to identify possible serious manifestations of SLE and its appropriate therapeutic intervention.

\section{P261}

LIPID PROFILE OF PATIENTS WITH JUVENILE IDIOPATHIC ARTHRITIS OF THE POLYARTICULAR AND SYSTEMIC SUBTYPES

Wellington Douglas Rocha Rodrigues ${ }^{1}$, Roseli Oselka Saccardo Sarni ${ }^{2}$, Fernando Luiz Affonso Fonseca², Claudio Arnaldo Len², Patricia Pontes Aires $^{2}$, Maria Teresa Terreri ${ }^{2}$

1 UNIVERSIDADE FEDERAL DE SAO PAULO, SÃO PAULO, SP, Brasil; ${ }^{2}$ UNIVERSIDADE FEDERAL DE SÃO PAULO, SÃO PAULO, SP, Brasil Advances in Rheumatology 2018, 58(Suppl 1):P261

Introduction: Juvenile idiopathic arthritis (JIA) is the most common chronic rheumatic disease in the pediatric population. The patients coexist chronically with the disease that presents negative outcomes such as osteoporosis and cardiovascular diseases

Objective: To describe the lipid profile of children and adolescents with JIA and to relate them to variables of the disease, lipid profile, nutritional status and food consumption.

Methods: Cross-sectional study of 62 patients with JIA of the polyarticular and systemic subtypes. The following variables were evaluated: disease activity, medications used, body mass index, height for age (z score), food consumption through a 24 hour recall, lipid profile (total cholesterol - TC, low density lipoprotein - LDL, high density lipoprotein - HDL, triglycerides - TG and non-HDL). The analyzed associations were stratified into: adequate $\mathrm{HDL}-\mathrm{c}$ and borderline/low $\mathrm{HDL}-\mathrm{C}$; adequate and borderline/high LDL-C, CT, TG and NHDL-C; levels of C-reactive protein (CRP) and erythrocyte sedimentation rate (ESR). For statistical analysis: Fischer's exact test, chi-square and Mann-Whitney; $a<0.05$ was adopted.

Results: The mean age and time since initial diagnosis was 7.7 years $( \pm 4.3$ ) and 5.0 years $( \pm 3.4)$, respectively. $74.2 \%$ belonged to the female sex and active disease was observed in $33.9 \%$ of the patients. The prevalence of dyslipidemia was $62.9 \%$ and when we compared the subtypes, the systemic subtype had a higher value and a higher frequency of altered LDL-c and NHDL-c when compared to the polyarticular subtype ( $p=0.017$ and 0.042 , respectively). 
Conclusion: We conclude that dyslipidemia is frequent in patients with JIA and the systemic subtype presents a higher value and a higher frequency of altered LDL-c and NHDL-c, suggesting a more atherogenic lipid profile when compared to the polyarticular subtype.

\section{P262}

\section{LIPID PROFILES IN RHEUMATOID ARTHRITIS: THE CONTRIBUTION} OF FACTORS

Nayara Mota Carvalho', Marina Laís Ramalho de Oliveira de Almeida², Cezar Augusto Muniz Caldas ${ }^{3}$, Ismari Perini Furlaneto ${ }^{4}$

'CENTRO UNIVERSITÁRIO DO ESTADO DO PARÁ - CESUPA, BELÉM, PA, Brasil; ${ }^{2}$ CENTRO UNIVERSITÁRIO DO ESTADO DO PARÁ, BELÉM, PARÁ,

Brasil; ${ }^{3}$ UNIVERSIDADE FEDERAL DO PARÁ, BELÉM, PARÁ, Brasil; ${ }^{4}$ CESUPA, BELÉM, PARÁ, Brasil

Advances in Rheumatology 2018, 58(Suppl 1):P262

Background: Rheumatoid Arthritis (RA) has been associated with atherogenic lipid profile. Based on this, the objective is to identify the contribution of several factors to the lipid profile of patients with RA.

Methods: Is a descriptive and analytical cross-sectional of the study entitled "The risks and benefits of immunosuppressive therapy for the treatment of RA in the Amazon Region". It was carried out during the period from February 2014 to June 2016 at a reference center in Rheumatology in Belém, PA. 77 female patients aged over 18 years, diagnosed with RA, without lipid-lowering drugs and enrolled in this service participated in the study. The questionnaire applied contained the following data: age, disease duration, comorbidities, presence of Rheumatoid Factor or anti-CCP, BMl, drugs in use, inflammatory marker (ESR), laboratory tests with lipid profile (HDL, LDL and triglycerides), DAS 28 and HAQ. The classification used to assess the lipid profile in RA was based on the Brazilian Update of Dyslipidemia. DAS 28 was applied in order to classify the disease activity. Results and conclusions: A total of 77 women with mean age of 52.1 \pm 12.7 years and mean BMI of $27.5 \pm 5.1 \mathrm{~kg} / \mathrm{m}^{2}$ were studied. The disease duration had an average of $11.1 \pm 8.7$ years. The majority had cardiovascular risk factors $(70.1 \%, 54 / 77, \mathrm{p}=0.0006)$, being the main Systemic Arterial Hypertension and Dyslipidemia. In the evaluation of the lipid profile, the mean value of total cholesterol was 193.9 $\pm 50.3 \mathrm{mg} / \mathrm{dL}$, the $\mathrm{HDL}$ fraction equal to $49.1 \pm 15.9 \mathrm{mg} / \mathrm{dL}$; the mean $\mathrm{LDL}$ was $118.6 \pm 38.6 \mathrm{mg} / \mathrm{dL}$ and the mean triglyceride concentration was $153.7 \pm 80.9 \mathrm{mg} / \mathrm{dL}$. In the disease activity, mean DAS was $4.2 \pm 1.4$; it was possible to observe that the majority $(42.9 \%, 33 / 74, \mathrm{p}=0.0039)$ of the patients had a disease with moderate activity. The concentration of triglycerides was on average $83.0 \mathrm{mg} / \mathrm{dL}$ higher among patients with two risk factors present. There were no significant differences between the means of the lipids considering the positivity or not for the RF. Correlations between total cholesterol and HAQ $(r=0.4552, p=0.0380)$ and between LDL and HAQ $(r=0.5455, p=0.0105)$ were significant, both being positive and moderate. The other correlations were not statistically significant. The higher the number of cardiovascular risk factors and the greater functional limitation evaluated by the $\mathrm{HAQ}$, the worse the lipid profile of the patients. Therefore we should have a better control of these factors present in patients with RA, as well as an early diagnosis with disease activity control.

\section{P263}

\section{LOW LUMBAR PAIN IN MEDICAL STUDENTS}

Camille Tavares, Cintia Sophia Salvi, Carla Luchese, Jhésyca Castaman Stédile, Thiago Alberto Fernandes Gomes dos Santos, Thelma Laroca Skare HOSPITAL UNIVERITÁRIO EVANGÉLICO DE CURITIBA, CURITIBA, PARANÁ, Brasil

Advances in Rheumatology 2018, 58(Suppl 1):P263

Background: Low lumbar pain is one of commonest problem in modern society, affecting $80 \%$ of individuals. It is the most frequent cause of physical limitation in people under 45 years of age. Medical students, taking into account the hours of sitting position to study, are considered to be at risk for this problem. We aimed to study the prevalence of low lumbar pain in medical students and the influence of epidemiological and life habits on its appearance.
Methods: Cross sectional study with online questionnaireswith questions on epidemiological data, presence and frequency of lumbar pain, treatment options, exerciseand posture habits. Students with back pain were invited to fill the Rolland questionnaire that measured the interference of back pain in daily activities (0 to 10). They were also asked to graduate in a VAS (visual analogic scale) from zero to 10 on how much they fell depressed and tired.

Results:Six hundred twenty nine students $(67.2 \%$ females, $32.7 \%$ males, median age of 23 years old) answered the forms. The median number of hours in which they remain seated was of $8 \mathrm{~h} /$ day; only $56.3 \%$ practiced regular physical activity ( 1 to 3 times/week). In this sample, $82.5 \%$ referred having lumbar pain: $7.5 \%$ every day, $34.1 \%$ in one to 4 times/week; $42.1 \%$ in one to 4 times a month and $16.1 \%$ only occasionally. About $31.7 \%$ used regular pain medication: the most common was AINHs (44.0\%) followed by paracetamol (20.1\%). Bad posture habits were declared by $89.0 \%$ (most common: $80.7 \%$ reading, using computer and watching TV in bed; $50.5 \%$ - sleeping on the stomach; $43.8 \%$ - sleeping with arms up). Those who believed had bad posture habits had more lumbar pain than the others $(\mathrm{OR}=2.29 ; 95 \% \mathrm{Cl}=1.33-3.9 ; \mathrm{p}=0.002)$. According to them, lumbar pain interfered with school achievement in $70.9 \%$ of those with this symptom. The VAS scale on depression showed a median value of $6.0(\mathrm{IQR}=4-8)$ and the VAS of fatigue, a median value of 2 $(\mathrm{IQR}=0-4)$. The median value of Rolland questionnaire was 1.87 . There was a positive correlation of Rolland scale with depression (Rho $=0.32 ; 95 \% \mathrm{Cl}=0.24-0.40 ; \mathrm{p}<0.0001)$ and the fatigue scale (Rho=0.20; $95 \% \mathrm{Cl}=0.11-0.28 ; \mathrm{p}<0.0001)$.

Conclusion:There is a high rate of low lumbar pain among medical students. Medical institutions should be aware of this health issue and preventive measures are warranted.

\section{References \\ 1. Smith DR, et al. Musculoskeletal disorders among Chinese medical} students. Kurume Med J. 2005; 52:139-146.

\section{P266}

LUPUS ERYTHEMATOSUS IN THE ELDERLY-CASE SERIES

Francisco Fellipe Claudino Formiga ${ }^{2}$, Jade Arruda de Carvalho Motta ${ }^{2}$, Alexia Lavinia Holanda Gama². Ana Valesca L. Carvalho ${ }^{2}$, Danielle Christinne Soares Egypto de Brito², Ana Karla Guedes de Melo², Eutilia Andrade Medeiros Freire ${ }^{2}$, Alessandra de Sousa Braz ${ }^{2}$

${ }^{1}$ UNIVERSIDADE FEDERAL DA PARAÍBA, JOÃO PESSOA, PB, Brasil; ${ }^{2}$ UFPB, JOÃO PESSOA, PB, Brasil

Advances in Rheumatology 2018, 58(Suppl 1):P266

Background: Systemic lupus erythematosus (SLE) is a complex multisystemic disease characterized by a spectrum of organic manifestations that reach the extreme of women of reproductive age (15-45 years). Studies point to a considerable increase in the life expectancy of these patients, reaching a survival (10 years) above $90 \%$ while in the 1950 s it was only $50 \%$. As a consequence, more patients with SLE are reaching advanced ages. However, it is still considered rare in the elderly. Several age-related manifestations, such as cardiovascular disease or increased susceptibility to infections, are often found in patients with SLE (sequelae of disease or resulting from medication).

Objective: To describe the clinical profile of patients diagnosed with SLE with an age of 60 years and older accompanied at a university hospital. Materials and methods: Observational study (series of cases), information obtained through the collection and analysis of medical records data of patients 60 years of age and older who meet the SLICC classification criteria (2012), after consent of the patients.

Results and conclusions: Out of a total of 110 patients with outpatient follow-up, $12(10.9 \%)$ were 60 years of age or older (mean age 65 years) and met SLICC criteria (2012). All patients are female and mostly brown. Regarding the SLICC criteria, $91 \%$ presented articular manifestation, $75 \%$ cutaneous manifestation, oral ulcers in $25 \%$ and $16 \%$ presented alopecia and hematological manifestations. None of the patients had renal or central nervous system (CNS) involvement. Arterial hypertension, fibromyalgia and osteoarthritis were the main comorbidities observed. Although there are no studies in the literature specifically related to the elderly, the data 
obtained in this population are in agreement with large population studies in patients with late onset SLE ( $\geq 50$ years), which indicate that the presence of mild to moderate manifestations are factors associated with better prognosis in SLE patients. The authors call attention to the follow-up of elderly patients with SLE, in whom cardiovascular comorbidities, osteoarthritis, osteoporosis and neoplasias should be part of the management. The occurrence of fibromyalgia as one of the most associated comorbidities was also an unusual finding, since it is of rare occurrence in the elderly. With the increasing burden of disease in these patients in the future, further research should be conducted to determine the clinical profile and direct appropriate therapy in elderly patients with SLE.

\section{P270 \\ LUPUS NEPHRITIS IN A RHEUMATOLOGIC CENTER IN MATO GROSSO DO SUL, BRAZIL}

Maikon Augusto V. A Florenciano', Maikon Augusto V. A Florenciano', Paulo César V. Souza Júnior', Marcelo O. Coutinho', Tatiana Galdino Leal ${ }^{2}$, Marcia Midori Shinzato ${ }^{3}$

'UNIVERSIDADE FEDERAL DA GRANDE DOURADOS, DOURADOS, MS, Brasil; ${ }^{2}$ HOSPITAL UNIVERSITÁRIO DA UNIVERSIDADE FEDERAL DA GRANDE DOURADOS, DOURADOS, MATO GROSSO DO SUL, Brasil; ${ }^{3}$ HOSPITAL UNIVERSITÁRIO DA UFGD, DOURADOS, MATO GROSSO DO SUL, Brasil

Advances in Rheumatology 2018, 58(Suppl 1):P270

Background: Nephritis is still one of the most devastating complications of systemic lupus erythematosus (SLE) despite the availability of new therapeutic regimens. Clinical features and response to treatment show wide variations in patients with different ethnic background. Therefore we aimed to described the characteristics of patients with lupus nephritis (LN) from a rheumatologic centre in Mato Grosso do Sul state, Brazil

Methods: Demographic, clinical and laboratory features of SLE patients, $\geq 18$ years old, diagnosis by rheumatologists were collected by chart review between march 2017 and march 2018. SLE Disease Activity Index (SLEDAI), Systemic Lupus Damage Index (SDI) were also obtained. Lupus nephritis (LN) was defined according to the definitions of the 2004 revised American College of Rheumatology (ACR) renal criteria in SLE. Patients with lupus nephritis was compared with those SLE without LN. We exclude patients with diseases onset before 16 years old. Categorical and continuous variables were analysed by qui-squared or Fisher' exact and t Student or Mann Whitney tests, respectively. Results were considered significant at two-tailed $p<$ 0.05.The study was approved by local ethic committe

Results: Seventy-one patients with SLE diagnosis were current attending the rheumatology outpatients clinic of the University Hospital of Grande Dourados University. Of these patients 4 (5.6\%) did not fulfilled the 1982 updated in 1997 ACR classification criteria for SLE but met the criteria of Systemic Lupus International Collaboration Clinics. The mean age of SLE patients was $37.96( \pm 12.81), 64(90.1 \%)$ were female and $32(45.1 \%)$ were white. LN was present in $35(49.3 \%)$ only $3 / 35(8,6 \%)$ underwent a kidney biopsy, 2 with class $V$ and 1 with class IV glomerulonephritis. One (2.9\%) developed chronic kidney diseases. Mean age $(34.23 \pm 12.14)$ and disease duration $(6.86$ \pm 4.76 ) of patients with LN were significantly lower than the mean age (41.58 \pm 12.55$), \mathrm{p}=0.014$, and diseases duration (10.33 $\pm 6.03 \%)$, $\mathrm{p}=0,0089$, of SLE without nephritis. LN patients also had a higher SLEDAI score before therapy $(11.76 \pm 5.34$ vs. $8.85 \pm 4.67 \%, p=0.019)$. More than a half $(55.5 \%)$ of $L N$ patients were white. While among SLE without $\mathrm{LN}, 34,3 \%$ were white, $\mathrm{p}=0,075$. We also observed a trend for the association of presence of anti-ds-DNA and diabetes mellitus with LN.

Conclusion: LN developed in quite a half of adult SLE patients. LN patients were younger and we observed a higher proportion of white subjects in SLE with LN than in the group without LN.
P271

LUPUS ORAL INJURIES: RESULTS FROM A PILOT STUDY

Rodrigo Rezende Reis Sepini, Rayla Cristina da Costa Ferreira, Maria das Graças Afonso Miranda Chaves, Antônio Scafuto Scotton, Viviane

Angelina Souza, Gisele Maria Campos Fabri

HOSPITAL UNIVERSITÁRIO DA UFJF, JUIZ DE FORA, MINAS GERAIS, Brasil Advances in Rheumatology 2018, 58(Suppl 1):P271

Background: Systemic lupus erythematosus (SLE) is a chronic and inflammatory autoimmune disease frequently associated with infectious factors, including some oral pathogens.

Methods: This pilot study aimed to identify the oral conditions of SLE patients, characterizing maxillofacial complaints and some oral diseases that could correlate with SLE activity and damage. The assessment of SLE patients included demographic data, SLE activity/ damage scores (SLEDAI and SLICC/ACR), dental history and systematic physical examination of stomatognatic structures, xerostomia and salivary flow evaluation.

Results: Eleven consecutive SLE patients were evaluated. The median age was 44 years (29-66) and all were female. The low socioeconomic status was prevalent $(72.73 \%)$. The time of diagnosis ranged from 1 to 20 years and all patients were in use of hydroxychloroquine. The most prevalent comorbidities were arterial hypertension $(45,45 \%)$ and depression (36, 36\%). The median SLEDAI was $2(0-16)$ and SLICC was $1(0-2)$. The laboratorial results showed median hemoglobin 12 , 68 (10, 40-13, 50), complement C3 103 (25-172) and C4 16 (5-43). Nine $(81.82 \%)$ patients had some kind of orofacial pain. The median of Decayed, Missing, and Filled Teeth (DMF-T) Index was 19.6 (7-32). The most prevalent gingival index (IG) was 0 in $5(45.45 \%)$ patients, followed by 1 in 4 (36.36\%). The salivary flow analysis revealed that 6 (54.6\%) patients had reduced salivary flow without stimulation, while $3(27.27 \%)$ presented reduced flow when stimulated, and $9(81.82 \%)$ individuals reported symptoms of xerostomia. Regarding the Helckimo index, $3(27,27 \%)$ patients had severe mandibular dysfunction and $4(36,36 \%)$ moderate dysfunction. The correlation analysis revealed an association between gingival index and SLICC ( $R=0,632$; $\mathrm{p}=0,048)$.

Conclusion: Despite the small sample size, it is evident that the SLE patients assessed showed critical oral damage with extensive dental loss (high DMF-T index), prevalent orofacial pain and moderate to severe mandibular dysfunction. In addition, SLE damage index was associated with gingival inflammation, which calls attention to the possible association between SLE and some oral pathogens that lead to oral infections. The continuation of the study is important to identify the bidirectional association of oral findings with SLE activity/ damage.

\section{P279}

\section{METHOTREXATE IN JUVENILE IDIOPATHIC ARTHRITIS:} VARIABLES ASSOCIATED WITH REMISSION AND TREATMENT INTOLERANCE

Ana Carolina Santos Londe, Roberto Marini, Simone Appenzeller UNICAMP, SP, SP, Brasil

Advances in Rheumatology 2018, 58(Suppl 1):P279

Background: Methotrexate is the first-choice disease-modifyng antirheumatic drug (DMARD) for the treatment of juvenile idiophatic arthritis (JIA). The objective of our study was to evaluate the reason for MTX interruption, through medical charts and the MTX Intolerance Severity Score questionnaire (MISS).

Methods: We included 60 patients with JIA (median age $15.4 \pm 7.5$ years), in use for least 3 months of MTX, followed at pediatric rheumatology unit. All the patients answered the MISS. Their medical charts were reviewed for age, sex, subtype of JIA, response to MTX (yes/no) and if there were adverse effects (yes/no) and the reason for discontinuation of MTX (intolerance/remission). The MISS was translated into Portuguese following the "Guidelines for the process of 
cross-cultural adaptation of self-report measures". The MISS consists of 4 domains: stomachache, nausea, vomiting, and behavioral complaints. Each domain includes three to four items, and for every item, four answers are possible: no complaints (0), mild complaints (1 point), moderate complaints ( 2 points), and severe complaints (3 points). The points are summed to give a total score from 0 to 36 MTX intolerance is defined by a score of 6 or more, with at least 1 point in the anticipatory and/or associated and/or behavioral symptoms.

Results: Sixty-eight (69\%) JIA patients reported the presence of sporadic episodes of adverse reactions during treatment. Episodes of adverse reactions related to MTX was mainly gastric intolerance (abdominal discomfort, nausea and vomiting). The reasons for JIA patients discontinue treatment with MTX was presence of side effects (35\%), interruption on their own decision (27\%), lost follow-up (12\%) and interruption of medication distribution (12\%), improvement of the disease (11\%), and inefficacy (6\%). All of patients answered MISS with less than 5 minutes. We also observed that patients taking MTX over a long term had more adverse reactions when compared to patients with only 6 months $(p<0.05)$. All patients who discontinued MTX on their own decision had additionally the presence of adverse reactions.

Conclusion: MISS is a good tool for physicians, because it can not only measure the intolerance, but also explore the different forms in which it manifests. Although MTX has a great therapeutic index, the adverse reactions are still seen as a major form of abandonment of this pharmacological treatment. Therefore a careful history is essential to identify side effects and adequate treatment to increase adherence.

\section{P280}

\section{MEVALONATE KINASE SECONDARY CARDIOMYOPATHY: NON-} RECOGNIZED COMPLICATION OR PHARMACOLOGICAL ADVERSE REACTION?

Luis Miguel Hincapié Rubio ${ }^{1}$, Christine Arango Slingsby²,

Lina María Saldarriaga Rivera

${ }^{1}$ CLINICA LOS ROSALES, PEREIRA, RISARALDA, Colômbia; ${ }^{2}$ CLINICA

COMFAMILIAR, PEREIRA, RISARALDA, Colômbia

Advances in Rheumatology 2018, 58(Suppl 1):P280

Background: Hyperimmunoglobulinemia-D syndrome (HIDS) is a rare disease of the mevalonate kinase deficiency spectrum, member of the autoinflammatory syndromes (AS). It is caused by a mutation in the MVK and characterizes by febrile episodes lasting 3-7 days, generalized cutaneous rash, oral-vaginal aphthous ulcers, cervical lymphadenopathy, severe headache, splenomegaly, conjunctivitis and arthritis. The most feared complication of the AS is the development of amyloidosis. There is an estimated of 200-400 cases reported to date, mostly observed in people from the Netherlands. Its occurrence in Latin-America is more than extraordinary.

Case Report: A 20-year-old female patient previously diagnosed with HIDS (patient's gene mutations p.lle298Thr and p.His197Asp presented to the emergency department with a 3 days history of progressive dyspnea, edema of the legs, face and abdomen, orthopnea, palpitations and fatigue. On physical examination a patient in bad general condition with mucocutaneous pallor, labial cyanosis, hypopigmented plaques on anterior thorax and scalp, low intensity- rhythmic cardiac sounds, lower extremity edema, diminished distal pulses, bilateral lung basal crackles on auscultation and hepatosplenomegaly was found. No other abnormalities were observed. An echocardiography was then performed in which large dilated right cavities, diminished global contraction, left ventricle ejection fraction of $40 \%$, discrete pericardial effusion and pulmonary artery systolic pressure of $43 \mathrm{mmHg}$ were found. Brain natriuretic peptide levels of 10.447 (pg/ $\mathrm{ml}$ ) was also obtained. The hypothesis of cardiac amyloidosis was the most obvious possible cause, but cardiac magnetic resonance didn't show signs of infiltration, and cardiac muscle biopsy was negative for the disease. Tuberculosis, HIV, thyroid and autoimmune disease were ruled out. Other potential causes were also ruled out. The possibility of a pharmacological adverse reaction to anakinra, which was being administered for the last 4 months to control the disease, was then looked for in the literature, retrieving only one case report with a temporal association between the substance and heart failure (no pathologic changes were found on that report). After medication adjustment for heart failure, she returned to her daily activities.

Conclusion: Occurrence of HIDS in Latin-America is more than rare. Possible complications such as amyloidosis should be looked for in patients who present with heart/renal failure. Infectious, endocrine, granulomatous and autoimmune causes should be ruled out. Drug adverse reactions should always be looked for, but correlation is often problematic. The p.His197Asp could be an explanation to this non-recognized complication since hasn't been yet reported nor characterized, so it shall remain as an hypothesis.

Consent for publication

The authors declare that they have obtained informed written consent from the patient's tutors for publication

\section{P282}

MICROSCOPIC ASPECTS OF MINOR SALIVARY GLANDS IN PATIENTS WITH RHEUMATOID ARTHRITIS AND SUSPECTED SJÖGREN'S SYNDROME

Camila Nunes Carvalho Sorgato, Luiz Alcino Monteiro Gueiros,

Jair Carneiro Leão, Marilia Lins E Silva, Andrea Tavares Dantas,

Aysa Cesar Pinheiro, Paula Regina Toche Dos Santos, Angela Luzia

Branco Pinto Duarte

UFPE, RECIFE, PE, Brasil

Advances in Rheumatology 2018, 58(Suppl 1):P282

Background: Sjögren's syndrome (SS) is characterized by the presence of focal lymphocytic inflammatory infiltrate in the salivary glands, resulting in reduction of secretory function and consequent oral dryness. The biopsy of minor salivary glands represents an important step for the diagnosis of SS, being considered a safe and high specificity method. The objective of this study was to evaluate the morphological characteristics of minor salivary glands of patients with rheumatoid arthritis (RA) and suspicion of SS, correlating them with clinical aspects.

Methods: We conducted an epidemiological and observational study of 55 individuals with RA and suspected SS. After the minor salivary gland biopsy and definition of the diagnosis, the patients were divided into 2 groups: RA group - Rheumatoid arthritis $(n=33)$ and AR/ SS group - Sjögren syndrome associated with rheumatoid arthritis $(n=22)$. In addition to the biopsy, the patients were submitted to salivary flow at rest and Schirmer's test.

Results and Conclusions: Individuals with SS presented higher age $(p=0.012)$. The AR/SS group had a lower Schirmer test mean and a higher frequency of positive microscopic SS biopsy. The markers of RA activity were not influenced by the presence of $S S(p>0.05)$. Ductal dilation and atrophy are common features in patients with SS. When the microscopic aspects were compared to the clinical criteria, there was an association between the presence of ductal dilatation and resting salivary flow $(p=0.033)$ and positive biopsy $(p=0.044)$. For the measures of accuracy, the following results were obtained: sensitivity $(45 \%)$, specificity $(87 \%)$, positive predictive value $(71 \%)$ and negative predictive value $(70 \%)$. The biopsy of the minor salivary glands presents high specificity, but low sensitivity. Morphological aspects alone do not seem to influence the clinical presentation of RA and SS.

\section{P287}

MORBIMORTALITY OF RHEUMATIC FEVER AND RHEUMATIC VALVULOPATHY IN THE PERIOD 2008 TO 2017 IN THE STATE OF PARÁ

Vitória Silva Rodrigues ${ }^{1}$, Leonardo Teixeira De Mendonça², João Pedro Nunes Aquime ${ }^{3}$, Caio Henrique De Souza Almeida ${ }^{4}$, Ana Carolina Tavares Da Fonseca ${ }^{5}$

${ }^{1}$ FACULDADE METROPOLITANA DA AMAZÔNIA-FAMAZ, BELÉM, PA, Brasil; ${ }^{2}$ FACULDADE METROPOLITANA DA AMAZÔNIA FAMAZ, BELÉM, PA, Brasil; ${ }^{3}$ CENTRO UNIVERSITÁRIO DO ESTADO DO PARÁ CESUPA, BELÉM, PA, Brasili ${ }^{4}$ CENTRO UNIVERSITÁRIO DO ESTADO DO PARÁ, BELÉM, PA, Brasil; ${ }^{5}$ FACULDADE METROPOLITANA DA AMAZONIA FAMAZ, BELÉM, PA, Brasil

Advances in Rheumatology 2018, 58(Suppl 1):P287 
Introduction: Rheumatic fever (RF) is an acute non-suppurative complication of pharyngotonsillitis, caused by group A beta-hemolytic streptococcus, being a late immune response in genetically predisposed populations. Carditis is the most severe manifestation, leading to chronic, highly debilitating and costly chronic rheumatic heart disease (CRHD).

Methods: The objective of this study was to evaluate the morbidity and mortality of acute rheumatic fever and rheumatic valvulopathy in the state of Pará from 2008 to 2017, through a descriptive and cross-sectional study. The online platform TABNET linked to DATASUS was used for data collection.

Results and conclusion: The total number of hospitalizations for RF in the period was 2,682, with a predominance of females (1451 cases -54\%). There was an increase in cases between 2008 (173) and 2013 (338), with a progressive decrease until 2017 (85). The range between 5-19 years was responsible for 662 cases (24\% of the total), with the remainder distributed among other age groups, especially within the range of 20 to 69 years (68\%).

CRHD hospitalizations were 1,524 , with a significant decrease from 2008 to 2013 (235 to 126 cases), with an increase from 2013 to 2016 (126 to 144 cases). Deaths by CRHD in this decade were 144 , with a mortality rate of 9.53 . The majority of admissions were female, 874 cases $(57.3 \%)$, and the predominant age group was between $20-59$ years, with no difference within this range. In relation to the number of deaths, there was a progressive increase according to the decade of life, 30-39 years with 22 cases (15.2\%), 27 cases between 40-49 years (18.7\%), 50-59 years with 28 cases (19.4\%) and 32 deaths in the range of $60-69$ years (22.2\%). Regarding the costs (in millions) of the FR, there was a progressive increase from 2008 to 2011 (42 to 127), with a decrease in the following years reaching 24 million in 2017, different from the cost of the CRHD, which showed a stable cost ranging from 160 to 210 million.

It was observed that RF and CRHD continue to present high morbimortality rates besides high cost. Epidemiological change in recent years, with fewer hospitalizations due to RF and increase in cases of chronic rheumatic valvulopathy especially until the age of 29 , demonstrates a return of undiagnosed cases without correct prophylaxis, showing the importance of applying the new modified criteria to the current reality.

\section{P292}

\section{MYTHS AND TRUTHS ABOUT OSTEOPOROSIS}

Laís Farrapo de Barros Leite, Denise Moraes Horiy, Bernardo Patrício Sequeira Dultra, Ana Clara Ribeiro, André Ozela Augusto, Dayrana Alves Lucena, Elaine de Azevedo, Rina Dalva Neubarth Giorgi HOSPITAL SERVIDOR PÚBLICO ESTADUAL-SP, SÃO PAULO, SÃO PAULO, Brasil

Advances in Rheumatology 2018, 58(Suppl 1):P292

Background: It is known that osteoporosis is a very prevalent disease in our population, especially in postmenopausal women, being the major cause of fractures in the elderly and being related to high morbimortality rates.

Patients' perception of this disease usually encompasses many popular myths and beliefs, making it difficult to adhere to long-term treatment.

Methods: In the present study, a questionnaire with 20 questions, called "QUIZ-Myths and Truths about Osteoporosis", was applied in 74 follow-up patients in the ambulatory of osteometabolic diseases of the Rheumatology service of a tertiary state hospital in São Paulo. The questionnaire includes topics related to popular beliefs about osteoporosis and aims to evaluate the understanding of patients who are undergoing treatment.

Results and Conclusions: More than $90 \%$ of interviwed know that having Osteoporosis does not contraindicate physical activity and that this habit brings strength to the bones. About $60 \%$ are aware that only calcium and vitamin $\mathrm{D}$ are not sufficient for treatment and that milk is not the only source of calcium. About $2 / 3$ of the patients understand that the disease has a family component, $70 \%$ know that it has no cure, but it can be prevented and more than $80 \%$ understand that sunbathing is one of the prophylactic factors.

In addition, more than $2 / 3$ of these people know that the disease is not exclusive to the elderly, and men and obese individuals may also have Osteoporosis. Almost $80 \%$ of the subjects are aware that women should not worry about osteoporosis only after menopause and that the diagnosis can be made before the occurrence of fractures. About $70 \%$ see that smoking is an important risk factor for the development of the disease.

However, it has been noted that just over half of the people surveyed believe that osteoporosis is related to pain and almost $2 / 3$ of them think that calcium brings relief to it. Nearly $66 \%$ think that all osteoporotic fractures are painful and $40 \%$ believe that they only occur after falls.

With the results obtained through the questionnaire, it was possible to conclude that although there are still many beliefs and myths about the subject in the general population, most patients interviewed have good knowledge and understanding about osteoporosis, probably because it is in a specialized service.

\section{P297}

OCCURRENCE OF GENERALIZED PAIN EXTENSION SYNDROME (FIBROMYALGIA) IN PATIENTS WITH SYSTEMIC ERITEMATOSUS LUPUS AND ASSESSMENT OF THEIR QUALITY OF LIFE

Jade Arruda de Carvalho Motta², Alexia Lavinia Holanda Gama², João Calvino Soares de Oliveira ${ }^{2}$, Matheus Vieira Falcão²,

Ana Karla Guedes de Melo², Danielle Christinne Soares Egypto de Brito², Eutilia Andrade Medeiros Freire ${ }^{2}$, Alessandra de Sousa Braz

${ }^{1}$ UNIVERSIDADE FEDERAL DA PARAÍBA, JOÃO PESSOA, PB, Brasil; ${ }^{2}$ UFPB, JOÃO PESSOA, PB, Brasil

Advances in Rheumatology 2018, 58(Suppl 1):P297

Introduction: The prevalence of Fibromyalgia (FM) in patients with Systemic Lupus Erythematosus (SLE) is higher compared to the general population, around $21 \%$. The presence of FM can represent a confounding factor both for diagnosis and for SLE therapy, due to the similarities of some clinical and epidemiological characteristics of both.

Objectives: To analyze the occurrence of fibromyalgia in patients with SLE and to evaluate the impact of this comorbidity on patients' quality of life.

Methods: This is a descriptive, observational and cross-sectional study. The sample consisted of patients diagnosed with SLE according to the classification criteria of Systemic Lupus International Collaborating Clinics (SLICC, 2012), followed in a Rheumatology outpatient clinic. Data collection was performed based on the active search for FM classification/diagnostic criteria according to the American College of Rheumatology (ACR) 1990 and 2010. The SF-36 Quality of Life Assessment questionnaire was applied to all participants, and the Fibromyalgia Impact Questionnaire (FIQ) in patients classified as having FM. The data were analyzed through descriptive statistics.

Results and conclusions: the sample consisted of 110 patients with SLE, composed of $95.4 \%$ women and mean age 54.1 years (SD: 12.1). After applying the criteria, 17 patients were classified as having FM (ACR-1990), as well as 21 by the 2010 criteria, totaling an FM occurrence of $19.1 \%$. All patients diagnosed with the syndrome were females and had a mean age of 45.6 years (SD: 9.6). The evaluation of the quality of life through SF-36 showed lower scores in patients with FM in all 8 domains, when compared to individuals with SLE without FM, especially in physical and emotional aspects. The mean FIQ score was 66.1 (SD: 12). The results were compatible with the literature, showing that FM is a common and underdiagnosed comorbidity among SLE patients and that it has an important negative impact on their quality of life. Considering the great sociocultural impact of chronic pain syndromes, the correct investigation of FM guides proper management of symptoms among SLE patients, especially pain, avoiding iatrogenic drug use and resulting in adequate therapy, which has modifying the quality of life of patients. 


\section{P299}

OLFACTORY DYSFUNCTION IN SYSTEMIC SCLEROSIS. A

LONGITUDINAL STUDY

Mariana Freschi Bombini, Fernando A. Peres, Nailu A. Sinicato, Aline

Tamires Lapa, Roberto Medeiros Souza, Paula Teixeira Fernandes,

Fernando Cendes, Ana Paula del Rio, João Francisco Marques-Neto,

Tiago Nardi Amaral, Leticia Rittner, Simone Appenzeller

UNICAMP, CAMPINAS, SP, Brasil

Advances in Rheumatology 2018, 58(Suppl 1):P299

Background: Several factors has been described as responsible for the association between olfactory impairment and brain regions. In systemic sclerosis (SSc) the olfactory interaction has been little explored.

To evaluate olfactory function in SSc over a 2-year period, and to determine the association of olfactory dysfunction with age, disease activity, disease damage, treatment, anxiety and depression symptoms and limbic structures volumes.

Methods: Consecutive SSc patients were enrolled in this study. Clinical, laboratory disease activity and damage were assessed according to diseases specific guidelines. Olfactory functions were evaluated using the Sniffin'Sticks test (TDI). Mood disorders were evaluated through Becks depression and anxiety Index. Volumetric magnetic resonance imaging (MRI) was obtained in a $3 \mathrm{~T}$ Phillips scanner. Amygdalae and hippocampi volumes were analyzed using FreeSurfer $^{\circledast}$ software.

Results: We observed that olfactory dysfunction was more frequent in patients than controls [21 (60\%) vs $12(30 \%)(p=0009)]$, remaining stable throughout this study. The presence of olfactory dysfunction was associated with age $(p<0.001)$, disease activity $(p=0.037)$ and its duration $(p=0.010)$, and left and right hippocampus $(p=0.014$; $p=0.01)$ and right amygdala's volumes $(p=0.04)$. Using linear regression, we observed that the TDI was correlated with anxiety symptoms $(p=0001)$ and left hippocampal volume $(p=0006)$ and right amygdala volume $(p=0050)$.

Conclusion: Olfactory dysfunction is more frequent in patients with SSc than controls. We observed that age, mood disorders, amygdala and hippocampal volumes were predictors for olfactory dysfunction.

\section{P302}

\section{ORTHOPEDIC SURGERIES IN PATIENTS WITH RHEUMATOID} ARTHRITIS: DATA OF A COHORT IN BRAZIL

Ana Paula Monteiro Gomides', Amanda Cristina de Souza ${ }^{2}$, Rebeca Naoum Lorga Roque 2 , Mariana Carvalho Gomes², Matheus Moreno de Oliveira ${ }^{2}$, Ana Carolina Gomes Siqueira ${ }^{2}$, Licia Maria Henrique da Mota ${ }^{3}$, Luciana Feitosa Muniz ${ }^{3}$, Talita Yokoy de Souza ${ }^{3}$, Viviane Cristina Uliana Peterle ${ }^{2}$, Luciano Junqueira Guimarães ${ }^{3}$, Regina Alice Fontes Von Kirchenheim ${ }^{4}$, Andressa Junqueira Osório ${ }^{3}$, Luciana Teófilo Lourençoni ${ }^{3}$, Isabela de Sousa Russo ${ }^{3}$, Tassiane Raquel Cunha Martins de Moraes ${ }^{3}$

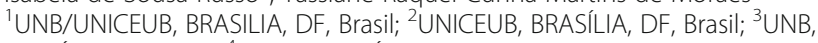
BRASÍLIA, DF, Brasil; ${ }^{4}$ SESDF, BRASÍLIA, DF, Brasil

Advances in Rheumatology 2018, 58(Suppl 1):P302

Introduction: Rheumatoid arthritis is a chronic, autoimmune inflammatory disease with a prevalence of approximately $1 \%$ in Brazil and worldwide. It affects mainly women and range in the age group of 30 to 50 years. The treatment can be medicated (symptomatic, anti-inflammatory, disease-modifying drugs, synthetic or biological), or non-medicated, consisting of physical therapy, exercise, occupational therapy and surgeries. Surgical treatment is necessary when conservative clinical and physiotherapeutic measures are no longer effective in controlling symptoms, or do not allow minimum levels of activities of daily living, such as working, domestic activities, walking for 30 minutes and independence. When instituted, surgical treatment should be done early, preventing the involvement of several joints.

Methods: Patients with rheumatoid arthritis were followed up at a University Hospital in an initial RA cohort.
The participants were submitted to clinical evaluation, analysis of complementary exams and medical records.

The work was approved by the Ethics Committee and the patients signed the informed consent form.

Results And Conclusions: 107 patients with RA, with a mean disease time of 12.8 years and 83 patients $(77.57 \%)$ had a positive rheumatoid factor. The mean SDAl was 8.64 (median 4.39).

The orthopedic surgeries performed are shown in Table 1.

Surgical intervention when indicated, if performed early, may prevent sequelae and cause pain and mobility improvement. The correct indication of these procedures should be remembered in the management of the severe patient so that, in many cases, they contribute with an improvement in the quality of life.

Table 1 (abstract P302). Surgeries performed in a cohort with initial AR

\begin{tabular}{lll}
\hline ORTHOPEDIC SURGERY & N & $\%$ \\
\hline SINOVECTOMY & 4 & $3,7 \%$ \\
TENOSIVECTOMY & 2 & $1,80 \%$ \\
ARTHROPLASTY & 5 & $4,6 \%$ \\
IMPLANTATION OF PROSTHESIS & 2 & $1,8 \%$ \\
OTHER SURGERY & 11 & $10,2 \%$ \\
NOT PERFORMED SURGERY & 83 & $77,5 \%$ \\
Total & 107 & $100,0 \%$ \\
\hline
\end{tabular}

\section{P306}

OVERLAPPING SYNDROME OF RHEUMATOID ARTHRITIS AND

\section{SYSTEMIC SCLERODERMA: A CASE REPORT}

Andreia Coimbra Sousa, Lynara Pinheiro Moraes, Raquel Moraes da

Rocha Nogueira, Thais Silva de Jesus, Tassia Oliveira Nunes da Silva,

Fernando César Costa da Silva, Adriana Medeiros Lima, Braulio Nunes da Souza Martins Filho

UNIVERSIDADE CEUMA, SÃO LUÍS, MA, Brasil

Advances in Rheumatology 2018, 58(Suppl 1):P306

Background: Rheumatoid arthritis (RA) and systemic scleroderma (ES) are autoimmune diseases, with a chronic and progressive inflammatory character. The presence of two or more connective tissue diseases in the same individual characterizes a superposition syndrome. These have been described in rheumatology, however, the coexistence of rheumatoid arthritis and scleroderma is not common in clinical practice.

Case Report: A.P.S., 43 years old, female, with rheumatoid arthritis diagnosed 9 years ago. On previous use of prednisone $20 \mathrm{mg} /$ day, but with irregular follow-up. For 1 year, the condition developed with worsening of arthralgia, morning stiffness, progressive dysphagia, and comsuptive syndrome with a $30-\mathrm{kg}$ weight loss in 10 months. She sought outpatient services at a referral hospital for oncological diseases where she was hospitalized for 6 days to investigate a possible neoplastic cause. Adequate investigation was performed, with no findings indicating neoplasia. She was referenced for a follow-up with a rheumatologist for diagnostic elucidation and treatment. At the physical examination, the patient had symmetrical cutaneous thickening in the proximal region of metacarpophalangeal, microstomia, Raynaud's phenônem, salt and pepper lesions in the palmar region, sclerodactyly and "swan fingers" in both hands. Laboratory tests: AntiCCP reagent (406.7); Anti-SCL 70 and Anti-Centromere negative. Left sided lesion biopsy compatible with scleroderma. Initiated pulse therapy with methylprednisone 1 $\mathrm{g} /$ day for 3 consecutive days, followed by the use of cyclophosphamide $1 \mathrm{~g} /$ month. It evolved with improvement of dysphagia, thickening of the skin and weight gain. Receiving discharge after clinical stabilization and referred to outpatient follow-up. 
Conclusion: Systemic sclerosis is the disease that is most often associated with other diffuse connective tissue disease. However, not always the diagnostic conclusion is obvious, as in the case reported, which evidenced an ES with Anti-Scl 70 and Anti-Centromere negative.

\section{Consent for publication}

The authors declare that they have obtained informed written consent from the patient's tutors for publication

\section{P309}

\section{PACHYDERMOPERIOSTOSIS: A CASE REPORT}

Bruna Laiza Fontes Almeida', Maria Angélica Sampaio Herculano', José Herculano Da Silva ${ }^{1}$, Anna Luísa Marinho De Andrade ${ }^{2}$

João Rodrigues De Araújo Neto ${ }^{3}$

${ }^{1}$ FACULDADE INTEGRADA DE PATOS, PATOS, PB, Brasil; ${ }^{2}$ UNIVERSIDADE FEDERAL DA PARAÍBA, JOÃO PESSOA, PB, Brasil; ${ }^{3}$ UNIVERSIDADE FEDERAL DE CAMPINA GRANDE, CAMPINA GRANDE, PB, Brasil Advances in Rheumatology 2018, 58(Suppl 1):P309

Introduction: Pachydermoperiostosis is a rare, idiopathic, benign, selflimiting genetic syndrome. It is characterized by progressive enlargement of the joints, leading to arthritis or arthralgia, associated with digital clubbing, periostosis in the distal diaphyses of long bones, pachydermia (thickening of the skin on the face and scalp), acrosteolysis, soft tissue and glands hypertrophy, mainly in face and scalp, seborrhea, hyperhidrosis and acne vulgaris.

Case report: A 32-year-old male patient, presenting polyarthralgia for 3 years associated with deformity, post-rest stiffness greater than 30 minutes, and edema in the knees, ankles, wrists and shoulders that did not yield to the use of anti-inflammatory drugs. He also complained of irregular skin thickening, especially in the face, associated with recurrent episodes of acneiform lesions and progressive exophthalmos. Physical examination revealed arthritis in knees, digital clubbing and skin thickening on the fingers (Fig. 1) and leonine facies (Fig. 2). The complementary evaluation had erythrocyte sedimentation rate (ESR) of $45 \mathrm{~mm} / \mathrm{h}$, normal thyroid function, normal growth hormone $(4 \mathrm{ng} / \mathrm{mL})$, negative VDRL and negative autoantibody (rheumatoid factor and antinuclear factor). The $x$-ray of the long bones showed thickening of the cortical layers with enlargement of the diaphysis. There was also a continuous lamellar periosteal reaction and preserved joint spaces (Figs. 3 and 4). The chest X-ray was normal, and Methotrexate (15mg/week) associated with Folic Acid (5mg/week) and Prednisone (10mg/day) were started for 6 months, with no satisfactory clinical response. Methotrexate was suspended and Colchicine was prescribed (1 mg/day) and intra-articular infiltration was performed on knees with triamcinolone. The patient developed a satisfactory response to Colchicine with improvement in relation to the joint complaints.

Conclusion: Because it is a rare pathology in which is difficult to diagnose and without specific treatment, it is important to know its clinical manifestations. Therefore, instruments such as detailed anamnesis, laboratory and radiological examinations are necessary to discard secondary causes and for the diagnosis to be made at an early stage, in order to alleviate clinical symptoms and improve their quality of life.

\section{Consent for publication}

The authors declare that they have obtained informed written consent from the patient's tutors for publication

\section{P310}

\section{PAIN, QUALITY OF LIFE AND COPING STRATEGIES IN} FIBROMYALGIC PATIENTS

Johnathan Gabriel Rodrigues Apolônio', André Gama Palone', Betânia Longo², Jhésyca Flávia Sousa Castaman Stédile², Sílvio Almeida de Lima², Clemilson Sombrio Gomes ${ }^{3}$, Cloves de Amissis Amorim ${ }^{3}$, Patricia Martin ${ }^{4}$ 'ESCOLA DE MEDICINA DA PUC, CURITIBA, PR, Brasil; ${ }^{2}$ HOSPITAL UNIVERSITÁRIO EVANGÉLICO DE CURITIBA, CURITIBA, PR, Brasil; ${ }^{3}$ FACULDADE DE PSICOLOGIA DA PUC, CURITIBA, PR, Brasil; ${ }^{4}$ ESCOLA DE MEDICINA DA PUC/HOSPITAL UNIVERSITÁRIO EVANGÉLICO DE CURITIBA, CURITIBA, PR, Brasil

Advances in Rheumatology 2018, 58(Suppl 1):P310
Introduction: Fibromyalgia is a chronic musculoskeletal disease with unknown etiology and is characterized by generalized pain, fatigue and other functional symptoms. Studies suggest that patients experiencing chronic pain develop ways to tolerate, minimize or reduce their pain, a process known as coping. Therefore, the present study aims to investigate whether the different coping strategies have an impact on the quality of life and pain intensity of patients with fibromyalgia.

Methods: Cross-sectional study evaluating 34 fibromyalgia female patients classified according to the criteria of the American College of Rheumatology (2011), randomly selected in outpatient rheumatologic public clinic. Demographic, social and clinical information was collected to better characterize the sample. The 12-Item Short-Form Health Survey (SF-12) and the Coping Strategies (CSQ) Questionnaires, already validated for Brazil, were applied. Pain intensity was also evaluated through visual analogue scale. All patients gave their informed consent to participate of the study.

Results: The median age was 54 years, ranging from 38 to 69 years. The family income is up to 2 minimum Brazilian wages for $42.9 \%$ of patients; from 2 to 4 for $38.1 \%$; and from 4 to 10 for $19 \%$ and $52.1 \%$ of the patients had completed the second phase of elementary education. Regarding domestic violence, $52.4 \%$ reported having suffered some type of violence and $23.8 \%$ reported living with a chemical dependent patient at home. In relation to pain intensity assessed by visual analogue scale (VAS), the median was $7.2 \mathrm{~mm}$, ranging from 1 to $10 \mathrm{~mm}$. The most used coping strategies were praying/waiting, followed by self-assertions of coping and ignoring painful sensations. There was a positive correlation between diversion and pray strategies with pain intensity $(r=0.41, p$-value 0,025 and $r=0.37, p=0.046$ respectively) and a negative correlation between catastrophizing and mental component of SF12 $(r=-0.40, p=0.03)$.

Discussion: Consistent with the literature, family violence and the presence of chemical dependents in the family were triggers for the development of the disease. Domains considered maladaptive as catastrophizing, did not result in greater pain intensity, however, it had a negative impact in quality of life. In our study, we found the pray domain associated with higher pain indexes, which may indicate less active behavior of the patients in the use of this coping resource. Unlike expected, the domain diversion was maladaptive in our sample because patients who use this coping strategy felt more pain.

\section{P312}

PANCREATITIS AND RHABDOMYOLYSIS AS INITIAL MANIFESTATIONS OF SYSTEMIC ERYTHEMATOSUS LUPUS: CASE REPORT

Gabriela Pereira Barros, Daniel Vitor Pereira De Lima, Lucas Rampazzo

Diniz, Arthur Frederico De Albuquerque Maranhã Filho, Andrea Tavares Dantas

HOSPITAL MIGUEL ARRAES, PAULISTA, PE, Brasil

Advances in Rheumatology 2018, 58(Suppl 1):P312

Background: Muscle involvement in systemic lupus erythematosus (SLE) is relatively common, with approximately half of the patients presenting with myalgias during disease activity. Myositis, which consists of muscular weakness and elevation of muscle enzymes, is much less frequent and its prevalence is around $2.6 \%$. Similarly, acute pancreatitis associated with SLE has a prevalence of less than $1 \%$ and is associated with higher mortality. The purpose of this report was to describe the case of a patient with SLE, whose onset of the disease was accompanied by myositis and acute pancreatitis simultaneously.

Case Report: A female patient, 18 years old, presented with high fever, myalgia and proximal muscle weakness in the four limbs started 30 days before hospital admission. She also complained of pain in the epigastric region, vomiting and weight loss of approximately $5 \mathrm{~kg}$ associated with asthenia and anorexia. On physical examination she had tachycardia, tachypnea and pain on the palpation of the epigastric region and proximal musculature. Initial laboratory tests revealed normocytic anemia $(\mathrm{Hb}=8.8 \mathrm{~g} / \mathrm{dl})$, leukopenia $(2,100 / \mu \mathrm{l})$, lymphopenia $(319 / \mu \mathrm{l})$, thrombocytopenia $(72,000 / \mu \mathrm{l})$ and 
elevated serum creatinine $(2.7 \mathrm{mg} / \mathrm{dl})$. She also presented elevation of CPK (up to 12000U/L), transaminases (AST 904 and ALT $277 \mathrm{U} / \mathrm{l}$ ) and pancreatic enzymes (amylase 2160 and lipase $4165 \mathrm{U} / \mathrm{L}$ ). Abdominal ultrasonography did not show signs of biliary lithiasis and the patient had no history of previous alcoholic libation. Computed tomography of the abdomen showed only signs of acute oedematous pancreatitis. The initial urine summary presented proteinuria, hematuria and granular casts. Screening for SLE demonstrated ANA $1 / 640$ nuclear homogenous pattern, anti-DNA positive $1 / 160$, and reduced complement (C3 and C4). The lgG4 dosage was within normal range. Patient underwent pulse therapy with methylprednisolone followed by oral corticosteroid and azathioprine. There was improvement of the muscular and systemic symptoms, and reduction of muscular and pancreatic enzymes.

Conclusion: Myositis and pancreatitis may represent initial manifestations of SLE, even in the absence of more characteristic clinical manifestations, such as cutaneous and articular manifestations. High suspicion is important for correct diagnosis and management of patients.

\section{Consent for publication}

The authors declare that they have obtained informed written consent from the patient's tutors for publication

\section{P319}

\section{PARSONAGE-TURNER SYNDROME - CASE REPORT}

Nelson Araujo Silva Filho ${ }^{1}$, Dayani Regina de Barros Freitas dos Santos², Camila Aguiar Lomônaco², Pedro Thiago Figueiredo Alves², Carolina Osorio Araujo Silva

${ }^{1}$ HOSPITAL CENTRAL DA AERONAUTICA, RIO DE JANEIRO, RIO DE JANEIRO, Brasil: ${ }^{2}$ HOSPITAL CENTRAL DA AERONÁUTICA, RIO DE JANEIRO, RIO DE JANEIRO, Brasil; ${ }^{3}$, RIO DE JANEIRO, RIO DE JANEIRO, Brasil

Advances in Rheumatology 2018, 58(Suppl 1):P319

Introduction: Parsonage-Turner Syndrome (SPT), also known as amyotrophic neuralgia of the shoulder girdle, is characterized by an acute pain of shoulder girdle, generally unilateral, with subsequent weakness and muscle atrophy. It has a predominance in men and the cause remains unknown, but some hypotheses are postulated as genetic, infections, immunological and biomechanical factors, with slow and progressive recovery that can last months to years.

Case report: A.P.S., male, 45 years old, previously healthy, started pharynxtonsillitis and high fever, progressing with pain in the shoulder girdle with strong intensity, located in the left shoulder subsequently, associated to movement limitation. He sought the emergency being medicated with analgesic, non-steroidal antiinflammatory and tramadol with partial improvement. Days later, he returned to the emergency room with persistent pain and was prescribed morphine and home tramadol use. Due to the nonimprovement of pain, he was referred to the rheumatology service for researching. At physical examination, good general condition, stable vital signs, but with intense pain to the mobilization of the glenohumeral joint and important functional limitation of the left shoulder, besides atrophy of the deltoid muscles (Fig. 1). Neurological examination with paresis to abduction of the left shoulder degree IV, without reflex and sensitivity alterations, and normal right shoulder, without other changes in the other systems. Laboratory tests with inflammatory activity tests and muscle enzymes within the limits of normality, non-reactive antinuclear factor and negative rheumatoid factor, serology for viral hepatitis, human immunodeficiency virus and syphilis negative. Electroneuromyography (ENMG) of the upper limbs demonstrated increased insertion activity, fibrillations and positive waves in the left deltoid muscle compatible with left axillary nerve mono neuropathy. Magnetic resonance imaging (MRI) of the shoulders showed left atrophy of the deltoid muscles (Fig. 2), with no other changes. A diagnosis of SPT was made, and treatment with analgesic, pregabalin and physiotherapy was started. Patient had an excellent response, evolving with recovery of muscle strength.
Conclusion: Although it is a rare condition, it is important to be alert to amyotrophic neuralgia, since this is included in the list of differential diagnosis with rheumatologic diseases, being the main ones: rheumatoid arthritis, rheumatic polymyalgia, gout and adhesive capsulitis. In addition, the correct diagnosis is of great importance for the treatment to be started as early as possible, thus avoiding axonal damage, its progression and associated sequelae.

\section{Consent for publication}

The authors declare that they have obtained informed written consent from the patient's tutors for publication

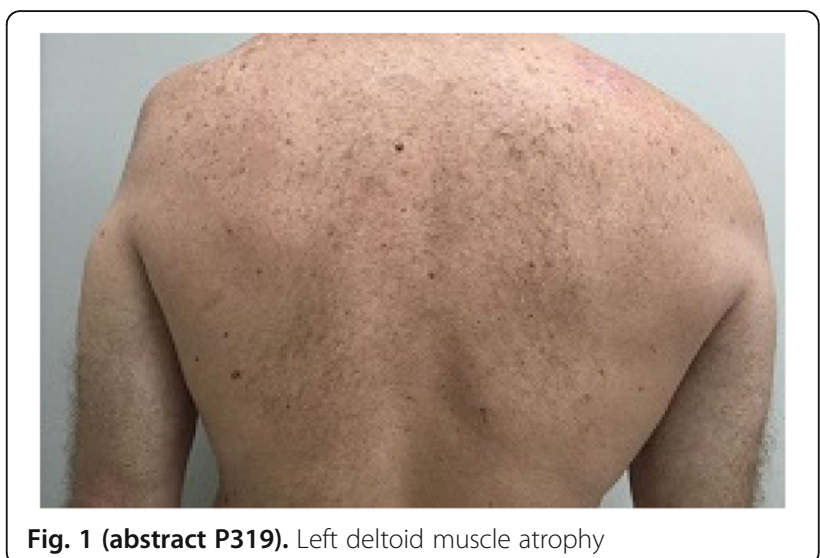

P321

PEDIATRIC LUPUS NEPHRITIS AND RITUXIMAB

Marcia Bandeira, Rafaela Wagner, Luana Ribeiro Carlos, Christina Feitosa

Pelajo, Ingrid Herta R Grein, Loris Lady Janz Junior

HOSPITAL PEQUENO PRINCIPE, CURITIBA, PR, Brasil

Advances in Rheumatology 2018, 58(Suppl 1):P321

Background: Systemic lupus erythematosus (SLE) is a heterogeneous autoimmune disease characterized by chronic inflammation that potentially leads to organ damage. Nonsteroidal antiinflammatory drugs, antimalarial drugs, glucocorticoids, immunosuppressive and citotoxic agents are the mainstays of treatment. For an amount of patients, these approaches arent enough to achieve disease activity control Rituximab (RTX) - a monoclonal antibody specific for human CD20 B-cell - has been widely prescribed. Although the clinical trials of RTX in SLE had controversial results, RTX seems to be a valuable option as an off-label drug for refractory patients.

Methods: We performed a retrospective chart review of patients with SLE that received RTX in some moment of their disease to treat lupus nephritis with previously unsatisfactory evolution and refractoriness to usual treatment with disease-modifying drugs (DMARDs) and corticosteroids.

The following variables will be evaluated: age at diagnosis, sex, SLE according to the SLICC (American College of Rheumatology) and ACR (American College of Rheumatology) criteria, evolution time to renal impairment, nephritis classification according to ISN/RPS (renal biopsy), medication prior to the use of the monoclonal antibody, medications in use at the time of onset of Rituximab, disease activity index (SLEDAl) at the time of initiation of the treatment to be analyzed, clinical response evaluated as responder, non responder and remission of disease.

Responder - patients with SLEDAI> 3 variation within 6 months after infusion of RTX.

Results: Nine patients were in use of the medication in the revised period. All of then were female and the indication of rituximab was basead in the kidney envolvment.

Patients achieved SLEDAl reduction of at least $50 \%$ after $4-6$ months of RTX, as well showed an important reduction of 
proteinuria $(<0.5 \mathrm{~g} / 24 \mathrm{~h})$ and corticoid tapering. One patient had improvement of her disease, however without achieving the paramethers of satisfactory response to the RTX. For this reason, this patient was considered to have a partial response to the RTX. The results are according to those found in similar studies. Conclusion: It is suggested that RTX continues to be used as a therapeutic option for patients with refractory SLE or with contraindication to traditional drugs. Prospected studies are still needed to evaluate efficacy and safety of RTX in pediatric patients.

\section{P322}

PEMBROLIZUMAB AND DEVELOPMENT OF SYMPTOMS OF RHEUMATIC DISEASES: A SYSTEMATIC REVIEW

Isabella Vargas De Souza Lima, Alberto Sérgio Saraiva Santiago UNIVERSIDADE FEDERAL DA BAHIA, SALVADOR, BA, Brasil

Advances in Rheumatology 2018, 58(Suppl 1):P322

Introduction: Pembrolizumab is an inhibitor of PD-1, the pro-apoptotic molecule expressed by diverse neoplasms, which allows them to escape immune surveillance. This same molecule is important in regulating self-tolerance and control of the immune response, central mechanisms in the physiopathology of rheumatology symptoms.

Aim: to describe and appreciate the frequency of rheumatic disease symptoms in users of Pembrolizumab

Methods: A systematic review of the literature from 2000 to 2017 in the MEDLINE, SCIELO, LILACS, and Portal Capes electronic databases, using the descriptor "Pembrolizumab" and excluding other reviews, case reports, studies that did not specify adverse reactions and that did not have a monotherapy group.

Results: In total 1494 articles were found, of which 67 were initially selected. After applying the exclusion criteria, the following were excluded: 1 review; 20 case reports; 9 articles without monotherapy with Pembrolizumab, and 7 that did not adequately record the adverse effects, so that 30 articles remained for final analysis. In total, 4566 patients participated in the studies, of whom 1737 (38.12\%) used $10 \mathrm{mg} / \mathrm{kg}$ ever 3 weeks; 1225 (26.82\%) $10 \mathrm{mg} / \mathrm{kg}$ every 2 weeks; $1113(24.37 \%) 2 \mathrm{mg} / \mathrm{kg}$ every 3 weeks; 474 (10.38\%) 200mg/kg every 3 weeks, and 17 (3.72\%) used other doses. Arthralgia was recorded in 363 patients in 18 studies, ranging from 0.3 to $21.1 \%$ when present. Arthritis was identified in 23 patients in 5 studies, ranging from 0.54 to $3.5 \%$ when present. Myalgia/Myositis was recorded in 137 patients in 13 studies, ranging from 0.27 to $21.10 \%$ when present.

Discussion: The most frequent rheumatology symptom in users of Pembrolizumab was arthralgia, followed by myalgia/myositis and arthritis. There was great variability among the nomenclatures and records used in the studies. Further studies are necessary to elucidate the prevalence and dose-adverse effect correlation.

\section{P323}

PENTRAXIN 3 GENE POLYMORPHISM IN ASSOCIATED WITH SUSCEPTIBILITY TO RHEUMATOID ARTHRITIS

Rui Medeiros Melo ${ }^{1}$, Marília Lins E Silva ${ }^{1}$, Rodrigo Feliciano Do Carmo', Luydson Richardson Silva Vasconcelos', Jair Carneiro Leão', Andrea Tavares Dantas', Angela Luzia Branco Pinto Duarte', Luiz Alcino Gueiros ${ }^{1}$ 'UFPE, RECIFE, PE, Brasil; ${ }^{2}$ UPE, RECIFE, PE, Brasil Advances in Rheumatology 2018, 58(Suppl 1):P323

Background: Recent lines of evidence gave pointed higher Pentraxin 3 (PTX3) levels in synovial fluid but not in serum of rheumatoid arthritis (RA) patients, being possibly related to inflammation maintenance. The aim of this study was to analyze two PTX3 single nucleotide polymorphisms (rs1840680 and rs2305619) and their association with RA susceptibility and clinical presentation. Methods: Two hundred and thirty eight patients were divided into two groups: Group RA $(n=134)$, and Group C - controls, $(n=124)$, in a convenience sample obtained from March to November/2014. For RA activity evaluation, the Disease Activity Score 28 (DAS28) was used. DNA was isolated from the blood and then stored at $-20^{\circ} \mathrm{C}$ until analysis. PCR reactions were prepared using a set of TaqMan ${ }^{\circledast}$ Universal PCR Master Mix and categorical variables were compared using the chi-squared test or Fisher's exact test when appropriate. Comparisons were performed using Student's t test or Mann-Whitney U-test. Stepwise logistic regression analysis was performed to identify predictors of RA.

Results and Conclusions: For the rs $1840680,46 \%$ of individuals in the control group had the GG genotype against $32.1 \%$ in the RA group $(p=0.022$ OR $0.55 \mathrm{Cl}$ 0.33-0.92). Regarding to the rs 2305619 , the GG genotype was carried by $32.3 \%$ of individuals in the control group against $20.9 \%$ in the RA group ( $p=0.038$ OR $0.55 \mathrm{Cl}$ 0.31-0.97). Stepwise logistic regression analysis showed, after adjustment, the PTX3 rs1840680 AG genotype remained independently associated with risk to RA (OR $2.11,95 \% \mathrm{Cl} 1.27$ 3.50, $\mathrm{P}=0.004)$ as well as female gender was also associated with RA (OR 2.66, 95\% Cl 1.04-6.80, P = 0.041. Our results support the idea that PTX3 AG (rs1840680) genotype confers susceptibility to RA. This is the first evidence of association between PTX3 polymorphisms and RA. Further studies are necessary to confirm this association in other populations.

\section{P325}

PERIPHERAL NEUROPATHIC PAIN IN CHIKUNGUNYA FEVER: DATA FROM A LONGITUDINAL COHORT

Ana Flávia Rocha de Oliveira', Hugo Deleon de Lima², Renan Mesel Correia', Alice Góes Liberato de Mattos' ${ }^{1}$ Flora Gomes Teles Vieira², Weidson Henrique Lira Borges ${ }^{2}$, Mariana Souza Pessoa de Luna ${ }^{2}$, Claudia Diniz Lopes Marques ${ }^{3}$, Angela Luzia Branco Pinto Duarte ${ }^{3}$, Aline Ranzolin², Paulo Roberto Sampaio de Melo², Laurindo Ferreira da Rocha Junior $^{2}$

${ }^{1}$ FACULDADE PERNAMBUCANA DE SAÚDE - FPS, RECIFE, PE, Brasil; ${ }^{2}$ INSTITUTO DE MEDICINA INTEGRAL PROF. FERNANDO FIGUEIRA - IMIP, RECIFE, PERNAMBUCO, Brasil; ${ }^{3}$ HOSPITAL DAS CLÍNICAS DA UNIVERSIDADE FEDERAL DE PERNAMBUCO - HC/UFPE, RECIFE,

PERNAMBUCO, Brasil

Advances in Rheumatology 2018, 58(Suppl 1):P325

Background: Chikungunya fever (CF) is associated with persistence of osteoarticular manifestations. In Brazil there was an outbreak of CF in 2016. Besides musculoskeletal symptoms, peripheral neuropathic pain (PNP) is associated with the clinical picture. This study aimed to determine the association between PNP and clinical and laboratory parameters in patients with $\mathrm{CF}$ and musculoskeletal manifestations.

Methods: Epidemiological, clinical and laboratory data were collected from patients from the cohort. Clinical diagnosis of PNP was made by applying Douleur Neuropathique 4 questionnaire (DN4), in which scores $\geq 4$ indicate PNP. The 12-Item Short-Form Health Survey (SF12) and Health Assessment Questionnaire (HAQ) were applied for assessment of quality of life and functional capacity, respectively. Statistical analysis was performed.

Results And Conclusions: Sixty-three patients were enrolled (52 females), mean age 51.65 years (range 20-79). In the first month after disease onset 24 patients were evaluated, the presence of PNP (50\%) was significantly associated with Visual Analogue Scale (VAS) for initial pain after disease onset $(p=0.0340)$; for stiffness $(p=0.0370)$ and for patient global assessment (PGA), $(p=0.0022)$. Correlation between DN4 score and VAS for Physician Global assessment (MDGA) was also significant ( $p=0.0190, r=0.4850$ ). From 2 nd to 4rth month, PNP (38 out of 63 ) was associated with painful $(p=0.0068)$ and swollen joints $(p=0.0143)$; VAS for pain $(p=0.0001)$, for morning stiffness $(p=0.0004)$, for fatigue $(p=0.0020)$, for PGA $(p=0.0068)$ and MDGA $(p<0.0001)$. Correlations between DN4 and HAQ ( $p=0.0015, r=0.4118)$, SF12 mental domain $(p=0.0174, r=-0.3196)$ and blood ferritin levels $(p<0.0001$, $r=-0.2985$ ) were found. From fifth to seventh month, PNP (14 out of 43) was associated with painful joints $(p=0.0036)$ and edema $(p=0,0052)$; VAS for pain $(p=0.0003)$, for stiffness $(p=0.0426)$, for fatigue $(p=0.0055)$, MDGA $(p=0.0008)$, with HAQ $(p=0.0080)$ and with physical $(p=0.0069)$ and mental $(p=0.0043)$ domains of SF12. From eighth to tenth month, PNP (12 out of 33) was associated with higher number of painful joints $(p=0.0209)$, VAS for stiffness $(p=0.0202)$, for fatigue $(p=0.0047), P G A(p=0.0247)$ and MDGA 
$(p=0.0002)$. From eleventh to thirteenth month, PNP (7 out of 24) was associated with the number of painful joints $(p=0.0115)$, VAS for pain $(p=0.0319)$, for morning stiffness $(p=0.0269)$, for fatigue $(p=0.0095)$ and MDGA $(p=0.0062)$. During the evaluation PNP was associated with symptoms referred in acute phase such as nausea, vomiting, anorexia and exanthema $(p<0.05)$. It is concluded that neuropathic pain is related to osteoarticular and clinical features of $\mathrm{CF}$, as well as alterations in laboratory tests.

\section{P328}

\section{PERIUNGUEAL CAPILLAROSCOPY IN PATIENTS WITH SYSTEMIC} SCLEROSIS SUBMITTED TO AUTOLOGOUS HEMATOPOIETIC STEM

\section{CELL TRANSPLANTATION}

Daniela Aparecida De Moraes², Juliana Bernardes Elias², Ana Beatriz

Pereira Lima Stracieri ${ }^{2}$, Fabiano Pieroni ${ }^{2}$, Belinda Pinto Simões ${ }^{2}$,

Cristiane Kayser $^{1}$, Maria Carolina de Oliveira ${ }^{2}$

${ }^{1}$ ESCOLA PAULISTA DE MEDICINA- UNIFESP, SÃO PAULO, SP, Brasil;

${ }^{2}$ FACULDADE DE MEDICINA DE RIBEIRÃO PRETO- USP, RIBEIRÃO PRETO,

$\mathrm{SP}$, Brasil

Advances in Rheumatology 2018, 58(Suppl 1):P328

Introduction: Systemic sclerosis (SSc) is associated with peripheral microvascular changes, which can be observed through periungueal capillaroscopy examination. These findings can change with disease course, starting as hemorrhages and giant capillaries, and later as avascular areas. Autologous hematopoietic stem cell transplantation (AHSCT) has been used as treatment for severe and progressive forms of SSc, and aims to reestablish the balance between autoreactivity and immunological tolerance, thereby promoting control of disease progression.

Objectives: To evaluate the outcomes of peripheral microvascular changes in patients with SSc who underwent AHSCT.

Methods: Twenty patients were included in the study. Patients were treated with high-dose cyclophosphamide $(200 \mathrm{mg} / \mathrm{kg})$ plus antithymocyte rabbit globulin, followed by infusion of unmanipulated autologous hematopoietic stem cells. Patients were evaluated with nailfold capillaroscopy (NC) before, and at 2, 6, 12, 18 and 24 months after AHSCT, under stereomicroscope (Olympus, USA) direct observation. For each patient, fingers 2 to 5 were analyzed for number of loops $/ \mathrm{mm}$, hemorrhages, dilated capillaries, shrubs and areas of devascularization. Data were analyzed by One-way ANOVA and significance was set at $p<0.05$.

Results: Median (range) of patient NC follow-up was 12 (2-24) months. After AHSCT, we observed significant increase in the number of loops $/ \mathrm{mm}(p=0.0051)$, reduction in hemorrhages $(p=0.0177)$ and periungual devascularization $(p=0.0255)$.

Conclusions: AHSCT can improve the structure of the periungual capillaries, but larger number of patients and longer follow-up are necessary for more definitive conclusions.

\section{P331}

\section{PICNODISOSTOSE SIMULATING SPONDILOARTRITES}

Bruno Meireles Brito De Souza', Sandra Lúcia Euzebio Ribeiro ${ }^{1}$, Rosana Barros De Souza', Helena Lucia Alves Pereira', Guilherme Andrade Bulbol', Juliana Buhring', Daniele Patricia Dal Bosco', Vania Mesquita Gadelha Prazeres', Diego De Souza Pereira²

${ }^{1}$ HOSPITAL UNIVERSITÁRIO GETULIO VARGAS - AM, MANAUS,

AMAZONAS, Brasil; ${ }^{2}$ FUNDAÇÃO DE MEDICINA TROPICAL - HVD, MANAUS, AMAZONAS, Brasil

Advances in Rheumatology 2018, 58(Suppl 1):P331

Background: Picnodysostosis (PYCD) is a rare autosomal recessive skeletal dysplasia due to a defect in the coding of the enzyme cathepsin K. It affects both sexes equally, as clinical features of short stature, bradydactyly, micrognathia, osteosclerosis, acrosteolysis, craniofacial deformities (micrognathia and late closure of the fontanelles), eruption delay and anomaly in the dentition and bone fragility. Patients with PYCD have a normal life expectancy, however. Due to poor vascularity and bone metabolism, the risk of postoperative complications increases, thus increasing the risk of osteomyelitis and pseudarthrosis.
Case Report: male, 37 years old, started three years ago with inflammatory lumbar pain, improved with NSAIDs. Referred to rheumatologist with diagnostic hypothesis of ankylosing spondylitis (AE). At the consultation he reported difficulty in learning, pain in the cervical spine, lumbar spine, knee and ankle joints. Physical examination: Emagretia, low implantation of the ears, micrognathia, 1st and 7th bilateral costochondral and right and left iliac crests in the compression of the sacriliaca, knees (flexion at $170^{\circ}$ ), decreased cervical mobility, genovalgo, upper limbs and lower limbs in flexion and shouber test $10 / 14,5 \mathrm{~cm}$. Laboratories: blood count anemia normonormo, HSV 8mm3, PCR 0,914 (Vr-0,5), Latex-negative and HLA-B27 absent. Radiograph of cervical spine: rectification of lordosis and reduction of vertebral bodies, reduction of $\mathrm{C} 4-\mathrm{C} 5$ space and rectification of lordosis, lumbosacral reduction of L5-S1 space. After the physical examination, anamnesis and radiological evaluation, a diagnosis of $A D$ was rejected and the hypothesis of genetic disease was raised, being referred to a geneticist. With clinical history and physical examination and radiological changes made diagnosis of PYCD.

Conclusion: We consider the PYCD due to deformities of the spine and knees can make differential diagnosis of spondyloarthritis, since the physical changes caused by the genetic condition can be a confusing factor at the time of diagnosis. The rheumatologist has to keep an eye on changes due to genetic diseases. radiographic study is necessary for the diagnosis of PYCD. The typical radiological findings allow the radiologist to infer the diagnosis with great accuracy.

\section{Consent for publication}

The authors declare that they have obtained informed written consent from the patient's tutors for publication.

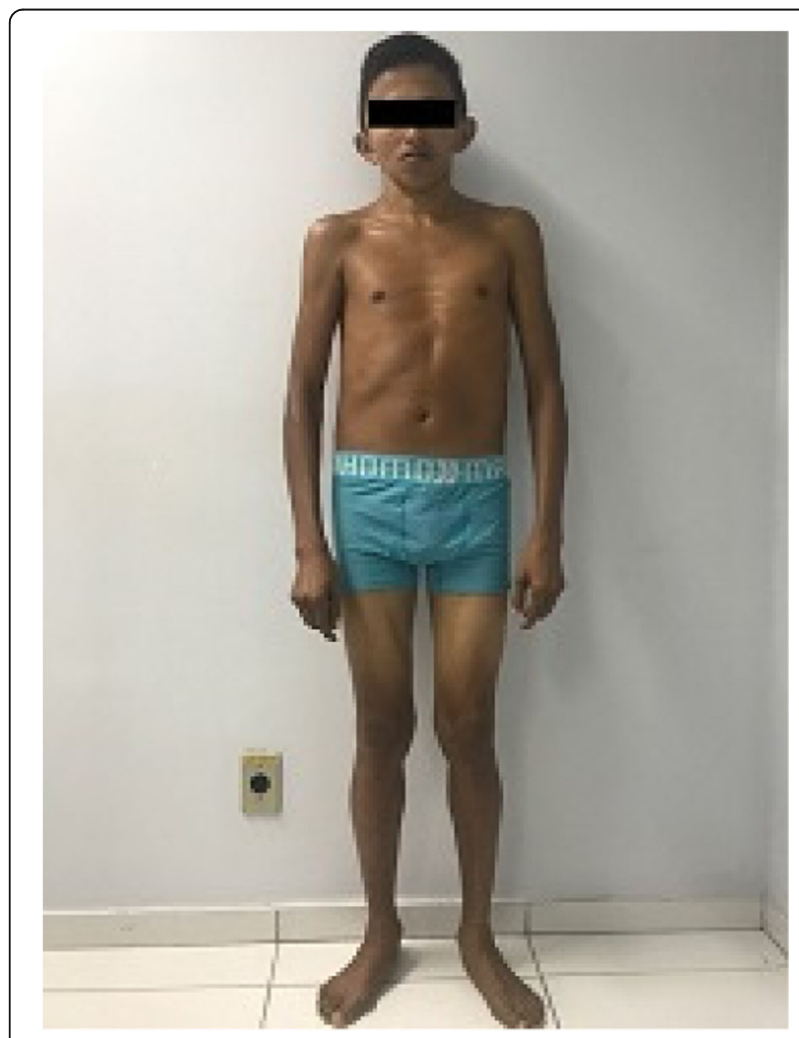

Fig. 1 (abstract P331). PATIENT WITH LOW IMPLANTATION OF THE EARS, MICROGNATISM, MUSCLE HYPOTROPY, ATROPHY OF QUADRICEPS AND UPPER AND LOWER MEMBRES IN FLEXION 
P337

POLYARTHRITIS ASSOCIATED WITH DISTAL SCLERODERMA AS A PARANEOPLASTIC MANIFESTATION OF BASAL CELL CARCINOMA

Rodrigo Antunes Da Silveira', Gabriela Gimenes Santos ${ }^{4}$, Gabriel Parise Ferreira Balbão ${ }^{4}$, Carlos Henrique Vidal ${ }^{4}$, Javan Alvaro Matera Turrini ${ }^{3}$, Cecilia Leon Calderon ${ }^{3}$, Rafael Amaro Silva ${ }^{3}$, Vanessa Rodrigues Dias Ferreira $^{3}$, Leonardo Rios Oliveira ${ }^{3}$, Philipe Sousa Silveira², Cecilia Davila Chambi $^{3}$, Priscila Nogueira Soares ${ }^{4}$, Gabrielle Quessada ${ }^{4}$, Gabriela Ereno ${ }^{4}$, Andressa Beber Castilho ${ }^{4}$, Amanda Corsini Leitao ${ }^{3}$

${ }^{1}$ HOSPITAL DAS CLINICAS LUZIA DE PINHO DE MELO (UMC), ARUJÁ, SP, Brasil; ${ }^{2}$ UNIVERSIDADE BELA VISTA, MOGI DAS CRUZES, SP, Brasil;

${ }^{3}$ HOSPITAL DAS CLINICAS LUZIA DE PINHO DE MELO, MOGI DAS

CRUZES, SP, Brasil; ${ }^{4}$ UNIVERSIDADE DE MOGI DAS CRUZES, MOGI DAS CRUZES, SP, Brasil

Advances in Rheumatology 2018, 58(Suppl 1):P337

Introduction: Skin neoplasms can mimic rheumatologic diseases and one of the main paraneoplastic manifestations is scleroderma.

Case report: A 72 year-old-man, natural of Mogi das Cruzes-SP, married, retired, resident of the city, referred to the rheumatology clinic due to pain and skin thickening in both hands 8 months ago, pain that responded well with prednisone, report to $6 \mathrm{~kg}$ weight loss in 3 months, denied symptoms related to systemic sclerosis such as raynaud phenomenon, dysphagia, diarrhea or other correlated symptoms. Patient reported that a $2 \times 2 \mathrm{~cm}$ lesion biopsy was scheduled in the left temporo-frontal region scheduled with a dermatology team. Past history of systemic arterial hypertension, dyslipidemia and melanoma. General survey: healthy-appearing, no cianosis, no jaundice, no fever, hydrated, conscious, alert, eupneic, BP 120/80 mmHg, HR $80 \mathrm{bpm}$, rhythmic and normophonetic sounds without murmurs, bilateral vesicular murmur without adventitious sounds, abdomen: unnoteworthy, absence of edema in lower limbs. Specific survey shows cutaneous thickening of both hands (Rodnan 18), presence of arthritis in proximal interphalangeals, metacarpals, bilateral positive squeeze maneuver. Laboratorial exams: Hemogram, antinuclear factor (ANF) negative, rheumatoid factor (RF) negative, uric acid normal, HSV: $98 \mathrm{~mm}$, PTH normal, ionized calcium normal, CA15.3, 72.3, 19.9, CEA and alpha-fetoprotein: normal. Hands X-Ray: no changes. High digestive endoscopic, chest $C T$ and abdomen: absence of changes, more specifically neoplasms. In this way, he underwent an empirical therapy with a low dose of corticoid (Prednisone 10mg 1x day) with partial response of the clinical picture, patient returned with biopsy of the lesion compatible with basal cell carcinoma, after excision, there was complete resolution of the condition in 2 months with improvement of the inflammatory tests and resolution of the anemia, but maintenance of the scleroderma in hands after 9 months follow up.

Discussion: Paraneoplastic syndromes are a challenge for the rheumatologist because of the complexity of their clinical manifestations, which involve neurological, hematological, endocrinological and dermatological manifestations, among others. In the present case the clinical investigation was guided by the fact that the patient already had a previous diagnosis of basal cell carcinoma raising the suspicion and correlating the time of onset of arthritis and skin thickening with the appearance of the neoplasia and its diagnosis.

Conclusion: Basal cell carcinoma has as its major rheumatologic paraneoplastic manifestation the cutaneous thickening and should always be remembered as a differential diagnosis of systemic sclerosis, whether in the limited or diffuse form.

\section{Consent for publication}

The authors declare that they have obtained informed written consent from the patient's tutors for publication
P345

PREDICTORS OF ADMISSION TO NEONATAL INTENSIVE CARE UNIT AMONG SYSTEMIC LUPUS ERYTHEMATOSUS PREGNANCIES

Evandro Mendes Klumb', Bruna Wafae², Camila Castro Silva², Bruna

Costa Rodrigues ${ }^{2}$, Marcela Ignacchiti Lacerda², Camila Pitasi Argueles², Guilherme Ribeiro Ramires De Jesús ${ }^{2}$, Nilson Ramires De Jesús², Roger Abramino Levy ${ }^{2}$, Evandro Mendes Klumb ${ }^{2}$

'UNIVERSIDADE DO ESTADO DO RIO DE JANEIRO, RIO DE JANEIRO, RJ, Brasil; ${ }^{2} U E R J, R J, R J$, Brasil

Advances in Rheumatology 2018, 58(Suppl 1):P345

Background: Systemic lupus erythematosus (SLE) is an autoimmune disorder that affects predominantly women in childbearing age and increases the risks of maternal and fetal complications. The most common fetal adverse outcomes are fetal loss, fetal growth restriction (FGR) and prematurity. The last two frequently require admission to neonatal intensive care unit (NICU). However, data regarding outcomes of newborns of SLE patients admitted to NICU are still scarce. Therefore, the aim of this study was to evaluate possible maternal predictors of NICU admission.

Methods: This is an observational study comprising 152 pregnancies among 140 SLE patients who were followed between 2011 and 2016, with singleton gestations and delivery after 22 weeks. The maternal variables studied as possible predictors of admission to NICU were maternal age at delivery, history of nephritis, permanent damage measured by SLICC/ACR damage index (SDI) $\geq 1$, antiphospholipid antibodies (aPL) or antiphospholipid syndrome (APS), SLE activity in pregnancy, medication during pregnancy, maternal hospitalization during pregnancy, hypertensive disorders of gestation. Results: After exclusion of 8 stillbirths among the patients studied, 32 out of 144 live newborns were admitted to NICU (22\%). Medical information was available for 26 newborns ( $18 \%$ of loss). Mean maternal age at delivery was $28.5 \pm 6$ years and mean time after diagnosis of SLE was 7.4 (3-11) years. Maternal variables associated with NICU admission were: SDI $\geq 1(p=0.04)$, active SLE during pregnancy $(p=0.02)$, especially active nephritis $(p=0.013)$, use of steroids at conception and during pregnancy ( $p=0.01$ and $p=0.005$, respectively), use of antihypertensive medication $(p=0.008)$ and maternal hospitalization unrelated to SLE $(p=0.008)$. The median time in NICU was of $8(1-123)$ days. There were 2 neonatal deaths (survival rate of 92\%).

Discussion and conclusions: For low-risk pregnancies the admission rate in NICU is around 7\% in Brazil (Datasus). Our frequency of NICU admission was 3 times higher, probably due to the impact of maternal SLE on fetal and newborn health, which highlights the importance of achieving disease remission before pregnancy in order to minimize risks and improve neonatal outcome.

Consent for publication

The authors declare that they have obtained informed written consent from the patient's tutors for publication

P346

PREGNANCY IN PATIENTS WITH SYSTEMIC ERITEMATOSUS LUPUS: AGREEMENT BETWEEN GYNECO-OBSTETRICIANS AND RHEUMATOLOGISTS CONCEPTS

Emily J. O. Carvalho, Letícia A. Peiter da Silva, Aiessa Zanchett Fedrigo,

Deborah Colombo, Thiago Alberto Fernandes Gomes dos Santos,

Thelma Laroca Skare

HOSPITAL UNIVERITÁRIO EVANGÉLICO DE CURITIBA, CURITIBA, PARANÁ, Brasil Advances in Rheumatology 2018, 58(Suppl 1):P346

Background: The good care of systemic lupus erythematosus (SLE) pregnant patients encompasses a good cooperation between rheumatologists and obstetricians. However, a great deal of disagreement 
between the ways they conduct such patients are noted. Herein, we tried to verify the degree of agreement between gynecologists/obstetricians and rheumatologists in the counseling, follow-up and treatment of lupus patients who are pregnant or wish to become pregnant

Methods: This is a cross-sectional and analytical study comprising a questionnaire for gynecologists/obstetricians with 5 items divided in 46 sub-items, covering: (a)-counseling on pregnancy; (b)-contraindications for pregnancy; (c)-use of anti-rheumatic drugs during pregnancy; (d)-specific manifestations of pregnancy that mimic SLE activity; (e)-indication oflaboratory work up; (f)-diagnosis of antiphospholipid antibody syndrome; (g)-use of contraceptives and (h)-delivery methods. Data from obstetricians regarding graduation time, age, and type of care that the professional performs (high and low risk patients) were collected. The questionnaires were answered by electronic platform or delivered in person.

Results:Eighty doctors answered the questions. Their graduation time varied from one to 55 years; $30 \%$ were males; $23.7 \%$ of the physicians worked in low risk care, $2.5 \%$ in high risk and $73.7 \%$ in both. Concordance in each of 46 sub-item ranged from $16.2 \%$ to $98.7 \%$. The two major areas with the greatest discrepancy were on the diagnosis of antiphospholipid antibody syndrome (concordance on $46.2 \%$ ), and those of on contraceptive indications (concordance on 57.5\%). The major areas with the highest concordance were about delivery (95\%) and laboratory work up (79\%). The concordance decreased as the interviewer's age increased $(p=0.006)$ with a tendency to decrease with an increase in graduation time $(p=0.055)$. No differences werefound when respondents performed high or low risk care.

Conclusion:There is a high level of disagreement among gynecologists - obstetricians and rheumatologists regarding the health of women with lupus. Interdisciplinary activities aiming continuing education in this issue are needed to provide better care for these patients.

\section{References}

1. Cavazzana I, Andreoli L, Limper M, Franceschini F, Tincani A. Update on Antiphospholipid Syndrome: Ten Topics in 2017. CurrRheumatol Rep. 2018;20(3):15.

2. Peart E, Clowse ME. Systemic lupus erythematosus and pregnancy outcomes: an update and review of the literature. CurrOpinRheumatol. 2014;26(2):118-23.

\section{P347}

PRESENCE OF ANTINEUTROPHIL CYTOPLASMATIC

\section{AUTOANTIBODIES IN A PATIENT WITH INFECTIVE ENDOCARDITIS}

Matheus Assunção De Holanda, Gabriela Pereira Barros, Fabio De Lima

Queiroga, Andréa Tavares Dantas

HOSPITAL MIGUEL ARRAES, PAULISTA, PE, Brasil

Advances in Rheumatology 2018, 58(Suppl 1):P347

Background: Antineutrophil cytoplasmatic autoantibodies (ANCAs) are commonly related to small-vessel vasculitic syndromes, such as granulomatosis with polyangiitis (GPA), microscopic polyangiitis (MPA) and eosinophilic granulomatosis with polyangiitis (GEPA). However, several infectious diseases can exhibit positive ANCA, and infective endocarditis is particularly one of major importance, because its clinical presentation can resemble that of a vasculitic syndrome in some cases.

Case Report: A female patient, 54 years old, presented with dyspnea and hypertension started two months before hospital admission, with worsening in the last week. Laboratory tests revealed elevated serum creatinine $(18.2 \mathrm{mg} / \mathrm{dL})$ and hemodialysis was started. During hospitalization the patient developed a high fever and a new cardiac murmur, with blood cultures revealing methicillin-sensitive $\mathrm{S}$. aureus. The diagnosis of infective endocarditis was established and antibiotic therapy with oxacillin was initiated. Transthoracic echocardiography showed no evidence of vegetation. She persisted febrile even after antibiotic therapy, and for better investigation a computed tomography of the thorax was requested, which revealed excavated pulmonary nodules. At this point the patient was presenting conjunctival hyperemia and she also had a previous history of chronic sinusitis with rhinorrhea and sporadic episodes of epistaxis. Tomography of paranasal sinus revealed chronic sinusopathy. On the suspicion of GPA, C-ANCA was requested, with positive result (1/30). However, dosage of anti-proteinase 3 (anti-PR3) was negative. No immunosuppression was initiated, and the fever ceased. After four weeks of antibiotic treatment, the blood cultures became negative, the pulmonary nodules at the tomography were resolved and the ANCA was repeated, the result of which was negative.

Conclusion: Infective endocarditis is a fatal disease that may present as systemic vasculitis, with ANCA positivity. It is important to remember this differential diagnosis to avoid the catastrophic consequences of an immunosuppression in these patients.

Consent for publication

The authors declare that they have obtained informed written consent from the patient's tutors for publication

P348

PREVALENCE OF JOINT MANIFESTATIONS IN SYSTEMIC LUPUS ERYTHEMATOSUS AND ITS RELATION WITH THE POSITIVITY OF RHEUMATOID FACTOR IN PATIENTS ATTENDED AT A MEDICAL SPECIALTIES CLINIC

Caio Henrique De Souza Almeida, Felipe Ruan Borges Rodrigues, Fabíola Brasil Barbosa Rodrigues, Dilma Costa de Oliveira Neves

CESUPA, BELÉM, PA, Brasil

Advances in Rheumatology 2018, 58(Suppl 1):P348

Background: Systemic Lupus Erythematosus (SLE) is an autoimmune disease that affects multiple organs and has a broad spectrum of clinical manifestations. It is more prevalent in women and musculoskeletal involvement occurs in most patients. Due to its high global prevalence and due to the low exploration of joint manifestations and the positivity of rheumatoid factor, characterizing the joint involvement will provide a better therapeutic management.

Method: A cross-sectional study that was carried out by analyzing the medical records of patients attended in a specialty ambulatory from March to December 2017. All data were presented as absolute and percentage values using non-parametric tests to evaluate the difference between groups.

Results and conclusions: A total of 58 records were analyzed, of which $53(91.4 \%)$ were female and $5(8.6 \%)$ were male. Only 28 records contained information about the positivity of the rheumatoid factor, being positive in 5 patients $(17.8 \%)$ and negative in $23(82.2 \%)$. On the initial clinical picture, arthralgia was the most prevalent symptom, occurring in $44(75.9 \%)$ patients, followed by non-cicatricial alopecia $34(58.6 \%)$ and acute cutaneous involvement $33(56.9 \%)$. The most affected joint was the fist, appearing in more than half of the patients $(55.1 \%)$, followed by knee joints (36.8\%) and shoulder (35.1\%). The onset of joint manifestation occurred at diagnosis in 52 of the 58 patients, representing almost $90 \%$ of the study population. About joint manifestations, arthralgia was present in $80 \%$ of the cases. Concerning the characteristics of joint impairment, the symmetrical pattern (70.2\%) and additive $(80.7 \%)$ predominated. There was no difference in the prevalence of rheumatoid factor in lupus patients with and without joint manifestation. There was a predominance of females and the age of onset of symptoms predominantly from 12 to 36 years of age and most the of the patients had no family history of autoimmune rheumatologic diseases. Regarding FAN, $86,2 \%$ of the patients had positive results. Most of the results are consistent with traditional literature about SLE. However, there were divergences, such as the characterization of joint involvement, which can be explained by the multifactorial etiology of the disease with interferences from genetics and environmental factors, and medical intervention that alters the course of the disease, explaining different characteristics in that. 


\section{P349}

PREVALENCE OF LOW BACK PAIN/NECK PAIN IN MEDICAL STUDENTS IN A PRIVATE COLLEGE IN TERESINA

Joelma Moreira De Noroes Ramos', Gleycianne da Silva Oliveira Dumont Vieira', Angelica Maria Assunção da Ponte Lopes', Jôyce Reis Costa², Gabriela Grabowski Amorim²

${ }^{1}$ FACID WYDEN, TERESINA, PIAUI, Brasil; ${ }^{2}$ UNIVERSIDADE ESTADUAL DO

PIAUÍ UESPI, TERESINA, PIAUÍ, Brasil

Advances in Rheumatology 2018, 58(Suppl 1):P349

Background: Pains in the spine are very common symptoms in modern society and affect a considerable amount of people. Medical students are quite susceptible to developing low back pain and neck pain due to the time they sit in front of a computer, in a classroom, or even studying, often with an inappropriate posture. Added to this, we can add as a risk factor for vertebral pain, weakening of the dorsal musculature due to lack of regular physical activity, which can cause imbalance and pain. Goals: To verify the prevalence of medical students suffering from neck pain and/or low back pain, as well as the factors related to such symptoms.

Methods: The research was developed obeying the ethical principles of Resolution 466/2012 of the National Health Council. To perform the same, questionnaires were applied on habits, posture, lifestyle and pain complaints in a portion of students enrolled from the first to the twelfth period of the medical course of a private college in Teresina-PI.

Results and conclusions: Of the students included in the study, $84.11 \%$ reported having felt or felt pain in the spine, with cervical and lumbar regions being the most prevalent, with $23.02 \%$ and $48.68 \%$, respectively. Among students with spinal pain, $40 \%$ reported that the pain started after they entered medical school and $49.53 \%$ reported worsening of symptoms over the periods. $71.63 \%$ of the sample reported cervical pain and $70.70 \%$ of the total reported low back pain after sitting for a long period of time performing academic activities. Through the study, it was found that $44.24 \%$ of the students do not practice any physical activity, and $70.37 \%$ do not perform due to lack of time. $27.57 \%$ of students who reported spinal pain use anti-inflammatory drugs to control pain. The research was of great importance, because it verified the percentage of students affected by neck pain and low back pain, the risk factors for such pathologies and whether they use analgesics and/or antiinflammatories to relieve symptoms.

\section{P350}

PREVALENCE OF METABOLIC SYNDROME IN PATIENTS WITH PSORIATIC ARTHRITIS AND PSORIASIS IN A RHEUMATOLOGY OUTPATIENT CLINIC

Isadora Emydio Gava ${ }^{1}$, Juliah Serraglio', Betânia Longo², Jhésyca Flávia

Sousa Castaman Stédile ${ }^{2}$, Sílvio Almeida de Lima², Carla Luchese de Almeida², Juliana Delfino², Deborah Cristyne Colombo², Ana Paula Beckhauser de Campos ${ }^{2}$

${ }^{1}$ FACULDADE EVANGÉLICA DE CURITIBA, CURITIBA, PR, Brasil; ${ }^{2}$ HOSPITAL UNIVERSITÁRIO EVANGÉLICO DE CURITIBA, CURITIBA, PR, Brasil

Advances in Rheumatology 2018, 58(Suppl 1):P350

BACKGROUND: The literature shows that both psoriatic arthritis and psoriasis present common comorbidities, among them the Metabolic Syndrome (MS). In this work, we aimed to verify the prevalence of MS in patients with Psoriatic Arthritis and Psoriasis, comparing them with the control group.

METHODS: A cross-sectional study was carried out recruiting patients with psoriasis ( $n=97)$, psoriatic arthritis $(n=95)$ and controls $(n=105)$. Comorbidities such as diabetes mellitus and hypertension were evaluated as well as their treatments. Epidemiological and clinical variables (gender, age, weight, height, waist circumference and blood pressure) and laboratory tests results (LDL cholesterol, triglycerides and fasting glucose) were collected. MS was considered using the National Cholesterol Education Program's Adult Treatment Panel III (NCEP-ACT III). The prevalence of overweight, obesity, diabetes and hypertension was calculated. The data obtained were collected in frequency and contingency tables and their distribution were analyzed by the Shapiro Wilks test. The Chi- square and Mann Whitney tests were used to compare data between groups. The level of significance was $5 \%-p<=0.5$.

RESULTS: The prevalence of MS in psoriasis was of $48 / 97$ (49.4\%), in psoriatic arthritis of 50/95 (52.6\%) and 36/105 (34.2\%) in controls of with $p=0,01$. No difference was found between patients with psoriasis and psoriatic arthritis $(p=0.60)$. Comparing controls with patients with psoriasis plus psoriatic arthritis, it was found that controls had lower BMI $(p<0.0001)$, systolic blood pressure $(p<0.01)$ and abdominal circumference $(p<0.0001)$. Comparing patients with psoriasis and psoriatic arthritis, significance was found for BMI $(p<0.01)$, systemic blood pressure $(p<0.04)$, dyslipidemia $(p<0.0043)$, fasting glycaemia $(p<0.003)$ and HDL $(p<0.002)$ all of them with higher values in psoriatic arthritis patients. CONCLUSION: There is a high prevalence in MS of patients with psoriasis and psoriatic arthritis than controls but the prevalence of MS in patients with psoriasis and psoriatic arthritis was the same. However, patients with psoriatic arthritis, has more hypertension, higher BMI and higher glucose serum levels than those with psoriasis. Medication used for control of arthritis symptoms may have contribute for these differences.

\section{References}

1. Caso, F. et al. Metabolic syndrome in psoriatic arthritis: the interplay with cutaneous involvement. Evidences from literature and a recent crosssectional study. Clin Rheumatol., 2018.

2. O'Neill S, O'Driscoll L. Metabolic syndrome: a closer look at the growing epidemic and its associated pathologies. Obes Rev. 2015;16:1-12.

\section{P351}

PREVALENCE OF MUSCULOSKELETAL SYMPTOMS IN A RURAL COMMUNITY IN MATO GRSSO DO SUL, BRAZIL

Marcelo Hitoshi Kinashi ${ }^{1}$, Marcia Midori Shinzato ${ }^{2}$

${ }^{1}$ HOSPITAL UNIVERSITÁRIO DA UNIVERSIDADE FEDERAL DA GRANDE

DOURADOS, DOURADOS, MATO GROSSO DO SUL, Brasil; ${ }^{2}$ HOSPITAL UNIVERSITÁRIO DA UFGD, DOURADOS, MATO GROSSO DO SUL, Brasil Advances in Rheumatology 2018, 58(Suppl 1):P351

Background: To the best of our knowledge, there is no musculoskeletal (MSK) diseases survey carried out in Mato Grosso do Sul (MS), Brazil. Therefore the objective of this study is to estimate the prevalence of musculoskeletal symptoms in a rural Brazilian community in MS.

Methods: The survey was carried out from August 2017 to January 2018 in a probabilistic sample of a rural district of Dourados city with a population of 3095 people (Instituto Brasileiro de Geografia e Estatística, 2010), Mato Grosso do Sul, Brazil. The phase 1 of the first stage questionnaire of the Program Oriented Community for the Control of Rheumatic Diseases (COPCORD) translated and validated to the Brazilian Portuguese language was applied by health professionals and medical students. Demographic data were collected and subjects with musculoskeletal (MSK) symptoms were identified. People above 15 years old, both gender, except for those who were not available or refuse to participate were included. The study was approved by local ethic committe. The prevalence of MSK symptoms, self-reported functional impairment and their categories are provided as percentage of the study sample. Student $t$ or qui-squared test were used to compare those with and without MSK pain, two-tailed $\mathrm{p}<0.05$ were considered statistically significant.

Results: 370 individual, mean age of 47.1 (standard deviation (SD): 18.34) years old, $210(56.76 \%)$ women were included. Overall 245 individuals $(66.2 \%), 95 \%$ confidence interval $(\mathrm{Cl}): 61.2-70.8 \%$, experienced musculoskeletal symptoms in the last 7 days. Of whom 239/ $245(97.5 \%, 95 \% \mathrm{Cl}=94.8-98.9 \%)$ reported that their symptoms was not related to a previous trauma. The pain intensity was reported as "strong" to "severe" in 89/245 $(36.33,95 \mathrm{Cl}=30.6-42.5)$. Functional impairment was reported by $117 / 245(47.8 \%, 95 \% \mathrm{IC}=41.6-54)$. The most common painful joint was the lumbar spine $(74.28 \%$; $95 \% \mathrm{Cl}, 68.5 \%$ $79.3 \%)$. Thirty seven out 245 (15.1\% 95\%Cl:11.2-20.1)) reported symptoms in only one region. The mean age of subjects with and without MSK symptoms were 49 (17.9) and 43.2 (18.6) respectively, $p=0.037$. The proportion of female was $59.9 \%$ in the group with MSK pain and $51.2 \%$ in the group without MSK pain, $p=0.15$. 
Conclusion: We emphasize the need to improve the health education process for the local population and improve the quality of health services provided in relation to the musculoskeletal diseases due to the high prevalence of musculoskeletal symptoms and functional impairment in this community.

\section{P354}

\section{PROFILE OF PATIENTS USING CYCLOPHOSPHAMIDE IN A PUBLIC} HOSPITAL OF SERGIPE

Ana Carolina Vilhena De Melo, Thiago Reis De Santana, Telma Rodrigues Santos Da Paixao, Maíra Carvalho Ivo, Lina Oliveira De Carvalho, Blenda Hilíria De Sousa Dias, Monica Valeria Siqueira Santana De Vechi, Regina Adalva De Lucena Couto Ocea, Jose Caetano Macieira UNIVERSIDADE FEDERAL DE SERGIPE, ARACAJU, SE, Brasil Advances in Rheumatology 2018, 58(Suppl 1):P354

Background: Cyclophosphamide is an immunosuppressant used in the treatment of several systemic autoimmune diseases, but it is associated with several adverse events.

Methods: a cross sectional and descriptive study, in a public hospital in Sergipe, reviewing charts of the last four years, to know the profile of patients using intravenous cyclophosphamide.

Results and conclusions: 80 patients were evaluated, $77.5 \%$ female and mean age 37.1 years. Systemic lupus erythematosus was the most frequent condition, $73.75 \%$ of cases $(71.25 \%$ due to nephritis), followed by systemic sclerosis (11.25\%), nephrotic syndrome (10\%), myopathy $(2.5 \%)$, and other diseases (2.5\%). The dose of the drug was $1 \mathrm{~g}$ in $68.9 \%$ of cases, $750 \mathrm{mg}$ in $21.3 \%$ and $500 \mathrm{mg}$ in $9.8 \%$ of patients. Patients used oral anti-emetic and venous hydration, but none used 2-mercaptoethanesulfonate. Only $15 \%$ continued on quarterly maintenance therapy for two years. Only $15 \%$ had mild infections, followed by cytopenia (5\%), nausea and vomiting $(2.5 \%)$ and no cases of hemorrhagic cystitis. Gestation occurred in three patients, with the drug suspended, but two had an abortion and one was followed up. Despite the new treatment modalities, cyclophosphamide continues to be an important option for the induction of remission of numerous systemic autoimmune disorders.

\section{P355}

\section{PROFILE OF PATIENTS WITH SPONDILOARTRITES IN}

\section{IMMUNOBIOLOGICAL USE IN A PUBLIC HOSPITAL OF SERGIPE}

Thiago Reis De Santana, Telma Rodrigues Santos Da Paixao, Maíra

Carvalho Ivo, Blenda Hilíria De Sousa Dias, Lina Oliveira De Carvalho,

Monica Valeria Siqueira Santana De Vechi, Regina Adalva De Lucena

Couto Ocea, Jose Caetano Macieira

UNIVERSIDADE FEDERAL DE SERGIPE, ARACAJU, SE, Brasil

Advances in Rheumatology 2018, 58(Suppl 1):P355

Background: spondyloarthritis encompasses a group of inflammatory rheumatic diseases affecting the axial, peripheral skeleton and the enthesis. The use of immunobiologicals is an important therapeutic tool in the good evolution of these diseases.

Methods: a cross sectional and descriptive study at a public hospital in Sergipe, analyzing patient charts with spondyloarthritis using immunobiologicals in the pulse therapy service.

Results and conclusions: 96 patients, $69.8 \%$ male, mean age 42.1 years, $75 \%$ had Ankylosing Spondylitis (AS), 21\% Psoriatic Arthritis (PA), 2\% Enteropathic Arthritis (EA) and 2\% Spondylarthritis Undifferentiated (SU). Of the patients with AS, 37.5\% were using etanercept, $36.1 \%$ adalimumab and $26.4 \%$ infliximab, with $12.5 \%$ being in the second immunobiological. Of those with PA, 50\% used adalimumab, 35\% etanercept and $15 \%$ infliximab, only $5 \%$ as the second immunobiological. The two patients with EA used infliximab and the two with SU used adalimumab, with no history of failure. Only $19.8 \%$ did prophylaxis with isoniazid for tuberculosis. As for complications, $8.3 \%$ had mild infections, $4.1 \%$ had an allergy to infliximab, $4.1 \%$ had uveitis and $2 \%$ had tuberculosis. It is concluded that, in this study, immunobiological therapy presented few complications and the great majority remained in use of the first immunobiological.
P358

PSEDOXANTHOMA ELASTICUM ASSOCIATED TO SEVERE SYSTEMIC LUPUS ERYTHEMATOSUS: CASE REPORT

Larissa Cristiane De Oliveira Souza, Francisco Alves Bezerra Neto, Joel

José Santos Filho, David Cavalcante Barbosa, José Hilton Nogueira Júnior UFRN, NATAL, RN, Brasil

Advances in Rheumatology 2018, 58(Suppl 1):P358

Background: Pseudoxanthoma Elasticum is a rare genetic disease that leads to the calcification of elastic fibers, associated with skin gastrointestinal tract, cardiovascular and ophthalmic manifestations.

The association of pseudoxanthoma elasticum and other connective tissue diseases is rare. However, there are reports of its association with systemic lupus erythematosus

(SLE), rheumatoid arthritis and ankylosing spondylitis.

Case Report:

A 49-year-old male experienced onset of symmetric polyarthritis 3 years ago, in which point he began treatment with methotrexate, prednisone and hydroxychloroquine. He discontinued such therapy without medical advice.

He was admitted due to hypertension, daily fever, asthenia and anasarca which initiated

2 months prior. Lab workup revealed positive ANA 1/2560 (nuclear homogeneous pattern), positive Anti-DNA 1/160 and positive Rheumatoid factor 4096 (RV: $<8 \mathrm{UI} / \mathrm{mL}$ ).

Urinalysis revealed proteinuria and hematuria. The urinary Protein/ Creatinin ratio was

1.3 , serum creatinine of 2.9 and serum urea of 188 .

The patient was diagnosed SLE and Lupus Nephritis with progressing uremia leading to the need for hemodialysis over 1 week.

Kidney biopsy revealed class IV diffuse proliferative glomerulonephritis IA: 6/12, IC:2/12 and non-inflammatory lupus vasculopathy.

He was given pulse therapy of $500 \mathrm{mg}$ Methylprednisolone daily and $1 \mathrm{~g}$ Cyclophosphamide daily, with good clinical response.

Upon physical examination he displayed yellowish papules on the cervical region bilaterally, which coalesced into two $8 \mathrm{~cm}$ diameter plaques laid parallel to skin cleavage lines, (Fig. 1. picture 1) as well as micro papules in the lower lip mucosa (Fig. 1. picture 2).

Both lesions are characteristic of pseudoxanthoma elasticum.

Due to complaint of visual blurring on the left eye, the patient had an ophthalmologic evaluation, which reported Angioid streaks in the right eye, posterior and intermediate uveitis in his left eye along with signs of vasculitis (Fig. 1. pictures 3 and 4).

Conclusion:

It is a rare association of two diseases. The pathophysiological mechanism of such association has not yet been identified. However, a rise in number of case reports over the last decades has taken place. Due to heightened patient morbidity related to lupus manifestations combined with serious ocular and vascular complications from pseudoxanthoma elasticum, special attention is required as to provide better clinical management.

\section{Consent for publication}

The authors declare that they have obtained informed written consent from the patient's tutors for publication.

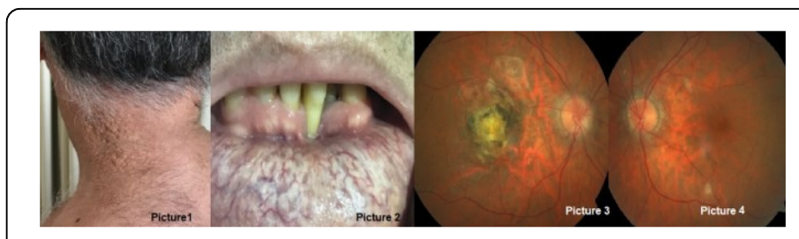

Fig. 1 (abstract P358). Picture 1, Picture 2 Picture 3 (Presence of angioid streaks with disciform scar and uncompromised vessels) Picture 4 [Presence of angioid streaks with vascular narrowing and cotton wool spots in upper and lower temporal vessels (vasculitis)] 


\section{P364}

PSORIATIC ARTHRITIS SINE PSORIASIS: CLINICAL AND

\section{ULTRASOUND DIAGNOSTIC EVALUATION}

Gabriela Miyuki Teodoro Ogawa' ${ }^{\text {}}$, Jacqueline Foelkel Pignatari ${ }^{2}$, Mateus de Miranda Moura Cortês ${ }^{2}$, Lara Ribeiro Teixeira Bonfim², Pamella de Paula Bellini ${ }^{2}$, Samuel de Oliveira Andrade ${ }^{2}$, Manoel de Barros Bertolo ${ }^{2}$, Michel Alexandre Yazbek ${ }^{2}$

${ }^{1}$ UNIVERSIDADE ESTADUAL DE CAMPINAS, CAMPINAS, SP, Brasil; ${ }^{2}$ UNIVERSIDADE ESTADUAL DE CAMPINAS (UNICAMP), CAMPINAS, SÃO PAULO, Brasil

Advances in Rheumatology 2018, 58(Suppl 1):P364

Background: The diagnosis of psoriatic arthritis (PA) is often challenging, since AP is a multifaceted disease with different clinical manifestations, in which skin involvement is not always present or is not recognized by the physician. Ultrasonography has proved to be a very useful tool in the diagnosis of this condition, even if the final diagnosis depends mainly on the clinical evaluation performed by the rheumatologist. High resolution ultrasonography with power doppler (PD) can aid in the evaluation of osteoarticular lesions, and response to treatment of the disease.

Case Report: A 63-year-old female patient, a retired washerwoman, under medical supervision for diffuse myalgia and bilateral calcaneodyne with initial fibromyalgia hypothesis and tendinopathy of calcaneus. He was treated with non-steroidal anti-inflammatory drugs (NSAIDs) and muscle relaxants with no satisfactory response. It envolved with symmetric arthritis of proximal interphalangeal, metacarpophalangeal and wrists. Leflunomide was prescribed for a probable diagnostic hypothesis of rheumatoid arthritis (RA), without improvement. Laboratory tests: negative rheumatoid factor, anti-CCP and FAN. Radiographs of hands and feet showed the findings of osteopenia and periarticular erosions; besides signs suggestive of perostitis and bone neoformations.

Due to the suspicion of AP, an ultrasound assessment with PD was requested, in which: signs of active synovitis in second and third metacarpophalangees bilaterally with PD positive and signs of paratendinitis throughout the extension of the extensor tendon of the third left chiropractor, characterizing dactylitis active.

At that moment, after the clinical, radiographic and ultrasonographic evaluations; we made the diagnostic hypothesis of PA, despite the absence of cutaneous psoriasis involvement, since the patient, according to the CASPAR criteria, presented arthritis, enthesitis and active dactylitis associated with negative rheumatoid factor tests and radiographic evidence of bone neoformation. Initiated therapy with adalimumab, the patient presented significant symptomatic and functional improvement, currently with low disease activity (DAPSA $=4.15$ ).

Conclusion: The case portrays the difficult diagnostic approach of a patient with AP without psoriasis skin involvement. Under these conditions, the radiographic findings associated with the ultrasound evaluation with PD were essential for the final diagnosis and management.

\section{Consent for publication}

The authors declare that they have obtained informed written consent from the patient's tutors for publication

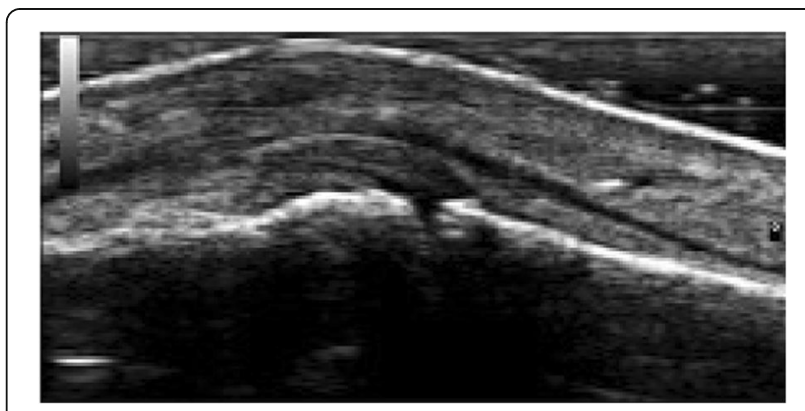

Fig. 1 (abstract P364). Signs of paratendinitis of the extensor tendon of the third left finger

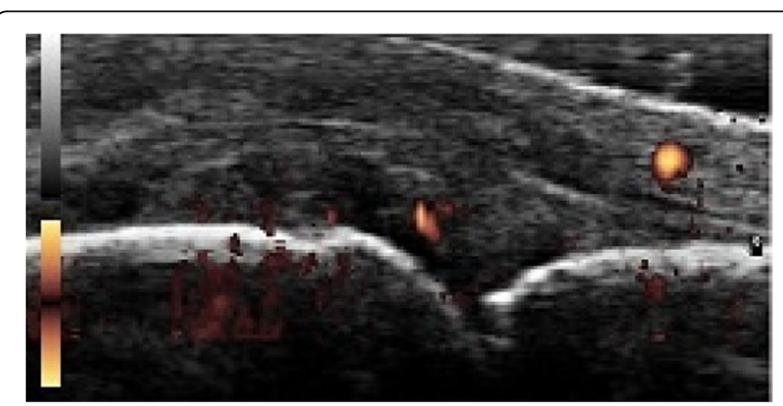

Fig. 2 (abstract P364). Signs of active synovitis (positive PD) in third left metacarpophalange

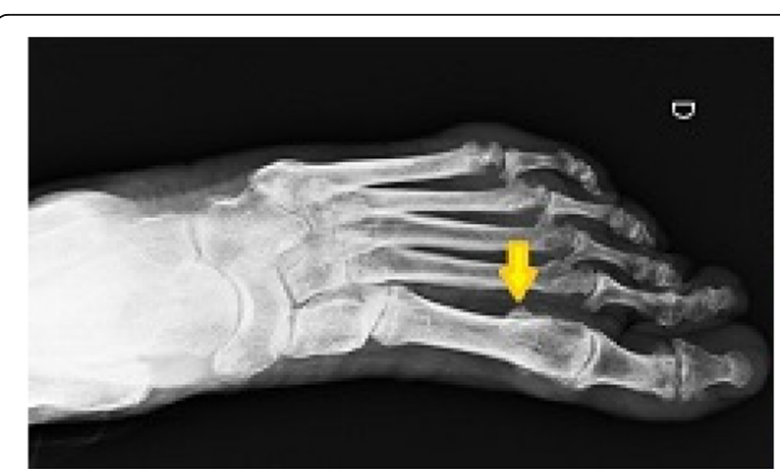

Fig. 3 (abstract P364). Signs of entesopathy of the third left finger nail insertion

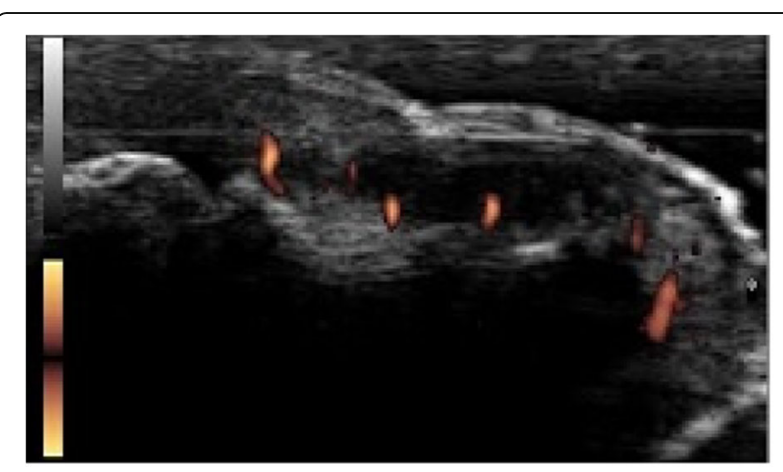

Fig. 4 (abstract P364). Radiographi

P370

PYODERMA GANGRENOSUM IN A PATIENT WITH RHEUMATOID ARTHRITIS AND SYSTEMIC LUPUS ERYTHEMATOSUS

Pamella de Paula Bellini ${ }^{1}$, Jacqueline Foelkel Pignatari ${ }^{2}$, Mateus de Miranda Moura Cortes², Gabriela Miyuki Teodoro Ogawa², Lara Ribeiro Teixeira Bonfim², Samuel de Oliveira Andrade 2 , Alisson Aliel Viagano Pugliesi ${ }^{2}$, Manoel Barros Bertolo ${ }^{2}$, Lilian Tereza Lavras Costallat ${ }^{2}$ ${ }^{1}$ UNIVERSIDA ESTADUAL DE CAMPINAS - UNICAMP, CAMPINAS, SP, Brasil; ${ }^{2}$ UNIVERSIDADE ESTADUAL DE CAMPINAS (UNICAMP), CAMPINAS, SP, Brasil

Advances in Rheumatology 2018, 58(Suppl 1):P370

Introduction: Pyoderma gangrenosum (PG) is a rare, chronic disease classified as a neutrophilic dermatosis. It has uncertain etiology and may be associated with systemic diseases in 50 to $70 \%$ of the cases, mainly: inflammatory bowel diseases, rheumatoid arthritis (RA), multiple myeloma, human immunodeficiency virus (HIV) infection, 
systemic lupus erythematosus (SLE), and leukemia. It is more common amongyoung adults (25-50 years of age) and predominates over the female sex. The clinical presentation is characterized by ulcerated (multiple or solitary) skin lesions evolving rapidly, with the lower limb being the most affectedsite. Glucocorticoids (GC) in high doses constitute first-line therapy.

Case report: A 38-year-old female patient diagnosed with RA -rheumatoid factor (RF) and anti-CCP positive-and SLE (rash, arthritis, anti-DNA, FAN 1/640 dotted thick reticulated), on treatment with hydroxychloroquine and methotrexate for 2 years, had a sudden rapid evolution of ulcer in the left forefoot (Photo 1), concomitant with worsening of the joint and consumption of complement. Based on the lesion characteristics and the histological appearance of neutrophilic dermatosis, a diagnosis of PG was made. Despite GC treatment, the lesion evolved for bone and tendon exposure, with PG complicated by osteomyelitis and requiring suprapatellar amputation. There was recurrence of the lesion in the amputated stump, and it was decided to associate infliximab with the treatment.

Discussion: As in the case reported, the association of PG with RA is more described in those with RF and anti-CCP positivity and, when associated with SLE, other elements of disease activity are commonly found. Amputation of areas affected by PG should be avoided, since there is frequentrecurrence in the amputated site. For patients not responsive to corticosteroid therapy, cytostatics or immunobiological agents may be used, all based on low levels of evidence. In the case reported, anti-TNF was chosen because of the need to treat the joint condition.

\section{Consent for publication}

The authors declare that they have obtained informed written consent from the patient's tutors for publication

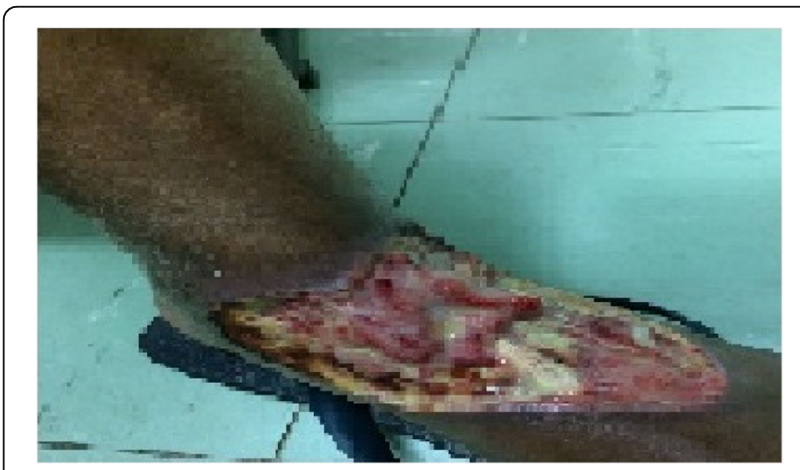

Fig. 1 (abstract P370). Ulcer in the left forefoot

\section{P371}

QUALITY OF LIFE OF CHILDREN AND ADOLESCENTS WITH AUTOIMMUNE RHEUMATIC DISEASES SEEN AT A REFERENCE CLINIC IN SALVADOR, BAHIA-BRAZIL

Teresa Cristina Martins Vicente Robazzi', Tayná Rangel Barreto', Ludmila Santiago Viana', Beatriz Oliveira Leão Carneiro', Jose

Cleosmaque Leite Júnior ${ }^{1}$, Cristiani Leal ${ }^{2}$, Leandra Chaves Silva Barros ${ }^{2}$, Regina Terse Ramos ${ }^{1}$

${ }^{1}$ FACULDADE DE MEDICINA DA UNIVERSIDADE FEDERAL DA BAHIA SALVADOR, BA, Brasil; ${ }^{2}$ HOSPITAL UNIVERSITÁRIO PROFESSOR EDGAR SANTOS, SALVADOR, BA, Brasil

Advances in Rheumatology 2018, 58(Suppl 1):P371

Background: Autoimmune rheumatic diseases are considered rare in the pediatric population. Diseases such as juvenile idiopathic arthritis (JIA), the most prevalent of this type, can present with symptoms that culminate in varying degrees of physical disability. In addition, it is known that chronic disease in general negatively affects various aspects of the quality of life (QoL) of their carriers.

Methods: A cross-sectional observational study employing an analytical base included children from 8 to 18 years of age. An interview was carried out to acquire clinical and sociodemographic data, and the Childhood Health Assessment Questionnaire (CHAQ) and Pediatric Quality of Life Inventory (PedsQL TM 4.0) Generic Core Scales were applied to evaluate participant functional capacity and QoL, respectively.

Results and Conclusions: The sample consisted of 59 children with autoimmune rheumatic disease; $61 \%$ were female; the median age and interquartile interval was 9 years and 9 months $(6.75 ; 12.83)$; $88 \%$ self-declared being non-white and most were diagnosed with JIA (42.4\%). The results of the CHAQ demonstrated that this population presents mild functional disability, with an mean score of 0.44 $( \pm 0.64)$. The social domain of quality of life was the least affected in both children and adolescents, while the psychosocial and emotional domains were the most affected in children aged between 8 and 12 years and adolescents aged between 13 and 18 years, respectively. The association between the domains of quality of life and functional capacity was negative and statistically significant in children, except with regard to the correlation between functional capacity and the emotional domain of quality of life and as regards pain and the emotional domain. We concluded that the population studied presented diminished QoL in all investigated domains. A negative association between the areas of QoL and the functional capacity of children in the physical, social, school and psychosocial areas and functional capacity was observed.

\section{P372}

RADAI PERFORMANCE (RHEUMATOID ARTHRITIS DISEASE ACTIVITY INDEX) IN PATIENTS WITH RHEUMATOID ARTHRITIS IN BRAZIL

Pedro Antônio Ramon Haddad, Ana Beatriz Vargas dos Santos, Geraldo da Rocha Castelar Pinheiro, Rodrigo Balbino Chaves Amorim, Leticia Rocha Pereira, Ricardo Azedo, Camila Souto Oliveira, Manuella Lima Gomes Ochtrop

UERJ, RIO DE JANEIRO, RJ, Brasil

Advances in Rheumatology 2018, 58(Suppl 1):P372

Background: Rheumatoid arthritis (RA) is an autoimmune systemic disease with multifactorial pathophysiology and affects about $1 \%$ of the population. It is characterized by inflammation of the peripheral joints which, if not properly controlled, can lead to destruction and deformity of these structures. Besides pain, progressive and permanent functional disability may significantly compromise the quality of life of these patients. The current concept of RA treatment emphasizes, among other aspects, the strict control of disease activity. In order to be controlled, disease activity must be measured, hence the creation of several disease activity composite indexes (DACls)-, using clinical and laboratory data. Among the most employed are DAS28 (Disease Activity Index-28 joints), SDAI (Simplified Disease Activity Index) and CDAl (Clinical Disease Activity Index). Although these traditional DACls take into account the patient's assessment of disease activity, they depend on and are based on the health professionals' evaluation and laboratory data. Alternatively, and exclusively valuing the patients' reports, a new DACl was created - the RADAI (Rheumatoid Arthritis Disease Activity Index), which does not depend on the participation of health professionals or complementary tests, being easy to perform and with low cost. There is no consistent prior data of RADAl in Brazil. The main objectives of the study were to evaluate the performance of RADAI in Brazilian patients with rheumatoid arthritis, analyzing their correlation with traditional DACls.

Methods: We analyzed data from the baseline of a prospective cohort of RA patients from a multicenter study "REAL - RA in real life in Brazil". A total of 1.115 patients were included, from 11 public institutions of the Unified Health System, from different Brazilian states. The performance of the RADAl was analyzed in a comparative way, 
using kappa test, to other tools already used in the evaluation of patients with RA, testing the agreement between them.

Results and conclusions: The correlation between RADAl and traditional DACls ranged from moderate to strong, 0.72 between RADAI and CDAl, 0.72 between RADAI and SDAl, 0.65 between RADAI and DAS28 CRP and 0.59 between RADAI and DAS28 ESR The preliminary analysis points to a good performance of RADAI in Brazilian patients affected by RA when compared to the most commonly used tools.

Table 1 (abstract P372). Agreement between the DACls - DACls: Disease Activity Composite Indexes; CDAl: Clinical Disease Activity Index; DAS28: Disease Activity Score 28 joints; SDAl: Simplified Disease Activity Index.

\begin{tabular}{|lcc|}
\hline & RADAI & $\mathrm{n}=1115$ \\
CDAI & 0.7235 & 1112 \\
SDAI & 0.7219 & 941 \\
DAS28 RCP & 0.6552 & 935 \\
DAS28 ESR & 0.5903 & 923 \\
\hline
\end{tabular}

\section{P380}

\section{REFRACTORY ASCITE IN THE SYSTEMIC LUPUS ERYTHEMATOSUS:} CASE REPORT

Eduarda Caroline Moreira Vieira, Emanuely Camargo Barbosa, Fernanda Miranda Caliani Sá, Izabella de Carvalho Sapia, Manoela Peluso Bucchi UNOESTE, PRESIDENTE PRUDENTE, SÃO PAULO, Brasil

Advances in Rheumatology 2018, 58(Suppl 1):P380

Background: Systemic lupus erythematosus (SLE) is a chronic autoimmune disease of multifactorial etiology (genetic, hormonal and environmental factors), which includes young women with involvement of multiple organs and systems, between periods of exacerbations and remissions. Serositis is a common disease in patients with SLE. About $16 \%$ of patients have pleuritis and/or pericarditis. However, peritoneal serositis presenting ascites is a rare manifestation.

Case report: Female patient, 24 years old, with diagnosis of SLE according to SLICC 2012 criteria: FAN nuclear homogeneous standard reagent $1 / 640$, anti-Sm reagent, complement consumption, polyarthritis, cicatricial alopecia and bilateral pleural effusion in hydroxychloroquine use and low dose of corticoid, evolute with pain complaint and progressive increase of abdominal volume. The production aprary globose abdomen, distended, dolorous and positive rap. Ultrasound of abdomen large ascites without other changes. Diagnostic and relief paracentesis was performed. Asthmatic liquid with standard exudate. Discarded with other causes of exudative ascites (such as peritoneal carcinomatosis, hepatocellular carcinoma, tuberculous peritonitis, fungal peritonitis, nephrotic syndrome, proteinlosing enteropathy ...), was launched with corticosteroid treatment with immunosuppressive dose. The persistence of ascites, the need for repeated paracenteses to alleviate the monthly pulse therapy of cyclophosphamide, evolving with the full picture of the fifth month of pulse therapy. Nowadays she is in outpatient follow-up, asymptomatic, using hydroxychloroquine and azathioprine.

Conclusion: Lupus peritonitis is a rare non-SLE manifestation and should be used as a diagnosis of exclusion. The prognosis is generally good, and the treatment is based on the use of nonhormonal anti-inflammatories and corticosteroids, obtaining a good response. In refractory cases, individual treatment measures are adopted.

\section{Consent for publication}

The authors declare that they have obtained informed written consent from the patient's tutors for publication
P383

RELATIONSHIP BETWEEN DENSE FINE SPECKLED ANTINUCLEAR ANTIBODIES AND MANIFESTATIONS CLINICAL AND LABORATORIAL OF SYSTEMIC LUPUS ERITHEMATOSUS

Fernanda Pulcheri Ramos, Andrey Tonetto Barbosa, Matheus Xavier Guimarães, Izaura Tereza Silva Guedes, Raissa Barbosa de Souza, Denise Moraes Horiy, Renata Ferreira Rosa, Rina Dalva Neubarth Giorgi, Nafice Costa Araujo

HOSPITAL DO SERVIDO PÚBLICO ESTADUAL DE SÃO PAULO, SÃO

PAULO, SÃO PAULO, Brasil

Advances in Rheumatology 2018, 58(Suppl 1):P383

Introduction: Systemic lupus erythematosus (SLE) is a multisystem inflammatory autoimmune disease of unknown etiology and with variable laboratory clinical manifestations. Antinuclear antibodies (ANA), for indirect immunofluorescence in Hep-2 substrate, were the firstline autoantibodies in suspicion of SLE. The pattern dense fine speckled (DFS) is most common in general population, and historical is not associated to SLE or other autoimmune disease. In SLE the most commons ANA patterns are homogeneous and speckled. The DFS ANA is less related with SLE in literature.

Objectives: To determine the relationship between DFS ANA and SLE in a tertiary hospital of São Paulo.

Methods: This retrospective study, conducted in 2018, included 236 patients with SLE according ACR 1997 criteria and followed at rheumatology clinic in a tertiary hospital. Eleven patients were excluded of this study due insufficiency of data. The samples were divided in two groups, group $1(\mathrm{~N}=94,40,44 \%)$ with DFS ANA not reagent and group $2(\mathrm{~N}=134,59,56 \%)$ DFS ANA reagent in some time of the evolution. For statistical analysis: mean, standard deviation, frequency (\%), t-student, Mann-Whitney and Chi-square tests were used. $\mathrm{P}<0,05$ was considered statistically significant.

Results: The mean age was $55,51 \pm 11,20$ and $52,97 \pm 13,39$ years, for group 1 and group 2 , respectively $(p=0,3928)$, and the time of followup not had statistical significance $(p=0,1574)$. Antiphospholipid syndrome (APS) was more common in group $2(\mathrm{~N}=24, \mathrm{p}=0,0312)$, and this group had more anti-DNAds, anticardiolipin IgG and lupic anticoagulant antibodies positives $(\mathrm{N}=54, \mathrm{p}=0,0014 ; \mathrm{N}=24, \mathrm{p}=0,0312$; $\mathrm{N}=17, \mathrm{p}=0,0379)$. Need of renal biopsy $(\mathrm{N}=41, \mathrm{p}=0.0065)$ were significantly higher in group 2. Not there were difference statistics regarding presence of nephritis $(p=0,1173)$. The majority of the patients in group 2 had DFS ANA titles largest 1/320 ( $N=122,91,04 \%)$.

Conclusion: The study demonstrated a relationship between DFS ANA and SLE with renal injury and APS association, not corroborating with the international medical literature. However, is not has been evaluated the DFS70, DFS ANA antibodie related. New studies are in progress for best elucidation the relationship between DFS ANA and SLE in our patients.

P384

RELATIONSHIP BETWEEN PARAMETERS OF METABOLIC SYNDROME AND PAIN, STIFFNESS AND PHYSICAL FUNCTION BY WOMAC QUESTIONNAIRE AND PAIN CATASTROPHIZING SCALE IN PATIENTS WITH KNEE OSTEOARTHRITIS

Aline Castilho de Almeida, Maria Gabriela Pedroso, Jéssica Bianca Aily, Gláucia Helena Gonçalves, Stela Márcia Mattiello

UNIVERSIDADE FEDERAL DE SÃO CARLOS (UFSCAR), SÃO CARLOS, SP, Brasil

Advances in Rheumatology 2018, 58(Suppl 1):P384

Background: Previous studies have suggested that metabolic syndrome (MS) is associated with osteoarthritis (OA). However, analyses have focus on pain level and radiographic severity and have not addressed other clinical and functional self-reported parameters as stiffness, physical function and the pain catastrophism. This study was undertaken to examine the relationship of MS and its components with pain, stiffness, function and pain catastrophism in patients with knee OA. 
Methods: Participants of both sexes, aged between 40 and 65 years with KOA were selected based on the American College of Rheumatology criteria (ACR) and classified by the Kellgren and Lawrence scale as grades 2 and 3 . Only participants with $B M I<30 \mathrm{~kg} / \mathrm{m} 2$ were included. Diagnostic Parameters for metabolic syndrome followed the National Cholesterol Education Program Adult Treatment Panel III. Subjects were considered to have metabolic syndrome if they met at least 3 of the following 5 criteria: 1 ) abdominal obesity (waist circumference $\geq 102 \mathrm{~cm}$ in men and $\geq 88 \mathrm{~cm}$ in women), 2) high triglyceride levels $(\geq 150 \mathrm{mg} / \mathrm{dl}), 3)$ low HDL cholesterol $(<40 \mathrm{mg} / \mathrm{dl}$ in men and $<50 \mathrm{mg} / \mathrm{dl}$ in women), 4) high blood pressure (systolic blood pressure $\geq 130 \mathrm{~mm} \mathrm{Hg}$ or diastolic blood pressure $\geq 85 \mathrm{~mm} \mathrm{Hg}$ or treatment for high blood pressure), and 5) high fasting glucose ( $\geq 110 \mathrm{mg} / \mathrm{dl}$ or diagnosis of diabetes). All patients completed the Western Ontario and McMaster Universities Osteoarthritis Index (WOMAC) questionnaires that measured self-reported pain, stiffness and physical function followed by the Pain Catastrophizing Scale. Initially, data were checked for normality by the Shapiro-Wilk test. To analyze the correlation index between the variables, the Pearson's test was used to correlate MS parameters WOMAC and PCS scores. For all analyzes was adopted a significance level of $5 \%(p \leq 0.05)$.

Results: Sixty participants (mean age of $53.08 \pm 5.92$ years, $1.65 \pm$ $0.06 \mathrm{~m}, 72.60 \pm 9.34 \mathrm{~kg}$ and $26.70 \pm 3.00 \mathrm{~kg} / \mathrm{m} 2$ ) were included in this study. The results of this study are shown in Table 1. A significant positive correlation was observed for the MS parameters triglycerides with pain, physical function and total scores. For systolic blood pressure, was observed a positive relationship only for pain, and for WOMAC pain, physical function and PCS.

Conclusion: It can be concluded that only triglyceride, SBP and DBP parameters of MS can influence on pain, physical function and total scores of the WOMAC questionnaire and only the DBP for PCS in the investigated population.

Table 1 (abstract P384). Pearson Correlation and $p$ values for Metabolic Syndrome parameters and self-reported Questionnaires (WOMAC and Pain Catastrophizing Scale)

\begin{tabular}{|c|c|c|c|c|c|c|}
\hline & IDL. & Trighucerbites & Glucese & SBP & DAP & $\begin{array}{l}\text { Abdominal } \\
\text { circumforeace }\end{array}$ \\
\hline $\begin{array}{l}\text { wousc } \\
\text { paia }\end{array}$ & $\begin{array}{l}-0.238 \\
\langle 0.070)\end{array}$ & $\begin{array}{l}0.279 \\
0.032 \gamma^{\circ}\end{array}$ & $\begin{array}{l}0.161 \\
(0.2221\end{array}$ & $\begin{array}{l}0.270 \\
0.0399^{\circ}\end{array}$ & $\begin{array}{l}0.390 \\
0.0025^{\circ}\end{array}$ & $\begin{array}{l}0.029 \\
10.827\end{array}$ \\
\hline $\begin{array}{l}\text { woxac } \\
\text {,tifness }\end{array}$ & $\begin{array}{l}0.027 \\
\langle 0.839\rangle\end{array}$ & $\begin{array}{l}0.234 \\
\langle 0.574\rangle\end{array}$ & $\begin{array}{l}0.163 \\
(0.216)\end{array}$ & $\begin{array}{l}0.166 \\
(0.208)\end{array}$ & $\begin{array}{l}0.162 \\
(0.221)\end{array}$ & $\begin{array}{l}0.064 \\
10.629)\end{array}$ \\
\hline $\begin{array}{l}\text { woslac } \\
\text { phissteal } \\
\text { fanction }\end{array}$ & $\begin{array}{l}-0,046 \\
\langle 0,728\rangle\end{array}$ & $\begin{array}{l}0.290 \\
\langle 0,026\rangle^{*}\end{array}$ & $\begin{array}{l}0,022 \\
(0,870)\end{array}$ & $\begin{array}{l}a, 130 \\
\langle 0,325\rangle\end{array}$ & $\begin{array}{l}0.298 \\
10.0225^{\circ}\end{array}$ & $\begin{array}{l}-0,015 \\
(0,910)\end{array}$ \\
\hline $\begin{array}{l}\text { woseac } \\
\text { Total }\end{array}$ & $\begin{array}{l}0,000 \\
\langle 0,600)\end{array}$ & $\begin{array}{l}0.309 \\
0.01720\end{array}$ & $\begin{array}{l}0.070 \\
0.5961\end{array}$ & $\begin{array}{l}0.175 \\
(0.185)\end{array}$ & $\begin{array}{l}0.331 \\
0.010)\end{array}$ & $\begin{array}{l}0.007 \\
10.961)\end{array}$ \\
\hline $\begin{array}{l}\text { Pain } \\
\text { Catsatrophysing } \\
\text { Scolk }\end{array}$ & $\begin{array}{l}-0,036 \\
\{0.787\rangle\end{array}$ & $0.217(0.099)$ & $\begin{array}{l}0.152 \\
(0.252)\end{array}$ & $\begin{array}{l}0.087 \\
\langle 0.512\rangle\end{array}$ & $\begin{array}{l}0.283 \\
0.030):\end{array}$ & $\begin{array}{l}-0.005 \\
(0.950)\end{array}$ \\
\hline
\end{tabular}

\section{P387}

RENAL TUBULAR INVOLVEMENT IN SYSTEMIC LUPUS

\section{ERYTHEMATOSUS}

Julia Soares Contador, Vanessa Jung, Aiessa Zanchett Fedrigo,

Betania Longo, Thiago Alberto Fernandes Gomes dos Santos,

Thelma Laroca Skare

HOSPITAL UNIVERITÁRIO EVANGÉLICO DE CURITIBA, CURITIBA, PARANÁ, Brasil

Advances in Rheumatology 2018, 58(Suppl 1):P387

Background:Although glomerulonephritis is well studied, little is known about tubular involvement in systemic lupus erythematosus (SLE). Here we studied the changes in renal tubules of SLE patients and their clinical associations.
Methods: We analyzed 32 records and tissue material of SLE patients who underwent renal biopsy. Epidemiological data, clinical and autoantibodies profile, and information on the disease course were collected. The biopsies werestudied for glomerular and tubular alterations. In the glomeruli we analyzed: quantity of glomeruli, cellularity in the Bowman's capsule, basement membrane and epithelial changes; in the renal tubules it was looked for presence of atrophy, inflammatory infiltrate and interstitial aspect. Each biopsy was inserted into the ISN/RPS classification (2003).

Results: In this sample, $54.8 \%$ were Afrodescendants, $41.9 \%$, Caucasian and $3.2 \%$ Asian. In relation to sex, $78.1 \%$ were women. Glomerulonephritis was found as class II in $6.25 \%$; class III in 18.7\%; class IV in $46.8 \%$; class V in $25 \%$ and class $\mathrm{VI}$ in $3.12 \%$. Of these patients: $56.2 \%$ had partial remission of nephritis; total remission wasseen in $21.8 \%$ and treatment failure in $21.8 \%$. Tubular findings showed fibrosis in $40.6 \%$, inflammatory infiltrate in $59.6 \%$ and mesangial expansion in $71.8 \%$. Only 11 patients had normal tubules. Patients with total remission had less tubular fibrosis $(p=0.002)$. The presence of mesangial expansion and inflammatory infiltrate did not change the remission rate. The glomerulonephritis class did not influence the tubular findings.

Conclusion: We concluded that the majority of patients with lupus glomerulonephritis in this sample had tubular involvement. Adverse treatment response was associated with the presence of tubular fibrosis.

\section{References}

1. Klum EM et al. Consenso da Sociedade Brasileira de Reumatologia para diagnóstico, manejo e tratamento da nefrite lúpica. Rev Bras Reumatol, 2015, 55(1):1-21

2. TEH, Cheng Lay et al. Causes and predictors of mortality in biopsyproven lupus nephritis: the Sarawak experience. Clin Kidney J. 2018; 11(1):56-61.

Table 1 (abstract P387). COMPARISON OF TREATMENT RESPONSE AND GLOMERULONEPHRITIS CLASSES ACCORDING TO PRESENCE OF TUBULAR FIBROSIS.

\begin{tabular}{|c|c|c|c|}
\hline & With fibrosis(n=13) & Without fibrosis(n=19) & p \\
\hline Total remission & $1 / 13-7.6 \%$ & $9 / 19-47.3 \%$ & $0.02\left(^{*}\right)(\#)$ \\
\hline Parcial remission & $9 / 13-69.2 \%$ & $6 / 19-31.5 \%$ & $0.07\left(^{*}\right)$ \\
\hline Treatmentfaillure & $3 / 13-23.07 \%$ & $4 / 19-21.05 \%$ & $1.00(* *)$ \\
\hline Anti-DNApositivity & $8 / 13-61.5 \%$ & $10 / 19-52.6 \%$ & $0.61(* *)$ \\
\hline \multirow[t]{5}{*}{ Glomerulonephritis classes } & Class $\|=1 / 13-7.6 \%$ & Class $\|=1 / 19-5.2 \%$ & $0.59(* *)$ \\
\hline & Class||I=2/13-15.3\% & Class III $=4 / 19-21.05 \%$ & \\
\hline & Class IV $=5 / 13-38.4 \%$ & Class IV $=10 / 19-52.6 \%$ & \\
\hline & Class V $=5 / 13-38.4 \%$ & Class $V=3 / 19-15.7 \%$ & \\
\hline & & Class VI $=1 / 19-5.2 \%$ & \\
\hline
\end{tabular}

\section{P388 \\ RESTRICTIVE CARDIOMYOPATHY IN SYSTEMIC LUPUS ERYTHEMATOSUS: CASE REPORT}

Fernanda Armond Castro ${ }^{1,2}$, Letícia Stivanin da Cunha Alves ${ }^{3}$,

Thaís Carvalho de Sousa ${ }^{3}$, Rafael Prado Colares ${ }^{3}$, Lara Maria Arantes Campos $^{3}$, Leila Patrícia Muniz ${ }^{3}$, Luiza Ferreira Ribeiro Tadeu,

Marina Costa Leite, Marcela Grobério Borba ${ }^{3}$, Cláudia Lopes Santoro Neiva ${ }^{3}$, Paulo Madureira de Pádua ${ }^{3}$, Gustavo Lamego de Barros Costa ${ }^{3}$,

Eduardo José do Rosário e Souza ${ }^{3}$

'SANTA CASA BH, BELO HORIZONTE, MINAS GERAIS, Brasil; ${ }^{2}$ SANTA

CASA DE BELO HORIZONTE - MG, BELO HORIZONTE, MINAS GERAIS,

Brasil; ' ${ }^{3}$ SANTA CASA DE BELO HORIZONTE, BELO HORIZONTE, MG, Brasil

Advances in Rheumatology 2018, 58(Suppl 1):P388

Background: Cardiac disease is common among patients with Systemic Lupus Erythematosus and can involve pericardium, myocardium, valves, conduction system and coronary arteries. Pericarditis is the most common manifestation. Myocarditis is uncommon but may be severe and it can appear as a dilated or restrictive cardiomiopathy. It should be suspected whenever there are resting tachycardia, 
electrocardiographic abnormalities or unexplained cardiomegaly. Echocardiography should help detecting pericardial effusion, enlarged left ventricular and atrial diameter, reduced left ventricular systolic function and diastolic function. Changes in echocardiographic parameters involving the cardiac structure and function abnormalities were associated with prolonged disease duration and high disease activity score. Although the mechanisms of these abnormalities remain unclear, it may relate to myocarditis or coronary artery disease. Echocardiographic assessment should be considered as a part of routine examination for SLE patients and parameters may predict clinical outcomes.

Case report: A 44-year-old female patient, healthy until December/ 2016, developed photosensitivity, discoid lesions on the trunk and upper limbs, malar rash and alopecia. In May/2017, she was admitted for investigation of paroxysmal nocturnal dyspnea, orthopnea, resting tachycardia and lower limb edema. Relevant exams: Homogenous nuclear ANA 1: 640 , lymphocyte count of $918 / \mathrm{mm}^{3}$, C3 $45 \mathrm{mg} / \mathrm{dL}(90-$ $180 \mathrm{mg} / \mathrm{dL}), \mathrm{C} 48 \mathrm{mg} / \mathrm{dL}$ (19-52 mg/dL), dsDNA 1:80, Ro 240. In this context she was diagnosed with SLE and SELENA-SLEDAI $=18$. Chest radiograph showed cardiomegaly and transthoracic echocardiogram showed preserved ejection fraction, biatrial increase (left atrium $=50 \mathrm{~mm}$ ) and left ventricular asymmetric hypertrophy (posterior wall $=11 \mathrm{~mm}$ and interventricular septum $=16 \mathrm{~mm}$ ), associated with diastolic dysfunction with restrictive pattern. Propaedeutic for hemochromatosis and amyloidosis including aspiration of the abdominal fat pad with congo red was negative. Myocardial involvement, in the form of restrictive cardiomyopathy, was attributed to SLE and treatment was initiated with methylprednisolone and monthly intravenous cyclophosphamide. After eleven months of follow-up, she had clinical improvement, but maintaining echocardiographic changes.

Conclusion: Restrictive cardiomyopathy is rare, characterized by biatrial increase without ventricular dilatation. Storage diseases, such as hemochromatosis and amyloidosis, are the main causes for this manifestation. After the complementary exams, they were ruled out. Although the duration of the disease was short until hospital admission, the activity index was high at the time of diagnosis. Studies suggest that echocardiographic evaluation should be considered as a part of routine examination for SLE patients, especially for those with prolonged history or active disease parameters.

\section{Consent for publication}

The authors declare that they have obtained informed written consent from the patient's tutors for publication

\section{P389}

\section{RETICULAR ERYTHEMATOUS MUCINOSIS ASSOCIATED WITH}

\section{SYSTEMIC LUPUS ERYTHEMATOSUS: A CASE REPORT}

Débora Rocha de Moura Aguiar, Ingrid Bottino, Paulo Cesar Meucci

Pereira Nogueira, Andressa Alexandre de Araujo, Luiz Felipe Dipe Prates Miranda, Carlos Baptista de Figueiredo

HOSPITAL FEDERAL DE BONSUCESSO, RIO DE JANEIRO, RJ, Brasil

Advances in Rheumatology 2018, 58(Suppl 1):P389

Background: The Reticular Erythematous Mucinosis (REM) is a rare cutaneous condition of unknown patophysiology, prevailing in women in the fifth decade of life. It can be associated to neoplastic, infectious and autoimmune diseases. REM is considered for some authors a primary idiopathic form of cutaneous mucinosis, but others consider it a disorder closely related, or associated, to chronic cutaneous lupus erythematosus. In general, this is not associated with laboratory findings or systemic diseases, although systemic lupus erythematosus, colon and breast cancer, diabetes mellitus, myxedema, hypothyroidism, Hashimoto thyroiditis, thrombocytopenia, monoclonal gammopathies and HIV infection have been reported in patients with REM.

Case Report: A 51-year-old male in investigation for weight loss, polyarticular pain, erythematous macules and papules coalescing into a reticulate pattern on the midline of the back, chest and arms; fever and interstitial lung disease. He complains of photosensitivity, Raynaud and recurrent oral ulcers. During investigation proteinuria of $1,3 \mathrm{~g} /$ day, anemia, elevated inflammatory tests, decreased levels of C3 and C4, positive ANA and anti-Ro antibodies in high titers were found. The cutaneous biopsy was compatible with reticular mucinosis and the renal histopathology with membranous nephropathy (class $V$ lupus nephropathy). In the light of the consumptive and febrile syndrome, plus the positive epidemiologic history for tuberculosis, a bronchoscopy with bronchoalveolar lavage was performed. , Mycobacterium tuberculosis DNA was not detected. Cultures and oncotic cytology were negatives. Infectious and neoplastic diseases were discarded. Once met the systemic lupus erythematosus diagnostic criteria, immunosuppressive therapy with glucocorticoids, mycophenolate mofetil and hydroxychloroquine was initiated, resulting in remission of fever and cutaneous lesions, in addition to controlling the renal and pulmonary conditions.

Comments: In this case, in addition to a rare disease in a male patient, when REM has a predilection for middle-aged women, we report REM as a clinical manifestation of systemic lupus erythematosus. Another highlight of our case is the presence of lupus nephritis, a rare association in the literature.

Hydroxychoroquine is first-line therapy, with complete remission in more than $50 \%$.

\section{Consent for publication}

The authors declare that the patient was oriented about the case report and signed the written informed consent form.

\section{Consent for publication}

The authors declare that they have obtained informed written consent from the patient's tutors for publication

\section{P391}

RETROORBITAL PSEUDOTUMOR IN A CHILD: A RARE CASE OF GRANULOMATOSIS WITH POLIANGIATIS

Guilherme Leví Tres, Fernando Schimidt Fernades, Luiza Rossi, Micheline Sulzbacher Batista, Bruna de Lima Porto, Afonso Papke, Joana Mattioni

Ourique, Nicole Pamplona Bueno de Andrade, Sandra Helena Machado, Ricardo Machado Xavier

HOSPITAL DE CLÍNICAS DE PORTO ALEGRE, PORTO ALEGRE, RIO GRANDE DO SUL, Brasil

Advances in Rheumatology 2018, 58(Suppl 1):P391

Background: Granulomatosis with poliangiatis presenting in the childhood is rare and there are few reports in the literature of pseudotumor retroorbital as an initial manifestation.

Case Report: We report a case of a 10 years and 4 month old girl referred to the pediatric rheumatology outpatient clinic by the ophthalmology department due to a bilateral, larger in the left eye with 2 years evolution. Patient had already undergone examination by several ophthalmologists and had a history of corticosteroid use in the previous year with partial improvement of proptosis. She was referred after a result of the ocular lesion biopsy that demonstrated chronic inflammation with vascular proliferation, thickening of the wall and foreign body type granuloma, suggesting vascular inflammation. Patient had a previous history of attention deficit hyperactivity disorder with previous use of risperidone and chlorpromazine. She also reported frequent rhinorrhea and epistaxis in the last 2 years and cough for 2 months without hemoptysis. Laboratory investigation was carried out and showed a positive antineutrophil cytoplasmic autoantibodies (ANCA), perinuclear standard, at the title of 1: 160, high inflammatory markers and negative antinuclear factor. Computed tomography of the thorax showed an excavated nodular opacity measuring $9 \mathrm{~mm}$ in the upper lobe of the left lung, optic bronchoscopy with no alterations and negative tuberculin test. Bronchoalveolar lavage excluded the possibility of associated infectious illness. Rhinoscopy showed an irregular ulcerated lesion on left anterior septal mucosa and biopsy was compatible with extensive lymphoplasmacytic infiltrate and sketching the formation of granulomas. Urine examination demonstrated dysmorphic hematuria, nephrotic proteinuria, granular and leukocyte cylinders and she had loss of renal function during the investigation. Urinary tract ultrasound showed normal size kidneys for her age with increased cortical echogenicity. Due to severe organic involvement, the patient started treatment with corticosteroids and cyclophosphamide with 
progressive improvement of the nephritis and upper airway symptoms, as well as partial regression of the retroorbital pseudotumor. Conclusion: ANCA-associated vasculitis does not usually present in childhood and more rarely initially presents with pseudotumor retroorbital, we bring attention to the two years delay for the diagnosis and importance of knowledge of ophthalmologists and rheumatologists for the possibility of this diagnosis to avoid permanent organic damage for these patients.

\section{Consent for publication}

The authors declare that they have obtained informed written consent from the patient's tutors for publication

\section{P393}

\section{RHEUMATIC DISEASE AUTOANTIBODIES IN PATIENTS WITH} AUTOIMMUNE THYROID DISEASES

Renato Nisihara', Yasmine G. Pigosso², Nathalia Prado², Shirley R. Utiyama ${ }^{2}$, Betania Longo ${ }^{3}$, Thiago Alberto Fernandes Gomes dos Santos ${ }^{3}$, Gisah A. de Carvalho2, Thelma Laroca Skare ${ }^{3}$

${ }^{1}$ HOSPITAL UNIVERITÁRIO EVANGÉLICO DE CURITIBA E HOSPITAL DE CLINICAS/UFPR, CURITIBA, PR, Brasil; ${ }^{2}$ HOSPITAL DE CLINICAS/UFPR, CURITIBA, PARANÁ, Brasil; ${ }^{3}$ HOSPITAL UNIVERITÁRIO EVANGÉLICO DE CURITIBA, CURITIBA, PARANÁ, Brasil

Advances in Rheumatology 2018, 58(Suppl 1):P393

Background: Patients with autoimmune thyroid diseases (ATD) such as Graves' disease (GD) and Hashimoto's thyroiditis (HT) may have nonorgan specific autoantibodies such as ANA (antinuclear antibodies) and $\mathrm{RF}$ (rheumatoid factor). In this study, we aimed to study the prevalence of rheumatic diseases autoantibodies in ATD patients without known rheumatic diseases and its association with the patient's clinical profile. We follow positive non-organ specific autoantibodies ATD individuals to see if they developed a rheumatic disorder.

Methods:154 ATD patients (70 HT and 84 GD; mean age 45.3 \pm 14.2 ) had determination of ANA by immunofluorescence (Hep-2 cells as substrate), ENA profile by ELISA kits and RF by latex agglutination. Clinical data was obtained through chart review. Patients were followed for the mean period of five years.

Results:Positive ANA was found in 17.5\%: anti Ro/SS-A in $4 / 154$ (2.5\%); anti RNP in 4/154 (2.5\%) and anti La/SS-B in 3/154 (1.9\%). None had anti-Sm antibodies. RF was detected in $7.7 \%$. All but one ANA positive patient were females. Tables 1 and 2 show the comparison of patients with and without autoantibodies. None of the autoantibody positive patients developed clinical rheumatic diseases during the observation.

Conclusion: We found $17.5 \%$ of prevalence of rheumatic autoantibodies in ATD patients without rheumatic diseases. None of them favored the appearance of clinical rheumatic disorder during the period of five years.

\section{References}

1. Tagoe $C E$, Zezon A, Khattri S. Rheumatic manifestations of autoimmune thyroid disease: the other autoimmune disease. J Rheumatol. 2012;39(6):1125-9

Table 1 (abstract P393). COMPARISON OF AUTOIMMUNE THYROID DISEASE PATIENTS WITH AND WITHOUT ANTINUCLEAR ANTIBODY (ANA)

TABLE 1- COMPARISON OF AUTOIMMUNE THYROID DISEASE PATIENTS WITH AND WITHOUT ANTINUCIFAR ANTIOODY (ANA)

\begin{tabular}{|c|c|c|c|}
\hline & $\begin{array}{c}\text { Positive ANA } \\
\text { Ne } 27\end{array}$ & $\begin{array}{l}\text { Negative ANA } \\
\qquad \begin{array}{l}N=127\end{array}\end{array}$ & p \\
\hline Median age (yeacs) & $49[41.57]$ & $46[34.55 .2]$ & 0.16 \\
\hline Caucasianethnic background (n) & $20 / 27[74 \%]$ & 98/127[77.1\%] & 0.82 \\
\hline Graves/Hashimoto (n) & $13 / 14$ & $71 / 56$ & 0.46 \\
\hline Positive Rheumatoidfactor (n) & $5 / 27(18.5 \%)$ & $7 / 127(5.5 \%)$ & $0.03(\xi)$ \\
\hline Smokers & $5 / 27[18.5 \%]$ & $22 / 127(17.3 \mathrm{~K})$ & 0.88 \\
\hline Arthragles (n) & $15 / 27(55.5 \%)$ & $79 / 127[62.2 \%]$ & 0.52 \\
\hline Tapazole and propylthiouracil users (n) & $3 / 27(11.1 \%)$ & $12 / 127[9,4 \%]$ & 1,00 \\
\hline Median dally $\mathrm{T4}$ dose $(\mu \mathrm{g} / \mathrm{Kg})$ & $1.45[1.12-1.81]$ & $1.58[1.20-1.93]$ & 0.91 \\
\hline
\end{tabular}

Table 2 (abstract P393). COMPARISON OF AUTOIMMUNE THYROID DISEASES PATIENTS WITH AND WITHOUT RHEUMATOID FACTOR (RF)

TABLE 2- COMPARISON OF AUTOIMMUNE THYROID DISEASES PATIENTS WITH AND WITHOUT RHEUMATOID FACTOR (RF)

\begin{tabular}{|c|c|c|c|}
\hline & $\begin{array}{c}\text { Positive Ri } \\
N=12\end{array}$ & $\begin{array}{l}\text { Negative RF } \\
\text { No14? }\end{array}$ & D \\
\hline Median age (years) & $55.8 \pm 14.5$ & $44.8 \pm 13.8$ & 0.007 \\
\hline Caucasianetheic background (n) & $10 / 12[83.3 \%]$ & $108 / 142[76.0 \%]$ & 0.83 \\
\hline Graves/Hashimoto & $8 / 4$ & $76 / 66$ & 0.54 \\
\hline Tobaccoexposure (n) & $5 / 12[41.6 \mathrm{~K}]$ & $22 / 142[15.4 \%]$ & $0.03(n)$ \\
\hline Arthralgias (n) & $9 / 12|75 \%|$ & $85 / 142(59.8 \%)$ & 0.56 \\
\hline Tapazole and propvlthiouracil users (n) & $2 / 12[16.1 \%]$ & $13 / 142[9.1 \%)$ & 0.33 \\
\hline 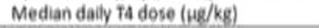 & $1.52[1,21 \cdot 1.79]$ & $1.54\{1.19-1.93\}$ & 0.70 \\
\hline
\end{tabular}

P394

RHEUMATOID ARTHRITIS AND METABOLIC SYNDROME: A PROSPECTIVE AND LONG-TERM COHORT OF ESTABLISHED RA PATIENTS

Nicole Pamplona Bueno de Andrade, Susana Ferreira Krampe, Penélope Esther Palominos, Vanessa Hax, Laura da Silva Alves, Filipe Abtibol, Brenda Steffani de Carvalho, Tauany Maria Ferraz Lopes, Claiton Viegas Brenol HOSPITAL DE CLÍNICAS DE PORTO ALEGRE, PORTO ALEGRE, RS, Brasil Advances in Rheumatology 2018, 58(Suppl 1):P394

Background: Epidemiologic studies suggest that rheumatoid arthritis (RA) is an independent risk factor for cardiovascular disease, and that the level of risk is influenced by the occurrence of metabolic syndrome (MS).

Methods: A prospective cohort of 283 RA patients was followed between 2008 and 2016. MS was defined according to the National Cholesterol Education Program (NCEP). Disease activity was assessed using the Disease Activity Score (DAS28). Clinical, anthropometric and biochemical evaluations were performed in both periods. A multivariate model was developed to investigate possible risk factors for the development of MS.

Results: Of 283 RA patients, 187 subjects were able to complete the evaluation in 2016. The sample consisted of 158 women (84.5\%) and the mean age was $61.4 \pm 10.9$ years. There was a significant increase in the prevalence of MS $(43.9 \%$ vs $59.4 \%, p<0.01)$. A significant reduction in DAS28 $(3.87 \pm 1.43$ vs $3.52 \pm 1.36, p=0.006), \mathrm{HDL}$ and systolic and diastolic blood pressure levels were also observed. ( $p$ $<0.001$ ). There was a significant association between the presence of MS and age, with the youngest patients not presenting MS at the first evaluation. Patients who maintained MS were those with the highest BMI. Of the 111 patients with MS in the second evaluation, $38(20.3 \%)$ did not have this condition at the beginning of the follow-up. After adjustment for the multivariate model, the increase in $\mathrm{BMI}$ (RR 1.12, 95\% Cl 1.02-1.23), CRP (RR 1.01, 95\% Cl 1-1.02), DAS 28 baseline (RR $2.1595 \% \mathrm{Cl} 1.03-4.48$ ) and higher prednisone dose in the 1st evaluation (RR $1.05,95 \% \mathrm{Cl} 1.02-1.08$ ) were factors independently associated with the occurrence of MS.

Conclusion: Over 8 years of follow-up, there was an increase in prevalence of MS in this cohort of patients with established RA, despite the improvement of DAS28. Other factors besides disease activity, such as the use of corticoticosteroids and weight, are associated to the development of MS over time.

\section{P395}

RHEUMATOID ARTHRITIS PATIENTS FAT MASS INDEX AND FUNCTIONAL CAPACITY ARE AFFECTED BY DISEASE ACTIVITY OVER 12 MONTHS

Rafaela Cavalheiro do Espírito Santo ${ }^{1,4}$, Bárbara Jonson Bartikoski ${ }^{4}$, Jordana Miranda de Souza Silva ${ }^{4}$, Priscila Schmidt Lora ${ }^{2}$, Lidiane Isabel Filippin $^{3}$, Ricardo Machado Xavier ${ }^{4}$ ${ }^{1}$ UNIVERSIDADE FEDERAL DO RIO GRANDE DO, PORTO ALEGRE, RIO GRANDE DO SUL, Brasil; ${ }^{2}$ UNIVERSIDADE DO VALE DO RIO DOS SINOS, SÃO LEOPOLDO, RIO GRANDE DO SUL, Brasil; ${ }^{3}$ UNIVERSIDADE LA SALLE, CANOAS, RIO GRANDE DO SUL, Brasil; ${ }^{4}$ UNIVERSIDADE FEDERAL DO RIO GRANDE DO SUL, PORTO ALEGRE, RIO GRANDE DO SUL, Brasil

Advances in Rheumatology 2018, 58(Suppl 1):P395 
Background: Rheumatoid cachexia (RC) is a condition characterized by adverse changes in body composition, specifically in muscle mass and fat mass components [1]. RA patients have life quality and expectative impacted by RC [2], but there are very few prospective data analyzing the evolution of this condition. Thus, our objective was to assess body composition, RC, clinical features and functional capacity in RA patients followed for 12 months.

Methods: Patients with RA, aged between 40 and 70 years, were recruited. Body composition was assessed by total body dual-energy $x-$ ray absorptiometry (DXA) for measurement of fat mass index (FMl; Kg/ $\mathrm{m} 2$ ) and fat free mass index (FFMl; $\mathrm{Kg} / \mathrm{m} 2$ ). Patients were categorized as rheumatoid cachectic if FFMI was below the 10th percentile and FMI above the 25th percentile [1], and if FFMI was below the 25th percentile and FMI above the 50th percentile [2]. Disease features assessed were disease activity score 28 (DAS28). Functional capacity was assessed by muscle strength (hand grip test;Kg) and gait speed (Time up and Go (TUG;m/s)). Frequency analysis, McNemar test and GEE analyses were used and statistical significance was considered as $p<0.05$.

Results: 81 patients analyzed, most were women $(88.9 \% ; 72 / 81)$, with mean age of $56.8 \pm 7.3$, mean disease duration time of $11.9 \pm 9.6$ years. Prevalence of RC did not change during the 12 month follow-up (13.3\% both times [1] and $30.0 \%$ at baseline $-24.4 \%$ at 12 months [2], p>0.05). FMI increased after 12 months and patients with moderate disease activity showed higher FMI when compared with other DAS28 categories $(p<0.05)$. Thus, over 12 months, DAS28 affected $\mathrm{FMl}$, and had no impact on FFMI ( $p>0.05$ ). Muscle strength decreased significantly after 12 months, and patients with high disease activity showed less muscle strength when compared with other DAS28 categories $(p<0.05)$. Gait speed increased after 12 months $(p<0.05)$. Conclusions: In this study, RC prevalence was similar to described data in literature. DAS28 score of our patients increased over 12 months, and it affected FMI, muscle strength and gait speed of RA patients. However, no effect was observed on FFMI. Our results show that the patients that are not in remission by DAS28 have decreased muscle strength and increased fat mass, possibly due the inflammatory process and the reduced physical activity level. This condition may negatively impact on life quality of RA patients.

\section{References}

[1] Engvall IL et al. Scand J Rheumatol. 2008. [2] Elkan AC et al. Arthritis Res Ther. 2009

\section{P397}

RHEUMATOID ARTHRITIS: RATIONAL USE OF HELMINTHS IN IMMUNOREGULATORY THERAPY

Thaís Rodrigues Roseno, Priscila Larcher Longo, Rômulo Tadeu Dias de

Oliveira

FACULDADE DAS AMÉRICAS, SANTOS, SP, Brasil

Advances in Rheumatology 2018, 58(Suppl 1):P397

Background: Rheumatoid arthritis (RA) is an autoimmune disease with inflammatory and chronic features, affecting approximately $1 \%$ of the world's adult population. AR has great physical, emotional and socioeconomic impact due to the high degree of synovial tissue involvement, bone loss and associated comorbidities. The most commonly used therapy is immunosuppression, which results in clinical improvement, but is associated with increase of opportunistic infections. The helminth therapy shows as an apparently safe, efficient and less debilitating alternative treatment.

Objective: This study aims to conduct a narrative review on the use of helminth therapy for Rheumatoid Arthritis. Methods: Search in database PUBMED performed for articles with the descriptors: arthritis and helminths.

Preliminary Results: A total of 61 articles were reached. 15 were excluded because they did not specifically treat about helminths or they just mentioned the pathology; in the end, we selected 46 studies including clinical trials and reviews. Based on the evolutionary history of humans and parasites, these studies indicate that helminth therapy is capable of modulate the host immune response, driving the immune response to $\mathrm{TH} 2$ profile, thus inhibiting the expression of inflammatory cytokines reactions, expressed in RA, and inducing the proliferation of regulatory $\mathrm{T}$ cells. Studies with experimental models show that treatment with helminths decreases inflammation and bone loss due change in activated T cells profile.

Conclusion: Up to now it is clear that the understanding of immunomodulation pathways promoted by helminths in RA is essential to explain its viability as a possible therapy with less harmful effects to the hosts of this debilitating autoimmune disease.

\section{P398}

\section{RHEUMATOLOGICAL INVOLVEMENT IN CHILDREN WITH} PSORIASIS

Thais Cugler Meneghetti ${ }^{1}$, Thais Mayumi Honda Padilha ${ }^{2}$ ${ }^{1}$ HOSPITAL DE CLINICAS DA UNIVERSIDADE FEDERAL DO PARANÁ, CURITIBA, PR, Brasil; ${ }^{2}$ HOSPITAL DE CLÍNICAS DA UFPR, CURITIBA, PARANÁ, Brasil

Advances in Rheumatology 2018, 58(Suppl 1):P398

Background: Recent evidences shows the role of enthesopathy in psoriatic disease and the high prevalence of musculoskeletal widespread pain in patients with psoriasis and psoriatic arthritis. Since limited data are available about the prevalence of rheumatological findings in children with psoriasis, our aim was to determine the frequency of musculoskeletal pain complaints, enthesitis and fibromyalgia tender points in a paediatric population with psoriasis and correlate it to the skin disease activity and quality of life.

Methods: 26 children and adolescents and with psoriasis and their parents were interviewed about musculoskeletal complaints, personal and familial history of rheumatic diseases. The physical examination included evaluation of active joint count, palpation of entheses and tender points of fibromyalgia. The PASI score and BSA assessed skin disease activity. Nails involvement where assessed by NAPSI. The functional capacity was assessed by CHAQ. The quality of life was assessed by CDLQI and by PedsQl 4.0 and the fatigue by PedsQI MFS.

Results: Participants were $69 \%$ female, with a mean age of $10.1 \pm 3.0$ years. PASI median was 4 (0.6-30.2), BSA 6 (2-39) and NAPSI 12 (3-36). PASI above 5 were present in 10 (38.5\%). Complaints of any kind of musculoskeletal recurrent pain were seen in $12(46.1 \%)$ of the patients and lumbar pain in $4(15.4 \%)$. Tenderness on palpation of sacroiliac joint was found in $5(19,2 \%)$ and tenderness on palpation of at least one site of enthesis was found in $8(30.7 \%)$ of the patients. The presence of enthesitis was associated to the skin disease activity scores of PASI $(p<0.006)$, BSA $(p<0.016)$ and nail index NAPSI $(p<0.05)$ (Fig. 1). Enthesitis was also associated to worse quality of life by CDLQI $(p<0,016)$. The median of fibromyalgia tender points was $5(0-16)$. Fatigue scores were higher for those with more than 11 tender points $(p<0,01)$. For those with a PASI $>5$, we found a higher number of tender points (Med 0 versus 10; $p<0.03$ ), a worse functional capacity by CHAQ (Med 0 versus $0.12 ; p<0.03$ ) and worse quality of life by CDLQI (Med 1 versus $9 ; p<0.05$ ).

Conclusions: High prevalence of musculoskeletal pain symptoms, enthesitis and fibromyalgia tender points was observed. This impairment generates fatigue has a negative impact on quality of life, and was associated with the severity of skin disease. Our data corroborate the literature findings that psoriasis is not a disease restricted to the skin, even in children.

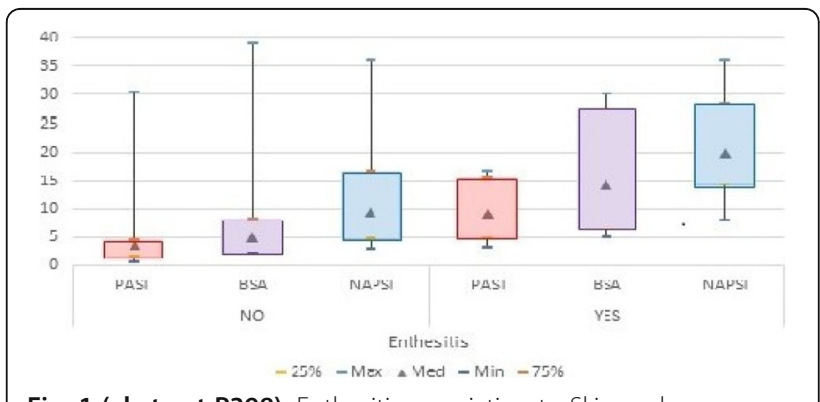

Fig. 1 (abstract P398). Enthesitis association to Skin and Nail involvement 
P399

RISK ASSESSMENT OF OSTEOPOROTIC FRACTURE IN PATIENTS FROM 65 YEARS ATTENDED IN A REUMATHOLOGY CLINIC

Alessandra de Sousa Braz', Matheus Vieira Falcãoº , Jade Arruda de Carvalho Motta², Alexia Lavínia Holanda Gama², João Calvino Soares de Oliveira², Karla Valéria Miranda de Campos ${ }^{2}$, Maria Roberta Melo P. Soares ${ }^{2}$, Eut́llia Andrade Medeiros Freire ${ }^{2}$, Alessandra de Sousa Braz ${ }^{2}$

${ }^{1}$ UNIVERSIDADE FEDERAL DA PARAÍBA, JOÃO PESSOA, PB, Brasil; ${ }^{2}$ UFPB, JOÃO PESSOA, PB, Brasil

Advances in Rheumatology 2018, 58(Suppl 1):P399

Introduction: Osteoporosis (OP) is defined as a decrease of the bone mass and deterioration of the tissue microarchitecture, with a greater predisposition to fractures. Its prevalence increases with aging, and bone densitometry is the diagnostic method that guides its treatment. However, in addition to bone mineral density (BMD), there are other factors that influence the risk of fracture in OP, such as age, use of corticoid or family history. The WHO Fracture Risk Assessment Tool (FRAX) was created to assess these variables and estimate the risk of fracture in 10 years, allowing a better stratification of patients more predisposed to this outcome. Studies conducted to assess fracture risk with FRAX were developed in women aged 50-64 years. Objectives: To assess the risk of osteoporotic fracture through FRAX ${ }^{\circledast}$ and determinate the prevalence of its risk factors in individuals aged 65 and over.

Methods: This is a cross-sectional, observational and analytical study of a quantitative approach performed in individuals $\geq 65$ years old, followed in a Rheumatology outpatient clinic. The data were collected through a clinical-epidemiological questionnaire and the FRAX, whose calculation was done with the tool validated for the Brazilian population. Data were analyzed through descriptive statistics.

Results and conclusions: The sample was 83 patients aged 65 years and above, 79 women and 4 men, with a mean age of 73 years (SD: 6). Among the patients, $81.9 \%$ performed densitometry in the last 2 years; $28.9 \%$ presented osteopenia and $38.5 \%$, osteoporosis. The mean age at menopause was 49.5 years (SD: 4.8), and a mean BMI of $26.7 \mathrm{~kg} / \mathrm{m} 2$ (SD: 4) was found. In the studied population, $26.1 \%$ had chronic use of glucocorticoids at some time in their lives and $10.7 \%$ presented a personal history of osteoporotic fracture after 40 years. The mean fracture score of the clinical FRAX was $8.5 \%$ (SD: 4.9) fracture at 10 years, and $7.9 \%$ (SD: 5.2) at 10 years when considered the $\mathrm{BMD}$. In relation to femoral neck fracture, the risk for clinical FRAX was $4.2 \%$ (SD: 4.2 ), and $3.4 \%$ (SD: 4) when associated to BMI, characterizing this sample as high risk for neck fracture. Considering the major impact of osteoporosis fracture, especially in more advanced ages, and the limitations of densitometry in the management of the patient with osteoporosis for this outcome, FRAX appears as an useful tool to complement the assess of patients $\geq 65$ years, as well as in follow-up treatment.

\section{P400}

\section{RISK FACTORS ASSOCIATED WITH MORTALITY IN PATIENTS WITH} JUVENILE SYSTEMIC LUPUS ERYTHEMATOSUS

Erica Naomi Naka Matos, Laura Loureiro de Sousa Rosa, Milena Foizer Leite, Elisa Paiva Barbosa, Solino de Matos Neto, Carolina Yume Arazawa UNIVERSIDADE FEDERAL DE MATO GROSSO DO SUL, CAMPO GRANDE, MS, Brasil

Advances in Rheumatology 2018, 58(Suppl 1):P400

Backgorund: Juvenile Systemic Lupus Erythematosus (JSLE) is a rare disease in children, with a more severe course compared to adults, requiring aggressive therapy. The objective of this study was to describe the risk factors associated with death in children with JSLE.

Methods: a retrospective analysis of files of JSLE patients treated in a pediatric rheumatologic practice who died between december 2000 and december 2017 was made. A clinical protocol with items such as demographic data, diagnostic criteria, treatment, complications and disease activity was created.

Results and Conclusions: In 17 years, 49 patients with a diagnosis of SLE were treated in this service, with average age at diagnosis of 10 years and 5 months and F:M ratio of $1.4: 1$. Five of the 49 patients died (10\%), the F:M ratio being $4: 1$ and average age at diagnosis of 8 years and of death at 12 years. The time from disease to death ranged between 12 months to 14 years, with an average of 7 years and 4 months. The mean number of criteria for diagnosis by the SICS was 7.7 and by the ACR of 5.7. The most common initial manifestations at diagnosis were fever and seizures. Nephritis was identified in three patients and in the course of the disease it culminated in chronic renal failure (CRF). Regarding therapeutics: corticoid (5), antimalarials (2), azathioprine (2) and rituximab (1) were used. Regarding the cause of death: 1 - One patient was diagnosed with visceral leishmaniasis and died due to septicemia and CRF (hemodialysis and refractory activity); 2- Another patient died after 34 months of diagnosis; she was also on CRI and the cause of death was renal disease activity and infection; 3- One patient died at home and was on CRI (death attributed to a complication); 4 - Another had no disease activity and died because complications of a congenital cardiopathy due to a Genetic Syndrome; 5- The male patient died 12 months after the diagnosis due to infection and complications of Antiphospholipid Syndrome. In conclusion, infections are frequently observed in children with JSLE, with high percentages of morbidity and mortality. In this study it caused $4 / 5$ deaths and these were also in activity of the disease. Lupus nephritis with CRF was also present in 3/5.

\section{P402}

RITUXIMAB USE IN PATIENTS WITH REFRACTORY SYSTEMIC SCLEROSIS SKIN AND LUNG INVOLVEMENT

Ana Clara Ribeiro, Caroline Almeida Oliveira, Fernanda Pulcheri Ramos, Lais Farrapo de Barros Leite, Marcelo Nora Resende, Natalia Carneiro dos Santos, Raissa Barbosa de Souza, Andrey Tonetto Barbosa, Silvia Carla Sousa Rodrigues, Mariana Silva Lima, Rina Dalva Neubarth Giorgi, Nafice Costa Araujo

HOSPITAL DO SERVIDOR PÚBLICO ESTADUAL DE SÃO PAULO, SÃO

PAULO, SÃO PAULO, Brasil

Advances in Rheumatology 2018, 58(Suppl 1):P402

Introduction: Systemic sclerosis (SSc) is a chronic systemic autoimmune disease characterized by progressive fibrosis, vasculopathy and immune $\mathrm{T}$ and $\mathrm{B}$ lymphocyte mediated alterations. The treatment of SSC is challenging, especially due to the complex pathophysiology presented. With the growing understanding of the Rituximab (RTX) effect on the pathophysiology of rheumatic diseases, it is necessary to study the efficacy of this medication in these patients.

Objective: Observe the response to RTX of skin and lung involvement refractory to previous treatment.

Methods: This retrospective descriptive analytical study, conducted in 2018, included 20 patients with SSc according ACR/EULAR 2013 criteria followed at Rheumatology clinic in a tertiary hospital of São Paulo and treated with RTX. For statistical analysis: mean, standard deviation, frequency (\%), t-student, Mann-Whitney and Chi-square tests were used. $\mathrm{P}<0,05$ was considered statistically significant.

Results: Of the patients evaluated, $90 \%$ were women $(\mathrm{N}=18)$, mean age $52.4 \pm 11,36$ years, with a mean diagnosis time of $6.3 \pm 7,9$ years. Interstitial lung disease was seen in $80 \%(\mathrm{~N}=16)$, all with nonspecified interstitial pneumonia (NSIP) pattern on computed tomography (CT), all with positive ANA, and anti-SCL70 positive in $45 \%(\mathrm{~N}=9)$ of cases. Overlap was observed with Systemic Lupus Erythematosus $(\mathrm{N}=4,20 \%)$, Sjogren's Syndrome $(\mathrm{N}=4,20 \%)$, Rheumatoid Arthritis $(\mathrm{N}=2,10 \%)$, Myopathies $(\mathrm{N}=2,10 \%)$. Previous treatment with cyclophosphamide occurred in $65 \%(\mathrm{~N}=13)$ of the cases, mycophenolate mofetil in $40 \%(\mathrm{~N}=8)$ and azathioprine in $15 \%(\mathrm{~N}=3)$. The indication of RTX therapy was concomitant skin and lung involvement in $15 \%(\mathrm{~N}=3)$ of cases, lung and skin only in $55 \%(\mathrm{~N}=11)$ and $25 \%(\mathrm{~N}=5)$ respectively. Indications for the overlap syndrome were $15 \%$. The mean duration of therapeutic with RTX was 2.03 $\pm 1,41$ years. Skin thickening, assessed with the Modified Rodnan Skin Score (MRSS), improved significantly after the use of RTX compared with the baseline score (14.8 \pm 9.5 vs. 9.65 \pm 6.31 , before vs after, respectively, $\mathrm{P}<0.05)$. Forced vital capacity (FVC) and diffusing capacity of carbon monoxide (DLCO) before and after the use of RTX, didn't present 
significant change (FVC: $76 \% \pm 16.96 \%$ vs. $77.33 \pm 18.28$, before vs. after, $\mathrm{p}=0.91$ and DLCO: $41.85 \% \pm 16.77 \%$ vs. $40.37 \% \pm 16.4 \%$, before vs after, $\mathrm{p}=0.83)$. Of the $\mathrm{CT}$ standards, $90 \%(\mathrm{~N}=18)$ had no alterations after the use of RTX, with 5\% showing improvement and 5\% worsening. Conclusion: Patients with refractory pulmonary and skin commitment due to SSc, who used RTX, showed significant improvement of skin, and pulmonary stability in both spirometric and imaging pattern. Stabilization in lung function may be the best result in these patients.

\section{P403}

RITUXIMAB: DOES IT INFLUENCE SYSTEMIC SCLEROSIS'S

\section{VASCULOPATHY?}

Gabriela Santiago Brum Marques Gabriela Brum, Camila Pitasi Arguelhes, Verônica Silva Vilela, Roger Abramino Levy, Rogério Lopes Rufino,

Cláudia Henrique da Costa

UERJ - HOSPITAL UNIVERSITÁRIO PEDRO ERNESTO, RIO DE JANEIRO, RIO DE JANEIRO, Brasil

Advances in Rheumatology 2018, 58(Suppl 1):P403

Objective: Systemic sclerosis (SSc) is an autoimmune disease that has vasculopathy as a central pathogenic mechanism. Capillaroscopy is an optimal method to evaluate peripheral microcirculation impairment and correlates with organ damage. Preliminary data suggested that rituximab may stabilize microcirculation in patients with early systemic SSc. Our objective is to evaluate if rituximab can influence capillaroscopy patterns in a series of patients with SSc.

Methods: we evaluated five patients that received rituximab (two times $1 \mathrm{~g}$ every 6 months for 24 months) for the treatment of refractory pulmonary and/or cutaneous disease. Nailfold videocapillaroscopy was performed before the first infusion and after the 24 months. The exams were performed with high magnification equipment $(200 \mathrm{x})$ on digits 2 to 5 on both hands. Four fields per digits were evaluated with semi-quantitative scale for the following parameters: capillary density, neoangiogenesis and architectural derangement.

Results and conclusions: Four female and one male patients were studied; mean age 43.6; mean disease duration 6.4 years; 3 caucasian and 2 black. Two female patients had early rapidly progressive diffuse SSc refractory to cyclophosphamid; one male and two female patients received rituximab due to pulmonary disease reactivated and refractory to cyclophosphamide and mycophenolate. One patient also received sildenafil for pulmonary hypertension treatment. The videocapillaroscopy patterns, capillary density and neoangiogenesis remained stable after 24 months of rituximab infusions. We observed some improvement on architectural derangement. The videocapillaroscopy scores before and after the infusions were respectively: (1) capillary density, 1.4 and 1.25 ; (2) neoangiogenesis 0.4 and 0.26 and, (3) architecture 0.89 and 0.59 . Based on current literature, SSc patients microcirculation is expected to worsen through time. According to recent data, we observed that rituximab could stabilize microcirculation in SSc patients after two years treatment. Once microcirculation correlates to disease pathogenesis and organ involvement, our findings may endorse that rituximab may be an emergent therapy for severe SSc.

\section{P405}

SARCOIDOSIS WITH OSTEO-SCLEROTIC LESIONS MIMICKING BONE PRIMARY NEOPLASMS AND METASTASIS: CASE REPORT

Amanda Terra de sá Koerich, Celine Yasmine Schweri, Ivânio Alves

Pereira, Adriana Fontes Zimmermann

UNIVERSIDADE FEDERAL DE SANTA CATARINA, FLORIANOPOLIS, SANTA

CATARINA, Brasil

Advances in Rheumatology 2018, 58(Suppl 1):P405

Introduction: Sarcoidosis is a non-caseous and idiopathic granulomatous disorder.[1] Bone involvement is rare, osteolytic lesions are most commonly observed.[1] We present a case of sarcoidosis with multiple osteo-sclerotic lesions.

Case report: A 27-year-old male had episodes of daily fever for the past two years, with diffuse lindonofomegalias, hepatosplenomegaly, weight loss of thirty kilos, intense low back pain and abdominal pain.
On previous investigation he had been submitted to eight biopsies, including axillary, abdominal, splenic, bone and bone marrow lymph nodes, all of them were negative for neoplasms or infectious diseases. He had microcytic anemia and a significant increase in inflammatory activity. Antinuclear factor, rheumatoid factor, IgG4 immunoglobulin, renal function, calcium, tuberculin test, complement, biomarkers of autoimmune hepatitis and serologies were normal. Serum angiotensin converting enzyme was increased. Ultrasonography of the testicles was normal. Thorax/abdomen/pelvis computed tomography revealed sclerotic lesions in T4, T7, T12, L4, L5, sacrum, pelvic bones and last right costal arch, irregular nodules in both lungs and liver, augmented spleen. Bone scintigraphy showed increased osteogenic activity in the thoracic spine, right costochondral joints and skull bones. Three additional biopsies were performed (hepatic, axillary lymph node, gastric). All of them showed chronic non-necrotizing granulomatous lymphadenitis, confirming the diagnosis of sarcoidosis with bone involvement. The patient was treated with glucocorticoids and methotrexate but no clinical response was observed. Anti TNF alpha agent (adalimumab) was then initiated and disease control was achieved.

Discussion and Conclusion: Bone sarcoidosis occurs in about 1-15\% of the cases, with cystic lesions being the most frequent.[2] Osteosclerotic lesions are rare and may mimic blastic metastasis.[1] Patients with bone sarcoidosis tend to have greater involvement of multiple organs, especially the spleen, liver and extrathoracic lymphnodes, when compared to those patients without bone involvement.[3] We report a rare case of sarcoidosis with osteo-sclerotic lesions and involvement of several organs, emphasizing the importance of differential diagnosis, since the disease may mimic other bone diseases, such as granulomatous conditions, primary neoplasms and metastasis.

\section{References}

1. Nessrine A, Zahra AF, Taoufik H. Musculoskeletal involvement in sarcoidosis. J Bras Pneumol. 2014 Mar-Apr;40(2):175-82

2. Bechman K, Christidis D, Walsh S, Birring SS, Galloway J. A review of the musculoskeletal manifestations of sarcoidosis. Rheumatology (Oxford). 2018 May 1;57(5):777-783.

3. Zhou Y, Lower EE, Li H, Farhey Y, Baughman RP. Clinical characteristics of patients with bone sarcoidosis. Semin Arthritis Rheum. 2017

Aug; $47(1): 143-148$

\section{P407}

SCLEREDEMA OF BUSCHKE: A SYSTEMIC SCLEROSIS MIMETIZER FOR RHEUMATOLOGIST KNOWLEDGE

Mateus de Miranda Moura Cortês ${ }^{1,2}$, Jacqueline Foelkel Pignatari ${ }^{2}$, Pamella de Paula Bellini², Gabriela Miyuki Teodoro Ogawa ${ }^{2}$, Lara Ribeiro Teixeira Bonfim², Samuel de Oliveira Andrade ${ }^{2}$, Alisson Aliel Vigano Pugliesi ${ }^{2}$, Ana Paula Toledo Del Rio ${ }^{2}$

${ }^{1}$ UNIVERSIDA ESTADUAL DE CAMPINAS-UNICAMP, CAMPINAS, SP, Brasil; ${ }^{2}$ UNIVERSIDADE ESTADUAL DE CAMPINAS-UNICAMP, CAMPINAS, SP, Brasil

Advances in Rheumatology 2018, 58(Suppl 1):P407

Background: Scleredema of Buschke (SB) is a rare fibromucinous connective tissue disease characterized by symmetrical and diffuse cutaneous thickening, with an insidious course. Occasionally, it affects the face and leads to periorbital edema, but it mainly involves posterior regions of upper trunk, neck and shoulders, sparing distal ends. Systemic manifestations of the disease are rare. Its etiology is unknown, but it is well established that diabetes mellitus (DM) is associated with half of the cases of SB. Because it is a sclerodermiform disease, the rheumatologist must be aware of it as a differential diagnosis for systemic sclerosis (SS).

Case report: A 51-year-old female patient underwent follow-up for rheumatoid arthritis for 12 years, in sustained remission of the disease by treatment with oral leflunomide and methotrexate. The patient underwent irregular treatment for other comorbidities: type 2 DM, diabetic neuropathy, systemic arterial hypertension, dyslipidemia, hypothyroidism and central obesity. In three months, she noticed restriction of shoulder girdle mobilization and progressive cutaneous thickening of the legs, nape of the neck, posterior face of 
the upper trunk and shoulders. On examination, the patient presented mild bilateral periorbital edema and diffuse, hyperchromic infiltration in orange peel appearance, located in the referred regions (Fig. 1). Thickened skin biopsy and panel of laboratory tests were requested. During the investigation, non-reactive antinuclear antibodies (ANA) was found and other tests proved normal (including hemogram, ASLO antibody, creatine phosphokinase, inflammatory markers, serum protein electrophoresis and renal, hepatic and thyroid functions), except for hypertriglyceridemia (855 mg/dL) and glycemic control (hemoglobin A1C 10.7\%). Histopathological examination of the skin evidenced dermis thickened by an increase of collagen fibers, dissociated by deposition of amorphous material. In view of the suggestive clinical picture and biopsy, SB was diagnosed associated with lack of glycemic control of DM. The patient was referred to Endocrinology for metabolic control, evolving with important improvement of the skin condition after optimization of glycemic control.

Conclusion: The absence of systemic involvement, the distribution of cutaneous involvement sparing hands and feet, suggestive cutaneous biopsy, negativity for autoantibodies, and the lack of other stigmas discouraged SS hypothesis, which is automatically thought of in a Rheumatology outpatient clinic, and corroborated the diagnosis of SB. Such entity may be idiopathic or associated with infections, paraproteinemias or DM. Suggestive clinic and biopsy lead to diagnosis. The therapy is difficult, controversial and with inconstant results, but, occasionally, glycemic control can lead to a partial cutaneous response.

\section{Consent for publication}

The authors declare that they have obtained informed written consent from the patient's tutors for publication

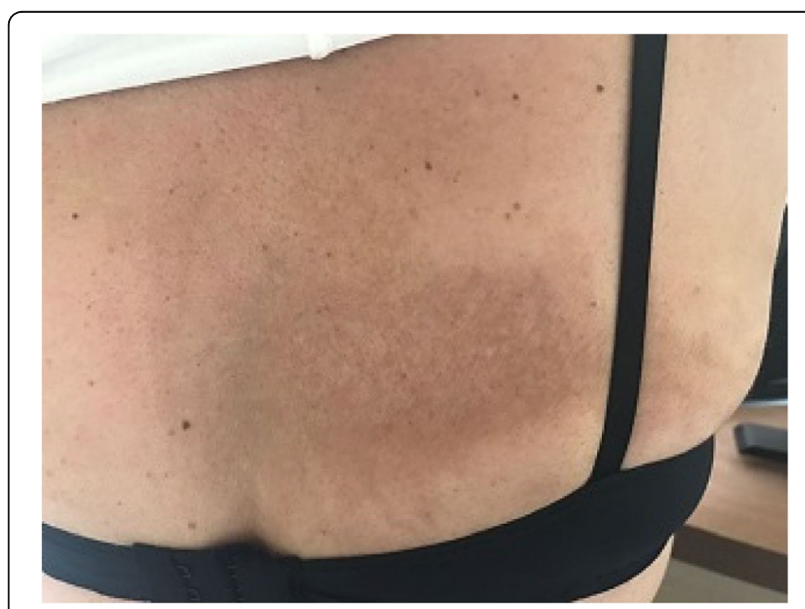

Fig. 1 (abstract P407). Cutaneous infiltration in orange peel appearance on the upper portion of back trunk

\section{P409}

SCREENING FOR DEPRESSION AND WORK DISABILITY IN PACIENTS WITH SYSTEMIC LUPUS ERYTHEMATOSIS

Thayana Ribeiro Kajitani Pacheco ${ }^{1}$, Mayara da Silva Cardoso², Renata Silva Forte ${ }^{1}$, Emanuelle de Matos Rodrigues ${ }^{1}$, Gabriele Barros de Aviz' Clara Coutinho Viana', Glenda Figueira Guimarães ${ }^{1}$

'CENTRO UNIVERSITÁRIO DO ESTADO DO PARÁ, BELÉM, PA, Brasil; ${ }^{2}$ HOSPITAL OPHIR LOYOLA, BELÉM, PARÁ, Brasil

Advances in Rheumatology 2018, 58(Suppl 1):P409

Introduction: Studies have demonstrated a high prevalence of depression in patients with systemic lupus erythematosus, often occurring as the first manifestation of the disease and can have a great impact on the employability, as permanent incapacity to perform the professional activities.

The severity of the disease, the autoimmune phenomena and the psychological stress of having a systemic disease are hypotheses to explain this prevalence.

Objectives: Analyze the incapacity to work and search for depressive symptoms in patients with SLE, treated at the Rheumatology clinic of the Jean Bitar Hospital Center, Belém - PA.

Methods: A descriptive, observational and cross-sectional study was carried out. The data were collected from interviews, according to research protocol, composed of a questionnaire designed to evaluate the labor situation and the CES-D scale for screening for depression. Statistical significance was established at $95 \%$ level ( $p$ value $<0.05$ )

Results: 51 patients participated in the study, with the majority of females $(92.2 \%)$, with a mean age of 33.9 years and income of up to 1 minimum wage (94.1\%). Depressive symptoms were found in $74.5 \%$ of the cases. There was a reduction in performance and workload due to the manifestations of SLE in $85.7 \%$, with skin changes (33.3\%) and nephritis (33.3\%) being the most implicated in the issue. $52.9 \%$ of the patients did not work, and SLE was responsible in $96.6 \%$ of the cases. Regarding social security situation, $45 \%$ of individuals receive some benefit from the INSS, with the "Desease Help" the most frequent (82.7\%). Among those who do not receive the benefit, $50 \%$ have already tried to obtain it.

Conclusion: the data from this study alert to the frequent presence of depressive symptoms, as well as the high labor incapacity, either partial or total, associated with SLE. It is necessary the adequate management of the disease and the valuation of psychological aspects to reduce the impact of SLE for the individual, his family and the community.

\section{P410}

SECONDARY DIABETIC QUIROARTROPATHY TO DM TYPE II: REVIEW OF LITERATURE

Bárbara Laise Guimarães Travassos ${ }^{1}$, Eduardo Souza Cruz'1 Igor Tadeu de Castro Nascimento ${ }^{1}$, Jéssyca Cristina Ramos do Nascimento ${ }^{1}$, Mirna Karen Botelho Figueiredo', Carolina Íris Pereira de Santana', Thaiana Pedrosa Azevedo', Thayana Ribeiro Kajitani Pacheco ${ }^{2}$, Glauce Leão Lima ${ }^{2}$ ${ }^{1}$ CENTRO UNIVERSITÁRIO DO PARÁ, BELÉM, PARÁ, Brasil; ${ }^{2}$ UNIVERSIDADE FEDERAL DO PARÁ, BELÉM, PARÁ, Brasil

Advances in Rheumatology 2018, 58(Suppl 1):P410

Background: Diabetic chiroartropathy, also called diabetic hand syndrome, is characterized by a limitation of joint movement, where thickening and tissue hardening occur, being a rheumatologic manifestation common to type II diabetes mellitus. It begins in the fifth quirodactyl and later affects the other interphalagian and metacarpophalangeal joints. This clinical condition prevents the patient from keeping the palms of the hands together, characterizing the "signal of prayer". Prevalence occurs in $76 \%$ of type II DM and is related to the duration of the disease.

Methods: A systematic review of the literature was carried out in 22 articles, without restriction of languages and period.

Results and conclusions: Regarding the impairment of joint mobility in diabetic patients, the main influence factor is increased stiffness of the joint capsule, ligaments and tendons. However, the exact pathophysiology of most musculoskeletal disorders in DM has not yet been fully elucidated. In diabetic chiroartropathy, there is a thickening and hardening of the skin, causing the flexion contracture of the fingers. As a result, the patient has difficulty in opposing the flexor surfaces of the fingers (signal of the speaker); rarely does the process affect large joints. The skin has a sclerodermic characteristic, but the histological study reveals preservation of the cutaneous appendages. It occurs in 55\% of type I DM and more than $76 \%$ of type II DM, and its incidence is associated with duration of DM. There is a positive association with the presence of renal and retinal microvascular disease. 


\section{P412}

SERIOUS AUTOIMMUNE ENCEPHALITIS WITH GOOD RESPONSE TO RITUXIMAB

Laís de Vico, João Paulo Canaan, Lívia Câmara Oliveira, Joely Cristina da

Silva Toledo, Sula Glaucia Lage Drumond Pacheco, Henrique Pereira

Sampaio, Luiz Eduardo Valente, Andrea de Almeida Peduti Batista,

Antonio Baccaro, Fabio Vicente Leite, Flavia Luiza Marin

UNESP, BOTUCATU, SP, Brasil

Advances in Rheumatology 2018, 58(Suppl 1):P412

Background: Autoimmune encephalitis (AD) is a rare clinical syndrome that encompasses subacute cognitive disorders such as amnesia and mental confusion, as well as movement disorders and epileptic seizures. Some cases may present without the classic symptoms of encephalitis described above. Although there are no randomized studies, immunomodulatory therapy is used to treat AD.

Case Report: A woman, 48 years old, who had previously had complaints of occipital headache, drowsiness in the morning and fever for 1 week, suffered sudden onset of fall and received a sleepy, noncontacting emergency room, with no verbal response to painful stimulus, convergent eye strabismus and absent Threat reflex in both eyes plus presence of signs of meningeal irritation. Initial Glasgow was 3, evolving with orotracheal intubation and intensive unit support. Performed CT scan without alterations, skull angiography without changes and Resonance (MRI), which evidenced an alteration of the extensive brainstem signal with associated lesions in the supratentorial compartment. Suggestive of infectious or autoimmune encephalitis (AD). Opted for treatment for viruses, fungus, and bacterium, worsening of the neurological condition, initiating protocol of brain death. Rheumatology evaluation was requested, which verified a family history of Systemic Lupus (SLE) and FAN + 1/320 fine dotted nuclear pattern, with no other criteria for SLE. Due to the severe neurological condition, we chose to immunosuppress the patient with methylprednisolone 1 gram (g) daily for 3 days, resulting in eyelid reflex on the right; followed by cyclophosphamide $0.8 \mathrm{~g} / \mathrm{m} 2$, with the reappearance of some trunk reflexes. Immunoglobulin $2 \mathrm{~g} /$ $\mathrm{kg}$ was performed without improvement of neurological status. Opposed by rituximab $1 \mathrm{~g}$ and patient presented spontaneous ocular opening and initiated left and hand movements; progressively showing right-hand movements, and improvement of the ventilatory part, evolving to extubation and discharge of the Intensive Unit.

Conclusion: In the present case, the patient had a severe autoimmune encephalitis and early onset of rituximab was a determinant of the clinical outcome, preventing permanent neurological damage.

Consent for publication

The authors declare that they have obtained informed written consent from the patient's tutors for publication

\section{P414}

\section{SERIOUS PURPURA FULMINANS ASSOCIATED WITH} SALMONELOSE

Fernanda Morello Nicole Batista', Luiza Corrêa Rodrigues ${ }^{2}$ Letícia Fonseca Favarato², Thais Chaves Belisário², Érica Vieira Serrano ${ }^{2}$, Ruben Horst Duque ${ }^{2}$, Ana Paula Espindula Gianordoli², Lidia Balarini da Silva², Maria Bernadete Renoldi de Oliveira Gavi ${ }^{2}$, Maria Carmen Ferreira Lopes Silva Santos ${ }^{2}$, Fabiola Assad Antunes ${ }^{2}$, Rafael Nunes Malta², Valquiria Garcia Dinis ${ }^{2}$, Sarah Lyra Firme ${ }^{2}$, Arthur Dalmaso Pinto ${ }^{2}$, Valeria Valim Cristo ${ }^{2}$

${ }^{1}$ UNIVERSIDADE FEDERAL DO ESPIRITO SANTO, VITÓRIA, ES, Brasil; ${ }^{2}$ UNIVERSIDADE FEDERAL DO ESPÍRITO SANTO, VITÓRIA, ES, Brasil Advances in Rheumatology 2018, 58(Suppl 1):P414

Background: Purpura fulminans is a sudden and fatal condition of dermal microvessels thrombosis that rapidly evolve into cutaneous hemorrhagic necrosis. It is associated/caused by Infectious diseases due to pneumococcus, meningococcus or other gram negative are well described in the literature as frequent causes of purpura fulminans in adults. This paper reports a rare case of a patient with typhoid fever caused by Salmonella typhi with a catastrophic evolution to purpura fulminans.
Case report: Female, 39 years old, previously healthy, cooker (suspicion of quality of water source not proper), hospitalized with progressive oedema and erythematous-violaceous macular lesions in the lower and upper limbs for 3 weeks, with progression to blisters, disabling pain and daily febrile peaks. She was submitted to imaging and laboratory tests, all initially negative (biochemistry, serology for hepatitis $B$ and $C$, HIV, syphilis, cytomegalovius, toxoplasmosis, Chikungunya, rheumatologic markers FAN, anti-DNA, complement, anti-Sm, anti-RNP, ANCA-p/c, cryoglobulins, anticardiolipins, beta2glycoprotein, investigation for thrombophilia, venous and arterial ultrasound of the lower limbs). She had received pulse therapy with methylprednisolone at another hospital. On admission to our service She started ceftriaxone IV based on blood culture results positive for Salmonella tiphy in three samples. Histopathological of the lesions showed arterial and venous thrombosis in the skin and subcutaneous, without evident inflammatory process. Patient evolved with severe skin and subcutaneous necrosis of legs and underwent surgical debridement. Culture of surgical collected material was positive for Salmonella typhi in the tissue. In the follow-up there was progressive improvement and stability of the clinical-laboratory parameters, but with intense pain in the lower limbs and need of opioid. Skin graft procedure was indicated.

Conclusion: Purpura fulminans usually result from a disorder in hemostasis, either due to an inherited or acquired anticoagulant protein deficiency or to a consumption coagulopathy. From our knowledge, this is the first case report of purpura fulminans associated to Salmonella typhi.

\section{Consent for publication}

The authors declare that they have obtained informed written consent from the patient's tutors for publication

\section{P415}

\section{SERUM LEVELS OF GALECTIN-9 ARE INCREASED IN PATIENTS WITH} RHEUMATOID ARTHRITIS

Kamila de Melo Vilar ${ }^{2}$, Nara Gualberto Cavalcanti ${ }^{1}$, Michelly Cristiny Pereira², Moacyr Jesus Barreto de Melo Rego ${ }^{2}$, Andréa Tavares Dantas",

Angela Luzia Branco Pinto Duarte ${ }^{1}$, Maira Galdino da Rocha Pitta ${ }^{2}$

${ }^{1}$ SERVICO DE REUMATOLOGIA - HC/UFPE, RECIFE, PE, Brasil;

${ }^{2}$ LABORATÓRIO DE IMUNOMODULAÇÃO E NOVAS ABORDAGENS

TERAPÊUTICAS -LINAT/NUPIT-SG/UFPE, RECIFE, PE, Brasil

Advances in Rheumatology 2018, 58(Suppl 1):P415

Background: RA is a heterogeneous disease, with variable clinical presentation and pathogenetic mechanisms. RA is a systemic inflammatory disease, with variable clinical presentation and complex pathogenetic mechanisms. The diversity of cytokines found in the circulation of RA patients is related to the pathophysiology of the disease, through mechanisms not yet completely elucidated. Galectin-9 (GAL-9) is an anti-inflammatory lectin, as suggested by studies in several diseases; it is expressed by T cells, macrophages, endothelial cells, and fibroblasts and plays an important role in regulating inflammation and immune responses. The aim of this study was to evaluate the serum levels of GAL-9 in RA patients and its association with clinical manifestations.

Methods: The study included 77 patients with the diagnosis of RA (1987 ACR criteria), being 72 females and 5 males, mean age 27.9yrs (28-77). The control group consisted of 62 female and 15 male individuals without the diagnosis of any autoimmune inflammatory disease, mean age 27.3yrs (28-77). GAL-9 serum levels were quantified by sandwich ELISA, with a detection limit of $46.87 \mathrm{pg} / \mathrm{ml}$, following the information recommended by the manufacturer (R\&D Systems). Results and Conclusions: Most patients had moderate $(46.8 \%)$ or severe $(45.5 \%)$ disease activity. $77.9 \%$ had positive rheumatoid factor and $61.0 \%$ had radiological erosions. GAL-9 levels were increased in RA patients compared to controls (mean $1477 \mathrm{pg} / \mathrm{ml}$ and $46.88 \mathrm{pg} /$ $\mathrm{ml}$, respectively; $\mathrm{p}<0.001$ ) (Fig. 1). We found no correlation between serum levels of galectin- 9 and clinical manifestations. Our results are preliminary and suggest a potential role of GAL-9 as a biomarker of RA. Further studies are needed to better understand the role of GAL9 in the pathogenesis of RA. 


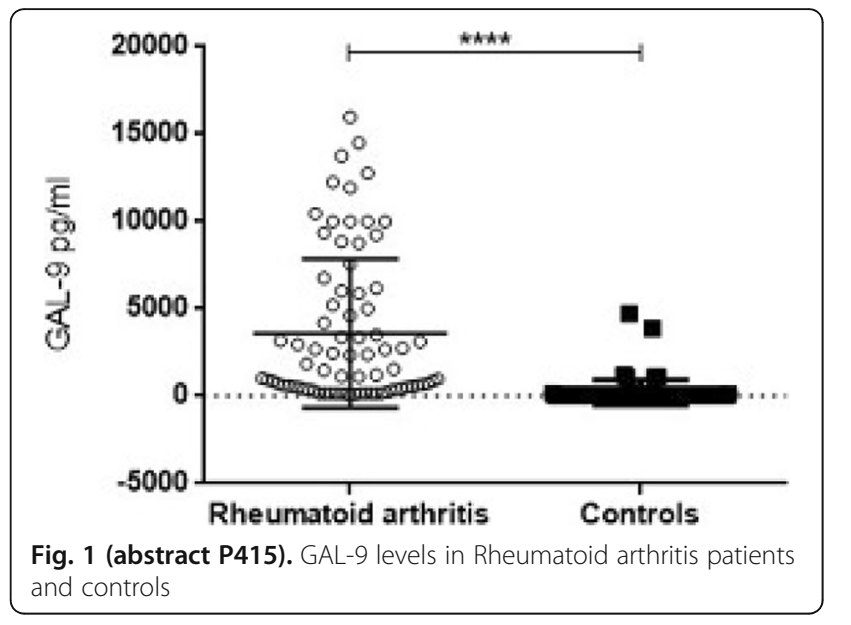

P423

SEVERE SYSTEMIC LUPUS ERYTHEMATOSUS: CLINICAL EVOLUTION AND THERAPEUTIC RESPONSE

Andreia Coimbra Sousa', Lynara Pinheiro Moraes², Tássia Oliveira Nunes da Silva ${ }^{3}$, Thais Silva de Jesus ${ }^{3}$, Fernando César Costa da Silva Junior ${ }^{3}$,

Adriana Medeiros Lima ${ }^{3}$, Braulio Nunes de Souza Martins Filho ${ }^{3}$, Raquel Moraes da Rocha Nogueira ${ }^{3}$

${ }^{1}$ UNIVERSIDADE CEUMA, SÃO LUÍS, MA, Brasil; ${ }^{2}$ UEMA, SÃO LUIS, MA, Brasil; ${ }^{3}$ CEUMA, SAO, MA, Brasil

Advances in Rheumatology 2018, 58(Suppl 1):P423

Background: Systemic lupus erythematosus (SLE) is an autoimmune, chronic, multisystemic and inflammatory disease of connective tissue. It evolves in outbreaks of varied activity, with periods of activity and remission that progresses with polymorphic clinical manifestations. Genetic and environmental factors are involved in the imbalance of the immune system, with the production of autoantibodies directed against nuclear proteins, some of which are proven to participate in the tissue injury.

Case report: C.S.R, female, 27 years old, with a single convulsive seizure for 3 years, without adequate etiological investigation and irregular use of phenobarbital $100 \mathrm{mg} /$ day since then. For 2 years, he complained of inflammatory polyarthralgia, evolving with joint edema and difficulty in walking. presented worsening of the condition, with a clear fall in general condition, evening fever, malar rash, anasarca and dyspnea at rest. He sought emergency care, where bilateral pleural effusion was found, and laboratory tests showed haemolytic anemia, platelet disease, high vhs and pcr, homogeneous homogeneous nuclear pattern and dotted cytoplasm. of the autoantibodies for SLE investigation, only anti-LA was negative, all other positives: anti-native DNA, anti-histone, anti-RNP, anti-SM and antiRO. It evolved with hypertension, worsening of nitrogenous slags and proteinuria of $24 \mathrm{~h}$ of $6.8 \mathrm{~g}$. In addition, he manifested 4 consecutive episodes of tonic-clonic seizure and pericardial effusion. An intravenous therapy with methylpredinilosone $1 \mathrm{~g}$ was instituted for 3 consecutive days followed by cyclophosphamide $1 \mathrm{~g}$ for 1 day and prednisone was maintained orally at a dose of $60 \mathrm{mg} / \mathrm{kg} /$ day, associated with hydroxychloroquine $400 \mathrm{mg} /$ day. He presented only partial improvement of the Sledai score and score after 2 weeks of the initial therapy showed very high activity of the disease. with a new dose of cyclophosphamide after one month of the first. The evolution was favorable and he was discharged from hospital, with referral to follow up in a rheumatology outpatient clinic.

Conclusion: SLE is a chronic inflammatory disease capable of affecting virtually any organ or tissue, but the occurrence of a neurological event as a primary manifestation is uncommon. In addition, the case report becomes private because it evolves with manifestations also infrequent, such as hemolytic anemia, present in only $10 \%$ of the cases. Despite the high degree of disease activity, the patient achieved significant improvement after conventional immunosuppressive therapy, thus highlighting the effectiveness of this therapy.

\section{Consent for publication}

The authors declare that they have obtained informed written consent from the patient's tutors for publication

\section{P425}

SEXUAL DISFUNCTION IN FEMALE RHEUMATOID ARTHRITIS PATIENTS: A CROSS-SECTIONAL STUDY

Flávia Jatobá de Barros', Bruna Ferraz Gutierrez Piola', Hugo Deleon de Lima$^{2}$, Rafael da Rocha Caminha', Pedro Arturo Bismara Carneiro Santos ${ }^{1}$, Amanda Calheiros Bertão ${ }^{1}$, Marina de Oliveira Stojanovic Gomes ${ }^{i}$, Amanda Fernandes Vieira Mendes da Silva', Felipe Lopes Torres da Silva ${ }^{2}$, Laurindo Ferreira da Rocha Junior ${ }^{2}$

${ }^{1}$ FACULDADE PERNAMBUCANA DE SAÚDE - FPS, RECIFE, PERNAMBUCO, Brasil; ${ }^{2}$ INSTITUTO DE MEDICINA INTEGRAL PROFESSOR FERNANDO

FIGUEIRA - IMIP, RECIFE, PERNAMBUCO, Brasil

Advances in Rheumatology 2018, 58(Suppl 1):P425

Background: Rheumatoid arthritis (RA) is an autoimmune debilitating disease that is most prevalent in women. The physical disability caused by RA can influence sexual function. This work aimed to evaluate whether RA interferes in sexual function of female patients. Methods: We conducted a cross-sectional study with 83 female patients classified as having RA according to the 1987 RA criteria of the American College of Rheumatology (ACR) or the ACR/EULAR (European League Against Rheumatism) of 2010. Clinical and sociodemographic data was collected. Sexual dysfunction (SD) was evaluated using Female Sexual Function Index (FSFI) questionnaire. The definition of SD was considered by a score less than or equal to 26.5. Statistical analysis was performed using GraphPad prism 6.0 software. Thirty age-matched healthy women were enrolled as a control group.

Results and conclusions: The median disease duration was 44 (iqr 20 - 89) months and median age was 54 (IQR 44-60) years. Seventy patients (84.3\%) had SD and $43(51.8 \%)$ were married. Seventeen $(56.66 \%)$ healthy women were married and $13(43,3 \%)$ had SD. The median FSFI score of RA patients was 5.6 (IQR 2.6-22.10) and of controls was 28.10 (IQR 3.125-30.78), $\mathrm{p}=0.0035$. Married patients had higher FSFI domains of arousal $(p=0.0252)$, lubrication $(p=0.0166)$, orgasm $(p=0.0198)$ and pain $(p=0.0102)$. Married patients with SD $(n=34)$ had greater scores in pain and lubrication, $p=0.0182$ and $p=0.0374$, respectively. Interestingly, patients with low disease activity compared to moderate disease activity according to CDAI had higher median FSFI score (19.80 [IQR 3.9-28.5] vs 3.4 [IQR 2 - 16.6], $\mathrm{p}=0.046$; pain (3.4 [IQR 0-5.9] vs. 0 [IQR 0-2], $\mathrm{p}=0.0469$ and satisfaction domain (4.4 [IQR 0.9-5.5] vs 0.8 [0.8-2], $\mathrm{p}=0.0344$ ). As for DAS28, patients with low disease activity had greater scores of lubrication (median 3.9 [IQR 0-5.7]), orgasm (median 3.6 [IQR 0-5.6]), satisfaction (median 3.6 (IQR 2.4-6) and pain (median 4 [IQR 0-6]) compared to moderate disease patients as follows: lubrication median 0 (IQR 03.3), orgasm median 0 (IQR 0-2.8), satisfaction median 0.8 (IQR 0.8-4) and pain median 0 (IQR $0-2.8), p<0.05$. When patients older or equal to 60 years old and younger were analyzed there was difference in all domains and in the total FSFI score $(p<0.05)$. There was significant negative correlation of desire domain with disease duration ( $r=-$ $0.292, p=0.0074$ ). Rheumatoid Arthritis has a significant negative impact on female sexual function. Sexual dysfunction in the patients studied might be associated with disease activity, marital status and older patients.

\section{P426}

SHRINKING LUNG SYNDROME: A CASE REPORT

Guilherme Brezenski Rodrigues, Bernardo Fróes Chedier Barreira,

Elicivaldo Lima Juvêncio, Ricardo Lopes Alves

UFRJ, RIO DE JANEIRO, RIO DE JANEIRO, Brasil

Advances in Rheumatology 2018, 58(Suppl 1):P426 
Background: Shrinking lung syndrome (SLS) is a rare and less known complication of systemic lupus erythematosus (SLE), although it has also been reported in patients with other connective tissue diseases (CTDs), such as primary Sjögren syndrome, scleroderma, rheumatoid arthritis, and undifferentiated connective tissue disorders. It is characterized by small lung volumes, elevation of the diaphragm and restrictive physiology without parenchymal involvement. The most common clinical manifestation is dyspnea, usually accompanied by pleuritic chest pain. The precise pathogenic mechanism underlying the SLS remains to be elucidated. Phrenic nerve involvement and myopathy were thought to be the cause of diaphragm dysfunction, but this has not been established until the present date. Corticosteroids are the most common method of treatment, but immunosuppressive therapy, beta-agonists and theophylline are used in those resistant to steroids. The prognosis is generally good. The great majority of patients described in the literature had significant clinical improvement and stabilization, as well as mild to moderate improvement on pulmonary function tests.

Case report: The authors report a case of a 17-year-old woman, with SLE previously diagnosed 8 years ago, presenting a 2-month history of dyspnea and chest pain.

Conclusion: SLS is a rare entity that can occur in individuals with SLE or others autoimmune disorders. Therefore, recognition of this unusual complication is of paramount importance, since early diagnosis and therapy can affect outcomes.

\section{Consent for publication}

The authors declare that they have obtained informed written consent from the patient's tutors for publication

\section{P433}

\section{SJÖGREN'S SYNDROME AFTER CHIKUNGUNYA: CASE REPORT}

Letícia Queiroga de Figueiredo, Evânia Claudino Queiroga de Figueiredo, João César Queiroga de Figueiredo

UFCG, CAMPINA GRANDE, PB, Brasil

Advances in Rheumatology 2018, 58(Suppl 1):P433

Background: Sjogren's syndrome (SS), an autoimmune systemic disease, is typically characterized by a secretory disfunction of the salivary and submandibulary glands, manifested as xerophthalmia and xerostomia. Autoimmunity induction in this affection is not know, being related in some studies to viral infections, such as EpsteinBarr (EBV), retroviruses and Coxsackie, as triggering factors within individuals with genetic and environmental predisposition. With the Chikungunya epidemic, caused by CHIKV, its clinical chronic and inflammatory related repercussions have been well reported, including as a possible initiating factor for rheumatic diseases. This study aims to report the case of a patient who presented SS after CHIKV infection.

Case report: A female patient, 18 years old, reported dry mouth complaint, making speech difficult, with thick saliva, with onset of days after a typical Chikungunya acute infection that was clinically and epidemiologically diagnosed. It evolved with progressive worsening of xerostomia and swelling of parotid and submandibular glands. She presented edema, pain and morning stiffness in proximal interphalangeal joints, and arthralgia on the knees, without edema. No further examination alterations were noticed. She presented a history of autoimmune thyroiditis at age 14, with anti-TPO higher than 1000, reaching the course of hypothyroidism. At the time of the consultation it was stable with the use of levothyroxine. Ultrasonography of the cervical region revealed submandibular and bilateral parotideal sialoadenitis. She had reactive FAN Hep2, nuclear speckled pattern of 1:320, reactive anti-Ro, with positive IgG and negative IgM Ab for Epstein-Barr. No other laboratory abnormalities including hemogram, renal or hepatic function, complement, and antibody test against Toxoplasmosis and Cytomegalovirus were noticed. The diagnosis of SS was made, in addition to the antecedent episode of Chikungunya, initiating therapy with chloroquine and corticoid in anti-inflammatory doses. In consultations with 20 days and 2 months, she presented a marked improvement in joint complaints and dry syndrome.

Conclusion: The reported case has its importance justified as being the first reported case in the literature, to our knowledge, of SS in patients after acute CHIKV infection. The patient had a history of predisposition to autoimmune diseases due to previous autoimmune thyroiditis, besides previous EBV infection, but only developed SS symptoms after CHIKV, what may suggest it as a triggering factor in predisposing situations. The relation between these two conditions is of great relevance to the knowledge still under construction regarding the association of autoimmune diseases and viral infections.

\section{Consent for publication}

The authors declare that they have obtained informed written consent from the patient's tutors for publication

\section{P436}

\section{SLEEP PATTERN CHANGE SCALE IN PATIENTS WITH RHEUMATOID ARTHRITIS}

Bruna Ferraz Gutierrez Piola', Flávia Jatobá de Barros ${ }^{1}$, Rafael da Rocha Caminha', Pedro Arturo Bismara Carneiro Santos ${ }^{1}$, Hugo Deleon de Lima², Mariana Souza Pessoa de Luna², Amanda Calheiros Bertão', Felipe Torres da Silva², Leia Teixeira de Andrade², Dennys Lapenda Fagundes², Laurindo Ferreira da Rocha Junior ${ }^{2}$

${ }^{1}$ FACULDADE PERNAMBUCANA DE SAÚDE - FPS, RECIFE, PERNAMBUCO, Brasil; ${ }^{2}$ INSTITUTO DE MEDICINA INTEGRAL PROF. FERNANDO FIGUEIRA IMIP, RECIFE, PERNAMBUCO, Brasil

Advances in Rheumatology 2018, 58(Suppl 1):P436

Background: Sleep disorders are very common complaints in patients with rheumatoid arthritis (RA). Intrinsic factors of the disease, such as chronic pain and low quality of life might be associated with sleep problems in patients with RA. The objective of this study was to evaluate the association of sleep disorders with functional capacity and clinical and laboratory parameters of AR.

Methods: Cross-sectional study with RA patients that were submitted to an interview, clinical evaluation and review of medical records for data collection. Epidemiological, clinical, serological data was collected. To assess sleep quality the Sleep pattern change scale of Beck Depression Inventory II (BDI-II) was used. The Scale identifies the type (hypersomnia and insomnia) and level of change in sleep pattern. Health Assessment Questionaire (HAQ) was used to evaluate the functional capacity. Statistical analysis was performed using Graph Pad Prism 6.01 software.

Results: Ninety-eight patients (95 women) were included in the study, of which $66(67.3 \%)$ had sleep problems. Patients with sleep disturbances had greater number of swollen joints median 2 (IQR 0-5) and lower Erythrocyte Sedimentation Rate (ESR) median 19.5 (IQR 8-31) compared with patients with no alterations of sleep median 1 (IQR $0-3$ ), $p=0,0156$ and $p=0,0207$, respectively. There was a trend to sleep problems being associated with higher CDAl scores $(0,0651)$. As for anthropometric measures, patients who have hypersomnia compared to those who have insomnia have greater height (mean $161.8 \mathrm{~cm}[+1.537]$ vs. mean 155.9 [+ 1.264], $\mathrm{p}=0,0181$ ) and lower BMI (median 24.37 [IQR 23.42-26.16] vs. median 29.06 [IQR 24.03-34.33], $p=0,025$ ). Comparing patients who report having insomnia with patients without insomnia there was a higher BMI in the former group (median 29.01 [IQR 23.2-34.2] vs. median 24.4 [IQR 21.4-29.21], $p=0,0008$ ). It was also identified the intensity of sleep problems had correlation with weight $(p=0,0251, r=0,2298), B M I(p=0,0465, r=0,2048)$, ESR ( $p=0,0151 r=-0,2473)$ and CDAI $(p=0,0466 ; r=0,2025)$. According to functional capacity, patients with HAQ score greater or equal than two had more intense sleep problems (median 2 
[IQR 1-2]) compared to patients with HAQ less than 2 (median 1 [IQR 0-2], $\mathrm{p}=0,0331$.

Conclusions: Sleep problems were present in the majority of patients studied. The disturbances of sleep were associated with anthropometric measures, edema and lower erythrocyte sedimentation rate. Further studies are necessary to better elucidate the association of anthropometric measures, acute phase reactants and clinical parameters of RA patients and sleep patterns.

\section{P437}

SLEEP QUALITY IN CHILDREN AND ADOLESCENTS WITH AUTOIMMUNE RHEUMATIC DISORDERS SEEN AT A REFERENCE OUTPATIENT CLINIC IN SALVADOR, BAHIA-BRAZIL

Teresa Cristina Martins Vicente Robazzi ${ }^{2}$, Ludmila Santiago Viana ${ }^{2}$, Tayná Rangel Barreto ${ }^{2}$, Jose Cleosmaque Leite Júnior ${ }^{2}$, Beatriz Oliveira Leão Carneiro $^{2}$, Leandra Chaves Silva Barros ${ }^{1}$, Cristiani Leal $^{1}$, Regina Terse Ramos $^{2}$

${ }^{1}$ HOSPITAL UNIVERSITÁRIO PROFESSOR EDGAR SANTOS, SALVADOR, BAHIA, Brasil; ${ }^{2}$ FACULDADE DE MEDICINA DA UNIVERSIDADE FEDERAL DA BAHIA, SALVADOR, BAHIA, Brasil

Advances in Rheumatology 2018, 58(Suppl 1):P437

Introduction: Rheumatic diseases can result in considerable physical limitations and changes in the development of children and adolescents. Here we highlight juvenile idiopathic arthritis (JIA), systemic lupus erythematosus (SLE), scleroderma and dermatomyositis as important potentially disabling conditions when these manifest in childhood. Growth, normal development, emotional health and immune function in general are closely related to the obtainment of adequate sleep in children and adolescents. Sleep disorders are especially prevalent in patients with acute and chronic illness, as is the case in rheumatologic disease.

Methods: This is an observational study with cross-sectional design and analytical base with 73 patients aged between 3 and 18 years. Sociodemographic data was collected by a trained staff using structured interviews, patient clinical information and the following specific instruments: Sleep Disturbance Scale for Children - SDSC, Epworth Sleepiness Scale - ESS, and the Childhood Health Assessment Questionnaire - CHAQ. For statistical analysis, the statistics Package for the Social Sciences for Windows, version 20.0 (SPSS Inc., Chicago, IL, USA), as well as descriptive statistics and correlation analyses were used. For correlation analysis, Pearson's test was used for variables with normal distribution, while Spearman's test was employed for non-parametric data. All tests were two-tailed. Descriptive statistics were used, with central tendency measures considered statistically significant when $\mathrm{p}<0.05$.

Results and conclusions: Regarding the frequency of sleep disorders in children and adolescents with autoimmune rheumatic disease, $30 \%$ of these patients showed signs of daytime sleepiness, evidenced by scores greater than 10 on the Epworth scale, and $62.5 \%$ had total sleep-related disturbances, demonstrated by the scores greater than or equal to 39 in the scale of sleep disorders in children. Regarding the association between sleep disorders and items on the Childhood Health Assessment Questionnaire (CHAQ), statistical significance was observed in 6 of 8 domains evaluated: "Arising", "Eating", "Walking", "Hygiene", "Reach" and "Dressing \& Grooming." In addition, we also detected significance in the association between the childhood functional disability index (CHAQ-DI) and sleep-related disorders with respect to the parameters of "difficulty initiating and maintaining sleep" - DIMS, "disorders of excessive sleepiness" - DOES and "sleeprelated disorders total" SRD total.
P438

SOCIAL ASPECTS OF AMBULATORY CARE IN A TERTIARY

HOSPITAL

Blanca E R G Bica', Francine Malinoski de Sousa², Juliana Ramos

Guimarães de Faria ${ }^{2}$

'UNIVERSIDADE FEDERAL DO RIO DE JANEIRO, RIO DE JANEIRO, RJ,

Brasil; ${ }^{2}$ UFRJ, R, RJ, Brasil

Advances in Rheumatology 2018, 58(Suppl 1):P438

Background: Patients who suffer from chronic diseases show psychosocial aspects that may compromise the treatment outcome. This descriptive cross-sectional study conducted in 2017 was performed in an educational and health care medical university located in Rio de Janeiro, Brazil.

Methods: The population of the study in this research included outpatients visiting the educational and learning hospital of our University in different clinics. A structured questionnaire was developed by investigators to obtain patient's information and data were collected by face-to-face interviews throughout the year of 2017. The study aimed to collect independent variables such as demographic features (age, education level, clinic in which the patient receives care; disease diagnosis; medicaments in use; means of transport used to get to the hospital; time taken to arrive at hospital; use of alternative therapies and which; aspects of the patient-physician relationship; whether the patient has ever abandoned treatment, how many times and why he abandoned the treatment.

Results and conclusions: Seventy (70) patients between 19 and 90 years old who go regularly to appointments of various medical specialties were interviewed. The largest group considering educational level has finished High School (37\%). Those who use some continuous medicaments represent $95 \%$ of the total and the largest part of this group (56\%) uses less than 5 medicines daily. Sixty-five percent $(65 \%)$ of the patients need public transportation to get to the hospital, the majority of them uses only one bus. Fifty-one percent $(51 \%)$ of the patients take less than an hour to arrive at the hospital, $37 \%$ spend more than an hour and less than two hours and $12 \%$ of the patients interviewed take more than two hours to reach the University Hospital. Seven percent (7\%) attend alternative therapies. Thirty percent (30\%) have some complaint about ambulatory attending and $22 \%$ have already abandoned treatment at least once. Among the ones who left medical care, $20 \%$ were due to lack of financial resources for transportation and $33 \%$ because of the long wait for rescheduling appointments and exams. In conclusion, most outpatients complained about difficulty of transport, financial difficulties to buy their medications and waiting time. According to our results, the social aspects of health care should be better understood to get better outcome.

\section{P439}

SOCIAL PHOBIA AND QUALITY OF LIFE IN CHILDHOOD-ONSET SYSTEMIC LUPUS ERYTHEMATOSUS

Cátia Maria Geralda dos Santos Nascimento, Paula Teixeira Fernandes,

Roberto Marini, Simone Appenzeller

UNICAMP, CAMPINAS, SP, Brasil

Advances in Rheumatology 2018, 58(Suppl 1):P439

Background: Low quality of life scores were observed in CSLE patients who presented lower scores SF-36 when compared to healthy children. Neuropsychiatric manifestations compromise the quality of life of patients with childhood-onset lupus. Anxiety disorders and mood are commonly reported in children with chronic diseases, such as rheumatic diseases. Anxiety when intense presents itself in many 
ways, becoming anxiety disorders. Among the various anxiety disorders, it has social phobia, which is characterized by intense and persistent fear of social situations. In social phobia, there is functional impairment and quality of life of the subject. The objective is to determine the frequency of social phobia and to avaluate the quality of life in patients with childhood-onset Systemic Lupus Erythematosus (cSLE) and to determine the associations with neuropsychiatric manifestations (NPM), disease activity, disease damage, and use of corticosteroids (CE).

Methods: In this cross-sectional study, we include 81 CSLE consecutive patients [mean age 17.61 years ( $\mathrm{DP} \pm 4.42$ ) from the Rheumatology outpatient unit and 59 healthy controls [mean age 18.58 years $(\mathrm{DP} \pm$ 3.89)]. Short-Form Health Survey (SF-36) assessed quality of life. Liebowitz Social Anxiety Scale (LSAS) assessed presence of symptoms of social phobia. Mood and anxiety disorders were determined through Beck Depression and Beck Anxiety Inventory. SLE patients were further assessed for clinical and laboratory SLE manifestations, disease activity [SLE Disease Activity Index (SLEDAI)], damage [Systemic Lupus International Collaborating Clinics/American College of Rheumatology Damage Index (SDI)] and current drug exposures. NPM were classified according to the criteria established by the American College of Rheumatology (ACR). Data were compared by non-parametric tests.

Results: Social phobia was observed in 34 (37.4\%) CSLE patients and in $6(10.2 \%)$ healthy controls $(p<0.001)$. Presence of anxiety symptoms, Depression symptoms were more rfequentlu on CSLE than controls $(p<0.05)$. There was significant association between social phobia and anxiety disorders $(p=0.023)$, mood disorders $(p=0.025)$ and age at disease onset $(\mathrm{p}=0.042)$. All SF-36 domains, physical and mental components scores were significantly lower in CSLE patients than healthy controls. Functional capacity domain was associated with the presence of social phobia in CSLE patients $(p=0.04)$. There was no significant association between social phobia and cumulative damage, activity disease, current dose prednisone, cumulative dose of corticosteroid and dose of corticosteroid-adjusted weight and other neuropsychiatric manifestations. In conclusion, social phobia should be screened routinely it can influence the quality of life of patients.

\section{P440}

SOCIAL SECURITY IMPACTS OF CHIKUNGUNYA FEVER AND RHEUMATOID ARTHRITIS IN 2016: ANALYSIS OF TIME LEAVE FROM WORK IN RIO GRANDE DO NORTE AND BRAZIL

Rayani Diógenes Umbelino Reis, Francisco Alves Bezerra Neto, Francisco Deoclecio Damasceno Rocha, Maethê Romero Frota Quinderé, Clara Edwiges Frota Moraes, Camila Fernandes da Cunha, Renata Bezerra de Miranda, Marcelo Augusto Firmino de Queiroz, Thaís Cav

UNP, NATAL, RN, Brasil

Advances in Rheumatology 2018, 58(Suppl 1):P440

Background: Chikungunya Fever (CF) is an arbovirosis that can occur with incapacitating polyarthritis of variable duration. In Brazil, in 2016, about 150 thousand CF cases were confirmed. Rheumatoid arthritis (RA) is a systemic inflammatory disease, characterized by symmetrical polyarthritis, determining impairment of mobility and joint function in affected individuals. Both nosological entities have an impact on the social security system due to the need to leave work in affected individuals.

Methods: A data collection was carried out for the period from January to December 2016, in the Unified Benefit Information System (SUIBE), referring to the total number of retired individuals, number of days of leave and amounts disbursed in sickness benefits for these patients.

Results: From the data obtained by SUIBE, a total of 1,020 beneficiaries were detected in Brazil as a result of the CF. The mean time to leave was 62 days with a total cost of 1,301,520.00 reais. In the same period, 3,154 beneficiaries with an average time of removal of 89 days at a cost of $3,596,289.86$ reais were removed by AR. In Rio
Grande do Norte (RN), 139 people were separated by CF for an average period of 57 days, at a total cost of 163,297.20 reais. In relation to RA, 67 people were taken apart, for an average of 79 days, generating a cost of $72,729.84$ reais.

Conclusions: The above values indicate a mean rate of significant days of absence, both for CF, and for RA, at the state and national levels. The amounts awarded to beneficiaries reflect the impact of the CF epidemic on revenue for aid and securities.

P443

SPONDYLOARTHRITIS AND RELAPSING POLYCHONDRITIS: A CASE REPORT

Bruna Laiza Fontes Almeida', Wládya Maria Diniz Mororó2, Anna Luiza Marinho de Andrade ${ }^{3}$, João Rodrigues de Araújo $\mathrm{Neto}^{4}$

${ }^{1}$ FACULDADE INTEGRADAS DE PATOS, PATOS, PB, Brasil; ${ }^{2}$ FACULDADE INTEGRADA DE PATOS, PATOS, PB, Brasil; ${ }^{3}$ UNIVERSIDADE FEDERAL DA PARAÍBA, JOÃO PESSOA, PB, Brasil; ${ }^{4}$ UNIVERSIDADE FEDERAL DE

CAMPINA GRANDE, CAMPINA GRANDE, PB, Brasil

Advances in Rheumatology 2018, 58(Suppl 1):P443

Introduction: Spondyloarthrites $(\mathrm{SpA})$ represent a group of chronic autoimmune diseases that lead to inflammatory involvement of entheses, and axial and peripheral joints, usually asymmetric oligoarthritis of large lower limb joints. It is also characterized by the absence of rheumatoid factor and association with HLA-B27 histocompatibility antigen. Recidivating Polychondritis (RP) is a rare and multisystemic autoimmune disease that presents recurrent episodes of inflammation of the cartilaginous tissues and it is associated with the human leukocyte antigen HLA-DR4. More than one-third of patients with RP are associated with some autoimmune disease, such as systemic vasculitis or connective tissue disease, or even with some malignant condition.

Case report: A 52-year-old female patient complaining of low back pain for 3 years, which worsened with rest and improved with the use of nonsteroidal anti-inflammatory drugs, associated with morning stiffness of 60 minutes, in addition to arthralgia and edema in the ankles and knees. She also complained of deformity in the auricular pavilion bilaterally, beginning 4 years ago, associated with episodes of pain and intermittent hyperemia. Physical examination showed right fist in arthritis and in both knees, clinical signs of bilateral sacroiliitis (FABER test positive) and infiltrative edema in auricular pavilions bilaterally (Fig. 1). Complementary tests showed HLA-B27 as positive, erythrocyte sedimentation rate $90 \mathrm{~mm} / \mathrm{h}$, autoantibodies research as negative (rheumatoid factor, antinuclear factor and anti-cyclic citrullinated peptide). The biopsy of the auricular pavilion cartilage evidenced perichondral inflammation, extensive fibrosis and vascular neoformation, histological findings compatible with Relapsing Polychondritis. Radiography of the sacroiliac joints did not show alterations, whereas the magnetic resonance imaging showed evidence of sclerosis and subchondral cortical thickening of both articular faces, bilaterally and thin subchondral bone edema, mainly in the sacrum (Fig. 2). A treatment with naproxen $(500 \mathrm{mg}$ every $12 \mathrm{~h})$ associated with sulphasalazine $(2 \mathrm{~g} /$ day) for 3 months was initiated, but there was no improvement in the condition, with sulfasalazine being suspended and Adalimumab prescribed. The patient remains on Adalimumab treatment, indicating important clinical improvement of the joint pattern, without new episodes of chondritis.

Conclusion: Autoimmune diseases such as rheumatoid arthritis, systemic lupus erythematosus, Hashimoto's thyroiditis and inflammatory bowel disease are commonly found in patients with relapsed polychondritis, but the association with axial spondyloarthritis is extremely uncommon, and the authors consider the description of this case to be of great relevance for Rheumatology.

\section{Consent for publication}

The authors declare that they have obtained informed written consent from the patient's tutors for publication 


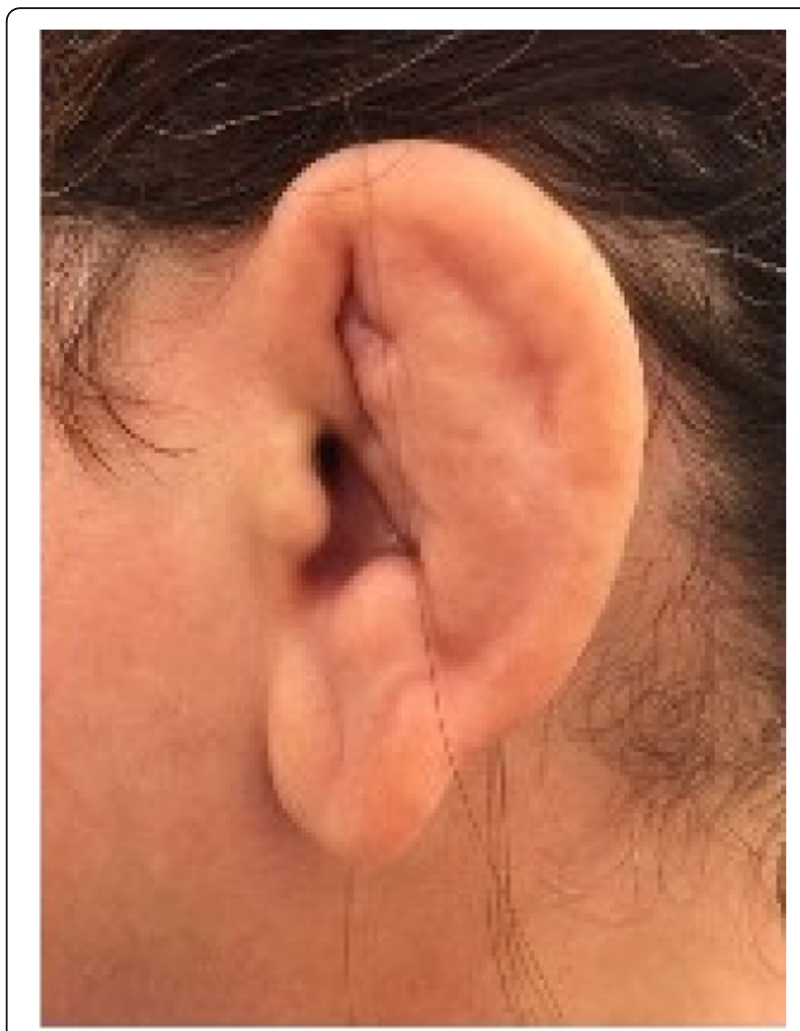

Fig. 1 (abstract P443). Infiltrative edema in auricular pavilions bilaterally

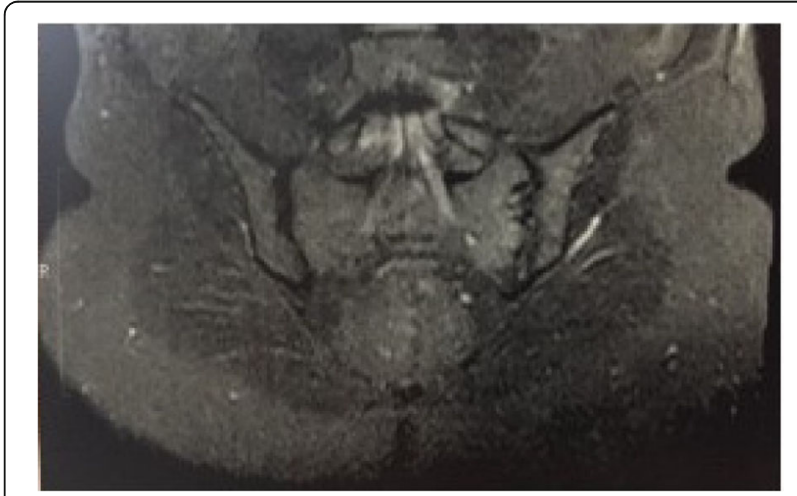

Fig. 2 (abstract P443). Magnetic resonance imaging showed evidence of sclerosis and subchondral cortical thickening of both articular faces, bilaterally and thin subchondral bone edema, mainly in the sacr

\section{P444}

\section{SPONDYLOARTHRITIS, ELECTROCARDIOGRAFIC CHANGES AND}

\section{ANTI-TNF-A TREATMENT}

Betânia Longo ${ }^{1}$, Luziel Andrei Kirchner², Jhésyca Flávia Sousa Castaman Stédile $^{3}$, Juliana Simioni ${ }^{3}$, Ana Paula Beckhauser de Campos ${ }^{3}$, Thelma Larocca Skare ${ }^{3}$

'HOSPITAL UNIVERSITÁRIO EVANGÉLICO DE CURITIBA (PR), CURITIBA, PR, Brasil; ' ${ }^{2}$ OSPITAL CARDIOLÓGICO CONSTANTINI, CURITIBA, PR, Brasil; ${ }^{3}$ HOSPITAL UNIVERSITÁRIO EVANGÉLICO DE CURITIBA, CURITIBA, PR, Brasil

Advances in Rheumatology 2018, 58(Suppl 1):P444
Introduction: Spondyloarthritis (SpAs) are chronic rheumatic diseases with several extra-articular manifestations. Among them are the cardiac manifestations that affect around $10 \%$ of the patients. Aortitis, conduction disorders and atrioventricular conduction block are prominent in this context. In this work we evaluate the prevalence of electrocardiographic changes in patients with SpA correlating them with the use of anti-TNF- $a$ and presence of HLA-B27.

Methods: Cross-sectional observational study with 100 patients diagnosed with SpA according to ASAS (Assessment of SpondyloArthritis International Society) criteria and 50 controls. Epidemiological and clinical data, inflammatory activity tests, presence of HLA-B27 and use of medications were collected in the patients. Disease activity was assessed by BASDAI (Bath Ankylosing Spondylitis Disease Activity Index). All individuals were submitted to electrocardiogram performed with a 12-lead device, being analyzed rhythm, heart rate, conduction disorders and QT interval corrected by the Bazett formula.

Results: Of the 100 patients with SpA, 49 did not use anti-TNF-a and 51 used this drug. HLA-B27 was present in $53.1 \%$ of the sample. Heart rate was lower $(p=0.06)$, the QT interval was longer $(p<0.0001)$, and there was a trend to longer QTc $(p=0.08)$ in SpA than controls. In addition, there was more RBCD (right bundle branch conduction disturbance; $p=0.014$ ) in patients with SpA. A modest correlation of disease duration with heart rate was found $(p=0.008, R h o=0.26,95 \%$ $\mathrm{Cl}=0.06-0.44)$. The presence of HLA-B27 $(p=0.03)$, but not the disease activity ( $p=0.19$ ) increased the prevalence of RBCD. None of the electrocardiographic parameters analyzed changed with anti-TNF-a use as seen in Table 1.

Conclusion: Patients with SpA showed a lower heart rate, longer QT interval and a higher prevalence of RBCD than controls. HLA B27 influences the prevalence of RBCD. The use of anti-TNF- $a$ did not influence the electrocardiographic findings.

\section{References}

1. Di Franco M, Paradiso M, Ceccarelli $F$, et al. Biological drug treatment of rheumatoid arthritis and spondyloarthritis: effects on QT interval and QT dispersion. J Rheumatol. 2012;39(1):41-5.

Table 1 (abstract P444). Electrocardiographic findings in SpA (SpondyloArthritis) patients with and without anti TNF-a treatment

\begin{tabular}{|c|c|c|c|}
\hline EKG & 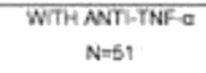 & $\begin{array}{l}\text { WIHOUT ANT1- } \\
\text { TNF-a } N=49\end{array}$ & $\mathrm{P}$ \\
\hline \multirow[t]{2}{*}{ Cardiac rate (bpm) } & $47-86$ & $47-97$ & 0.77 \\
\hline & Madian=66 (58-77) & Madian=68 (60-75) & \\
\hline Cardiac rate & $0.61-128$ & 0.52 .128 & 0.71 \\
\hline (duration'sec) & Masn $=0.90 \pm 0.15$ & Mean $=0.89 \pm 0.14$ & \\
\hline \multirow[t]{3}{*}{ aT (sec) interval } & 0.32 .0 .49 & $0.32 \cdot 0.46$ & 0.62 \\
\hline & Modian $=0.40(0.37$ & Modian=0.40 10.37. & \\
\hline & $0.40)$ & $0.40)$ & \\
\hline \multirow[t]{2}{*}{ ate $(s e c)$ interval } & $0.36-0.48$ & $0.38 .0 .49^{\circ}$ & 0.53 \\
\hline & Mean $=0.41 \pm 0.02$ & Mean $=0.41 \pm 0.025$ & \\
\hline $\operatorname{RBCD}\{a\}$ & 20151 & $17 / 49$ & 0.63 \\
\hline
\end{tabular}

P446

SPONDYLOEPIPHYSEAL DYSPLASIA: DESCRIPTION OF A FAMILY WITH INFLAMMATORY MANIFESTATIONS MIMICKING RHEUMATOID ARTHRITIS

Larissa Aniceto Moreira, Bernardo Matos da Cunha HOSPITAL SARAH, BRASÍLIA, DF, Brasil

Advances in Rheumatology 2018, 58(Suppl 1):P446 
Background: Osteochondrodysplasia are a group of hereditary disorders in which bones and cartilages grows abnormally. There is a great clinical variability between bone/cartilaginous dysplasias. Spondyloepiphysial dysplasia is one subtype that causes platyspondyly and widening of epiphyses, causing disproportionate short stature and early osteoarthritis.

We describe family members with a unique phenotype: normal stature, shortening of the fourth metatarsal, positive inflammatory tests and ultrasonographic findings similar to rheumatoid arthritis. The condition was inherited as a dominant trait. Marik, I et al. [1] described a family with similar manifestations - a pseudorheumatoid form, but without inflammatory findings.

Case series: Twelve patients from the same family with spondyloepiphyseal dysplasia were evaluated. All our patients had normal height. Ten patients presented with mechanical hand symptoms. Clinical synovitis of the hands (wrists and metacarpophalangeal) was identified in six patients. Five patients presented with synovitis by ultrasonography. Eight patients presented increased inflammatory tests (C-reactive protein and erythrocyte sedimentation rate). Rheumatoid factor and anti CCP were negative in all patients.

Conclusions: This family presents a similar phenotype to a spondyloepiphyseal dysplasia subtype called progressive pseudorheumatoid dysplasia associated with hypoplastic toes.

This phenotype was described in only 11 families, without publications in Brazil. However, a different feature in our patients is an inflammatory component, with high inflammatory tests and synovitis, turning into an important differential diagnosis of rheumatoid arthritis.

The mutation described in patients with pseudorheumatoid dysplasia is present in the COL2A1 gene, which encodes type 2 collagen. The family reported in this study have not had the mutation identified yet. Our research aims to identify if we are facing a new mutation or if it is a phenotypic variation of the same mutation.

\section{References}

1. Marik at all - Dominantly inherited progressive pseudorheumatoid dysplasia with hypoplastic toes - Skeletal Radiol (2004) 33:157-164DOI 10.1007/s00256-003-0708-z

\section{P448}

SPOROTRICHOSIS PRESENTING AS A RHEUMATOID SYNDROME: A CASE REPORT

Nicole Pamplona Bueno de Andrade ${ }^{2}$, Bruna de Lima Porto ${ }^{2}$, Micheline Sulzbacher Batista', Afonso Papke², Luiza Rossi², Guilherme Leví Tres², Fernando Schmidt Fernandes ${ }^{2}$, Vanessa $\mathrm{Hax}^{2}$, Claiton Viegas Brenol ${ }^{2}$ ${ }^{1}$ HOSPITAL DE CLINICAS DE PORTO ALEGRE, PORTO ALEGRE, RS, Brasil; ${ }^{2}$ HOSPITAL DE CLÍNICAS DE PORTO ALEGRE, PORTO ALEGRE, RS, Brasil Advances in Rheumatology 2018, 58(Suppl 1):P448

Background: Sporotrichosis is a subacute to chronic infection caused by the dimorphic fungus Sporothrix schenckii. Clinical manifestations most commonly include cutaneous and lymphocutaneous forms of the infection. Occasionally the infection involves the joints, most commonly the knee. Rare forms of sporotrichosis include osteomyelitis occurring after local or hematogenous spread. The diagnosis of sporotrichosis is established by culture or histopathology findings. In cases of joint involvement, synovial fluid parameters often show relatively low white cell counts with a neutrophil predominance. We report a case of disseminated sporotrichosis presenting as a rheumatoid syndrome with characteristic skin lesions.

Case Report: A 56-year-old African-Brazilian woman, with history of kidney transplantation, presented to the emergency department because of a 4-month complaints of arthralgia, cutaneous lesions, weight loss and fatigue. Physical examination on presentation revealed polyarthritis of elbows, wrists, metacarpophalangeal and proximal interphalangeal joints, also multiple hyperkeratotic and hyperpigmented plaques. She had no history of fever or lymphadenopathy. Laboratory evaluation showed a white blood cell count of 6140 cells $/ \mathrm{mm} 3$, erythrocyte sedimentation rate (ESR) of $120 \mathrm{~mm} / \mathrm{h}$, C-reactive protein (CRP) of $118 \mathrm{mg} / \mathrm{L}$; rheumatoid factor (RF) and anticitrullinated protein antibody (ACPA) were negative. Radiographs of hands and elbows were normal, though magnetic resonance of these joints demonstrated synovitis with small joint effusion and intense bone marrow edema, suggestive of septic arthritis and osteomyelitis. Cutaneous biopsy revealed granulomatous inflammation. Fungal culture of skin lesions showed Sporothrix schenckii. Bacterial and acidfast bacilli (AFB) cultures were negative. US-guided arthrocentesis could not retrieve synovial fluid. The patient was treated with intravenous amphotericin B for 2 weeks and then with oral itraconazole. Because there was significant clinical and laboratory improvement, no surgical debridement was needed.

Conclusion: We reported an unusual case of osteoarticular sporotrichosis, presenting as a rheumatoid syndrome. Fungal infections, including sporotrichosis, should be considered as a differential diagnosis especially in immunosuppressed and alcoholics patients.

\section{Consent for publication}

The authors declare that they have obtained informed written consent from the patient's tutors for publication

\section{P449}

STUDY OF CARDIOVASCULAR REPERCUSSIONS IN CHIKUNGUNYA FEVER IN PATIENTS OF A UNIVERSITY HOSPITAL

Camila Rocha Vieira Torres², Camila Amorim Polonio², Luan Fernandes Gonçalves de Oliveira², Tâmara Tamiris Rocha Vleira', Eutilia Andrade Medeiros Freire², Ana Karla Guedes de Melo², Alessandra de Sousa Braz², Danielle Christinne Soares Egypto², Sandra Rejane Cabral Batista², Benedito Begnailson Ribeiro2, Mattheus Dos Santos Mousinho ${ }^{2}$ ${ }^{1}$ UERN, JOÃO PESSOA, PB, Brasil; ${ }^{2}$ UFPB, JOÃO PESSOA, PB, Brasil Advances in Rheumatology 2018, 58(Suppl 1):P449

Background: Chikungunya fever (CF) is a seasonal infection caused by arbovirus Chikungunya (CHIKV). It can affect cardiac tissue and promote arrhythmias, hemodynamic instability, myocarditis, pericarditis, heart failure, coronary ischemic disease and dilated cardiomyopathy. The objective of this study is to evaluate the prevalence, clinical and epidemiological data of the cardiological alterations found in patients diagnosed with CF attended at a university hospital.

Methods: This is a descriptive, observational and cross-sectional study. It is based on the information contained in medical records and epidemiological notification sheets from January 2016 to January 2018. The studied population is the patients with laboratory confirmation for chikungunya fever, above 18 years of age, resident in Paraíba and that do not present coinfection with other arboviruses. During the collection, a survey of all reported cases of chikungunya in the hospital was carried out, then cases from other states, younger than 19 years and cases that did not have laboratory confirmation were excluded. Such information was used to build the database in Excel spreadsheets. Statistical analysis was performed using statistical software Statistical Package for Social Science (SPSS).

Results and conclusions: The research is still going on, and partial results have shown that of the 143 cases reported at Lauro Wanderley University Hospital, 45 cases were laboratory confirmed for Chikungunya. Of these, $6.67 \%$ of the individuals, all of them female, presented cardiovascular alterations with lipotimia and transient arrhythmias in the acute or subacute phases of the $\mathrm{CF}$, requiring 01 hospital admission. Therefore, it is not possible to reach conclusions based only on these partial results, but with this sample, it is predicted that the prevalence of cardiovascular repercussions in chikungunya fever is low, and requires larger studies to conclude data on the prevalence of cardiovascular manifestations in patients of CF.

\section{P450}

STUDY ON THE EFFECTS OF STRENGHT TRAINING IN PAIN AND DISEASE ACTIVITY IN WOMEN WITH PRIMARY SJOGREN'S

\section{SYNDROME}

Dardin ${ }^{1}$, Virginia Fernandes Moça Trevisani ${ }^{2}$, Ana Beatriz Andreo Garcia ${ }^{2}$

'UNIVERSIDADE FEDERAL DE SAO PAULO, SÃO PAULO, SÃO PAULO,

Brasil; ${ }^{2}$ UNIFESP, SÃO PAULO, SÃO PAULO, Brasil

Advances in Rheumatology 2018, 58(Suppl 1):P450 
Background: Muskuloeskeletal manifestations such as myalgias and arthralgias occur in $40 \%-75 \%$ in patients with pSS, and commonly run with pain. The purpose of this study was investigating the effects of strength training on pain and disease activity in women with primary Sjogren's Syndrome (pSS)

Metodology: Blind randomized controlled clinical trial, including 59 women diagnosed with pSS according to "European-American Consensus Group Criteria", allocated in two groups: control (30) and exercise (29). Both maintained drug treatment and the exercise group added sixteen weeks of strenght training for one hour, twice a week. The evaluation was performed using the Visual Analogue Scale of Pain (VAS) and the Sjogren's Syndrome Disease Activity Index (ESSDAI).

Results: We did not find significant differences between the means of the groups at the initial moment ( $p$ intergroup $=0.778$ ), but the mean of the control group at the final moment was significantly higher than the mean of the intervention group (p-intergroup $<0.001$ ). The exercise group achieved a $50 \%$ improvement in pain. ESSDAl: we did not find significant differences between the behaviors of the two groups over time ( $p$-interaction $=0.399)$, nor between the means at the initial and final moments ( $p$-intragroup $=0.059$ ). Conclusion: Based on the stability of disease activity during training and significant improvement of pain, we can conclude that strenght training was efficient and safe improving pain in women with pSS.

\section{P451}

SUBCLINICAL DIFFUSE ALVEOLAR HAEMORRHAGE (DAH) IN SYSTEMIC LUPUS ERYTHEMATOSUS (SLE) PATIENT: CASE REPORT

Larissa Cristiane de Oliveira Souza, Francisco Alves Bezerra Neto,

Alexandre Guimarães Gouveia, Jéssica Mayara de Figueirêdo Oséas,

Renata Bezerra Menezes, Ana Luísa Souza Leandro de Carvalho, Péricles de Sousa Cardoso, Bruna Aparecida Queiróz Oliveira, Maria Jose Pereira

Vilar, José Hilton Nogueira Junior

UFRN, NATAL, RN, Brasil

Advances in Rheumatology 2018, 58(Suppl 1):P451

Background: DAH is a rare manifestation with high mortality rate (70$90 \%$ ) in SLE patients. DAH pathogenesis is not clearly defined, but it is related to SLE disease activity, involving immune complex deposits and cellular infiltrate in pulmonary vessels leading to pulmonary capillaritis. It may present itself with or without hemoptysis and the treatment consists in pulse therapy with methylprednisolone, plasmapheresis, immunoglobulin, rituximab and cyclophosphamide, which has its use linked to controversy.

Case report: Female, 26 years old, was admitted to the hospital with a 1 month history of dyspnea at rest, dry cough, anasarca, anemia and butterfly rash. She had been experiencing asthenia, hair loss, arthralgia and oral ulcers for one year. Laboratory workup on admission were $6.3 \mathrm{~g} / \mathrm{dL}$ hemoglobin, negative direct coombs test, positive ANA 1/640 (mixed, nuclear, nucleolar and cytoplasmic pattern), positive anti-DNA and anti-RNP, 3+ dipstick urine protein, and urinary protein/creatinine ratio of 9.4. HIV, VDRL and hepatitis-related serology tested negative. She was initially diagnosed with SLE and Lupus Nephritis.

Chest CT scan (Fig. 1) was performed with resulting images suggestive of Non-Specific Interstitial Pneumonia (NSIP). The patient then progressed to severe anemia ( $3.8 \mathrm{~g} / \mathrm{dL}$ Hemoglobin) without presenting hematemesis or melena, being managed with blood transfusion and a 3-day pulse therapy of $1 \mathrm{~g}$ methylprednisolone plus cyclophosphamide. On the 20th day from admission, the patient displayed hemoptisis of roughly $20 \mathrm{~mL}$, in spite of our therapeutic measures. Hematic fluids were observed on the performed Bronchoscopy.

She was then, transferred to ICU and treated with $0.4 \mathrm{mg} / \mathrm{Kg}$ human immunoglobulin daily for 5 days, with good response and stabilization of hemoglobin levels around $6.5 \mathrm{~g} / \mathrm{dL}$. However, the patient progressed to massive hemoptysis, respiratory and renal insufficiency within 1 week of immunoglobulin infusion, leading to the need for mechanical ventilation and hemodialysis. She also experienced enterococcus septic shock, passing away while in ICU.

Conclusion: DAH is considered a severe complication of SLE. We warn the possibility of its initial clinical presentation consisting mainly of dyspnea associated with anemic syndrome, without hemoptisis. DAH is known to be associated with active lupus nephritis and high morbidity and mortality rates, as reported on this case.

\section{Consent for publication}

The authors declare that they have obtained informed written consent from the patient's tutors for publication

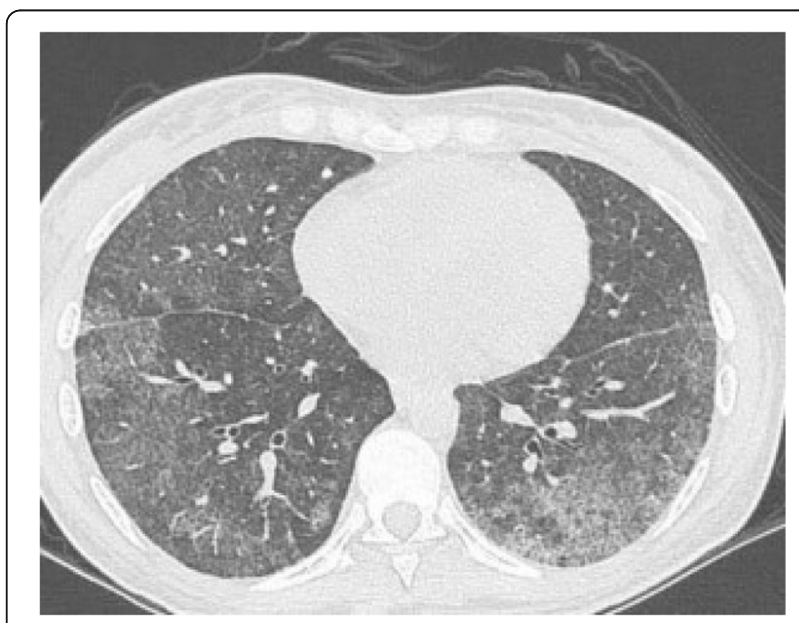

Fig. 1 (abstract P451). Interstitial Infiltrate, suggestive of NSIP

P453

SYPHILITIC SCLERITIS AS DIFFERENTIAL DIAGNOSIS OF RHEUMATIC DISEASES

Flávio Ribeiro Pereira', Ingrid Chagas Vilela Borges², Maria Carolina Faria da Costa $^{2}$

'SECRETARIA DE SAÚDE DE MACAÉ, MACAE, RJ, Brasil; ${ }^{2}$ UNIVERSIDADE FEDERAL DO RIO DE JANEIRO, MACAÉ, RJ, Brasil

Advances in Rheumatology 2018, 58(Suppl 1):P453

Background: the interface between rheumatology and ophthalmology is well known. Several ophthalmological manifestations associated with various diffuse collagen diseases are documented. One of the most severe and threatening ocular changes is scleritis, whose presentation and evolution are related to severe ways of various rheumatic diseases. The differential diagnosis with other pathologies, especially infectious, is necessary for the correct diagnosis and treatment of this complication.

Case report: M.S.A, 66 years old, female, housewife, admitted to the rheumatology outpatient clinic due to hyperemia and severe pain in the left eye in the last three months, with progressive worsening, associated with decreased visual acuity, strongly suggestive of scleritis (Fig. 1). She had musculoskeletal complaints such as arthralgia in small joints of hands and wrists, knee pain, diffuse myalgia and cyanosis of extremities suggestive of Raynaud's phenomenon. 
Ophthalmologic evaluation confirmed anterior and superior diffuse scleritis. Laboratory tests showed erythrocyte sedimentation rate (ESR) $45 \mathrm{~mm}$, antinuclear antibodies (ANA) $1 / 160$ nuclear fine dotted, with other negative autoimmunity markers such as Rheumatoid Factor, anti cyclic citrullinated peptide (anti-CCP) and antineutrophil cytoplasmic antibodies (p-ANCA and c-ANCA). The initial infection investigation was inconclusive, with non-reactor PPD, negative serologies for HIV, hepatitis B and C. Chest X-ray without changes and hands and wrists with no erosions or deformities. Due to the severity of the condition, we opted to initiate therapy with Prednisone $60 \mathrm{mg} /$ day and insisted on the investigation for syphilis (initially not accepted by the patient, but being authorized later), revealing VDRL 1/64 and FTA-abs IgM and IgG positive. The diagnosis of syphilitic scleritis was promptly done and the patient was submitted to therapy with crystalline penicillin $G$ and initiated the gradual withdrawal of the steroid, evolving with complete improvement of the lesion and visual acuity.

Conclusion: scleritis is one of the most frightening ophthalmologic complications in the context of autoimmune rheumatic diseases, and its fast identification and appropriate treatment are imperative. However, one should not forget other pathologies that make differential diagnosis and that often mimic collagen diseases.

\section{Consent for publication}

The authors declare that they have obtained informed written consent from the patient's tutors for publication

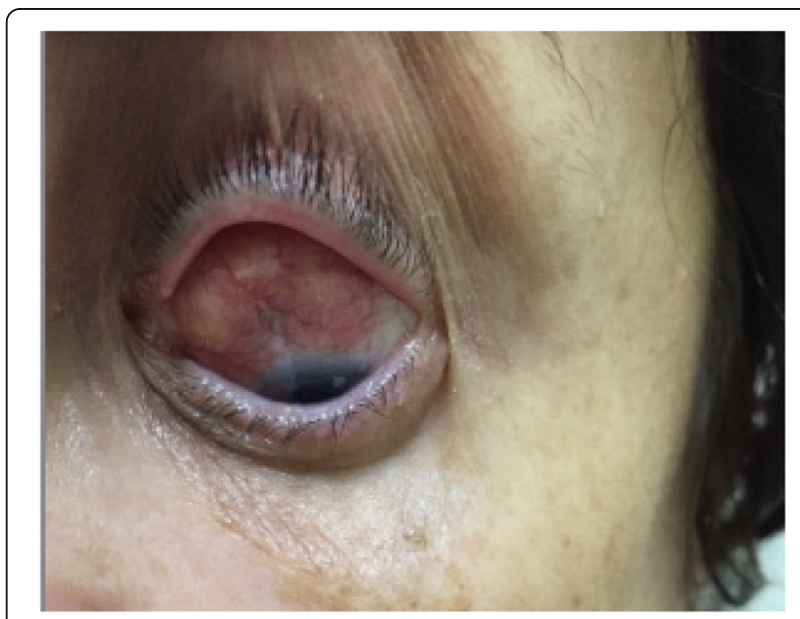

Fig. 1 (abstract P453). Hyperemia in the left eye

\section{P457}

SYSTEMIC LUPUS ERYTHEMATOSUS WITH SEVERE PANCYTOPENIA AFTER CITOMEGALOVIRUS INFECTION: CASE REPORT

Aderbal Gaulino Galassi Neto ${ }^{1}$, Ellen Tiyemi Tsutsumoto da Silva², Fernanda Carla Salesse Cunha ${ }^{2}$. Fernanda Miranda Caliani ${ }^{2}$

${ }^{1}$ UNIVERSIDADE DO OESTE PAULISTA - UNOESTE, PRES.PRUDENTE, SÃO PAULO, Brasil; ${ }^{2}$ UNOESTE-UNIVERSIDADE DO OESTE PAULISTA, PRESIDENTE PRUDENTE, SÃO PAULO, Brasi

Advances in Rheumatology 2018, 58(Suppl 1):P457

Background: Infectious diseases are one of the major causes of complications that threaten the lives of patients with rheumatic diseases. Cytomegalovirus (CMV) is the major cause of morbidity and mortality in immunosuppressed individuals, causing fever and damage to organs such as lung, liver, gastrointestinal tract, bone marrow and retina. Patients with Systemic Lupus Erythematosus (SLE) use immunosuppressants and are at risk of reactivating latent CMV infection.

Case report: Female patient, Caucasian, 23 years old, diagnosed with SLE for 4 years. The diagnosis was according to the criteria of the American College of Rheumatology (ACR) presenting: FAN thick dotted reagent 1/640, anti-Sm reagent, hematological changes (leukopenia and thrombocytopenia), mucocutaneous lesions (malar rash, oral canker sores) and polyarthritis. Initially treated with hydroxychloroquine and prednisone evolved with total stabilization of her clinical condition, with progressive weaning of prednisone. After two days of treatment, developed lupus nephritis treated with pulse therapy with methylprednisolone for 3 days and monthly cyclophosphamide for 6 months with control. After 6 months of the end of pulse therapy presented low fever, nausea, vomiting, palpable lymph nodes in the cervical chain. Laboratory tests showed severe pancytopenia, low inflammatory tests, renal and hepatic function without alterations, urine I without alteration and normal complement. Normal chest radiographs, abdominal ultrasonography, and echocardiography. Before the clinical and laboratory study, an investigation was started. Serological tests were made for HIV, $B$ virus, $C$ virus, cytomegalovirus, Epstein-Baar, toxoplasmosis and bone marrow puncture with immunohistochemistry. Discarded the possibility of haematological neoplasm, negative serologies, except for the positivity of IgM serology for cytomegalovirus. PCR was performed for CMV that had a positive result. Initiated ganciclovir treatment for 21 days. After this period the patient was asymptomatic, with normal hemogram and PCR negative for CMV.

Conclusion: Although active CMV infection is not frequent in patients with SLE, when it exists is extremely serious and can lead to death. The early diagnosis of active CMV infection is very important, because it allows an earlier and more effective treatment in relation to the serological diagnosis, especially in patients with immunological failure as the patient of the described case.

Consent for publication

The authors declare that they have obtained informed written consent from the patient's tutors for publication

P458

SYSTEMIC LUPUS ERYTHEMATOSUS (SLE) AND ANTIPHOSPHOLIPID ANTIBODY SYNDROME (AAS) WITH EXTENSIVE NECROTIC LESIONS: CASE REPORT

Larissa Cristiane de Oliveira Souza, Maria Jose Pereira Vilar, Roberta

Gadelha Peixoto, Henrique de Paula Bedaque, Priscilla Brenda Fonseca

Dantas, Ana Márcia Azevedo de Sousa, Layssa Carolinne de Sousa

Carvalho

UFRN, NATAL, RN, Brasil

Advances in Rheumatology 2018, 58(Suppl 1):P458

Background: AAS is a systemic autoimmune disorder characterized by a combination of arterial and/or venous thrombosis, recurrent fetal loss and persistently high titers of antiphospholipid antibodies (APL). The AAS can be secondary (AASs) when associated with other diseases, such as Systemic Lupus Erythematosus (SLE). Approximately $40 \%$ of patients with SLE are positive for APL. However, it is estimated that AASs can develop in up to $50-70 \%$ of patients with SLE.

Case report: Female, 22 years-old, married, reports arthritis of knees and wrists, associated with prolonged morning stiffness, started 4 months ago. She evolved with hyporexia, nausea and vomiting, lasting a few days, progressing with loss of consciousness associated with tremors in the body, suggestive of seizures. She attended to emergency care where has been hospitalized for 1 week, when purple skin lesions appeared, which progressed to a necrotic and exudative aspect, painful, measuring from 2.0 to $5.0 \mathrm{~cm}$, in the lateral region of the thighs, lateral of the left arm, right ankle and some toes of both feet, which prevented ambulation. She denied photosensitivity, other skin lesions, Raynaud's phenomenon, hypertension and edema before this condition. There was a history of pre-eclampsia in 
the two previous pregnancies, with no miscarriages. She reported cocaine use, abstain for 3 years. Considering LES as hipoteses, some complementary examinations were asked: Hematocrit: $21.5 \%$, leukocytes: 7,930 / mm3, lymphocytes: $795 \mathrm{~mm} 3$, platelets: $137,000 / \mathrm{mm} 3$, FAN: 1: 640 (thick nuclear core) and chest tomography with pleural effusion. Made pulse therapy with methylprednisolone and sent to the rheumatology service. At admission, hemodynamically stable, lower limb edema $++/ 4+$, with the described necrotic lesions and arterial hypertension of difficult control. Exams: Creatinine $=0.9 \mathrm{mg} /$ dl, Protein/Creatinine ratio: 2.6, C3: $36.1 \mathrm{mg} / \mathrm{dl}, \mathrm{C} 4: 6.0 \mathrm{mg} / \mathrm{dl}$, transthoracic echocardiogram: mild pericardial effusion, electroencephalogram: brief frontal region, skull tomography: area of hypoatenation of the subcortical parenchyma in the frontal lobe suggestive of subacute ischemic infarction. In the same week, new necrotic lesions appeared when we started full anticoagulation with AAS in mind. Conclusion: This case demonstrates the challenge to the general clinician, in an adverse scenario of prompt care, faced with the need for immunosuppression. The association between SLE and AAS seems to be a possibility in this case, since the improvement of the patient after heparinization. Awaiting examinations for the conclusion of the diagnostic association.

\section{Consent for publication}

The authors declare that they have obtained informed written consent from the patient's tutors for publication

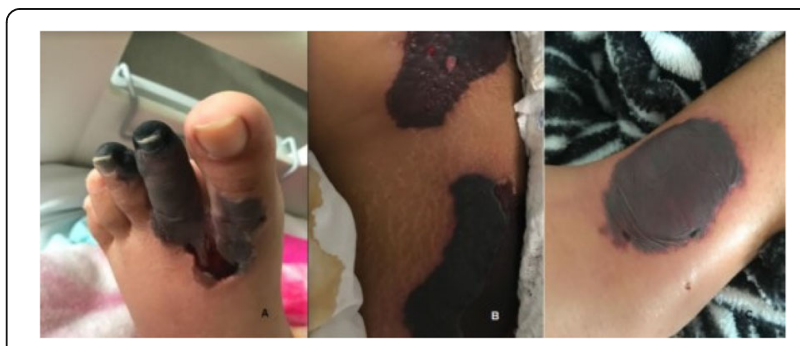

Fig. 1 (abstract P458). Lesões necróticas (A: pé esquerdo B: quadril direito C: perna direita)

\section{P459}

\section{SYSTEMIC SARCOIDOSIS: CASE REPORT}

Paula Meirelles Silva², Guilherme Martins Guzman', Virna ${ }^{2}$, Nicole Oliveira Alves $^{2}$

${ }^{1}$ UNIFESP, SÃO PAULO, SP, Brasil; ${ }^{2}$ HOSPITAL DO SERVIDOR PÚBLICO MUNICIPAL, SÃO PAULO, SP, Brasil

Advances in Rheumatology 2018, 58(Suppl 1):P459

Background: Sarcoidosis is a systemic inflammatory disorder that can potencially affect any organ system, notable for great variabillity in clinical presentation. Etiological factors leading to sarcoidosis are not clear yet. The lung and intrathoracic lymph nodes are almost invariably affected. The typical histopathologic lesion of sarcoidosis is granuloma without caseous necrosis. This study aims to report a typical sarcoidosis frame with presentation systemic involvement, reviewing the clinical features, diagnosis and treatment of disease Case report: A 36-year-old female white patient began a sporadic coughing in December 2016, self-medicating with natural syrups. She presented at the Emergency Care Service in December 2017 with asthenia and adinamia reporting erythematous and painful spots with five days of evolution that began in both knees associated with pain, increased articular volume. When it started, axilar temperature was $38-39{ }^{\circ} \mathrm{C}$. Physical examination revealed arthritis of both knees and ankles, erythematous lesions painful on both legs and arms. Chest Xray was performed and showed mediastinal widening. Chest CT scans showed in paratracheal lymphadenopathy. As in all cases of hilar adenopathy, corticosteroids were avoided until infectious or malignant causes could be excluded. A skin biopsy showing the lymphocytic vasculitis of small vessels with focal interstitial granuloma without caseous necrosis. Infectious and neoplastic causes were ruled out. Morphological findings associated with clinical and imaging suggest that sarcoidosis is the main diagnostic hypothesis. Patient was referenced for a rheumatologist who started treatment with Prednisone with symptomatic improvement.

Conclusion: Sarcoidosis is a chronic, granulomatous condition with unknown cause. Diagnosis is based on tissue biopsies, demonstrating granulomas without caseous necrosis in the presence of clinical and radiological findings, consistent with sarcoidosis, whereas other causes which might be associated with a similar manifestation are ruled out. Presence of granulomas alone is not diagnostic. From the histological perspective, same lesions may also be observed in chronic berylliosis, tuberculosis, histoplasmosis, coccidioidomycosis, lymphoma, Hodgkin's disease, bronchogenic carcinoma, foreign body granuloma, schistosomiasis, syphilis, and leprosy. Lungs are the most frequently involved organ. Bilateral hilar lymphadenopathy is characteristic and usually accompanied by paratracheal lymphadenopathy. Our patient was diagnosed upon put a way infection and cancer and identifying noncaseous granuloma in skin biopsy. Sarcoidosis has a variable clinical course and prognosis. One-third of the patients undergo spontaneous remission. In $10-30 \%$ of the patients, however, the disease takes a chronic course. Corticosteroids remain as the main therapeutic choice.

Consent for publication

The authors declare that they have obtained informed written consent from the patient's tutors for publication

P460

SYSTEMIC SCLEROSIS AFTER CHIKUNGUNYA VIRUS INFECTION A CASE REPORT

Ana Carla Fernandes Oliveira, Ana Valeska Lisboa Carvalho, Francisco

Fellipe Claudino Formiga, Sandra Rejane Cabral Batista, Ana Karla Guedes de Melo, Danielle Christinne Soares Egypto de Brito, Alessandra de

Sousa Braz, Eutília Andrade Medeiros Freire

HOSPITAL UNIVERSITÁRIO LAURO WANDERLEY, JOÃO PESSOA, PARAÍBA, Brasil

Advances in Rheumatology 2018, 58(Suppl 1):P460

Background: Systemic Sclerosis (SS) is a connective tissue disease characterized by diffuse vascular damage and fibrotic changes, which affect both skin and internal organs. ES pathogenesis is complex and little understood. The main disturbances associated to the clinical phenotype are endothelial damage, activation of immune response and tissue fibrosis. Vascular injury and endothelial damage are noticed in an early stage, causing tissue hypoxia and activation of the innate and adaptive immune system. Physical and chemical agents, oxidative stress, toxins and anti-endothelial cell antibodies are some factors involved in vascular insult. Viral agents, given its tropism by many cellular subtypes, including immune cells, and its capacity to virtually transform any cell in an antigen presenter, are considered potential triggers for SS. We reported the case of a 34-year-old female that developed SS symptoms after Chikungunya virus infection. It was found a similar case described in Brazil.

Case report: A 34-year-old female reported to the department with complaint of tender and swollen hands and feet for one year and 7 months, due to Chikungunya fever. One year after this arbovirus infection, she noticed thickened skin, especially on hands, face and chest; hypochromic macules on the chest, heartburn, regurgitation, Raynaud's phenomenon and fingertip ulcers. Examination revealed: hard and thickened face skin, microstomia, photosensitivity in face and chest, pitting scars on fingertips, hypochromic macules on chest and back, diffuse hand swelling. No disturbances were found on cardiovascular and pulmonary auscultating. Main serum exams: IgG ELISA serology for Chikungunya was positive, ANA 1/1280 nuclear homogeneous, non-reagent anti-centromere antibody. Chest Computed Tomography: subpleural ground glass opacities, predominantly on basal segments. No abnormalities were found at the transthoracic echocardiogram. 
Conclusion: At the present time, the evidence in favor of viral etiopathogenesis in SS is limited to Citomegalovirus and Parvovirus B19. However, considering the high number of viral pathogens capable of infecting human tissues, and the viral capacity to cause immune system activation, the role of Chikungunya virus as a trigger for SS must be studied. Furthermore, an early diagnosis must be stablished, once it's not possible to foresee how many patients who had Chikungunya fever will develop SS and how the autoimmune disease will behave in those patients.

\section{Consent for publication}

The authors declare that they have obtained informed written consent from the patient's tutors for publication

\section{P465}

TAKAYASU'S ARTERITIS: CLINCAL EXAM AND INTERVIEW IMPORTANCE IN DIAGNOSIS

Martins $^{1}$, Thais Campos de Paula Martins², Maria do Carmo Lacerda

Barbosa ${ }^{2}$, Adriana Lima dos Reis Costa ${ }^{2}$, Alessandra Porto Pereira Galdez ${ }^{2}$ ${ }^{1}$ UFMA, SAO LUIS, MA, Brasil; ' ${ }^{2}$ UNIVERSIDADE FEDERAL DO MARANHÃO, BELO HORIZONTE, MA, Brasil

Advances in Rheumatology 2018, 58(Suppl 1):P465

Background: With high imaging technologies, is clinical examination still matters? Despite the physician's graduation difficulty of the fragmentation of clinical knowledge, communication and physical exam in a technological era, are essential to patient be seen as an individual person. Thus, vital signs and patient history are important to the diagnosis of diseases, especially as autoimmune pathologies. Takayasu's arteritis is an example of clinical pathology that constantly has delay diagnosis, with signs and symptoms are not perceived at exam by some doctors.

Objectives: Reaffirm the importance of physical examination to proper diagnosis in Takayasu's Arteritis.

Methods: This was a retrospective, descriptive study, with a sample of 11 patients with Takayasu arteritis in Maranhão. Data were collected from medical records of a private hospital and HUUFMA in São Luís, Maranhão, Brazil, comprising the period from 2000 to 2011. Data were analyzed using Microsoft Office Excel $2010^{\circ}$. The study was approved by the Ethics Committee of Research of HUUFMA. Results: The disease evolution between the first clinical signs and the diagnosis varied from 2 months to 12 years. The most frequent manifestations were headaches (63.6\%), lightheadedness (63.6\%), arterial bruit (54.5\%) and decrease or absence of pulses (54.5\%). The survey reported rare presentation of Takayasu's Arteritis with abdominal pain, skin lesions, visual impairciment and chorea.

Conclusion: Even though with rare inicial signs there was a delay diagnosis with Takayasu's disease. Therefore, patient's clinical exam and trials are critically important to a proper diagnostic investigation.

\section{Consent for publication}

The authors declare that they have obtained informed written consent from the patient's tutors for publication

\section{P466}

TAKAYASU'S ARTERITIS: EPIDEMIOLOGICAL, CLINICAL AND ANGIOGRAPHIC PROFILE AT DIAGNOSIS FROM A SINGLE CENTER

\section{IN RIO DE JANEIRO}

Matheus Vieira Gonçalves', Gustavo Guimarães Moreira Balbi², Camila Souto Oliveira', Manuella Lima Gomes Ochtrop'로 Ana Beatriz Santos Bacchiega'

'DEPARTMENT OF RHEUMATOLOGY, HOSPITAL UNIVERSITÁRIO PEDRO ERNESTO, UNIVERSIDADE DO ESTADO DO RIO DE JANEIRO, RIO DE JANEIRO, BRAZIL, NITERÓI, RJ, Brasil; '2DEPARTMENT OF RHEUMATOLOGY, HOSPITAL UNIVERSITÁRIO, UNIVERSIDADE FEDERAL DE JUIZ DE FORA, JUIZ DE FORA, MINAS GERAIS, BRAZI, JUIZ DE FORA, MINAS GERAIS, Brasil Advances in Rheumatology 2018, 58(Suppl 1):P466
Background: Takayasu's Arteritis (TA) is a rare disease, classified as a large-vessel vasculitis that affects primarily aorta and its major branches. The marjority of patients are females under the age of 40 . The incidence is higher in Asia, and clinical profile differs among different regions of the world. There are few epidemiological data from Brazil to date. The aim of this study is to evaluate epidemiological, clinical and angiographic profile from TA patients from a single center in Rio de Janeiro.

Methods: We included 30 consecutive outpatients that fulfilled the 1990 ACR classification criteria for TA. Clinical, laboratorial and imaging data were collected from medical charts. We analyzed the profile at the beginning of the disease and during a follow-up period.

Results: Thirty patients were analyzed, with 28 females (93\%), mean age at first symptom $28,7+12$ years, mean age at diagnosis $33.9+14.6$ years, with a mean follow-up period of $87.6+59.5$ months. Extremities claudication was present in $83,3 \%$, blood pressure difference in $76,7 \%$, new arterial bruit in $73,3 \%$, and carotidynia in $13,3 \%$. Fever, fatigue and anemia were observed in $20 \%$ of patients, and weight loss was present in $16,7 \%$. Headache and vertigo were observed in $16,7 \%$. Ten percent presented with erythema nodosum. The prevalence of the most frequent cardiovascular comorbidities were dyslipidemia in $70 \%$, hypertension in $53,3 \%$ and obesity in $36,7 \%$, with cerebrovascular and cardiovascular events occurring in $26,7 \%$ and $6,7 \%$, respectively. Frequency of angiographic types at diagnosis were $16.7 \%, 6.7 \%, 10 \%, 3.3 \%$ and $63.3 \%$ for types I, Ila, Illb, IV and V, respectively. We found no type III. Two patients (types I and Illb) changed their angiographic type over time to type $\mathrm{V}$, totaling $70 \%$ of the whole population; the remaining patients maintained their previous angiographic type. There was no difference between type $\mathrm{V}$ patients and other types patients regarding epidemiological, clinical, and angiographic data.

Conclusions: Angiographic type $\mathrm{V}$ was by far the most frequent among patients from our center. Similar results were reported in another Brazilian cohort (Sato et al.) and in other parts of the world, including Japan, China, India, France, Italy and Mexico. Approximately one in four TA patients had cerebrovascular events in our cohort, most of them as an initial symptom, reflecting the severity at the time of diagnosis.

\section{P467}

\section{TAKAYASU'S-LIKE SYPHILITIC AORTITIS: CASE REPORT}

Déborah Lobato Guimarães', Filipe Augusto Carvalho de Paula', Ana Julia Bicalho', Ihan Bruno Lopes Rabelo', Maria Raquel da Costa Pinto', Flávia Patrácia Sena Teixeira Santos ${ }^{1}$, Raul Silva Filho², Leandro Augusto Tanure', Gustavo Gomes Resende', Gilda Aparecida Ferreira ${ }^{3}$

${ }^{1}$ HC UFMG, BELO HORIZONTE, MG, Brasil; ${ }^{2}$ INSTITUTO HERMES PARDINI,

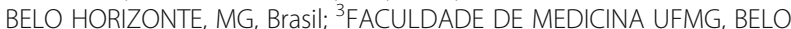

HORIZONTE, MG, Bósnia Herzegovínia

Advances in Rheumatology 2018, 58(Suppl 1):P467

Background: Syphilis is an infectious disease caused by Treponema pallidum with a broad clinical presentation. The aorta is vulnerable to spirochetes and, preferably, the upper segments (aortic root) are reached, being less attacked the thoracic and, very rarely, the abdominal segment. Syphilis may also involve the coronary arteries, resulting in coronary artery narrowing and thrombosis causing a true coronary insufficiency, with ischemic phenomena.

Case report: a 53-year-old woman, previously hospitalized (at 44 years of age) for dyspnea investigation. A coronary lesion was detected in diagonal anterior descending artery undergoing myocardial revascularization. Currently, there were no major risk factors for atherosclerosis. After six years, she evolved with severe supra-aortic stenosis and severe obstructions of coronary and multiple thoracic aortic branches. It was requested rheumatology advice for differential diagnosis in view of the atypical presentation. Patient had not had jaw or limbs claudication nor blood pressure variations between the 
limbs and presented symmetrical pulses. Exhibited low erythrocyte sedimentation rate and low levels of C-reactive protein. VDRL and treponemal test were positive. The patient was treated with benzathine penicillin but also started prednisone $1 \mathrm{mg} / \mathrm{kg}$ due to diagnostic uncertainty, since Takayasu arteritis may present with coronary stenosis in up to $25 \%$ of the cases. It was decided that aortic supravalvular stenosis should be corrected on second time due to the thickening of the vessel wall. Imaging with angiotomography of cervical vessels, thorax and abdomen also generated dubiety and was later assessed by a radiologist with great expertise in Takayasu arteritis. He considered the imaging changes suggestive of syphilis. Indeed, post-surgical aortic valve anatomopathological was consistent with syphilitic aortitis. The patient has died after complications of the surgical procedure.

Conclusion: Syphilitic aortitis represents a chronic, progressive and harmful process. Clinical manifestations typically occur 15 to 30 years from initial infection in the untreated patient. Therefore, in most cases, its diagnosis is challenging and sometimes may even simulates primary vasculitis. The differential diagnosis with atherosclerosis and vasculitis should be considered and there is a need for an expert professional to analyze imaging methods. The prognosis is generally poor, and sudden death may occur.

\section{Consent for publication}

The authors declare that they have obtained informed written consent from the patient's tutors for publication

\section{P468}

TEMPOROMANDIBULAR JOINT (TMJ) INVOLVEMENT AND QUALITY OF LIFE IN JUVENIL IDIOPATHIC ARTHRITIS (JIA)

Jaqueline Serra Martins', Andre Luiz Ferreira Costa², Sérgio Luiz Marques dos Santos ${ }^{1}$, Fernanda Cardoso Santos ${ }^{1}$, Roberto Marini ${ }^{1}$, Simone

Appenzeller ${ }^{1}$

${ }^{1}$ UNICAMP, SP, SP, Brasil; ${ }^{2}$ UNICID, CAMPINAS, SP, Brasil

Advances in Rheumatology 2018, 58(Suppl 1):P468

Objective: To investigate the types of temporomandibular joint (TMJ) involvement in patients with juvenile idiopathic arthritis (JIA) and to determine its relationship to quality of life.

Methods: A total of $29 \mathrm{JIA}$ patients ( 23 female and 6 male, median age: min-max, 14: 7-21) were selected, paired with a control group (7 females and 8 males, median 12: 8-19) and evaluated for signs and symptoms of temporomandibular dysfunction (TMD). All individuals performed a 1.5T MRI of bilateral TMJ that were analyzed according to Omeract consensus for JIA MRI). In addition, all individuals were screened for mood disorders with Beck's depression and anxiety inventory (BAI, BDI), quality of life with SMILEY questionaries. A trained rheumatologists evaluated articular and extra-articular damge through JADI-A and JADI-E. The chi-square test and Fisher's exact test were used for the categorical variables, and the Mann-Whitney test was used to evaluate the numerical variables. The significance level adopted for the study was $5 \%$ ( $P$ significant $\leq 0,05$ ).

Results: Fourteen (46\%) JIA patients and $3(20 \%)$ controls $(p=0.05)$ had inflammatory features and $26(89 \%) \mathrm{JIA}$ and $8(53 \%)$ controls $(\mathrm{p}=0.01)$ had damage feature either uni or bilaterally. There parafunctional habits were more frequently observed in JIA when compared to controls $(p=0.0033)$, and associated with headache $(p=0.0017)$ and joint noise $(p=0.0001)$. Effusion was more frequently observed in polyarticular subtype $(p=0.0084)$. However, no significant correlation was found between TMJ involvement and quality of life scores ( $P>0.05)$.

Conclusion: Patients with degenerative TMJ changes did not present a decline in quality of life, since they adapted to the structural conformation of the condyle and their limitations.

\section{P469}

TENOSYNOVITIS BY MYCOBACTERIUM AVIUM IN THE PATIENT ON ANTI-TNF-ALPHA USE

Renan de Souza Santana Soares ${ }^{1}$, Yuka Nakamura², Vinicius Costa Vieira ${ }^{2}$ Lara Milleri Francischetto ${ }^{2}$, Felicia Maria da Rocha ${ }^{2}$

${ }^{1}$ HOSPITAL FEDERAL DOS SERVIDORES DO ESTADO, RIO DE JANEIRO, RJ, Brasil; ${ }^{2}$ HOSPITAL FEDERAL SERVIDORES DO ESTADO, RIO DE JANEIRO,

RIO DE JANEIRO, Brasil

Advances in Rheumatology 2018, 58(Suppl 1):P469

Background: Tenosynovitis due to Mycobcaterium avium is rare, with the germ being ubiquitous in the middle. It should be considered in immunocompromised patients after peri or intraarticular infiltration. Case report: Male, white, 60 years old with diagnosis of axial and radiographic spondyloarthritis in 2013. In use of adalimumab and methotrexate since diagnosis. Persistent dactylitis for three years on the third right finger. Infiltrated with cortiocoide for three consecutive years due to annual recurrences. After five months of the last infiltration he developed signs of local phlogose. Suspense immunosuppression and treated with empirical antibiotic therapy without improvement. Magnetic resonance imaging (MRI) of the right hand with signs of soft tissue and bone edema. Surgical approach and debridement on the eighth day of antibiotics. Material sent to the histopathological was inconclusive. Research for fungi and common germs was negative. BAAR positive and geneXpert negative for tuberculosis. Treated for atypical mycobacteriosis with coverage of $80 \%$ of the different types. After 45 days there was growth of Mycobacterium avium sensitive to the previous regimen with significant improvement after two months of treatment, which was maintained for 1 year.

Conclusion: In cases in which an infectious process during the use of anti-TNF, low virulence germs should be considered as mycobacteria. Obtained written informed consent with patient for publication of this study

Consent for publication

The authors declare that they have obtained informed written consent from the patient's tutors for publication

\section{P470}

\section{TERAPIA ANTI-TNF EM PACIENTES HIV}

Lucas Merfa E Silva, Themis Mizerkowski Torres

PINDAMONHANGABA, SP, Brasil

Advances in Rheumatology 2018, 58(Suppl 1):P470

The management of patients with autoimmune disease and concomitant HIV infection requiring anti-TNF therapy is a paradigm in medicine. Although the role of TNF and its receptors in the inflammatory response and pathogenesis of HIV infection has been the subject of numerous studies, such topics are still not fully understood. The use of agents that block TNF represents a major advance in the treatment of several rheumatologic diseases, but may present high risk for opportunistic infections due to its possibly immunosuppressive effect. If added to the infectious risk of a patient with AIDS, anti-TNF therapy may theoretically be catastrophic. In this work, a review of the literature was made in search of papers that provide scientific data to support the indication of anti-TNF for HIV-infected patients. We analyzed 33 case reports of patients with autoimmune diseases and HIV infection and their outcomes. Opportunistic infections occurred in only 4 of the cases, and death from the infectious condition occurred in only one of them, with CD4 + of 20. Of the followed patients, only 7 were not using antiretroviral therapy at the 
start of anti-TNF and 5 of them did not treat for HIV. Two patients presented worsening viral load and CD4 + counts, having improved after an exchange of the antiretroviral theraty regimen. Data analysis suggests that anti-TNF therapy in HIV-controlled and antiretroviral therapy patients may be safe. The lack of randomized clinical trials or other study models of greater statistical significance limits the conclusions of this study, requiring more significant work in the area.

\section{P471}

THALAMIC ATROPHY AND MOOD CHANGES IN CHILDHOOD-ONSET SYSTEMIC LUPUS ERYTHEMATOUS (CSLE)

Wolfgang Thyerre Pinto, Cátia Maria Geralda dos Santos Nascimento,

Aline Tamires Lapa, Paula Teixeira Fernandes, Fernando Cendes, Roberto Marini, leticia Rittner, Simone Appenzeller

UNICAMP, SP, SP, Brasil

Advances in Rheumatology 2018, 58(Suppl 1):P471

Background: The thalamus is a neuroanatomic structure that performs many connections with the cerebral cortex and limbic structures. It is associated with sensorial, motor, emotional and motivational processes. In CSLE, the casualty and the diversity of neuropsychiatric symptoms are not fully established. The objective of this work is to evaluate the existence of structural changes in thalamus and its relation to depression, anxiety, and cognitive impairment in CSLE.

Methods: In this cross-sectional study, a total of 72 patients were selected with diagnostic of cSLE. The control group was composed of 27 healthy people, age and sex matched. All patients and the control group were submitted to magnetic resonance imaging. The thalamus was segmented using software "Freesurfer". Atrophy was defined when $z$-score $\leq-2$ standard deviations of the mean obtained from structures of the control individuals. Mood disorders were evaluated through Beck depression and anxiety index. The cognitive impairment was evaluated using Montreal Cognitive Assessment. Medical records were reviewed for clinical, immunological and treatment related variables. Statistical analyzes were performed according to the nature of the variables with $p \leq 0.05$ statistically significant.

Results and Conclusions: The thalamic volume was significantly smaller in cSLE than in controls $(p \leq 0,001)$. Thalamic atrophy was observed in $18(25 \%)$ and $1(3.7 \%)(p<0.05)$. Thalamic atrophy was associated with anxiety $(p=0.047)$ and history of seizures $(p=0.002)$. A correlation between thalamic volume and cognitive evaluation scores was observed $(p=0.007 ; r=0.364)$. No association between thalamic volumes and autoantibodies and corticosteroid use was observed. We concluded that reduction in thalamic volume may contribute to neuropsychiatric manifestation, requiring further investigations.

\section{P472}

THE CHALLENGE OF DIFFERENTIAL DIAGNOSIS IN LIFETHREATENING ADMISSIONS OF SYSTEMIC LUPUS ERYTHEMATOSUS PATIENTS IN THE PEDIATRIC INTENSIVE CARE UNIT OF A TERTIARY HOSPITAL AT RIO DE JANEIRO

Rodrigo Moulin Silva, Flavio Roberto Sztajnbok, Raquel de Seixas Zeitel, Rosana de Andrade Flintz, Bernardo Considera Vogas, Pedro Henrique Nunes Costa Silami, Bruno Bohme HOSPITAL UNIVERSITÁRIO PEDRO ERNESTO, RIO DE JANEIRO, RJ, Brasil Advances in Rheumatology 2018, 58(Suppl 1):P472

The objective of this study was to assess causes, clinical presentations, laboratory findings, organ dysfunctions, prognosis and outcomes of critically ill systemic lupus erythematosus (SLE) patients requiring pediatric intensive care unit (PICU) management, given the limited literature data on this particular group.

Twelve SLE patients amounting 18 total admissions were identified in this retrospective case series over a seven-year period (from January 2010 to June 2017). All patients fullfilled diagnostic criteria for lupus. We aim to discuss the differential diagnosis of sepsis, disease activity and disease-related complications.

The majority of our patients were female, within the first year of disease. Four patients were admitted to the PICU without a previously established SLE diagnosis. Disease flares and disease-related complications (eg. macrophage activation syndrome) were the most common causes for admission
(61\%), with the mean disease activity score using SLEDAI2K consistent with severe activity (27.7). Immunosupression treatment during PICU stay was used in $62,5 \%$ of the admissions. During PICU stay, all of them had at least one organ dysfunction, most frequently respiratory (55\%) and neurologic (38\%). The most frequent PICU interventions were invasive mechanical ventilation ( $81 \%)$, vasoactive amines $(66 \%)$ and hemodialysis $(37,5 \%)$. Overall mortality rate during PICU stay was $27 \%$, which was higher than the predicted deaths by Pediatric Risk of Mortality (PRISM) III (8,0\%).

Our findings suggest that our group of SLE patients presented with severe disease activity from the beginning as well as the need of aggressive immunosupression therapy in the PICU. Also, in the context of organ dysfunction, being able to distinguish between infection and disease activity remains a challenge.

\section{P473}

THE DANCING GIRL - ONE CASE OF CHOREA IN A PATIENT WITH JUVENILE SYSTEMIC LUPUS ERYTHEMATOUS

Guilherme Leví Tres, Juliana Beirao De Almeida Guaragna, Sara Kvitko de Moura, Nicole Pamplona Bueno De Andrade, Lygia Ohlweiler, Sandra Helena Machado

HOSPITAL DE CLÍNICAS DE PORTO ALEGRE, PORTO ALEGRE, RS, Brasil Advances in Rheumatology 2018, 58(Suppl 1):P473

Background: Chorea, from the Latin choreus, means "dance", is characterized by involuntary, brief, random and irregular movements. Chorea is an uncommon presentation in systemic lupus erythematous (SLE) occurring in about $4 \%$ of pediatric patients.

Case Report: We report a case of an eleven years old girl that came into de emergency room with involuntary limb movements. She had been diagnosed with SLE about two years before with the predominance of articular and skin involvement. She was medicated with methotrexate $20 \mathrm{mg}$ subcutaneous, folic acid, hydroxychloroquine $5 \mathrm{mg} / \mathrm{kg}$, prednisone $5 \mathrm{mg}$, calcium carbonate and vitamin D supplementation. At first evaluation, she reported difficulty holding objects with her right hand for two days that evolved to involuntary movements first of the fingers, progressively worsening until involving the entire upper limb and right lower limb on the day before. The movements did not occur when she was in deep sleep, were more intense in the morning and worse with anxiety. She denied and did not presented signs of fever or infection and had no history of taking any new drugs. Her family history was unremarkable for genetic diseases. On physical examination she was in good general condition, normal vital signs, active, with hemichorea on the right body and without other neurological disfunctions. Workup showed signs of a serological active SLE, with reduction of complement and high title antiDNAds antibodies. We performed magnetic resonance imaging of the brain showing only hyperintensity signal foci in the deep white matter of the frontal lobes on T2/FLAIR. Lumbar puncture and other laboratory test were performed to rule out other causes of chorea. The spinal fluid analyses were unremarkable and none other laboratory abnormalities were found, with negative ASLO and negative antiphospholipid antibodies. She received a pulse of methylprednisolone 1 gram per day for 3 days and showed improvement of the movements after the second dose of methylprednisolone. Later she was initiated in azathioprine $2 \mathrm{mg} / \mathrm{kg} / \mathrm{day}$ and high dose prednisone that dose was progressively tapered.

Conclusion: Chorea is rare in juvenile SLE, but it is a well-recognized manifestation, that is often associated with other neuropsychiatric manifestations and requires recognition and appropriate treatment by the rheumatologist.

\section{Consent for publication}

The authors declare that they have obtained informed written consent from the patient's tutors for publication

\section{P474}

THE IMPACT OF FEED IN THE EVOLUTION OF SYMPTOMS PRESENTED IN FIBROMYALGIA PATIENTS

Wallace Dorneles Gomes, Juliana Morini Bevilacqua, Lais Roberta Garcia Bernardini, Luis Henrique Savoi de Almeida, José Eduardo Martinez PUC SOROCABA, SP, SP, Brasil

Advances in Rheumatology 2018, 58(Suppl 1):P474 
Background: this research seeks to relate fibromyalgia and nutritional factors. Objective: to determine the influence of the food habits on the intensity of the symptoms of fibromyalgia patients.

Methods: we interviewed 40 female patients, aged 15 to 59 , in the pain outpatient clinic of Sorocaba Hospital Complex (CHS) through about the fibromyalgia clinical picture and their eating habits.

Results: the mean duration of symptoms of fibromyalgia among the interviewed patients was 6.75 years. The average impact of fibromyalgia on the quality of life was about 64 measured by the Fibromyalgia Impact Questionnaire. The only food that was related to symptom improvement by the patients was the banana (17.5\%). The majority of respondents did not noticed relationship between such foods and modification of disease symptoms.

Conclusion: there was not observed association between food intake and fibromyalgia symptom severity.

\section{P475}

THE MANAGEMENT OF REFRACTORY BLUE TOE SYNDROME AS A MANIFESTATION: A SUSPECTED CASE OF NON-SMOKING THROMBOANGIITIS OBLITERANS

Surian Clarisse da Costa Rocha Ribeiro', Samara da Silva Gavinier², Lila Morena Bueno da Silva ${ }^{2}$, Dilson Marreiros Nunes Filho ${ }^{2}$, Taysa Cristiane Moreira da Silva ${ }^{2}$, Eduardo Borba Ferreira Neto ${ }^{2}$, Lissiane Karine Noronha Guedes$^{2}$, Michelle Remião Ugolini Lopes², Rosa Maria Rodrigues Pereira ${ }^{2}$ 'HCFMUSP, SÃO PAULO, SP, Brasil; ${ }^{1}$ DISCIPLINA DE REUMATOLOGIA, HOSPITAL DAS CLINICAS DA FACULDADE DE MEDICINA DA UNIVERSIDADE DE SÃO PAULO (FMUSP), SÃO PAULO, SP, Brasil Advances in Rheumatology 2018, 58(Suppl 1):P475

Background: Blue toe syndrome is a small vessel disease, characterized by development of painful and blue discolouration in one or more toes. The most common etiology is atheroembolic disease; however, it can also appear in hypercoagulability disorders, vasculitis or autoimmune diseases. Thromboangiitis obliterans (TAO) is a vascular inflammatory thrombotic and segmental disease affecting distal small and medium-sized arteries of the limbs. Less than $5 \%$ of TAO patients are non-smokers. The authors describe a case of a young non-smoker woman who presented with blue toe syndrome without underlying atheroembolic disease. Treatment using high-potency vasodilators, anticoagulation and hyperbaric oxygen, based on thromboangiitis obliterans pathophysiology, allowed regression of purplish appearance of the toes and fingers.

Case report: A 32-years old woman presented with a one-year history of pain and blue appearance of both feet and purple discoloration of fourth and fifth left fingers. She has never smoked. The skin lesions became worse after cold exposition. Laboratory tests, including blood count, biochemistry, protein electrophoresis, tests for human immunodeficiency virus (HIV), hepatitis C virus, and hepatitis B virus, and hypercoagulability study were normal or negative. Urinalysis shows hematuria and kidney biopsy revealed poststreptococcalglomerulonephritis. Antinuclear antibodies, extractable nuclear antigen antibodies, cryoglobulins, anti-phospholipid and lupus anticoagulant antibodies were also negative. A possible source of systemic embolism was excluded by transthoracic echocardiogram and carotid-vertebral doppler. The angiography (abdominal and lower limbs) was normal. The first toe's skin biopsy shows superficial perivascular lymphomononuclear infiltrate in the dermis with neutrophils. Even with glucocorticoid and cyclophosphamide pulse-therapy, rituximab, amlodipine, AAS, cilostazol, sildenafil and analgesic therapy her blue toes had worsened in the last 4 months and bilateral second and third toes and fifth right toe became necrotic. The new toe's skin biopsy shows a thrombosing vasculopathy. Anticoagulation with enoxaparin was begun, bosentan and hyperbaric oxygen were added. Finally, the thrombotic injury was controlled.

Conclusion: The knowledge of disease's pathology contributes to exploring several alternative treatments for refractory cases. That is a case of Thromboangiitis obliterans and its pathology consists of thrombosis, angiitis, vascular inflammation, obliteration, and vasospasm. There is some evidence for the use of bosentan, iloprost, sildenafil, glucocorticoid and cyclophosphamide pulse-therapy and hyperbaric oxygen, but more randomized studies with blinded outcomes are necessary. In this case the addition of anticoagulation, bosentan and hyperbaric oxygen made the difference for the treatment.

\section{Consent for publication}

The authors declare that they have obtained informed written consent from the patient's tutors for publication

\section{P477}

THE PREVALENCE OF LATENT AND ACTIVE TUBERCULOSIS IN PATIENTS UNDERGOING IMMUNOBIOLOGICAL THERAPY WITH TNF INHIBITORS IN A TERTIARY CARE PUBLIC HOSPITAL

Pedro Felipe de Almeida Vianna', Helena de Almeida Tupinambá ${ }^{2}$ Laíssa Cristina Alves Alvino², Manuella Lima Gomes Ochtrop²,

Ana Beatriz Vargas dos Santos ${ }^{2}$

${ }^{1}$ HOSPITAL UNIVERSITÁRIO PEDRO ERNESTO - UERJ, RIO DE JANEIRO, RJ, Brasil; ${ }^{2}$ HOSPITAL UNIVERSITÁRIO PEDRO ERNESTO, RIO DE JANEIRO,

RIO DE JANEIRO, Brasil

Advances in Rheumatology 2018, 58(Suppl 1):P477

Background: Inhibitors of tumor necrosis factor alpha (TNF-a) represent important treatment advances in a number of inflammatory conditions, such as Rheumatoid Arthritis (RA) and Juvenile Idiopathic Arthritis (JIA), including the capacity to change the natural history of these diseases. However, some potential complications have been identified, like an increased risk of mycobacterial infections. Latent Tuberculosis infection (LTBI) has the potential to develop into active infection at any time; hence identification and treatment of LTBI greatly reduces the likelihood of reactivation. Our study aimed to demonstrate the prevalence of LTBI and active tuberculosis in a tertiary care public hospital patient population using TNFa-inhibitors.

Methods: A cross-sectional study was carried out, with data obtained from medical chart review of patients with RA or JIA treated from April 2013 until March 2018 at the Biologic Outpatient Clinic of a tertiary care public hospital in Rio de Janeiro. A total of 146 patients were selected for analysis. LTBI, active disease and Tuberculin Skin Test (TST), either positive or negative were analyzed using simple frequencies.

Results: Before the initiation of immunobiological therapy, $10 \%$ of patients had active Tuberculosis (TB), 15\% of the population had a positive TST, $58 \%$ had a negative TST and $26 \%$ did not have these data in the records. Among the patients with a past history of active TB, there was a median interval of 132 months between the infection and start using the TNF-inhibitor. $22,6 \%$ of all patients were treated for LTBI, with one patient of this group evolving to active TB. Another two patients without LTBI treatment presented active TB. After the initiation of immunobiological, only three patients presented active TB, from a group of 146 patients. Discussion: The use of immunobiological medications may be associated with an increased risk of mycobacterial infection. Our study, however, evidenced a low prevalence for this type of infection after initiation of immunobiological therapy. Only $2 \%$ of patients developed active TB, after using certolizumab (2 patients) 
and adalimumab (1 patient), reinforcing data from the literature, which showed $<5 \%$ of disease manifestation with this therapy. Conclusions: Immunological therapy represents a breakthrough in the treatment of diseases such as RA and JIA. Even with the risks of developing mycobacterial infection, this therapy is safe, since there is effort to identify $L T B I$, reducing the risk of active disease.

\section{P480}

THROMBOTIC THROMBOCYTOPENIC PURPURA ASSOCIATED WITH SYSTEMIC LUPUS ERYTHEMATOSUS: CASE REPORT

Fernanda Armond Castro, Leila Patrícia Muniz, Gustavo Lamego de Barros Costa, Thais Carvalho de Sousa, Letícia Stivanin da Cunha Alves, Lara Maria Arantes Campos, Rafael Prado Colares, Ana Flávia Madureira de Paula Dias, Eduardo José do Rosário e Souza, Ana Carolina Duarte Couto, Paulo Madureira de Pádua

SANTA CASA BH, BELO HORIZONTE, MINAS GERAIS, Brasil

Advances in Rheumatology 2018, 58(Suppl 1):P480

Background: Thrombotic thrombocytopenic purpura (TTP) is rare and threatening syndrome that requires prompt management. It is characterized by fever, microangiopathic hemolytic anemia, severe thrombocytopenia and organ ischemia linked to disseminated microvascular platelet rich-thrombi (specially renal and neurological alterations). TTP may be idiopathic or secondary to malignant hypertension, drugs (cyclosporine, quinine, clopidogrel, ticlopidine and chemotherapy), bone marrow or solid organ transplantation or autoimmune diseases such as systemic lupus erythematosus (SLE) and rheumatoid arthritis. TTP and SLE rarely occur simultaneously and generally this association imposes a difficult diagnosis. The finding of fragmented red blood cells or schizocytes in a peripheral blood smear indicates microangiopathic hemolytic anemia and, when associated with severe thrombocytopenia, it's strongly suggestive of TTP. This condition is also related to a severe deficiency in ADAMTS13 (a specific von Willebrand factor cleaving protease) and its activity less than $10 \%$ is specific for TTP. ADAMTS13 deficiency is most frequently acquired via ADAMTS13 autoantibodies.

Case report: RSS, 47 years old, female, started with oral ulcers, inflammatory arthralgia, leukopenia and lymphopenia. Laboratory tests showed positive antinuclear antibodies (fine speckled pattern with titer 1: 640), low C3: 40mg/dL (reference: $90-170 \mathrm{mg} / \mathrm{dL}$ ), normal C4: $30 \mathrm{mg} / \mathrm{dL}$ (reference: $12-36 \mathrm{mg} / \mathrm{dL}$ ) and anti-dsDNA positive. Concomitantly, the patient presented neurological involvement with right hemiparesis, headache and mental confusion, without alterations to cranial computed tomography. She also presented with hemolytic anemia, Hb: $4.3 \mathrm{~g} / \mathrm{dL}$, LDH: 1.800U/L (normal: 135-214U/L), mild renal impairment (creatinine: $1.4 \mathrm{mg} / \mathrm{dL}$ with baseline creatinine: $0.8 \mathrm{mg} / \mathrm{dL}$ ) and thrombocytopenia of 18.000/L. In view of the clinical context, antibody dosage and consumption of complements, the diagnosis of SLE was established. The activity level of ADAMTS-13 was measured and showed low activity (5\%), confirming the rare association of SLE and TTP. The patient was submitted to five plasmapheresis sessions, with an unsatisfactory response, and Rituximab was initiated, with improvement after four weekly doses of $500 \mathrm{mg}$. She was discharged with prednisone, orally $60 \mathrm{mg}$ a day in one take and Hydroxychloroquine $400 \mathrm{mg}$ a day. The patient remained stable, in an outpatient setting, with difficult weaning from prednisone.

Conclusion: The occurrence of TTP in patients with SLE can be difficult to diagnose due to the overlap of characteristics of the two entities. It is known that the combination of TTP and SLE has an unfavorable prognosis compared to SLE or idiopathic TTP alone. Therefore, an accurate diagnosis and an immediate treatment are necessary, leading to an aggressive management with plasmapheresis and immunosuppression.
Consent for publication

The authors declare that they have obtained informed written consent from the patient's tutors for publication

\section{P482}

TOXIC EPIDERMAL NECROLYSIS TRIGGERED BY THE USE OF HYDROXYCHLORQUINE IN A SYSTEMIC LUPUS ERYTHEMATOSUS PATIENT

Michelle Tenório de Andrade ${ }^{1}$, Igor Henrique Balandino Silva², Luiza Villar de Andrade e Silva², Laís Soares Almada², Fernanda Gomes Gonçalves Chaer ${ }^{2}$, Dawton Yukito Torigoe ${ }^{2}$, Rogerio Castro Reis ${ }^{2}$

${ }^{1}$ IRMANDADE SANTA CASA DE MISERICÓRDIA DE SÃO PAULO, SÃO

PAULO, SP, Brasil; ${ }^{2}$ RMAANDADE SANTA CASA DE MISERICORDIA DE SÃO

PAULO, SAO PAULO, SP, Brasil

Advances in Rheumatology 2018, 58(Suppl 1):P482

Introduction: The antimalarial agents chloroquine (CQ) and hydroxychloroquine (HCQ) are considered effective in the treatment of cutaneous lupus erythematosus (CEL) and symptoms associated with systemic lupus erythematosus (SLE). The main activities of antimalarials involve inhibition of Toll-like receptor activation, resulting in the negative regulation of interferon expression and decreased inflammation. Stevens-Johnson syndrome (SJS) and toxic epidermal necrolysis (TEN) are two of the main idiosyncratic reactions, mainly induced by drugs and characterized by fever and mucocutaneous lesions. Some cases of toxic epidermal necrolysis/Stevens-Johnson Syndrome have been reported following the use of antimalarials, especially HCQ.

Case report: ASS, 45 years old, female, evolving for 7 months with erythematous body lesions, thrombocytopenia, leucopenia and arthritis in the hands and knees, alopecia and weight loss.

The requested exams revealed fine speckled pattern ANA 1/1280, anti-RO and anti-LA reagents, urine 1 with protein $1+$, leucocytes 1390 , platelets 106000 , hemoglobin 12.9 .

Once the diagnosis of systemic lupus erythematosus was concluded, Hydroxychloroquine $400 \mathrm{mg} / \mathrm{day}$ and prednisone $20 \mathrm{mg}$ were introduced. Three days later, she presented macules and papules on the trunk and arms that coalesced into plaques. $\mathrm{Pa}$ tient denied use of medications other than those prescribed.

Dermatological examination revealed disseminated dermatosis, characterized by papules and erythematous-violet plaques with target appearance, many topped by blisters, several confluents, oral and anal mucosa lesions and broken blisters on the shoulders and gluteal region.

Biopsy revealed Erythema multiforme of keratocytes, superficial perivascular dermatitis of lichenoid interface with necrosis, compatible with Stevens-Johnson syndrome. HCQ was suspended and cyclosporine $125 \mathrm{mg} /$ day was introduced, with improvement of the lesions after 1 month of treatment.

Conclusion: TEN is a serious illness that can lead to death. There is extensive involvement of skin detachment (>30\% of the surface area), characterized by necrosis of total epidermal thickness. Causes include drugs, especially antibiotics, antiepileptics, allopurinol and NSAIDs, as well as infections, more rare causes such as graft versus host disease, SLE and vaccines have been reported. The description of cases triggered by $\mathrm{HCQ}$ is rare. The few cases reported in the literature show a variation between introduction of $\mathrm{HCQ}$ and emergence of NET up to 3 years. There are no reports of its incidence. In a study conducted by Auqier-Dunant et al., 2002, SLE seems to confer a greater risk for the development of druginduced TEN and has been attributed as a cofactor.

Consent for publication

The authors declare that they have obtained informed written consent from the patient's tutors for publication 


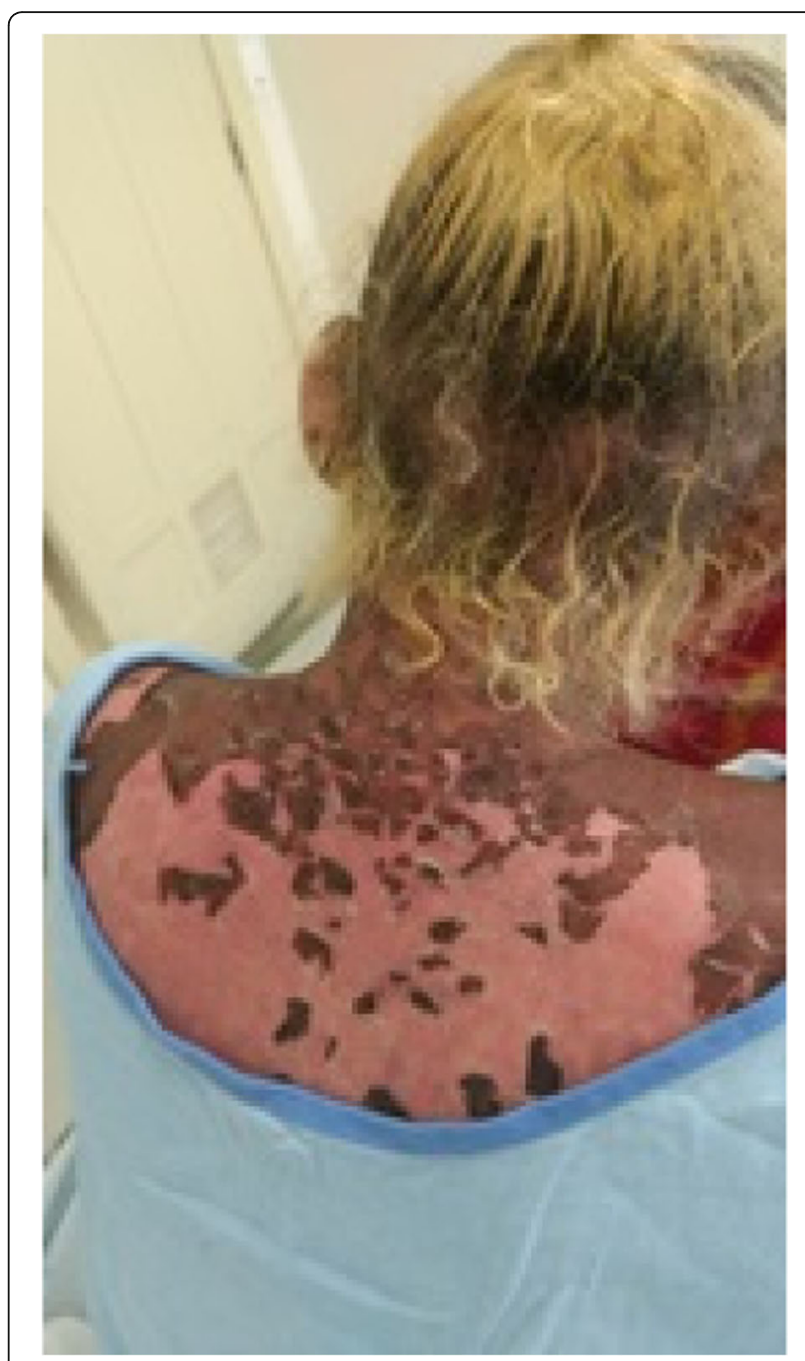

Fig. 1 (abstract P482). Toxic epidermal necrolysis triggered by the use of Hydroxychlorquine in a systemic lupus erythematosus patient

\section{P486 \\ TREATMENT OF STILL'S DISEASE IN A TERTIARY HOSPITAL IN SÃO PAULO: CASE SERIES \\ Bernardo Patricio Sequeira Dultra, Caroline Almeida Oliveira, Lisa Mielke de Oliveira, Matheus Xavier Guimares, Fernanda Pulcheri Ramos, Denise Moraes Horiy, Rina Dalva Neubarth Giorgi, Andrey Tonetto Barbosa HOSPITAL DO SERVIDOR PÚBLICO ESTADUAL DE SAO PAULO, SAO PAULO, SAO PAULO, Brasil \\ Advances in Rheumatology 2018, 58(Suppl 1):P486}

Introduction: Adult Still's disease (AOSD) is an autoinflammatory disorder characterized by fever, arthritis, and skin rash. The treatment aims at controlling symptoms, reducing inflammatory activity, preventing organ damage, and minimizing adverse treatment events. The low prevalence of this disease makes it difficult to carry out clinical trials to study the best therapeutic approach, which in general can be started with NSAIDs, corticoterpics and later, diseasemodifying antirheumatic drugs, synthetic or not. Objective Evaluate the different forms of treatment and therapeutic response of AOSD in a tertiary hospital in the State of São Paulo.

Methods: This retrospective and descriptive study was carried out in 2018 with patients attended at the Rheumatology outpatient clinic of a tertiary hospital in the state of São Paulo.
Results: The study included 07 patients with the diagnosis of AOSD, these $71.4 \%(\mathrm{~N}=5)$ female and $28.6 \%(\mathrm{~N}=2)$ male. Patients had a mean age $57.5 \pm 12$ years, with an average disease duration of $5.6 \pm 7.1$ years. One patient underwent bariatric surgery about 10 years before diagnosis and another two had psoriasis. All patients started treatment with Corticotherapy and Methotrexate, but only $43 \%(n=3)$ presented a good response. Subsequently those who maintained disease activity started the use of anti-IL-6 receptor (Tocilizumabe - TCZ), with good clinical and laboratory response. One of the patients treated with IL-6 inhibitor had an adverse reaction to the medication and was then switched to Adalimumab, evolving with remission of disease.

Conclusion: The initial therapeutic choice of AOSD depends on the presentation of the disease, usually starting with NSAIDs and low doses of corticosteroid in the mildest forms, as was done in our series. In cases where there is no response after 02 months and in patients with moderate to severe involvement, more aggressive strategies are used, usually with high dose corticosteroids and immunobiological use. Because of the scarcity of data and studies with immunobiological treatment in Still's disease, the use of these medicamentions are essentially empirical. The first bDMARDs used were the anti-TNF, which showed benefit. More recently, with the proven efficacy in the literature of the use of IL- 6 and IL-1 antagonists in the systemic form of juvenile idiopathic arthritis, these medications are also being used for still adults, showing a good response, as in the present study, in which all patients achieved disease's remission.

\section{P488}

TUBERCULOSIS RECURRENCE IN A RHEUMATOID ARTHRITIS PATIENT ON ANTI-TNF THERAPY: ENDOGENOUS REACTIVATION OR EXOGENOUS REINFECTION?

Natalia Pereira Machado, Luan Felipe Lückmann, Luiza Bueno Zeni, Maiara Bastiani Bisognin, Ana Beatriz Artigas Guimarães, Chayanne Rossetto, Keoma Azevedo Sabião, Isadora Welter Pioresan, Eduardo Santos Paiva HOSPITAL DE CLÍNICAS - UFPR, CURITIBA, PARANÁ, Brasil Advances in Rheumatology 2018, 58(Suppl 1):P488

Backgound: The anti-TNF therapy in rheumatoid arthritis (RA) has shown satisfactory clinical outcomes, but its use implies in enhancing the risk of producing multiple infections, especially tuberculosis (TB). TB reactivation leads to clinical active infection and tends to occur early following initiation of TNF-alpha blocker. There is a 4.7-fold increase of TB reactivation risk during anti-TNF therapy as compared to placebo has been shown and this risk is increased to 13-fold when using anti-TNF therapy in combination with metothrexate or azathioprine as compared with monotherapy.

Case report: We report a 46 years old-female patient with rheumatoid arthritis for 12 years with positive rheumatoid factor. Seven years ago, she was gave adalimumab as she had failed oral DMARDS (metothrexate and leflunomide), with remission of disease activity. In that occasion, the tuberculin skin test (TST) was negative. After 2 years on adalimumab, she had active pulmonary TB and completed 6 months-treatment for TB. Afterwards she was given abatacept for her RA treatment, without response and adalimumabe was reintroduced three years ago. After 2 years, she had new diagnosis of pulmonar TB, based on microbiological test and chest computed tomography (CT) (Fig. 1).

Conclusion: Recurrence of tuberculosis (TB) can be due to a regrowth of the same strain of Mycobacterium tuberculosis that caused the previous TB episode, known as relapse, or reinfection through a different strain, diagnosed by molecular tests. The recurrence rate in countries of high TB incidence, like Brazil, is elevated and reinfection seems to be the principal cause. We can conclude in this case that TB reactivation risk should be assessed in all patients, and in case of positive results, non-anti-TNF targeted biologics for RA represent the safest option. It has been suggested that patients on anti-TNF therapy with diagnosis of active TB should receive an extended period of 9 months of TB treatment, and the biological drug should be switched to a more safety one. 


\section{Consent for publication}

The authors declare that they have obtained informed written consent from the patient's tutors for publication

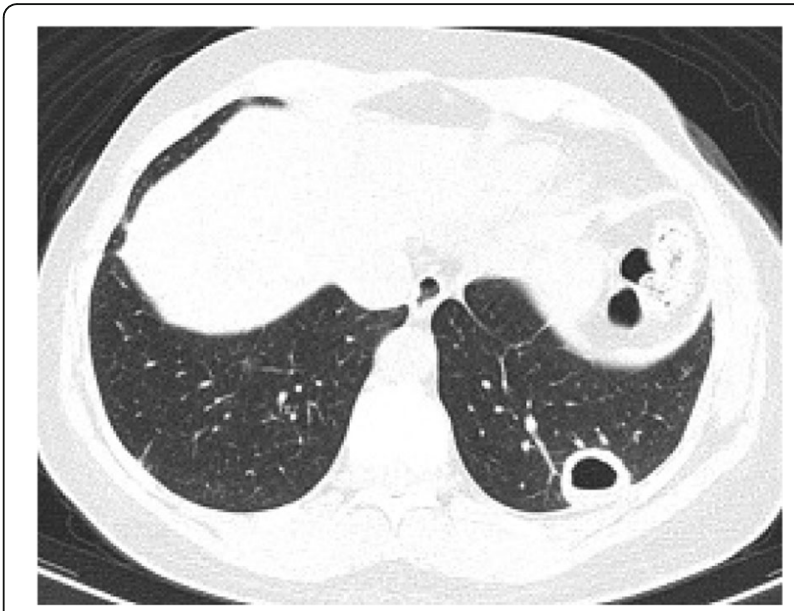

Fig. 1 (abstract P488). Thorax tomography showing cavitated lesion
(4 kg). The symptoms did not abate even with retreatment with colchicine and prednisone. A new chest radiograph at this time showed indeterminate enlargement in the right pulmonary hilum. To assess the possibilities of differential diagnosis including large vessel vasculitis (e.g., pulmonary artery aneurysm), lymphoproliferative disorder, and infectious disease, a computed tomography (CT) of the chest with intravenous contrast material was performed. The CT scan showed mediastinal and hilar lymphadenopathy with extrinsic compression of the right main bronchus. A lymph node biopsy revealed granulomatous lymphadenitis with extensive areas of necrosis and no signs of malignancy. Ziehl-Neelsen staining was positive for acidfast bacilli, and culture of lymph node tissue was positive for Mycobacterium tuberculosis, leading to the diagnosis of tuberculous lymphadenopathy. Antimycobacterial treatment with rifampicin, isoniazid, pyrazinamide, and ethambutol was prescribed for 2 months, followed by consolidation therapy with rifampicin and isoniazid for 7 months. She evolved with remarkable clinical and radiological improvement in the following months. Regarding Behcet's disease, she is currently in remission with colchicine $2 \mathrm{mg} /$ day and prednisone 5 $\mathrm{mg} /$ day.

Conclusion: This case illustrates the importance of evaluate recalcitrant BD patients for infections, including tuberculosis. A complete work-up study for mycobacterial infection may be worth in patients with frequent exacerbations despite appropriate therapy.

Consent for publication

The authors declare that they have obtained informed written consent from the patient's tutors for publication

P492

TWIN PREGNANCY IN PATIENTS WITH TAKAYASU ARTERITIS: A CASE REPORT

Samuel de Oliveira Andrade ${ }^{1,2}$, Jacqueline Foelkel Pignatari², Gabriela Miyuki Teodoro Ogawa², Pamella de Paula Bellini²,

Lara Ribeiro Teixeira Bonfim², Mateus De Miranda Moura Cortês²,

Allison Aliel Vigano Pugliesi ${ }^{2}$, Zoraida Sachetto ${ }^{3}$

'UNIVERSIDADE ESTADUAL DE CAMPINAS (UNICAMP) UNIVERSIDADE

ESTADUAL DE CAMPINAS (UNICAMP), CAMPINAS, SP, Brasil;

${ }^{2}$ UNIVERSIDADE ESTADUAL DE CAMPINAS (UNICAMP), CAMPINAS, SP, Brasil; ${ }^{3}$ UNIVERSIDA ESTADUAL DE CAMPINAS - UNICAMP, CAMPINAS, SP, Brasil

Advances in Rheumatology 2018, 58(Suppl 1):P492

Background: Takayasu arteritis (TA) is a vasculitis of indeterminate origin that affects large vessels, predominantly aorta and its branches. As the disease affects young women, gestation during treatment is not uncommon. During pregnancy, there may be a worsening of the disease and of preexisting arterial lesions, leading to massive hemoptysis and eclampsia in more severe cases. Twin pregnancy in this context is a high risk.

Case report: A patient diagnosed with TA with infrarenal aorta, subclavian and right common artery lesions, using infliximab since 2009 (previous diagnosis of Crohn's disease) and methotrexate. The patient refused to use corticosteroids. Methotrexate was discontinued and, after six months, pregnancy was allowed. Infliximab was maintained until the third trimester of gestation. The patient progressed well, without obstetric or clinical complications. After the end of breastfeeding, we chose to resume the medication.

Conclusion: Takayasu arteritis is the most common vasculitis during pregnancy. It is well established in the literature that gestation in patients with TA is of high risk, with the largest study in this topic demonstrating that complications may increase tenfold mainly due to worsening of arterial hypertension and consequent preeclampsia.[1] The preferred route of delivery is the vaginal route.[2] The oscillation of blood pressure during delivery in patients with TA is higher than the mean, being this the main cause of maternal mortality. Although treatment with corticosteroids and 
anti-TNF does not prevent obstetric complications, there is a tendency of lower incidence with the use of these medications. No reports of twin pregnancy have been found in this context in the literature.

\section{References}

1. C. Comarmond et al; Takayasu Arteritis and Pregnancy; Arthritis \& Rheumatology Vol. 67, No. 12, December 2015, pp 3262-3269

2. Hidaka N. et al; Clinical manifestations of pregnancy in patients with Takayasu arteritis: experience from a single tertiary center Arch Gynecol Obstet (2012) 285:377-385

\section{P495}

ULTRASOUND AS AN IMPORTANT DIAGNOSTIC AND EVALUATION TOOL OF THE OS ACROMIALE

Rubia Carla Cappellari Tolentino, Leonardo Michaelis Schmidt, Diogo

Cunha Lacerda, Polyana Klomfass Piati, Fernando Henrique Cappellari

Tolentino, Leandro Silva Pivato

CENTRO UNIVERSITÁRIO FAG, CASCAVEL, PR, Brasil

Advances in Rheumatology 2018, 58(Suppl 1):P495

Background: The os acromiale is a congenital defect in the mature skeleton resulting from the lack of fusion of the centers of ossification of the acromion between each other or of the spine of the scapula, often being the site of degenerative changes that imply the pathology of some painful shoulders and rotator cuff tendon lesion. Os acromiale is a relatively common anatomic variation in which the acromial apophysis fails to completely fuse with the scapula. Prevalence ranges from 1.3 to $18.2 \%$ in the general population, reaching bilaterally 33 to $62 \%$. Although typically regarded as an incidental finding, os acromiale may produce symptoms arising from the hypermobile nonunion site itself or as a result of downward impingement on the underlying rotator cuff. Identifying symptomatic os acromiale can be clinically challenging when a patient has shoulder pain, resulting in delayed diagnosis.

Report: We describe some cases of os acromiale diagnosed by ultrasonography. We also evaluated dynamically the presence of mobility between the os acromiale and the acromial by the arm flexion.

Conclusion: Ultrasonography is an important method of evaluation of musculoskeletal structures. The evaluation of the acromion and the presence of the os acromiale is very important in the routine exam of the shoulder by ultrasonography, because we can easily detect possible fusion fails in the acromial epiphysis, as well as perform dynamic maneuvers during the evaluation, facilitating the diagnosis of os acromiale, that may be associated with shoulder pain and rotator cuff diseases. In addition, ultrasound also can be used to safely guide infiltrations on acromion

\section{P500}

\section{VALUE OF HOSPITAL SERVICES WITH INTERVENTION FOR} SYSTEMIC DISEASES OF THE CONJUNCTIVE TISSUE IN THE CITIES OF SERGIPE

Henrique Gouveia Borba E Souza, Bruno José Santos Lima, Angela Santos Lima, Larissa sá dos Santos, Juliana Monroy Leite, Nicole Santiago Leite, Meyling Belchior de sá Menezes, Luíza Brito Nogueira, Yasmin Oliveira Santos, João Victor de Andrade Carvalho, Denison Santos Silva UNIVERSIDADE TIRADENTES, ARACAJU, SE, Brasil

Advances in Rheumatology 2018, 58(Suppl 1):P500

Background: Systemic diseases of the connective tissue refer primarily to autoimmune or collagen conditions with generalized organism involvement, such as systemic lupus erythematosus (SLE). The management of those diseases by means of treatments and hospital care demand expenditure and an organized Primary Health Care. Thus, for example, to attend 15 millions of people affected by rheumatism, Brazil made available, in 2011, 3, 84\% of the Gross Domestic Product (GDP), less investment than european countries. That's why to evaluate and compare the costs of inpatient care for patients with systemic connective tissue diseases in states as Sergipe is so relevant.
Methods: This is a retrospective observational study performed through the analysis of all hospital admissions occurred in SUS in cities of Sergipe from November 2008 to November 2017. Data were obtained from the hospital information system (SIH/SUS) by the value in hospital services and understood together with the population estimate of each city by the Brazilian Institute of Geography and Statistics (IBGE).

Results and conclusions: The total cost of Sergipe in hospital services with hospitalizations for systemic connective tissue diseases was $\mathrm{R} \$ 220,762.50$. Of these, $98 \%$ are only from the capital Aracaju, which is the most populous city in Sergipe. Although it occupies the sixth demographic position, Estância is followed with $0.6 \%$ of the costs. The same concession occurs with Nossa Senhora do Socorro, which despite being the second most populous city, obtained the most economic position next to Riachuelo, both with values of $R \$ 33,34$. Some other cities, such as Itabaiana, Lagarto, Neópolis, Nossa Senhora da Glória and Propriá, share an average of $0.2 \%$ of the total value. The detailed value of them, excluding the capital Aracaju, is shown in the Fig. 1. Through the analyzed values, it can be concluded that the disparity between the amount used by the small cities and the capital is due, in addition to the demographic values, to the concentrations of more complex levels of health in the most populous places and the high rates of migration to the capital in search of better services. In this context, in order to reduce the impacts on population health and to achieve economic paths to the Unified Health System (SUS), the Basic Care still needs to make important preventive measures and therapeutic follow-ups to rheumatic diseases.

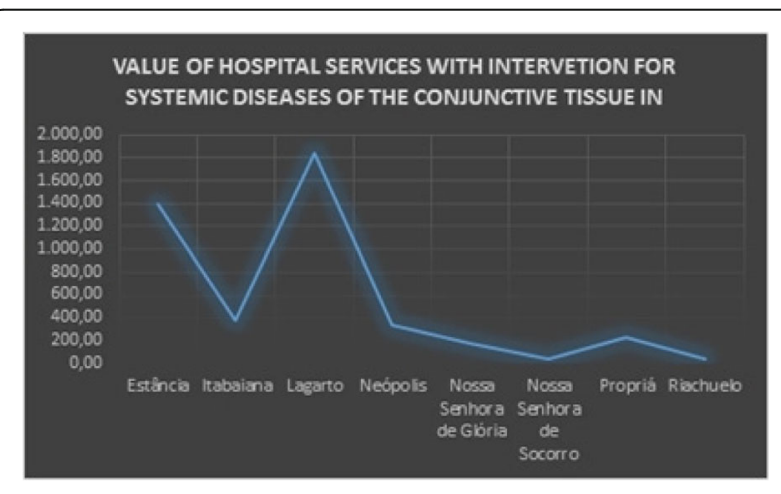

Fig. 1 (abstract P500). VALUE OF HOSPITAL SERVICES WITH INTERVETION FOR SYSTEMIC DISEASES OF THE CONJUNCTIVE TISSUE IN

P501

VALUE OF HOSPITAL SERVICES WITH INTERVENTION OF ELDERLY FOR DEGENERATIVE DISC DISEASES IN BRAZILIAN REGIONS IN THE LAST 10 YEARS

Meyling Belchior de sá Menezes, Henrique Gouveia Borba e Souza, Luíza Brito Nogueira, Bruno José Santos Lima, Angela Santos Lima, Larissa Sá dos Santos, Juliana Monroy Leite, Nicole Santiago Leite, Yasmin Oliveira Santos, João Victor de Andrade Carvalho, Denison Santos Silva UNIVERSIDADE TIRADENTES, ARACAJU, SE, Brasil

Advances in Rheumatology 2018, 58(Suppl 1):P501

Background: Degenerative Disc Disease is a condition resulting from a natural aging process that affects the discs of the spine. As the individual ages, the intervertebral discs (especially the Central Pulp) lose water. This progressive dehydration causes the disc to stiffen, which reduces its ability to absorb and cushion the impacts, increasing pressure under the walls that constitute the Annulus Fibrosus, which can cause cracks in the disc. As a result, the contents of the disc ruptured or the projection of it can press the spinal cord and nerve roots, causing pain, whose 
intensity is often decisive for situations of urgency. However, hospitalizations due to discopathies have a significant economic impact on the Unified Health System (SUS), and its analysis is relevant, since such hospital services can be avoided by preventive measures.

Methods: This is a retrospective observational study performed through the analysis of all hospital admissions of people onwards 60 years of age occurred in SUS in brazilian regions from January 2008 to January 2018. Data were obtained from the hospital information system (SIH/SUS) by the value in hospital services.

Results and conclusions: The total amount invested in hospital services was $\mathrm{R} \$ 159,511,654.61$ over a period of 10 years. Of these, approximately $70.6 \%$ refers to the age group from 60 to 69 years. In all regions, this age group holds the largest investment. The lowest investment is in patients from the age of 80 , with $3 \%$ of the total value. The Southeast region has the highest value with $\mathrm{R} \$ 66,916,901.54$; close to the South region with $\mathrm{R} \$ 58,337,212.07$. The lowest amount is invested in the North region, with R\$ 2,356,023.80; followed by the Northeast region, with $\mathrm{R} \$ 12,970,623.51$. The Midwest region has a comparatively intermediate value of $\mathrm{R} \$ 18,930,893.69$. Thus, it is concluded that, in Brazil, millions of reais are invested in support of urgencies when, although being natural of the aging process, many preventive practices could avoid the early appearance or intense affection of the Degenerative Disc Disease. Examples of these are tips on reducing spine impact, such as avoiding stains and sprains. This information could be better disseminated by the Basic Health Care in order to save large amounts of money and ensure a better quality of life for the elderly population.

\section{P509}

WELCOME TO FAMILIARS IN THE WAITING ROOM IN THE RHEUMATOLOGY AMBULATORY

Carlos Eduardo Gil Sarzi, Uriah Carolina Bataglia Ferreira, Lidiane Ferreira Marinho, Glauce Cerqueira Corrêa da Silva, Luciana Mateus Pinto, Katia Maria da Cunha Ferrari, Washington Alves Bianchi, Morgana Ohira Gazzeta, Dante Valdetaro Bianchi, Breno Valdetaro Bianchi SANTA CASA DA MISERICORDIA DO RIO DE JANEIRO, RIO DE JANEIRO, RIO DE JANEIRO, Brasil

Advances in Rheumatology 2018, 58(Suppl 1):P509

Introduction: The hospitalization of a person is a very difficult time for the family, even more, when it comes to rheumatologic diseases. It is a period marked by suffering, pain, sadness, anguish and fear. Therefore, the reception is essential to prepare the family for the encounter with their sick family member. The waiting room has proved to be a rich field for psychological interventions. It is a space for conversation and exchange of experiences, in which patients and companions can reflect on the health-illness process and feelings, in an autonomous and active way. We can say that the waiting room softens the physical and emotional wear and tear associated with waiting time.

Objectives: The objective of this research is to support patients who use rheumatology services in the outpatient clinic, to show the importance of welcoming the family member who expects news about the health status of their relative and to provide emotional support to patients and their families about the current situation of the hospitalized. Method: review of integrative literature and qualitative approach. Data were collected in a semi-open questionnaire and using the Rosenberg Self-Esteem Questionnaire.

Results: all literature evaluated makes clear that family members and patients present symptoms of anxiety, sadness and fear due to living with different situations on a daily basis. It is necessary for the professional to empathize with their suffering and to help them in the best possible way. Discussion: According to Rodrigues et al., It is through dialogues that take place in the waiting room that one can interact, demystify taboos and understand certain beliefs that permeate disease and treatment. Conclusions: it is indispensable to welcome relatives to be informed in detail about the operation in the outpatient clinic. It is believed that these feelings would be minimized if the space of the waiting room were occupied by multiprofessionals of health, especially Psychologists, who offered the welcome to these relatives, preparing them to visit their loved one, listening to them, clarifying doubts and meeting their needs.
P512

WORSENING OF CUTANEOUS MANIFESTATION IN SYSTEMIC LUPUS ERYTHEMATOSUS (SLE) CAUSED BY EMERGENCY CONTRACEPTIVE METHOD ("MORNING AFTER PILL")

Larissa Cristiane de Oliveira Souza, Maria Jose Pereira Vilar, Raquel Holanda de Lima, Maria Clara Coutinho Carlos de Lima, João Paulo Praxedes de Sales, Natalie Christine Braz Fernandes, Francisco Alves Bezerra Neto, José Hilton Nogueira Júnior

UFRN, NATAL, RN, Brasil

Advances in Rheumatology 2018, 58(Suppl 1):P512

Background:SLE pathogenesis is composed of genetic, environmental and hormonal factors. Epidemiological, clinical and experimental data alongside frequent disease relapses during pregnancy, emphasize the influence of feminine sexual hormones in lupus activity. The disease activity associated with hormonal contraceptives has been the focus of attention. Several available evidences have indicated that the mere emergency use of combined oral contraceptives or progesterone alone are not responsible for the worsening of inflammations or disease activity in women with SLE. However, this is still in discussion. Evidences regarding cardiovascular outcomes suggest a possible increase in the risk of thromboembolic events in patients with positive antiphospholipid antibodies who take hormonal contraceptives. Relapse after oral contraceptive use was first reported by Pimstone in 1966, in which remission was achieved by the discontinuation of such associated with short treatment course of corticosteroids.

Case report: Female, 23 years old, diagnosed SLE from the age of six with skin, joint, neurologic (seizures) and kidney involvement (nephritis). The patient was currently on azathioprine, hydroxychloroquine, prednisone, topiramate and phenobarbital.

She had been under outpatient monitoring with partial remission from cutaneous manifestations for two years. One week following use of Levonorgestrel as emergency contraceptive, she experienced acute cutaneous manifestation of SLE consisting of scaly erythematous lesions on her face, chest and torso, as well as crusty lip lesions and painful oral ulcerations presenting with granulation tissue and erythematous halo in palate (Fig. 1). She was treated with oral and topical corticosteroids along with sun block, with good response. Conclusion: Existing studies in this area are limited, once they lack specific information such as type and use duration of hormonal contraceptives, and are based on isolated reports. As for the present case, we witnessed a severe case of SLE in which the use of emergency contraceptive lead to flaring of skin disease activity. This reinforces the need for additional studies as to promote safety when prescribing contraceptive drugs to SLE patients, as not to expose the them to possible complications such as the one described.

\section{Consent for publication}

The authors declare that they have obtained informed written consent from the patient's tutors for publication

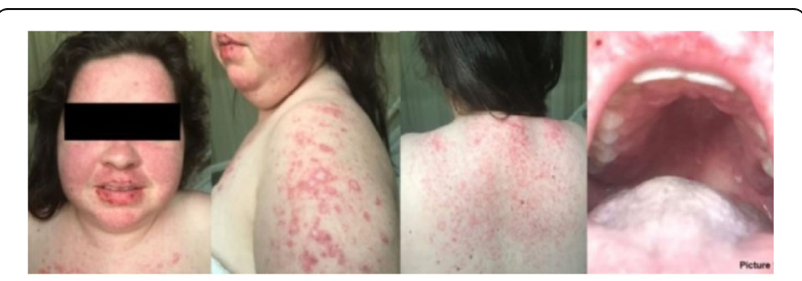

Fig. 1 (abstract P512). Scaly erythematous lesions on her face, chest and torso, as well as crusty lip lesions and oral ulcerations presenting with granulation tissue and erythematous halo in palate

\section{Publisher's Note}

Springer Nature remains neutral with regard to jurisdictional claims in published maps and institutional affiliations. 